\title{
Sobre Ações Parciais, Fibrados de Fell, e Grupóides
}

\author{
Fernando Abadie - Vicens ${ }^{1}$
}

\author{
TESE APRESENTADA \\ $\mathrm{AO}$ \\ INSTITUTO DE MATEMÁTICA E ESTATÍSTICA \\ DA \\ UNIVERSIDADE DE SÃO PAULO \\ PARA \\ OBTENÇÃO DO GRAU DE DOUTOR \\ EM \\ MATEMÁTICA
}

Área de Concentração: Análise

Orientador: Prof. Dr. Ruy Exel

1 de Setembro de 1999

${ }^{1}$ Durante a elaboração deste trabalho o autor recebeu apoio financeiro da Fapesp, Processo No. 95/04097-9 
Sobre Ações Parciais, Fibrados de Fell, e Grupóides

Este exemplar corresponde à redação final devidamente corrigida e defendida por Fernando Abadie-Vicens,

$\epsilon$ aprovada pela comissão julgadora

São Paulo, 1 de Setembro de 1999

Comissão Julgadora:

Prof. Dr. Ruy Exel Filho (Presidente)

UFSC, IME-USP

Prof. Dr. Paulo Piccione

IME-USP

Prof. Dr. Antonio Roberto Da Silva

IM-UFRJ

Prof. Dr. Cassio Sigaud

IF-UFRJ

Prof. Dr. Gustavo Corach

UBA 


\section{AGRADECIM(I)ENTOS}

A tese é longa, os agradecimentos também.

Antes que nada, agradezco a mi esposa, Daniella, y a mi hijo Marcos, que muchas veces me prestaron un tiempo que no se puede devolver, $y$ me apoyaron en todo momento. Gracias a ellos concluí este trabajo, y a ellos también les pertenece.

Quero agradecer também ao Ruy pela dupla oportunidade de ter aceito ser meu orientador de mestrado primeiro, e de doutorado depois. Sua paixão pela matemática foi um permanente estímulo para mim, e seu profissionalismo um exemplo invalorável. Além do mais, o valor desta tese teria sido muito menor sem suas sugestōes e correções.

A nuestras familias, que siempre nos alentaron y apoyaron.

A Jorge y Marcelo, que me hospedaron en su casa cuando llegué a San Pablo, y con quienes compartimos muy buenos momentos, conversaciones delirantes, cine, fútbol, pan con requesón y jamón, café con leche, cerveza, chocolates Bis, y otras mil cosas, incluyendo matemática. Con Marcelo fuimos, también. compañeros de carcajes y semillas de girasol.

Aos meus colegas da USP, de várias nacionalidades: Juaci, Ana Cláudia, Lúcia, Jair, Orlando. Claus, Pablo, Marcela, Ayumi, Marcelo, Saraiva, Bárbara, Irene, Zé, Sidney, Raul, Márcio, Cristian. Carolina, Nelson. Jorge, Jocirei, Ronaldo, Jorge.

A Celso, Silviane, Mara, Vera, Cláudia, David, Ademir, Lúcia, Ruy, Gui e Karin.

A Carlos, Susana, Carlos César, Sandra y Carla. Dancona ellos, que nos recibieron con los brazos abiertos y con quienes pasamos hermosos momentos. Gracias a Luis también.

À Universidade Federal de Santa Catarina em Florianópolis, pela hospitalidade com que fui recebido e por ter me facilitado o uso de suas instalações.

A Martha, que siempre estuvo generosamente a nuestro lado.

Aos meus companheiros de seminário de Álgebras de Operadores en São Paulo e Florianópolis: Cristina, Paolo. Marcela, Ruy, Ayumi, Ariel, José e Luiz; cada um deles me ensinou alguma coisa.

A la familia Merklen, siempre dispuesta a dar una mano.

A Paolo e Djacui, pela aconchegante hospitalidade com que me receberam em sua casa.

Pela mesma razào a Jorge, Jocirei, Ronaldo e Ramiro.

E também por isso a Claus e Fátima.

A la Universidad de la República, Uruguay, por el apoyo recibido.

A Bea, el Leva y Walter, con quienes le agarré el gustito a esto.

A Cristina e Paolo, pelos vários favores que fizeram à distância para mim.

A la querida Marcela, por hacer varios trámites por mí durante mi ausencia en San Pablo, por haber recibido, guardado y comunicado mi correspondencia, y por otros múltiples favores.

A nuestra comunidad uruguaya y afín en San Pablo, por los momentos vividos: Jorge, Marcelo. Ana, Marcela, Daniel, Irene, Zé, Cecilia, Nelson, Jorge, Leticia, Fanny, Pablo.

A Nelson, que entre otras cosas le preparó y le dio la mema a Marcos varias veces, y nos dejó dormir un rato más.

A la gran Cecilia, siempre primera a la hora de mudanzas y hospitales. Che Ceci, sabés que ahora nos vamos para Montevideo a buscar donde vivir, y como nuestras cosas están en Paysandú, en una de esas nos podés dar una manito ...

A Jorge, con quien tanto ejercitamos el bello idioma castellano.

A Ayumi, nossa companheira e amiga em São Paulo e em Floripa. Vamos dizer o que? Te esperamos em Liruguay.

A nuestros compañeros chilenos, rivales en la cancha de fútbol. No se amarguen che, no siempre se gana; i pero podrían haberlo hecho por lo menos una vez! Igual, lo importante es jugar, ¿. no?

À Fundaçào de Amparo à Pesquisa do Estado de São Paulo, pelo apoio financeiro recebido.

Ao Brasil e ao povo brasileiro. 
Este trabajo está dedicado a la tripulación de mi barco en este viaje:

Marcos y Daniella 


\begin{abstract}
This thesis has three parts. In the first one, we study the tensor products between Fell bundles and their applications. The main result of this part relates the maximal and minimal tensor products of the full and reduced cross sectional (" ${ }^{*}$-algebras of two Fell bundles, with the full and reduced cross sectional $C^{*}$-algebras of the corresponding maximal and minimal tensor products of the bundles. As an application we see, for instance, that the cross sectional $C^{*}$-algebra of a Fell bundle with the approximation property and exact fiber is also exact. In particular, the crossed product of an exact $C^{*}$-algebra by a partial action of an amenable group is exact.

In the second part. we study and solve in three categories the following problem: when is a partial action the restriction of a global one? Such an action is called enveloping action, and the space where it acts is the enveloping space. In the category of sets and maps, the enveloping action exists and is unique, and it is characterized by a universal property. The same happens in the category of topological spaces and continuous functions. In this case, however, the enveloping action of a partial action that acts on a Hausdorff space does not act in general on a Hausdorff space. We show that the enveloping space is Hausdorff if and only if the original one is Hausdorff, and the graph of the partial action is closed. We show that the flow of a vector field on a manifold is a partial action, whose graph may be closed or not. There exists one and only one enveloping action in the category of $C^{*}$-algebras and Morita equivalence bimodules as morphisms. In this context, the problem of the enveloping action turns out to be intimately related with Takai duality. In fact, our study of the enveloping action shows the form that Takai duality takes for crossed products by partial actions. As applications, we mention two. We prove that any partial action of a connected group on a unital $C^{*}$-algebra must be a global action. This generalizes the well known fact that the solutions of a differential equation on a compact manifold are defined on all of the real line. We also prove that the crossed product of the reduced cross sectional $C^{*}$-algebra of a Fell bundle by the dual coaction is liminal, postliminal, or nuclear, if and only if the unit fiber of the bundle is, respectively, liminal, postliminal, or nuclear.

In the third part we show that any partial action on a commutative $C^{*}$-algebra has naturally associated a grupoid with a Haar system, and that the crossed product by the partial action is isomorphic to the $C^{*}$-algebra of the grupoid.
\end{abstract}




\section{Resumo}

Esta tese tem tres partes. Na primeira, estudamos os produtos tensoriais de fibrados de Fell e suas aplicações. O resultado principal desta parte relaciona os produtos tensoriais máximo e mínimo das $C^{*}$-álgebras seccionais plena e reduzicla de dois fibrados de Fell, com as $C^{*}$-álgebras plena e reduzida. dos correspondentes produtos tensoriais máximo e mínimo dos fibrados. Como aplicação vemos, por exemplo, que se $\mathrm{um}$ fibrado de Fell satisfaz a propriedade de aproximaçào e tem fibra exata, então sua $C^{*}$-álgebra seccional é exata. Em particular, o produto cruzado de uma $C^{*}$-álgebra exata por uma ação parcial de um grupo amenable também é exato.

Na segunda parte estudamos e resolvemos em tres categorias o seguinte problema: toda açào parcial é a restriçào de uma açào global? Uma tal ação global é chamada açào envolvente, e o espaço onde age é o espaço envolvente. Na categoria de conjuntos e funçōes a ação envolvente existe e é única, e ela está caracterizada por uma propriedade universal. Na categoria de espaços topológicos e funções contínuas também. Porém, neste caso a ação envolvente de uma açào parcial sobre um espaço de Hausdorff pode estar agindo sobre um espaço que nào é de Hausdorff. Vemos então que o espaço envolvente é de Hausdorff se e somente se o espaço inicial é de Hausdorff e o gráfico da ação parcial é fechado. Mostramos que o fluxo de um campo vetorial sobre una variedade é uma ação parcial, cujo gráfico é fechado em alguns casos e em outros não. Na categoria de $C^{*}$-álgebras com os bimódulos de equivalencia Morita como morfismos, a ação envolvente existe e é única. Nest.e contexto, o problema da ação envolvente está intimamente ligado com a dualidade de Takai. De fat.o, através do nosso estudo da ação envolvente mostramos qual é a forma que a dualidade de Takai toma para produtos cruzados por açōes parciais. Como aplicações, mencionamos duas a seguir. Provamos que toda ação parcial de um grupo conexo sobre uma $C^{*}$-álgebra com unidade deve ser uma açào global, o que generaliza o conhecido fato de que as soluções de uma equação diferencial sobre uma variedade compacta estào definidas em toda a reta real. Também provamos que o produto cruzado da $C^{*}$-álgebra reduzida de um fibrado de Fell pela coação dual é liminal, postliminal ou nuclear, se e somente se a fibra sobre a identidade é respectivamente liminal, postliminal, ou nuclear.

Na terceira parte mostramos que toda ação parcial sobre uma $C^{*}$-álgebra comutativa tem naturalmente associado um grupóide com sistema de Haar, e que o produto cruzado pela ação parcial é isomorfo à $C^{*}$-álgebra do grupóide. 


\section{Conteúdo}

Prefácio

xiii

Parte I. Produtos Tensoriais de Fibrados de Fell 1

Introdução à Parte I

Capítulo 1. Produtos Tensoriais de ('* -anéis Ternários 9

1.1. $\mathrm{C}^{*}$-anéis ternários 11

1.2. Produtos tensoriais de $C^{*}$-anéis ternários $\quad 19$

1.3. C. -anéis ternários exatos 28

Capítulo 2. Produtos Tensoriais de Fibrados de Fell 31

2.1. Produtos tensoriais de fibrados sobre grupos discretos 32

2.2. Produtos tensoriais de fibrados sobre grupos contínuos 36

2.3. Representações de produtos tensoriais de fibrados de Fell 40

Capítulo 3. $\quad \mathrm{C}^{*}$-álgebras secionais de produtos tensoriais de fibrados de Fell $4 \bar{\imath}$

3.1. $C^{*}$-álgebra reduzida de um fibrado de Fell. Amenabilidade 49

3.2. $C^{*}$-álgebras seccionais de produtos tensoriais de fibrados de Fell $\quad 54$

3.3. Algumas aplicaçòes $\quad 59$

3.4. Comentários finais 69

Parte II. Ações Envolventes de Ações Parciais

Introdução à Parte II

Capítulo 4. Ações Envolventes na Categoria de Espaços Topológicos

4.1. Ações envolventes de açōes parciais sobre conjuntos 80

4.1.1. Ações parciais sobre conjuntos $\quad 80$

4.1.2. Ações envolventes 86

4.2. Ações envolventes de ações parciais topológicas 90

4.2.1. Uma construção alternativa do espaço e da ação envolventes

4.2.2. Alguns exemplos $\quad 96$

4.2.3. Propriedades dinamicas da ação envolvente 100

Capítulo 5. Açòes Envolventes na Categoria de C**álgebras 103

5.1. Sobre a unicidade da ação envolvente $10 \tau$

5.2. Ação parcial sobre o espectro e o espaço de ideais primitivos 108

5.3. Propriedades da álgebra envolvente 113

Capítulo 6. Produtos Cruzados Parcial e Envolvente 11 i

6.1. Equivaléncia Morita dos produtos cruzados parcial e envolvente 118 
6.2. Aplicações e exemplos 122

Capítulo 7. Açōes Morita Envolventes 133

7.1. Ações parciais equivalentes Morita. 134

7.2. $C^{*}$-álgebras de núcleos associadas a um fibrado de Fell 140

7.2.1. Operadores de Hilbert-Schmidt e núcleos de um fibrado de Fell 141

7.2.2. Ação sobre os núcleos 144

7.2.3. A $C^{*}$-álgebra reduzida dos núcleos de um fibrado de Fell 145

7.2.4. $\mathbb{E}(\mathcal{B})=\mathbb{R}_{r}(\mathcal{B})$, e algumas aplicaçōes $\quad 151$

7.3. Sobre a existencia da açào envolvente a menos de equivalencia Morita 153

7.4. Sobre a unicidade da açào envolvente a menos de equivalencia Morita 158

7.5. Açòes envolventes e produtos cruzados por coaçòes $\quad 160$

$\begin{array}{ll}\text { 7.6. Algumas aplicaçòes } & 163\end{array}$

Parte III. Produtos Cruzados Parciais e C*-álgebras de Grupóides 165

$\begin{array}{lr}\text { Introdução à Parte III } & 167\end{array}$

Capítulo 8. Girupóide Associado a uma Ação Parcial 169

8.1. Ações parciais sobre . Y e açòes parciais sobre $C_{0}(X) \quad 170$

8.2. Grupóide associado a uma açào parcial 172

8.3. Sistema de Haar sobre $\mathcal{G} \quad 174$

8.4. O Produto Cruzado Parcial e a C*-álgebra do Grupóide 176

$\begin{array}{lr}\text { Bibliografia } & 181\end{array}$ 
Prefácio 
Nesta tese estudamos diferentes aspectos das açòes parciais e suas relações com as açōes globais, os fibrados de Fell, e os grupóides.

A seguir descrevemos de forma bastante esquemática o trabalho. Maiores detalhes podem ser progressivamente achados nas introduçòes a cada parte. capítulo e seção.

Na Parte I estudamos os produtos tensoriais de fibrados de Fell. Os resultados obtidos, aplicados às açôes parciais, permitem concluir que todo produto cruzado de uma $C^{*}$-álgebra nuclear por uma ação parcial contínua e torcida de um grupo localmente compacto amenable é também nuclear. Um resultado análogo é obticlo para $C^{*}$-álgebras exatas.

Na Parte II consideramos o seguinte problema: quando e em que sentido uma ação parcial é a restriçào de uma açào global? As respostas dependem da categoria onde ocorre a ação parcial, e também do que entendamos por restrição de uma ação parcial. Como veremos no Capítulo $\bar{\tau}$, na classe das $C^{*}$-álgebras é conveniente considerar estas restriçòes "a menos de equivalência Morita". Neste sentido, mostramos que se $\alpha$ é uma ação parcial contínua de um grupo localmente compacto $G$ sobre uma $C^{*}$-álgebra $A$, então existe uma ação global 3 sobre uma $C^{*}$-álgebra $B$, que tem um ideal $I$ cuja $\beta$-órbita linear fechada é $B$, tal que $\alpha$ é equivalente Morita $\left.\operatorname{com} \beta\right|_{I}$. Provamos também que esta ação $\beta$ é única a menos de equivalencia Morita. Vemos também que este problema está intimamente relacionado com a dualidade de Takesaki-Takai de produtos cruzados. Ao longo do desenvolvimento da segunda parte, surgem alguns resultados sobre produtos cruzados por coaçōes que acreditamos são novos; por exemplo, se $\mathcal{B}$ é um fibrado de Fell sobre o grupo localmente compacto $G$, com fibra $B_{\epsilon}$ sobre a identidade de $G$, e se $\delta$ é a coação dual de $G$ sobre $C_{r}^{*}(\mathcal{B})$, entào $C_{r}^{*}(\mathcal{B}) \rtimes_{\delta, r} \hat{G}$ é nuclear, liminal, postliminal, se e somente se $B_{\epsilon}$ é respectivamente nuclear, liminal, postliminal.

A última parte é praticamente um apendice desta tese. Nela vemos que toda ação parcial $\alpha$ de um grupo localmente compacto $G$ sobre uma $C^{*}$-álgebra comutativa $A$ tem associado naturalmente um grupóide localmente compacto $\mathcal{G}$, com um sistema de Haar canonico, e tal que a $C^{*}$-álgebra do grupóide $C^{*}(\mathcal{G})$ coincide com o produto cruzado $A \rtimes_{\alpha} G$.

Os produtos cruzados por grupos localmente compactos foram gradualmente definidos e estudados durante a década dos anos sesenta. Para açōes de grupos discretos, foram introduzidas por Turumaru em [62], e estudados em profundidade por Zeller-Mleyer em [67]. Os produtos cruzados de $C^{*}$ álgebras comutativas por grupos localmente compactos, também chamados $C^{*}$-álgebras de grupos de transformações, foram introduzidas por Glimm em [38]. e estudados com detalhe por Effros e Hahn ([22]). A definição geral se deve a Doplicher. Kastler e Robinson ([21]). A idéia é codificar, na estrutura do produto cruzado, a ação do grupo sobre uma $C^{*}$-álgebra. Os produtos cruzados sào usados tanto para estudar estas ações como para produzir novos exemplos de $C^{*}$-álgebras, ou descrever outras já conhecidas em termos de elementos mais simples.

Posteriormente, no final dos anos sesenta e durante os anos setenta e oitenta, foram introduzidas e estudadas outras construçòes relacionadas com a noção de produto cruzado. Talvez as principais sejam os fibrados de Fell e as $C^{*}$-álgebras de grupóides.

Em [34],[35], e [36], tentando generalizar a "análise do subgrupo normal" de Mackey, Fell desenvolveu a teoria de fibrados de Banach $C^{*}$-algébricos, hoje chamados fibrados de Fell, que de uma certa forma podem ser considerados como uma generalização dos produtos cruzados: cada produto cruzado induz um fibrado de Fell, cuja $C^{*}$-álgebra seccional coincide com o produto cruzado. A forma final de boa parte da obra de Fell se encontra em [37].

No ano 1980 apareceu o trabalho de J. Renault sobre $C^{* *}$-álgebras de grupóides ([58]). As $C^{*}$ álgebras de grupóides constituem uma generalizaçào das $C^{*}$-álgebras de grupos, dos produtos cruzados de $C^{*}$-álgebras comutativas por grupos localmente compactos. e das $C^{*}$-álgebras de folheaçòes. Em muitos casos constituem um substituto adequado ao espaço de funções contínuas sobre espaços topológicos quociente não Hausdorff (ver por exemplo [15]). 
Já nos anos noventa. uma generalizaçào dos produtos cruzados, mais próxima da idéia original que os fibrados de Fell e as ( ${ }^{*}$-álgebras de grupóides, começou a ser desenvolvida por Exel em [23]. Em lugar de tomar uma açào dos inteiros sobre uma $C^{*}$-álgebra, o que é equivalente a dar um automorfismo da $C^{*}$-álgebra, se considera apenas um automorfismo parcial, isto é, um isomorfismo entre dois ideais da $C^{\prime *}$-álgebra. e se consegue fazer uma construção análoga à de produto cruzado. assim como descrever algumas classes amplas de $C^{*}$-álgebras através desta construção ([25], [24]). Logo depois, esta construçào foi generalizada, primeiro para o caso de açōes parciais de grupos discretos $([47])$, e depois para o caso geral de açôes parciais torcidas de um grupo localmente compacto ([27]). Os produtos cruzados parciais estào intimamente ligados com os fibrados de Fell: em [27] se mostra que, sob certas condiçòes nào muito restritivas, todo fibrado de Fell provém de uma ação parcial torcida do grupo base. Posteriormente, outras $C^{*}$-álgebras foram descritas como produtos cruzados parciais, e inclusive generalizadas, como as $C^{*}$-álgebras de Cuntz-Kirieger para matrizes infinitas ([31]).

Assim, as teorias de produtos cruzados, produtos cruzados parciais. (**álgebras de grupóides e fibrados de Fell, são teorias fortemente ligadas, de forma que resultados em uma delas podem produzir resultados em outra. O objetivo geral deste trabalho é achar novas relaçòes entre todas estas construçòes, guiados pela teoria de açòes parciais. 


\section{Parte I}

Produtos Tensoriais de Fibrados de Fell 

Introdução à Parte I 
Esta primeira parte da tese está dedicada a desenvolver uma teoria de produtos tensoriais de fibrados de Fell sobre grupos localmente compactos, e aplicar esta teoria aos produtos tensoriais de $C^{*}$-álgebras. Está constituída por tres capítulos que generalizam os resultados obtidos em [3] ao caso contínuo, e além disso inclui um resultado sobre $C^{*}$-álgebras exatas.

As motivações para iniciar estes estudos foram várias e de diferente teor. Por um lado, houve a intenção, menor, de generalizar a teoria de produtos tensoriais de $C^{*}$-álgebras a produtos tensoriais de $C^{*}$-anéis ternários (as fibras de um fibrado de Fell são $C^{*}$-anéis ternários), visando aplicações às $C^{*}$-álgebras. Por outro, dois artigos de Exel, [27] e [29], colocavam o problema da "estabilização" de um fibrado de Fell como uma questào de releváncia. Com efeito, em [27], um amplo espectro de fibrados de Fell são classificados: todo fibrado de Fell $\mathcal{B}$ sobre um grupo localmente compacto $G$, tal que $\mathcal{B}$ satisfaz o segundo axioma de enumerabilidade, e tem fibra $B_{e}$ estável sobre a identidade de $G$, é isomorfo ao fibrado de Fell de alguma ação parcial contínua torcida de $G$ sobre $B_{e}$. Lembrar que $B_{e}$ é chamada estável se $B_{\epsilon} \cong B_{\epsilon} \otimes \mathcal{K}$, onde $\mathcal{K}$ é a $C^{*}$-álgebra dos operadores compactos sobre um espaço de Hilbert separável. Supondo que $\mathcal{B}$ tem o segundo axioma de enumerabilidade, mas $B_{e}$ não é estável, pode ser tentado "estabilizar" o fibrado $\mathcal{B}$. Isto implica definir algum tipo de produto tensorial do fibrado de Fell $\mathcal{B}$ por $\mathcal{K}$. sendo o resultado um novo fibrado de Fell sobre o grupo $G$, cuja fibra sobre a identidade de $G$ seja $B_{e} \otimes K$. . De ter sucesso uma tal construção, o fibrado de Fell $\mathcal{B} \otimes \mathcal{K}$ seria isomorfo a um fibrado de Fell originado por uma ação parcial contínua torcida de $G$ sobre $B_{e} \otimes \mathcal{K}$, e desta forma poderíamos obter informação sobre o próprio fibrado $\mathcal{B}$. $\mathrm{Em}$ [29], é feita uma estabilização ad hoc de um fibrado de Fell $\mathcal{B}$, via uma representaçào de $\mathcal{B}$; neste caso, nosso propósito era fornecer uma construção de $\mathcal{B} \otimes \mathcal{K}$ independente de toda representação de $\mathcal{B}$.

Para explicar nossa motivação principal, devemos ainda citar outros trabalhos. Por um lado, já em 1997 a $K K$-teoria de Kasparov permitia classificar, a menos de isomorfismos, as $C^{*}$-álgebras que são simples, puramente infinitas. e nucleares (os principais nomes ligados com esta classificação são Kirchberg, Phillips e Rørdam). A nuclearidade de uma $C^{*}$-álgebra é uma propriedade relativa a produtos tensoriais. Dadas $C^{*}$-álgebras $A$ e $B$, sobre o produto tensorial algébrico $A \odot B$ é possível definir, em geral, mais de uma $C^{*}$-norma. Entre elas, existem uma que é máxima e outra que é mínima, sendo os correspondentes completamentos denotados por $A \bigotimes_{\text {máx }} B$ e $A \bigotimes_{\text {min }} B$. Uma $C^{*}$ álgebra $A$ é nuclear se $A \bigotimes_{\text {máx }} B=-\bigotimes_{\text {min }} B$, para toda $C^{*}$-álgebra $B$. Os teoremas de classificação tornaram o problema cla nuclearidade de uma $C^{*}$-álgebra mais importante do que nunca. Entre as $C^{*}$-álgebras nucleares se encontram as $C^{*}$-álgebras comutativas, as $C^{*}$-álgebras aproximadamente finitas, e as $C^{*}$-álgebras de Cuntz-Kirieger. Além disso, a classe de $C^{*}$-álgebras nucleares é fechada por ideais, quocientes, extensōes, limites indutivos, equivalência Morita, e produtos cruzados por grupos amenable. Em [4], C. Anantharaman-Delaroche generalizou o conceito de grupo amenable para o de ação amenable, e provou que o produto cruzado de uma $C^{*}$-álgebra nuclear por uma ação amenable de um grupo discreto é nuclear. Em particular, mostrou que se $A$ é comutativa, então a ação é amenable sempre que certa propriedade de aproximação fosse satisfeita. Mais tarde, foi introduzida por Exel ([28]) a noção de fibrado de Fell amenable sobre um grupo discreto, que generaliza o conceito de amenabilidade de uma ação. Exel provou também que, se o fibrado tem certa propriedade de aproximação, cuja relação com a propriedade de aproximação de AnanatharamanDelaroche ainda não está esclarecida ${ }^{2}$, então o fibrado é amenable. Surgiu então a conjectura de que todo fibrado de Fell sobre um grupo discreto, com fibra nuclear sobre a identidade do grupo e com a propriedade de aproximação de Exel, teria $C^{*}$-álgebra seccional nuclear. Provar este resultado foi a razão definitiva para desenvolver este trabalho (os resultados iniciais se encontram em [3]). Para completar a descrição do momento em que este trabalho foi emprendido, deveríamos mencionar ainda um outro antecedente. Por esta época. Exel e Laca estavam generalizando as $C^{*}$-álgebras de CuntzKirieger para o caso de matrizes infinitas, usando os métodos de ações parciais. Eles mostraram que estas álgebras são $C^{*}$-álgebras seccionais de fibrados de Fell sobre grupos discretos livres, em geral em

\footnotetext{
${ }^{2}$ Esta situação mudou recentemente; consultar [33], 4.5 e 4.6
} 
infinitos geradores. Mostraram também que, sob determinadas condiçōes na matriz, estas álgebras são simples e puramente infinitas; além disso, os fibrados de Fell correspondentes tém a propriedade de aproximação de Exel, e fibra comutativa sobre a identidade, em particular nuclear. Provando a conjectura citada, se concluia então que estas álgebras são nucleares. e portanto seriam novos exemplos das $C^{*}$-álgebras classificáveis através da $K$-teoria.

Descrevamos de maneira geral esta Parte I. No início de cada capítulo e cada seção podem ser achadas introduções mais detalhadas.

Para definir os produtos tensoriais entre fibrados de Fell, consideramos primeiro os produtos tensoriais entre as fibras correspondentes dos fibrados. Estas fibras sào $C^{\prime *}$-anéis ternários positivos. Um $C^{*}$-anel ternário positivo é uma versão abstrata de um anel ternário de operadores fechado, ou seja, um subespaço de Banach $E \subseteq B(H, K)$, onde $H$ e $K$ são espaços de Hilbert, tal que $T_{1} T_{2}^{*} T_{3} \in E$, sempre que $T_{1}, T_{2}, T_{3}$ pertençam a $E$. Por exemplo, toda $C^{*}$-álgebra é um $C^{*}$-anel ternário positivo. Por outro lado, se $E \subseteq B\left(H . K^{\circ}\right)$ é um anel ternário de operadores fechado, então $E$ é um módulo de Hilbert pleno à direita sobre a $\left(^{*}\right.$-álgebra $B=\overline{\operatorname{span}}\left\{T^{*} S: T . S \in E\right\} \subseteq B(H)$. Inversamente, todo módulo de Hilbert tem uma representaçào como anel ternário de operadores. Assim vemos que o estudo de produtos tensoriais entre $C^{*}$-anéis ternários é conveniente nào somente como estágio intermediário antes de considerar produtos tensoriais entre fibrados de Fell, senão também porque generaliza os produtos tensoriais entre (:*álgebras ao contexto de módulos de Hilbert. Em particular. isto é importante do ponto de vista da equivaléncia Morita de ("'-álgebras. Como a equivalência Morita de $C^{*}$-álgebras se expressa através de módulos de Hilbert, é razoável esperar que obtenhamos algumas conclusões interessantes ao considerar produtos tensoriais entre objetos que incluem tanto às $C^{*}$-álgebras quanto aos módulos de Hilbert. De fato, veremos que as propriedades das $C^{*}$-álgebras que são definidas a partir de produtos tensoriais, como a nuclearidade e a exatidão, são preservadas por equivalência Morita, devido a que estas propriedades são comuns aos próprios módulos de equivalência. Para ser um pouco mais precisos, notemos que sobre o produto tensorial algébrico $E \odot F$ entre dois $C^{*}$-anéis ternários $E$ e $F$ poderia existir mais de uma $C^{*}$-norma sobre $E \odot F$, ou seja, uma norma tal que o correspondente completamento fosse um $C^{*}$-anel ternário: pensar, por exemplo, que $E$ e $F$ poderiam ser $C^{*}$-álgebras. Veremos que, como no caso de $C^{*}$-álgebras, existem um produto tensorial máximo $E \bigotimes_{\text {máx }} F$ e um produto tensorial mínimo $E \bigotimes_{\text {mín }} F$ entre os $C^{* *}$-anéis ternários $E$ e $F$. Como provou Zettl em [68], todo $C^{*}$-anel ternário tem associada uma $C^{*}$-álgebra $B$ que satisfaz determinadas condiçōes. Veremos que, se $E_{1}, E_{2}$ são $C^{*}$-anéis ternários com $C^{*}$-álgebras associadas $B_{1}$ e $B_{2}$ respectivamente, entào existe uma bijeção entre a família de $C^{*}$-normas sobre $E_{1} \odot E_{2}$ e a família de $C^{*}$-normas sobre $B_{1} \odot B_{2}$; além disto, esta bijeção preserva às ordens.

Para construir produtos tensoriais entre os fibrados de Fell $\mathcal{A}=\left(. A_{t}\right)_{t \in G}$ e $\mathcal{B}=\left(B_{s}\right)_{s \in H}$, começamos por definir produtos tensoriais entre cada fibra de $\mathcal{A}$ e cada fibra de $\mathcal{B}$. Estes produtos tensoriais devem ser feitos de uma maneira coerente, pois esperamos que a família de produtos tensoriais assim obtidos formem as fibras de um fibrado de Fell sobre $G \times H$. Assim, por exemplo, não devemos considerar o produto tensorial máximo num caso, e o mínimo em outro caso. Como as fibras de $\mathcal{A}$ são módulos de Hilbert à direita sobre $A_{e}$, e as de $\mathcal{B}$ sobre $B_{e}$, escolhido um produto tensorial $A_{e} \otimes_{\alpha} B_{\epsilon}$ entre $A_{\epsilon}$ e $B_{\epsilon}$ existe uma única $C^{*}$-norma sobre cada $A_{t} \odot B_{s}$ tal que o completamento $A_{t} \bigotimes_{\alpha} B_{s}$ correspondente é um módulo de Hilbert à direita sobre $A_{e} \bigotimes_{\alpha} B_{e}$, devido à correspondência entre as $C^{*}$-normas sobre produtos tensoriais de $C^{*}$-anéis ternários e de $C^{*}$-álgebras mencionada anteriormente. Aqui surge um problema: na verdade. a fibra de um fibrado de Fell é um bimódulo de Hilbert sobre a fibra da identidade, e nós construímos apenas uma família de "fibras" que são módulos de Hilbert á direita sobre a fibra da identidade. Deveríamos ver, então, que a mesma coleção de "fibras" seria obtida se fizéssemos a mesma construção considerando cada $A_{t}$ e cada $B_{s}$ como módulos de Hilbert à esquerda sobre $A_{e}$ e $B_{e}$ respectivamente. Verificamos este fato para o caso das normas máxima e mínima, que são as interessantes para os nossos fins. Não sabemos se vale também para qualquer outra $C^{*}$-norma sobre $A_{e} \odot B_{e}$. Uma vez feito isto, temos ainda que definir 
INTRODUÇĀO À PARTE I

uma topologia sobre $\mathcal{A} \otimes \mathcal{B}$. Fazer isto equivale essencialmente a declarar quais devem ser as seçōes contínuas do fibrado. Nós requerimos o que parece o mais natural: se $f$ é uma seção contínua de $\mathcal{A}$ e $g$ é uma seçào contínua de $\mathcal{B}$, entào a função $G \times H \longrightarrow \mathcal{A} \otimes \mathcal{B}$ tal que $(t, s) \longmapsto f(t) @ g(s)$ deve ser uma seção contínua de $\mathcal{A} \otimes \mathcal{B}$. Provamos então que esta família de seçōes determina realmente uma topologia sobre $\mathcal{A} \otimes \mathcal{B}$. que o transforma num fibrado de Banach sobre $G \times H$. Vemos depois que, com as operaçōes de produto e involuçào naturais, $\mathcal{A} \otimes \mathcal{B}$ é um fibrado de Fell sobre $G \times H$.

$\mathrm{O}$ seguinte passo é estudar as representaçòes de $\mathcal{A} \bigotimes_{\text {máx }} \mathcal{B}$ e $\mathcal{A} \bigotimes_{\text {mín }} \mathcal{B}$. No primeiro caso vemos que as representaçòes nào degeneradas de $\mathcal{A} \bigotimes_{\text {máx }} \mathcal{B}$ sobre um módulo de Hilbert $\mathcal{H}$ estão em correspondencia biunívoca com as representaçōes de $\mathcal{A}$ e $\mathcal{B}$ sobre $\mathcal{H}$ cujas imagens comutam. No segundo caso, mostramos que se $\mathcal{A}$ se representa sobre o módulo de Hilbert $\mathcal{H}$ através de $\pi_{\mathcal{A}}$, e $\mathcal{B}$ se representa sobre o módulo de Hilbert $\mathcal{\Lambda}$ através de $\pi_{\mathcal{B}}$, então existe uma única representação $\pi_{\mathcal{A}} \odot \pi_{\mathcal{B}}$ de $\mathcal{A} \bigotimes_{\min } \mathcal{B}$ sobre $\mathcal{H} \bigotimes_{\min } \mathcal{K}$ tal que $\left(\pi_{\mathcal{A}} \pi_{\mathcal{B}}\right)(a \odot b)=\pi_{\mathcal{A}}(a) \odot \pi_{\mathcal{B}}(b) \in \mathcal{L}\left(\mathcal{H} \bigotimes_{\min } \mathcal{K}\right), \forall a \in \mathcal{A}$, $b \in \mathcal{B}$.

A seguir analizamos as $C^{* *}$-álgebras seccionais dos produtos tensoriais, a $C^{*}$-álgebra seccional plena e a $C^{*}$-álgebra seccional reduzida.

Dado um fibrado de Fell $\mathcal{B}$, existem duas $C^{*}$-álgebras seccionais importantes associadas a $\mathcal{B}$. A primeira é a $C^{*}$-álgebra seccional (plena) $C^{*}(\mathcal{B})$, definida por Fell, cuja teoria de representações é equivalente à teoria de representaçòes de $\mathcal{B}$. A segunda é $C_{r}^{*}(\mathcal{B})$, a $C^{*}$-álgebra reduzida de $\mathcal{B}$, concretamente definida através de uma representação de $C^{*}(\mathcal{B})$. A $C^{*}$-álgebra reduzida de um fibrado de Fell foi definida por Exel em [28] para o caso de fibrados de Fell sobre grupos discretos. Em [50], $\mathrm{Ng}$ generalizou a definiçào para o caso contínuo. Eis rapidamente a definição de $C_{r}^{*}(\mathcal{B})$. Se $C_{c}(\mathcal{B})$ denota o espaço de seçòes contínuas de suporte compacto do fibrado $\mathcal{B}$, entào $C_{\mathfrak{c}}(\mathcal{B})$ é um pré- $B_{e}$ módulo de Hilbert à direita com o produto interno $\langle f, g\rangle=\int_{G} f(t)^{*} g(t) d t$. O completamento correspondente é denotado $L^{2}(\mathcal{B})$, e é um módulo de Hilbert pleno à direita sobre $B_{\epsilon}$. Agora. se $b_{t} \in B_{t}$, a transformação $\Lambda_{b_{t}}$ tal que $\left.\Lambda_{b_{t}}(f)\right|_{s}=b_{t} f\left(t^{-1} s\right)$, para $f \in C_{c}(\mathcal{B})$, se estende a um operador adjuntável sobre $L^{2}(\mathcal{B})$, cujo adjunto é $\Lambda_{b_{i}}$, e a aplicação $\mathcal{B} \longrightarrow \mathcal{L}\left(L^{2}(\mathcal{B})\right)$, tal que $b \longmapsto \Lambda_{b}$, é uma representação de $\mathcal{B}$, que se integra a uma representação $\Lambda: C^{\prime *}(\mathcal{B}) \longrightarrow \mathcal{L}\left(L^{2}(\mathcal{B})\right)$, chamada representação regular. Define-se então $C_{r}^{*}(\mathcal{B}):=\Lambda\left(C^{*}(\mathcal{B})\right)$. Quando $\perp$ é injetiva, diz-se que $\mathcal{B}$ é amenable.

Comparamos então as $C^{*}$-álgebras seccionais plenas e reduzidas de $\mathcal{A} \bigotimes_{\text {máx }} \mathcal{B}$ e $\mathcal{A} \otimes_{\text {mín }} \mathcal{B}$ com os produtos tensoriais das respectivas $C^{*}$-álgebras seccionais de $\mathcal{A}$ e $\mathcal{B}$. Consideramos que nosso principal resultado na Parte 1 , sem contar as aplicaçōes posteriores, é o seguinte: existe um diagrama comutativo natural:

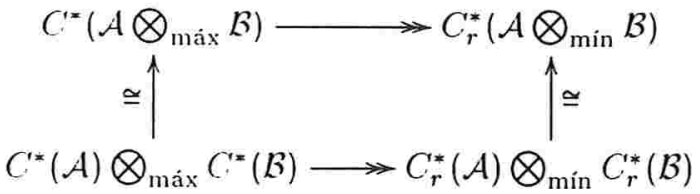

Os isomorfismos verticais refletem uma bela harmonia entre construções universais (flecha da esquerda) e construções espaciais (flecha da direita). As flechas horizontais são produzidas por epimorfismos naturais: a superior é a composiçào da aplicação natural $C^{*}\left(\mathcal{A} \otimes_{\operatorname{máx}} \mathcal{B}\right) \longrightarrow C^{*}\left(\mathcal{A} \bigotimes_{\min } \mathcal{B}\right)$, que existe porque $\|\cdot\|_{\text {máx }} \geq\|\cdot\|_{\text {mín, }}$ com a representação regular $C^{*}\left(\mathcal{A} \bigotimes_{\min } \mathcal{B}\right) \longrightarrow C_{r}^{*}\left(\mathcal{A} \bigotimes_{\min } \mathcal{B}\right)$; a flecha inferior é a extensào contínua da funçào identidade sobre $C^{*}(\mathcal{A}) \bigodot C^{*}(\mathcal{B})$, que existe porque $\|\cdot\|_{\text {máx }} \geq\|\cdot\|_{\text {mín }}$, composta com o produto tensorial das representações regulares de $C^{*}(\mathcal{A})$ e $C^{*}(\mathcal{B})$. Junto com a propriedade de aproximação de um fibrado de Fell, o diagrama acima é a ferramenta fundamental nas aplicações posteriores.

$\mathrm{Na}$ seção correspondente às aplicaçòes, última da Parte I, começamos estendendo a propriedade de aproximação, introduzida por Exel para fibrados de Fell sobre grupos discretos, ao caso contínuo. $\mathrm{Na}$ verdade, damos duas versões da propriedade de aproximação que generalizam a de Exel. Uma, formalmente mais parecida à original. nào parece ser de grande utilidade no caso contínuo. A segunda, 
que chamamos propriedade de aproximaçào em $L^{1}$, permite obter resultados análogos aos obtidos em [28] para o caso discreto: toclo fibrado de Fell com a propriedade de aproximação em $L^{1}$ é amenable; se $\mathcal{B}$ é um fibrado de Fell sobre um grupo amenable, então $\mathcal{B}$ tem a propriedade de aproximação em $L^{1}$, e em particular é amenable. No entanto. um fibrado de Fell sobre um grupo nào amenable pode t.er a propriedade de aproximaçào. e em particular ser amenable ([29], [31]). O fato de que um fibrado de Fell sobre um grupo amenable é. ele também, amenable, parece ter sido provado primeiro para grupos localmente compactos por $\mathrm{Ng}$ em [50].

A propriedade de aproximaçào de Exel é expressada em termos de redes definidas sobre o grupo e a valores na fibra $B_{f}$. L'ma variaçào pequena que introduzimos, porém útil para o caso de um fibrado de Fell sobre um grupo amenable. é permitir que estas redes tomem valores em $M\left(B_{\epsilon}\right)$. a $C^{*}$-álgebra dos multiplicadores de $B_{e}$. É necessário dizer que, antes que nós, o próprio $\mathrm{Ng}$ deu uma definição de propriedacle de aproximaçào para o caso contínuo em [50]. A pesar de que tomamos um aspecto formal da definiçào dele (permitir duas redes em lugar da única presente na definição de Exel), achamos que a definiçào por ele proposta não é adequada. e portanto decidimos desenvolver a nossa própria versào.

O fato fundamental é que a propriedade de aproximação em $L^{1}$ é preservada por produtos tensoriais. Seguem deste fato nossas aplicaçòes principais: se $B$ é um fibrado de Fell com a propriedade de aproximação em $L^{1}$ e fibra $B_{t}$ nuclear. entào $C^{*}(\mathcal{B})$ é nuclear; se $\mathcal{B}$ é um fibrado de Fell com a propriedade de aproximaçào em $L^{1}$ c fibra $B_{e}$ exata, então $C^{*}(\mathcal{B})$ é exata. 


\section{CAPíTULO 1}

Produtos Tensoriais de C*-anéis Ternários 
Neste capítulo estudaremos produtos tensoriais de $C^{*}$-anéis ternários $(1.1 .1 .8)$, como passo prévio antes de estudar os produtos tensoriais de fibrados de Fell. Embora seja este último nosso objetivo final, o tratamento dado aos ( ${ }^{* *}$-anéis ternários é feito num contexto abstrato e independente dos fibrados de Fell: consideraremos os $C^{\prime *}$-anéis ternários e seus homomorfismos como uma categoria que contém à categoria de ('*álgebras e seus homomorfismos como subcategoria plena. É sob este ponto de vista que investigaremos os produtos tensoriais destes objetos e, portanto. os resultados obtidos são paralelos aos conhecidos para $C^{*}$-álgebras. Por exemplo, provamos que existem um produto tensorial máximo e um produto tensorial mínimo entre $C^{* *}$-anéis ternários (1.51). L lm outro aspecto eventualmente considerado dos $C^{*}$-anéis ternários é a função que eles tem como bimódulos de imprimitividade de $C^{*}$-álgebras ([59]), pelo menos no caso de $C^{*}$-anéis ternários positivos (1.27). Assim. seguem facilmente da teoria desenvolvida neste capítulo resultados já conhecidos: a nuclearidade e a exatidào são propriedades invariantes por equivalencia Morita de $C^{*}$-álgebras (1.53 e 1.58 ).

O material está organizado da seguinte maneira. A primeira seção começa com a introdução de alguns conceitos básicos: ('-anéis ternários, homomorfismos de ('-anéis ternários, $C^{*}$-normas sobre um *-anel ternário. Também está dedicada a relembrar os principais resultados de Zettl em [68], o primeiro deles sobre a decomposiçào de um $C^{*}$-anel ternário como soma direta de um $C^{*}$ anel ternário positivo e um ('*-anel ternário negativo (ver 1.27 e a página 12), e o segundo sobre a existencia e unicidade de certa $C^{\prime *}$-álgebra ligada ao $C^{*}$-anel ternário (ver página 12). Posteriormente são estudados os homomorfismos. ideais e quocientes de $C^{*}$-anéis ternários. Igual ao que acontece com os homomorfismos de $C^{*}$-álgebras, todo homomorfismo de $C^{*}$-anéis ternários é uma contração, uma isometria quando é injetivo, e tem imagem fechada (1.18, 1.32). Estes resultados sobre homomorfismos de $C^{*}$-anéis ternários são obtidos a partir dos já conhecidos para $C^{*}$-álgebras, usando o fato de que a cada homomorfismo de $C^{{ }^{*}}$-anéis ternários está associado um único homomorfismo entre as $C^{*}$ álgebras que Zettl associa a cada um dos $C^{\prime *}$-anéis ternários. Este processo de associação de uma $C^{* *}$-álgebra a cada $C^{*}$-anel ternário, e de um homomorfismo de $C^{*}$-álgebras a cada homomorfismo de $C^{*}$-anéis ternários, é de fato um funtor, que denotamos por Z em honra a Zettl (ver a página 12 e 1.19). Se $E$ é um módulo de Hilbert à esquerda sobre uma $C^{*}$-álgebra $A$, a ação de $A$ sobre $E$ e o produto interno de $E$ a valores em $A$ definem uma estrutura de $C^{*}$-anel ternário sobre $E$; neste caso, tem-se que $Z(E)$ é a $C^{*}$-álgebra dos operadores adjuntáveis compactos $\mathcal{K}_{A}(E)$. Na verdade, seria suficiente desenvolver toda a teoria apresentada neste capítulo apenas para módulos de Hilbert, objetos nos quais serào aplicados clepois os resultados obtidos. Porém, acreditamos que o ponto de vista adotado clarifica o panorama e é mais sugestivo para os nossos fins, que são os de generalizar a teoria de produtos tensoriais de $C^{*}$-álgebras ao contexto de $C^{*}$-anéis ternários: de maneira um pouco esquemática, um $C^{*}$-anel ternário é um objeto muito parecido com uma $C^{*}$-álgebra: no entanto, um módulo de Hilbert é um espaço onde age uma $C^{*}$-álgebra.

As noções de ideais à esquerda, à direita, e bilaterais de um $C^{* *}$-anel ternário são introduzidas em 1.20. A nossa definição de ideal bilateral parece, a princípio, mais fraca que a definida em [27] para o caso de anéis ternários de operadores. Porém, elas são equivalentes (1.22). Em 1.23 e 1.24, mostramos que o funtor $Z$ é um isomorfismo entre os reticulados de ideais bilaterais do $C^{*}$-anel ternário $E$ e da $C^{*}$-álgebra $Z(E)$. Posteriormente, mostramos que o quociente de um $C^{*}$-anel ternário por um ideal bilateral é um $C^{*}$-anel ternário com a norma quociente, e que $Z$ é um funtor exato (1.29 e 1.31).

O núcleo do capítulo está na segunda seção. onde se encontra o resultado principal: cada $C^{*}$-norma sobre o produto tensorial algébrico $E \odot F$ entre $C^{*}$-anéis ternários $E$ e $F$ define uma única $C^{*}$-norma sobre o produto tensorial algébrico $Z(E) \odot Z(F)$ entre as $C^{*}$-álgebras $Z(E)$ e $Z(F)$. e viceversa; além disso. esta correspondencia preserva as ordens entre as $C^{*}$-normas (1.50). Como conseqüencia, tem-se que sempre existem uma $C^{* *}$-norma máxima e uma $C^{*}$-norma mínima sobre $E \odot F(1.51)$. Também deduzimos que um $C^{*}$-anel ternário $E$ é nuclear se e somente se $Z(E)$ é nuclear, e que se $A$ e $B$ são $C^{*}$-álgebras equivalentes Morita. entào $A$ é nuclear se e somente se $B$ é nuclear $(1.52,1.53)$. Esta seçào é encerrada com um resultado que generaliza o bem conhecido para espaços de Hilbert, que diz 
que $B(H) \bigotimes_{\min } B\left(K^{\circ}\right) \hookrightarrow B\left(H \otimes K^{\circ}\right)$. Mostramos, em 1.54 , que se $E$ e $F$ são módulos de Hilbert plenos sobre $C^{\prime *}$-álgebras $A$ e $B$ respectivamente, e $\gamma$ é uma $C^{*}$-norma sobre $E \odot F$, então existe uma (única) $C^{\prime *}$-norma $\dot{\gamma}$ sobre $\mathcal{L}(E) \bigcirc \mathcal{L}(F)$ tal que $\mathcal{L}(E) \bigotimes_{\hat{\gamma}} \mathcal{L}(F) \hookrightarrow \mathcal{L}\left(E \bigotimes_{\gamma} F\right)$. Em particular. tem-se que $\mathcal{L}(E) \bigotimes_{\min } \mathcal{L}(F) \subseteq \mathcal{L}\left(E \bigotimes_{\min } F\right.$ ). (aqui $\mathcal{L}(E)$ representa, como é usual, a $C^{* *}$-álgebra dos operadores adjuntáveis sobre $E$ ).

A última seçào está dedicada ao estudo de $C^{*}$-anéis ternários exatos, e os resultados principais são análogos aos obticlos para $C^{*}$-anéis ternários nucleares: $E$ é exato se e somente se $Z(E)$ é exato (1.57). Se $A$ e $B$ sào $\left(^{*}\right.$-álgebras equivalentes Morita, então $A$ é exata se e somente se $B$ é exata (1.58).

\section{1. ('”-anéis ternários}

DEFIniçÃo 1.1. Im *-ancl ternário ó um espaço vetorial complexo $E$ com uma transformaçào $\mu: E \times E \times E \longrightarrow E$. chamado ${ }^{*}$-produto ternário sobre $E$, que é linear nas variáveis ímpares e conjugado linear na segunda, $e$ tal que:

$$
\mu\left(\mu(x, y, z), u, \imath^{\prime}\right)=\mu(x, \mu(u, z, y), v)=\mu(x, y, \mu(z, u, v)) . \forall x, y, z, u, v \in E
$$

Um homomorfismo de *-anéis ternários é uma transformação linear $0:(E . \mu) \longrightarrow(F, \nu)$ tal que

$$
\nu(\phi(x), o(y), \phi(z))=\phi(\mu(x, y, z)), \forall x, y, z \in E .
$$

Às vezes escreveremos simplesmente $(x, y, z)$ ou $(x, y, z)_{E}$ em lugar de $\mu(x, y, z)$. Usaremos a expressào abreviada *-ater em lugar de *-anel ternário.

Observação 1.2. Estamos chamando aqui *-aters aos mesmos objetos que aparecem em [44] sob o nome de "associative triple systems of the second kind". Pensamos que a nossa terminologia é mais coerente com a noçào de (** -anel ternário devida a Zettl (Definition 0.1 de [68]), porque um $C^{*}$-anel ternário é um certo tipo de completamento de um *ater (ver Definiçào 1.8).

Exemplo 1.3. Seja $E=M_{m \times n}(\mathbb{C})$ o espaço de todas as matrizes $m \times n$ com entradas en $\mathbb{C}$. e seja $\mu(x, y, z)=x \cdot y^{*} . z$. onde $y^{*}$ é a matriz transposta e conjugada de $y$. e onde $x . y$ denota o produto usual de matrizes. Entào, é fácil verificar que $(E, \mu)$ é um *ater.

ExEmplo 1.4. Suponhamos que $(E, \mu)$ é um ${ }^{*}$-ater. Sejam $E^{a}=E$ e $\mu^{a}=-\mu$. Então $\left(E^{a}, \mu^{a}\right)$ também é um *-ater. como se verifica fácilmente. Diremos que $(E,-\mu)$ é o anti-*anel ternário de $(E, \mu)$. Se $\phi$ é um homomorfismo de um *-ater $E$ no anti- ${ }^{*}$-ater de $F$. diremos que $\phi$ é um antihomomorfismo de $E$ em $F$, e que $E$ e $F$ sào anti-isomorfos no caso de ser 0 um isomorfismo.

Exemplo 1.5. Se $E$ é um espaço vetorial complexo, denotemos por $E^{*}$ o espaço vetorial complexo que é igual a $E$ como grupo abeliano, e no qual a $\mathbb{C}$-ação está dada por $\lambda . x=\bar{\lambda} x, \forall x \in E, \lambda \in \mathbb{C}$, onde no termo direito da igualdade a açào de $\bar{\lambda}$ é a ação original $\mathbb{C}$ sobre $E$. Agora. se $(E, \mu)$ é um ${ }^{*}$-ater, então $\mu^{*}: E^{*} \times E^{*} \times E^{*} \longrightarrow E^{*}$ dada por $\mu^{*}(x, y, z)=\mu(z, y, x), \forall x, y . z \in E^{*}$, é um *-produto ternário sobre $E^{*}$. Chamaremos o *-ater $\left(E^{*}, \mu^{*}\right){ }^{*}$-anel ternário adjunto de $(E, \mu)$.

DEFiniçÃo 1.6. Um anel ternário de operadores ([68]) é um subespaço $E$ of $B(H, K)$, onde $H$, $K$ são espaços de Hilbert complexos, e tal que $T_{1} T_{2}^{*} T_{3} \in E, \forall T_{1}, T_{2}, T_{3} \in E$. 
Observaçào 1.T. É claro que um anel ternário de operadores é também um *ater; por exemplo. o *ater do Exemplo 1.3 é isomorfo ao anel ternário de operadores $B\left(\mathbb{C}^{n}, \mathbb{C}^{m}\right)$.

DEFiniçÃo 1.8. Uma $C^{* *}$-norma sobre um *-anel ternário $(E, \mu)$ é uma norma que satisfaz as seguintes duas propriedades:

1. $\|\mu(x, y, z)\| \leq\|x\|\|y\|\|z\| . \forall x, y, z \in E$.

2. $\|\mu(x, x, x)\|=\|x\|^{3}, \forall x \in E$.

Dizemos então que $(E .\|\cdot\|)$ é um pré-('*anel ternário, e se é um espaço de Banach, dizemos que é um $C^{*}$-anel ternário. Como no caso de *-aters. também usaremos as formas abreviadas pre- $C^{*}$-ater e $C^{*}$-ater.

OBSERVAÇÃo 1.9. É claro que $\|\cdot\|$ é uma $C^{* *}$-norma sobre o *ater $E$ se e somente se ela é uma $C^{* *}$-norma sobre $E^{a}(1.4)$, e também se e somente se ela é uma $C^{*}$-norma sobre $E^{*}(1.5)$.

Exemplo 1.10. Se $E$ é um módulo de Hilbert à direita sobre a $C^{\prime *}$-álgebra $B$, com produto interno $\langle\cdot, \cdot\rangle$, entào $(E, \mu)$ é um ("*anel ternário, onde $\mu(x, y, z)=x\langle y, z\rangle, \forall x, y, z \in E$.

Exemplo 1.11. Se $(E, \mu),(F, \nu)$ sào $C^{* *}$-anéis ternários, entào $(E \oplus F, \mu \oplus \nu)$ também é um $C^{*}$ anel ternário, onde consideramos a norma do máximo sobre $E \oplus F$, e $\mu \oplus \nu$ é dada por $\mu \oplus \nu\left(x_{1} \oplus\right.$ $\left.y_{1}, x_{2} \oplus y_{2}, x_{3} \oplus y_{3}\right)=\mu\left(x_{1}, x_{2}, x_{3}\right) \pitchfork \nu\left(y_{1}, y_{2}, y_{3}\right), \forall x_{1}, x_{2}, x_{3} \in E, y_{1}, y_{2}, y_{3} \in F$.

Exemplo 1.12. O *ater adjunto (cf. Exemplo 1.5) de um $C^{*}$-ater é ele mesmo um $C^{*}$-ater, e o anti-*-ater de um $C^{* *}$-ater é um $C^{* *}$-ater.

Exemplo 1.13. Suponhamos que $E_{1} \subseteq B(H), E_{2} \subseteq B\left(K^{\circ}\right)$ são anéis ternários de operadores completos, e consideremos $E=E_{1} \bigoplus E_{2}$ com a norma $\left\|\left(x_{1}, x_{2}\right)\right\|=\operatorname{máx}\left\{\left\|x_{1}\right\|,\left\|x_{2}\right\|\right\}$. Se $\mu: E \times E \times$ $E \longrightarrow E$ está dada por $\mu\left(\left(x_{1}, x_{2}\right),\left(y_{1}, y_{2}\right),\left(z_{1}, z_{2}\right)\right)=\left(x_{1} y_{1}^{*} z_{1},-x_{2} y_{2}^{*} z_{2}\right)$, entào $(E, \mu)$ é um $C^{*}$-ater.

Os $C^{*}$-anéis ternários foram definidos e estudados por Zettl em [68]. Ele provou que se $E$ é um $C^{*}$-anel ternário, entào existe. a menos de isomorfismos, um único par $\left(Z_{r}(E),\langle\cdot, \cdot\rangle_{r}\right)$ tal que:

1. $Z_{r}(E)$ é uma $C^{*}$-álgebra e $E$ é um $Z_{r}(E)$ módulo de Banach à direita.

2. $\langle\cdot, \cdot\rangle_{r}: E \times E \longrightarrow Z_{r}(E)$ é sesquilinear. conjugada linear na primeira variável, com $\|\langle\cdot, \cdot\rangle\|_{r} \leq 1$. e

- $\langle x, y b\rangle_{r}=\langle x, y\rangle_{r} b . \forall x, y \in E, b \in Z_{r}(E)$.

- $\langle x, y\rangle_{r}^{*}=\langle y, x\rangle_{r}, \forall x, y \in E$.

3. $\mu(x, y, z)=x\langle y, z\rangle_{r}, \forall x, y, z \in E$.

4. $\operatorname{span}\langle E, E\rangle_{r}$ é denso em $Z_{r}(E)$.

Além disso, temos que

$$
\|x\|^{2}=\|\langle x, x\rangle\|, \forall x \in E .
$$

Entào ele definiu $E^{+}=\left\{x \in E:\langle x, x\rangle_{r} \in Z_{r}(E)^{+}\right\}$e $E^{-}=\left\{x \in E:\langle x, x\rangle_{r} \in-Z_{r}(E)^{+}\right\}$, e mostrou que eles são sub- $C^{*}$-anéis ternários de $E$, tais que $E^{+}$é isomorfo a um anel ternário de operadores, e $E^{-}$é anti-isomorfo a um anel ternário de operadores (ver 1.4 e 1.6 acima). De fato, tem-se que $E=E^{+} \oplus E^{-}$como $C^{* *}$-anéis ternários, e esta é a uńica decomposição de $E$ como soma direta de dois sub- $C^{*}$-anéis ternários, um deles isomorfo a um anel ternário de operadores e o outro anti-isomorfo a um anel ternário de operadores. Chamaremos esta decomposição a decomposiçcio fundamental de $E$.

Naturalmente, temos uma versào à esquerda destes resultados: existe um par $\left(Z_{l}(E),\langle\cdot, \cdot\rangle_{l}\right)$ satisfazendo propriedades análogas às de $\left(Z_{r}(E),\langle\cdot, \cdot\rangle_{r}\right)$, só que enunciadas da forma óbvia do lado 
esquerdo. Assim, E. é um $Z_{l}(E)-Z_{r}(E)$ bimódulo de Banach. Notar que a decomposição de $E$ com respeito à forma $\langle\cdot, \cdot\rangle_{l}$ coincide com a feita com respeito a $\langle\cdot, \cdot\rangle_{r}$, por causa da unicidade de tal decomposição. Em particular, concluímos que o Exemplo 1.13 é de fato o exemplo genérico de $C^{*}$-ater. Observar também que temos

$$
\langle x, y\rangle_{1} z=\mu(x, y, z)=x\langle y, z\rangle_{r}, \forall x, y, z \in E .
$$

Exemplo 1.14. Sejam $E$ um $C^{*}$-anel ternário, $x \in E$, e $E_{x}$ o sub-C*anel ternário gerado por $x$ dentro de $E$. Para cada $n \geq 0$, seja $x_{n}=x\left(\langle x, x\rangle_{r}\right)^{n}$. Então é claro que $x_{n} \in E_{x}, \forall n \geq 0$. Por outro lado, é imediato que $\left(x_{n}, x_{m}, x_{k}\right)=x_{n+m+k+1}$, donde segue que $E_{x}=\overline{\operatorname{span}}\left\{x_{n}: n \geq 0\right\}$. Além disso, como $\left\langle x_{n}, x_{m}\right\rangle_{r}=\langle x, x\rangle_{r}^{n+m+1}$. concluímos que $\mathrm{Z}_{r}\left(E_{x}\right)=C^{*}\left(\langle x, x\rangle_{r}\right)=C^{\prime}\left(\sigma\left(\langle x, x\rangle_{r}\right)\right)$.

ProposiçÃo 1.15. Ś jam $(E, \mu)$ um $C^{* *}$-anel ternário, $e\left(E^{*}, \mu^{*}\right)$ o seu $C^{*}$-anel ternário adjunto (1.5). Então $Z_{l}\left(E^{*}\right)=Z_{r}(E) . e\langle\hat{x}, \bar{y}\rangle_{l}^{\sim}=\langle x, y\rangle_{r}, \forall x, y \in E$. onde estamos indicando por $\langle\cdot, \cdot\rangle_{\hat{l}}$ o $\mathrm{Z}_{1}\left(E^{*}\right)$-produto interno esquerdo associado a $E^{*}$ como acima, e por $\bar{x}, \bar{y}$ os elementos $x, y \in E$ vistos dentro de $E^{*}$.

Demonstraçáo. Seja $[\cdot, \cdot]: E^{*} \times E^{*} \longrightarrow Z_{r}(E)$ dada por $[\tilde{x}, \tilde{y}]=\langle x, y\rangle_{r}, \forall \tilde{x}, \tilde{y} \in E^{*}$ Como $\langle\cdot, \cdot\rangle_{r}$ é conjugada linear na primeira variável, e como $E^{*}$ é o $\mathbb{C}$-espaço vetorial conjugado de $E$, entào $[\cdot, \cdot]$ será uma forma sesquilinear conjugada linear na segunda variável. É claro que $[\tilde{x}, \tilde{y}]^{*}=[\tilde{y}, \tilde{x}]$, e que $\|[\cdot, \cdot]\| \leq 1$. Notar que, como $E$ é um módulo de Banach à direita sobre $Z_{r}(E)$, então $E^{*}$ é um módulo de Banach à esquerda sobre $Z_{r}(E)$, onde consideramos a açào de $Z_{r}(E)$ sobre $E^{*}$ dada por $b \cdot \tilde{x}=x b^{*}$. Se $b \in Z_{r}(E), \tilde{x}, \dot{y} \in E^{*}$, temos:

$$
[b \cdot \tilde{x}, \tilde{y}]=\left\langle x b^{*}, y\right\rangle_{r}=\left(\left\langle y, x b^{*}\right\rangle_{r}\right)^{*}=\left(\langle y, x\rangle_{r} b^{*}\right)^{*}=\left([\tilde{x}, \tilde{y}]^{*} b^{*}\right)^{*}=b[\tilde{x}, \tilde{y}]
$$

Por outro lado:

$$
\langle\tilde{x}, \tilde{y}\rangle_{l} \tilde{z}=\mu^{*}(\tilde{x}, \bar{y}, \bar{z})=\mu(z, y, x)=z\langle y, x\rangle_{r}=z\langle x, y\rangle_{r}^{*}=\langle x, y\rangle_{r} \cdot z=[\tilde{x}, \tilde{y}] \cdot z,
$$

o que encerra a prova (Na verdade, esta última igualdade já implica que $[\because$. $]$ é uma forma sesquilinear com as propriedades requeridas).

ObservaÇÃo 1.16. Suponhamos que $(E, \mu)$ é um $C^{*}$-anel ternário, e seja $\left(E^{a}, \mu^{a}\right)$ o anti- $C^{*}$-anel ternário de $(E, \mu)$, i.e.: $E^{a}=E$ como espaço de Banach, e $\mu^{a}=-\mu$ (ver 1.4). Então temos um resultado similar ao da Proposiçào 1.15: $Z_{r}\left(E^{a}\right)=Z_{r}(E)$, e $\langle\cdot, \cdot\rangle_{r}^{a}=-\langle\cdot, \cdot\rangle_{r}$, com a notação óbvia. Também, se $(F, \nu)$ é um outro $C^{*}$-ater, temos que (de novo com a notação óbvia) $Z_{r}(E \bigoplus F)=$ $\mathbf{Z}_{r}(E) \oplus \mathbf{Z}_{r}(F)$, e $\left\langle x_{1} \oplus y_{1}, x_{2} \oplus y_{2}\right\rangle_{r}^{E \oplus F}=\left\langle x_{1}, x_{2}\right\rangle_{r}^{E} \oplus\left\langle y_{1}, y_{2}\right\rangle_{r}^{F}, \forall x_{1}, x_{2} \in E, y_{1}, y_{2} \in F$ (ver o Exemplo 1.11). As demonstraçōes são muito fáceis e serão omitidas.

Notar que $E$ e $E^{*}$ sào basicamente o mesmo objeto como $C^{*}$-anéis ternários, e portanto, pela Proposição 1.15 , decorre que as propriedades de $Z_{r}(E)$ e $Z_{l}(E)$ deduzidas de propriedades de $E$ como $C^{*}$-anel ternário serão as mesmas. Por esta razão, no que segue consideraremos apenas a versão direita da situação. e usaremos a notaçào $Z$ e $\langle\cdot, \cdot\rangle$ em lugar de $Z_{r}$ e $\langle\cdot, \cdot\rangle_{r}$. a menos que seja necessário mencionar explicitamente o lado considerado. Porém, o leitor deve levar em conta que todos os resultados obtidos a seguir para $Z_{r}$ valem também para $Z_{l}$. 
Lema 1.17. Sejam I um *-ideal bilateral. não necessariamente fechado, de uma *álgebra de Banach $A, B$ uma $C^{*}$-álgebra $\in \rho: I \longrightarrow B$ um *-homomorfismo. Então $\rho$ é contrativa, e portanto pode ser estendido. de forma única. a um homomorfismo de *álgebras de Banach $\rho: \bar{I} \longrightarrow B$.

Demonstra̧̧ào. Seja $\pi: \overline{\rho(I)} \longrightarrow B(H)$ uma representação fiel e nào degenerada. Então $\pi \rho$ é uma representação nào degenerada de $I$. Por [37], VI-19.11, $\pi \rho$ é contrativa, e como $\pi$ é uma isometria, tem-se que $\rho$ também deve ser contrativa.

Proposiçào 1.18. Seja $\pi: E \longrightarrow F$ um homomorfismo de *aters (1.1), onde E e $F$ são $C^{*}$ aters. Então $\pi$ é uma contraçào, $\epsilon$ existe um único *-homomorfismo $Z(\pi): Z(E) \longrightarrow Z(F)$ tal que $\mathrm{Z}(\pi)\left(\langle x, y\rangle_{E}\right)=\langle\pi(x), \pi(y)\rangle_{F}, \forall x, y \in E$. Tem-se que $\pi(x b)=\pi(x) Z(\pi)(b), \forall x \in E, b \in Z(E)$. Se $\pi \dot{\epsilon}$ injetivo (sobrejetivo. isomorfismo). entào $\pi \dot{\epsilon}$ uma isometria, $\epsilon Z(\pi)$ é injetivo (sobrejetivo. isomorfismo).

Demonstraçào. Se $Z(\pi)$ existe, deve ser $Z(\pi)\left(\langle x, y\rangle_{E}\right)=\langle\pi(x), \pi(y)\rangle_{F}, \forall x, y \in E$. Portanto. temos que verificar que $\sum_{i}\left\langle x_{i}, y_{i}\right\rangle_{E}=0$ implica que $\sum_{i}\left\langle\pi\left(x_{i}\right), \pi\left(y_{i}\right)\right\rangle_{F}=0$. Agora, se $\sum_{i}\left\langle x_{i}, y_{i}\right\rangle_{E}=0$ :

$$
\begin{aligned}
\left(\sum_{i}\left\langle\pi\left(x_{i}\right), \pi\left(y_{i}\right)\right\rangle_{F}\right)^{*}\left(\sum_{j}\left\langle\pi\left(x_{j}\right), \pi\left(y_{j}\right)\right\rangle_{F}\right) & =\sum_{i}\left(\left\langle\pi\left(y_{i}\right), x_{i}\right\rangle_{F}\right)\left(\sum_{j}\left\langle\pi\left(x_{j}\right), \pi\left(y_{j}\right)\right\rangle_{F}\right) \\
& \left.=\sum_{i}\left\langle\pi\left(y_{i}\right), \pi\left(x_{i}\right) \sum_{j}\left\langle\pi\left(x_{j}\right), \pi\left(y_{j}\right)\right\rangle_{F}\right)\right\rangle_{F} \\
& =\sum_{i}\left\langle\pi\left(y_{i}\right), \pi\left(x_{i} \sum_{j}\left\langle x_{j}, y_{j}\right\rangle_{E}\right)\right\rangle_{F} \\
& =0
\end{aligned}
$$

Portanto, temos definido $Z(\pi): \operatorname{span}\langle E, E\rangle_{E} \longrightarrow \mathrm{Z}(F)$, que é um homomorfismo de *-álgebras. Como $\operatorname{span}\langle E, E\rangle_{E}$ é um *-ideal bilateral denso de $Z(E), Z(\pi)$ tem uma única extensão a um homomorfismo $\mathrm{Z}(\pi): \mathbf{Z}(E) \longrightarrow \mathrm{Z}(F)$, pelo Lema 1.17 acima.

Agora, se $x \in E$, tem-se:

$$
\|\pi(x)\|^{2}=\left\|\langle\pi(x), \pi(x)\rangle_{F}\right\|=\left\|Z(\pi)\left(\langle x, x\rangle_{E}\right)\right\| \leq\left\|\langle x, x\rangle_{E}\right\|=\|x\|^{2},
$$

já que $Z(\pi)$ é uma contração: portanto $\pi$ é também uma contraçào. Em particular, $\pi$ é contínuo, e portanto $\pi(x b)=\pi(x) Z(\pi)(b), \forall x \in E, b \in Z(E)$, pois isto é válido para todo $b \in \operatorname{span}\langle E, E\rangle_{E}$, que é denso em $Z(E)$

Se $\pi$ é sobrejetiva, é claro que $Z(\pi)$ também será sobrejetiva. Suponhamos que $\pi$ é injetiva, e seja $b \in \operatorname{ker} Z(\pi)$. Entào $\pi(x b)=0, \forall x \in E$. e como $\pi$ é injetiva, tem-se que $x b=0, \forall x \in E$, e portanto $\operatorname{span}\langle E, E\rangle_{E} b=0$, donde $Z(E) b=0$; em particular $b^{*} b=0$, e então $b=0$. Assim, concluímos que $Z(\pi)$ é injetiva. Em particular, $Z(\pi)$ é uma isometria, e portanto

$$
\|\pi(x)\|^{2}=\left\|\langle\pi(x), \pi(x)\rangle_{F}\right\|=\left\|\mathrm{Z}(\pi)\left(\langle x, x\rangle_{E}\right)\right\|=\left\|\langle x, x\rangle_{E}\right\|=\|x\|^{2},
$$

donde segue que $\pi$ é uma isometria.

OBservação 1.19. Junto ao resultado do Zett.l, a Proposição 1.18 mostra que a cada $C^{*}$-anel ternário podemos associar uma $C^{\prime *}$-álgebra. única a menos de isomorfismos, e que a cada homomorfismo $\pi: E \longrightarrow F$ de $C^{*}$-anéis ternários podemos associar um único homomorfismo $Z(\pi): Z(E) \longrightarrow$ $Z(F)$ de $C^{*}$-álgebras. Suponhamos que $\rho: F \longrightarrow G$ é um outro homomorfismo de $C^{*}$-aters. Então $Z(\rho \pi)=Z(\rho) Z(\pi)$, pela condição de unicidade. Na verdade, o homomorfismo $Z(\pi)$ depende das $C^{*}$ álgebras escolhidas para representar $Z(E)$ e $Z(F)$. Por esta razão, $Z$ não é exatamente um funtor na 
categoria de $C^{* *}$-álgebras. Seria um funtor se escolhermos um representante de cada classe de isomorfismos de $C^{*}$-álgebras, e definíssemos o valor de $Z$ dentro dessa família de representantes. Porém. perder flexibilidade na escollı de $u m$ representante para $Z(E)$ nào é muito conveniente: o melhor é escolher o representante mais adequaclo ao contexto. Por exemplo, se $F \subseteq E$, sempre suporemos que $\mathrm{Z}(F)=\overline{\operatorname{span}}\langle F, F\rangle_{E} \subseteq \mathrm{Z}(E)$.

DEFiniçÃo 1.20. Sejam $E$ um ("x-ater. e $F \subseteq E$ um subespaço fechado. Diremos que $F$ é um ideal à esquerda (à direita) de $E$ sc $(E, E . F) \subseteq \bar{F}$ (respectivamente: se $(F, E, E) \subseteq F)$. Se $F$ é um ideal à esquerda è à direita de $E$. diremos que é um ideal de $E$. Denotaremos os conjuntos de ideais, ideais à esquerda, icleais à direita de $E$. respectivamente por $I(E), L(E), R(E)$. Usaremos a notação $F \triangleleft E$ para indicar que $F \dot{c}$ un ideal bilateral de $E$.

ObSERVAÇÃo 1.21. Em [27]. o autor introduziu a noção de ideal (bilateral) de um anel ternário de operadores, através de uma definiçào que, aparentemente, é mais restritiva que a nossa. Porém. elas são equivalentes, como se deduz de 1.22 a seguir.

Observaçào 1.22 . Todo ideal à esquerda ou à direita é em particular um sub- $C^{*}$-ater. Por outro lado, $F$ é um ideal bilateral de $E$ se e somente se ele satisfaz a condiçào $(E, F, E) \subseteq F$. Com efeito. suponhamos primeiro que $F$ é um ideal bilateral de $E$. Então, como $\operatorname{span}(F, F, F)$ é denso em $F$, e o produto ternário é contínuo:

$$
\begin{aligned}
(E, F, E) & \subseteq \overline{\operatorname{span}}(E,(F, F, F), E) \\
& =\overline{\operatorname{span}}(E, F,(F, F, E)) \\
& \subseteq \overline{\operatorname{span}}(E, F,(F, E, E)) \\
& \subseteq \overline{\operatorname{span}}(E, F, F) \\
& \subseteq \overline{\operatorname{span}}(E, E, F) \\
& \subseteq F .
\end{aligned}
$$

Inversamente, suponhamos que $(F . F . E) \subseteq F$, e mostremos que $F$ é um ideal à direita de $E$ :

$$
\begin{aligned}
(E . E, F) & \subseteq \overline{\operatorname{span}}(E, E,(F, F, F)) \\
& \subseteq \overline{\operatorname{span}}(E,(F, F, E), F) \\
& \subseteq \overline{\operatorname{span}}(E,(E, F, E), F) \\
& \subseteq \overline{\operatorname{span}}(E, F, F) \\
& \subseteq \overline{\operatorname{span}}(E, F, E) \\
& \subseteq F .
\end{aligned}
$$

De forma análoga se mostra que $F$ também é um ideal à esquerda de $E$.

Proposição 1.23. Sejam E um ('*ater, $\in A:=Z(E)$. Consideremos as famílias $H(A)$ de sub$C^{*}$-álgebras hereditárias de $A$. I $I(A)$ de ideais de A. Então, $Z: L(E) \longrightarrow H(A)$ é uma bijeçào com inversa $T: H(A) \longrightarrow L(E)$ dada por $T(B)=E B$ (levar em conta 1.19). Quando restrita a $I(E)$. Z é uma bijeçào sobre $I(A)$. Além disto. $Z$ e $T$ preservam as relaçôs de inclusão. 
Demonstração. Se $B$ é uma sub- $C^{*}$-álgebra de $A$. então:

$$
(E, E, E B)=E\langle E, E B\rangle=E\langle E, E\rangle B=(E, E, E) B \subseteq E B,
$$

donde $E B$ é um ideal à esquerda de $E$. Inversamente, se $F$ é um ideal à esquerda de $E$, temos:

$$
\langle F, F\rangle\langle E, E\rangle\langle F, F\rangle=\langle E\langle F, F\rangle, E\langle F, F\rangle\rangle=\langle(E, F, F),(E, F, F)\rangle \subseteq\langle F, F\rangle,
$$

donde tomando os fechos dos correspondentes espaços vetorias gerados, vemos que $\mathrm{Z}(F) \mathrm{Z}(E) \mathrm{Z}(F)=$ $\mathrm{Z}(F)$, isto é, $\mathrm{Z}(F)$ hereditária. Para ver que as correspondencias são mutuamente inversas, note que se $F$ é um $C^{*}$-anel ternário, entào $F=Z(F) F$; por outro lado, se $B$ é uma sub-C:*álgebra hereditária de $A$, então $Z(E B)=\overline{\operatorname{span}}\langle E B, E B\rangle=\overline{\operatorname{span}} B\langle E, E\rangle B=B A B=B$.

Se $I$ é um ideal de $Z(E)$. temos que $(E I, E, E)=E I\langle E, E\rangle \subseteq E I A=E I$, e portanto $E I$ é também um ideal à direita de $E$, e portanto um ideal de $E$. Inversamente, se $F$ é um ideal de $E$, ele é em particular um ideal à esquerda, c portanto $Z(F)$ é um ideal à direita de $Z(E)$. Mas como $F$ é também um ideal à direita de $E$, temos: $\langle E, E\rangle\langle F, F\rangle=\langle F\langle E, E\rangle, F\rangle=(F, E, E) \subseteq\langle F, F\rangle$, e entào $Z(F)$ é um ideal à esquerda de $A$.

Proposiçào 1.24. Seja E um $C^{*}$-anel ternário e seja $\left(E_{j}\right)_{j \in J}$ uma família de ideais de E. Então $\mathrm{Z}\left(\bigcap_{j \in J} E_{j}\right)=\bigcap_{i \in J} \mathrm{Z}\left(E_{j}\right), \epsilon \mathrm{Z}\left(\overline{\text { span }} \bigcup_{j \in J} E_{j}\right)=\overline{\operatorname{span}} \bigcup_{j \in J} \mathrm{Z}\left(E_{j}\right)$.

Demonstraçäo. Sejam $I=\bigcap_{j \in J} \mathrm{Z}\left(E_{j}\right), J=\mathrm{Z}\left(\bigcap_{j \in J} E_{j}\right)$. (omo $\bigcap_{j \in J} E_{j} \subseteq E_{j_{0}}, \forall j_{0} \in J$, e como Z preserva as inclusōes, tem-se $I \subseteq J$. Então:

$$
\bigcap_{j \in J} E_{j}=E I \subseteq E J \subseteq E Z\left(E_{j_{0}}\right)=E_{j_{0}}, \forall j_{0} \in J,
$$

donde segue que $E I \subseteq E J \subseteq E\left(\bigcap_{j \in J} \mathrm{Z}\left(E_{j}\right)\right)=E I$, e portanto $I=J$.

A outra afirmaçào é conseqüencia da já provada, pois o ideal gerado por uma família de ideais é a interseção de todos os ideais que contem a união dos ideais da família.

Corolário 1.25. Seja $\pi: E \longrightarrow F$ um homomorfismo de ${ }^{*}$-aters entre os $C^{*}$-aters $E \in F$. Então tem-se que $\mathrm{Z}(\operatorname{ker}(\pi))=\operatorname{ker}(\mathrm{Z}(\pi))$. Em particular. $\pi \dot{\epsilon}$ injetiva se e somente se $\mathrm{Z}(\pi) \dot{\epsilon}$ injetiva.

Demonstração. Suponhamos que $x, y \in \operatorname{ker}(\pi)$. Então $Z(\pi)(\langle x, y\rangle)=\langle\pi(x), \pi(y)\rangle=0$, e portanto $\langle x, y\rangle \in \operatorname{ker}(Z(\pi)) ; \log o, Z(\operatorname{ker}(\pi)) \subseteq \operatorname{ker}(Z(\pi))$. Seja agora $G=E \operatorname{ker}(Z(\pi))$, e seja $x=y a \in G$, onde $y \in E, a \in \operatorname{ker}(Z(\pi))$. Temos: $\pi(x)=\pi(y a)=\pi(y) Z(\pi)(a)=0$, donde segue que $x \in \operatorname{ker}(\pi)$, e portanto que $G \subseteq \operatorname{ker}(\pi)$. Assim, como $Z$ é um isomorfismo de reticulados segundo 1.24 , temos: $\operatorname{ker}(Z(\pi))=Z(G) \subseteq Z(\operatorname{ker}(\pi)) \subseteq \operatorname{ker}(Z(\pi))$, e concluímos que $Z(\operatorname{ker}(\pi))=\operatorname{ker}(Z(\pi))$.

OBSERVAÇÃo 1.26. Na Proposiçào 1.18 vimos que se $\pi: E \longrightarrow F$ é um homomorfismo sobrejetivo entre $C^{*}$-anéis ternários. então $Z(\pi): Z(E) \longrightarrow Z(F)$ também é sobrejetivo. Porém, a sobrejetividade de $Z(\pi)$ não implica a sobrejetividade de $\pi$. Basta considerar a inclusão $\mathbb{C} \stackrel{\prime}{\hookrightarrow} \mathbb{C}^{2}$ como espaços de Hilbert sobre $\mathbb{C}: \iota$ não é sobrejetiva, mas $Z(\iota)$ é a identidade sobre $\mathbb{C}$.

Como já foi observado, todo $C^{*}$-anel ternário admite uma única decomposição $E=E^{+} \oplus E^{-}$, com $E^{+}$isomorfo a um anel ternário de operadores e $E^{-}$anti-isomorfo a um anel ternário de operadores (página 12). $E^{+}$e $E^{-}$sào ideais de $E$, totalmente independentes um do outro. Por esta razão, será conveniente (e suficiente como veremos depois), considerar $\left(^{*}{ }^{*}\right.$-anéis ternários nos quais $E^{-}=0$. 
Definição 1.27. Dizemos que o $C^{*}$-anel ternário $E$ é positivo (negativo) se $E=E^{+}$(respectivamente se $\left.E=E^{-}\right)$. Uma representaçào de um $C^{*}$-anel ternário positivo $E$ sobre os espaços de Hilbert. $H_{1}$ e $H_{2}$ é um homomorfismo de *-anéis ternários $\pi: E \rightarrow B\left(H_{1}, H_{2}\right)$, onde o produto ternário sobre $B\left(H_{1}, H_{2}\right)$ está definido por: $\left(T_{1}, T_{2}, T_{3}\right) \longmapsto T_{1} T_{2}^{*} T_{3}$. $\pi$ é dita fiel se ela é injetiva (por $1.18, \pi$ é automaticamente contrativa, e ela é uma isometria se for fiel)

Dado um $B$-módulo de Hilbert pleno à direita $\left(E,\langle\cdot, \cdot\rangle_{B}\right)$, vimos no exemplo 1.10 que $E$ é um $C^{*}$-ater com o produto ternário dado por $\mu(x, y, z)=x\langle y, z\rangle_{B}$. É claro que teremos $Z_{r}(E)=B$, e $\langle\cdot \cdot\rangle_{r}=\langle\cdot, \cdot\rangle_{B}$. Em particular, $(E, \mu)$ é um $C^{*}$-ater positivo. Portanto, $C^{*}$-anéis ternários positivos e módulos de Hilbert plenos são essencialmente o mesmo tipo de objetos. Porém, do ponto de vista da teoria de módulos, o usual é considerar homomorfismos entre módulos de Hilbert sobre a mesma $C^{*}$-álgebra $B$, e estes sào certo tipo de transformaçōes lineares contínuas que "interlaçam" as açōes da álgebra $B$. Estes homomorfismos diferem dos homomorfismos que nós estamos considerando (1.1. 1.18). Um comentário similar cabe sobre os anéis ternários de operadores (1.6): um anel ternário de operadores pode ser entendido como uma representação concreta, espacial, de um $C^{*}$-ater positivo, o de um módulo de Hilbert. Mas, como objetos espaciais, talvez a noçào de homomorfismo entre eles deveria também ser espacial: por exemplo, se $E_{1} \subseteq B\left(H_{1}, K_{1}\right), E_{2} \subseteq B\left(H_{2}, K_{2}\right)$, onde $H_{1}, H_{2}, K_{1}, K_{2}$ sào espaços de Hilbert, poderíamos desejar como homomorfismos pares $\left(\rho_{1}, \rho_{2}\right)$ tais que $\rho_{1}: H_{1} \longrightarrow$ $H_{2}, \rho_{2}: K_{1} \longrightarrow K_{2}$ são isometrias, e $\rho_{2} E_{1} \rho_{1}^{*} \subseteq E_{2}$.

Portanto, podemos pensar em $C^{\prime *}$-anéis ternários positivos, módulos de Hilbert plenos e anéis ternários de operadores como objetos que se diferenciam apenas pelo ponto de vista adotado para estudá-los, o que se reflete nos homomorfismos escolhidos.

ObservaçÃo 1.28. Seja $(E, \mu)$ um $C^{* *}$-anel ternário, e seja $E=E^{+} \bigoplus E^{-}$a decomposição fundamental de $E$ (definida na página 12 ). Seja $P_{E}: E \longrightarrow E^{+}$a projeçào de $E$ sobre $E^{+}$ao longo de $E^{-}$ (i.e.: $\operatorname{ker} P_{E}=E^{-}$). Entào temos a simetria fundamental $J_{E}: E \longrightarrow E$, dada por $J_{E}=2 I d_{E}-P_{E}$. A simetria fundamental é o único operador sobre $E$ que satisfaz ([68], 3.1):

- $J_{\bar{E}}^{2}=I d_{E}$

- $J_{E}(x, y, z)=\left(J_{E} x, y, z\right)=\left(x, J_{E} y, z\right)=\left(x, y, J_{E} z\right), \forall x, y, z \in E$.

- $\left(E, J_{E} \mu\right)$ é isomorfo a um anel ternário de operadores.

Portanto, se $E^{p}:=E$ como espaço de Banach, $\mu^{p}:=J_{E} \mu$, temos que $\left(E^{p}, \mu^{p}\right)$ é um $C^{*}$-anel ternário positivo. De fato, se $x_{1}, x_{2}, x_{3} \in E^{+}, y_{1}, y_{2}, y_{3} \in E^{-}$, então

$$
\begin{aligned}
\mu^{P}\left(x_{1} \oplus y_{1}, x_{2} \oplus y_{2}, x_{3} \oplus y_{3}\right) & =J_{E} \mu\left(x_{1} \oplus y_{1}, x_{2} \oplus y_{2}, x_{3} \oplus y_{3}\right) \\
& =J_{E} \mu\left(x_{1}, x_{2}, x_{3}\right)+J_{E} \mu\left(y_{1}, y_{2}, y_{3}\right) \\
& =\mu\left(x_{1}, x_{2}, x_{3}\right)-\mu\left(y_{1}, y_{2}, y_{3}\right),
\end{aligned}
$$

donde $\left(E^{p}, \mu^{p}\right)=\left(E^{+}, \mu_{\left.\right|_{E^{+}}}\right) \oplus\left(E^{-},-\mu_{\left.\right|_{E^{-}}}\right)$.

Notar que $J_{E}$ é um automorfismo de $(E, \mu)$, e em particular uma isometria, e que cada sub- $C^{*}$ ater $F$ de $E$ é invariante por $J_{E}$ pois, segundo a Proposição 1.18 , temos $F^{+}=F \cap E^{+}, F^{-}=F \cap E^{-}$, e portanto $P_{F}=P_{\left.E\right|_{F}}, J_{F}=J_{\left.E\right|_{F}}$. Em particular, $F$ é um sub-C $C^{*}$-ater (ideal) de $E$ se e somente se $F^{P}$ é um sub-C $C^{*}$-ater (resp.: ideal) de $E^{p}$

Proposição 1.29. Seja $F$ um ideal de um $C^{*}$-anel ternário $E$, e consideremos a estrutura algébrica natural de *anel ternário do espaço quociente $E / F$. Com a norma quociente. E/F $\varepsilon$ um $C^{*}$-anel ternário. $\epsilon \mathrm{Z}(E / F)=\mathrm{Z}(E) / \mathrm{Z}(F)$. 
Demonstraçào. Como $F$ é um ideal de $E$, ele é invariante pelo produto ternário, e portanto o produto ternário sobre $E$ induz um produto ternário $\bar{\mu}$ sobre $E / F$ da forma natural, de maneira que $E / F$ é um *-anel ternário. A dificuldade está em verificar que $\|\cdot\|_{q}$, a norma quociente sobre $E / F$, é uma $C^{*}$-norma. Na verdade, também é imediato que, se $\bar{x}$ é a imagem de $x \in E$ em $E / F$ pela aplicação quociente, entào $\|\bar{\mu}(\bar{x}, \bar{y}, \bar{z})\|_{q} \leq\|\bar{x}\|_{q}\|\bar{y}\|_{q}\|\bar{z}\|_{q}$. Já a condição 2. da Definição 1.8 é menos evidente.

Suponhamos primeiro que $E$ é um $C^{* *}$-anel ternário positivo.

Sejam $A=Z(E) . I=Z(F)$. Se $a \in A$, denotamos por $\bar{a}$ a imagem de $a$ em $A / I$. Suponhamos que $\bar{a}=\bar{b}, \bar{x}=\bar{y}$, e $\bar{u}=\bar{v}$, para $a, b \in A, x, y, u, v \in E$. Entào:

$$
\begin{gathered}
x a-y b=x(a-b)+(x-y) b \in E I+F A=F, \\
\langle x, u\rangle-\langle y \cdot v\rangle=\langle x, u-v\rangle+\langle x-y, v\rangle \in\langle E . F\rangle+\langle F, E\rangle \subseteq\langle F, F\rangle \subseteq I .
\end{gathered}
$$

Assim, temos umas funçòes $: E / F \times A / I \longrightarrow E / F$ e $[\because]:, E / F \times E / F \longrightarrow A / I$ dadas por $\bar{x} \cdot \bar{a}=\overline{x a}$, e $[\bar{x}, \bar{y}]=\overline{\langle x, y\rangle}, \forall a \in A, x, y \in E$. É rotineiro verificar que $(E / F, \cdot,[\cdot, \cdot])$ é um $A / I$-módulo com semi-produto interno (ver [43]. página 3). De fato. [., $]$ é um produto interno: se $[\bar{x}, \bar{x}]=0$, entào $\langle x, x\rangle \in I$, e portanto $Z\left(E_{r}\right) \subseteq I$, onde $E_{x}$ é o sub-C' $C^{\prime *}$-anel ternário de $E$ gerado por $x$ (ver o Exemplo 1.14). Segue que $x \in E_{r}=E_{r} Z\left(E_{x}\right) \subseteq E I=F$. e portanto $\bar{x}=0$. Além disso, temos:

$$
\|[\bar{x}, \bar{x}]\|_{A / I}=\|\overline{\langle x, x\rangle}\|_{A / I}=\inf _{b \in I}\|\langle x, x\rangle+b\|_{A} \leq \inf _{y \in F}\|\langle x+y, x+y\rangle\|_{A}=\inf _{y \in F}\|x+y\|_{A}^{2}=\|\bar{x}\|_{q}^{2}
$$

Temos então que $E / F$ é um pré-módulo de Hilbert sobre $t / I$, e que $\|\cdot\| \leq\|\cdot\|_{q}$, onde a primeira norma é a induzida pelo produto interno $[\cdot, \cdot]$. Queremos provar a desigualdade contrária.

Seja $\left(u_{i}\right)$ uma identidade aproximada de $I$. Se $x \in E$ temos, $\forall y \in F$ :

$\|\bar{x}\|_{q} \leq \limsup _{i}\left\|x-x u_{i}\right\|=\limsup _{i}\left\|x-y+y u_{i}-x u_{i}\right\|=\limsup _{i}\left\|(x-y)\left(1-u_{i}\right)\right\| \leq\|x-y\| \leq\|\bar{x}\|_{q}$, donde segue que $\|\bar{x}\|_{q}=\lim _{i}\left\|x-x u_{i}\right\| . \forall x \in E$. Agora:

$$
\begin{aligned}
\left\|x-x u_{i}\right\|^{2} & =\left\|\langle x, x\rangle-\langle x, x\rangle u_{i}-u_{i}\langle x, x\rangle+u_{i}\langle x, x\rangle u_{i}\right\| \\
& =\left\|\left(1-u_{i}\right)\left(\langle x, x\rangle-\langle x, x\rangle u_{i}\right)\right\| \\
& \leq\left\|\langle x, x\rangle-\langle x, x\rangle u_{i}\right\| .
\end{aligned}
$$

Temos entào que:

$$
\|\bar{x}\|_{q}^{2}=\lim _{i}\left\|x-x u_{i}\right\|^{2} \leq \lim _{i}\left\|\langle x, x\rangle-\langle x, x\rangle u_{i}\right\|=\|\overline{\langle x, x\rangle}\|=\|\bar{x}\|^{2},
$$

e portanto $\|\bar{x}\|_{q} \leq\|\bar{x}\|$. e segue entào que $\|\bar{x}\|_{q}=\|\bar{x}\|$. Isto mostra que $\left(E / F,\|\cdot\|_{q}\right)$ é um módulo de Hilbert pleno sobre $\mathbf{Z}(E) / Z(F)$, e portanto $\mathbf{Z}(E / F)=\mathbf{Z}(E) / Z(F)$.

Uma vez provado o resultado para $C^{*}$-anéis ternários positivos. é fácil estendé-lo para o caso geral. Como $F$ é um ideal de $E$, entào $F^{p}$ é um ideal de $E^{p}$ (ver 1.28 acima). Além disso, como $J_{E}(F)=F$, temos que $J_{E}$ se fatora no quociente definindo um isomorfismo isométrico $\bar{J}_{E}: E / F \longrightarrow E / F$. Por outro lado, $E^{p} / F^{p}$ e $E / F$ sào o mesmo espaço de Banach com as respectivas normas quocientes. Portanto:

$$
\begin{aligned}
\|\overline{\mu(x, x, x)}\|_{E / F} & =\left\|\overline{J_{E} \mu(x, x, x)}\right\|_{E / F} \\
& =\left\|\overline{J_{E} \mu(x, x, x)}\right\|_{E^{p} / F^{p}} \\
& =\left\|\overline{\mu\left(J_{E} x, J_{E} J^{p} J_{E^{x}}\right)}\right\|_{E^{p} / F^{p}} \\
& =\left\|\overline{J_{E} x}\right\|_{E^{p} / F^{p}}^{3} \\
& =\left\|\overline{J_{E} x}\right\|_{E / F}^{3} \\
& =\|\bar{x}\|_{E / F}^{3},
\end{aligned}
$$


o que encerra a prova.

Corolário 1.30. Sejam $E \in G^{*} C^{*}$-anéis ternários, e $\pi: E \rightarrow G$ um homomorfismo de *aters. Sejam $F=\operatorname{ker}(\pi)$. $f,: Z(E) \longrightarrow Z(E) / Z(F)$ a aplicaçào quociente. Então existe um único homomorfismo de ('* -álgebras $\overline{Z(\pi)}: Z(E) / Z(F) \longrightarrow Z(G)$ tal que $\overline{Z(\pi)} p=Z(\pi)$ : este homomorfismo $\overline{Z(\pi)}$ é injetivo. Em particular. se $\pi: E \longrightarrow E / F$ é a aplicação quociente, onde $F$ é um ideal de $E$. entcio $\overline{\mathrm{Z}(\pi)}: \mathrm{Z}(E) / \mathrm{Z}(F) \longrightarrow \mathrm{Z}(E / F)$ i um isomorfismo natural.

Demonstraçào. Pela Proposiçào 1.18, existe um único homomorfismo de $C^{*}$-álgebras $Z(\pi)$ : $Z(E) \longrightarrow Z(G)$ tal que $\langle\pi(x), \pi(y)\rangle=Z(\pi)(\langle x, y\rangle), \forall x, y \in E$. A existencia e unicidade de $\overline{Z(\pi)}$, como também a sua injetividade, seguem agora da propriedade universal do quociente, junto com o fato de que ker $(Z(\pi))=Z(F)$ pelo Corolário 1.25 .

Finalmente, se $F \dot{~}$ um ideal de $E$. pela Proposiçào 1.29 acima, temos que $E / F$ é um $C^{* *}$-anel ternário, e a projeção $\pi: E \longrightarrow E / F$ é um homomorfismo de *aters.

Corolário 1.31. Z e $Z_{1}$ sào "funtores" (ver 1.19) exatos da categoria de $C^{*}$-anéis ternários

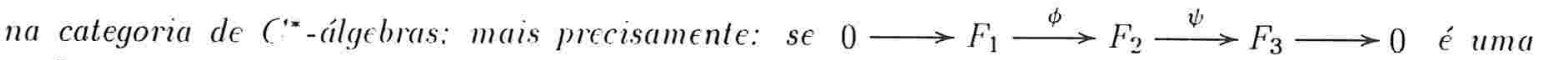
seqüência exata de $C^{*}$-anéis ternários, então também são exatas as seqüèncias de $C^{*}$-álgebras:

$$
0 \longrightarrow Z_{r}\left(F_{1}\right) \stackrel{Z_{r}(o)}{\longrightarrow} Z_{r}\left(F_{2}\right) \stackrel{Z_{r}(\psi)}{\longrightarrow} Z_{r}\left(F_{3}\right) \longrightarrow 0
$$

$e$

$$
0 \longrightarrow \mathrm{Z}_{l}\left(F_{1}\right) \stackrel{\mathrm{z}_{1}(0)}{\longrightarrow} \mathrm{Z}_{l}\left(F_{2}\right) \stackrel{\mathrm{z}_{1}(\psi)}{\longrightarrow} \mathrm{Z}_{l}\left(F_{3}\right) \longrightarrow 0
$$

Demonstraçào. Pelo Corolário 1.30 acima temos o seguinte diagrama comutativo:

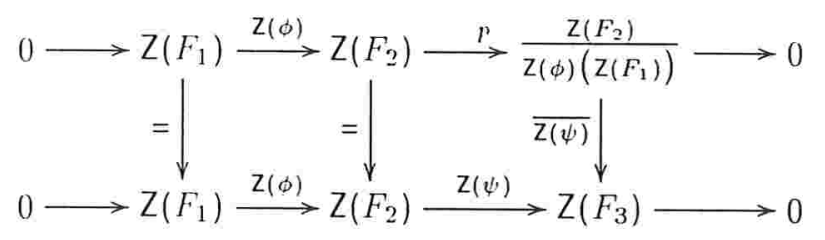

Como a primeira fila é exata e os homomorfismos verticais são isomorfismos, concluímos que a segunda fila também é exata.

Corolário 1.32. Ś $\pi: E \longrightarrow F$ é um homomorfismo de $C^{*}$-anéis ternários, então $\pi(E) \dot{\epsilon}$ fechado em F. Os ideais de um $C^{\star *}$-anel ternário E são exatamente os núcleos dos seus homomorfismos.

\subsection{Produtos tensoriais de $C^{*}$-anéis ternários}

No Lema seguinte. o produto tensorial algébrico dos espaços $E_{1}, \ldots, E_{n}$ será denotado por $E_{1} \bigodot \ldots \odot E_{n}$, ou simplesmente por $\bigodot_{j=1}^{n} E_{j}$. Usaremos a mesma notação ao longo de todo o trabalho. 
Lema 1.33. Sejam $E_{i, j} . F_{i}$ espaços retoriais complexos, $\forall i=1, \ldots, m, j=1, \ldots, n$, e suponhamos que $a_{i}: \prod_{j=1}^{n} E_{i, j} \longrightarrow F_{i}$ é uma transformação n-linear, para cada $i=1 \ldots$ m. Então existe uma única transformaşio $n$-linear $a:=\alpha_{1} \Leftrightarrow \ldots \alpha_{m}$,

$$
n: \prod_{i=1}^{n} \bigodot_{i=1}^{m} E_{i j} \longrightarrow \bigodot_{i=1}^{m} F_{i}
$$

tal que $\alpha\left(\odot_{i=1}^{m} e_{i 1}, \ldots, \Theta_{i=1}^{m} \epsilon_{i n}\right) \mapsto m_{n=1}^{m} \alpha_{i}\left(e_{i 1}, \ldots, e_{i n}\right)$

Demonstração. A existencia da transformação a segue do uso reiterado dos isomorfismos naturais $L\left(V_{1}, \ldots, V_{n} ; H^{\prime}\right) \cong L\left(\bigodot_{j=1}^{t} V_{j}, I^{\circ}\right)$, onde $L\left(V_{1}, V_{2}, \ldots V_{n} ; W\right)$ denota o espaço vetorial de transformaçōes $n$-lineares sobre $V_{1} \times \ldots \times V_{n}$ a valores em $W$. A unicidade é evidente, porque a toma valores pré-estabelecidos sobre um conjunto gerador de seu domínio.

Proposição 1.34. Si $(E, \mu),(F, \nu)$ saio *anéis ternários, entio $(E \odot F, \mu, \nu)$ é um *anel ternário.

Demonstração. Śeja $E^{*}$ o espaço vetorial conjugado de $E$, como no exemplo 1.5. Como as transformações

$$
\mu: E \times E^{*} \times E \longrightarrow E \quad \text { e } \quad \nu: F \times F^{*} \times F \longrightarrow F
$$

são trilineares, também $\mu, \cdots:(E \odot F) \times\left(E^{*} \odot F^{*}\right) \times(E \odot F) \longrightarrow E \odot F$ é trilinear. Mas $E^{*} \odot F^{*}=(E \odot F)^{*}$, donde $\mu \cdot \nu:(E \odot F) \times(E \odot F)^{*} \times(E \odot F) \longrightarrow E \bigodot F$ é trilinear e, com respeito às identidades algébricas da definiçào de ${ }^{*}$-ater $(1.1)$, sobre tensores elementares temos: se

$$
(\star)=\mu \odot \nu\left(\mu \odot \nu\left(x_{1} \odot y_{1}, x_{2} \odot y_{2}, x_{3} \odot y_{3}\right), x_{4} \odot y_{4}, x_{5} \odot y_{5}\right)
$$

então:

$$
\begin{aligned}
(\star) & =\mu \nu \nu\left(\mu\left(x_{1}, x_{2}, x_{3}\right) \odot \nu\left(y_{1}, y_{2}, y_{3}\right), x_{4} \odot y_{4}, x_{5} \odot y_{5}\right) \\
& =\mu\left(\mu\left(x_{1}, x_{2}, x_{3}\right), x_{4}, x_{5}\right) \odot \nu\left(\nu\left(y_{1}, y_{2}, y_{3}\right), y_{4}, y_{5}\right) \\
& =\mu\left(\left(x_{1}, \mu\left(x_{4}, x_{3}, x_{2}\right), x_{5}\right) \odot \nu\left(y_{1}, \nu\left(y_{4}, y_{3}, y_{2}\right), y_{5}\right)\right. \\
& =\mu \odot \nu\left(\left(x_{1}, \mu\left(x_{4}, x_{3}, x_{2}\right), x_{5}\right) \odot\left(y_{1}, \nu\left(y_{4}, y_{3}, y_{2}\right), y_{5}\right)\right) \\
& =\mu \odot \nu\left(x_{1} \odot y_{1}, \mu \nu\left(x_{4} \odot y_{4}, x_{3} \odot y_{3}, x_{2} \odot y_{2}\right), x_{5}-y_{5}\right)
\end{aligned}
$$

A outra identidade é verificada com cálculos análogos.

Definição 1.35. Uim $C^{* *}$-produto tensorial de dois $C^{*}$-anéis ternários $(E \cdot \mu \cdot\|\cdot\|)$ e $(F, \nu,\|\cdot\|)$ é um completamento do respectivo produto tensorial algébrico $(E \odot F . \mu \nu)$ com respeito a uma $C^{*}$-norma. Se $\gamma$ é uma tal $C^{* *}$-norma, denotamos $E \bigotimes_{\gamma} F$ o correspondente $C^{* *}$-produto tensorial.

DEFINiçÃo 1.36. Diremos que o $C^{*}$-anel ternário $E$ é nuclear se para todo $C^{*}$-anel ternário $F$ existe um único $C^{*}$-produto tensorial $E \otimes F$.

Observação 1.37. Sejam $(E, \mu),(F, \nu)$ dois $C^{*}$-aters. Então

$$
E \bigodot F=\left(E^{+} \bigodot F^{+}\right) \bigoplus\left(E^{-} \bigodot F^{-}\right) \bigoplus\left(E^{+} \bigodot F^{-}\right) \bigoplus\left(E^{-} \bigodot F^{+}\right)
$$

Se $\gamma$ é uma $C^{*}$-norma sobre $E \odot F$. entào ela define por restriçào $C^{*}$-normas sobre cada um dos somandos diretos $F^{+} \odot F^{+}, E^{-} \odot F^{-}, E^{+} \odot F^{-}, E^{-} \odot F^{+}$. Inversamente, se temos dada uma 
$C^{*}$-norma sobre cada somando direto, entào podemos definir unicamente uma $C^{*}$-norma sobre $E \odot F$ de forma tal que restringindo esta norma a cada somando reobtemos as normas dadas: basta definir. para $x=x^{++} \oplus x^{--}+x^{+-}+x^{-+}$segundo a decomposição acima, $\gamma(x)$ igual ao máximo das normas respectivas em cada somando de $x$. Em particular, e lembrando também a Observação 1.9, ̧ é uma $C^{*}$-norma sobre $E \odot F$ se e somente se ela é uma $C^{* *}$-norma sobre $E^{r} \bigodot F^{r}$. Portanto, é suficiente estudar os produtos tensoriais de $C^{*}$-aters positivos. Notar que $E$ é nuclear se e somente se $E^{+}$e $E^{-}$ são nucleares.

A seguir vemos uma prova simples de que sempre existe pelo menos um $C^{*}$-produto tensorial entre dois $C^{*}$-aters. Se $E$ é um $C^{*}$-ater positivo, ele é um $\left(Z_{l}(E)-Z_{r}(E)\right)$-bimódulo de Hilbert pleno (pleno significa aquí que ele é pleno como módulo à esquerda e também como módulo à direita). Para simplificar a notaçào, vamos por $A$ em lugar de $Z_{l}(E)$, e $B$ em lugar de $Z_{r}(E)$. Então podemos formar a álgebra vinculante $E(E)$ de $E$ (em ingles: linking algebra). Lembrar que $\mathbb{L}(E)=\left(\begin{array}{cc}A & E \\ E^{*} & B\end{array}\right)$, com o produto:

$$
\left(\begin{array}{ll}
a_{1} & \epsilon_{1} \\
f_{1}^{*} & b_{1}
\end{array}\right)\left(\begin{array}{ll}
a_{2} & \epsilon_{2} \\
f_{2}^{*} & b_{2}
\end{array}\right)=\left(\begin{array}{cc}
a_{1} a_{2}+\left\langle\epsilon_{1}, f_{2}\right\rangle_{1} & a_{1} \epsilon_{2}+\epsilon_{1} b_{2} \\
f_{1}^{*} a_{2}+b_{1} \cdot f_{2}^{*} & \left\langle f_{1}, \epsilon_{2}\right\rangle_{r}+b_{1} b_{2}
\end{array}\right)
$$

e a involução:

$$
\left(\begin{array}{cc}
a & \epsilon \\
f^{*} & b
\end{array}\right)^{*}=\left(\begin{array}{cc}
a^{*} & f \\
\epsilon^{*} & b^{*}
\end{array}\right)
$$

Falta definir a norma sobre $\mathbb{L}(E)$. Consideremos o $A$-módulo de Hilbert à esquerda pleno $A \oplus E$, onde o produto interno $[\because \cdot]_{l}:(A \oplus E) \times(A \oplus E) \rightarrow A$ é dado por: $\left[\left(a_{1}, \epsilon_{1}\right),\left(a_{2}, e_{2}\right)\right]_{l}=a_{1} a_{2}^{*}+\left\langle\epsilon_{1}, e_{2}\right\rangle_{l}$. Definamos $[\cdot, \cdot]_{r}:(A \bigoplus E) \times(A \oplus E) \longrightarrow \mathbb{L}(E)$ como:

$$
\left[\left(a_{1}, \epsilon_{1}\right),\left(a_{2}, \epsilon_{2}\right)\right]_{r}=\left(\begin{array}{cc}
a_{1} & e_{1} \\
0 &
\end{array}\right)^{*}\left(\begin{array}{cc}
a_{2} & e_{2} \\
0 & 0
\end{array}\right)=\left(\begin{array}{cc}
a_{1}^{*} a_{2} & a_{1}^{*} e_{2} \\
\epsilon_{1}^{*} \cdot \epsilon_{2} & \left\langle\epsilon_{1}, e_{2}\right\rangle_{r}
\end{array}\right) .
$$

$A \oplus E$ é um módulo à direita sobre $\mathbb{L}(E)$, através de:

$$
\left(\left(a^{\prime}, c^{\prime}\right),\left(\begin{array}{ll}
a & e \\
f^{*} & b
\end{array}\right)\right) \longmapsto\left(a^{\prime} a+\left\langle\epsilon^{\prime}, f\right\rangle_{l}, a^{\prime} \epsilon+\epsilon^{\prime} b\right) .
$$

Tem-se então, $\forall x . y . z \in A \oplus E$, que $[x, y]_{l} z=x[y, z]_{r}$. Portanto, temos uma inclusão

$$
\operatorname{span}[A \bigoplus E, A \bigoplus E]_{r} \hookrightarrow Z_{r}(A \bigoplus E) .
$$

Pelo teorema de Cohen-Hewitt (em 2.1 incluímos o enunciado deste teorema), sabemos que cada elemento de $E$ é da forma $a \epsilon$, para alguns $a \in A, e \in E$. Analogamente, $a=a_{1} a_{2}, b=b_{1} b_{2}, \forall a \in A$. $b \in B$, e alguns $a_{1}, a_{2} \in A, b_{1}, b_{2} \in B$. Portanto, temos que $[A \oplus E, A \oplus E]_{r}=\mathbb{L}(E)$. Por outro lado, as restriçōes da norma sobre $Z_{r}(A \oplus E)$ aos cantos $\left(\begin{array}{ll}A & 0 \\ 0 & 0\end{array}\right),\left(\begin{array}{ll}0 & E \\ 0 & 0\end{array}\right),\left(\begin{array}{cc}0 & 0 \\ E^{*} & 0\end{array}\right)$ e $\left(\begin{array}{ll}0 & 0 \\ 0 & B\end{array}\right)$ coincidem, através das identificaçòes óbvias, com as normas sobre $A, E, E^{*}$ e $B$ respectivamente. Logo. $\mathbb{L}(E)$ é completa com respeito a esta norma, donde concluímos que $Z_{r}(A \oplus E)=\mathbb{L}(E)$. Consultar [10] e [11] para mais detalhes. Se $E$ é um $C^{*}$-ater positivo, também chamaremos álgebra vinculante de $E$ a $\mathbb{L}(E)$, a álgebra vinculante do $\left(Z_{l}(E)-Z_{r}(E)\right)$-bimódulo de Hilbert $E$.

Proposição 1.38. Sejam $E \in F$ dois $C^{*}$-anéis ternários positivos. Então cada $C^{*}$-produto tensorial $\mathbb{L}(E) \bigotimes_{-}-(F)$ entre as álgebras vinculantes de $E \in F$ define um $C^{*}$-produto tensorial $E \bigotimes_{\gamma} F$, por restriçcio da norma $\gamma$ à imagem da inclusào natural $\iota: E \odot F \hookrightarrow \mathbb{L}(E) \bigotimes_{\gamma} \mathbb{L}(F)$ tal que $\sum_{i} e_{i} \odot f_{i} \longmapsto \sum_{i}\left(\begin{array}{ll}0 & \epsilon_{i} \\ 0 & 0\end{array}\right)=\left(\begin{array}{ll}0 & f_{i} \\ 0 & 0\end{array}\right)$. 
Demonstraçào. A inclusão $\iota: E \odot F \hookrightarrow \mathbb{L}(E) \bigotimes_{\gamma} \mathbb{L}(F)$ tal que $\sum_{i} e_{i} \odot f_{i} \longmapsto \sum_{i}\left(\begin{array}{cc}0 & e_{i} \\ 0 & 0\end{array}\right) \ominus$ $\left(\begin{array}{ll}0 & f^{\prime} \\ 0 & 0\end{array}\right)$ é claramente uma inclusào de ${ }^{*}$-aters. Portanto, a restriçào da $C^{*}$-norma de $\mathbb{L}(E) \bigotimes_{\gamma} \mathbb{L}(F)$ a $\iota(E \odot F)$ induz uma $C^{*}$-norma sobre $E \odot F$, como queríamos.

Corolário 1.39. Dados dois $C^{* *}$-améis ternários E e F. sempre existe um $C^{*}$-produto tensorial entre eles.

Demonstração. Pela Observaçào $1.3 \bar{\tau}, \hat{i}$ é uma $C^{*}$-norma sobre $E \odot F$ se e somente se ela é uma $C^{*}{ }^{*}$-norma sobre $E^{p} \odot F^{p}$. Portanto. o Corolário é uma conseqüencia direta da Proposição 1.38 acima.

DEFINIÇȦo 1.40. O produto tensorial espacial entre dois $C^{*}{ }^{*}$-anéis ternários $E$ e $F$ é o produto tensorial induzido pelo produto tensorial espacial $\mathbb{L}\left(E^{p}\right) \otimes \mathbb{L}\left(F^{p}\right)$, segundo a Proposição 1.38 e o Corolário 1.39

ObservaÇão 1.41. Quando $E$ e $F$ sào $C^{1 *}$-aters positivos, eles podem ser pensados como módulos de Hilbert. Deste ponto de vista. o produto tensorial espacial que acabamos de definir é conhecido como produto tensorial exterior ([43]).

Observação 1.42. A Proposiçào 1.38 implica que se $x=\sum_{i=1}^{n} \epsilon_{i} \odot f_{i} \in E \odot F$, e $x \neq 0$, entào $0 \neq \iota(x)^{*} \iota(x)=\sum_{i, j=1}^{n}\left\langle\epsilon_{i}, \epsilon_{j}\right\rangle_{E} \circlearrowleft\left\langle f_{i}, f_{j}\right\rangle_{F}$. Note-se que esta é uma maneira bem simples de ver que $\sum_{i, j=1}^{n}\left\langle e_{i}, e_{j}\right\rangle_{E} \odot\left\langle f_{i}, f_{j}\right\rangle_{F} \neq 0$, enquanto que a prova fornecida em [43] usa o teorema altamente não trivial de estabilizaçào de Liasparov ([41]).

Queremos ver que as $C^{* *}$-normas sobre $E \odot F$ estão em bijeção natural com as $C^{*}$-normas sobre $Z(E) \odot Z(F)$. Precisaremos de alguns resultados preliminares, entre eles o seguinte Lema, cuja demonstração incluímos apenas para conveniencia do leitor, já que consiste da repetiçào dos argumentos usados na prova do Lemma 4.3 de [43]. onde somente a $C^{*}$-norma espacial foi considerada.

Lema 1.43. Sejam $A \in B C^{*}$-álgebras, e suponhamos que $\mathfrak{a}=\left(a_{i j}\right), \mathfrak{c}=\left(c_{i j}\right) \in M_{n}(A), \mathfrak{b}=$ $\left(b_{i j}\right), \mathfrak{o}=\left(d_{i j}\right) \in M_{n}(B)$. Seja $A \otimes_{,} B$ algum $C^{*}$-produto tensorial de $A \in B$. Então:

1. Se $0 \leq \mathfrak{a} \leq \mathfrak{c} \epsilon 0 \leq \mathfrak{b} \leq \mathfrak{o}$. temos $\left(a_{i j} \otimes b_{i j}\right) \leq\left(c_{i j} \otimes d_{i j}\right) \epsilon m M_{n}(A \otimes, B)$.

2. Se $\mathfrak{a}, \mathfrak{b} \geq 0$, entào $\sum_{i, j=1}^{n} a_{i j} \odot b_{i, j} \geq 0 \mathrm{em} A \bigotimes_{\gamma} B$.

DemonstraçÃo. Mostremos primeiro que se $\mathfrak{a}=\left(a_{i j}\right) \in M_{n}(A)^{+}$e $\mathfrak{b}=\left(b_{i j}\right) \in M_{n}(B)^{+}$, então $\left(a_{i j} \otimes b_{i j}\right) \in M_{n}\left(A \otimes_{\gamma} B\right)^{+}$.

Como $\mathfrak{a}=\mathfrak{s}^{*} \mathfrak{s}$ em $M_{n}(A)$, e $\mathfrak{b}=\mathfrak{t}^{*} \mathfrak{t} \operatorname{em} M_{n}(B)$, teremos que, se $\mathfrak{s}=\left(s_{i j}\right)$ e $\mathfrak{t}=\left(t_{i j}\right)$, então

$$
a_{i, j}=\sum_{k=1}^{n} s_{k i}^{x} s_{k, j} \text {. and } b_{i, j}=\sum_{h=1}^{n} t_{h i}^{*} t_{h, j}, \forall i, j=1 \ldots n \text {. }
$$

Portanto,

$$
\left(a_{i, j} \odot b_{i, j}\right)=\left(\sum_{k, h=1}^{n} s_{k i}^{*} s_{k j} \odot t_{h i}^{*} t_{h j}\right)=\sum_{h, k=1}^{n}\left(s_{k i}^{*} s_{k, j} \odot t_{h i}^{*} t_{h j}\right) .
$$


Assim, é suficiente mostrar que cada somando na última soma é um elemento positivo de $M_{n}\left(A \otimes_{\gamma} B\right)$. o que equivale a provar que se $a_{1} \ldots a_{n} \in A$ e $b_{1} \ldots, b_{n} \in B$, entào $\left(a_{i}^{*} a_{j} \odot b_{i}^{*} b_{j}\right)$ é positivo em $M_{n}\left(A \otimes_{\gamma} B\right)$. Mas $a_{i}^{*} a_{j} b_{i}^{*} b_{j}=\left(a_{i} \odot b_{i}\right)^{*}\left(a_{j}-b_{j}\right)$, donde segue que $\left(a_{i}^{*} a_{j} \odot b_{i}^{*} b_{j}\right)=u^{*} u \in$ $M_{n}\left(A \bigotimes_{\gamma} B\right)^{+}$, onde $u=\left(u_{i, j}\right)$ é a matriz em $M_{n}\left(A \bigotimes_{\gamma} B\right)$ tal que $u_{1 i}=a_{i} \odot b_{i}$, e $u_{i j}=0$ para $i>0$. Agora, 1. segue do fato que $\left(\left(c_{i, j}-a_{i, j}\right) \odot b_{i, j}\right)$ e $\left(a_{i, j} \_\left(d_{i, j}-b_{i j}\right)\right)$ são elementos positivos pela primeira parte da demonstraçào, e então também sua soma é um elemento positivo.

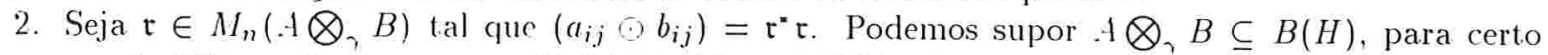
espaço de Hilbert $H$, e portanto $M_{n}\left(A \otimes_{\gamma} B\right) \subseteq B\left(H^{n}\right)$. Considere o operador $\mathrm{i}: H \longrightarrow H^{n}$ t.al que $\mathrm{i} h=(h, \ldots h), \forall h \in H$. Entào $\sum_{i, j=1}^{n} a_{i j} b_{i j}=\mathrm{i}^{*} u^{*} u \mathrm{i} \in B(H)^{+}$. Como $\left(A \otimes_{\gamma} B\right)^{+}=$ $\left(A \bigotimes_{\gamma} B\right) \cap B(H)^{+}$. concluímos que $\sum_{i, j=1}^{n} a_{i j} \odot b_{i j}$ é positivo em $A \otimes, B$.

Suponhamos que $B_{0}$ é uma sub-*-álgebra de uma $C^{*}$-álgebra $B$, e $E_{0}$ é um $B_{0}$-módulo à direita com uma forma sesquilinear definida positiva $\langle\cdot, \cdot\rangle: E_{0} \times E_{0} \longrightarrow B_{0}$, ou seja:

1. $\langle x, \alpha y+\beta z\rangle=\alpha\langle x, y\rangle+\beta, x z, \forall x, y, z \in E_{0} . \alpha . \beta \in \mathbb{C}$.

2. $\langle x, y b\rangle=\langle x, y\rangle\rangle, \forall x, y \in E_{0}, b \in B_{0}$.

3. $\langle y, x\rangle=\langle x, y\rangle^{*}, \forall x, y \in E_{0}$.

4. $\langle x, x\rangle \geq 0, \forall x \in E_{0}$. e $\langle x, x\rangle=0 \Longleftrightarrow x=0$.

Então, $\|\cdot\| \longrightarrow \mathbb{E}$ tal que $\|x\|=\sqrt{\|\langle x, x\rangle\|}$ é uma norma sobre $E_{0}$. Seja $E$ o completamento de $E_{0}$ com respeito a esta norma. É possível estender a ação de $B_{0}$ sobre $E_{0}$ a una açào de $B$ sobre $E$, e também a forma sesquilinear a um $B$-produto interno sobre $E$. obtendo assim um $B$-módulo de Hilbert. Nos referiremos a esta construçào como o processo de duplo completamento para $\left(E_{0}, B_{0},\langle\cdot \cdot\rangle\right)$ (ver [43] para mais informaçào).

Proposiçào 1.44. Sejam $(E, \mu) \in(F, \nu) C^{*}$-anéis ternários positivos. e suponhamos que $\gamma$ é uma $C^{*}$-norma sobre $\mathbf{Z}(E) \odot \mathrm{Z}(F)$.

1. Entào existe uma única forma sesquilinear definida positiva $\langle\cdot, \cdot\rangle:(E \odot F) \times(E \odot F) \longrightarrow$ $\mathrm{Z}(E) \odot \mathrm{Z}(F)$ tal que $\left\langle e \odot f, \epsilon^{\prime} \odot f^{\prime}\right\rangle=\left\langle e, \epsilon^{\prime}\right\rangle_{E} \odot\left\langle f, f^{\prime}\right\rangle_{F}, \forall e, \epsilon^{\prime} \in E . f, f^{\prime} \in F$.

2. A norma $\bar{i}: E \odot F \rightarrow \mathbb{P}$ tal que $\tilde{\gamma}(x)=\sqrt{\gamma(\langle x, x\rangle)}$ é uma $\left(^{(x}\right.$-norma sobre $E \odot F$. e a função $\gamma \longmapsto \hat{\gamma}$, definida no conjunto $\mathcal{N}_{1}$ de $C^{*}$-normas sobre $\mathrm{Z}(E) \odot \mathrm{Z}(F)$ e a valores no conjunto $\mathcal{N}_{2}$ de $C^{*}$-normas sobre $E \odot F$, preserva a ordem.

3. $E \bigotimes_{\hat{\gamma}} F$ é um $C^{*}$-anel ternário positivo, $\epsilon \mathbf{Z}\left(E \bigotimes_{\dot{\gamma}} F\right)=\mathrm{Z}(E) \bigotimes_{\gamma} \mathrm{Z}(F)$.

DemonstraÇào. O Lema 1.33 implica a existencia e unicidade de tal forma sesquilinear $\langle\cdot, \cdot\rangle$. Vejamos que $\langle x, x\rangle \geq 0, \forall x=\sum_{i=1}^{n} e_{i} \odot f_{i} \in E \odot F$ :

$$
\langle x, x\rangle=\sum_{i, j=1}^{n}\left\langle e_{i} \odot f_{i}, e_{j} \odot f_{j}\right\rangle=\sum_{i, j=1}^{n}\left\langle e_{i}, e_{j}\right\rangle_{E} \odot\left\langle f_{i}, f_{j}\right\rangle_{F}
$$

Agora, $\left(\left\langle e_{i}, e_{j}\right\rangle_{E}\right)_{i, j=1}^{n}$ e $\left(\left\langle f_{i}, f_{j}\right\rangle_{F}\right)_{i, j=1}^{n}$ são elementos positivos de $M_{n}(A)$ e $M_{n}(B)$ respectivamente ([43], Lemma 4.2). e portanto $\sum_{i, j=1}^{n}\left\langle e_{i}, e_{j}\right\rangle_{E} \odot\left\langle f_{i}, f_{j}\right\rangle_{F}$ é positivo em $Z(E) \otimes_{\gamma} Z(F)$ pelo Lemma 1.43. Finalmente. lembremos que, pela Observação $1.42,\langle x, x\rangle=0$ implica que $x=0$. Logo, $\langle\cdot, \cdot\rangle$ é uma forma sesquilinear definida positiva . e então 1 . fica provado.

Pelo processo de duplo completamento descrito acima, obtemos um $Z(E) \otimes_{\gamma} Z(F)$-módulo de Hilbert $G$, com norma $\dot{\gamma}$. Para provar que $\bar{\gamma}$ é uma $C^{*}$-norma sobre $E \odot F$, mostremos primeiro que $\mu \odot \nu(x, y, z)=x\langle y, z\rangle, \forall x, y, z \in E \odot F$; é claramente suficiente ver isto para $x=e \odot f, y=e^{\prime} \odot f^{\prime}$. 
$z=\epsilon^{\prime \prime} \odot f^{\prime \prime}$. Agora:

$$
\begin{aligned}
\mu \cdots \nu(x, y, z) & =\mu \odot\left(e \odot f, e^{\prime} \odot f^{\prime}, \epsilon^{\prime \prime} \odot f^{\prime \prime}\right) \\
& =\mu\left(\epsilon, \epsilon^{\prime}, e^{\prime \prime}\right) \odot \nu\left(f, f^{\prime}, f^{\prime \prime}\right) \\
& =\left(\epsilon\left\langle\epsilon^{\prime}, e^{\prime \prime}\right\rangle_{E}\right) \odot\left(f\left\langle f^{\prime}, f^{\prime \prime}\right\rangle_{F}\right) \\
& =(\epsilon \odot f)\left(\left\langle e^{\prime}, \epsilon^{\prime \prime}\right\rangle_{E} \odot\left\langle f^{\prime}, f^{\prime \prime}\right\rangle_{F}\right) \\
& =(\epsilon \odot f)\left\langle e^{\prime} \odot f^{\prime}, e^{\prime \prime} \odot f^{\prime \prime}\right\rangle \\
& =r\langle y, z\rangle
\end{aligned}
$$

Portanto, usando a teoria de módulos de Hilbert, podemos concluir que ì é uma $C^{* *}$-norma:

$$
\begin{aligned}
\dot{\gamma}(\mu, \nu(x, y, z)) & =\dot{\gamma}(x\langle y, z\rangle) \\
\leq & \tilde{\gamma}(x) \gamma(\langle y, z\rangle) \\
\leq & \dot{\gamma}(x) \bar{\gamma}(y) \dot{\gamma}(z)
\end{aligned}
$$

E:

$$
\begin{aligned}
\dot{i}(\mu \cdots \nu(x, x, x)) & =\tilde{\gamma}(x\langle x, x\rangle) \\
& =\sqrt{\gamma(\langle x\langle x, x\rangle}, x\langle x, x\rangle)) \\
& =\sqrt{\gamma\left(\langle x, x\rangle^{3}\right)} \\
& =\sqrt{\gamma(\langle x, x\rangle)^{3}} \\
& =\tilde{\gamma}(x)^{3}
\end{aligned}
$$

Além disto, é óbvio que $\hat{i} \longmapsto \dot{i}$ preserva a ordem.

Finalmente, segue de 1. e 2. que $E \bigotimes_{\gamma} F$ é um $Z(E) \bigotimes_{\gamma} \mathrm{Z}(F)$-módulo de Hilbert pleno, e portanto $\mathrm{Z}\left(E \bigotimes_{\dot{\gamma}} F\right)$ é precisamente $\mathrm{Z}(E) \bigotimes_{,} \mathrm{Z}(F)$.

Corolário 1.45. Sejam $(E, \mu) \in(F, \nu) C^{*}$-anéis ternários, e suponhamos que $\gamma$ é uma $C^{*}-$ norma sobre $\mathrm{Z}(E) \odot \mathrm{Z}(F)$.

1. Então existe uma única forma sesquilinear $\langle\cdot, \cdot\rangle:(E \odot F) \times(E \odot F) \longrightarrow Z(E) \odot Z(F)$ tal que $\left\langle e \odot f, e^{\prime} \odot f^{\prime}\right\rangle=\left\langle\epsilon, \epsilon^{\prime}\right\rangle_{E} \curvearrowright\left\langle f, f^{\prime}\right\rangle_{F}, \forall e, \epsilon^{\prime} \in E, f, f^{\prime} \in F$.

2. A norma $\dot{\gamma}: E \odot F \longrightarrow \mathbb{F}$ tal que $\bar{\gamma}(x)=\sqrt{\gamma(\langle x, x\rangle)}$ é uma $C^{+x}$-norma sobre $E \odot F$, $e$ a função $\gamma \longmapsto \bar{\gamma}$. definida no conjunto $\mathcal{N}_{1}$ de $C^{*}$-normas sobre $Z(E) \odot Z(F)$ e a valores no conjunto $\mathcal{N}_{2}$ de $C^{*}$-normas sobre $E \odot F$, preserva a ordem.

3. $\mathrm{Z}\left(E \otimes_{\gamma} F\right)=\mathrm{Z}(E) \otimes_{\gamma} \mathrm{Z}(F)$.

Demonstração. Basta aplicar a Proposição 1.44 aos $C^{*}$-aters positivos $E^{p}$ e $F^{r}$, e lembrar que $\mathrm{Z}\left(E^{p}\right)=\mathrm{Z}(E), \mathrm{Z}\left(F^{p}\right)=\mathrm{Z}(F)(1.28)$, e que $\tilde{\gamma}$ é uma $C^{*}$-norma sobre $E \odot F$ se e somente se é uma $C^{*}$-norma sobre $E^{p} \odot F^{r}(1.3 T)$.

A seguir, queremos ver que vale o resultado recíproco, quer dizer: todo produto tensorial $E \otimes_{\alpha} F$ induz um produto tensorial $Z(E) \bigotimes_{\bar{a}} Z(F)$. Os resultados seguintes nos levam nessa direção.

O Lema a seguir é uma ligera modificação de T.6.1 de [65]. Denotamos por $M(A)$ a álgebra dos multiplicadores da $C^{*}$-álgebra $A$. e. se $I, J$ são pré- $C^{*}$-álgebras, e ; é uma $C^{*}$-norma sobre a *-álgebra $I \odot J$, então indicamos o correspondente completamento por $I \otimes . J$.

Lema 1.46. Suponhamos que $I$. J são *-ideais bilaterais (nào necessariamente fechados) das $C^{*}$ álgebras $A \in B$ respectivamente. $є$ que, é uma $C^{*}$-norma sobre $I \odot J$. Entào: 
1. Existem aplicações $\iota_{A}: A \longrightarrow M\left(I \bigotimes_{\gamma} J\right) \in \iota_{B}: B \longrightarrow M(I \otimes, J)$ tais que

$$
\iota_{A}(x) \iota_{B}(y)=\iota_{B}(y) \iota_{A}(x)=x \otimes y . \forall x \in I, y \in J .
$$

2. Se $I$ é denso em A $\mathrm{e} J$ é denso $\mathrm{em} B, \iota_{A}$ e $\iota_{B}$ são injetivas.

DEmonstraçÃo. Faremos a construção de $\iota_{A}$, sendo análoga a construção de $\iota_{B}$. Se $\iota: I \odot J \longrightarrow$ $I \bigotimes_{\gamma} J$ é a inclusão canonica, definimos, para $a \in A$, as funçōes $L_{a}, R_{a}: I \odot J \longrightarrow I \otimes_{\gamma} J$ através das fórmulas $L_{a}=\imath\left(l_{a} \odot i d_{J}\right), R_{a}=\imath\left(r_{a} \odot i d_{J}\right)$, onde $l_{a}, r_{a}: I \longrightarrow I$ são as multiplicações à esquerda e à direita pelo elemento a respectivamente; isto é, $L_{a}(x \odot y)=a x \odot y$. and $R_{a}(x \odot y)=x a \odot y$. Queremos ver $\left(L_{a}, R_{a}\right)$ como um centralizador duplo de $I \bigotimes_{\gamma} J$. Notar que se $x=\sum_{i} a_{i} \odot b_{i}$, $y=\sum_{j} c_{j} \odot d_{j} \in I \odot J$, entào:

$$
\begin{aligned}
L_{a}(x y) & =L_{a}(x) y \\
R_{a}(x y) & =x R_{a}(y) \\
x L_{a}(y) & =\sum_{i, j} a_{i}\left(a c_{j}\right) \subset b_{i} d_{j} \\
& =\sum_{i, j}\left(a_{i} a\right) c_{j} \odot b_{i} d_{j} \\
& =R_{a}(x) y
\end{aligned}
$$

Se provamos que $L_{a} . R_{a}$ sào limitados, poderemos estendé-los a operadores sobre $I \otimes_{\gamma} J$, obtendo um centralizador duplo.

Então, seja $t=\sum_{i=1}^{n} a_{i} \rho b_{i} \in I \odot J$, e consideremos

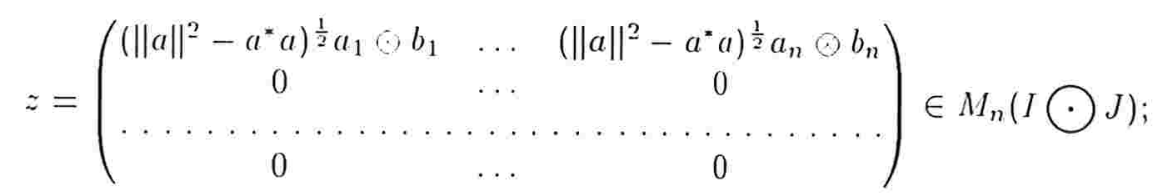

Calculando, se verifica que $z^{*} z=\left(z_{i j}\right)$, onde $z_{i j}=a_{j}^{*}\left(\|a\|^{2}-a^{*} a\right) a_{i} b_{j}^{*} b_{i}$. Agora, segue do Lema 1.43, que:

$$
\sum_{i, j=1}^{n} a_{j}^{*}\left(\|a\|^{2}-a^{*} a\right) a_{i} \otimes b_{j}^{*} b_{i} \geq 0
$$

e portanto que:

$$
\sum_{i, j=1}^{n} a_{j}^{*} a^{*} a a_{i} \odot b_{j}^{*} b_{i} \leq\|a\|^{2} \sum_{i, j=1}^{n} a_{j}^{*} a_{i} \otimes b_{j}^{*} b_{i}
$$

Assim, $L_{a}(t)^{*} L_{a}(t) \leq\|a\|^{2} t^{*} t$ e, tomando normas, concluímos que $\left\|L_{a}(t)\right\| \leq\|a\|\|t\|$. Logo $\left\|L_{a}\right\| \leq$ $\|a\|$, e podemos estender $L_{a}: I \bigotimes_{\gamma} J \longrightarrow I \bigotimes_{\gamma} J$. De forma similar, podemos ver que também $\left\|R_{a}\right\| \leq\|a\|$, e desta maneira obtivemos um centralizador duplo de $I \otimes_{\gamma} J$. É claro que a aplicação $\iota_{A}: a \longmapsto\left(L_{a}, R_{a}\right)$ é um homomorfismo de $C^{*}$-álgebras. É de verificação fácil que se $\iota_{B}$ é construída da mesma forma. teremos $\iota_{A}(x) \iota_{B}(y)=\iota_{B}(y) \iota_{A}(x)=x \odot y, \forall x \in I, y \in J$. Isto encerra a prova de 1 . .

Vejamos agora que se $I$ é denso em $A$, então $\iota_{A}$ é injetivo: se $\left(L_{a}, R_{a}\right)=0$, então $L_{a}(t)=0$, $\forall t \in I \odot J$, donde $a x \odot y=0, \forall x \in I, y \in J$; isto implica que $a x=0 . \forall x \in I$, e entào $a=0$, porque $I$ é denso em $A$. Isto prova 2 . . 
Proposiçào 1.4T. Sejam I. J *-ideais bilaterais densos das $C^{*}$-álgebras $A \in B$ respectivamente. Então cada $C^{*}$-norma sobre $I \odot J$ pode ser estendida a uma $C^{*}$-norma (nào necessariamente única) sobre $M(A) \odot M(B)$. Além disto. os completamentos de $I \odot J$ e $A \odot B$ com respeito a esta $C^{*}$ norma coincidem.

Demonstração. Seja ᄀ uma $\left(^{\prime *}\right.$-norma sobre $I \odot J$, e consideremos as aplicaçōes $\iota_{A}: A \longrightarrow$ $M\left(I \otimes_{\gamma} J\right)$ e $\iota_{B}: B \longrightarrow M\left(I \bigotimes_{\gamma} J\right)$ fornecidas pelo Lema 1.46. Suponhamos que $t=\sum_{i} a_{i} \odot$ $b_{i} \in \operatorname{ker}\left(\iota_{A} \odot \iota_{B}\right)$. Como $\operatorname{ker}\left(\iota_{A} \cdots \iota_{B}\right)$ é um ideal bilateral de $A \odot B$, temos em particular que $(x \odot y) t$, $t(x \odot y) \in \operatorname{ker}\left(\iota_{A} \odot \iota_{B}\right), \forall x \in I, y \in J$. Isto implica que $\sum_{i} x a_{i} \odot y b_{i}=0=\sum_{i} a_{i} x \odot b_{i} y, \forall x \in I$, $y \in J$, porque $\iota_{A} \odot \iota_{B}$ é injetiva sobre $I \odot J$ : mas estas igualdades valem também em qualquer $C^{*}$ completamento de $A \odot B$, por exemplo em $A \bigotimes_{\min } B$. Como $I \odot J$ é denso em $A \bigotimes_{\min } B$, concluímos que $t=0$. Assim, $\iota_{A} \iota_{B}: A \odot B \longrightarrow M\left(I \bigotimes_{\gamma} J\right)$ é injetiva. Portanto, o fecho de $\left(\iota_{A} \odot \iota_{B}\right)(A \odot B)$ em $M\left(I \otimes_{\gamma} J\right)$ é um ('*-produto tensorial de $A$ e $B$, que contém $I \odot J$ como subconjunto denso: deduzimos então que coincide com $I \bigotimes_{,}, J$. O resto da prova decorre agora de T.6.3 de [65], que afirma que toda $C^{*}$-norma sobre $A \odot B$ pode ser estendida a $M(A) \odot M(B)$.

Com respeito a última afirmação, é so levar em conta que, como $I$ é denso em $A$ e $J$ é denso em $B$, entào $I \odot J$ deve ser denso em $A \odot B$, pois toda $C^{*}$-norma $\|\cdot\|$ sobre $A \odot B$ satisfaz $\|a \odot b\|=$ $\|a\|_{A}\|b\|_{B}$.

Proposiçào 1.48. Sejam $(E, \mu) \in(F, \nu) C^{*}$-anéis ternários. Suponhamos que a é uma $C^{*}$ norma sobre $E \odot F$. Entioo $Z\left(E \otimes_{n} F\right)$ é um $C^{*}$-produto tensorial de $Z(E) \in Z(F)$, ou seja, existe uma $C^{*}$-norma $\bar{\alpha}$ sobre $\mathbf{Z}(E) \odot Z(F)$ (necessariamente única) tal que $\mathbf{Z}\left(E \bigotimes_{\alpha} F\right)=\mathbf{Z}(E) \bigotimes_{\bar{\alpha}} \mathbf{Z}(F)$.

Demonstraçào. Sejam $\langle\cdot, \cdot\rangle_{a} \cdot\langle\cdot, \cdot\rangle_{E},\langle\cdot \cdot\rangle_{F}$ as correspondentes formas sesqulineares de $E \otimes_{\alpha} F$, $E$ e $F$ sobre $Z\left(E \bigotimes_{a} F\right), Z(E)$ e $Z(F)$ respectivamente. Lembrar que se $E$ é um $C^{*}$-ater, entào $\operatorname{span}\langle E, E\rangle_{E}$ é um *-icleal bilateral denso em $Z(E)$. Então, pela Proposição 1.4i. é suficiente provar que $Z\left(E \bigotimes_{\alpha} F\right)$ é um $\left(^{*}\right.$-completamento de $Z_{E}^{\prime} \odot Z_{F}^{\prime}$, onde $Z_{E}^{\prime}=\operatorname{span}\langle E, E\rangle_{E}$. e $Z_{F}^{\prime}=\operatorname{span}\langle F, F\rangle_{F}$. Como $E$ é um $Z_{E}^{\prime}$ módulo à direita e $F$ é um $Z_{F}^{\prime}$-módulo à direita, $E \odot F$ é um $Z_{E}^{\prime} \odot Z_{F}^{\prime}$-módulo à direita. Pelo Lema 1.33, existe uma única forma sesquilinear [., $]:(E \odot F) \times(E \odot F) \longrightarrow Z_{E}^{\prime} \odot Z_{F}^{\prime}$ tal que $\left[e_{1} \odot f_{1}, e_{2} \odot f_{2}\right]=\left\langle\epsilon_{1}, \epsilon_{2}\right\rangle_{E}:\left\langle f_{1}, f_{2}\right\rangle_{F} \in Z_{E}^{\prime} \odot Z_{F}^{\prime}, \forall e_{1}, e_{2} \in E, f_{1}, f_{2} \in F$. Por outro lado, se $e \odot f \in E \odot F$, tem-se:

$$
\begin{aligned}
(\epsilon \odot f)\left\langle\epsilon_{1} \odot f_{1}, \epsilon_{2} \odot f_{2}\right\rangle_{a} & =\mu \odot \nu\left(e \odot f, e_{1} \odot f_{1}, e_{2} \odot f_{2}\right) \\
& =\mu\left(e, e_{1}, e_{2}\right) \odot \nu\left(f, f_{1}, f_{2}\right) \\
& =e\left\langle e_{1}, e_{2}\right\rangle_{E} \odot f\left\langle f_{1}, f_{2}\right\rangle_{F} \\
& =(e \odot f)\left[e_{1} \odot f_{1}, e_{2} \odot f_{2}\right]
\end{aligned}
$$

Assim, temos uma função $\phi: Z_{E}^{\prime} \odot Z_{F}^{\prime} \longrightarrow C^{\prime}:=\operatorname{span}\langle E \odot F, E \odot F\rangle_{\alpha}$. Notar que $C$ é uma sub-*álgebra de $Z\left(E \bigotimes_{a} F\right)$, que é densa em $Z\left(E \bigotimes_{\alpha} F\right)$ porque $E \odot F$ é denso em $E \bigotimes_{\alpha} F$. Agora, $\phi$ é um *-homomorfismo injetivo, pois a ação de $\phi(x)$ sobre $E \bigotimes_{\alpha} F$ é a extensão, necessariamente única. da ação de $x$ sobre $E \odot F$. Na verdade, é claro também que a imagem de o é toda a subálgebra $C$. Portanto, $\phi\left(Z_{E}^{\prime} \odot Z_{F}^{\prime}\right)$ é denso em $Z\left(E \bigotimes_{\alpha} F\right)$. Em outras palavras, $Z\left(E \bigotimes_{\alpha} F\right)=Z_{E}^{\prime} \bigotimes_{\bar{\alpha}} Z_{F}^{\prime}$, para alguma $C^{*}$-norma $\bar{\alpha}$ sobre $Z_{E}^{\prime} \odot Z_{F}^{\prime}$. Pela Proposição 1.47 , concluímos que $Z\left(E \bigotimes_{\alpha} F\right)=$ $\mathrm{Z}(E) \bigotimes_{\bar{\alpha}} \mathrm{Z}(F)$.

Observaçào 1.49. Notar que a Proposição 1.48 implica que se $\alpha$ é uma $C^{* *}$-norma sobre $E \odot F$, então $\alpha(x \odot y)=\|x\|\|y\|$. 
Teorema 1.50, Ścjam E $E F C^{* *}$-anéts temários. Consideremos os seguintes conjuntos:

E as funçòes

$$
\begin{aligned}
& \Lambda_{1}=\left\{C^{\prime *}-\text { normas sobre } \mathrm{Z}(E) \odot \mathrm{Z}(F)\right\} \\
& \therefore_{2}=\left\{C^{\prime *}-\text { normas sobre } E \bigodot F\right\}
\end{aligned}
$$

$$
\begin{aligned}
& \text { o: } \hat{N}_{1} \longrightarrow \hat{N}_{2}: \quad i \longmapsto j \text { definido no Corolário } 1.45 \\
& \text { ¿ : } \hat{N}_{2} \longrightarrow \bar{N}_{1}: \quad a \longrightarrow \bar{\alpha} \text { definido na Proposiçào } 1.4 \delta
\end{aligned}
$$

Consideramos $\mathcal{N}_{1} \in \mathcal{N}_{2}$ ordenados pela ordem usual de funçóes reais. Entào. $\phi$ é t' são isomorfismos de conjuntos ordenados. muluamente imersos.

Demonstraçào. Suponhamos que $\hat{j} \in \mathcal{N}_{1}$. Então, por 3 . do Corolário 1.45 , tem-se que $\mathrm{Z}\left(E \otimes_{\dot{\gamma}} F\right)$ é precisamente $\mathrm{Z}(E) \otimes_{\gamma} \mathrm{Z}(F)$ : isto é: $\overline{\bar{\gamma}}=\gamma$, e portanto $\psi \mathrm{\sigma}=i d_{\mathcal{N}_{1}}$.

Consideremos agora $a \in \underline{1}_{2}$. $\bar{a} \dot{e}$ a $C^{\prime *}$-norma sobre $Z(E) \odot Z(F)$ tal que $Z(E) \bigotimes_{\bar{\alpha}} Z(F)=$ $Z\left(E \bigotimes_{\alpha} F\right)$. Agora, lembrar que $\bar{a}: E \odot F \longrightarrow \mathbb{F}$. é definida como $\bar{a}(x)=\sqrt{\bar{\alpha}(\langle x, x\rangle)}, \forall x \in E \odot F$. e $\bar{a}(\langle x, x\rangle)=\alpha(x)^{2}$ pela equação (1) na página 12. Logo, $\tilde{\bar{a}}=\alpha$, e isto mostra que $\dot{\phi} \psi=i d_{\mathcal{N}_{2}}$.

Como $\phi$ preserva a ordem (2. do ('orolário 1.45), só falta provar que $\iota^{\prime}$ também preserva a ordem para completar a prova. Sejam entào $a, 3 \in \mathcal{\Lambda}_{2}$. com $\alpha \geq \beta$. Entào a identidade sobre $E \odot F$ se estende por continuidade a um homomorfismo sobrejetivo $\pi: E \bigotimes_{\alpha} F \longrightarrow E \bigotimes_{\beta} F$, e portanto temos um homomorfismo sobrejetivo $\mathrm{Z}(\pi): \mathrm{Z}\left(E \bigotimes_{\alpha} F\right) \longrightarrow \mathrm{Z}\left(E \bigotimes_{\beta} F\right)$. Como $\mathrm{Z}(\pi)$ é sobrejetivo e $E \bigotimes_{\alpha} F=\mathrm{Z}(E) \bigotimes_{\bar{\alpha}} \mathrm{Z}(F)$ e $E \bigotimes_{\beta} F=\mathrm{Z}(E) \bigotimes_{\bar{\beta}} \mathrm{Z}(F)$, concluímos que $\bar{\alpha} \geq \bar{\beta}$.

Daqui em diante, escreveremos a em lugar de $\bar{\alpha}$ e $\gamma$ em lugar de $\bar{\gamma}$.

Corolário 1.51. Sejam $E \in F$ ('*-anéis ternários. Então existem uma $C^{*}$-norma máxima $\|$.

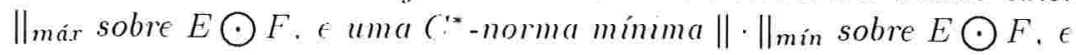

$$
\begin{aligned}
& \mathrm{Z}\left(E \bigotimes_{\text {maix }} F\right)=\mathrm{Z}(E) \bigotimes_{\text {má }} \mathrm{Z}(F) . \\
& \mathrm{Z}\left(E \bigotimes_{\text {min }} F\right)=\mathrm{Z}(E) \bigotimes_{\text {min }} \mathrm{Z}(F) .
\end{aligned}
$$

A norma mínima é a norma espacial.

Corolário 1.52. Itm $C^{*}$-anel ternário E é nuclear (1.36) se e somente se $\mathrm{Z}(E)$ é uma $C^{*}$ álgebra nuclear.

Obtemos também como Corolário o resultado seguinte, já bem conhecido ([7]). Salientamos que estamos usando, e usaremos ao longo de todo o trabalho, a expressão "equivalencia Morita" em lugar da expressào "equivalencia forte de Morita”. Notar que não se trata do mesmo conceito de equivalencia Morita da teoria de módulos.

Corolário 1.53. Sejam A $\in B$ duas ( ${ }^{\times}$-álgebras equivalentes Morita. Então A é nuclear se $\epsilon$ somente se $B$ é nuclear. 
Para concluir esta seçào, provamos um resultado que será útil mais tarde. Lembrar que se $E$ é um $B$-módulo de Hilbert à direita. $\mathcal{L}(E)=\mathcal{L}_{B}(E)$ denota a $C^{*}$-álgebra dos operadores adjuntáveis sobre $E$, e $\mathcal{K}(E)$ é a sua sub-('*álgebra dos operadores adjuntáveis compactos.

Proposição 1.54. Śjam E $\in$ F módulos de Hilbert à direita plenos sobre as $C^{*}$-álgebras $B_{E} \epsilon$ $B_{F}$ respectivamente. $\epsilon$ suponhamos que $\gamma$ é uma $C^{*}$-norma sobre $E \odot F$. Entrio $\gamma$ pode ser "estendida", de maneira única. a uma ("* -norma ̀े sobre $\mathcal{L}(E) \odot \mathcal{L}(F), \epsilon$ temos as seguintes inclusôes:

$$
\kappa(E) \bigotimes \mathcal{\Lambda}(F) \hookrightarrow \mathcal{L}(E) \bigotimes_{\dot{\gamma}} \mathcal{L}(F) \hookrightarrow \mathcal{L}\left(E \bigotimes_{\gamma} F\right)
$$

Se y é a norma espacial. j̀ também é a norma espacial.

Demonstraşào. A unicidade segue do fato de que $\bar{\gamma}$ sobre $\mathcal{L}(E) \odot \mathcal{L}(F)$ deve ser a restrição a este álgebra da $C^{*}$ norma de $\mathcal{L}(E \otimes, F)$. Portanto, só temos que provar a existencia de $\bar{\gamma}$. Pensando $E$ como $C^{*}$-ater, temos $Z_{r}(E)=B_{E}, Z_{r}(F)=B_{F}$ (ver o Exemplo 1.10), e entào $Z_{l}(E)=\mathcal{K}(E), Z_{l}(F)=$ $\mathcal{K}(F)$. Observar que, pela versào à esquerda do Teorema 1.50 , temos $\mathcal{\Lambda}(E) \bigotimes_{,} \mathcal{K}(F)=\mathcal{K}\left(E \bigotimes_{\gamma} F\right)$. Por outro lado, temos que $M(\mathcal{K}(E))=\mathcal{L}(E), M(\mathcal{K}(F))=\mathcal{L}(F)$, e $M\left(\mathcal{K}\left(E \bigotimes_{\gamma} F\right)\right)=\mathcal{L}\left(E \bigotimes_{\gamma} F\right)$ [41]). Agora. a prova se encerra usando T.6.3 de [65], que diz que existe uma $C^{*}$-norma $\bar{\gamma}$, que é a $C^{*}$-norma espacial se $;$ for a $\left(^{* *}\right.$-norma espacial, tal que se tem:

$$
\mathrm{Z}_{l}(E) \bigotimes \mathrm{Z}_{l}(F) \subseteq M\left(\mathrm{Z}_{l}(E)\right) \bigotimes_{\dot{\gamma}} M\left(\mathrm{Z}_{l}(F) \subseteq M\left(\mathrm{Z}_{l}(E) \bigotimes_{\gamma} \mathrm{Z}_{l}(F)\right)\right.
$$

\section{3. $C^{*}$-anéis ternários exatos}

Nesta seção introduzimos a noção de $C^{*}$-anel ternário exato, e provamos um resultado análogo ao Corolário 1.52. Lima boa introdução à teoria das $C^{*}$-álgebras exatas pode ser achada em [64].

Suponhamos que $0 \longrightarrow F_{1} \stackrel{\phi}{\longrightarrow} F_{2} \stackrel{\psi^{\prime \prime}}{\longrightarrow} F_{3} \longrightarrow 0$ é uma seqüencia exata de $C^{*}$-aters, isto é, $\phi$ e $\psi$ são homomorfismos de *aters, $\phi$ é injetiva, $\psi$ é sobrejetiva, e ker $\psi^{*}=\phi\left(F_{1}\right)$. Seja $E$ um $C^{*}$ ater. Então a seqüencia $0 \longrightarrow E \odot F_{1} \stackrel{i d @ \phi}{\longrightarrow} E \odot F_{2} \stackrel{i d \odot \psi}{\longrightarrow} E \odot F_{3} \longrightarrow 0$ também é exata. Por outro lado, temos uma inclusào $\left(E \odot F_{2}\right) /\left(E \odot F_{1}\right) \hookrightarrow\left(E \bigotimes_{\min } F_{2}\right) /\left(E \bigotimes_{\min } F_{1}\right)$, e este quociente é um $C^{*}$-ater. Portanto. existe uma $C^{\prime *}$-norma $\gamma$ sobre $E \odot F_{3}$ tal que

$$
0 \longrightarrow E \otimes_{\min } F_{1} \stackrel{i d @ \phi}{\longrightarrow} E \otimes_{\min } F_{2} \stackrel{i d \otimes \psi}{\longrightarrow} E \otimes_{\gamma} F_{3} \longrightarrow 0
$$

é exata. Como $\gamma$ é maior ou igual à norma mínima, a identidade sobre $E \odot F_{3}$ se estende por continuidade a um homomorfismo sobrejetivo $E \otimes, F_{3} \longrightarrow E \otimes_{\min } F_{3}$.

Definiçào 1.55. Dizemos que um $C^{* *}$-anel ternário $E$ é exato se para toda seqüencia exata

$$
0 \longrightarrow F_{1} \longrightarrow F_{2} \longrightarrow F_{3} \longrightarrow 0
$$

de $C^{*}$-anéis ternários, tem-se que

$$
0 \longrightarrow E \bigotimes_{\min } F_{1} \longrightarrow E \bigotimes_{\min } F_{2} \longrightarrow E \bigotimes_{\min } F_{3} \longrightarrow 0
$$


também é exata.

Proposiçào 1.56. Ścjam E e F C**anéis ternários, e suponhamos que $G$ é um ideal de $F$. Entào

$$
0 \longrightarrow E \otimes_{\min }\left(i \longrightarrow E \bigotimes_{\min } F \longrightarrow E \otimes_{\min }(F / G) \longrightarrow 0\right.
$$

é exata se e somente st

$$
0 \longrightarrow \mathrm{Z}(E) \otimes_{\text {min }} \mathrm{Z}(G) \longrightarrow \mathrm{Z}(E) \otimes_{\min } \mathrm{Z}(F) \longrightarrow \mathrm{Z}(E) \otimes_{\min }(\mathrm{Z}(F) / \mathrm{Z}(G)) \longrightarrow 0
$$

é exata.

Demonstraçio. Suponhamos primeiro que

$$
() \longrightarrow E \otimes_{\text {min }}\left(i \longrightarrow E \otimes_{\min } F \longrightarrow E \otimes_{\min }(F / G) \longrightarrow 0\right.
$$

é exata. Pelo ('orolário 1.5l e o fato que Z é um funtor exato, obtemos o seguinte diagrama comutativo:

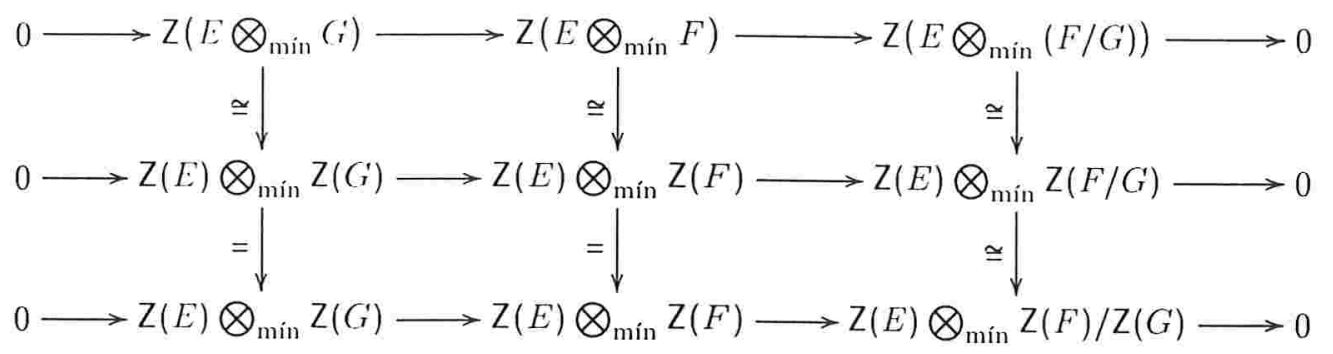

Como a primeira e a segunda filas são exatas, a terceira também é exata.

Para provar o recíproco, notemos primeiro que:

$$
0 \longrightarrow E \bigotimes_{\text {min }} G \longrightarrow E \bigotimes_{\text {min }} F \longrightarrow\left(E \bigotimes_{\min } F\right) /\left(E \bigotimes_{\min } G\right) \longrightarrow 0
$$

é exata, e $\left(E \bigotimes_{\text {min }} F\right) /\left(E \bigotimes_{\text {mín }} G\right)$ é um $C^{*}$-completamento do *ater $E \odot(F / G)$. Denotando a correspondente $C^{*}$-norma por $\uparrow$, temos um homomorfismo sobrejetivo $\phi: E \bigotimes_{\gamma}(F / G) \longrightarrow E \bigotimes_{\text {mín }}(F / G)$ que estende a identidade sobre $E \odot(F / G)$. Aplicando o funtor exato Z, obtemos o seguinte diagrama comutativo com filas exatas:

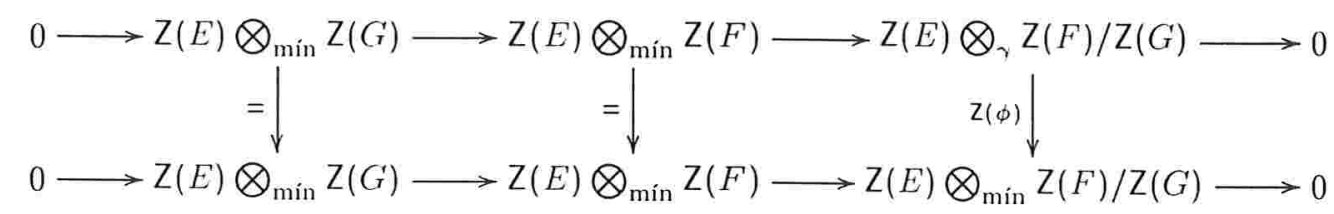

Como o diagrama comuta e as filas sào exatas, o homomorfismo sobrejetivo $Z(\phi)$ é um isomorfismo. exata.

Corolário 1.5T. U'm C**anel ternário E é exato (1.55) se e somente se $\mathrm{Z}(E)$ é uma $C^{*}$-álgebra

Demonstraçào. Imediato da Proposiçào 1.56

O seguinte resultado é conheciclo ([48]): 
Corolário 1.58. Sejam $A \in B$ duas $C^{*}$-álgebras equivalentes Morita (cf. página 27). Então $A$ é exata se e somenté se $B$ é exata. 
CAPíTULO 2

Produtos Tensoriais de Fibrados de Fell 
O nosso principal propósito neste capítulo é o de introduzir uma noçào conveniente de produto tensorial de fibrados de Fell (2.8). I $\mathrm{m}$ produto tensorial dos fibrados $\mathcal{A}=\left(A_{t}\right)_{t \in G}$ e $\mathcal{B}=\left(B_{s}\right)_{s \in H}$ sobre os grupos $G$ e $H$ será um fibrado de Fell $\mathcal{C}=\left(C_{r}^{\prime}\right)_{r \in G \times H}$ sobre $C_{r} \times H$, e teremos que $C_{e}$ é um produto tensorial de $A_{\epsilon}$ e $B_{\epsilon}$ (denotaremos por $\epsilon$ o elemento neutro de qualquer grupo). Mostraremos que existem, a menos de isomorfismos, produtos tensoriais únicos $\mathcal{C}_{\text {max }}$ e $\mathcal{C}_{\text {min }}$ de $\mathcal{A}$ e de $\mathcal{B}$, tais que $\left(\mathcal{C}_{\text {máx }}\right)_{\epsilon}=A_{\epsilon} \bigotimes_{\text {máx }} B_{\epsilon}$ e $\left(\mathcal{C}_{\text {minn }}\right)_{\epsilon}=A_{\epsilon} \otimes_{\text {min }} B_{\epsilon}$.

Na primeira seçào estudaremos o caso dos fibrados sobre grupos discretos. e na segunda seçào o caso geral. Na terceira seçào estudamos as representaçòes dos produtos tensoriais.

Ao longo deste trabalho usaremos reiteradamente dois result ados muito importantes, cujos enunciados será conveniente incluir. O primeiro deles nào é estritamente necessário para desenvolver o trabalho com sucesso. mas é útil para economizar tempo e espaço. evitando repetir várias vezes os mesmos argumentos de aproximaçào. Lembrar que se $B$ é uma álgebra de Banach, e um espaço de Banach $E$ é um $B$-módulo à direita. entào $E$ é chamado $B$-módulo de Banach se $\|x b\| \leq\|x\|\|b\|$. $\forall x \in E, b \in B$. O módulo é dito nào degenerado se span $E B$ é denso em $L$. Temos entào:

Teorema 2.1 (Cohen-Hewitt). Tejam B uma álgebra de Banach rom undade aproximada. $e E$ um B-módulo de Banach nào degenerado. Entào. dado $x \in E$. extstem $y \in E$. $b \in B$. tais que $x=y b$.

Uma demonstraçào do teorema de Cohen-Hewitı pode ser achada en [37] ou em [57].

Já o segundo resultado é fundamental na teoria de fibrados de Banach (incluímos a definição de fibrado de Banach na página 36). Trata-se do teorema de Douady-dal Soglio Hérault, sobre a existencia de suficientes seçōes contínuas de um fibrado de Banach. Para uma demonstração deste teorema, consultar o Apendice C' de [37], especialmente C'16 e ('1 $t$.

Teorema 2.2 (Douady-dal Soglio Hérault). Seja .' um espaço topológıro de Hausdorff, e seja $(B, p)$ um fibrado de Banach sobre $\mathrm{X}$. Se $\mathrm{X}$ é paracompacto ou localmente compacto, então para todo $b \in \mathcal{B}$ existe uma seçio contínua de suporte compacto $\xi$ de $\mathcal{B}$. tal que $\xi(p(b))=b$.

\subsection{Produtos tensoriais de fibrados sobre grupos discretos}

Sejam $\mathcal{A}=\left(A_{t}\right)_{t \in G}$ e $\mathcal{B}=\left(B_{s}\right)_{s \in H}$ fibrados de Fell sobre os grupos ( $i$ e $H$ respectivamente. Consideremos, para $t \in G, s \in H$, o produto tensorial algébrico $A_{t} \odot B_{s}$. Fazendo variar $t, s$ sobre $G$ e $H$, obtemos uma família $\mathcal{A} \odot B=\left\{A_{t} \odot B_{s}\right\}_{(t, s) \in G \times H}$ de espaços vetoriais complexos, indexados por $G \times H$ (pensaremos também $\mathcal{A} \odot \mathcal{B}$ como a uniào disjunta dos espaços $A_{t} \odot B_{s}$ ). O Lema 1.33 mostra que, se $(t, s)$. $\left(t^{\prime}, s^{\prime}\right) \in G \times H$, temos aplicaçōes $\left(A_{t} \odot B_{s}\right) \times\left(A_{1} \cdot \odot B_{s^{\prime}}\right) \longrightarrow A_{t t^{\prime}} \odot B_{s s^{\prime}}$ tais que $\left(a_{t} \odot b_{s}, a_{t^{\prime}} \odot b_{s^{\prime}}\right) \longmapsto a_{t} a_{t^{\prime}} \odot b_{s} b_{s^{\prime}}$, e $A_{t} \odot B_{s} \longrightarrow A_{t-1} \odot B_{s-1}$ tais que $a_{t}\left(b_{s} \longmapsto a_{t}^{*} \odot b_{s}^{*}\right.$. Combinadas, estas famílias de aplicaçòes produzem um produto : $(\mathcal{A} \odot \mathcal{B}) \times(\mathcal{A} \odot \mathcal{B}) \longrightarrow(\mathcal{A} \odot \mathcal{B})$ e uma involuçào $*:(\mathcal{A} \odot \mathcal{B}) \longrightarrow(\mathcal{A} \odot \mathcal{B})$ tais que o produto é associativo, bilinear sobre cada $\left(A_{t} \odot B_{s}\right) \times\left(A_{t^{\prime}} \odot B_{s^{\prime}}\right) \longrightarrow A_{t t^{\prime}} \odot B_{s^{\prime}}{ }^{*}{ }^{*}$ é antilinear quando restrita a $A_{t} \odot B_{s} \longrightarrow A_{t-1} \odot B_{s-1}$. e $(x \cdot y)^{*}=y^{*} \cdot x^{*}, \forall x, y \in \mathcal{A} \odot \mathcal{B}$. Diremos que $\mathcal{A} \odot \mathcal{B}$ é o produto tensorial algébrico de $\mathcal{A}$ e $\mathcal{B}$. Em outras palavras, $\mathcal{A} \odot \mathcal{B}$ é um *-fibrado algébrico, no sentido da seguinte Definição.

DEFiniÇÃo 2.3. Seja $G$ um grupo discreto, e suponhamos que $\mathcal{A}=\left(\mathcal{A}_{t}\right)_{t \in G}$ é uma família de espaços vetoriais complexos. Dizemos que $\mathcal{A}$ é um *-fibrado algébrico sobre $G$ com produto : : $\mathcal{A} \times \mathcal{A} \longrightarrow \mathcal{A}$ e involuçào $*: \mathcal{A} \longrightarrow \mathcal{A}$ se, $\forall a, b \in \mathcal{A}$. $1 . s \in G$. tem-se:

1. $A_{t} A_{s} \subseteq A_{t s}$

2. O produto é bilinear sobre $A_{t} \times A_{s} \longrightarrow A_{t s}$

3. O produto sobre $\mathcal{A}$ é associativo.

4. $\left(A_{t}\right)^{*} \subseteq A_{t-1}$ 
5. * é antilinear de $A_{1}$ em $A_{t-1}$.

6. $(a b)^{*}=b^{*} a^{*}$.

i. $a^{* *}=a$.

Se, além disso, existe uma norma $\|\cdot\|: \mathcal{A} \longrightarrow \mathbb{R}$. tal que:

8. $\left(A_{t},\|\cdot\|\right)$ é um espaço normado.

9. $\|a b\| \leq\|a\|\|b\|$

10. $\left\|a^{*} a\right\|=\|a\|^{2}$.

11. $a^{*} a \geq 0$ no completamento da pré-( ${ }^{*}$-álgebra $\left(A_{\epsilon},\|\cdot\|\right)$,

dizemos que $(\mathcal{A}, \cdot, *,\|\cdot\|)$ é um pré-fibrado de Fell sobre o grupo discreto $(i$, e dizemos que $\|\cdot\|$ é uma $C^{* *}$-norma sobre $\mathcal{A}$. Notar que 9. e 10. implicam que $\left\|a^{*}\right\|=\|a\| . \forall a \in \mathcal{A}$. Lembrar que, se $\left(\mathcal{A}_{t},\|\cdot\|\right)$ é completo para todo $t \in G$, entào $(\mathcal{A}, \cdot *,\|\cdot\|)$ é chamado fibrado de Fell.

Proposiçào 2.4. Séja $\mathcal{A}^{0}=\left(.1_{1}^{0}\right)_{t \in G}$ um pré-fibrado de Fell sobre o grupo discreto $G$, com $C^{*}$ norma \|.\|. Para cada $t \in G$. seja $A_{t}$ o rompletamento de $A_{t}^{0}$, e consideremos a família de espaşos de Banach $\left(A_{t}\right)_{t \in G}$. com a norma estendida. que ainda denotamos $\|\cdot\|$. Entcio o produto $\epsilon$ a involuçcio sobre $\mathcal{A}^{0}$ podem ser estendidos de maneira única a $\mathcal{A}$, de tal forma que $\mathcal{A}$ i um fibrado de Fell sobre $G$ com as operaçóes estendidas. Dizemos entào que $\mathcal{A}$ é um completamento do pré-fibrado de Fell $\mathcal{A}^{0}$.

Demonstraçào. A clemonstraçào é imediata, porque as condiçòes 9. e 10. da Definição 2.3 implicam que $* \mathrm{e} \cdot$ sào contínuas sobre $A_{t}^{0} \longrightarrow A_{t-1}^{0}$ e $A_{t}^{0} \times A_{s}^{0} \longrightarrow A_{t s}^{0}$ respectivamente. para todos $t, s \in G$, e portanto podem ser estendidas unicamente a aplicaçòes contínuas $A_{t} \longrightarrow A_{t-1} \mathrm{e}$ $A_{t} \times A_{s} \longrightarrow A_{t s}$, definindo assim $: \mathcal{A} \times \mathcal{A} \longrightarrow \mathcal{A} \mathrm{e} *: \mathcal{A} \longrightarrow \mathcal{A}$. Pela continuidade das operações estendidas, estas satisfazem as condiçōes algébricas 1.-7., e também 8.-11. de 2.3

Definiçào 2.5. Sejam $\mathcal{A}=\left(A_{t}\right)_{t \in G}$ e $\mathcal{B}=\left(B_{t}\right)_{t \in G}{ }^{*}$-fibrados algébricos sobre o grupo discreto $G$. Um homomorfismo $\theta: \mathcal{A} \longrightarrow \mathcal{B}$ é uma aplicaçào tal que $\phi\left(A_{t}\right) \subseteq B_{t}, \forall t \in G$, e, $\forall a, b \in A, t \in G$ :

1. $\phi$ é linear sobre $A_{t} \longrightarrow B_{t}$

2. $\phi(a b)=\phi(a) \phi(b)$

3. $\phi\left(a^{*}\right)=\phi(a)^{*}$

Se $\mathcal{A}, \mathcal{B}$ são pré-fibrados de Fell. também requerimos que $\phi$ seja contínua sobre cada $\mathcal{A}_{t}$.

DefiniçÃo 2.6. Seja $\mathcal{A}=\left(A_{t}\right)_{t \in G}$ um *-fibrado algébrico sobre o grupo discreto $G$. Uma representação $\pi: \mathcal{A} \longrightarrow \mathcal{L}(\mathcal{H})$ de $\mathcal{A}$ sobre o módulo de Hilbert $\mathcal{H}$ é uma aplicação $\pi$ tal que $\mathcal{A} \longrightarrow G \times \mathcal{L}(\mathcal{H})$ dada por $a_{t} \longmapsto\left(t, \pi\left(a_{t}\right)\right)$ é um homomorfismo de ${ }^{*}$-fibrados algébricos, onde o produto sobre $G \times \mathcal{L}(\mathcal{H})$ é $(t, T)(s, S)=(t s, T S)$, e a involuçào é $(t, T)^{*}=\left(t^{-1}, T^{*}\right) . \pi$ é dita não degenerada se $\operatorname{span} \pi(\mathcal{A}) \mathcal{H}$ é denso em $\mathcal{H}$.

Observaçào 2.i. Seja $\mathcal{A}=\left(A_{t}\right)_{t \in G}$ um fibrado de Fell dobre o grupo discreto $G$. e sejam

$$
\begin{aligned}
C_{c}(\mathcal{A}) & =\left\{f: G \longrightarrow \mathcal{A}: f(t) \in A_{t}, \forall t \in G, \text { e } \sup (f) \text { é finito }\right\} \\
L^{1}(\mathcal{A}): & =\left\{f: G \longrightarrow \mathcal{A}: f(t) \in A_{t}, \forall t \in G, \text { e } \sum_{t \in G}\|f(t)\|<\infty .\right\}
\end{aligned}
$$

$C_{c}(\mathcal{A})$ e $L^{1}(\mathcal{A})$ sào ${ }^{*}$-álgebras com as operaçòes:

$$
f * g(t)=\sum_{t \in G} f(r) g\left(r^{-1} t\right)
$$




$$
f^{*}(t)=f\left(t^{-1}\right)^{*} \text {. }
$$

Além disso, $L^{1}(\mathcal{A})$ é uma *álgebra de Banach com a norma: $\|f\|_{1}=\sum_{t \in G}\|f(t)\|$. A $C^{*}$-álgebra envolvente de $L^{1}(\mathcal{A})$ é chamada $C^{\prime *}$-álgebra seccional (plena) do fibrado de Fell $\mathcal{A}$, e é denotada por $C^{*}(\mathcal{A})$. Por [37], VIII-1T.3, exist.e uma bijeção entre as representações de $\mathcal{A}$ sobre o espaço de Hilbert. $H$ e as representaçòes de $C^{\prime *}(\mathcal{A})$ sobre $H$, tal que se $\pi: \mathcal{A} \longrightarrow B(H)$ é uma representação, a representação correspondente de $C^{*}(\mathcal{A})$ asigna o elemento $\sum_{t} \pi(f(t)) \in B(H)$ a todo elemento $f \in C_{c}(\mathcal{A})$. Voltaremos sobre este ponto quando consideremos o caso contínuo, no começo do Capítulo 3 .

Notar que se $\mathcal{A}$. $\mathcal{B}$ sào pré-fibrados de Fell e $\phi: \mathcal{A} \longrightarrow \mathcal{B}$ é um homomorfismo de *-fibrados algébricos, então $\phi$ é contínua se e somente se $\phi: A_{\epsilon} \longrightarrow B_{\epsilon}$ é contínua, porque se $x \in \mathcal{A}$, então

$$
\|\phi(x)\|^{2}=\left\|\varphi(x)^{*} \phi(x)\right\|=\left\|\phi\left(x^{*} x\right)\right\| \leq\left\|\left.\phi\right|_{A_{e}}\right\|\left\|x^{*} x\right\|=\left\|\left.\phi\right|_{A_{e}}\right\|\|x\|^{2} .
$$

Em particular, todo homomorfismo de *-fibrados algébricos entre fibrados de Fell sobre grupos discretos é contínuo.

Note-se que com a noçào de homomorfismo que acabamos de introduzir, dois completamentos de um pré-fibrado de Fell dado sempre são isomorfos, e portanto o completamento de um pré-fibrado de Fell é essencialmente único.

Seja $\phi: \mathcal{A} \longrightarrow \mathcal{B}$ um homomorfismo entre dois fibrados de Fell, e suponhamos que $C^{*}(\mathcal{B}) \hookrightarrow$ $B(H)$, como sub- $C^{*}$-álgebra nào degenerada. A composiçào de $\phi$ com esta inclusão define uma representação sobre $H$ do fibrado de Fell $\mathcal{A}$. Portanto, pela propriedade universal da $C^{*}$-álgebra de um fibrado de Fell ([37]. VIII-17.3), existe uma única representação $C^{*}(\phi): C^{*}(\mathcal{A}) \longrightarrow B(H)$, que estende $\phi$. Como a imagem de $C_{c}^{\prime}(\mathcal{A})$ está incluída em $C_{c}(\mathcal{B}) \subseteq C^{*}(\mathcal{B})$, e como span $C_{c}(\mathcal{A})$ é denso em $C^{*}(\mathcal{A})$, vemos que de fato tem-se $C^{*}(\phi): C^{*}(\mathcal{A}) \longrightarrow C^{*}(\mathcal{B})$. Em conclusão, temos um funtor covariante $C^{*}$, da categoria dos fibrados de Fell sobre o grupo discret.o $G$ e seus homomorfismos (2.5), na categoria de $C^{*}$-álgebras.

Finalmente, observar que segue dos comentários acima que se $\mathcal{A}$ é um fibrado de Fell e $\pi$ é uma representação de $\mathcal{A}$, entào temos que $\|\pi(a)\| \leq\|a\|, \forall a \in \mathcal{A}$.

Voltemos para os produtos tensoriais.

DEFINIÇÃo 2.8. Sejam $\mathcal{A}=\left(A_{t}\right)_{t \in G}$ e $\mathcal{B}=\left(B_{s}\right)_{s \in H}$ fibrados de Fell sobre os grupos discretos $G$ e $H$, e consideremos o produto tensorial algébrico $\mathcal{A} \odot \mathcal{B}$. Se a é uma $C^{*}$-norma sobre $\mathcal{A} \odot \mathcal{B}$, chamaremos o correspondente completamento $\mathcal{A} \otimes_{\alpha} \mathcal{B}$ de $(\mathcal{A} \odot \mathcal{B}, \alpha)$ um produto tensorial de $\mathcal{A}$ e $\mathcal{B}$.

Observação 2.9. Se $\mathcal{A} \bigotimes_{\alpha} \mathcal{B}$ é um produto tensorial entre $\mathcal{A}$ and $\mathcal{B}$, então $\left(\mathcal{A} \bigotimes_{\alpha} \mathcal{B}\right)_{e}$ deve ser um produto tensorial entre $A_{\epsilon}$ e $B_{\epsilon}$. De fato, se conhecermos a $C^{*}$-norma determinada por $\left(\mathcal{A} \otimes_{\alpha} \mathcal{B}\right)$ sobre $A_{\epsilon} \odot B_{\epsilon}$, então conhecemos a norma de qualquer elemento $x \in \mathcal{A} \bigotimes_{\alpha} \mathcal{B}$, porque deve ser igual a $\sqrt{\alpha\left(x^{*} x\right)}$. Portanto, dois produtos tensoriais serão isomorfos se e somente se suas fibras sobre a identidade é o mesmo produto tensorial de $A_{e} \operatorname{com} B_{e}$. Uma primeira questão que surge é se cada produto tensorial de $A_{\epsilon}$ e $B_{\epsilon}$ determina um produto tensorial entre os fibrados de Fell $\mathcal{A}$ e $\mathcal{B}$. A pesar de que não estudaremos com profundidade este problema, veremos que este é verdadeiramente o caso para os produtos tensoriais que são realmente importantes para os nossos propósitos: o produt.o tensorial minimal e o produto tensorial maximal (Proposiçào 2.12). 
Proposição 2.10. Sejam $\mathcal{A}=\left(A_{t}\right)_{t \in G} \in \mathcal{B}=\left(B_{t}\right)_{s \in H}$ fibrados de Fell, e seja $\alpha$ uma $C^{*}$-norma sobre $A_{\epsilon} \odot B_{\epsilon}$. Entcio a pode ser estendida a uma $C^{*}$-norma (ver 2.3) sobre todo $\mathcal{A} \odot \mathcal{B}$ se e somente se $\alpha\left(x^{*} x\right)=\alpha\left(x x^{*}\right), \forall x \in \mathcal{A} \odot \mathcal{B}$. Neste caso. a extensào é única.

Demonstraçìo. A condiçào é obviamente necessária devido à condição 10. da Definição 2.3.

Vejamos que também é suficiente. Para $x \in \mathcal{A} \odot \mathcal{B}$, definamos $\|x\|=\sqrt{\alpha\left(x^{*} x\right)}$. Queremos ver que esta é uma $C^{*}$-norma sobre o ${ }^{*}$-fibrado algébrico $\mathcal{A} \odot \mathcal{B}$, isto é, que satisfaz as condições 8.-11. da Definição 2.3. É claro. pela própria definiçào de $\|\cdot\|$, que a condição 10 . é satisfeia e, como estamos supondo que $\alpha\left(x^{*}, x\right)=a\left(x, x^{*}\right), \forall \cdot x \in \mathcal{A} \odot \mathcal{B}$, também temos:

$$
\|x\|=\left\|x^{*}\right\| . \forall x \in \mathcal{A} \bigodot \mathcal{B}
$$

Definamos $\langle\cdot\rangle_{r}:(\mathcal{A} \odot \mathcal{B}) \times(\mathcal{A} \odot \mathcal{B}) \longrightarrow \mathcal{A} \odot \mathcal{B}$ como $\langle x, y\rangle_{r}=x^{*} y$. Por 1.44 sabemos que $\langle\cdot,\rangle_{r}$ é um produto interno sobre cada $A_{t} \odot B_{s}$, e é claro que $\|x\|^{2}=\alpha(\langle x, x\rangle), \forall x \in \mathcal{A} \odot \mathcal{B}$. Logo, as condiçòes 8 . e 11. também sào satisfeitas. Com respeito à condiçào 9., observemos que o completamento de cada $A_{1} \odot B_{s}$ com respeito a $\|\cdot\|$ é um módulo de Hilbert à direita sobre $A_{e} \bigotimes_{\alpha} B_{\epsilon}$ : em particular, temos:

$$
\|a x\| \leq\|a\|\|x\|, \forall a \in \mathcal{A} \bigodot B, x \in A_{\epsilon} \bigodot B_{\epsilon}
$$

Entào:

$$
\begin{aligned}
\|a b\|^{2} & =\left\|b^{*} a^{*} a b\right\| & & \\
& =\left\|\left\langle b, a^{*} a b\right\rangle_{r}\right\| & & \\
& \leq\|b\|\left\|a^{*} a b\right\| & & \text { por Cauchy-Schwarz } \\
& =\|b\|\left\|b^{*} a^{*} a\right\| & & \text { por (2) } \\
& \leq\|b\|\left\|b^{*}\right\|\left\|a^{*} a\right\| & & \text { por (3) } \\
& =\|b\|^{2}\|a\|^{2} & & \text { por (2) }
\end{aligned}
$$

Em conclusão, $\|\cdot\|$ é uma $C^{*}$-norma sobre $\mathcal{A} \odot \mathcal{B}$.

Finalmente, a validacle da última afirmaçào se deve a 2.9 .

Nossa próxima tarefa é mostrar que $\|\cdot\|_{\text {máx }} \mathrm{e}\|\cdot\|_{\min }$ sobre $A_{\epsilon} \odot B_{\epsilon}$ podem ser estendidas a $\mathcal{A} \odot \mathcal{B}$. Começamos com o resultado seguinte, com certeza bem conhecido, a pesar de que não conseguimos achar nenhuma referencia concreta para citar.

Lema 2.11. Séjam I $\in$ J ideais bilaterais fechados das $C^{*}$-álgebras $A$ e $B$ respectivamente. Então $I \bigotimes_{\text {máx }} J$ é o fecho de $I \odot J$ em $A \bigotimes_{\text {max }} B$.

Demonstraçào. Seja $\pi: I \bigotimes_{\text {máx }} J \longrightarrow B(H)$ uma representação fiel e não degenerada de $I \bigotimes_{\operatorname{máx}} J$. Então, ela se decompõe em representações fieis e não degeneradas $\pi_{I}: I \longrightarrow B(H)$ e $\pi_{J}: J \longrightarrow B(H)$, tais que $\pi_{I}(x) \pi_{J}(y)=\pi(x \otimes y)=\pi_{J}(y) \pi_{I}(x), \forall x \in I, y \in J$ (ver [65]. T6.4, por exemplo). (Como sào não degeneradas, existem extensōes únicas $\pi_{A}: A \longrightarrow B(H)$ e $\pi_{B}: B \longrightarrow B(H)$ a representaçōes de $A$ e $B$ respectivamente ([37]. VI-19.11). Se $a \in A, x \in I$. $b \in B$ and $y \in J$. entào $\pi_{A}(a x) \pi_{B}(b y)=\pi_{B}(b y) \pi_{A}(a x)$, porque $a x \in I$ e $b y \in J$. Como $\pi_{I}$ e $\pi_{J}$ sào não degeneradas, concluímos que $\pi_{A}(a) \pi_{B}(b)=\pi_{B}(b) \pi_{A}(a), \forall a \in A, b \in B$. Portanto, existe uma representaçào $\tilde{\pi}: A \bigotimes_{\text {máx }} B \longrightarrow B(H)$ tal que $\tilde{\pi}(a \bigcirc b)=\pi_{A}(a) \pi_{B}(b), \forall a \in A, b \in B$. Assim, $\tilde{\pi}$ é uma extensão de $\pi$. Como toda representação é contrativa, concluímos que se $x \in I \odot J$, sua norma em $A \bigotimes_{\text {máx }} B$ é maior o igual à sua norma maximal; portanto coincidem. 
Proposiçào 2.12. Śjam $\mathcal{A}=\left(A_{t}\right)_{t \in G} \in \mathcal{B}=\left(B_{s}\right)_{s \in H}$ fibrados de Fell sobre os grupos discretos $G \in H$. Então, as normas $\|\cdot\|_{\text {min }} \epsilon\|\cdot\|_{\text {max }}$ sobre $A_{e} \bigcirc B_{e}$ podem ser estendidas a $C^{*}$-normas sobre $\mathcal{A} \odot \mathcal{B}$. Se $\mathcal{A} \otimes_{a} B \in \mathcal{A} \otimes_{3,} \mathcal{B}$ sào produtos tensorias de $\mathcal{A} \in \mathcal{B}$. com $a \geq B$. então existe um único homomorfismo

$$
\mathcal{A} \bigotimes_{a} \mathcal{B} \longrightarrow \mathcal{A} \bigotimes_{\beta} \mathcal{B}
$$

que é a identidade quando restrito a cada $A_{t} \odot B_{s}$; este homomorfismo é sobrejetivo.

Demonstraçào. Seja $A_{t}^{*} A_{t}$ o fecho em $A_{e}$ de $\operatorname{span}\left\{a_{t}^{*} a_{t}: a_{t} \in A_{t}\right\}$. e similarmente $B_{s}^{*} B_{s}$. Então $A_{t}^{*} A_{t}$ e $B_{s}^{*} B_{s}$ ideais bilaterais fechados de $A_{\epsilon}$ e $B_{\epsilon}$ respectivamentce e $A_{t}$ pode ser visto como um $C^{* *}$-anel ternário positivo $\operatorname{com} Z_{r}\left(A_{t}\right)=A_{t}^{*} A_{t}$ e $Z_{l}\left(A_{t}\right)=A_{t} A_{t}^{*}$. e similarmente com $B_{s}$. Lembremos que existe uma ("-norma maximal $\|\cdot\|_{\text {máx }}$ sobre $A_{t} \bigotimes_{\text {máx }} B_{s}$. Pelo Teorema 1.50 , deve ser $Z_{r}\left(A_{t} \bigotimes_{\text {máx }} B_{s}\right)=A_{t}^{*} A_{t} \bigotimes_{\text {máx }} B_{s}^{*} B_{s}$ e $Z_{l}\left(A_{t} \bigotimes_{\text {máx }} B_{s}\right)=A_{t} A_{t}^{*} \bigotimes_{\text {máx }} B_{s} B_{s}^{*}$. Como estamos em presença de várias normas maximais, fixemos a seguinte notaçào para evitar confusòes: com $\|\cdot\|_{\text {máx }}, \|$. $\left\|_{\text {máx }}^{r},\right\| \cdot \|_{\text {máx }}^{l}$ e $\|\cdot\|_{\mu}$ denotaremos respectivamente as normas maximais sobre $A_{t} \odot B_{s}, A_{t}^{*} A_{t} \odot B_{s}^{*} B_{s}$, $A_{t} A_{t}^{*} \odot B_{s} B_{s}^{*}$, e $A_{t} \odot B_{t}$. (omo cada $A_{t} \bigotimes_{\text {máx }} B_{s}$ é um $\left(A_{t} A_{t}^{*} \bigotimes_{\text {máx }} B_{s} B_{s}^{*}-A_{t}^{*} A_{t} \bigotimes_{\text {máx }} B_{s}^{*} B_{s}\right)$ bimódulo de Hilbert sabemos que:

$$
\left\|x \cdot x^{*}\right\|_{\text {máx }}^{l}=\|x\|_{\text {máx }}^{2}=\left\|x^{*} x\right\|_{\text {máx }}^{r}, \forall x \in A_{t} \bigodot B_{s} .
$$

Finalmente, pelo Lema $2.11,\|\cdot\|_{\mu}$ coincide com $\|\cdot\|_{\text {máx }}^{r}$ sobre $A_{t}^{*} A_{t} \odot B_{s}^{*} B_{s}$ e com $\|\cdot\|_{\text {máx }}^{\prime}$ sobre $A_{t} A_{t}^{*} \odot B_{s} B_{s}^{*}$. Deduzimos que

$$
\left\|x x^{*}\right\|_{\mu}=\|x\|_{\text {máx }}^{2}=\left\|x^{*} x\right\|_{\mu}, \forall x \in \mathcal{A} \bigodot B \text { B. }
$$

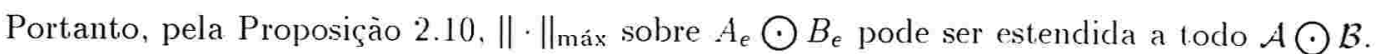

Os mesmos argumentos usados acima mostram que $\|\cdot\|_{\min }$ pode ser estendida, porque a norma mínima é a norma espacial, e. pela construçào do produto tensorial espacial, é claro que a restrição da $\|\cdot\|_{\text {min }}$ sobre $t_{\digamma} \odot B_{\epsilon}$ a $I \odot J$ coincide com a norma espacial sobre $I \odot J$, onde $I$ e $J$ são sub-C $C^{*}$-álgebras de $A_{\varepsilon}$ e $B_{\varepsilon}$ respectivamente.

A prova da última afirmaçào é trivial: o homomorfismo é a extensão contínua da identidade sobre $\mathcal{A} \odot \mathcal{B}$ (ver a Observaçào 2.7).

\subsection{Produtos tensoriais de fibrados sobre grupos contínuos}

Nesta seção estendemos a construção feita na primeira parte do capítulo ao caso de fibrados de Fell sobre grupos localmente compactos.

Um fibrado de Fell $B=\left(B_{t}\right)_{t \in G}$ sobre um grupo localmente compacto $G$ é um fibrado de Banach $\mathcal{B}$ sobre $G$, com fibra $B_{t}$ sobre $t \in G$, e existem sobre $\mathcal{B}$ um produto e uma involução contínuos que satisfazem as condiçòes 1-11 da Definição 2.3. Em [37], os fibrados de Fell são chamados $C^{*}$-fibrados algébricos

Lembrar que um fibrado de Banach ([37], II-13.4) $\mathcal{B}$ sobre um espaço topológico de Hausdorff $X$ é um par $(B, p)$ formado por um espaço topológico de Hausdorff $B$ e uma aplicação contínua, aberta e sobrejetiva $p: B \longrightarrow X$, junto com operaçōes e normas que fazem com que cada fibra $B_{x}=p^{-1}(\{x\})$ seja um espaço de Banach complexo, e satisfazendo as seguintes quatro propriedades:

1. $b \longmapsto\|b\|$ é contínua de $B$ a $\mathbb{E}$.

2. A soma $+:\left\{\left(b, b^{\prime}\right) \in B \times B: p(b)=p\left(b^{\prime}\right)\right\} \longrightarrow B$ é contínua.

3. Para cada $\lambda \in \Xi$ a a aplicaçào $B \longrightarrow B$ tal que $b \longmapsto \lambda b$ é contínua.

4. Se $x \in X$ e $\left(b_{i}\right) \subseteq B$ é uma rede tal que $p\left(b_{i}\right) \longrightarrow x$ e $\left\|b_{i}\right\| \longrightarrow 0$. entào $b_{i} \longrightarrow 0_{x} \in B$. 
Nós denotaremos o espaço total também por $\mathcal{B}$.

Para futura referencia, introduzimos a seguir tres exemplos de fibrados de Fell.

Exemplo 2.13 (Fibrados triviais). Sejam $G$ um grupo localmente compacto e $A$ uma $C^{*}$-álgebra. Seja $\mathcal{B}_{G, A}$ o fibrado de Banach sobre $G$ cujo espaço total é $G \times A$, com a projeçào $p: G \times A \longrightarrow G$ tal que $p(t, a)=t$. Sobre cada fibra tomamos a estrutura de espaço de Banach de $A$. O produto e a involução são definidos assim: $(s, a)(t, b)=(s t, a b)$, e $(t, b)^{*}=\left(t^{-1} \cdot b^{*}\right)$. Diremos que $\mathcal{B}_{G, A}$ é o

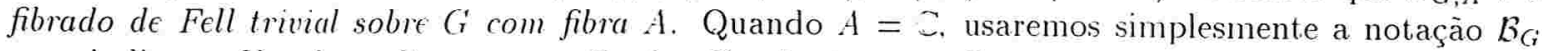
para indicar o fibrado, e diremos que $\mathcal{B}_{G}$ é o fibrado do grupo $G$.

Exemplo 2.14 (Fibrado produto). Se $\mathcal{A}=\left(A_{t}\right)_{t \in G}$ é um fibrado de Fell sobre o grupo localmente compacto $G$, e $H$ é um outro grupo localmente compacto, podemos formar o fibrado produto $\mathcal{A} \times H$. que é um fibrado sobre $G \times H$, sendo $\left(A_{t}, s\right)$ a fibra sobre $(t, s) \mathrm{com}$ a estrutura de espaço de $A_{t}$. Sobre $\mathcal{A} \times H$ colocamos a topologia produto. As operações sobre $\mathcal{A} \times H$ sào definidas como: $\left(a_{t}, s\right)\left(a_{t^{\prime}}, s^{\prime}\right)=$ $\left(a_{t} a_{t^{\prime}}, s s^{\prime}\right)$, e $\left(a_{t}, s\right)^{*}=\left(a_{t}^{*}, s^{-1}\right)$. Notar que 2.13 é um caso particular desta construção. Os dois exemplos podem ser vistos como casos particulares de produtos tensoriais de fibrados de Fell.

Exemplo 2.15 (Fibrado associado a uma açào parcial torcida). Iima açcio parcial torcida de $G$ sobre a $C^{*}$-álgebra $A$ é uma tripla

$$
a=\left(\left\{D_{t}\right\}_{t \in G},\left\{\alpha_{t}\right\}_{t \in G},\left\{w\left(t, t^{\prime}\right)\right\}_{\left(t, t^{\prime}\right) \in G^{*} \times G^{\prime}}\right)
$$

tal que, $\forall t \in G, D_{t}$ é um ideal bilateral fechado de $A, a_{t}: D_{t-1} \longrightarrow D_{t}$ é um isomorfismo, e, $\forall\left(t, t^{\prime}\right) \in G \times G, w\left(t, t^{\prime}\right)$ é um multiplicador unitário de $D_{t} \cap D_{t t^{\prime}}$, satisfazendo, para todos $t, t^{\prime}, t^{\prime \prime}$ pertencentes a $G$, as seguintes condições:

1. $D_{e}=A, a_{t}=i d_{A}$.

2. $\alpha_{t}\left(D_{t^{-1}} \cap D_{t^{\prime}}\right)=D_{t} \cap D_{t t^{\prime}}$.

3. $\alpha_{t}\left(\alpha_{t^{\prime}}(a)\right) w\left(t, t^{\prime}\right)=\alpha_{t t^{\prime}}(a) w\left(t, t^{\prime}\right)^{*}, \forall a \in D_{t^{\prime-1}} \cap D_{t^{\prime-1} t-1}$.

4. $w(e, t)=1=w(t, e)$.

5. $\alpha_{t}\left(a w\left(t^{\prime}, t^{\prime \prime}\right)\right) w\left(t, t^{\prime} t^{\prime \prime}\right)=\alpha_{t}(a) w\left(t^{\prime}, t^{\prime \prime}\right) w\left(t t^{\prime} . t^{\prime \prime}\right), \forall a \in D_{t^{-1}} \cap D_{t^{\prime}} \cap D_{t^{\prime} t^{\prime \prime}}$

Se, além disso, $\alpha$ satisfaz certas condiçōes de continuidade (que não precisaremos aqui: consultar [27]. Section 3, e ver a Definiçào 5.3 para o caso não torcido), ela é chamada açào parcial torcida contínua de $G$ sobre $A$.

Suponhamos que $\alpha=\left(\left\{D_{t}\right\}_{t \in G},\left\{\alpha_{t}\right\}_{t \in G},\left\{w\left(t . t^{\prime}\right)\right\}_{\left(t, t^{\prime}\right) \in G \times G}\right)$ é uma açào parcial torcida contínua de $G$ sobre $A$. Então $\alpha$ tem associado um fibrado de Fell $\mathcal{A}$ sobre $G$, definido como segue. $\mathcal{A}=\{(t, a)$ : $\left.t \in G, a \in D_{t}\right\} \subseteq G \times A$ com a topologia produto. e a projeção $p: \mathcal{A} \longrightarrow G$ é dada por $p((t, a))=t$. Simbolizamos o elemento $(t, a)$ como $a \delta_{t}$, de forma que $A_{t}=D_{t} \delta_{t}$. O produto e a involução sobre $\mathcal{A}$ são definidos assim:

$$
\begin{gathered}
\left(a_{t} \delta_{t}\right)\left(a_{t^{\prime}}^{\prime} \delta_{t^{\prime}}\right)=\alpha_{t}\left(a_{t-1}\left(a_{t}\right) a_{t^{\prime}}^{\prime}\right) w\left(t, t^{\prime}\right) \delta_{t t^{\prime}} \\
\left(a_{t} \delta_{t}\right)^{*}=\alpha_{t-1}\left(a_{t}^{*}\right) w\left(t^{-1} \cdot t\right)^{*} \delta_{t-1}
\end{gathered}
$$

Este exemplo é bem importante, porque, por $\bar{\imath} .3$ de [27]. todo fibrado de Fell $\mathcal{B}=\left(B_{t}\right)_{t \in G} \operatorname{com}$ o segundo axioma de enumerabilidade e fibra $B_{\epsilon}$ estável é isometricamente isomorfo a um fibrado de Fell que provém de uma açào parcial contínua torcida. Neste capítulo veremos que todo fibrado de Fell pode ser "estabilizado", isto é, sempre existe $\mathcal{B} \otimes \mathcal{K}$. onde $\mathcal{K}$ é a $C^{*}$-álgebra dos operadores compactos sobre um espaço de Hilbert separável. Se $\mathcal{B}$ é um fibrado de Fell que tem o segundo axioma de enumerabiliclade, entào $\mathcal{B} \otimes \mathcal{K}$ também o tem, e pelo resultado mencionado acima, concluímos que $\mathcal{B} \otimes \mathcal{K}$ é isomorfo ao fibrado de Fell definido por certa ação parcial contínua torcida. 
Se $X$ é um espaço topológico e $(B, p)$ é um fibrado de Banach sobre $X$, representaremos por $C_{c}(X)$ o espaço vetorial das seçòes contínuas de suporte compacto de $X$. Indicaremos por $X_{d}$ o conjunto $X$ com a topologia discreta. e por $B_{d}$ o fibrado de Banach sobre $\mathrm{Y}_{d}$, cuja fibra sobre $x \in X$ é idêntica como espaço de Banach à fibra correspondente de $B$, e a topologia sobre $B_{d}$ é tal que $p: B_{d} \longrightarrow X_{d}$ é contínua, ou seja, $B_{d}$ é a uniào disjunta clas fibras $B_{x}, x \in \mathcal{X}$. Notar que se $\mathcal{A}=\left(A_{t}\right)_{t \in G}$ é um fibrado de Fell, então $\mathcal{A}_{d}$ é um fibrado de Fell sobre $G_{d}$. Suponhamos que $\mathcal{A}=\left(A_{t}\right)_{t \in G}$ e $\mathcal{B}=\left(B_{s}\right)_{s \in H}$ são fibrados de Fell sobre os grupos localmente compactos $G$ e $H$, e suponhamos que $\mathcal{A}_{d} \bigotimes_{\alpha} \mathcal{B}_{d}$ é algum produto tensorial de $\mathcal{A}_{d}$ e $\mathcal{B}_{d}$. como na seçào anterior. Introduziremos sobre $\mathcal{A}_{d} \otimes \mathcal{B}_{d}$, de maneira canonica, uma topologia tal que $\mathcal{A}_{d} \bigotimes_{a} \mathcal{B}_{d}$ será um fibrado de Fell sobre $G \times H$.

Para cada $f \in C_{c}(\mathcal{A}) . g \in C_{c}(\mathcal{B})$, seja $f \odot g: G \times H \longrightarrow \mathcal{A} \otimes \mathcal{B}$ tal que $(f \circ g)(t, s)=f(t) \otimes g(s)$, $\forall t \in G, s \in H$. Como cada $f$ é uma seção de $\mathcal{A} \otimes_{a} \mathcal{B}$ (de suporte compacto), podemos considerar o espaço vetorial $\Gamma=\operatorname{span}\left\{f, g: f \in C_{c}(\mathcal{A}), g \in C_{c}^{\prime}(\mathcal{B})\right\}$. A topologia que queremos definir sobre $\mathcal{A} \otimes_{a} \mathcal{B}$ fica determinada pela exigencia de que todo elemento de $\Gamma$ seja uma seção contínua.

ProposiçÃo 2.16. Tem-se:

1. Para cada $l \in \Gamma$. a funçcio $G \times H \longrightarrow \mathbb{P}$ tal que $(t, s) \longmapsto\|l(t, s)\|$ é contínua.

2. Para cada $(t, s) \in G \times H$. o conjunto $\Gamma(t, s):=\{l(t, s): l \in \Gamma\}$ é denso em $A_{t} \bigotimes_{\alpha} B_{s}$.

3. Existe uma úmica topologia sobre $\mathcal{A}_{d} \bigotimes_{\alpha} \mathcal{B}_{d}$ que faz com que $\mathcal{A}_{d} \bigotimes_{\alpha} \mathcal{B}_{d}$ seja um fibrado de Banach sobre $(G \times H)$. e tal que $\Gamma$ esteja incluído no espaço de seções contínuas do fibrado $\mathcal{A}_{d} \otimes \mathcal{B}_{d}$ com esta topologia.

$O$ fibrado de Banach sobre $G \times H$ assim obtido será denotado por $\mathcal{A} \otimes_{\alpha} \mathcal{B}$.

Demonstrạ̧ào. É suficiente provar 1. e 2., porque entào 3 . é apenas uma conseqüência ([37], II-13.18). Comecemos por 2.: se $x=\sum_{i=1}^{n} a_{i} \odot b_{i} \in A_{t} \odot B_{s}$, existem seçōes contínuas $f_{i}, g_{i}$ de $\mathcal{A}$ e $\mathcal{B}$ respectivamente, tais que $f_{i}(t)=a_{i}, g_{i}(s)=b_{i}, \forall i=1 \ldots n([37]$, C.1 $1 \tau)$. Se $l=\sum_{i=1}^{n} f_{i} \oslash g_{i}$, então $l \in \Gamma, \mathrm{e}:$

$$
l(t, s)=\sum_{i=1}^{n} f_{i}(t) \ominus g_{i}(s)=\sum_{i=1}^{n} a_{i} \odot b_{i}=x .
$$

Isto demonstra 2., porque $A_{t} \odot B_{s}$ é denso em $A_{t} \bigotimes_{a} B_{s}$. Para provar 1 ., fixemos $l=\sum_{i=1}^{n} f_{i} \oslash g_{i} \in \Gamma$, e suponhamos que $(t, s) \longrightarrow\left(t_{0}, s_{0}\right)$. Então:

$$
\|l(t, s)\|^{2}=\left\|\sum_{i=1}^{n} f_{i}(t) \circlearrowleft g_{i}(s)\right\|^{2}=\left\|\sum_{i, j=1}^{n} f_{i}(t)^{*} f_{j}(t) \odot g_{i}(s)^{*} g_{j}(s)\right\|
$$

Notar que $t \longmapsto f_{i}(t)^{*} f_{j}(t)$ e $s \longmapsto g_{i}(s)^{*} g_{j}(s)$ são contínuas, porque os $f_{i}$ e os $g_{i}$ são seções contínuas, e as involuções de $\mathcal{A}$ e $\mathcal{B}$ são contínuas também. Agora, a propriedade de "cross-norm" (i.e.: $\|a \otimes b\|=$ $\|a\|\|b\|)$ das $C^{*}$-normas sobre produtos tensoriais, implica que $a \otimes b \longrightarrow a_{0} \otimes b_{0}$ quando $a \longrightarrow a_{0} \mathrm{e}$ $b \longrightarrow b_{0}$. Portanto,

$$
\sum_{i, j=1}^{n} f_{i}(t)^{*} f_{j}(t) \circlearrowleft g_{i}(s)^{*} g_{j}(s) \longrightarrow \sum_{i, j=1}^{n} f_{i}\left(t_{0}\right)^{*} f_{j}\left(t_{0}\right) \circlearrowleft g_{i}\left(s_{0}\right)^{*} g_{j}\left(s_{0}\right)
$$

quando $(t, s) \longrightarrow\left(t_{0}, s_{0}\right)$. Assim. $\|l(t, s)\| \longrightarrow\left\|l\left(t_{0}, s_{0}\right)\right\|$ se $(t, s) \longrightarrow\left(t_{0}, s_{0}\right)$.

Lema 2.17. A involuçào * : $\mathcal{A} \bigotimes_{\mathrm{a}} B \longrightarrow \mathcal{A} \bigotimes_{\alpha} \mathcal{B}$ é contínua. 
Demonstraçào. Suponhamos que $G$ é um grupo, $\mathrm{Y}$ é um conjunto com uma involução $*$. e $l: G \longrightarrow \mathrm{X}$ é uma função: definimos uma nova função $i: G \longrightarrow \mathrm{X}$ como $\tilde{l}(t)=l\left(t^{-1}\right)^{*}$. Em particular. se $l$ é uma seçào contínua de suporte compacto de um fibrado de Fell, $\tilde{l}$ também é.

Demonstremos agora o Lema. Provar que a involução é contínua equivale a mostrar que ela é aberta. Sabemos que um aberto básico da topologia definida na Proposição 2.16 é da forma

$$
\mathcal{W}(l, l, c)=\left\{w \in \mathcal{A} \bigotimes_{\mathrm{a}} \mathcal{B}: p(w) \in U \text {, e }\|l(p(w))-w\|<\epsilon\right\} .
$$

onde $p: \mathcal{A} \otimes_{\alpha} B \longrightarrow G \times H$ é a projeçào do fibrado, $I \subseteq G \times H$ é aberto, $l=\sum_{i} f_{i} \oslash g_{i}$. com $f_{i} \in C_{c}(\mathcal{A}), g_{i} \in C_{c}(\mathcal{B})$, e $c>0([37]$. II-13.18). Entào:

$$
\begin{aligned}
\mathcal{W}(l, l, c)^{*} & =\left\{w^{*} \in \mathcal{A} \bigotimes_{a} B: p(w) \in U, \text { e }\|I(p(w))-w\|<\epsilon\right\} \\
& =\left\{u^{*} \in \mathcal{A} \bigotimes_{a} B: p\left(w^{*}\right) \in U^{-1} \text {, e }\left\|I\left(p\left(w^{*}\right)^{-1}\right)^{*}-u^{*}\right\|<\epsilon\right\} \\
& =\left\{z \in \mathcal{A} \bigotimes_{a} B: p(z) \in U^{-1} \text {, e }\|\bar{l}(p(z))-z\|<\epsilon\right\} \\
& =\mathcal{W}\left(i, I^{-1}, \epsilon\right),
\end{aligned}
$$

o que prova que *é aberta, e portanto contínua.

Proposição 2.18. O produto $\left(\mathcal{A} \otimes_{\alpha} \mathcal{B}\right) \times\left(\mathcal{A} \bigotimes_{\alpha} \mathcal{B}\right) \longrightarrow \mathcal{A} \bigotimes_{\alpha} \mathcal{B}$ é contínuo.

Demonstraçio. Afimamos primeiro que se $a \longrightarrow a_{0}$ em $\mathcal{A}$, e $b \longrightarrow b_{0}$ em $\mathcal{B}$, então $a @ b \longrightarrow$ $a_{0} \odot b_{0}$ em $\mathcal{A} \otimes_{a} B$. Sejam $W \subseteq \mathcal{A} \otimes_{\alpha} B$ aberto, tal que $a_{0} \odot b_{0} \in W$. e $f \in C_{c}(\mathcal{A}), g \in C_{c}(\mathcal{B})$ tais que $f\left(t_{0}\right)=a_{0}$ e $g\left(s_{0}\right)=b_{0}$. Então $(f \odot g)\left(t_{0}, s_{0}\right)=a_{0} \odot b_{0}$. Como $f \odot g \in C_{c}\left(\mathcal{A} \otimes \bigotimes_{0} \mathcal{B}\right)$. e como a norma é contínua, existem $\epsilon>0, U \subseteq G$ e $V \subseteq H$ abertos tais que $\left(t_{0}, s_{0}\right) \in U \times V$. e $W \cap\left(\mathcal{A} \bigotimes_{a} \mathcal{B}\right)_{(t, s)} \supseteq B((f, g)(t, s), \epsilon), \forall(t, s) \in U \times \bar{V}$. Consideremos agora $W\left(f, U, \epsilon^{1 / 2}\right) \mathrm{e}$ $W\left(g, V, \epsilon^{1 / 2}\right)$, abertos de $\mathcal{A}$ e $B$ que contém $a_{0}$ e $b_{0}$ respectivamente. Temos:

$$
\begin{aligned}
& W\left(f, L \cdot \epsilon^{1 / 2}\right) W\left(g, V, \epsilon^{1 / 2}\right)=\left\{a_{t} \odot b_{s} \in \mathcal{A} \bigotimes_{a} B:(t, s) \in U \times V, \mathrm{e}\right. \\
& \left.\left\|f(t)-a_{t}\right\|<\epsilon^{1 / 2},\left\|g(s)-a_{s}\right\|<\epsilon^{1 / 2}\right\} \\
& \subseteq\left\{x_{(t, s)} \in \mathcal{A} \bigotimes_{\alpha} \mathcal{B}:(t, s) \in U \times V \text {, e }\left\|(f \bigcirc g)(t, s)-x_{(t, s)}\right\|<\epsilon\right\} \\
& =W(f \circ g, U \times V, \epsilon) \\
& =\bigcup_{(t, s) \in U \times V} B((f \bigcirc g)(t, s), \epsilon) \\
& \subseteq W \text {, }
\end{aligned}
$$

e portanto $a \odot b \longrightarrow a_{0} \rho b_{0}$ quando $a \longrightarrow a_{0}, b \longrightarrow b_{0}$.

Observar que $\forall f, f^{\prime} \in C_{c}(\mathcal{A}), g, g^{\prime} \in C_{c}^{\prime}(\mathcal{B})$, tem-se que a função $\mu:(G \times H) \times(G \times H) \longrightarrow \mathcal{A} \bigotimes_{a} \mathcal{B}$ tal que $\left((t, s),\left(t^{\prime}, s^{\prime}\right)\right) \longmapsto(f \circ g)(t, s) \odot\left(f^{\prime} \odot g^{\prime}\right)\left(t^{\prime}, s^{\prime}\right)$ é contínua, porque os produtos sobre $\mathcal{A}$ e $\mathcal{B}$ são contínuos. $f . f^{\prime}, g, g^{\prime}$ são contínuas, e como $\mu\left(t, s, t^{\prime}, s^{\prime}\right)=f(t) f^{\prime}\left(t^{\prime}\right) \odot g(s) g^{\prime}\left(s^{\prime}\right)$, a continuidade de $\mu$ segue do fato provado no começo.

Consideremos agora $x_{0} \in(\mathcal{A} \otimes \mathcal{B})_{\left(t_{0}, s_{0}\right)}, x_{0}^{\prime} \in(\mathcal{A} \otimes \mathcal{B})_{\left(t_{0}^{\prime}, s_{0}^{\prime}\right)}$, e sejam $m \in \Gamma, 1>\epsilon>0$. tais que $x_{0} x_{0}^{\prime} \in W(m, Z, \epsilon)$. onde $Z$ é algum subconjunto aberto de $G \times H$ que contém $\left(t_{0} t_{0}^{\prime}, s_{0} s_{0}^{\prime}\right)$. Sejam $M>1+\left\|x_{0}\right\|+\left\|x_{0}^{\prime}\right\| . l^{\prime} l^{\prime} \in \Gamma$ tais que $\left\|l\left(t_{0}, s_{0}\right)-x_{0}\right\|<\epsilon / 2 M,\left\|l\left(t_{0}^{\prime}, s_{0}^{\prime}\right)-x_{0}^{\prime}\right\|<\epsilon / 2 . M$. Então temos que $\left\|l\left(t_{0}, s_{0}\right) l^{\prime}\left(t_{0}^{\prime}, s_{0}^{\prime}\right)-x_{0} x_{0}^{\prime}\right\|=d<\epsilon$. Seja $d<\epsilon^{\prime}<\epsilon$. Pelo visto acima, a funçào 
$l l^{\prime}:(G \times H) \times(G \times H) \longrightarrow \mathcal{A} \otimes B$ tal que $\left((t, s),\left(t^{\prime}, s^{\prime}\right)\right) \longmapsto l(t, s) l^{\prime}\left(t^{\prime} . s^{\prime}\right)$ é contínua, e portanto existem vizinhanças abertas $U$. $I^{\prime}$. $I^{\prime \prime}$ e $I^{\prime \prime}$ de $t_{0}, s_{0}, t_{0}^{\prime}$ e $s_{0}^{\prime}$ respectivamente, tais que $l l^{\prime}((U \times$ $\left.V) \times\left(U^{\prime} \times V^{\prime}\right)\right) \subseteq W^{\prime}\left(m, Z, \epsilon^{\prime}\right)$. Sejam agora $N>1+\|l\|_{\infty}+\left\|l^{\prime}\right\|_{\infty} .0<\delta<\left(\epsilon-\epsilon^{\prime}\right) / 4 N$. Então temos que $\left.W(l, U \times V, \delta) W\left(l^{\prime}, U^{\prime} \times I^{\prime \prime}, \delta\right)\right) \subseteq W(m, Z, \epsilon)$. Com efeito. se $x_{(t, s)} \in W(l, U \times V, \delta)$, $x_{\left(t^{\prime}, s^{\prime}\right)}^{\prime} \in W\left(l, U^{\prime} \times I^{\prime \prime}, \delta\right):$

$$
\begin{aligned}
\left\|x_{(t, s)} x_{\left(t^{\prime}, s^{\prime}\right)}^{\prime}-m\left(t t^{\prime}, s s^{\prime}\right)\right\| & \leq\left\|x_{(t, s)} x_{\left(t^{\prime}, s^{\prime}\right)}^{\prime}-l(t, s) l^{\prime}\left(t^{\prime}, s^{\prime}\right)\right\|+\left\|l(t . s) l^{\prime}\left(t^{\prime}, s^{\prime}\right)-m\left(t t^{\prime}, s s^{\prime}\right)\right\| \\
& \leq \epsilon^{\prime}+\left\|x_{(t, s)}\left(x_{\left(t^{\prime}, s^{\prime}\right)}^{\prime}-l^{\prime}\left(t^{\prime}, s^{\prime}\right)\right)\right\|+\left\|\left(x_{(t, s)}-l(t, s)\right) l^{\prime}\left(t^{\prime}, s^{\prime}\right)\right\| \\
& <\epsilon^{\prime}+\left(\epsilon-\epsilon^{\prime}\right) / 4 N\left(\left\|x_{(t, s)}-l(t, s)\right\|\|l(t, s)\|\right)+\left(\epsilon-\epsilon^{\prime}\right) / 4 \\
& <\epsilon^{\prime}+\left(\epsilon-\epsilon^{\prime}\right) / 4 N\left(\left(\epsilon-\epsilon^{\prime}\right) / 4+N\right)+\left(\epsilon-\epsilon^{\prime}\right) / 4 \\
& =\epsilon^{\prime}+\left(\epsilon-\epsilon^{\prime}\right)\left(1 / 2+\left(\epsilon-\epsilon^{\prime}\right) / 4 N\right) \\
& <\epsilon
\end{aligned}
$$

Definição 2.19. Sejam $\mathcal{A}$ e $\boldsymbol{B}$ fibrados de Fell sobre os grupos localmente compactos $G$ e $H$, e a uma $C^{*}$-norma sobre $\mathcal{A} \odot \mathcal{B}$. O produto tensorial $\mathcal{A} \otimes_{\mathrm{a}} \mathcal{B}$ de $\mathcal{A}$ e $\mathcal{B} \mathrm{com}$ respeito a $\alpha$ é o fibrado de Fell obtido completando o produto tensorial algébrico $\mathcal{A} \odot \mathcal{B}$ com respeito à $C^{*}$-norma $\alpha$, e provisto da topologia definida pela Proposição 2.16.

Proposição 2.20. Sejam $\mathcal{A}=\left(A_{t}\right)_{t \in G} \in \mathcal{B}=\left(B_{s}\right)_{s \in H}$ fibrados de Fell solve os grupos localmente compactos $G$ e H. Se $\alpha \geq \beta$ sào $C^{*}$-normas sobre $\mathcal{A} \odot \mathcal{B}$, entio canste um único homomorfismo de fibrados de Fell

$$
\sigma_{\beta}^{a}: \mathcal{A} \bigotimes_{\alpha} B \longrightarrow \mathcal{A} \bigotimes_{\beta} B
$$

tal que $\sigma_{\beta}^{\alpha}(a \odot b)=a \odot b . \forall a \in \mathcal{A}, b \in \mathcal{B}$. Este homomorfismo é sobrejetivo.

DemonstraçÃo. Por 2.12 sabemos que existe e é única tal aplicaçào $\sigma_{3}^{\alpha}$. Também sabemos que é sobrejetiva. Só falta provar que é contínua. Mas as seçōes que definem as duas topologias em jogo são as mesmas, e a aplicaçào $\sigma_{\beta}^{a}$ calculada em uma tal seção da como result ado a mesma seção, donde. por [37] VIII-2.4, concluímos que $\sigma_{\beta}^{a}$ é contínua.

\subsection{Representaçōes de produtos tensoriais de fibrados de Fell}

Os seguintes resultados serão úteis no próximo capítulo. O primeiro diz como obter uma representaçào de $\mathcal{A} \bigotimes_{\text {mín }} \mathcal{B}$ a partir de representações de $\mathcal{A}$ e de $\mathcal{B}$. O segundo mostra que existe uma correspondencia bijetiva entre as representaçôes não degeneradas de $\mathcal{A} \bigotimes_{\text {máx }} \mathcal{B}$ e as representaçôes não degeneradas de $\mathcal{A}$ e $\mathcal{B}$ que comutam.

DEFINIÇÃo 2.21. Seja $\mathcal{A}=\left(A_{t}\right)_{t \in G}$ um fibrado de Fell sobre o grupo localmente compacto $G$. Uma representação de $\mathcal{A}$ sobre o módulo de Hilbert $\mathcal{H}$ é uma aplicaçào $\pi: \mathcal{A} \longrightarrow \mathcal{L}(\mathcal{H})$ tal que $\pi\left(a a^{\prime}\right)=\pi(a) \pi\left(a^{\prime}\right) . \pi\left(a^{*}\right)=\pi(a)^{*}, \forall a, a^{\prime} \in \mathcal{A},\left.\pi\right|_{A_{t}}$ é linear (ou seja: $\pi$ é uma representação do fibrado de Fell $\mathcal{A}_{d}$ ) que é contínua, no sentido de que $\forall h \in \mathcal{H}$ a funçào $\mathcal{A} \longrightarrow \mathcal{H}$ dada por $a \longmapsto \pi(a) h$ é contínua. A representação $\pi$ é dita nào degenerada se $\operatorname{span} \pi(\mathcal{A}) \mathcal{H}$ é denso em $\mathcal{H}$; isto é equivalente 
ao fato de ser nào degenerada a restriçào $\left.\pi\right|_{A_{e}}$, que é uma representaçào de $A_{e}$ sobre o módulo de Hilbert $\mathcal{H}$.

Se $\mathcal{A}=\left(A_{t}\right)_{t \in G}$ é um fibrado de Fell (ou um *-fibrado algébrico), e $\mathcal{H}$ é um módulo de Hilbert. indicaremos por $R(\mathcal{A}, \mathcal{H})$ a família de representações não degeneradas de $\mathcal{A}$ sobre o módulo de Hilbert $\mathcal{H}$. Se $\mathcal{B}=\left(B_{s}\right)_{s \in H}$ é outro fibrado de Fell (ou *-fibrado algébrico), indicaremos por $R(\mathcal{A}, \mathcal{B}, \mathcal{H})=$ $\left\{\left(\pi_{1}, \pi_{2}\right) \in R(\mathcal{A}, \mathcal{H}) \times R(\mathcal{B}, \mathcal{H}): \pi_{1}(a) \pi_{2}(b)=\pi_{2}(b) \pi_{1}(a), \forall a \in \mathcal{A}, b \in \mathcal{B}\right\}$, e por $R(\mathcal{A}, \mathcal{B})$ a uniào de todos os $R(\mathcal{A}, \mathcal{B}, \mathcal{H})$; em outras palavras, $R(\mathcal{A}, \mathcal{B})$ está formada pelos pares de representações não degeneradas de $\mathcal{A}$ e $\mathcal{B}$ que comutam. Se $A$ e $B$ são *álgebras, também usaremos as notações $R(A, \mathcal{H})$. $R(A), R(A, B, \mathcal{H}), R(A, B)$ com o mesmo significado.

Proposição 2.22. Śjam $\mathcal{A} \in \boldsymbol{B}$ fibrados de Fell sobre os grupos localmente compactos $G \in$ $H, \epsilon \operatorname{sejam} \pi_{\mathcal{A}} \in R\left(\mathcal{A}, \mathcal{H}_{\mathcal{A}}\right) . \pi_{B} \in R\left(\mathcal{B}, \mathcal{H}_{B}\right)$ Então existe uma única representação $\pi_{\mathcal{A}} \sigma \pi_{B} \in$ $R\left(\mathcal{A} \otimes_{\min } \mathcal{B}, \mathcal{H}_{\mathcal{A}} \bigotimes_{\min } \mathcal{H}_{\mathcal{L}}\right)$ tal que $\left(\pi_{\mathcal{A}} \pi_{\mathcal{B}}\right)(a \odot b)=\pi_{\mathcal{A}}(a) \bigcirc \pi_{\mathcal{B}}(b)$. $\forall a \in \mathcal{A}$ e $\forall b \in \mathcal{B}$. Se $\left.\pi_{\mathcal{A}}\right|_{\mathcal{A}}$, $\left.\pi_{\mathcal{B}}\right|_{B_{e}}$ são fiéis. entioio $\left.\left(\pi_{\mathcal{A}}, \pi_{\mathcal{B}}\right)\right|_{\left(\mathcal{A} \otimes_{m i n} \mathfrak{B}\right)_{e}}$ também é fiel.

Demonstração. Pela Proposição 1.54, temos uma inclusão de $\mathcal{L}\left(\mathcal{H}_{\mathcal{A}}\right) \bigotimes_{\text {mín }} \mathcal{L}\left(\mathcal{H}_{\mathcal{B}}\right)$ dentro de $\mathcal{L}\left(\mathcal{H}_{\mathcal{A}} \bigotimes_{\text {min }} \mathcal{H}_{\mathcal{B}}\right)$, tal que, $\forall T \in \mathcal{L}\left(\mathcal{H}_{\mathcal{A}}\right), S \in \mathcal{L}\left(\mathcal{H}_{\mathcal{B}}\right), h_{\mathcal{A}} \in \mathcal{H}_{\mathcal{A}}, h_{\mathcal{B}} \in \mathcal{H}_{\mathcal{B}}$ tem-se $(T \odot S)\left(h_{\mathcal{A}} \odot h_{\mathcal{B}}\right)=$ $T\left(h_{\mathcal{A}}\right) \odot S\left(h_{\mathcal{B}}\right)$. Podemos considerar entào, para cada $(t, s) \in G \times H$. a aplicaçào $A_{t} \times B_{s} \longrightarrow$ $\mathcal{L}\left(\mathcal{H}_{\mathcal{A}} \bigotimes_{\min } \mathcal{H}_{\mathcal{B}}\right)$ tal que $\left(a_{t}, b_{s}\right) \longmapsto \pi_{\mathcal{A}}\left(a_{t}\right) \odot \pi_{\mathcal{B}}\left(b_{s}\right)$. Ela é bilinear, e portanto existe uma única transformação linear $\left(\pi_{\mathcal{A}} \pi_{\mathcal{B}}\right)_{(t, s)}: \mathcal{A}_{t} \odot B_{s} \longrightarrow \mathcal{L}\left(\mathcal{H}_{\mathcal{A}} \otimes_{\min } \mathcal{H}_{\mathcal{B}}\right)$ tal que $\left(\pi_{\mathcal{A}} \odot \pi_{\mathcal{B}}\right)_{(t, s)}\left(a_{t} \odot b_{s}\right)=$ $\pi_{\mathcal{A}}\left(a_{t}\right) \odot \pi_{\mathcal{B}}\left(b_{s}\right), \forall a_{t} \in A_{t}, b_{s} \in B_{s}$. Esta coleçào de transformaçōes lineares constitui uma *. representação $\pi_{\mathcal{A}} \odot \pi_{\mathcal{B}}$ do pré-fibrado de Fell $\mathcal{A} \odot \mathcal{B}$ (com a norma minimal); como restrita a $A_{\epsilon} \odot B_{\epsilon}$ é contínua, ela é contínua sobre cada fibra, e portanto se estende unicamente a uma representação de $\mathcal{A} \otimes_{\min } \mathcal{B}$, como fibrado de Fell sobre o grupo discreto $G_{d} \times H_{d}$. Falta mostrarmos que esta representação é contínua na topologia de $\mathcal{A} \bigotimes_{\min } \mathcal{B}$ definida na Proposição 2.16.

Sejam $h_{\mathcal{A}} \in \mathcal{H}_{\mathcal{A}}, h_{\mathcal{B}} \in \mathcal{H}_{\mathfrak{B}}$, e consideremos o fibrado de Banach $G \times H \times\left(\mathcal{H}_{\mathcal{A}} \bigotimes_{\min } \mathcal{H}_{\mathfrak{B}}\right)$ sobre $G \times H$, e a aplicaçào $\Phi: \mathcal{A} \otimes_{\min } \mathcal{B} \longrightarrow G \times H \times\left(\mathcal{H}_{\mathcal{A}} \otimes_{\min } \mathcal{H}_{\mathcal{B}}\right)$ dada por

$$
\Phi\left(c_{t, s}\right)=\left(t, s,\left(\pi_{\mathcal{A}} \odot \pi_{\mathcal{B}}\right)\left(c_{t, s}\right)\left(h_{\mathcal{A}} \odot h_{\mathcal{B}}\right)\right), \forall c_{t, s} \in \mathcal{A}_{t} \bigotimes_{\text {mín }} B_{s}
$$

Seja $\Gamma$ como na Proposição 2.16. Para ver que $\Phi$ é um homomorfismo contínuo de fibrados de Banach. basta mostrar, segundo [37] II-13.16. que $\forall l \in \Gamma$ se tem que $\Phi l$ é uma seção contínua do fibrado $G \times H \times\left(\mathcal{H}_{\mathcal{A}} \bigotimes_{\operatorname{mín}} \mathcal{H}_{\mathcal{B}}\right)$. Para isto, basta apenas considerar seções $l$ da forma $f \bigcirc g$, com $f \in C_{c}(\mathcal{A}), g \in C_{c}(\mathcal{B})$. Suponhamos então que $(t, s) \longrightarrow\left(t_{0}, s_{0}\right)$ em $G \times H$; temos que mostrar que $\Phi(f(t) \ominus g(s)) \longrightarrow \Phi\left(f\left(t_{0}\right) \subseteq g\left(s_{0}\right)\right)$. o que é equivalente a provar que $\pi_{\mathcal{A}}(f(t)) h_{\mathcal{A}} \ominus \pi_{\mathcal{B}}(g(s)) h_{\mathcal{B}}$ converge para $\pi_{\mathcal{A}}\left(f\left(t_{0}\right)\right) h_{\mathcal{A}} \odot \pi_{\mathcal{B}}\left(g\left(s_{0}\right)\right) h_{\mathcal{B}}$. Agora, se $\varepsilon(t, s)=\| \pi_{\mathcal{A}}(f(t)) h_{\mathcal{A}} \odot \pi_{\mathcal{B}}(g(s)) h_{\mathcal{B}}-\pi_{\mathcal{A}}\left(f\left(t_{0}\right)\right) h_{\mathcal{A}} \odot$ $\pi_{\mathcal{B}}\left(g\left(s_{0}\right)\right) h_{\mathcal{B}} \|$, temos:

$$
\begin{gathered}
\varepsilon(t, s) \leq\left\|\pi_{\mathcal{A}}(f(t)) h_{\mathcal{A}} \odot \pi_{\mathcal{B}}(g(s)) h_{\mathcal{B}}-\pi_{\mathcal{A}}(f(t)) h_{\mathcal{A}} \otimes \pi_{\mathcal{B}}\left(g\left(s_{0}\right)\right) h_{\mathcal{B}}\right\| \\
\quad+\left\|\pi_{\mathcal{A}}(f(t)) h_{\mathcal{A}} \oslash \pi_{\mathcal{B}}\left(g\left(s_{0}\right)\right) h_{\mathcal{B}}-\pi_{\mathcal{A}}\left(f\left(t_{0}\right)\right) h_{\mathcal{A}} \otimes \pi_{\mathcal{B}}\left(g\left(s_{0}\right)\right) h_{\mathcal{B}}\right\| \\
\leq\left\|\pi_{\mathcal{A}}(f(t))\right\|\left\|h_{\mathcal{A}}\right\| \\
\quad\left\|\pi_{\mathcal{B}}(g(s)) h_{\mathcal{B}}-\pi_{\mathcal{B}}\left(g\left(s_{0}\right)\right) h_{\mathcal{B}}\right\| \\
\quad+\left\|\pi_{\mathcal{A}}(f(t)) h_{\mathcal{A}}-\pi_{\mathcal{A}}\left(f\left(t_{0}\right)\right) h_{\mathcal{A}}\right\|\left\|\pi_{\mathcal{B}}\left(g\left(s_{0}\right)\right) h_{\mathcal{B}}\right\| \\
\leq\|f\|_{\infty}\left\|h_{\mathcal{A}}\right\|\left\|\pi_{\mathcal{B}}(g(s)) h_{\mathcal{B}}-\pi_{\mathcal{B}}\left(g\left(s_{0}\right)\right) h_{\mathcal{B}}\right\| \\
\quad+\left\|\pi_{\mathcal{A}}(f(t)) h_{\mathcal{A}}-\pi_{\mathcal{A}}\left(f\left(t_{0}\right)\right) h_{\mathcal{A}}\right\|\|g\|_{\infty}\left\|h_{\mathcal{B}}\right\|,
\end{gathered}
$$

que converge a zero porque $\pi_{\mathcal{A}}$ e $\pi_{\mathcal{B}}$ são representações contínuas.

$\mathrm{O}$ fato de ser $\Phi$ contínua implica que $\forall h_{\mathcal{A}} \in \mathcal{H}_{\mathcal{A}}, h_{\mathcal{B}} \in \mathcal{H}_{\mathcal{B}}$, a aplicaçào $\mathcal{A} \bigotimes_{\text {mín }} \mathcal{B} \longrightarrow \mathcal{H}_{\mathcal{A}} \bigotimes_{\text {mín }} \mathcal{H}_{\mathcal{B}}$ tal que $c \longmapsto\left(\pi_{\mathcal{A}} \odot \pi_{\mathcal{B}}\right)(c)\left(h_{\mathcal{A}} \mathcal{B} h_{\mathcal{B}}\right)$ é contínua; como $\left\|\left(\pi_{\mathcal{A}} \odot \pi_{\mathcal{B}}\right)(c)\right\| \leq\|c\| . \forall c \in \mathcal{A} \bigotimes_{\text {min }} \mathcal{B}$. temos 
também que $c \longmapsto\left(\pi_{\mathcal{A}} \quad \pi_{\mathcal{B}}\right)(c)(h) \dot{c}$ contínua, $\forall h \in \mathcal{H}_{\mathcal{A}} \bigotimes_{\min } \mathcal{H}_{\mathcal{B}}$, donde segue que $\pi_{\mathcal{A}} \otimes \pi_{\mathcal{L}}$ é uma representação contínua.

Se $\pi_{\mathcal{A}}, \pi_{\mathcal{B}}$ são nào degeneradas. então $\left.\pi_{\mathcal{A}}\right|_{A_{\mathcal{C}}}$ e $\left.\pi_{\mathcal{B}}\right|_{B_{c}}$ também são nào degeneradas, e portanto. pelo teorema de Cohen-Hewit. dados $h_{\mathcal{A}} \in \mathcal{H}_{\mathcal{A}}, h_{\mathcal{B}} \in \mathcal{H}_{\mathcal{B}}$. existem $a \in A_{e}, b \in B_{e}, h_{\mathcal{A}}^{\prime} \in \mathcal{H}_{\mathcal{A}}$. $h_{\mathcal{B}}^{\prime} \in \mathcal{H}_{\mathcal{B}}$, tais que $\pi_{\mathcal{A}}(a) h_{\mathcal{A}}^{\prime}=h_{\mathcal{A}}, e \pi_{\mathcal{B}}(b) h_{\mathcal{B}}^{\prime}=h_{\mathcal{B}}$. Portanto. $\left(\pi_{\mathcal{A}} \odot \pi_{\mathcal{B}}\right)(a \odot b)\left(h_{\mathcal{A}}^{\prime} \odot h_{\mathcal{B}}^{\prime}\right)=h_{\mathcal{A}} \odot h_{\mathcal{B}}$, donde deduzimos que span $\left\{\left(\pi_{\mathcal{A}} \pi_{\mathcal{B}}\right)\left(\mathcal{A} \bigotimes_{\min } \mathcal{B}\right)\left(\mathcal{H}_{\mathcal{A}} \bigotimes_{\min } \mathcal{H}_{\mathcal{B}}\right)\right\}$ é denso em $\mathcal{H}_{\mathcal{A}} \bigotimes_{\min } \mathcal{H}_{\mathcal{B}}$.

Por último, $\left.\left(\pi_{\mathcal{A}} \pi_{\mathcal{B}}\right)\right|_{\left(\mathcal{A} \otimes_{\min } \boldsymbol{B}\right)_{c}}=\left.\left.\pi_{\mathcal{A}}\right|_{A_{e}} \odot \pi_{\mathcal{B}}\right|_{B_{\mathcal{c}}}$, que é injetiva se e somente se $\left.\pi_{\mathcal{A}}\right|_{A_{e}}$ e $\left.\pi_{\mathcal{B}}\right|_{B_{c}}$ são injetivas.

Suponhamos que $\mathcal{A}=\left(A_{t}\right)_{t \in G}$ í um fibrado de Fell, e $L . R: \mathcal{A} \longrightarrow \mathcal{A}$. sào aplicaçōes contínuas e existe $t \in G$ tal que $L\left(A_{s}\right) \subseteq A_{t s}, R(B s) \subseteq B_{s t}, \forall s \in G,\left.L\right|_{A_{s}}: A_{s} \longrightarrow A_{t s},\left.R\right|_{A_{s}}: A_{s} \longrightarrow A_{s t}$ sào lineares limitadas, e $\|L\|:=s u p p_{s}\left\|\left.L\right|_{A_{s}}\right\|<\mathcal{C}_{,}\|R\|:=\sup _{s}\left\|\left.R\right|_{A_{s}}\right\|<\chi$. Entào $(L, R)$ é chamado multiplicador de ordem $t$ de $\mathcal{A}$ se, $\forall a_{1}, a_{2}, \in \mathcal{A}$, vale:

$$
\begin{aligned}
& a_{1} L\left(a_{2}\right)=R\left(a_{1}\right) a_{2} \\
& L\left(a_{1} a_{2}\right)=L\left(a_{1}\right) a_{2} \\
& R\left(a_{1} a_{2}\right)=a_{1} R\left(a_{2}\right)
\end{aligned}
$$

Simbolizamos o conjunto dos multiplicadores de $\mathcal{A}$ de ordem $t$ por $M_{t}(\mathcal{A})$, e por $M(\mathcal{A})=\bigcup_{t \in G} M_{t}(\mathcal{A})$ o conjunto de todos os multiplicadores de $\mathcal{A}$ (a notação é diferente da usada em [37], VIII-2.14).

Cada $M_{t}(\mathcal{A})$ é um espaço de Banach com as operaçòes óbrias e a norma

$$
\|(L, R)\|_{0}=\operatorname{máx}\{\|L\|,\|R\|\} .
$$

De fato, tem-se $\|L\|=\|R\|$. Além disso, podemos definir um produto e uma involução sobre $M(\mathcal{A})$ :

$$
\begin{gathered}
\left(L_{1}, R_{1}\right)\left(L_{2}, R_{2}\right)=\left(L_{1} L_{2}, R_{2} R_{1}\right) \\
(L, R)^{*}=\left(R^{*}, L^{*}\right),
\end{gathered}
$$

onde $L^{*}(a)=L\left(a^{*}\right)^{*}$, c $R^{*}(a)=R\left(a^{*}\right)^{*}$. Com estas operaçòes e norma, $M(\mathcal{A})$ é um fibrado de Fell sobre o grupo discreto $G_{d}$.

Além disto, $M(\mathcal{A})$ tem uma topologia "forte": se $u_{i}=\left(L_{i}, R_{i}\right), u=(L, R)$, então $u_{i} \longrightarrow u$ se $\forall a \in \mathcal{A}$ tem-se que $L_{i}(a) \longrightarrow L(a) . R_{i}(a) \longrightarrow R(a)$. Por analogia ao caso das $C^{*}$-álgebras. chamaremos esta topologia de estrita (em [37]. VIII-15.2, esta topologia é chamada forte). Se $u=$ $(L, R) \in M(\mathcal{A})$, escreveremos ua e au em lugar de $L(a)$ e $R(a)$ respectivamente.

Há uma inclusão isométrica e contínua $\mathcal{A} \hookrightarrow M(\mathcal{A})$, dada por $a \longmapsto\left(L_{a}, R_{a}\right)$, onde $L_{a}$ é a multiplicação por $a$ à esquerda, e $R_{a}$ é a multiplicação por a à direita. Em particular, a topologia de $\mathcal{A}$ é mais forte que a herdada da topologia estrita de $M(\mathcal{A})$. Se $\mathcal{A}_{\epsilon}$ tem unidade, elas coincidem; se $A_{e}$ não tem unidade, são diferentes: toda unidade aproximada de $A_{\epsilon}$ é convergente estritamente a $1=(i d, i d) \in M(\mathcal{A})$, mas não é convergente em $A_{e}$. Também temos uma inclusão isométrica $M\left(A_{\epsilon}\right) \hookrightarrow M_{e}(\mathcal{A})$, porque, sendo cada $A_{t}$ um bimódulo de Hilbert sobre $A_{\epsilon}$, ele é também, de forma natural, um bimódulo de Hilbert sobre $M\left(A_{\epsilon}\right)$, e é fácil mostrar que as ações por multiplicação à esquerda e à direita por elementos de $M\left(A_{\ell}\right)$ sobre $\mathcal{A}$ definem multiplicadores de ordem $e$ (Ver 2.23 abaixo).

Se $\pi: \mathcal{A} \longrightarrow \mathcal{L}(\mathcal{H})$ é uma representaçào nào degenerada de $\mathcal{A}$. entào existe uma única extensão ([37], VIII-15.3) de $\pi$ a uma representaçào $\pi^{\prime}: M(\mathcal{A}) \longrightarrow \mathcal{L}\left(\mathcal{H}\right.$ i tal que $\forall h \in \mathcal{H}$, a função $u \longmapsto \pi^{\prime}(u) h$ é estritamente contínua sobre cilindros de $\mathcal{A}$ (o cilindro de raio $r$ de $\mathcal{A}$ é $C_{r}=\{a \in \mathcal{A}:\|a\| \leq r\}$ )

Lema 2.23. A aplicaçcio $M\left(B_{\epsilon}\right) \times B \longrightarrow B$ tal que $(u, b) \longrightarrow u b$ é contínua. 
Demonstraçào. Vejamos primeiro que. fixado $u \in M\left(B_{\epsilon}\right)$, a função $\mathcal{B} \longrightarrow \mathcal{B}$ tal que $b \longmapsto u b$ é contínua. Para isto, basta provar que se $f \in C_{c}(\mathcal{B})$, então $u f: G \longrightarrow \mathcal{B}$ tal que $\left.(u f)\right|_{t}=u f(t)$ é contínua. Notemos que $B_{f} C_{c}(\mathcal{B})$ é denso na topologia do limite indutivo de $C_{c}(\mathcal{B})$, por [37], II-14.6 (ou 3.14), e portanto $B_{\epsilon} C_{c}(\mathcal{B})$ é denso em $C_{0}^{\prime}(\mathcal{B})$. Logo, $C_{0}(\mathcal{B})$ é um $B_{\epsilon}$-módulo de Banach não degenerado. Portanto, pelo teorema de Cohen-Hewitt, existem $b \in B_{e}, g \in C_{0}(\mathcal{B})$, tais que $f=b g$, e é claro que podemos supor que $g \in C_{c}^{\prime}(\mathcal{B})$ (em outras palavras: $B_{e} C_{c}^{\prime}(\mathcal{B})=C_{c}(\mathcal{B})$ ). Mas entào $u f=(u b) g \in C_{c}(\mathcal{B})$, pois o produto é contínuo sobre $\mathcal{B}$.

Por último, suponhamos que $b_{j} \longrightarrow b \mathrm{em} \mathcal{B}, \operatorname{com} b_{j} \in B_{t_{j}}, b \in B_{t}$, e que $u_{i} \longrightarrow u \operatorname{em} M\left(B_{\epsilon}\right)$. Como $\|\cdot\|: \mathcal{B} \longrightarrow \mathbb{F}$ é contínua, existe $j_{0}$ tal que $\forall j \geq j_{0}$ tem-se que $\left\|b_{j}\right\| \leq M$. Então, se $j \geq j_{0}$ : $\left\|u_{i} b_{j}-u b_{j}\right\| \leq M\left\|u_{i}-u\right\| \longrightarrow 0$, donde segue que $\left(u_{i} b_{j}-u b_{j}\right) \longrightarrow 0_{t}$ quando $(i, j) \longrightarrow \infty$. Por outro lado, vimos no comeşo que $u b_{j} \longrightarrow u b$, e portanto concluímos que $u_{i} b_{j} \longrightarrow u b$ se $(i, j) \longrightarrow \infty$.

O seguinte resultado é análogo ao Lema 1.46 .

LEMA 2.24. Sejam $\mathcal{A}=\left(\mathcal{A}_{1}\right)_{t \in G} \in \mathcal{B}=\left(B_{s}\right)_{s \in H}$ fibrados de Fell $\in \mathcal{A} \otimes B$ um produto tensorial entre $\mathcal{A}$ e $\mathcal{B}$. Entio cristem inclusies únicas $\iota_{\mathcal{A}}: M(\mathcal{A}) \longrightarrow M(\mathcal{A} \otimes \mathcal{B}) \in \iota_{\mathfrak{B}}: M(\mathcal{B}) \longrightarrow M(\mathcal{A} \otimes \mathcal{B})$ tais que $\iota_{\mathcal{A}}(u) \iota_{\mathcal{B}}(v)=\iota_{\mathfrak{B}}(v) \iota_{\mathcal{A}}(u), \forall u \in M(\mathcal{A}), v \in M(\mathcal{B})$, e tais que $\iota_{\mathcal{A}}(a) \iota_{\mathcal{B}}(b)=a \odot b, \forall a \in \mathcal{A}$. $b \in \mathcal{B}$. Estas inclusóes saio isométricas e contínuas nas topologias estritas quando restritas a cilindros.

Demonstração. Seja $u \in M_{t}(\mathcal{A})$. Para cada $r \in G, s \in H$, a transformação $A_{r} \times B_{s} \longrightarrow$ $A_{t r} \otimes B_{s}$ tal que $\left(a_{r}, b_{s}\right) \longmapsto\left(u a_{r}, b_{s}\right)$ é bilinear, e portanto existe una única transformação linear $L_{u}: A_{t} \odot B_{s} \longrightarrow A_{t} \otimes B_{s}$ tal que $a_{r} \odot b_{s} \longmapsto u a_{r} \odot b_{s}$. Analogamente, existe $R_{u}: A_{t} \odot B_{s} \longrightarrow$ $A_{r t} \otimes B_{s}$ tal que $R_{u}\left(a_{r}, b_{s}\right)=a_{r} u \odot b_{s}$. A coleção destas transformaçōes define duas aplicaçōes $L_{u}, R_{u}: \mathcal{A} \odot \mathcal{B} \longrightarrow \mathcal{A} \otimes \mathcal{B}$ tais que $\forall x, y \in \mathcal{A} \odot \mathcal{B} \subseteq \mathcal{A} \otimes \mathcal{B}$ satisfazem: $L_{u}(x y)=L_{u}(x) y, R_{u}(x y)=$ $x R_{u}(y), x L_{u}(y)=R_{u}(x) y$. Se provarmos que $L_{u}, R_{u}$ são limitados, entào se estendem unicamente sobre cada fibra a operadores contínuos, que continuarào satisfazendo as relaçòes algébricas acima; em outras palavras, o par formado por estas extensōes será um multiplicador de ordem $(t, \epsilon)$ de $(\mathcal{A} \otimes \mathcal{B})_{d}$.

Seja $x=\sum_{i=1}^{n} a_{i} \fallingdotseq b_{i} \in A_{r} \otimes B_{s}$. Então:

$$
\left\|L_{u} x\right\|^{2}=\left\|\sum_{i=1}^{n} u a_{i} \circlearrowright b_{i}\right\|^{2}=\sum_{i, j=1}^{n} a_{i}^{*} u^{*} u a_{j} \odot b_{i}^{*} b_{j}
$$

Sejam $\mathfrak{u}=\left(u_{i j}\right) \in M_{n}\left(M\left(A_{\epsilon}\right)\right), \mathfrak{a}=\left(a_{i j}\right) \in M_{n}\left(A_{r}\right)$, dados por: $u_{i j}= \begin{cases}\sqrt{u^{*} u} & \text { se } i=j, \\ 0 & \text { em outro caso }\end{cases}$ e $a_{i j}=\left\{\begin{array}{ll}a_{j} & \text { se } i=1, \\ 0 & \text { em outro caso }\end{array}\right.$. Como $A_{r}$ é um bimódulo de Hilbert sobre $M\left(A_{e}\right), M_{n}\left(A_{r}\right)$ é um $M_{n}\left(M\left(A_{e}\right)\right)$-bimódulo de Hilbert: à direita com o produto $\langle\mathfrak{r}, \mathfrak{y}\rangle_{r}=\mathfrak{r}^{*} \mathfrak{n}$. onde o produto é o produto usual de matrizes e, se $\mathfrak{r}=\left(x_{i, j}\right)$ e $\mathfrak{r}^{*}=\left(y_{i j}\right)$, então $y_{i j}=x_{j i}^{*}$; o produto à esquerda é $\langle\mathfrak{r}, \mathfrak{y}\rangle_{l}=\mathfrak{r} \mathfrak{h}^{*} ;$ as ações são dadas pelo produto de matrizes. Em particular temos $\langle\mathfrak{u a}, \mathfrak{u a}\rangle_{r} \leq \mathfrak{u}^{*} \mathfrak{u}\langle\mathfrak{a}, \mathfrak{a}\rangle_{r} \leq\|u\|^{2}\langle\mathfrak{a}, \mathfrak{a}\rangle_{r}$, donde $\mathfrak{c}:=\|u\|^{2}\langle a, a\rangle_{r}-\langle u a, u a\rangle_{r} \geq 0$. Um cálculo rápido mostra que, se $\mathfrak{c}=\left(c_{i j}\right)$, então $c_{i j}=$ $a_{i}^{*}\left(\|u\|^{2}-u^{*} u\right) a_{j}$.

Por outro lado, também $M_{n}\left(B_{s}\right)$ é um bimódulo de Hilbert sobre $M_{n}\left(M\left(B_{e}\right)\right)$. Em particular, se $\mathfrak{b}=\left(b_{i, j}\right) \in M_{n}\left(B_{s}\right)$ está dado por $b_{i, j}=\left\{\begin{array}{ll}b_{j} & \text { se } i=1 \\ 0 & \text { em outro caso }\end{array}\right.$. o elemento $b^{*} \mathfrak{b}=\left(b_{i}^{*} b_{j}\right)$ é positivo em $M_{n}\left(M\left(B_{e}\right)\right)$. Agora, pelo Lema 1.43, temos que c $@ b^{*} \mathfrak{b}=\left(a_{i}^{*}\left(\|u\|^{2}-u^{*} u\right) a_{j} \odot b_{i}^{*} b_{j}\right)$ é um elemento positivo em qualquer $C^{* *}$-completamento de $M_{n}\left(M\left(f_{e}\right)\right) \odot M_{n}\left(M\left(B_{e}\right)\right)$. e $\sum_{i, j=1}^{n} a_{i}^{*}\left(\|u\|^{2}-u^{*} u\right) a_{j}$. 
$b_{i}^{*} b_{j}$ é positivo em qualquer $C^{\prime *}$-completamento de $M\left(A_{e}\right) \bigodot M\left(B_{e}\right)$. Em particular,

$$
\left\|\sum_{i, j=1}^{n} a_{i}^{*} u^{*} u a_{j} \odot b_{i}^{*} b_{j}\right\| \leq\|u\|^{2}\left\|\sum_{i, j=1}^{n} a_{i}^{*} a_{j} \odot b_{i}^{*} b_{j}\right\| .
$$

para qualquer $C^{*}$-norma sobre $A_{\epsilon} \odot B_{\mathrm{t}}$. Isto mostra que $\left\|L_{u} x\right\|^{2} \leq\|u\|^{2}\|x\|^{2}$, como queríamos. Analogamente, vemos que $\left\|R_{u} x\right\|^{2} \leq\|u\|^{2}\|x\|^{2}$, e portanto $\left(L_{u}, R_{u}\right)$ se estende a um multiplicador $\iota_{\mathcal{A}}(u)$ sobre $(\mathcal{A} \otimes \mathcal{B})_{d}$, e $\left\|\iota_{\mathcal{A}}(u)\right\| \leq\|u\|$. Na verdade, $\left\|\iota_{\mathcal{A}}(u)\right\|=\|u\|:$ se $a \in \mathcal{A}, b \in \mathcal{B}$ são tais que $\|a\|,\|b\| \leq 1$, então

$$
\left\|\iota_{\mathcal{A}}(u)\right\| \geq\left\|\iota_{\mathcal{A}}(u)(a \odot b)\right\|=\|u a\|\|b\|=\|u a\| .
$$

donde segue que $\left\|\iota_{\mathcal{A}}(u)\right\| \geq\|u\|$ : assim, $\left\|\iota_{\mathcal{A}}(u)\right\|=\|u\|$, e portanto $\iota_{\mathcal{A}}$ '́ una isometria.

Para ver que $\iota_{\mathcal{A}}(u) \in M(\mathcal{A} \otimes \mathcal{B})$. falta mostrar que ele é contímuo. Para isto, consideremos $f \in C_{c}^{\prime}(\mathcal{A}), g \in C_{c}^{\prime}(\mathcal{B})$; entào as funçòes $G \times H \longrightarrow \mathcal{A} \otimes \mathcal{B}$ tais que $(t . s) \longmapsto u f(t) \bigcirc g(s)$ e $(t, s) \longmapsto f(t) u \circlearrowleft g(s)$ são contínuas. Lim argumento igual ao de [37], 11-13.16 (que é sobre a continuidade de aplicações que preservam as fibras). mostra que a continuidade das funções acima implica a continuidade de $L_{u}$ e $R_{u}$ : de qualquer forma apresentamos a prova a seguir. Suponhamos que $x_{i} \longrightarrow x$ em $\mathcal{A} \otimes \mathcal{B}$, e seja $l=\sum_{i} f_{i} g_{i}$ tal que $\|l(t, s)-x\|<\epsilon$, onde $x \in A_{1} \otimes B_{s}, x_{i} \in \mathcal{H}_{1} \otimes B_{s_{1}}$. Como $x_{i} \longrightarrow x$ e $l\left(t_{i}, s_{i}\right) \longrightarrow l(t, s)$. a continuidade da norma e da soma implicam que existe $i_{0}$ tal que $\forall i \geq i_{0}$ deve ser $\left\|l\left(t_{i}, s_{i}\right)-x_{i}\right\|<c$. Agora, $\left\|L_{u} l\left(t_{i}, s_{i}\right)-L_{u} x_{i}\right\| \leq \mathrm{c}\|u\|$. e $\left\|L_{u} l(t, s)-L_{u} x\right\| \leq \epsilon\|u\|$, e como $L_{u} l\left(t_{i}, s_{i}\right) \longrightarrow L_{u}(t, s)$. concluímos que $L_{u} x_{i} \longrightarrow L_{u} x$.

Vejamos que $\iota_{\mathcal{A}}$ é estritamente contínua sobre cilindros. Se $a \in \mathcal{A}, b \in \mathcal{B}$. e $\left(u_{i}\right) \subseteq \mathcal{A}$ é uma rede que converge estritamente a $u \in \mathcal{A}$, com $\left\|u_{i}\right\|,\|u\| \leq C$, então:

$$
\begin{aligned}
& l_{\mathcal{A}}\left(u_{i}\right)(a \odot b)=u_{i} a \odot b \longrightarrow u a \odot b=\iota_{\mathcal{A}}(u)(a \odot b) \\
& (a \odot b) \iota_{\mathcal{A}}\left(u_{i}\right)=a u_{i} \odot b \longrightarrow a u \odot b=(a \odot b) \iota_{\mathcal{A}}(u)
\end{aligned}
$$

Então $\iota_{\mathcal{A}}\left(u_{i}\right) x \longrightarrow \iota_{\mathcal{A}}(u) x$ e $x \iota_{\mathcal{A}}\left(u_{i}\right) \longrightarrow x \iota_{\mathcal{A}}(u), \forall x \in \mathcal{A} \odot \mathcal{B}$; como $\left\|\iota_{\mathcal{A}} u_{i}\right\| .\left\|\iota_{\mathcal{A}} u\right\| \leq C$, concluímos que $\iota_{\mathcal{A}}\left(u_{i}\right) x \longrightarrow \iota_{\mathcal{A}}(u) x$ e $x \iota_{\mathcal{A}}\left(u_{i}\right) \longrightarrow x \iota_{\mathcal{A}}(u), \forall x \in \mathcal{A} \otimes \mathcal{B}$, donde $\iota_{\mathcal{A}} u_{i}$ converge estritamente a $\iota_{\mathcal{A}}(a)$.

Analogamente construímos $\iota_{\mathfrak{B}}: M(\mathcal{B}) \longrightarrow M(\mathcal{A} \otimes \mathcal{B})$ : se $v \in M(\mathcal{B})$ e $a \in \mathcal{A}, b \in \mathcal{B}$, então $\iota_{\mathfrak{B}}(v)(a \otimes b)=a \odot v b$, e $(a @ b) \iota_{\mathcal{B}}(v)=a \odot b v$. É claro que $\iota_{\mathcal{A}}(u) \iota_{\mathfrak{B}}(v)=\iota_{\mathfrak{B}}(v) \iota_{\mathcal{A}}(u), \forall u \in M(\mathcal{A})$, $v \in M(\mathcal{B})$, e também que $\iota_{\mathcal{A}}(a) \iota_{\mathcal{B}}(b)=a \odot b, \forall a \in \mathcal{A}, b \in \mathcal{B}$.

Assim obtemos una aplicaçào $M(\mathcal{A}) \times M(\mathcal{B}) \longrightarrow M(\mathcal{A} \otimes \mathcal{B})$, dada por $(u, v) \longmapsto \iota_{\mathcal{A}}(u) \iota_{\mathcal{B}}(v)$, que é bilinear sobre cada $M_{t}(\mathcal{A}) \times M_{s}(\mathcal{B})$, e portanto conseguimos uma aplicação $M(\mathcal{A}) \odot M(\mathcal{B}) \longrightarrow$ $M(\mathcal{A} \otimes \mathcal{B})$, que é linear sobre cada $M_{t}(\mathcal{A}) \odot M_{s}(\mathcal{B})$, e que é um homomorfismo de fibrados de Fell porque $\iota_{\mathcal{A}}(u)$ e $\iota_{\mathcal{B}}(v)$ comutam. $\forall u \in M(\mathcal{A}), v \in M(\mathcal{B})$.

Proposiçào 2.25. Sejam $\mathcal{A}=\left(A_{t}\right)_{t \in G_{i}} \in \mathcal{B}=\left(B_{s}\right)_{s \in H}$ fibrados de Fell sobre os grupos localmente compactos $G$ e H, e seja $\mathcal{H}$ um módulo de Hilbert. Então, para cada $\left(\pi_{1}, \pi_{2}\right) \in R(\mathcal{A}, \mathcal{B}, \mathcal{H})$, existe uma única $\pi \in R\left(\mathcal{A} \bigotimes_{\text {máx }} \mathcal{B}, \mathcal{H}\right)$ tal que $\pi(a \odot b)=\pi_{1}(a) \pi_{2}(b), \forall a \in \mathcal{A}, b \in \mathcal{B} . \epsilon$ a aplicação $\left(\pi_{1}, \pi_{2}\right) \longmapsto \pi$ assim definida é uma bijeçào entre $R(\mathcal{A}, \mathcal{B}, \mathcal{H})$ e $R\left(\mathcal{A} \otimes_{\text {máx }} \mathcal{B}, \mathcal{H}\right)$.

Demonstração. Seja $\left(\pi_{1}, \pi_{2}\right) \in R(\mathcal{A}, \mathcal{B}, \mathcal{H})$. A aplicação $\mathcal{A} \times \mathcal{B} \longrightarrow \mathcal{L}(\mathcal{H})$ tal que $\left(a_{t}, b_{s}\right) \longmapsto$ $\pi_{1}\left(a_{t}\right) \pi_{2}\left(b_{s}\right)$ é bilinear sobre cada $A_{t} \times B_{s}$, e portanto existe uma única $\pi: \mathcal{A} \odot \mathcal{B} \longrightarrow \mathcal{L}(\mathcal{H})$ tal que $\pi\left(a_{t} \otimes b_{s}\right)=\pi_{1}\left(a_{t}\right) \pi_{2}\left(b_{s}\right)$. Como $\pi_{1}(a)$ e $\pi_{2}(b)$ comutam, $\forall a \in \mathcal{A} . b \in \mathcal{B}$, tem-se que $\pi$ : $\mathcal{A} \odot \mathcal{B} \longrightarrow \mathcal{L}(\mathcal{H})$ é uma representaçào do pré-fibrado de Fell $\mathcal{A} \odot \mathcal{B}$. Votar que $x \longmapsto\|x\|^{\prime}=$ máx $\left\{\|x\|_{\text {máx }},\|\pi(x)\|\right\}$ é uma $C^{\prime *}$-norma sobre $A_{l} \odot B_{s}$, donde segue que $\|\cdot\|^{\prime}=\|\cdot\|_{\text {máx }}$, e portanto $\|\pi(x)\| \leq\|x\|_{\text {máx }}, \forall x \in \mathcal{A}_{t} \odot B_{s}$. Assim, $\pi$ tem uma única extensão a uma representação $\pi$ : 
$\left(\mathcal{A} \otimes_{\text {máx }} \mathcal{B}\right)_{d} \longrightarrow \mathcal{L}(\mathcal{H})$. Usando o teorema de Cohen-Hewitt, é fácil ver que esta representação é nào degenerada: como $\pi_{1}$ é nào degenerada, para todo $h \in \mathcal{H}$ existem $h^{\prime} \in \mathcal{H}$ e $a \in A_{e}$ tais que $\pi_{2}(a) h^{\prime}=h$; como $\pi_{2}$ é nào degenerada, existem $h^{\prime \prime} \in \mathcal{H}$ e $b \in B_{e}$ tais que $\pi_{1}(b) h^{\prime \prime}=h^{\prime}$; portanto $\pi(a \odot b) h^{\prime \prime}=\pi_{1}(a) \pi_{2}(b) h^{\prime \prime}=\pi_{1}(a) h^{\prime}=h$.

Falta mostrar ainda que $\pi$ é contínua. Por [37] II-13.16. para isto é suficiente provar que $\forall f \in$ $C_{c}(\mathcal{A}), g \in C_{c}(\mathcal{B})$. e $h \in \mathcal{H}$, a funçào $G \times H \longrightarrow \mathcal{H}$ tal que $(t, s) \longmapsto \pi((f, g)(t, s)) h$ é contínua. Mas, se $(t, s) \longrightarrow\left(t_{0}, s_{0}\right):$

$$
\begin{aligned}
\left\|\pi((f \odot g)(t, s)) h-\pi\left((f \circ g)\left(t_{0}, s_{0}\right)\right) h\right\|= & \left\|\pi_{1}(f(t)) \pi_{2}(g(s)) h-\pi_{1}\left(f\left(t_{0}\right)\right) \pi_{2}\left(g\left(s_{0}\right)\right) h\right\| \\
\leq & \left\|\pi_{1}(f(t))\left(\pi_{2}(g(s))-\pi_{2}\left(g\left(s_{0}\right)\right)\right) h\right\| \\
& +\left\|\left(\pi_{1}(f(t))-\pi_{1}\left(f\left(t_{0}\right)\right)\right) \pi_{2}\left(g\left(s_{0}\right)\right) h\right\| \\
\leq & \|f\|_{\infty} \|\left(\pi_{2}(g(s)) h-\pi_{2}\left(g\left(s_{0}\right)\right) h \|\right. \\
& +\left\|\pi_{1}(f(t)) \pi_{2}\left(g\left(s_{0}\right)\right) h-\pi_{1}\left(f\left(t_{0}\right)\right) \pi_{2}\left(\left(s_{0}\right)\right) h\right\| \\
& \longrightarrow 0, \quad 0 \quad \text { pois } \pi_{1} \rho \pi_{2} \text { sào contínuas. }
\end{aligned}
$$

Portanto, $\pi \in R\left(\mathcal{A} \bigotimes_{\text {máx }}\right.$ B. $\left.\mathcal{H}\right)$.

Inversamente. suponhamos que $\pi \in R\left(\mathcal{A} \bigotimes_{\text {máx }} \mathcal{B}, \mathcal{H}\right)$. Por [37] VIII-15.3, $\pi$ se estende de forma única a uma representaçào $\pi^{\prime}$ de $M\left(\mathcal{A} \bigotimes_{\max } \mathcal{B}\right)$, tal que $x \longmapsto \pi^{\prime}(x) h$ é estritamente contínua sobre cilindros, $\forall h \in \mathcal{H}$. Sejam $\pi_{1}=\left.\pi^{\prime} \iota_{\mathcal{A}}\right|_{\mathcal{A}}: \mathcal{A} \longrightarrow \mathcal{L}(\mathcal{H})$, e $\pi_{2}=\left.\pi^{\prime} \iota_{\mathcal{B}}\right|_{\mathfrak{L}}: \mathcal{B} \longrightarrow \mathcal{L}(\mathcal{H})$, onde $\iota_{\mathcal{A}}$ e $\iota_{\mathcal{B}}$ sào as inclusòes fornecidas pelo Lema 2.24. Como $\pi^{\prime}, \iota_{\mathcal{A}}$ e $\iota_{\mathfrak{L}}$ sào contínuas sobre cilindros temos imediatamente que $\forall h \in \mathcal{H}$ as funçōes $\mathcal{A} \longrightarrow \mathcal{H}$ e $\mathcal{B} \longrightarrow \mathcal{H}$ dadas por $a \longmapsto \pi_{1}(a) h$ e $b \longmapsto \pi_{2}(b) h$ respectivamente sào contínuas. donde segue que $\pi_{1}$ e $\pi_{2}$ são representaçòes contínuas. Como $\forall a \in \mathcal{A}$, $b \in \mathcal{B}$ se tem que $\iota_{\mathcal{A}}(a)$ e $\iota_{\mathfrak{B}}(b)$ comutam, então também comutam $\pi_{1}(a)$ e $\pi_{2}(b)$. Finalmente, as representações $\pi_{1}$ e $\pi_{2}$ são nào degeneradas: basta mostrar que todo $\pi(a ; b) h$ está na imagem de $\pi_{1}$ e na imagem de $\pi_{2}$. Mas, por Cohen-Hewitt, $a$ e $b$ podem ser fatorizados como $a=a_{1} a_{2}, b=b_{1} b_{2}$, e portanto:

$$
\begin{aligned}
& \pi_{1}\left(a_{1}\right) \pi\left(a_{2} \odot b\right) h=\pi\left(a_{1} a_{2} \odot b\right) h=\pi(a \odot b) h \\
& \pi_{2}\left(b_{1}\right) \pi\left(a \odot b_{2}\right) h=\pi\left(a \odot b_{1} b_{2}\right) h=\pi(a \odot b) h
\end{aligned}
$$

Temos construído assim correspondencias $\left(\pi_{1}, \pi_{2}\right) \longmapsto \pi$ e $\pi \longmapsto\left(\left.\pi \iota_{\mathcal{A}}\right|_{\mathcal{A}},\left.\pi \iota_{\mathcal{B}}\right|_{\mathcal{B}}\right)$, que sào claramente mutuamente inversas.

OBservaçÃo 2.26. Na demonstração da Proposição 2.25, vimos na verdade que se $\pi_{1}: \mathcal{A} \longrightarrow$ $\mathcal{L}(H)$ e $\pi_{2}: \mathcal{B} \longrightarrow \mathcal{L}(H)$ são representaçōes tais que $\pi_{1}(a) \pi_{2}(b)=\pi_{2}(b) \pi_{1}(a), \forall a \in \mathcal{A}, b \in \mathcal{B}$, então existe uma representação $\pi: \mathcal{A} \bigotimes_{\text {máx }} \mathcal{B} \longrightarrow \mathcal{L}(H)$ tal que $\pi(a \oslash b)=\pi_{1}(a) \pi_{2}(b), \forall a \in \mathcal{A}, b \in \mathcal{B}$. A hipótese de não degeneração de $\pi_{1}$ e $\pi_{2}$ foi usada apenas para ver que $\pi$ também é não degenerada. 
2. PRODUTOS TENSORIAIS DE FIBRADOS DE FELL 
CAPíTULO 3

C*-álgebras secionais de produtos tensoriais de fibrados de Fell 
A organizaçào deste capítulo é a seguinte. Na primeira seçào introduzimos a $C^{* *}$-álgebra reduzida de um fibrado de Fell sobre um grupo localmente compacto, e também a noção de "amenabilidade" de um fibrado de Fell. Na segunda seçào comparamos as $C^{\prime \prime}$-álgebras seccionais de produtos tensoriais de fibrados de Fell com os produtos tensoriais das correspondentes $C^{* *}$-álgebras seccionais. Finalmente, na última seçào, utilizamos os resultados obtidos nas seçòes e capítulos anteriores para obter aplicaçōes à teoria geral de $C^{\star x}$-álgebras, especialmente na área de produtos cruzados. ('oncretamente mostramos que: um fibrado de Fell com fibra nuclear (exata) e certa propriedade de aproximação, que generaliza ao caso contínuo a propriedade de aproximaçào introduzida em [28], tem ( ${ }^{*}$-álgebra seccional nuclear (respectivamente: exata). Estes resultados sào obtidos essencialmente graças aos seguintes dois fatos: por um lado, as propriedades de aproximação são preservadas sob produtos tensoriais $(3.2 \bar{T})$, e por outro tem-se que $C^{* *}(\mathcal{A}) \bigotimes_{\operatorname{máx}} C^{* *}(\mathcal{B}) \cong C^{* *}\left(\mathcal{A} \bigotimes_{\operatorname{máx}} \mathcal{B}\right)$, e $C_{r}^{* *}(\mathcal{A}) \bigotimes_{\min } C_{r}^{\prime *}(\mathcal{B}) \cong C_{r}^{*}\left(\mathcal{A} \bigotimes_{\min } \mathcal{B}\right)$, resultado que mostra uma bela coerencia entre construçōes universais e construçòes espaciais (3.21).

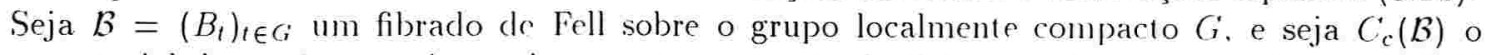
espaço vetorial das seçòes contínuas de suporte compacto de $\mathcal{B}$ (a notação rorrespondente em [37] é $\mathfrak{L}(\mathcal{B})$ ). Consideremos, para cada subconjunto compacto $K$ de $C$. o subespaço $C_{K}(\mathcal{B})$ de $C_{c}(\mathcal{B})$, formado pelas seçòes contínuas de $B$ cujo suporte está contido em $K$. ('om a norma do supremo, $C_{K}(\mathcal{B})$ é um espaço de Banach. Denotemos por $i_{K}: C_{K}(\mathcal{B}) \hookrightarrow C_{C}(\mathcal{B})$ a inclusão canonica. O sistema indutivo $\left(C_{K}(B), i_{K}\right)_{K}$ define sobre $C_{C}(B)$ uma topologia localmente convexa, chamada topologia do limite indutivo. Ela está caracterizada pela seguinte propriedade universal: se ł́ é um espaço vetorial topológico localmente convexo. e $\phi:(C(B) \longrightarrow \mathfrak{F}$ é uma transformaçào linear, entào $\phi$ é contínua na topologia do limite indutivo se e somente se $\phi i_{K}: C_{K}(B) \longrightarrow \mathfrak{F} \dot{c}$ contínua, para todo subconjunto compacto $K$ de $G([37]$, II-14.3).

Sobre $C_{c}(\mathcal{B})$ podemos considerar outras topologias, induzidas pelas normas $\|\cdot\|_{p}$, para todo $p \in[1, \infty)$ : se $f \in C_{c}(\mathcal{B})$. entào $\|f\|_{r}=\left(\int_{G}\|f(t) d t\|^{p}\right)^{1 / r}$. O completamento de $C_{c}(\mathcal{B})$ com respeito à norma $p$ é denotado por $\mathfrak{L}^{\prime}(\mathcal{B})$. No caso $p=1$, o denotaremos também por $L^{1}(\mathcal{B})$. Os espaços $\mathfrak{L}^{p}(\mathcal{B})$ podem ser definidos para qualquer fibrado de Banach, e seus elementos podem ser vistos como seçōes do fibrado. De fato. $\mathfrak{L}^{\prime}(\mathcal{B})$ está formado pelas seçōes localmente mensuráveis $f$ de $\mathcal{B}$ que se anulam fora de subconjuntos $\sigma$-compactos de $G$, e tais que $\int_{G}\|f(t)\|^{r} d t<x$ : naturalmente, seções que diferem apenas sobre um conjunto de medida nula devem ser identificadas (consultar [37], II-15.i e II-15.9).

Como é claro, a convergencia em $C_{C}(B)$ com a topologia do limite indutivo implica a convergencia em $\mathfrak{L}^{p}(\mathcal{B}), \forall p$.

$C_{c}(\mathcal{B})$ e $L^{1}(\mathcal{B})$ são $*$-álgebras com as operações:

$$
\begin{gathered}
f * g(t)=\int_{G} f(r) g\left(r^{-1} t\right) d r \\
f^{*}(t)=\Delta(t)^{-1} f\left(t^{-1}\right)^{*}
\end{gathered}
$$

onde $\Delta: G \longrightarrow \mathbb{P}$ é a funçào modular sobre o grupo $G$ (sempre consideraremos medidas de Haar à esquerda). $L^{1}(\mathcal{B})$ é de fato uma *-álgebra de Banach com estas operaçōes ([37], VIII-5.6). A $C^{*}$-álgebra envolvente de $L^{1}(\mathcal{B})$ é chamada a $C^{*}$-álgebra seccional (plena) do fibrado de Fell $\mathcal{B}$, e denotada por $C^{*}(\mathcal{B})$. Tem-se que $L^{1}(\mathcal{B}) \subseteq C^{* *}(\mathcal{B})$. Por [37], VIII-17.3. cada representação $\pi$ sobre um espaço de Hilbert $H$ (ver Definição $2.2 \overline{1}$ ) pode ser "integrada" a uma representação $\int \pi$ de $C^{*}(\mathcal{B})$, de forma tal que para toda $f \in L^{1}(\mathcal{B}), h, k \in H$ se tem:

$$
\left\langle\left.\left(\int \pi\right)(f)\right|_{h} \cdot k\right\rangle=\int_{G}\langle\pi(f(t)) h, k\rangle d t .
$$


Inversamente, cada representação de $C^{* *}(\mathcal{B})$ pode ser obtida como a representação integrada de uma única representaçào do fibrado $\mathcal{B}$. Assim, temos que $\mathcal{B}$ e $C^{*}(\mathcal{B})$ tem a mesma teoria de representaçōes.

\section{1. ("-álgebra reduzida de um fibrado de Fell. Amenabilidade}

Em [50], Ng definiu a ('*álgebra reduzida de um fibrado de Fell sobre um grupo localmente compacto, generalizando a definiçào de Exel para fibrados sobre grupos discretos dada en [28]. A definiçào é a seguinte: $C_{r}^{*}(\mathcal{B})$ é o fecho em $\mathcal{L}\left(L^{2}(\mathcal{B})\right)$ de $\Lambda\left(L^{1}(\mathcal{B})\right)$, onde $\Lambda_{f} \xi=f * \xi, \forall f \in C_{c}(\mathcal{B}) \subseteq L^{1}(\mathcal{B})$, $\xi \in C_{c}(\mathcal{B}) \subseteq L^{2}(\mathcal{B})$. No caso de ser $G$ um grupo localmente compacto nào discreto, não é imediato que $\Lambda$, definida por convolução entre funções contínuas, possa ser estendida a uma representação de $L^{1}(\mathcal{B})$. No que segue, daremos uma demonstração deste fato, diferente da fornecida por $\mathrm{Ng}$, e que nós achamos mais apropriada do ponto de vista da teoria dos fibrados de Fell. Posteriormente provamos que se $\mathcal{A}$ é um subfibrado de Fell cle $B$, entào $C_{r}^{*}(\mathcal{A})$ é uma sub-('*álgebra de $C_{r}^{* *}(\mathcal{B})$.

Se $\mathcal{B}=\left(B_{t}\right)_{t \in G}$ é um fibrado de Fell sobre o grupo localmente compacto $G$, com fibra $B_{e}$ sobre a unidade e de $G_{i}$, entào $L^{2}(\mathcal{B})$ é o $B_{\epsilon}$-módulo de Hilbert à direita obtido completando $C_{c}(\mathcal{B})$ com respeito à norma definida pelo $B_{\epsilon}$-produto interno: $\langle\xi, \eta\rangle=\int_{G} \xi(s)^{*} \eta(s) d s$. Se $b_{t} \in B_{t}$, seja

$$
\left.\left(\Lambda_{l, t} \xi\right)\right|_{s}=b_{t} \xi\left(t^{-1} s\right), \forall \xi \in C_{c}(\mathcal{B}) \text {. }
$$

Temos que $\Lambda_{b_{t}} \xi \in C_{c}(\mathcal{B})$. e $\sup \left(\Lambda_{b_{t}} \xi\right) \subseteq t \sup (\xi)$, pois se $\xi(s)=0$, entào $0=b_{t} \xi\left(t^{-1} t s\right)=\left(\Lambda_{b_{t}} \xi\right)(t s)$. Por outro lado:

$$
\begin{aligned}
\left\langle\Lambda_{b_{t}} \xi . \Lambda_{b_{t}} \xi\right\rangle & =\int_{G}\left(\Lambda_{b_{t}} \xi\right)(s)^{*}\left(\Lambda_{b_{t}} \xi\right)(s) d s \\
& =\int_{G}\left(b_{t} \xi\left(t^{-1} s\right)\right)^{*}\left(b_{t} \xi\left(t^{-1} s\right)\right) d s \\
& =\int_{G} \xi\left(t^{-1} s\right)^{*} b_{t}^{*} b_{t} \xi\left(t^{-1} s\right) d s \\
& \leq \int_{G} \xi\left(t^{-1} s\right)^{*}\left\|b_{t}^{*} b_{t}\right\| \xi\left(t^{-1} s\right) d s \\
& =\left\|b_{t}\right\|^{2} \int_{G} \xi(s)^{*} \xi(s) d s \\
& =\left\|b_{t}\right\|^{2}\langle\xi, \xi\rangle
\end{aligned}
$$

donde concluímos que $\Lambda_{b_{t}}$ pode ser estendido a todo $L^{2}(B)$; de fato, é fácil verificar que $\Lambda_{b_{t}}$ é adjuntável, e $\Lambda_{b_{i}}^{*}=\Lambda_{b_{i}}$.

Definamos $\Lambda: \mathcal{B} \longrightarrow \mathcal{L}\left(L^{2}(\mathcal{B})\right)$ dado por $b \longmapsto \Lambda_{b}$. É imediato que $\Lambda$ é uma representação algébrica de $\mathcal{B}$. Queremos ver que $\Lambda$ é também contínua.

Lema 3.1. Se $\xi \in C_{c}(\mathcal{B}) \in b_{t} \in \mathcal{B}$, dado $\varepsilon>0$ existe $U \subseteq \mathcal{B}$ aberto, com $b_{t} \in U$. tal que se $b \in U$, entâo $\left\|\Lambda_{b} \xi-\Lambda_{b_{t}} \xi\right\|_{\infty}<\Xi$.

Demonstração. Suponhamos que o resultado é falso. Então existe $\varepsilon>0$ tal que para toda vizinhança aberta $l^{i}$ de $b_{t}$ existem $b_{r_{U}} \in U$ e $s_{U} \in G$ tais que $\left\|\left(\Lambda_{b_{r_{U}}} \xi\right)\left(s_{U}\right)-\left(\Lambda_{b_{t}} \xi\right)\left(s_{U}\right)\right\| \geq \varepsilon$, ou seja, $\left\|b_{r_{U}} \xi\left(r_{U}^{-1} s_{U}\right)-b_{t} \xi\left(t^{-1} s_{U^{*}}\right)\right\| \geq \Xi$. Notemos que $\sup \left(\Lambda_{b_{r_{U}}} \xi\right) \subseteq r_{U} \sup (\xi), \sup \left(\Lambda_{b_{t}} \xi\right) \subseteq t \sup (\xi)$. Como $b_{r_{u}} \longrightarrow b_{t}$, então $r_{t} \longrightarrow t$ (pois $\pi: \mathcal{B} \longrightarrow G$ é contínua). Portanto existem um compacto $K \subseteq G$ e uma vizinhança $l_{0}$ de $b_{t}$ tais que $\sup \left(\Lambda_{b_{r_{U}}} \xi\right) \subseteq K, \forall U \geq U_{0}$ (aqui $U \geq U_{0}$ significa que $U \subseteq \bar{U}_{0}$ ). 
Entào $s_{U} \in K, \forall U \geq U_{0}$. e deve ter uma subrede convergente a certo $s_{0} \in K$. Podemos supor que a própria rede $s_{U} \longrightarrow s_{0}$. Mas entào:

$$
0=\left\|b_{t} \xi\left(t^{-1} s_{0}\right)-b_{t} \xi\left(t^{-1} s_{0}\right)\right\|=\lim _{U}\left\|b_{r_{U}} \xi\left(r_{U^{U}}^{-1} s_{U}\right)-b_{t} \xi\left(t^{-1} s_{U}\right)\right\| \geq \varepsilon
$$

o que é uma contradiçào.

Lembrar que $\mathfrak{L}^{2}(\mathcal{B})$ se obtém completando $C_{c}(\mathcal{B})$ com respeito à norma

$$
\|\xi\|_{2}=\left(\int_{G}\|\xi(s)\|^{2} d s\right)^{1 / 2} .
$$

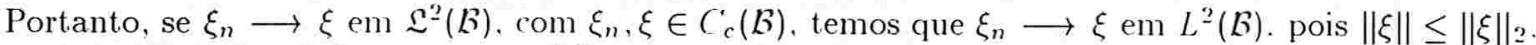
Com efeito, $\|\xi\|=\left\|\int_{G} \xi(s)^{*} \xi(s) d s\right\|^{1 / 2}$. e como $\int_{G} \xi(s)^{*} \xi(s) d s$ é um elemento positivo de $B_{\epsilon}$, existe um estado $\varphi$ de $B_{\text {f }}$ tal que $\left\|\int_{G} \xi(s)^{*} \xi(s) d s\right\|=\varphi\left(\int_{G} \xi(s)^{*} \xi(s) d s\right)$. Mas então temos:

$$
\begin{aligned}
\left\|\int_{G_{i}} \xi(s)^{*} \xi(s) d s\right\| & =\varphi\left(\int_{G} \xi(s)^{*} \xi(s) d s\right) \\
& =\int_{G} \varphi\left(\xi(s)^{*} \xi(s)\right) d s \\
& \leq \int_{G}\left\|\xi(s)^{*} \xi(s)\right\| d s \\
& =\|\xi\|_{2}^{2}
\end{aligned}
$$

Por outro lado, é claro que se $b_{r} \longrightarrow b_{t}$, então $\Lambda_{b_{r}} \xi \longrightarrow \Lambda_{b_{t}} \xi$ em $\|\cdot\|$, pois $\|\xi\|_{2} \leq \lambda(\sup (\xi))\|\xi\|_{\infty}$ (sendo $\lambda$ a medida de Haar à esquerda em $G$ ), e então pelo Lema $3.1 \Lambda_{b_{r}} \xi \longrightarrow \Lambda_{b_{t}} \xi$ em $\|\cdot\|_{\infty}$, e portanto $\Lambda_{b_{r}} \xi \longrightarrow \Lambda_{b_{t}} \xi \mathrm{em}\|\cdot\|_{2}$ e $\|\cdot\|$.

Proposiçào 3.2. Ś ja $\xi \in L^{2}(B)$. Entrio $B \longrightarrow L^{2}(\mathcal{B})$ tal que $b \longmapsto \Lambda_{b} \xi \epsilon$ contínua.

Demonstração. Fixemos $b \in \mathcal{B}$. e seja $b_{j} \rightarrow b$ em $\mathcal{B}$. Dado $\varepsilon>0$. seja $\xi_{\varepsilon} \in C_{c}(\mathcal{B})$ tal que $\left\|\xi-\xi_{\varepsilon}\right\|<\varepsilon$, e seja $j_{0}$ tal que $\|b, j\| .\|b\| \leq c, \forall j \geq j_{0}$ e certa constante $c$. Entào, se $j \geq j_{0}$ :

$$
\begin{aligned}
\left\|\Lambda_{b_{j}} \xi-\Lambda_{b} \xi\right\| & \leq\left\|\Lambda_{b} \xi-\Lambda_{b}, \xi_{\varepsilon}\right\|+\left\|\Lambda_{b_{j}} \xi_{\varepsilon}-\Lambda_{b} \xi_{\varepsilon}\right\|+\left\|\Lambda_{b} \xi_{\varepsilon}-\Lambda_{b} \xi\right\| \\
& \leq\left\|b_{j}\right\|\left\|\xi-\xi_{\varepsilon}\right\|+\left\|\Lambda_{b_{j}} \xi_{\varepsilon}-\Lambda_{b} \xi_{\varepsilon}\right\|+\|b\|\left\|\xi_{\varepsilon}-\xi\right\| \\
& <2 c \Xi+\left\|\Lambda_{b j} \xi_{\varepsilon}-\Lambda_{b} \xi_{\varepsilon}\right\|
\end{aligned}
$$

Segue que $\lim \sup _{i}\left\|\Lambda_{b_{j}} \xi-\Lambda_{b} \xi\right\| \leq 2 c \varepsilon . \forall \varepsilon>0$, e portanto $\Lambda_{b j} \xi \longrightarrow \Lambda_{b} \xi \mathrm{em}\|\cdot\|$.

DEFiniçÃo 3.3. A representação $\Lambda: \mathcal{B} \longrightarrow \mathcal{L}\left(L^{2}(\mathcal{B})\right)$ definida acima é chamada representação regular do fibrado de Fell $\mathcal{B}$. $\Lambda_{b}$ s é a única extensào contínua a todo $L^{2}(\mathcal{B})$ da aplicaçào $C_{c}(\mathcal{B}) \longrightarrow$ $C_{c}(\mathcal{B})$ tal que, se $\xi \in C_{c}(\mathcal{B}), t \in G$, entào $\left.\Lambda_{b_{s}}(\xi)\right|_{t}=b_{s} \xi\left(s^{-1} t\right)$.

TEOREMA 3.4. Existe uma única representação não degenerada

$$
\text { A : } L^{1}(\mathcal{B}) \longrightarrow \mathcal{L}\left(L^{2}(\mathcal{B})\right)
$$

sobre o módulo de Hilbert $L^{2}(\mathcal{B})$. dada por $f \longmapsto \Lambda_{f}$. onde $\Lambda_{f}(\xi)=f * \xi . \forall f \in C_{c}(\mathcal{B}) \subseteq L^{1}(\mathcal{B})$, $\xi \in C_{c}(\mathcal{B}) \subseteq L^{2}(\mathcal{B})$. 
Demonstraçào. A Proposição 3.2 nos diz que $\Lambda: \mathcal{B} \rightarrow \mathcal{L}\left(L^{2}(\mathcal{B})\right)$ é uma representaçào de Fréchet, no sentido da Definiçào VIII-8.2 de [37]. Podemos aplicar então a Proposição VIII-11.3 de [37] para concluir que $\Lambda$ é integrável, quer dizer, existe uma representaçào $1: C_{c}(\mathcal{B}) \longrightarrow B\left(L^{2}(\mathcal{B})\right)$ tal que:

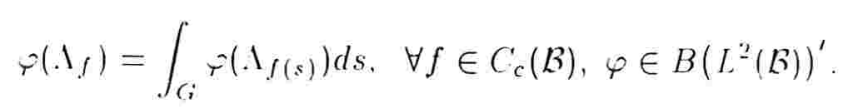

Além do mais, $\Lambda$ é única. Pomos simplesmente $\Lambda_{f}=\int_{G} \Lambda_{f(s)} d s$.

Ainda queremos ver que $\forall f \in C^{\prime}(\mathcal{B})$. tem-se $\Lambda_{f} \in \mathcal{L}\left(L^{2}(\mathcal{B})\right)$. Agora. dados $\xi, \eta \in L^{2}(\mathcal{B})$, a

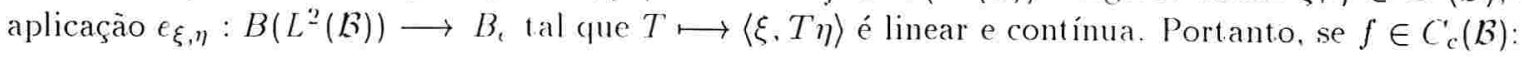

$$
\epsilon_{\xi, \eta \eta}\left(\Lambda_{f}\right)=\epsilon_{\xi, \eta)}\left(\int_{G} \Lambda_{f(s)} d s\right)=\int_{G} \epsilon_{\xi, \eta}\left(\Lambda_{f(s)} d s\right)=\int_{G}\left\langle\xi \ldots \Lambda_{f(s)}(\eta)\right\rangle d s,
$$

ou seja:

$$
\left\langle\xi, \Lambda_{f}(\eta)\right\rangle=\int_{G}\left\langle\xi, \Lambda_{f(s)}(\eta)\right\rangle d s
$$

Em particular:

$$
\begin{aligned}
\left\langle\xi, \Lambda_{f} \cdot(\eta)\right\rangle & =\int_{G}\left\langle\xi, \Lambda_{f} \cdot(s)(\eta)\right\rangle d s \\
& =\int_{G}\left\langle\xi, \Lambda_{\left.\left.\Delta\left(s^{-1}\right) f\left(s^{-1}\right) \cdot(\eta)\right)\right\rangle d s}\right. \\
& =\int_{G} \Delta\left(s^{-1}\right)\left\langle\xi, \Lambda_{f\left(s^{-1}\right)} \cdot(\eta)\right\rangle d s \\
& =\int_{G} \Delta\left(s^{-1}\right)\left(\Delta(s)\left\langle\Lambda_{f(s)}(\xi), \eta\right\rangle\right) d s \\
& =\int_{G}\left\langle\eta, \Lambda_{f(s)}(\xi)\right\rangle^{*} d s \\
& =\left(\int_{G}\left\langle\eta, \Lambda_{f(s)}(\xi)\right\rangle d s\right)^{*} \\
& =\left(\left\langle\eta, \Lambda_{f}(\xi)\right\rangle\right)^{*} \\
& =\left\langle\Lambda_{f}(\xi), \eta\right\rangle
\end{aligned}
$$

$\operatorname{Assim} \Lambda_{f}^{*}=\Lambda_{f}$, , e portanto $\Lambda_{f} \in \mathcal{L}\left(L^{2}(\mathcal{B})\right)$

A representaçào $\Lambda: C_{c}(\mathcal{B}) \longrightarrow \mathcal{L}\left(L^{2}(\mathcal{B})\right)$ é contínua na norma $\|\cdot\|_{1}$ : pela equação (4) temos:

$$
\left\|\left\langle\xi, \Lambda_{f}(\eta)\right\rangle\right\| \leq \int_{G}\|\xi\|\left\|\Lambda_{f(t)} \eta\right\| d t \leq \int_{G}\|f(t)\|\|\xi\|\|\eta\| d t=\|f\|_{1}\|\xi\|\|\eta\| .
$$

Em particular:

$$
\left\|\Lambda_{f} \xi\right\|^{2}=\left\|\left\langle\Lambda_{f} \xi \ldots \Lambda_{f} \xi\right\rangle\right\|=\left\|\left\langle\Lambda_{f \cdot * f} \xi, \xi\right\rangle\right\| \leq\left\|f^{*} f\right\|_{1}\|\xi\|^{2} \leq\|f\|_{1}^{2}\|\xi\|^{2},
$$

donde $\left\|\Lambda_{f}\right\| \leq\|f\|_{1}$. Portanto, $\Lambda$ se estende de forma única por continuidade a uma representação de $L^{1}(\mathcal{B})$. Esta representaçào é nào degenerada, pois $C_{c}(\mathcal{B}) * C_{c}(\mathcal{B})$ é denso em $C_{c}(\mathcal{B})$ na topologia do limite indutivo, e portanto também em $L^{2}(\mathcal{B})$. 
DEFiniçÃo 3.5. A representação $\Lambda: L^{1}(\mathcal{B}) \longrightarrow \mathcal{L}\left(L^{2}(\mathcal{B})\right)$ definida no Teorema 3.4 é chamada representação regular (à esquerda) de $L^{1}(\mathcal{B})$. e $C_{r}^{*}(\mathcal{B}):=\overline{\Lambda\left(L^{1}(\mathcal{B})\right)} \subseteq \mathcal{L}\left(L^{2}(\mathcal{B})\right)$ é chamada $C^{*}$-álgebra reduzida de $\mathcal{B}$. Se o fibrado de Fell provém de uma ação parcial torcida $\alpha$ de $G$ sobre a $C^{*}$-álgebra $A$, então a correspondente $C^{\prime *}$-álgebra reduzida é chamada produto cruzado reduzido, e é denotada por $A \rtimes_{\alpha, r} G$.

ObservaÇão 3.6. Em [50]. Ng mostrou que se a é uma ação global de $G$ sobre $A$, então o produto cruzado reduzido usual coincide com o produto cruzado reduzido que acabamos de definir (ver também [28], 3.8). Recentemente. Exel generalizou este resultado, provando que, se $\pi: \mathcal{B} \longrightarrow$ $B(H)$ é uma representaçào de $B$ tal que $\left.\pi\right|_{B_{c}}$ é fiel, e consideramos a representação $\pi_{\lambda}: \mathcal{B} \longrightarrow$ $B\left(L^{2}(G, H)\right)$, dacla por $\pi_{\lambda}\left(b_{t}\right)=\lambda_{t} \pi\left(b_{t}\right)$. onde $\lambda$ é a representação regular à esquerda de $G$ sobre $L^{2}(G)$, entào a representaçào integrada de $\pi_{\lambda}$ define uma representaçào de $C^{*}(\mathcal{B})$, que se fatoriza por uma representação fiel de $C_{r}^{*}(\mathcal{B})$. Portanto. $C_{r}^{*}(\mathcal{B}) \cong \pi_{\lambda}\left(C^{*}(\mathcal{B})\right)$.

Lembrar que um grupo ( $i$ é dito "amenable" se existe um estado $\mu$ da $C^{\prime *}$-álgebra $L^{\infty}(G)$ invariante à esquerda, ou seja tal que $\mu(f)=\mu\left(\lambda_{t}(f)\right) . \forall f \in L^{\infty}(G), t \in G$, onde $\left.\lambda_{t}(f)\right|_{s}=f\left(t^{-1} s\right)$. Esta condição de $G$ é equivalente à seguinte: a representaçào regular $\lambda: L^{1}(G) \longrightarrow B\left(L^{2}(G)\right)$ se estende a um homomorfismo injetivo $\lambda: C^{\prime *}(G) \longrightarrow B\left(L^{2}(G)\right)$; lembrar também que a $C^{*}$-álgebra reduzida do grupo $G$ é definicla precisamente como $C_{r}^{*}(G):=\lambda\left(C^{*}(G)\right) \subseteq B\left(L^{2}(G)\right)$. Portanto, $G$ é amenable exatamente quando $\lambda: C^{*}(G) \longrightarrow C_{r}^{*}(G)$ é um isomorfismo. Entre os exemplos de grupos amenable estão todos os grupos abelianos e os grupos compactos. Entre os grupos discretos, a classe de grupos amenable é fechada por subgrupos, quocientes, extensões e limites indutivos. Exemplos de grupos que não são amenable são os grupos livres em dois ou mais geradores. A seguinte definição generaliza esta noção a fibrados de Fell.

DEFINiÇÃo 3.T. A representação regular 1 induz uma representação, também chamada regular e denotada por $\Lambda$, de $C^{*}(\mathcal{B})$. Quando

$$
\Lambda: C^{*}(\mathcal{B}) \longrightarrow C_{r}^{*}(\mathcal{B})
$$

é um isomorfismo, dizemos que $B$ é "amenable".

Encerrando esta seção, mostramos que se $\mathcal{A}$ é um subfibrado de Fell do fibrado $\mathcal{B}$, então $C_{r}^{*}(\mathcal{A})$ é uma sub- $C^{*}$-álgebra de $C_{r}^{*}(\mathcal{B})$.

DEFINiÇÃo 3.8. Se $\mathcal{B}=\left(B_{r}\right)_{x \in \lambda}$ é um fibrado de Banach sobre o espaço topológico de Hausdorff $\mathrm{X}$, dizemos que $\mathcal{A} \subseteq \mathcal{B}$ é um subfibrado de Banach de $\mathcal{B}$ se $\mathcal{A}$ é fechado pelas operações de $\mathcal{B}$, e a projeção $p$ de $\mathcal{B}$ restrita a $\mathcal{A}$ é aberta, ou seja, para todo aberto $U \subseteq \mathcal{B}, p(U \cap \mathcal{A})$ é um aberto de $X$. Um subfibrado de Fell $\mathcal{A}$ de um fibrado de Fell $\mathcal{B}$ sobre o grupo localmente compacto $G$ é um subfibrado de Banach de $B$ que também é fechado sob as operações de produto e involução. Um subfibrado de Banach $\mathcal{A}$ de um fibrado cle Fell $\mathcal{B}$ é chamado ideal à direita se $\mathcal{A} \mathcal{B} \subseteq \mathcal{A}$, e à esquerda se $\mathcal{B} \mathcal{A} \subseteq \mathcal{A}$. Se $\mathcal{A}$ é ideal à esquerda e à direita de $\mathcal{B}$, diremos que ele é um ideal bilateral, ou simplesmente um ideal de $\mathcal{B}$, e indicaremos esta relação por $\mathcal{A} \triangleleft \mathcal{B}$.

Observaçào 3.9. É imediato verificar que se $\mathcal{A}$ é um subfibrado de Banach (de Fell) de $\mathcal{B}$, entào ele é um fibrado de Banach (respectivamente: de Fell) sobre $p(\mathcal{A})$, que é um subespaço aberto de $X$ 
(respectivamente: um subgrupo aberto de $G$ ). No caso em que $\mathcal{B}$ é um fibrado de Fell sobre $G$, a medida de Haar sobre $p(\mathcal{A})$ é a restrição a $p(\mathcal{A})$ da medida de Haar de $G$. Assim, temos uma inclusào natural $L^{1}(\mathcal{A}) \hookrightarrow L^{1}(\mathcal{B})$.

Proposiçào 3.10. Ś Á é um subfibrado de Fell de $\mathcal{B}$, então $C_{r}^{*}(\mathcal{A}) \subseteq C_{r}^{*}(\mathcal{B})$.

Demonstração. Seja $\pi: B \longrightarrow B(H)$ uma representação de $\mathcal{B}$ sobre o espaço de Hilbert $H$, tal que $\left.\pi\right|_{B_{\varepsilon}}$ é fiel. Então $\rho:=\left.\pi\right|_{\mathcal{A}}: \mathcal{A} \longrightarrow B(H)$ é uma representaçào de $\mathcal{A}$, tal que $\left.\rho\right|_{A_{c}}$ é fiel. Seja $\pi_{\lambda}: \mathcal{B} \longrightarrow B\left(L^{2}\left(G^{\prime}\right) \otimes H\right)$ tal que $\pi_{\lambda}\left(b_{t}\right)=\lambda_{t} \odot \pi\left(b_{t}\right)$, onde $\lambda: G \longrightarrow B\left(L^{2}(G)\right)$ é a representaçào regular à esquerda de $G^{\prime}$. ou seja: $\forall \xi \in L^{2}(G),\left.\lambda_{t}(\xi)\right|_{s}=\xi\left(t^{-1} s\right)$. Da mesma forma, definamos $\rho_{\lambda}: \mathcal{A} \longrightarrow B\left(L^{2}(G) \otimes H\right)$. É claro que $\rho_{\lambda}=\left.\pi_{\lambda}\right|_{\mathcal{A}}$. Integrando $\pi_{\lambda}$ e $\rho_{\lambda}$, obtemos representaçòes de $C^{*}(\mathcal{B})$ e $C^{*}(\mathcal{A})$. que também chamamos $\pi_{\lambda}$ e $\rho_{\lambda}$ respectivamente, e é claro de novo que $\left.\rho_{\lambda}\right|_{L^{1}(\mathcal{A})}$ coincide com $\left.\pi_{\lambda}\right|_{L^{1}(\mathcal{A})}$. Agora, por 3.6 de [30], temos que $\pi_{\lambda}$ se fatoriza através de um isomorfismo $\tilde{\pi}_{\lambda}: C_{r}^{*}(\mathcal{B}) \longrightarrow \overline{\pi_{\lambda}\left(C^{*}(\mathcal{B})\right)}$, e $\rho_{\lambda}$ através de um isomorfismo $\dot{\rho}_{\lambda}: C_{r}^{*}(\mathcal{A}) \longrightarrow \overline{\rho_{\lambda}\left(L^{1}(\mathcal{A})\right)} \cong C_{r}^{*}(\mathcal{A})$. Portanto, $\tilde{\pi}_{\lambda}^{-1} \tilde{\rho}_{\lambda}: C^{* *}(\mathcal{A}) \longrightarrow \overline{C_{C}(\mathcal{A})} \subseteq C_{r}^{*}(\mathcal{B})$ é um isomorfismo. Assim, concluímos que $C_{r}^{*}(\mathcal{A})$ se identifica naturalmente $\operatorname{com} \overline{C_{c}(\mathcal{A})}$ dentro de $C_{r}^{*}(\mathcal{B})$.

Observação 3.11. No caso em que o grupo $G$ seja discreto, há uma demonstração bem mais direta da Proposiçào 3.10. que comentaremos a seguir.

Se $A$ e $B$ são *álgebras, e $A$ é um $B$-módulo à direita, dizemos que $E: A \longrightarrow B$ é uma $(A-B)$ esperança condicional, se $E$ é um homomorfismo de $B$-módulos à direita que preserva a involução, i.e.

- $E(a b)=E(a) b, \forall a \in A, b \in B$.

- $E\left(a^{*}\right)=E(a)^{*}, \forall a \in A$.

Por [28], existe uma esperança condicional fiel $E_{\mathcal{B}}: C_{r}^{*}(\mathcal{B}) \longrightarrow B_{\epsilon}$, ou seja. $E\left(a^{*} a\right)=0 \Longleftrightarrow a=0$. Suponhamos que $B$ é uma $C^{*}$-álgebra, $G$ é um grupo discreto, e que $\left(B_{t}\right)_{t \in G}$ é uma graduação de $B$. isto é ([28], 3.1):

- Os $B_{t}$ sào uma coleçào linearmente independente de subespaços de Banach de $B$, e tais que $B=\overline{\bigoplus_{t \in G} B_{t}}$.

- $B_{t}^{*}=B_{t-1}, \forall t \in G$.

- $B_{s} B_{t} \subseteq B_{s t}, \forall s, t \in G$.

A graduação é chamada topológica se existe uma esperança condicional $F: B \longrightarrow B_{\epsilon}$ tal que $\left.F\right|_{B_{\epsilon}}=$ $i d_{B_{e}},\left.F\right|_{B_{t}}=0, \forall t \neq e$.

A coleção $\left(B_{t}\right)$ define naturalmente um fibrado de Fell $\mathcal{B}$ sobre $G$. Por [37], VIII-16.11, existe um epimorfismo natural $C^{* *}(\mathcal{B}) \longrightarrow B$ e. se a graduação for topológica, existe um outro epimorfismo $B \longrightarrow C_{r}^{*}(\mathcal{B})$; este epimorfismo é um isomorfismo exatamente quando a esperança condicional $F$ é fiel, o que permite caracterizar facilmente a $C^{\prime *}$-álgebra reduzida de um fibrado de Fell entre as $C^{*}$-álgebras que têm uma graduaçào topológica dada pelas fibras do fibrado e certa esperança condicional.

Voltando à Proposiçào 3.10, $C_{r}^{*}(\mathcal{B})$ possui uma esperança condicional fiel $E: C_{r}^{*}(\mathcal{B}) \longrightarrow B_{e}$. Se $A$ é o fecho de $L^{1}(\mathcal{A})$ em $C_{r}^{*}(\mathcal{B})$. e $F=\left.E\right|_{A}$, então o par $\left(\left(A_{t}\right)_{t \in G}, F\right)$ é uma graduação topológica de $A$ e $F$ é fiel, donde concluímos que $A \cong C_{r}^{*}(\mathcal{A})$ naturalmente. 
DEFiniÇÃo 3.12. Sejam $\mathcal{A}=\left(A_{t}\right)_{t \in G}$ e $\mathcal{B}=\left(B_{t}\right)_{t \in G}$ fibrados de Fell sobre o grupo localmente compacto $G$, e seja $\phi: \mathcal{A} \longrightarrow \mathcal{B}$, tal que $\phi: \mathcal{A}_{d} \longrightarrow \mathcal{B}_{d}$ é um homomorfismo de fibrados de Fell sobre grupos discretos (ver Definiçào 2.5). Sé $\phi$ é contínua, dizemos que $\phi$ é um homomorfismo de $\mathcal{A}$ em $\mathcal{B}$.

Observaçào 3.13. Suponhamos que $\dot{\phi}: \mathcal{A} \longrightarrow \mathcal{B}$ é um homomorfismo de fibrados de Fell. Então, se $f \in L^{1}(\mathcal{A})$, temos que $\phi^{1}(f): G \longrightarrow \mathcal{B}$ tal que $\phi^{1}(f)(t)=\phi(f(t))$ está em $L^{1}(\mathcal{B})$, e $\left\|\phi^{1}(f)\right\|_{1} \leq\|f\|_{1}$. Por outro lado, é imediato que $\phi^{1}$ é um homomorfismo de *álgebras, e portanto se estende de forma única a um homomorfismo $C^{*}(\phi): C^{*}(\mathcal{A}) \longrightarrow C^{* *}(\mathcal{B})$. Assim, obtemos um funtor da categoria dos fibrados de Fell sobre $G$ na categoria das $C^{*}$-álgebras; de fato, este funtor é a composição do funtor $A \longmapsto C^{* *}(A)$ da categoria das *álgebras com unidade aproximada e contraçōes na categoria de $C^{\prime *}$-álgebras. com o funtor: $\mathcal{A} \longmapsto L^{1}(\mathcal{A}),(\mathcal{A} \stackrel{0}{\longrightarrow} \mathcal{B}) \longmapsto\left(L^{1}(\mathcal{A}) \stackrel{\phi^{1}}{\longrightarrow} L^{1}(\mathcal{B})\right)$.

\section{2. ('*álgebras seccionais de produtos tensoriais de fibrados de Fell}

O objetivo desta seçào é de comparar os produtos tensoriais de $C^{*}$-álgebras associadas a fibrados de Fell com as $C^{*}$-álgebras associadas aos produtos tensoriais dos fibrados de Fell. O principal resultado é o Teorema 3.21. Começamos com uma ligeira generalizaçào de [37]. II-14.6:

LEMA 3.14. Sejam B um fibrado de Banach sobre o espaço topológico $\mathrm{X}$. $€ F \subseteq C_{c}(\mathcal{B})$ um subespaço vetorial de $C_{c}(B)$ tal que existe um subespaço vetorial $\Theta \subseteq C_{c}\left(\mathrm{Y}^{*}\right)$. denso na topologia do limite indutivo, $e$ tal que:

1. $\forall f \in F, \theta \in \Theta$. tem-se que $\theta f \in F$.

2. $F(x)=\{f(x): f \in F\}$ é denso em $B_{x}, \forall x \in \mathrm{X}$.

Entào $F$ é denso $\mathrm{\epsilon m} C_{c}(B)$ na topologia do limite indutivo (II-14.6 de [37] tem exatamente o mesmo enunciado, exceto que $\Theta$ é o espaço letorial de todas as funçōes complexas contínuas sobre $X)$.

Demonstraçào. Seja $\bar{F}$ o fecho de $F$ em $C_{c}(B)$ com respeito à topologia do limite indutivo. Queremos provar que $\bar{F}=C_{c}(B)$. e portanto basta mostrar que $\bar{F}$ é denso em $C_{c}(B)$. Para isto usamos [37], II-14.6. Em primeiro lugar: $B_{x}=\overline{F(x)} \subseteq \overline{\bar{F}(x)}$, donde segue que $\bar{F}(x)$ é denso em $B_{x}$. $\forall x \in X$. Só resta provar que $\forall g \in \Theta$ e $\forall \theta: Y \longrightarrow \mathbb{Y}$ contínua, tem-se que $\theta g \in \bar{F}$. Podemos supor $\theta \in C_{c}(X)$ (porque $g \in C_{c}(B)$ ). e também que $\theta \neq 0, g \neq 0$. Agora, dado $\epsilon>0$, sejam $f \in F$ tal que $f \neq 0$ e $\|f-g\|_{\infty}<\epsilon / 2\|\theta\|_{\infty}$, e $\phi \in \Theta$ tal que $\|\theta-\phi\|_{\infty}<\epsilon / 2\|f\|_{\infty}$. Entào:

$$
\|\phi f-\theta g\|_{\infty} \leq\|(\phi-\theta) f\|_{\infty}+\|\theta(f-g)\|_{\infty} \leq\|\phi-\theta\|_{\infty}\|f\|_{\infty}+\|\theta\|_{\infty}\|f-g\|_{\infty}<\epsilon
$$

Portanto, como $\phi f \in F$, temos que $\theta g \in \bar{F}$.

Lema 3.15. Sejam $\mathcal{A}=\left(A_{t}\right)_{t \in G_{i}} \in B=\left(B_{s}\right)_{s \in H}$ fibrados de Fell, $D=C_{c}(\mathcal{A}) \odot C_{c}(\mathcal{B})$, e suponhamos que $\mathcal{A} \otimes \mathcal{B}$ é um produto tensorial de $\mathcal{A} \in \mathcal{B}$. Então existe um único homomorfismo injetivo de álgebras $j^{\prime}: D \longrightarrow C_{c}(\mathcal{A} \otimes \mathcal{B})$. tal que $j^{\prime}(f \odot g)=f \oslash g$. ou seja: $\left.j^{\prime}(f \odot g)\right|_{(t, s)}=f(t) \otimes g(s)$. $\forall f \in C_{c}(\mathcal{A}), g \in C_{c}^{\prime}(\mathcal{B}) . t \in G$. e $s \in H$. Além disso. $j^{\prime}(D)$ é denso $e m C_{c}(\mathcal{A} \otimes \mathcal{B})$ na topologia do limite indutivo.

Demonstraçào. Pela propriedacle universal do produto tensorial, existe uma única transformaçào linear $j^{\prime}: C_{c}(\mathcal{A}) \odot C_{c}(\mathcal{B}) \longrightarrow C_{c}(\mathcal{A} \odot \mathcal{B})$ tal que $j^{\prime}(f \odot g)(t, s)=f(t) \odot g(s)$. $j^{\prime}$ é um 
homomorfismo de *álgebras, como se verifica facilmente. Além disto, ele é injetivo: suponhamos que $l=\sum_{i=1}^{n} f_{i} \odot g_{i}$ é tal que $j^{\prime}(l)=0$. Entào $\left\langle j^{\prime}(l), j^{\prime}(l)\right\rangle=0$, ou seja:

$$
\int_{G \times H} \sum_{i, j=1}^{n} f_{i}(t)^{*} f_{j}(t) \odot g_{i}(s)^{*}, g_{j}(s) d(t, s)=0
$$

Mas

$$
\int_{G \times H} \sum_{i, j=1}^{n} f_{i}(t)^{*} f_{j}(t) g_{i}(s)^{*} g_{j}(s) d(t, s)=\left(\int_{G} \sum_{i, j=1}^{n} f_{i}(t)^{*} f_{j}(t) d t\right)\left(\int_{H} \sum_{i, j=1}^{n} g_{i}(s)^{*} g_{j}(s) d s\right),
$$

e portanto $\left\langle j^{\prime}(I), j^{\prime}(I)\right\rangle=\langle I . I\rangle$, onde $l$ está sendo visto como elemento de $L^{2}(\mathcal{A}) \odot L^{2}(\mathcal{B})$, e o produto interno $\langle l, l\rangle$ toma valores sobre $A_{\epsilon} \odot B_{c}$. (Como $\langle l, l\rangle=0$, tem-se que $l=0$.

Para ver que $j^{\prime}(D) \dot{e}$ denso em $(\cdot(\mathcal{A} \otimes B)$ na topologia do limite indutivo, usamos o Lema 3.14 acima. É claro que $j^{\prime}(D)(t, s)$ é denso em $(\mathcal{A} \otimes \mathcal{B})_{(t, s)}, \forall(t, s) \in(i \times H$; por outro lado, se $\Theta=C_{c}(G) \odot C_{c}^{\prime}(H)$, sejam $\theta \in \Theta, c l \in D$. digamos $\theta=\sum_{i} \phi_{i} \odot \psi_{i}, l=\sum_{j} f_{j} \odot g_{j}$. Então:

$$
\left.\theta j^{\prime}(l)\right|_{(t, s)}=\left(\sum_{i} \phi_{i}(l) \iota_{i}(s)\right)\left(\sum_{i} f_{j}(t) \quad g_{j}(s)\right)=\sum_{i} \sum_{j}\left(\phi_{i} f_{j}\right)(t) \quad\left(\iota_{i}, g_{j}\right)(s)=j^{\prime}\left(l^{\prime}\right)(t, s),
$$

onde $l^{\prime}=\sum_{i} \sum_{j} \phi_{i} f_{j} \iota_{i} \eta_{j} \in D$. Assim. concluímos que $j^{\prime}(D)$ é denso em $\left({ }_{c}(\mathcal{A} \otimes \mathcal{B})\right.$.

Proposiçào 3.16. Sejam $\mathcal{A}=\left(A_{t}\right)_{t \in G} \in B=\left(B_{s}\right)_{s \in H}$ fibrados de Fell. Então existe um único isomorfismo $j: C^{\prime *}(\mathcal{A}) \bigotimes_{\text {max. }} C^{\prime *}(\mathcal{B}) \longrightarrow C^{* *}\left(\mathcal{A} \bigotimes_{\text {max. }} \mathcal{B}\right)$, tal que $\left.j(f \otimes g)\right|_{(t, s)}=f(t) \otimes g(s), \forall f \in$ $C_{c}(\mathcal{A}), g \in C_{c}(\mathcal{B}), \epsilon(t, s) \in G \times H$

Demonstração. Se $H$ é um espaço de Hilbert e $\mathcal{C}=\left(C_{t}\right)_{t \in G}$ é um fibrado de Fell, então existe uma bijeção $R(\mathcal{C}, \mathcal{H})$ e $R\left(C^{*}(\mathcal{C}), \mathcal{H}\right)$ (notação introduzida na página 41 ), que a cada $\pi \in R(\mathcal{C}, \mathcal{H})$ associa a representaçào integrada $\int_{C} \pi$ de $C^{*}(\mathcal{C})$. determinada pelos seus valores em $C_{c}(\mathcal{C})$ : se $f \in$ $C_{c}(\mathcal{C})$ e $h \in \mathcal{H}$, então $\left.\left(\int_{G_{i}} \pi\right) f\right|_{h}=\int_{G i} \pi(f(t)) h d t$ (ver [37]. VIII-17.3. e a introdução ao presente capítulo). Notar também que se $C^{\prime \prime}=\left(C_{s}^{\prime \prime}\right)_{s \in H}$ é um outro fibrado de Fell, então $R\left(\mathcal{C}, \mathcal{C}^{\prime}, \mathcal{H}\right) \longrightarrow$ $R\left(C^{*}(\mathcal{C}), C^{*}\left(\mathcal{C}^{\prime}\right), \mathcal{H}\right)$ tal que $\left(\pi, \pi^{\prime}\right) \longmapsto\left(\int_{G} \pi, \int_{H} \pi^{\prime}\right)$ também é uma bijeção. pois os correspondentes integrandos comutam.

Por outro lado, lembremos que. pela Proposição 2.25 , temos uma bijeção entre $R\left(\mathcal{C}, \mathcal{C}^{\prime}, \mathcal{H}\right)$ e $R\left(\mathcal{C} \otimes_{\text {máx }} \mathcal{C}^{\prime}, \mathcal{H}\right)$, dada por $\left(\pi_{1}, \pi_{2}\right) \longmapsto \pi_{1} \times \pi_{2}$, onde $\left(\pi_{1} \times \pi_{2}\right)(a @ b)=\pi_{1}(a) \pi_{2}(b)$ (em particular, isto é válido quando $G$ e $H$ sào o grupo trivial, e então a correspondencia acima vira uma correspondencia entre representações de $C^{*}$-álgebras).

Sejam $D$ e $j^{\prime}: D \longrightarrow C_{c}\left(\mathcal{A} \bigotimes_{\text {máx }} \mathcal{B}\right)$ como no Lema 3.15 . Os comentários acima implicam que $C^{*}(\mathcal{A}) \bigotimes_{\text {máx }} C^{*}(\mathcal{B})$ é um completamento de $D$ com respeito à norma:

$$
\left\|\sum_{i} f_{i} \odot g_{i}\right\|=\sup \left\{\left\|\sum_{i} \int_{G_{i}} \pi_{1}\left(f_{i}\right) \int_{H} \pi_{2}\left(g_{i}\right)\right\|:\left(\pi_{1}, \pi_{2}\right) \in R(\mathcal{A}, \mathcal{B}, \mathcal{H})\right\},
$$

e $C^{*}\left(\mathcal{A} \otimes_{\text {máx }} \mathcal{B}\right)$ é o completamento de $j(D)$ com respeito à norma:

$$
\left\|j^{\prime}\left(\sum_{i} f_{i} g_{i}\right)\right\|=\sup \left\{\left\|\int_{G \times H}\left(\pi_{1} \times \pi_{2}\right)\left(\sum_{i} f_{i} \odot g_{i}\right)\right\|:\left(\pi_{1}, \pi_{2}\right) \in R(\mathcal{A}, \mathcal{B}, \mathcal{H})\right\}
$$


Agora, se $h \in \mathcal{H}$ :

$$
\begin{aligned}
\left(\int_{G \times H}\left(\pi_{1} \times \pi_{2}\right)\left(\sum_{i} f_{i} \odot g_{i}\right)\right) h & =\int_{G \times H} \sum_{i} \pi_{1}\left(f_{i}(t)\right) \pi_{2}\left(g_{i}(s)\right) h d(t, s) \\
& =\sum_{i} \int_{G} \int_{H} \pi_{1}\left(f_{i}(t)\right) \pi_{2}\left(g_{i}(s)\right) h d s d t \\
& =\sum_{i} \int_{G} \pi_{1}\left(f_{i}(t)\right) \int_{H} \pi_{2}\left(g_{i}(s)\right) h d s d t \\
& =\sum_{i} \int_{G} \pi_{1}\left(f_{i}(t)\right)\left(\int_{H} \pi_{2}\left(g_{i}\right)\right) h d t \\
& =\sum_{i}\left(\int_{G} \pi_{1}\left(f_{i}\right) \int_{H} \pi_{2}\left(g_{i}\right)\right) h,
\end{aligned}
$$

o que mostra que $j^{\prime}: D \longrightarrow j^{\prime}(D)$ é uma isometria com estas normas, e portanto, como $D$ é denso em $C^{*}(\mathcal{A}) \bigotimes_{\text {máx }} C^{\prime *}(\mathcal{B}), j^{\prime}$ tem uma única extensão a um homomorfismo $j: C^{*}(\mathcal{A}) \bigotimes_{\text {máx }} C^{*}(\mathcal{B}) \longrightarrow$ $C^{*}\left(\mathcal{A} \otimes_{\text {máx }} \mathcal{B}\right)$, que é um isomorfismo porque $j^{\prime}(D)$ é denso em $C^{* *}\left(\mathcal{A} \otimes_{\text {máx }} \mathcal{B}\right)$, já que, por 3.15 , é denso em $C_{c}\left(\mathcal{A} \otimes_{\operatorname{máx}} \mathcal{B}\right)$ na topologia do limite indutivo, e portanto em $L^{1}\left(\mathcal{A} \bigotimes_{\text {máx }} \mathcal{B}\right)$.

Proposiçào 3.1T. Sejam $\mathcal{A}=\left(A_{t}\right)_{t \in G} \in \mathcal{B}=\left(B_{s}\right)_{s \in H}$ fibrados de Fell. $\epsilon$ suponhamos que a $\geq 3$ sào $C^{*}$-normas sobre $\mathcal{A} \odot \mathcal{B}$. Entióo existe um único homomorfismo

$$
\sigma_{\beta}^{a}: C^{* *}\left(\mathcal{A} \bigotimes_{a} \mathcal{B}\right) \longrightarrow C^{*}\left(\mathcal{A} \bigotimes_{\beta} \mathcal{B}\right)
$$

tal que $\sigma_{\beta}^{\alpha}(f \circ g)=f \bigcirc g, \forall f \in C_{c}(\mathcal{A}), g \in C_{c}(\mathcal{B})$. Além disso, $\sigma_{\beta}^{\alpha}$ é sobrejetivo.

Demonstração. Por 2.20 existe $\sigma_{\beta}^{a}: \mathcal{A} \otimes_{a} \mathcal{B} \longrightarrow \mathcal{A} \otimes_{\beta} \mathcal{B}$, homomorfismo sobrejetivo de fibrados de Fell. Agora, por 3.13, $\sigma_{\beta}^{\alpha}$ induz um homomorfismo $C^{*}\left(\mathcal{A} \otimes_{\alpha} \mathcal{B}\right) \longrightarrow C^{*}\left(\mathcal{A} \otimes_{\beta} \mathcal{B}\right)$, que continuamos chamando $\sigma_{\beta}^{a}$. Se $f \in C_{c}(\mathcal{A}), g \in C_{c}^{\prime}(\mathcal{B}),\left.\sigma_{\beta}^{\alpha}(f \otimes g)\right|_{(t, s)}=\sigma_{\beta}^{\alpha}(f(t) \otimes g(s))=f(t) \otimes g(s)=$ $f \bigcirc g(t, s)$, donde segue que $\sigma_{\beta}^{a}(f \bigcirc g)=f \bigcirc g$. Como $\operatorname{span}\left\{f \bigcirc g: f \in C_{c}(\mathcal{A}), g \in C_{c}(\mathcal{B})\right\}$ é denso em $C^{*}\left(\mathcal{A} \otimes_{a} \mathcal{B}\right)$, vemos que $\sigma_{\beta}^{\alpha}$ é único.

Para o próximo resultado, notar que $L^{2}(\mathcal{A})$ e $L^{2}(\mathcal{B})$ são módulos de Hilbert plenos sobre $A_{\epsilon}$ e $B_{e}$ respectivamente, e portanto, se $\alpha$ é uma $C^{*}$-norma sobre $A_{e} \odot B_{\epsilon}$, existe uma única $C^{*}$ norma sobre $L^{2}(\mathcal{A}) \odot L^{2}(\mathcal{B})$, que também chamamos $\alpha$, tal que o completamento $L^{2}(\mathcal{A}) \bigotimes_{\alpha} L^{2}(\mathcal{B})$ de $L^{2}(\mathcal{A}) \odot L^{2}(\mathcal{B})$ com respeito a esta norma é um módulo de Hilbert pleno sobre $A_{e} \bigotimes_{\alpha} B_{e}$, de forma que $\forall \xi_{1}, \xi_{2} \in L^{2}(\mathcal{A}), \eta_{1}, \eta_{2} \in L^{2}(\mathcal{B})$ seja (ver 1.44$)$ :

$$
\left\langle\xi_{1} \odot \eta_{1}, \xi_{2} \odot \eta_{2}\right\rangle=\left\langle\xi_{1}, \xi_{2}\right\rangle \otimes\left\langle\eta_{1}, \eta_{2}\right\rangle .
$$

Lema 3.18. Sejam $\mathcal{A}=\left(A_{t}\right)_{t \in G_{i}} \in \mathcal{B}=\left(B_{s}\right)_{s \in H}$ fibrados de Fell, e $\alpha$ uma $C^{*}$-norma sobre $\mathcal{A} \odot \mathcal{B}$. Então existe um único isomorfismo

$$
j_{2}: L^{2}(\mathcal{A}) \bigotimes_{a} L^{2}(\mathcal{B}) \longrightarrow L^{2}(\mathcal{A} \bigotimes \underset{a}{\bigotimes} \mathcal{B})
$$

tal que $j_{2}(\xi \bigcirc \eta)=\xi \subseteq \eta . \forall \xi \in C_{c}(\mathcal{A}) \subseteq L^{2}(\mathcal{A}) . \eta \in C_{c}(\mathcal{B}) \subseteq L^{2}(\mathcal{B})$. 
Demonstraçào. Seja $j^{\prime}$ a aplicação definida no Lema 3.15. Se $\xi_{1}, \xi_{2} \in C_{c}(\mathcal{A}), \eta_{1}, \eta_{2} \in C_{c}(\mathcal{B})$, $j^{\prime}\left(\xi_{1} \odot \eta_{1}\right), j^{\prime}\left(\xi_{2} \odot \eta_{2}\right) \in C_{c}^{\prime}\left(\mathcal{A} \otimes_{\alpha} \mathcal{B}\right) \subseteq L^{2}\left(\mathcal{A} \otimes_{\alpha} \mathcal{B}\right)$. Temos:

$$
\begin{aligned}
\left\langle j^{\prime}\left(\xi_{1} \odot \eta_{1}\right), j^{\prime}\left(\xi_{2} \odot \eta_{2}\right)\right\rangle & =\int_{G \times H}\left(\xi_{1} \odot \eta_{1}\right)(t, s)^{*}\left(\xi_{2} \odot \eta_{2}\right)(t, s) d(t, s) \\
& =\int_{G} \int_{H} \xi_{1}(t)^{*} \xi_{2}(t) \otimes \eta_{1}(s)^{*} \eta_{2}(s) d s d t \\
& =\left\langle\xi_{1}, \xi_{2}\right\rangle \odot\left\langle\eta_{1}, \eta_{2}\right\rangle
\end{aligned}
$$

Por outro lado, se $a \in \mathcal{A}_{\epsilon}, b \in B_{\epsilon}, \xi \in C_{c}^{\prime}(\mathcal{A})$. e $\eta \in C_{c}(\mathcal{B})$ :

$$
\left.\left(j^{\prime}(\xi \odot \eta)\right)(a \odot b)\right|_{(t, s)}=(\xi(t) \odot \eta(s))(a \odot b)=\xi(t) a \odot \eta(s) b=\left.j^{\prime}((\xi \odot \eta)(a \odot b))\right|_{(t, s)} .
$$

Portanto, $j^{\prime}$ é um homomorfismo de pré-módulos de Hilbert sobre $A_{e} \bigotimes_{a} B_{e}$, que é injetivo (3.15) e de imagem densa em $L^{2}(\mathcal{A} \otimes, B)$ : por 3.15, a imagem de $j^{\prime}$ é densa na topologia do limite indutivo de $C_{c}\left(\mathcal{A} \otimes_{\Omega} \mathcal{B}\right)$, e portanto denso em $L^{\prime 2}\left(\mathcal{A} \otimes_{\Omega} \mathcal{B}\right)$. Logo, $j^{\prime}$ se estende de forma única a um isomorfismo $j_{2}: L^{2}(\mathcal{A}) \otimes_{\alpha} L^{2}(\mathcal{B}) \longrightarrow L^{2}\left(\mathcal{A} \otimes_{\alpha} \mathcal{B}\right)$.

Seja $\alpha$ uma $C^{*}$-norma sobre $\mathcal{A} \odot \mathcal{B}$. Como temos inclusões $C_{r}^{*}(\mathcal{A}) \subseteq \mathcal{L}\left(L^{2}(\mathcal{A})\right)$ e $C_{r}^{*}(\mathcal{B}) \subseteq$ $\mathcal{L}\left(L^{2}(\mathcal{B})\right)$, então $C_{r}^{*}(\mathcal{A}) \odot \mathcal{C}_{r}^{*}(\mathcal{B}) \subseteq \mathcal{L}\left(L^{2}(\mathcal{A})\right) \odot \mathcal{L}\left(L^{2}(\mathcal{B})\right)$. Pela Proposição 1.54 , temos uma inclusão $\mathcal{L}\left(L^{2}(\mathcal{A})\right) \odot \mathcal{L}\left(L^{2}(\mathcal{B})\right) \hookrightarrow \mathcal{L}\left(L^{2}(\mathcal{A}) \bigotimes_{\alpha} L^{2}(\mathcal{B})\right)$, e portanto temos uma inclusão:

$$
C_{r}^{*}(\mathcal{A}) \bigodot C_{r}^{*}(\mathcal{B}) \hookrightarrow \mathcal{L}\left(L^{2}(\mathcal{A}) \bigotimes_{\alpha} L^{2}(\mathcal{B})\right)
$$

DefiniçÃo 3.19. Se a é uma $C^{*}$-norma sobre $\mathcal{A} \odot \mathcal{B}$, definimos $C_{r}^{*}(\mathcal{A}) \bigotimes_{\dot{\alpha}} C_{r}^{*}(\mathcal{B})$ como sendo o fecho de $C_{r}^{*}(\mathcal{A}) \odot C_{r}^{*}(\mathcal{B})$ dentro de $\mathcal{L}\left(L^{2}(\mathcal{A}) \bigotimes_{\alpha} L^{2}(\mathcal{B})\right)$ segundo a inclusào acima.

Proposiçào 3.20. Sejam $\mathcal{A}=\left(A_{t}\right)_{t \in G} \in \mathcal{B}=\left(B_{s}\right)_{s \in H}$ fibrados de Fell sobre os grupos localmente compactos $G \in H, \epsilon$ seja $j_{2}: L^{2}(\mathcal{A}) \bigotimes_{\alpha} L^{2}(\mathcal{B}) \longrightarrow L^{2}\left(\mathcal{A} \bigotimes_{\alpha} \mathcal{B}\right)$ o isomorfismo de 3.18 . Então $\operatorname{Ad}\left(j_{2}\right)\left(C_{r}^{*}(\mathcal{A}) \bigotimes_{\bar{a}} C_{r}^{*}(\mathcal{B})\right)=C_{r}^{*}\left(\mathcal{A} \bigotimes_{\alpha} \mathcal{B}\right)$, ou seja: se $T \in \mathcal{L}\left(L^{2}(\mathcal{A}) \bigotimes_{\alpha} L^{2}(\mathcal{B})\right)$, então $T \in$ $C_{r}^{*}(\mathcal{A}) \bigotimes_{\tilde{\alpha}} C_{r}^{*}(\mathcal{B})$ se $\epsilon$ somente se $j_{2} T j_{2}^{*} \in C_{r}^{*}\left(\mathcal{A} \bigotimes_{\alpha} \mathcal{B}\right)$. Assim, obtemos um isomorfismo

único tal que

$$
j_{r}: C_{r}^{*}(\mathcal{A}) \underset{\dot{\alpha}}{\bigotimes} C_{r}^{*}(\mathcal{B}) \longrightarrow C_{r}^{*}\left(\mathcal{A} \bigotimes_{\alpha} \mathcal{B}\right)
$$

$$
j_{r}\left(\Lambda_{\mathcal{A}}(f) \odot \Lambda_{\mathcal{B}}(g)\right)=\Lambda_{\mathcal{A}} \otimes_{\alpha} \mathfrak{B}(f \odot g)
$$

$\forall f \in C_{c}(\mathcal{A}), g \in C_{c}(\mathcal{B})$.

DemonstraçÃo. A unicidade de $j_{r}$ é clara, pois está determinada sobre um conjunto denso pela equaçào (5). Temos que provar que $j_{r}$ existe. Em lugar de $\Lambda_{\mathcal{A}} \otimes_{\mathrm{a}} \mathfrak{b}$ escreveremos simplesmente $\Lambda$. Pela propriedade universal do produto tensorial, existe uma única transformação $\Lambda_{\mathcal{A}} \odot \Lambda_{\mathcal{B}}$ : $\mathcal{A} \odot \mathcal{B} \longrightarrow \mathcal{L}\left(L^{2}(\mathcal{A}) \otimes_{\Omega} L^{2}(\mathcal{B})\right)$ tal que $\left(\Lambda_{\mathcal{A}} \odot \Lambda_{\mathcal{B}}\right)(a \otimes b)=\Lambda_{\mathcal{A}}(a) \otimes \Lambda_{\mathcal{B}}(b), \forall a \in \mathcal{A}, b \in \mathcal{B}$. Por 3.18 temos um isomorfismo $j_{2}: L^{2}(\mathcal{A}) \otimes_{\alpha} L^{2}(\mathcal{B}) \longrightarrow L^{2}\left(\mathcal{A} \otimes_{\alpha} \mathcal{B}\right)$, único tal que $j_{2}(\xi \otimes \eta)=\xi @ \eta$. $\forall \xi \in C_{c}(\mathcal{A}) \subseteq L^{2}(\mathcal{A}), \eta \in C_{c}(\mathcal{B}) \subseteq L^{2}(\mathcal{B})$. Assim, $j_{2}$ induz um isomorfismo

$$
\operatorname{Ad}\left(j_{2}\right): \mathcal{L}\left(L^{2}(\mathcal{A}) \bigotimes_{a} L^{2}(\mathcal{B})\right) \rightarrow \mathcal{L}\left(L^{2}\left(\mathcal{A} \bigotimes_{a} \mathcal{B}\right)\right)
$$


dado por $T \longmapsto j_{2} T j_{2}^{*}$. Em particular, se $T=\Lambda\left(a_{t} \otimes b_{s}\right)$, temos:

$$
\begin{aligned}
\left.\Lambda\left(a_{t} b_{s}\right)\left(j_{2}(\xi \eta)\right)\right|_{\left(t_{0}, s_{0}\right)} & =\left(a_{t} \odot b_{s}\right)(\xi \odot \eta)\left((t, s)^{-1}\left(t_{0}, s_{0}\right)\right) \\
& =a_{t} \xi\left(t^{-1} t_{0}\right) \odot b_{s} \eta\left(s^{-1} s_{0}\right) \\
& =\left(\Lambda_{\mathcal{A}}\left(a_{t}\right) \xi\right)\left(t_{0}\right) \odot\left(\Lambda_{\mathcal{B}}\left(b_{s}\right) \eta\right)\left(s_{0}\right) \\
& =\left(\Lambda_{\mathcal{A}}\left(a_{t}\right) \xi \odot \Lambda_{\mathcal{B}}\left(b_{s}\right) \eta\right)\left(t_{0}, s_{0}\right) \\
& =j_{2}\left(\Lambda_{\mathcal{A}}\left(a_{t}\right) \xi \odot \Lambda_{\mathcal{B}}\left(b_{s}\right) \eta\right)\left(t_{0}, s_{0}\right) \\
& =\left.j_{2}\left(\left(\Lambda_{\mathcal{A}} \odot \Lambda_{\mathcal{B}}\right)\left(a_{t} \odot b_{s}\right)(\xi \odot \eta)\right)\right|_{\left(t_{0}, s_{0}\right)},
\end{aligned}
$$

donde segue que $\Lambda\left(x^{*}\right)=j_{2}\left(\Lambda_{\mathcal{A}} \quad \Lambda_{B^{*}}\right)(x) j_{2}^{*}, \forall x \in \mathcal{A} \odot \mathcal{B}$, e também que $\Lambda_{\mathcal{A}} \Lambda_{\mathcal{B}}$ se estende a todo $\mathcal{A} \otimes_{\alpha} \mathcal{B}$. Depois de integrar as representaçōes, segue que também é $\Lambda(f \cdot g)=j_{2}\left(\Lambda_{\mathcal{A}} \Lambda_{\mathcal{B}}\right)(f \bigcirc g) j_{2}^{*}$, $\forall f \in C_{c}(\mathcal{A}), g \in C_{c}(B)$.

Finalmente, como $j^{\prime}(D)=\operatorname{span}\left\{f \quad g: f \in C_{c}(\mathcal{A}), g \in C_{c}(\mathcal{B})\right\} \dot{\rho}$ denso em $C_{r}^{*}\left(\mathcal{A} \bigotimes_{a} \mathcal{B}\right)$. concluímos que $C_{r}^{*}\left(\mathcal{H} \otimes_{a} \mathcal{B}\right)=j_{2}\left(C_{r}^{*}(\mathcal{A}) \bigotimes_{\bar{\alpha}} C_{r}^{*}(\mathcal{B})\right) j_{2}^{*}$, como queríamos demonstrar.

Em particular, $j_{r}: C_{r}^{\prime *}(\mathcal{A}) \bigotimes_{i} C_{r}^{\prime *}(\mathcal{B}) \longrightarrow C_{r}^{*}\left(\mathcal{A} \bigotimes_{0} \mathcal{B}\right)$ tal que $x \longmapsto j_{2}, r j_{2}^{*}$ é um isomorfismo que satisfaz a equaçào (j).

Teorema 3.21. Sejam $\mathcal{A}=\left(A_{t}\right)_{t \in r_{i}} \in \mathcal{B}=\left(B_{s}\right)_{s \in H}$ fibrados de Fell sobre os grupos localmente compactos $G$ e H. Entio. para toda $\left(^{* *}\right.$-norma a sobre $\mathcal{A} \odot \mathcal{B}$, temos o seguinte diagrama comutativo:

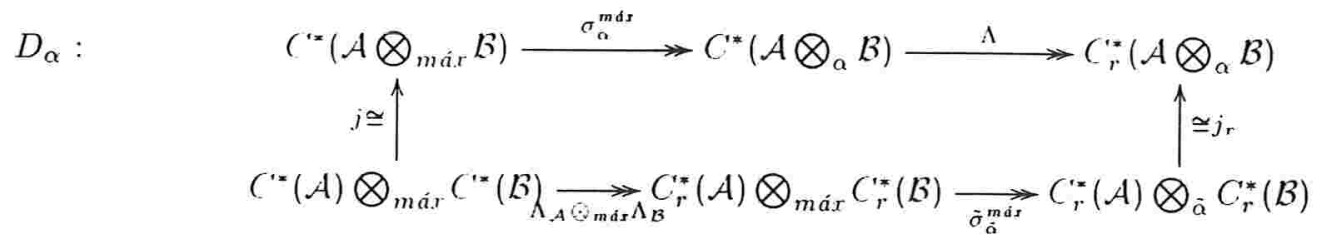

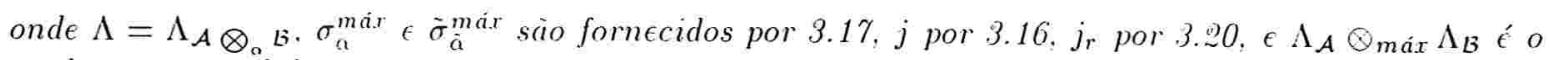
produto tensorial das representaçöes regulares de $C^{*}(\mathcal{A})$ e $C^{*}(\mathcal{B})$ respectivamente.

Demonstração. Sejam $f \in C_{c}(\mathcal{A}), g \in C_{c}(\mathcal{B})$. Então:

$$
\begin{aligned}
I \sigma_{a}^{\operatorname{máx}} j(f \circlearrowleft g) & =\Lambda \sigma_{\alpha}^{\text {máx }}(f \bigcirc g) & \text { por } 3.16 \\
& =\Lambda(f \oslash g) & \text { por } 3.15
\end{aligned}
$$

Por outro lado:

$$
\begin{array}{rlr}
j_{r} \tilde{\sigma}_{\alpha}^{\operatorname{máx}}\left(\Lambda_{\mathcal{A}} \bigcirc_{\text {máx }} \Lambda_{\mathcal{B}}\right)(f \odot g) & =j_{r} \tilde{\sigma}_{\alpha}^{\operatorname{máx}}\left(\Lambda_{\mathcal{A}}(f) \odot \Lambda_{\mathcal{B}}(g)\right) & \\
& =j_{r}\left(\Lambda_{\mathcal{A}}(f) \otimes \Lambda_{\mathcal{B}}(g)\right) & \text { por } 3.15 \\
& =\Lambda(f \oslash g) \quad \text { por } 3.20 &
\end{array}
$$

Como $C_{c}(\mathcal{A}) \odot C_{c}(\mathcal{B})$ é denso em $C^{*}(\mathcal{A}) \bigotimes_{\text {máx }} C^{*}(\mathcal{B})$, concluímos que

$$
\Lambda_{\mathcal{A}} \otimes_{\mathfrak{a}} \mathfrak{b} \sigma_{a}^{\text {máx }} j(x)=j_{r} \tilde{\sigma}_{a}^{\text {máx }}\left(\Lambda_{\mathcal{A}} \Theta_{\text {máx }} \Lambda_{\mathcal{B}}\right)(x), \quad \forall x \in C^{*}(\mathcal{A}) \bigotimes_{\text {máx }} C^{*}(\mathcal{B}),
$$

e portanto o diagrama comuta.

Corolário 3.22. O fibrado de Fell $\mathcal{A} \otimes_{\text {máx }} \mathcal{B}$ é amenable se e somente se $\mathcal{A}$, B são fibrados de Fell amenable. $\epsilon C^{*}(\mathcal{H}) \bigotimes_{\text {máx }} C^{*}(\mathcal{B})=C^{* *}(\mathcal{A}) \bigotimes_{\widetilde{m a x}} C^{*}(\mathcal{B})$. 
Demonstraçào. Para $\alpha=$ máx, o correspondente diagrama $D_{\text {máx fica: }}$

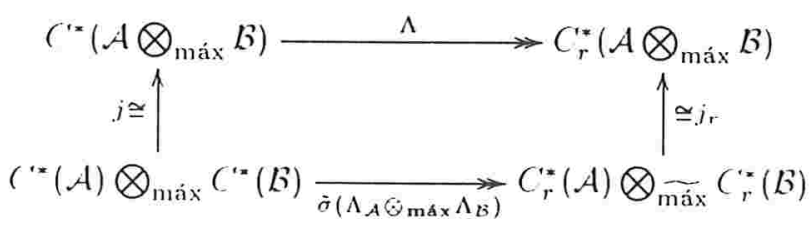

Assim, $\Lambda$ é um isomorfismo se e somente se $\tilde{\sigma}\left(\Lambda_{\mathcal{A}} \odot \Lambda_{\mathcal{B}}\right)$ também é. ou seja, $C^{*}\left(\mathcal{A} \bigotimes_{\text {máx }} \mathcal{B}\right)=$ $C_{r}^{*}\left(\mathcal{A} \otimes_{\text {máx }} \mathcal{B}\right)$ se e só se $\left(^{* *}(\mathcal{A})=C_{r}^{* *}(\mathcal{A}), C^{* *}(\mathcal{B})=C_{r}^{*}(\mathcal{B})\right.$, e $\|\cdot\|_{\text {máx }}=\|\cdot\|_{\text {máx }}$

\subsection{Algumas aplicações}

Nesta seção estudaremos algumas conexòes entre a amenabilidade de fibrados de Fell e produtos tensoriais de fibrados de Fell. Daremos duas versões da propriedade de aproximação para fibrados de Fell sobre grupos localmente compactos que generalizam, ambas, a definicào de Exel em [28]. Uma delas, a propriedade de aproximaçào em $L^{1}$, implica que o fibrado que a possui é amenable. A demonstração deste fato é exatamente igual à original para grupos discretos ([28], 4.6). válida no contexto contínuo pelo recente resultado em [30] que permite construir uma imagem isomorfa de $C_{r}^{*}(\mathcal{B})$ a partir de qualquer representaçào de $\mathcal{B}$ que seja fiel quando restrita à fibra sobre a unidade. Veremos que estas propriedades de aproximação são preservadas por produtos tensoriais, e deste fato obteremos as nossas principais aplicações: (a) se $\mathcal{B}$ é um fibrado de Fell com a propriedade de aproximação em $L^{1}$ e com fibra nuclear, então $C^{*}(\mathcal{B})$ é nuclear (3.29). (b) se $\mathcal{B}$ é um fibrado de Fell com a propriedade de aproximaçào em $L^{1}$ e com fibra exata, entào $C^{*}(\mathcal{B})$ é exata (3.35). Veremos também que todo fibrado de Fell sobre un grupo amenable tem a propriedade de aproximação em $L^{1}$, e em particular é amenable.

Em [28]. 4.4. Exel introduziu a noçào de fibrado de Fell com a proprifdade de aproximaçào: um fibrado de Fell $B=\left(B_{t}\right)_{t \in G}$ sobre um grupo discreto $G$ tem a propriedacle de aproximaçào se existe uma rede $\left(\phi_{i}\right)_{i \in I}$ de funçòes de suporte finito $\phi_{i}: G \longrightarrow B_{\epsilon}$ tal que:

1. A rede está limitada, no sentido de que: $\sup _{i \in I}\left\|\sum_{t \in G_{i}} \phi_{i}(t)^{*} \phi_{i}(t)\right\|=M<\infty$.

2. Para cada $t \in G_{i}$ e $b_{t} \in B_{t}$ tem-se: $\lim _{i} \sum_{r \in G} \phi_{i}(t r)^{*} b_{t} \phi_{i}(r)=b_{t}$.

A relevancia deste conceito está no fato de que um fibrado de Fell sobre um grupo discreto com a propriedade de aproximaçào é necessariamente amenable, ou seja, a $C^{\prime *}$-álgebra do fibrado coincide com a $C^{*}$-álgebra reduzida do fibrado ([28]. 4.6). Posteriormente. $\mathrm{Ng}$ sugeriu uma definição de propriedade de aproximaçào para o caso de um fibrado sobre um grupo localmente compacto qualquer. parecida com a de Exel, embora nào mais geral, e provou que, se o fibrado tem esta propriedade de aproximação e satisfaz a condição de ser "próprio", então ele é amenable ([50]). Recentemente. Exel provou que todo fibrado de Fell é próprio ([30]), razào pela qual a hipótese adicional do fibrado de Fell ser próprio colocada por Ng virou supérflua. Por outro lado, nào conhecemos nenhum exemplo não trivial de um fibrado de Fell que satisfaça a propriedade de aproximação introduzida em [50]. $\mathrm{Ng}$ afirma que se o fibrado de Fell é sobre um grupo amenable, entào ele satisfaz automaticamente sua propriedade de aproximaçào. Porém. nós achamos que a demonstraçào deste fato providenciada por $\mathrm{Ng}$ é, no mínimo. incompleta. Por esta razào, nào tentaremos usar os resultados de [50] para generalizar os nossos ao caso contínuo. Em lugar disso, consideraremos uma outra propriedade de aproximaçào no caso contínuo, na verdade duas, que generalizam quase diretamente a definição de Exel. mas que tomam também um elemento da definição de $\mathrm{Ng}$, no sentido de permitir duas redes 
em lugar da única rede $\left(\phi_{i}\right)$ considerada acima. Também admitimos que as redes envolvidas tomem valores na álgebra $M\left(B_{\epsilon}\right)$ dos multiplicadores de $B_{\epsilon}{ }^{1}$

Parte da terminologia ( $M$-propriedade de aproximação, propriedade de aproximação positiva) é tomada da definiçào de $\mathrm{Ng}$ :

DefiniçÃo 3.23 . Seja $\mathcal{B}=\left(B_{t}\right)_{t \in G}$; um fibrado de Fell sobre o grupo localmente compacto $G$.

1. Dizemos que $B$ tem a $M$-propriedade de aproximação (enumerável) se existem redes (respectivamente: seqüencias). $\left(\phi_{i}\right) \cdot\left(\iota_{i}\right) \subseteq C_{c}\left(G, M\left(B_{e}\right)\right) \subseteq L^{2}(G) \otimes M\left(B_{e}\right)$ tais que:

(a) $\sup _{i}\left\{\left\|\phi_{i}\right\|\left\|\psi_{i}\right\|\right\} \leq M$ (a norma é como elementos de $L^{2}\left(C_{i}\right) \otimes M\left(B_{\epsilon}\right)$ ).

(b) Se $\Phi_{i}: \mathcal{B} \longrightarrow \mathcal{B}$ está definido como: $\Phi_{i}\left(b_{t}\right)=\int_{G} \phi_{i}(s)^{*} b_{t} v_{i}\left(t^{-1} s\right) d s$, então $\Phi_{i}\left(b_{t}\right) \longrightarrow b_{t}$. $\forall b_{t} \in \mathcal{B}$. Diremos que $\left(o_{i}\right)$ e $\left(\psi_{i}\right)$ sào redes aproximantes para $\mathcal{B}$.

2. $\mathcal{B}$ tem a propriedade de aproximaçào (enumerável) se tem a.$/$-propriedade de aproximaçào (enumerável) para algum.$/ />0$.

3. Se em (a) podemos escolher $o_{i}=\psi_{i}$. dizemos que $\mathcal{B}$ tem a $.1 /$-propriedade de aproximaçào positiva, ou a propriedade de aproximaçào positiva.

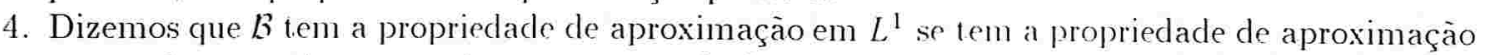
$\mathrm{com}$ redes aproximantes tais que, para toda funçào $f \in C_{c}(\mathcal{B})$. tent-se que as funçòes $\Phi_{i}(f)$ definidas como $\left.\Phi_{i}(f)\right|_{t}=\Phi_{i}(f(t))$ convergem a $f$ em $L^{1}(\mathcal{B})$

ObservaÇÀo 3.24. Notar que $L^{2}(G)$ é um $C^{*}$-anel ternário nuclear, pois é um espaço de Hilbert, de forma que o produto tensorial $L^{2}(G) \otimes M\left(B_{e}\right)$ é único. Por outro lado, $L^{2}(G) \otimes M\left(B_{e}\right)$ se identifica naturalmente com $L^{2}\left(G, M\left(B_{\epsilon}\right)\right)$. o completamento de $C_{c}\left(C, M\left(B_{t}\right)\right)$ rom respeito ao produt.o interno: $\langle f, g\rangle=\int_{G} f(t)^{*} g(t) d t$, ou seja, $L^{2}\left(G, M\left(B_{e}\right)\right)=L^{2}\left(M\left(B_{c}\right) \times(i)\right.$ (ver 2.14).

Como vimos no Capítulo 2 (página 42), cada multiplicador de $B_{\text {t }}$ define um multiplicador de $\mathcal{B}$ de ordem $e$, e portanto a fórmula que define $\Phi\left(b_{t}\right)$ faz sentido, e tem-se que $\Phi\left(b_{t}\right) \in B_{t}, \forall b_{t}$. Com respeito a 4 .. observar que, pelo Lema 2.23 . o integrando na integral que define $\Phi(f)$ é uma função contínua de suporte compacto. e portanto está nas condições de [37]. 11-15.19, o que implica que $\Phi(f) \in C_{c}(\mathcal{B})$.

Notar, por último, que no caso de ser $G$ um grupo discreto, as duas propriedades de aproximaçào são equivalentes, ou seja. a propriedade de aproximação implica a propriedade de aproximação em $L^{1}$. Veremos em 3.26 outros casos em que ambas propriedades são equivalentes.

O seguinte resultado é uma generalizaçào direta de [28], 4.3, ao caso contínuo.

Teorema 3.25. Se $\mathcal{B}$ é um fibrado de Fell com a propriedade de aproximação em $L^{1}$, entào $\mathcal{B}$ é amenable.

Demonstraçào. Suponhamos que $\pi: \mathcal{B} \longrightarrow B(H)$ é uma representaçào não degenerada tal que $\left.\pi\right|_{B_{e}}$ é fiel. Também chamamos $\pi$ à representação integrada de $\pi$, e a sua única extensão a $M(\mathcal{B})$ (ver página 42). Como $\left.\pi\right|_{B_{e}}$ é fiel, $\left.\pi\right|_{M\left(B_{e}\right)}$ também é fiel. Dado $\phi \in C_{c}\left(G, M\left(B_{e}\right)\right) \subseteq L^{2}(G) \otimes M\left(B_{e}\right)$,

\footnotetext{
${ }^{1}$ Este fato, que sugere maior flexibilidade para um fibrado de Fell ter a propriedade de aproximação. na verdade não modifica nada sustancialmente: basta multiplicar à direita cada elemento da rede por uma unidade aproximada de $B_{e}$; contudo, no caso de um fibraclo de Fell sobre um grupo amenable achamos uma rede aproximante (ver Definição 3.23 ) que toma valores no centro de $M\left(B_{\epsilon}\right)$. o que talvez tenha relaçào com a propriedade de aproximaçào de AnantharamanDelaroche
} 
consideremos o operador $V_{\phi}: H \longrightarrow L^{2}(G) \otimes H$ tal que $\left.V_{\phi} h\right|_{t}=\pi(\phi(t)) h$. Temos então:

$$
\begin{aligned}
\left\|V_{\phi}\right\|^{2} & =\sup _{\|h\|=1} \int_{G}\langle\pi(\phi(t)) h, \pi(\phi(t)) h\rangle d t \\
& =\sup _{\|h\|=1} \int_{G}\left\langle\pi\left(\phi(t)^{*} \phi(t)\right) h, h\right\rangle d t \\
& =\sup _{\|h\|=1}\left\langle\int_{G} \pi\left(\phi(t)^{*} \phi(t)\right) d t h, h\right\rangle \\
& =\sup _{\|h\|=1}\langle\pi(\langle\phi, \phi\rangle) h, h\rangle \\
& =\|\pi(\langle\phi, \phi\rangle)\| \quad \text { porque } \pi(\langle\phi, \phi\rangle) \text { é positivo } \\
& =\|\langle\phi, \phi\rangle\| \quad \text { porque }\left.\pi\right|_{M\left(B_{c}\right)} \text { é fiel } \\
& =\|\phi\|^{2}
\end{aligned}
$$

Calculemos $I_{0}^{*}:$ se $h \in H, \xi \in L^{2}\left(\left(^{\prime}\right)\right.$, então

$$
\left\langle V_{\phi} h, \xi\right\rangle=\int_{G}\langle\pi(\phi(t)) h, \xi(t)\rangle d t=\int_{G}\left\langle h, \pi\left(\phi(t)^{*}\right) \xi(t)\right\rangle d t=\left\langle h, \int_{G} \pi\left(\phi(t)^{*}\right) \xi(t) d t\right\rangle
$$

donde concluímos que $I_{\phi}^{*}(\xi)=\int_{G} \pi\left(\phi(t)^{*}\right) \xi(t) d t$. Notar que se $\psi \in C_{c}\left(G, M\left(B_{e}\right)\right)$, então $V_{\phi}^{*} V_{\psi} h=$ $\left.\int_{G} \pi\left(\phi(t)^{*}\right) V_{i} h\right|_{t} d t=\int_{G_{i}} \pi\left(\phi(t)^{*}\right) \pi(\psi(t)) h d t=\pi(\langle\phi, \psi\rangle) h$. Além disso, se $\phi_{1}, \phi_{2}, \phi_{3} \in C_{c}\left(G, M\left(B_{\epsilon}\right)\right)$. $h \in H$, tem-se:

$$
\begin{aligned}
\left.V_{\phi_{1}\left\langle\phi_{2}, \phi_{3}\right\rangle} h\right|_{t} & =\pi\left(\phi_{1}(t)\left\langle\phi_{2}, \phi_{3}\right\rangle\right) h \\
& =\pi\left(\phi_{1}(t)\right) \pi\left(\left\langle\phi_{2}, \phi_{3}\right\rangle\right) h \\
& =\left.V_{\phi_{1}} V_{\phi_{2}}^{*} V_{\phi_{3}} h\right|_{t}
\end{aligned}
$$

Portanto, como $\circ \longmapsto V_{\phi}$ é uma isometria sobre o subespaço denso $C_{c}\left(G, M\left(B_{\epsilon}\right)\right)$, então se estende a uma aplicação $\pi_{2}: L^{2}(G) \otimes M\left(B_{e}\right) \longrightarrow \pi_{2}\left(L^{2}(G) \otimes M\left(B_{e}\right)\right) \subseteq B\left(H, L^{2}(G) \otimes H\right)$, que é um isomorfismo de $C^{*}$-anéis ternários positivos ${ }^{2}$.

Consideremos agora a representação $\pi_{\lambda}: \mathcal{B} \longrightarrow B\left(L^{2}(G) \otimes H\right)$, tal que $\pi_{\lambda}\left(b_{t}\right)=\lambda_{t} \otimes \pi\left(b_{t}\right)$ e sua representaçào integrada, que continuaremos chamando $\pi_{\lambda}: C^{*}(\mathcal{B}) \longrightarrow B\left(L^{2}(G) \otimes H\right.$ ) (ver 3.6). Definamos, para $0 . \psi \in L^{2}(G) \otimes M\left(B_{e}\right)$, a aplicaçào completamente limitada $\Psi: \pi_{\lambda}\left(C^{*}(\mathcal{B})\right) \longrightarrow$ $B(H)$ tal que $\Psi(x)=V_{\omega}^{*} x V_{\psi}, \forall x \in \pi_{\lambda}\left(C^{*}(\mathcal{B})\right)$. Temos então que $\|\Psi(x)\| \leq\|\phi\|\|\psi\|\|x\|$, e portanto $\|\Psi\| \leq\|\phi\|\|\psi\|$. Consideremos também, para $f \in C_{c}(\mathcal{B})$, a função $\Phi(f): G \longrightarrow \mathcal{B}$ tal que $\left.\Phi(f)\right|_{t}=$ $\int_{G} \phi(s)^{*} f(t) \psi^{\prime}\left(t^{-1} s\right) d s$. Seja $F(t, s)=\phi(s)^{*} f(t) \psi\left(t^{-1} s\right)$. Então, por 2.23, $F: G \times G \longrightarrow \mathcal{B}$ é contínua de suporte compacto, e tal que $F(t, s) \in B_{t}, \forall t \in G$. Entào, por [37], II-15.19, temos que a funçào $t \longmapsto \int_{G} F(t, s) d s$ é uma seçào contínua de suporte compacto de $\mathcal{B}$. Em outras palavras, $\Phi(f) \in C_{c}(\mathcal{B})$. De fato, é claro que sup $(\Phi(f)) \subseteq \sup (f)$. Além disto, temos que $\pi(\Phi(f))=\Psi\left(\pi_{\lambda}(f)\right)$. Com efeito.

\footnotetext{
${ }^{2}$ Curiosamente, ou nem tanto. a mesma fórmula utilizada para definir $\pi_{2}$ pode ser utilizada para obter um homomorfismo injetivo de $C^{* *}$-anéis ternários positivos $L^{2}(B) \longrightarrow B\left(H, L^{2}(G) \otimes H\right)$ : ver 7.22
} 
se $h, k \in H:$

$$
\begin{aligned}
& \langle\pi(\Phi(f)) h, k\rangle=\left\langle\int_{G} \pi\left(\left.\Phi(f)\right|_{t}\right) h, k\right\rangle \\
& =\int_{G i}\left\langle\pi\left[\int_{G} \phi(s)^{*} f(t) \psi\left(t^{-1} s\right) d s\right] h, k\right\rangle \\
& =\int_{G} \int_{G^{i}}\left\langle\pi\left(o(s)^{*} f(t) \psi\left(t^{-1} s\right)\right) h, k\right\rangle d s d t \\
& =\int_{G} \int_{G^{\prime}}\left\langle\pi\left(\varphi(s)^{*}\right) \pi(f(t)) \pi\left(\psi\left(t^{-1} s\right)\right) h, k\right\rangle d s d t \\
& =\int_{G_{i}}\left\langle\left.\pi\left(o(s)^{*}\right) \int_{G} \pi(f(t)) V_{\psi} h\right|_{t^{-1} s} d t, k\right\rangle d s \\
& =\int_{C_{i}}\left\langle\left.\pi\left(o(s)^{*}\right)\left[\int_{G}\left(\lambda_{t} \odot \pi\right)(f(t))\left(V_{\psi}, h\right) d t\right]\right|_{s}, k\right\rangle d s \\
& =\int_{G}\left\langle\pi\left(o(s)^{*}\right) \pi_{\lambda}(f)\left(V_{\psi}, h\right)(s) d s, k\right\rangle \\
& =\left\langle\int_{G_{i}} \pi\left(\sigma(s)^{*}\right)\left(\pi_{\lambda}(f) V_{\psi}, h(s)\right) d s, k\right\rangle \\
& =\left\langle\left(V_{0}^{*} \pi_{\lambda}(f) V_{\psi}\right) h, k\right\rangle \\
& =\left\langle\Psi\left(\pi_{\lambda}(f)\right) h, k\right\rangle
\end{aligned}
$$

donde segue que $\pi(\Phi(f))=\Psi\left(\pi_{\lambda}(f)\right)$. Em particular, temos que $\Psi\left(\pi_{\lambda}\left(C_{c}(\mathcal{B})\right)\right) \subseteq \pi\left(C_{c}(\mathcal{B})\right) \subseteq$ $\pi\left(C^{*}(\mathcal{B})\right)$, que é fechado, e portanto $\Psi\left(\pi_{\lambda}\left(C^{*}(\mathcal{B})\right)\right) \subseteq \pi\left(C^{*}(\mathcal{B})\right)$. Desta forma, temos que $\Psi$ : $\pi_{\lambda}\left(C^{*}(\mathcal{B})\right) \longrightarrow \pi\left(C^{* *}(\mathcal{B})\right)$. Suponhamos que $\left(\phi_{i}\right),\left(\psi_{i}\right)$ são redes aproximantes como na Definição 3.23 , $\mathrm{com}\left\|\phi_{i}\right\|\left\|\psi_{i}\right\| \leq M, \forall i$, de forma que temos $\Phi_{i}: C_{c}(\mathcal{B}) \longrightarrow C_{c}(\mathcal{B})$, e sabemos que $\Phi_{i}(f)$ converge em $L^{1}(\mathcal{B})$, para toda $f \in C_{c}^{\prime}(\mathcal{B})$. Sejam $\Psi_{i}: \pi_{\lambda}\left(C^{*}(\mathcal{B})\right) \longrightarrow \pi\left(C^{*}(\mathcal{B})\right)$ as correspondentes transformações completamente limitadas, ou seja: $\Psi_{i}(x)=I_{\phi_{1}}^{*} x V_{\psi_{1}}, \forall x \in \pi_{\lambda}\left(C^{* *}(\mathcal{B})\right)$. Como $\Phi_{i}(f) \longrightarrow f$ em $L^{1}(\mathcal{B})$, entào $\Phi_{i}(f) \longrightarrow f$ em $C^{* *}(\mathcal{B})$. e portanto $\pi\left(\Phi_{i}(f)\right) \longrightarrow \pi(f)$ em $\pi\left(C^{*}(\mathcal{B})\right)$. Conseqüéntemente, $\left\|\pi\left(\Phi_{i}(f)\right)\right\| \longrightarrow\|\pi(f)\|$. Por outro laclo, $\pi\left(\Phi_{i}(f)\right)=\Psi_{i}\left(\pi_{\lambda}(f)\right)$, donde segue que:

$$
\|\pi(f)\|=\lim _{i}\left\|\pi\left(\Phi_{i}(f)\right)\right\|=\lim _{i}\left\|\Psi_{i}\left(\pi_{\lambda}(f)\right)\right\| \leq \limsup _{i}\left\|\Psi_{i}\right\|\left\|\pi_{\lambda}(f)\right\| \leq M\left\|\pi_{\lambda}(f)\right\| .
$$

Como $C_{c}(\mathcal{B})$ é denso em $L^{1}(\mathcal{B})$, segue que $\|\pi(y)\| \leq M\left\|\pi_{\lambda}(y)\right\|, \forall y \in C^{*}(\mathcal{B})$.

Em particular, se $\pi$ é uma representação fiel de $C^{*}(\mathcal{B})$, concluímos que $\pi_{\lambda}$ também é fiel, o que, por [30], implica que $C^{*}(\mathcal{B})=C_{r}^{*}(\mathcal{B})$.

ProposiçÃo 3.26. Seja $\mathcal{B}$ um fibrado de Fell sobre o grupo localmente compacto $G$. Tem-se:

1. Se $G$ é discreto $\in \mathcal{B}$ tem a propriedade de aproximação, entào $\mathcal{B}$ tem a propriedade de aproximação $\mathrm{em} L^{1}$.

2. Se $\mathcal{B}$ tem a propriedade de aproximaçào enumerável, então $\mathcal{B}$ tem a propriedade de aproximação em $L^{1}$.

3. Se $G$ é amenable, entào $\mathcal{B}$ tem a 1-propriedade de aproximação positiva em $L^{1}$.

DemonstraçÃo. A primeira afirmaçào, como já foi observado, é claramente verdadeira, porque se $f$ é uma função de suporte compacto sobre o grupo discreto $G$, então $f$ é de suporte finito, e como $\sup (\Phi(f))$ está incluído em $\sup (f)$. as integrais envolvidas são de fato somas finitas sobre $\sup (f)$, e portanto a convergencia pontual implica a convergencia destas somas. 
Com respeito à segunda afirmaçào, se $\mathcal{B}$ tem a $M$-propriedade de aproximação enumerável, temos que cada função $t \longmapsto\left\|\left.\left(\Phi_{n}(f)-f\right)\right|_{t}\right\|$ t.em suporte compacto contido em $\sup (f)$. Por outro lado, esta seqüencia converge pontualmente a zero e está limitada em $\|\cdot\|_{\infty}$. Com efeito, na demonstraçào de 3.25 , vimos que para toda $f \in C_{c}(\mathcal{B})$ tem-se que $\pi(\Phi(f))=\Psi\left(\pi_{\lambda}(f)\right)=V_{\phi}^{*} \pi_{\lambda}(f) V_{\psi^{\prime}}$, onde $\pi$ é uma representaçào de $C^{\prime *}(B)$. A mesma conta mostra que $\pi\left(\Phi\left(b_{t}\right)\right)=\Psi\left(\pi_{\lambda}\left(b_{t}\right)\right)=V_{\phi}^{*} \pi_{\lambda}\left(b_{t}\right) V_{\psi}$, para toda representaçào $\pi$ de $\mathcal{B}$. e para todo $b_{t} \in \mathcal{B}$. Em particular, se $\left.\pi\right|_{B_{c}}$ é injetiva, tem-se que:

$$
\|\Phi(f(t))\|=\|\pi(\Phi(f(t)))\|=\left\|V_{\phi}^{*} \pi_{\lambda}(f(t)) V_{\psi}\right\| \leq\|\varphi\|\|\psi\|\|f(t)\|,
$$

donde segue que $\|\Phi(f)\|_{\infty} \leq\|\circ\|\|\imath \cdot\|\|f\|_{\infty}$ Entào temos que $\left\|\Phi_{n}(f)-f\right\|_{\infty} \leq\left(\left\|\phi_{n}\right\|\left\|\psi_{n}\right\|+1\right)\|f\|_{\infty} \leq$ $(M+1)\|f\|_{\infty}$. Segue entào, pelo teorema de convergencia dominada de Lebesgue, que $\Phi_{n}(f)$ converge a $f$ en $L^{1}(\mathcal{B})$.

Por último, consideremos a terceira afirmação. Por [52], 7.3 .8 , existe uma rede $\left(\varphi_{i}\right)_{i \in I}$ na esfera unitária de $L^{2}(G)$, tal que a rede $\left(\sigma_{i}\right)_{i \in I}$ converge uniformemente sobre compactos a 1 , onde $\sigma_{i}: G \longrightarrow$ $\mathbb{C}$ está definida como $\sigma_{i}(t)=\int_{G i} \overline{r_{i}(s)} \varphi_{i}\left(t^{-1} s\right) d s$. Vejamos que podemos supor que $\varphi_{i}$ é contínua de suporte compacto. Para cada $n \in Z^{+}$, e cada $i \in I$, existe $\varphi_{i, n} \in C_{c}\left(G^{\prime}\right)$ tal que $\left\|\varphi_{i, n}\right\|_{2} \leq 1$, e $\left\|\varphi_{i}-\varphi_{i, n}\right\|_{2}<1 / 2 n$. Temos entào:

$$
\begin{aligned}
\int_{G}\left|\bar{\varphi}_{i, n}(s)_{\hat{r}_{i, n}}\left(t^{-1} s\right)-\overline{\varphi_{i}(s)} \varphi_{i}\left(t^{-1} s\right)\right| d s & \leq \int_{G i}\left|\varphi_{i, n}\left(t^{-1} s\right)\right|\left|\overline{\varphi_{i, n}(s)}-\overline{\varphi_{i}(s)}\right| d s \\
& \quad+\int_{G}\left|\overline{\varphi_{i}(s)}\right|\left|\hat{\varphi}_{i, n}\left(t^{-1} s\right)-\varphi_{i}\left(t^{-1} s\right)\right| d s \\
& \leq\left\|\varphi_{i, n}\right\|_{2}\left\|\varphi_{i, n}-\varphi_{i}\right\|_{2}+\left\|\varphi_{i}\right\|_{2}\left\|\varphi_{i, n}-\varphi_{i}\right\|_{2} \\
& \leq 2\left\|\varphi_{i, n}-\varphi_{i}\right\|_{2} \\
& <1 / n
\end{aligned}
$$

Consideremos agora a rede $\left(\hat{\varphi}_{i, n}\right)$ indexada em $I \times \mathbb{Z}^{+}$com a ordem produto, e sejam $\sigma_{i, n}: G \longrightarrow \mathbb{C}$ tais que $\sigma_{i, n}(t)=\int_{G} \bar{\varphi}_{i, n}(s) \hat{y}_{i, n}\left(t^{-1} s\right) d s$. Então $\sigma_{i, n}$ converge uniformemente a 1 sobre compactos. Com efeito, sejam $K \subseteq G$ um compacto, $\epsilon>0$. Como $\sigma_{i}$ converge a 1 uniformemente sobre compactos. existe $i_{0} \in I$ tal que para todo $i \geq i_{\text {n }}$ e para todo $t \in K$ se tem: $\left|1-\sigma_{i}(t)\right|<\epsilon / 2$. Seja $n_{0}>2 / \epsilon$; entào, se $i \geq i_{0}, n \geq n_{0} . t \in K$. pelo cálculo acima temos:

$$
\left|1-\sigma_{i, n}(t)\right| \leq\left|1-\sigma_{i}(t)\right|+1 / n<\epsilon / 2+\epsilon / 2=\epsilon .
$$

Pelo provado acima sabemos entào que existe uma rede $\left(\varphi_{i}\right)_{i \in I}$ incluída na bola unidade de $L^{2}(G)$, formada por funçōes contínuas de suporte compacto, e tais que $\sigma_{i}$ converge uniformemente sobre compactos à função 1 , onde $\sigma_{i}(t)=\int_{G} \overline{\varphi_{i}(s)} \varphi_{i}\left(t^{-1} s\right) d s$. Para cada $i \in I$, definamos $\phi_{i}$. $\psi_{i}: G \longrightarrow M\left(B_{\epsilon}\right)$ tais que $\phi_{i}(t)=\iota_{i}(t)=\varphi_{i}(t) 1$, onde 1 é a unidade de $M\left(B_{e}\right)$. Então:

$$
\left\langle\phi_{i}, \phi_{i}\right\rangle=\int_{G}\left(\hat{\varphi}_{i}(s) 1\right)^{*} \varphi_{i}(s) 1 d s=\int_{G_{i}}\left|\varphi_{i}(s)\right| d s 1=\left\|\varphi_{i}\right\| 1,
$$

o que mostra que $\phi_{i} \in L^{2}\left(G^{\prime}\right) \otimes M\left(B_{\epsilon}\right)$, e que $\left\|\phi_{i}\right\| \leq 1, \forall i \in I$. Por outro lado, se $b_{t} \in \mathcal{B}$,

$$
\Phi_{i}\left(b_{t}\right)=\int_{G_{i}} \phi_{i}(s)^{*} b_{t} \phi\left(t^{-1} s\right) d s=\sigma_{i}(t) b_{t} \longrightarrow b_{t},
$$

e se $f \in C_{c}(\mathcal{B})$, entào:

$$
\left\|\Phi_{i}(f)-f\right\|_{1}=\int_{\sup (f)}\left\|\sigma_{i}(t) f(t)-f(t)\right\| d t \leq\|f\|_{\infty} \int_{\sup (f)}\left|\sigma_{i}(t)-1\right| d t \longrightarrow 0,
$$

porque $\sup (f)$ é compacto, e portanto $\sigma_{i} \longrightarrow 1$ uniformemente sobre $\sup (f)$, que por sua vez implica que a integral correspondente converge a zero. Concluímos assim que $\mathcal{B}$ tem a 1 -propriedade de aproximação positiva em $L^{1}$. 
ProposiçÃo 3.27. Sejam $\mathcal{A}=\left(A_{t}\right)_{t \in G} \in \mathcal{B}=\left(B_{s}\right)_{s \in H}$ fibrados de Foll sobre os grupos localmente compactos $G$ e $H$, e a uma $\left(^{\prime *}\right.$-norma sobre $\mathcal{A} \odot \mathcal{B}$. Tem-se:

1. Se $\left(\phi_{i}\right)_{i \in I},\left(\psi_{i}\right)_{i \in I}$ são redes aproximantes para $\mathcal{A}, \epsilon\left(\phi_{j}^{\prime}\right)_{j \in J} .\left(\imath_{j}^{\prime}\right)_{j \in J}$ sào redes aproximantes para $\mathcal{B}$, então $\left(\phi_{i} \phi_{j}^{\prime}\right)_{(i, j) \in I \times J .}\left(\psi_{i} \odot l_{j}^{\prime}\right)_{(i, j) \in I \times J} \subseteq C_{c}\left(G \times H . . M\left(\cdot, \bigotimes_{a} B_{\epsilon}\right)\right)$ é uma rede aproximante para $\mathcal{A} \otimes_{0} \mathcal{B}$.

2. Se $\mathcal{A}$ tem a M-propriedade de aproximação (positiva. enumeráiel), Bi tem a $N$-propriedade

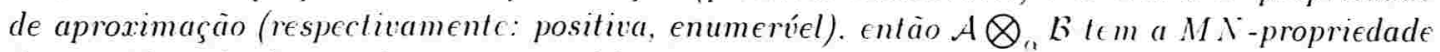
de aproximaşào (respectivamente: positiva, enumerável).

3. Se $\mathcal{A} \in \mathcal{B}$ tém a propriedade de aproximação. entào $\mathcal{A} \bigotimes_{n} B$ te m a propredade de aproximação.

4. Se $\mathcal{A}$ e $B$ tém a propriedade de aproximaçào em $L^{1}$ então $\mathcal{A} \bigotimes_{1}$ B lem a propriedade de

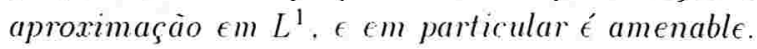

Demonstraçào. Por [65], T.6.3. existe uma inclusão $M\left(\cdot A_{\epsilon}\right) \odot . M\left(B_{\epsilon}\right) \hookrightarrow M\left(\cdot A_{\epsilon} \bigotimes_{\alpha} B_{\epsilon}\right)$. Sejam $\bar{\alpha}$ a $C^{*}$-norma obtida sobre $M\left(A_{\epsilon}\right) \odot M\left(B_{\epsilon}\right)$ através desta inclusão. e $M\left(A_{\epsilon}\right) \bigotimes_{\bar{\alpha}} M\left(B_{\epsilon}\right)$ o correspondente produto tensorial. Podemos ver $L^{2}(G) \otimes M\left(A_{\epsilon}\right)$ e $L^{2}(H) \otimes 1 /\left(B_{t}\right)$ como $L^{2}\left(. M\left(A_{\epsilon}\right) \times G\right)$ e $L^{2}\left(M\left(B_{\epsilon}\right) \times H\right)$ respectivamente, onde $M\left(A_{\epsilon}\right) \times G$ e $M\left(B_{\epsilon}\right) \times I I$ são os fibrados de Fell produto (ou trivias com fibras $M\left(A_{\epsilon}\right)$ e $M\left(B_{\epsilon}\right)$ respectivamente; ver 2.13 e 2.14 ). ('omo $L^{2}(G) \otimes M\left(A_{\epsilon}\right)$ e $L^{2}(H) \otimes M\left(B_{\epsilon}\right)$ sào módulos de Hilbert à direita plenos sobre $M\left(A_{\epsilon}\right)$ ค $M\left(B_{\epsilon}\right)$ respectivamente, então existe uma única $C^{\prime *}$-norma sobre $\left(L^{2}(G) \otimes M\left(A_{t}\right)\right) \odot\left(L^{2}(H) \otimes M\left(B_{t}\right)\right)$. que também chamamos $\bar{\alpha}$, e tal que $\left(L^{2}\left(G^{\prime}\right) \otimes M\left(A_{\epsilon}\right)\right) \bigotimes_{\bar{a}}\left(L^{2}(H) \otimes M\left(B_{e}\right)\right)$, o correspondente completamento, é um módulo de Hilbert à direita pleno sobre $M\left(A_{\epsilon}\right) \bigotimes_{\bar{a}} M\left(B_{\epsilon}\right)$. De fato, em 3.18 vimos que este módulo de Hilbert é naturalmente isomorfo a $L^{2}(G \times H) \otimes\left(M\left(A_{\epsilon}\right) \bigotimes_{\bar{a}} M\left(B_{\epsilon}\right)\right)$. Assim, nas condiçōes de 1 ., obtemos redes $\left(\phi_{i} \bigcirc \phi_{j}^{\prime}\right),\left(\psi_{i} \odot \psi_{j}^{\prime}\right) \subseteq L^{2}\left(G \times H . M\left(A_{\epsilon}\right) \bigotimes_{\bar{a}} M\left(B_{\epsilon}\right)\right) \subseteq L^{2}\left(\left(i \times H, M\left(A_{i} \bigotimes_{a} B_{\epsilon}\right)\right)\right.$, e é claro que $\phi_{i} \odot \phi_{j}^{\prime}$ e $\psi_{i} \odot \psi_{j}^{\prime}$ sào contínuas de suporte compacto. Além disso. $\left\|\phi_{i} \odot \phi_{j}^{\prime}\right\|=\left\|\phi_{i}\right\|\left\|\phi_{j}^{\prime}\right\|$, e $\left\|\psi_{i} \odot \psi_{j}^{\prime}\right\|=\left\|\psi_{i}\right\|\left\|\psi_{j}^{\prime}\right\|$ (1.49). Em particular. se $\left\|\phi_{i}\right\|\left\|\psi_{i}\right\| \leq M$ e $\left\|\phi_{j}^{\prime}\right\|\left\|\iota_{j}^{\prime}\right\| \leq N$, entào

$$
\left\|\phi_{i} \odot \phi_{j}^{\prime}\right\|\left\|\psi_{i} \odot \psi_{j}^{\prime}\right\|=\left\|\phi_{i}\right\|\left\|\phi_{j}^{\prime}\right\|\left\|\psi_{i}\right\|\left\|\psi_{j}^{\prime}\right\| \leq M \Lambda
$$

Notar também que se $\phi_{i}=\psi_{i}$ e $\phi_{j}^{\prime}=\psi_{j}^{\prime}$, então $\phi_{i} \otimes \phi_{j}^{\prime}=\psi_{i} \otimes \psi_{j}^{\prime}$

Suponhamos que as redes acima são aproximantes, de forma que $\mathcal{A}$ tem a $M$-propriedade de aproximação e $\mathcal{B}$ tem a $N$-propriedade de aproximação. Sejam $\Phi_{i}: \mathcal{A} \longrightarrow \mathcal{A}$ tal que $a_{t} \longmapsto$ $\int_{G} \phi_{i}\left(t^{\prime}\right)^{*} a_{t} \psi_{i}\left(t^{-1} t^{\prime}\right) d t^{\prime}$, e $\Phi_{j}^{\prime}: \mathcal{B} \longrightarrow \mathcal{B}$ tal que $b_{s} \longmapsto \int_{H} \phi_{j}^{\prime}\left(s^{\prime}\right)^{*} b_{s} t_{j}^{\prime}\left(s^{-1} s^{\prime}\right) d s^{\prime}$. Sejam $a_{t_{0}} \in \mathcal{A}$ e $b_{s_{0}} \in \mathcal{B}$. Como $\Phi_{i}$ e $\Phi_{j}^{\prime}$ convergem pontualmente às respectivas identidades de $\mathcal{A}$ e $\mathcal{B}$. existem $i_{0} \in I, j_{0} \in J$ tais que, $\forall i \geq i_{0}, j \geq j_{0}$, se tem $\left\|\Phi_{i}\left(a_{t_{0}}\right)-a_{t_{0}}\right\|<\epsilon / 2 \mathcal{N}^{\circ} \circ\left\|\Phi_{j}^{\prime}\left(b_{s_{0}}\right)-b_{s_{0}}\right\|<\epsilon / 2$. Consideremos $\Phi_{i, j}: \mathcal{A} \otimes_{\Omega} \mathcal{B} \longrightarrow \mathcal{A} \otimes_{\Omega} \mathcal{B}$ tal que

$$
\Phi_{i, j}\left(x_{(t, s)}\right)=\int_{G \times H}\left(\phi_{i} \odot \phi_{j}^{\prime}\right)\left(t^{\prime} . s^{\prime}\right) x_{(t, s)}\left(\psi_{i} \otimes \psi_{j}^{\prime}\right)\left(t^{-1} t^{\prime} \cdot s^{-1} s^{\prime}\right) d\left(t^{\prime}, s^{\prime}\right) .
$$


Se $(i, j) \geq\left(i_{0}, j_{0}\right)$ temos:

$$
\begin{aligned}
\left\|\Phi_{i, j}\left(a_{t_{0}} \otimes b_{s_{0}}\right)-\left(a_{t_{0}} \odot b_{s_{0}}\right)\right\| & =\left\|\int_{G \times H}\left(\phi_{i} \odot \phi_{j}^{\prime}\right)(t, s)\left(a_{t_{0}} \odot b_{s_{0}}\right)\left(\psi_{i} \odot \psi_{j}^{\prime}\right)\left(t_{0}^{-1} t, s_{0}^{-1} s\right) d(t, s)-\left(a_{t_{0}} \odot b_{s_{0}}\right)\right\| \\
& =\left\|\int_{G} \int_{H} \phi_{i}(t) a_{t_{0}} \psi_{i}\left(t_{0}^{-1} t\right) \odot \phi_{j}^{\prime}(s) b_{s_{0}} \iota_{j}^{\prime}\left(s_{0}^{-1} s\right) d t d s-\left(a_{t_{0}} \odot b_{s_{0}}\right)\right\| \\
& =\left\|\Phi_{i}\left(a_{t_{0}}\right) \odot \Phi_{j}^{\prime}\left(b_{s_{0}}\right)-\left(a_{t_{0}} \odot b_{s_{0}}\right)\right\| \\
& \leq\left\|\left(\Phi_{i}\left(a_{t_{0}}\right)-a_{t_{0}}\right) \odot \Phi_{j}^{\prime}\left(b_{s_{0}}\right)\right\|+\left\|a_{t_{0}}-\left(\Phi_{j}^{\prime}\left(b_{s_{n}}\right)-b_{s_{0}}\right)\right\| \\
& \leq\left\|\Phi_{i}\left(a_{t_{0}}\right)-a_{t_{0}}\right\|\left\|\Phi_{j}^{\prime}\left(b_{s_{0}}\right)\right\|+\left\|a_{t_{0}}\right\|\left\|\Phi_{j}^{\prime}\left(b_{s_{n}}\right)-b_{s_{0}}\right\| \\
& \leq \epsilon \quad \text { porque }\left\|\Phi_{j}^{\prime}\left(b_{s_{0}}\right)\right\| \leq N\left\|b_{s_{0}}\right\|
\end{aligned}
$$

Como vimos na demonstração de 3.25 , tem-se que $\left\|\Phi_{i, j}(x)\right\| \leq M \Lambda\|x\| . \forall x \in \mathcal{A} \otimes_{\alpha} \mathcal{B}$, e conseqüentemente $\Phi_{i, j}(x) \longrightarrow x . \forall x \in \mathcal{A} \otimes_{0} \mathcal{B}$. Isto prova $1 ., 2$. e 3 . .

Suponhamos agora que as redes acima são tais que $\mathcal{A}$ e $\mathcal{B}$ tem a propriedacle de aproximaçào em

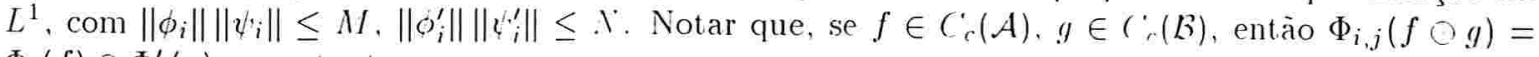
$\Phi_{i}(f) \bigcirc \Phi_{j}^{\prime}(g)$, e portanto,

$$
\begin{aligned}
\left\|\Phi_{i, j}(f, g)-f, g\right\|_{1} & =\left\|\Phi_{i}(f) \oslash \Phi_{j}^{\prime}(g)-f \bigcirc g\right\|_{1} \\
& \leq\left\|\Phi_{i}(f) \oslash\left(\Phi_{j}^{\prime}(g)-g\right)\right\|_{1}+\left\|\left(\Phi_{i}(f)-f\right)-g\right\|_{1} \\
& \leq\left\|\phi_{i}\right\|\left\|\psi_{i}\right\|\|f\|_{1}\left\|\Phi_{j}^{\prime}(g)-g\right\|_{1}+\left\|\Phi_{i}(f)-f\right\|_{1}\|g\|_{1} \\
& \longrightarrow 0 \quad \text { quando } i, j \longrightarrow \infty
\end{aligned}
$$

Logo, temos também que $\Phi_{i, j}(l) \longrightarrow l$ em $L^{1}\left(\mathcal{A} \bigotimes_{\alpha} \mathcal{B}\right)$, para toda $l \in \Gamma=\left\{\sum_{k} f_{k} \bigcirc g_{k}\right\}$. Como $\Gamma$ é denso em $C_{c}\left(\mathcal{A} \bigotimes_{\alpha} \mathcal{B}\right)$ na topologia do limite indutivo, também é denso em $L^{1}\left(\mathcal{A} \bigotimes_{\alpha} \mathcal{B}\right)$. Logo. dados $\epsilon>0, m \in C_{c}\left(\mathcal{A} \otimes_{\alpha} \mathcal{B}\right)$, existe $l \in \Gamma$ tal que $\|m-l\|_{1}<\left(/ 3(M N+1)\right.$. e existem $i_{0}, j_{0}$ tais que $\left\|\Phi_{i, j}(l)-l\right\|_{1}<\epsilon / 3, \forall i \geq i_{0}, j \geq j_{0}$. Portanto, se $i \geq i_{0}, j \geq j_{0}$ :

$$
\begin{aligned}
\left\|\Phi_{i, j}(m)-m\right\|_{1} & \leq\left\|\Phi_{i, j}(m-l)\right\|_{1}+\left\|\Phi_{i, j}(l)-l\right\|_{1}+\|l-m\|_{1} \\
& <M N \epsilon / 3(M N+1)+\epsilon / 3(M N+1)+\epsilon / 3 \\
& <\epsilon .
\end{aligned}
$$

donde concluímos que $\mathcal{A} \otimes_{a} \mathcal{B}$ tem a propriedade de aproximação em $L^{1}$.

Antes de passar às aplicaçòes provamos um outro resultado, sobre limites indutivos de fibrados de Fell, que pode resultar útil em algumas situações (ver por exemplo 3.31).

Proposiçào 3.28. Seja $\mathcal{B}=\left(B_{t}\right)_{t \in G}$ um fibrado de Fell sobre o grupo localmente compacto $G, \epsilon$ suponhamos que $\left(G_{i}\right)_{i \in I}$ é uma família dirigida de subgrupos abertos de $(i$ (i.e.: para todos $i, j$ existe $k \geq i, j$, tal que $\left.G_{i}, G_{j} \subseteq G_{k}\right)$. tal que $G=\bigcup_{i \in I} G_{i}$. Seja $\mathcal{B}_{i}=\mathcal{B}_{G}$,. a retração do fibrado $\mathcal{B}$ a $G_{i}$. Então $\lim _{\longrightarrow} C_{r}^{*}\left(\mathcal{B}_{i}\right)=C_{r}^{*}(\bar{B})$. e se cada $\mathcal{B}_{i}$ é amenable, então também $\mathcal{B}$ é amenable.

Demonstração. Como cada $\mathcal{B}_{i}$ é um subfibrado de Fell de $\mathcal{B}$. temos por 3.10 , que $C_{r}^{*}\left(\mathcal{B}_{i}\right)$ é uma sub- $C^{*}$-álgebra de $C_{r}^{*}(\mathcal{B})$; como $C_{c}(\mathcal{B}) \subseteq \bigcup_{i} C_{r}^{*}\left(\mathcal{B}_{i}\right)$, concluímos que $\bigcup_{i} C_{r}^{*}\left(\mathcal{B}_{i}\right)$ é denso em $C_{r}^{*}(\mathcal{B})$, e portanto $\underline{\lim _{r}} C_{r}^{*}\left(\mathcal{B}_{i}\right)=C_{r}^{*}(\mathcal{B})$.

Suponhamos agora que cada $\mathcal{B}_{i}$ é amenable, e denotemos por $\|\cdot\|_{i}$ a norma sobre $C^{* *}\left(\mathcal{B}_{i}\right)=C_{r}^{*}\left(\mathcal{B}_{i}\right)$. Denotemos por $\|\cdot\|$ e $\|\cdot\|_{r}$ as normas sobre $C^{*}(\mathcal{B})$ e $C_{r}^{*}(\mathcal{B})$ respectivamente. Seja $f \in C_{c}(\mathcal{B})$; para provar que $\mathcal{B}$ é amenable, é suficiente mostrar que $\|f\|=\|f\|_{r}$. Como $f$ tem suporte compacto e os $G_{i}$ são uma família de abertos que cobrem $G$, existe uma subfamília finita $G_{i_{1}}, \ldots, G_{i_{n}}$ que cobre o 
suporte de $f$. Como os $G_{i}$ sào uma família dirigida, existe $j \in I$ tal que $j \geq i_{l}$ e $G_{j} \supseteq G_{i_{l}}, \forall l=1, \ldots n$. Então $f \in C_{c}\left(\mathcal{B}_{j}\right)$. Consideremos uma representação $\pi: L^{1}(\mathcal{B}) \longrightarrow B(H)$. Por restrição, temos uma representação $\pi_{j}: L^{1}\left(B_{j}\right) \longrightarrow B(H)$, e portanto $\|\pi(f)\|=\left\|\pi_{j}(f)\right\| \leq\|f\|_{j}$, donde segue, depois de tomar supremo na família das representaçōes de $L^{1}(\mathcal{B})$, que $\|f\| \leq\|f\|_{j}$. Conseqüêntemente, $\|f\|=\|f\|_{r}$, já que $\|f\|_{r}=\|f\|_{j}$.

Corolário 3.29. Se $\mathcal{A} \in \mathcal{B}$ sáo fibrados de Fell que tém a propriedade de aproximação em $L^{1}$, então $A_{\epsilon} \odot B_{e}$ admite exatamente uma $C^{* *}$-norma se $\epsilon$ somente se $C^{*}(\mathcal{A}) \odot C^{*}(\mathcal{B})$ admite exatamente uma $C^{-}{ }^{-}$-norma. Em particular. se $\mathcal{A}$ é um fibrado de Fell com a propriedade de aproximaçào $\mathrm{\epsilon m} L^{1}$ e fibra nuclear $A_{\epsilon}$. entio $C^{*}(\mathcal{A})$ também é nuclear (lembrar que por 3.26. basta que o grupo de base seja amenable para que o fibrado tenha a propriedade de aproximação em $L^{1}$ ).

Demonstração. ('omo $\mathcal{A}, \mathcal{B}$ são fibrados de Fell com a propriedade de aproximação em $L^{1}$, então $\mathcal{A} \bigotimes_{\text {mín }} \mathcal{B}$ tem também a propriedade de aproximaçào em $L^{1}$ por $3.2 \bar{\tau}$, e assim o diagrama $D_{\text {min fica: }}$

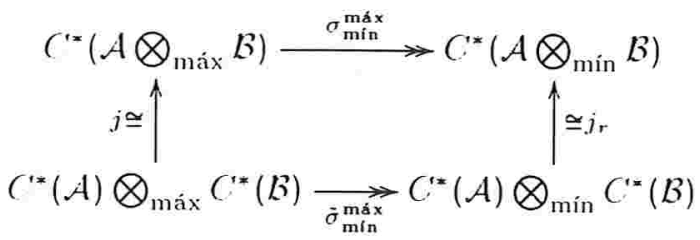

Se $A_{e} \odot B_{e}$ admite apenas uma $C^{* *}$-norma, entào $\mathcal{A} \bigotimes_{\text {máx }} \mathcal{B}=\mathcal{A} \bigotimes_{\text {mín }} \mathcal{B}$ o que implica que $\sigma_{\text {mín }}^{\text {máx }}=i d$, e portanto que $\tilde{\sigma}_{\operatorname{mí}}^{\max }=i d$, donde segue que $C^{*}(\mathcal{A}) \bigotimes_{\operatorname{máx}} C^{*}(\mathcal{B})=C^{*}(\mathcal{A}) \bigotimes_{\min } C^{*}(\mathcal{B})$. Na otra direção. suponhamos que $C^{*}(\mathcal{A}) \odot C^{* *}(\mathcal{B})$ admite apenas uma $C^{*}$-norma. Então $\tilde{\sigma}_{\text {míx }}^{\text {máx }}=i d$, e portanto $\sigma_{\text {min }}^{\text {máx }}=i d$. Logo, $\mathcal{A} \bigotimes_{\text {máx }} B=\mathcal{A} \bigotimes_{\text {min }} \mathcal{B}$, donde segue que $A_{\epsilon} \bigotimes_{\text {máx }} B_{e}=A_{\epsilon} \bigotimes_{\text {mín }} B_{\epsilon}$.

Com respeito à última afirmação, observar que toda $C^{*}$-álgebra $B$ pode ser enxergada como um fibrado de Fell sobre o grupo trivial, e é claro que toda unidade aproximada de $B$ fará o serviço de rede aproximante para este fibrado de Fell (ou mesmo a função que vale $1 \in M(B)$ !). Conseqüentemente, pela primeira parte do Corolário temos $C^{*}(\mathcal{A}) \bigotimes_{\text {máx }} B=C^{*}(\mathcal{A}) \bigotimes_{\min } B$, i.e., $C^{*}(\mathcal{A})$ é uma $C^{*}-$ álgebra nuclear.

Corolário 3.30. Se A é um fibrado de Fell com a propriedade de aproximação em $L^{1} \epsilon$ fibra nuclear sobre a unidade, $e$ se $\mathcal{B}$ é um fibrado de Fell amenable. entào $\mathcal{A} \otimes \mathcal{B}$ também é amenable.

Demonstraçào. Pelo Corolário 3.29 , as nossas suposições sobre $\mathcal{A}$ implicam que $C^{*}(\mathcal{A})$ é nuclear. Portanto, deve ser $C^{*}(\mathcal{A}) \bigotimes_{\max } C^{*}(\mathcal{B})=C^{*}(\mathcal{A}) \bigotimes_{\overline{\max }} C^{*}(\mathcal{B})$, e o resultado segue do Corolário 3.22 .

Corolário 3.31. Suponhamos que o fibrado de Fell $\mathcal{B}$ está nas hipóteses da Proposição 3.2S. Se $B_{e}$ é nuclear e cada $\mathcal{B}_{i}$ tem a propriedade de aproximação em $L^{1}$, então $C^{*}(\mathcal{B})$ é nuclear.

DemonstraçÃo. Como $B_{\epsilon}$ é nuclear e cada $\mathcal{B}_{i}$ tem a propriedade de aproximação em $L^{1}$, então por 3.29 temos que $C^{* *}\left(\mathcal{B}_{i}\right)$ é nuclear, $\forall i \in I$, e daqui $\underset{\lim }{\longrightarrow} C^{* *}\left(\mathcal{B}_{i}\right)$ também é nuclear; mas $C^{* *}(\mathcal{B})=$

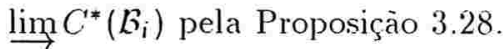


Corolário 3.32. Todo produto cruzado parcial torcido de uma $C^{*}$-álgebra nuclear por um grupo amenable é nuclear. Em particular. a $C^{* *}$-álgebra parcial $C_{p}^{*}(G)$ de um grupo discreto amenable $e ́$ nuclear.

Demonstraçào. Lembrar de [27] (ver também 2.15) que uma ação parcial torcida de uma $C^{*}$ álgebra $A$ por um grupo localmentc compacto define um fibrado de Fell sobre o grupo. cuja fibra sobre a identidade é $A$. Agora, se o grupo é amenable, 4.7 de [28] mostra que o fibrado de Fell em questào tem a propriedade de aproximaçào é á amenable, e portanto, se além disso $A$ é nuclear. o Corolário 3.29 implica que também é nuclear a ('* -álgebra seccional do fibrado, que é precisamente o produto cruzado pela açào parcial.

Com respeito à última afirmaçào, ela é verdadeira porque $C_{p}^{*}(G)$ é por definição um produto cruzado parcial de uma ('*álgebra comutativa pelo grupo $G$ (ver [26], 6.4).

Definiçào 3.33. Sejam $\mathcal{A}, \mathcal{B}$ e C' fibrados de Fell sobre o grupo localmente compacto $G$, e sejam $\circ: \mathcal{A} \longrightarrow \mathcal{B}, \psi: B \longrightarrow \mathcal{C}$ homomorfismos de fibrados de Fell. Dizemos que a seqüencia

$$
() \longrightarrow \mathcal{A} \stackrel{\phi}{\longrightarrow} \mathcal{B} \stackrel{\dot{*}}{\longrightarrow} \mathrm{C} \longrightarrow 0
$$

é exata se $\phi$ é injetiva. $v$ é sobrejetiva, e ker $\psi=\operatorname{Im} \phi$. Equivalentemente, a seqüencia acima é exata se e somente se a seqülencia

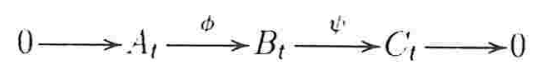

é exata, $\forall t \in G$.

A seguinte é uma generalização de [1], 3.1, ao caso contínuo.

ProposiçÃo 3.34. Seja $(\longrightarrow \mathcal{A} \stackrel{\phi}{\longrightarrow} B \stackrel{\psi}{\longrightarrow} \longrightarrow \longrightarrow 0$ uma seqüéncia exata de fibrados de Fell sobre o grupo localmente compacto $G$. Então:

1. $0 \longrightarrow L^{1}(\mathcal{A}) \stackrel{\dot{o}^{1}}{\longrightarrow} L^{1}(\mathcal{B}) \stackrel{l^{\prime \prime 1}}{\longrightarrow} L^{1}(\mathcal{C}) \longrightarrow 0$ é exata.

2. $0 \longrightarrow C^{*}(\mathcal{A}) \stackrel{C^{* *}(\phi)}{\longrightarrow} C^{* *}(B) \stackrel{C^{*}(*)}{\longrightarrow} C^{* *}(\mathcal{C}) \longrightarrow 0$ é exata.

Demonstração. Como toda representação não degenerada de $L^{1}(\mathcal{A})$ tem uma única extensão a uma representação de $L^{1}(\mathcal{B})$, temos que $C^{* *}(\mathcal{A})$ é o fecho de $L^{1}(\mathcal{A})$ dentro de $C^{*}(\mathcal{B})$. e portanto basta provar 1., porque 2. segue de [67], 2.29, e do fato de $L^{1}(\mathcal{F})$ ter uma unidade aproximada, para todo fibrado de Fell $\mathcal{F}$.

- $\phi^{1}$ é isométrica:

Como $\varphi$ é injetiva, ela é uma isometria, e portanto, se $f \in L^{1}(\mathcal{A})$ :

$$
\left\|\phi^{1}(f)\right\|_{1}=\int_{G}\left\|\phi^{1}(f)(t)\right\| d t=\int_{G}\|\phi(f(t))\| d t=\int_{G}\|f(t)\| d t=\|f\|_{1} .
$$

- $\operatorname{ker} \psi^{1}=\operatorname{Im} \varphi^{1}$ :

A inclusão Im ${ }^{1} \subseteq \operatorname{ker} \iota^{1}{ }^{1}$ é clara; provemos a inclusão inversa. Seja $g \in \operatorname{ker} \psi^{1}$. Então $\left\|\psi^{1}(g)\right\|_{1}=0$. e protanto $\psi^{\prime}(g(t))=0$, para quase todo $t$ de $G$; podemos supor que $\psi(g(t))=0$. para todo $t$ em $\left(i\right.$. Assim, $g(t) \in \operatorname{ker} \psi=\operatorname{Im} \phi, \forall t \in G$, e portanto existe um único $f(t) \in A_{t}$ tal que $\phi(f(t))=g(t), \forall t \in G$. Como $\phi$ é um isomorfismo contínuo isométrico entre $\mathcal{A}$ e $\phi(\mathcal{A})$ ([37], II-13.1T), e temos que $f=\left(\phi^{1}\right)^{-1}(g)$, então $f \in L^{1}(\mathcal{A})$, e portanto $g \in \operatorname{Im} \phi^{1}$. 
- $\psi^{1}$ é sobrejetiva:

Podemos supor que $\mathcal{A} \subseteq \mathcal{B}$. $L^{1}(\mathcal{A})$ é um *-ideal bilateral fechado de $L^{1}(\mathcal{B})$, portanto existe um isomorfismo de *álgebras $\frac{L^{1}(\boldsymbol{B})}{L^{1}(\mathcal{A})} \stackrel{\bar{\psi}}{\longrightarrow} \psi^{, 1}\left(L^{1}(\mathcal{B})\right) \subseteq L^{1}\left(\mathcal{C}^{\prime}\right)$. Notar que a imagem de $\bar{\psi}$ contém $\psi^{1}\left(C_{c}^{\prime}(\mathcal{B})\right)$, que é denso em $C_{c}^{\prime}\left(\mathcal{C}^{\prime}\right)$ na topologia do limite indutivo porque. sendo $\psi$ sobrejetiva, $\psi^{1}{ }^{1}\left(C_{c}(\mathcal{B})\right)$ satisfaz a hipótese de 3.14. Portanto $\operatorname{Im} \chi^{\prime}$ é denso em $L^{1}\left(C^{2}\right)$. Basta provar então que $\bar{\psi}$ é uma isometria, onde $\frac{L^{1}(\mathcal{L})}{L^{1}(\mathcal{A})}$ tem a norma quociente. Sejam $f \in C_{C}(B), \bar{f}$ sua projeção no quocient.e. Pelo teorema do quociente, temos que $\|\iota \cdot\|=\left\|\iota^{1}\right\|$, e portanto $\|\bar{l}(\bar{f})\| \leq\left\|\iota^{, 1}\right\|\|\bar{f}\| \leq$ $\|\bar{f}\|$. Para provar a desigualdade contrária, consideremos c $>0$ arbitrário, e seja $M$ a medida de uma vizinhança compacta $I^{\prime}$ clo suporte de $f$. Para cada $s \in I^{\prime}$. existe $g_{s} \in C_{c}(\mathcal{A})$ tal que $\left\|f(s)-g_{s}(s)\right\|<\|\overline{f(s)}\|+\epsilon / M$. e podemos supor que $\sup \left(g_{s}\right) \subseteq \mathrm{I}$. ('omo $f, g_{s}$. e $t \longmapsto \overline{f(t)}$ sào contínuas, para cada $s \in \sup (f)$ deve existir uma vizinhança $l_{\text {: }}$ de $s$, que podemos supor conticla em $I$. e tal que $\left\|f(t)-y_{s}(t)\right\|<\|\overline{f(t)}\|+\epsilon / M, \forall t \in I_{s}$. Agora. $\left\{I_{s}: s \in \sup (f)\right\}$ é uma cobertura aberta do compacto $\sup (f)$. e portanto existe uma subcobertura finita $V_{s_{1}}, \ldots V_{s_{n}}$. Seja $G_{\star}$ a compactificaşào com um ponto de $G$. e definamos $s_{n+1}=\star$. $I_{s_{n+1}}=G_{\star} \backslash \sup (f)$ e $g_{s_{n+1}}=0$. onde $\star$ representa o ponto acrescentado. Assim. $\left\{I_{s}\right\}_{i=1}^{n+1} \dot{i}$ uma cobertura aberta de $G_{\star}$. Seja $\left(O_{i}\right)_{i=1}^{n+1}$ uma partiçào da unidade de $G_{\star}$ subordinada a $\left\{I_{s_{r}}\right\}_{i=1}^{n+1}$, e definamos $g=\sum_{i=1}^{n+1} \phi_{i} g_{s_{1}}$. Entào $g \in C_{c}(\mathcal{A}), \sup (g) \subseteq V^{\prime}, \mathrm{e}$

$$
\begin{aligned}
\|\bar{f}\| & \leq \int_{C_{i}}\|f(t)-g(t)\| d t \\
& =\int_{1} \| \sum_{i=1}^{n+1}\left(\phi_{i}(t) f(t)-o_{i}(t) g_{s_{,}}(t) \| d t\right. \\
& \leq \sum_{i=1}^{n+1} \int_{\Gamma_{s_{i}}} \phi_{i}(t)\left\|f(t)-g_{s,}(t)\right\| d t \\
& \leq \int_{1} \sum_{i=1}^{n+1} \phi_{i}(t)(\|\overline{f(t)}\|+\epsilon / . M) d t \\
& \leq \int_{C^{i}}\left\|\psi^{\prime}(f(t))\right\| d t+\epsilon \\
& =\left\|l^{1}(f)\right\|_{1}+\epsilon .
\end{aligned}
$$

Como esta desigualdade vale para todo $\epsilon>0$, concluímos que $\|f\| \leq\left\|\psi^{1}(f)\right\|_{1}$, e portanto $\|\bar{f}\|=\|\bar{\psi}(f)\|_{1}$. Como, além disso, $\bar{\psi}$ tem imagem densa em $L^{1}(\mathcal{C})$, entào $\operatorname{Im}(\bar{\psi})=L^{1}(\mathcal{C})$, e como $\operatorname{Im}\left(\psi^{1}\right)=\operatorname{Im}(\bar{\psi})$, concluímos que $\psi^{1}$ é sobrejetiva.

TeOrema 3.35. Se $\mathcal{A}=\left(A_{t}\right)_{t \in G} \dot{\epsilon}$ um fibrado de Fell com fibra exata $\epsilon$ a propriedade de aproximação $\mathrm{em} L^{1}$, então $C^{* *}(\mathcal{A})$ é uma $C^{* *}$-álgebra exata.

Demonstração. Sejam $B$ uma $C^{*}$-álgebra, $I \triangleleft B$. Como $A$, é exalo $\forall t \in G$, a seqüencia de fibrados de Fell

$$
0 \longrightarrow \mathcal{A} \otimes_{\min } I \longrightarrow \mathcal{A} \otimes_{\min } B \longrightarrow \mathcal{A} \otimes_{\min }(B / I) \longrightarrow 0
$$

é exata, e cada um dos fibrados da seqüencia tem a propriedade de aproximação em $L^{1},(3.2 \tau)$. Como $C^{*}$ é um funtor exato (da categoria de *álgebras com unidade aproximada na categoria das 
$C^{*}$-álgebras), e neste caso é $C^{\prime *}=C_{r}^{* *}$, a seqüencia de $C^{* *}$-álgebras

$$
0 \longrightarrow C_{r}^{*}\left(\mathcal{A} \otimes_{\min } I\right) \longrightarrow C_{r}^{*}\left(\mathcal{A} \otimes_{\min } B\right) \longrightarrow C_{r}^{*}\left(\mathcal{A} \otimes_{\min }(B / I)\right) \longrightarrow 0
$$

também é exata.

Finalmente, 3.20 fornece um isomorfismo natural entre $C_{r}^{*}\left(\mathcal{A} \otimes_{\min }\left(^{\prime}\right)\right.$ e $C_{r}^{* *}(\mathcal{A}) \bigotimes_{\min } C^{\prime}$, para toda $C^{* *}$-álgebra $C$. Assim, obtemos o seguinte diagrama comutativo:

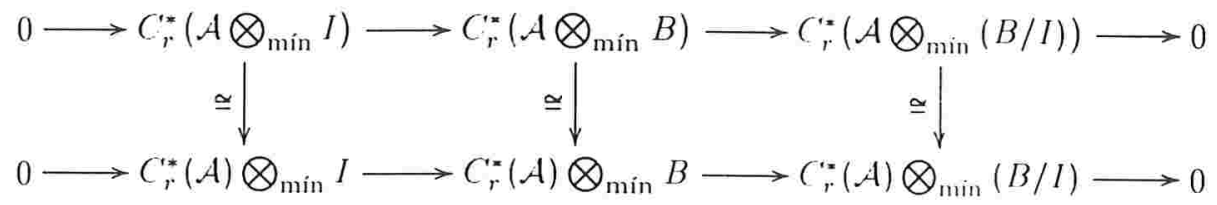

Como a primeira fila é exata e o diagrama comuta, a segunda fila também é exata. Segue daqui que $C^{*}(\mathcal{A})$. que é igual a $C_{r}^{*}(\mathcal{A})$, é uma $\left(^{* *}\right.$-álgebra exata.

Corolário 3.36. Todo produto cruzado parcial torcido de uma (")-álgebra exata por um grupo amenable é exato.

Demonstraçào. Um produto cruzado parcial torcido é por definiçào a $C^{* *}$-álgebra seccional de um certo fibrado de Fell sobre o grupo que age $([27])$, e com fibra sobre a identidade igual à $C^{*}$-álgebra onde a ação ocorre. Se o grupo é amenable. o correspondente fibrado de Fell tem a propriedade de aproximação em $L^{1}$ por 3.26: se a $C^{*}$-álgebra é exata, o fibrado tem fibra exata, e portanto o Corolário decorre do Teorema 3.35 acima.

\subsection{Comentários finais}

Como já foi mencionado na Introduçào à Parte I. os resultados do Capítulo 3 foram provados primeiro para fibrados sobre grupos cliscretos em [3] (exceto. é claro, o Teorema 3.25 e a Proposiçào 3.26, provados em [28]). Em particular, 3.2. foi provado neste caso. Quando o grupo é discreto. a $C^{*}$-álgebra seccional reduzida de um fibrado de Fell tem uma caracterização muito conveniente em termos de uma esperança condicional (3.11), caracterização que já nào existe no caso contínuo. Logo depois de divulgarmos [3], recebemos de parte de $\mathrm{Ng}$ o seu pré-artigo [50]. Neste artigo, ele considerava uma certa $C^{*}$-álgebra seccional $C_{\epsilon}^{*}(\mathcal{B})$ do fibrado $\mathcal{B}$, e chamava "próprio" ao fibrado $\mathcal{B}$ se $C_{\epsilon}^{*}(\mathcal{B})=C_{r}^{*}(\mathcal{B})$. Entào mostrava que, se $\mathcal{B}$ é próprio e $G$ é amenable (ou se $\mathcal{B}$ satisfaz uma certa propriedade de aproximaçào), então $\mathcal{B}$ é amenable. Em avril deste ano, Exel publicou [30], onde prova que $C_{\epsilon}^{*}(\mathcal{B})=C_{r}^{*}(\mathcal{B})$ para todo fibrado $\mathcal{B}$. Quando apareceu [30], Exel e $\mathrm{Ng}$ decidiram combinar os seus respectivos resultados em [30] e [50]. O resultado é [33]. que foi divulgado em junho.

$\mathrm{Em}[30]$, mostra-se como obter uma imagem isomorfa de $C_{r}^{*}(\mathcal{B})$ usando representações do fibrado sobre espaços de Hilbert. Com este resultado na mão, nós tentamos generalizar os nossos resultados de [3] ao contexto contínuo (ainda sabendo que [33] estava em elaboraçào). Em particular, achávamos que a versào da propriedade de aproximação introduzida por $\mathrm{Ng}$ em [50] para o caso contínuo era inadequada. Os autores de [33] também consideraram uma outra propriedade de aproximação no caso contínuo, igual à nossa exceto que a convergencia de $\Phi_{i}(f)$ a $f$ é na topologia do limite indutivo de $C_{c}(\mathcal{B})$. Em [33], a convergencia de $\Phi(f)$ a $f$ na topologia do limite indutivo para toda $f \in$ $C_{c}(\mathcal{B})$ é expressada dizendo que $\Phi$ converge à identidade $i d_{\mathcal{B}}$ sobre fatias compactas de $\mathcal{B}$ ("compact slices"). Naturalmente que a convergencia $\Phi_{i} \longrightarrow i d_{\mathcal{B}}$ sobre fatias compactas implica a propriedade de aproximação em $L^{1}$; nào sabemos se vale também a recíproca. Em todo caso, a convergencia 
sobre fatias compactas considerada em [33] é utilizada apenas para ver que $\Phi(f) \longrightarrow f$ em $L^{1}(\mathcal{B})$, e portanto pode ser substituida pela propriedade de aproximação em $L^{1}$.

Antes de encerrar estes comentários, gostaríamos de acrescentar uma última observação. Nossa propriedade de aproximação implica a existencia de umas redes aproximantes que tomem valores na álgebra dos multiplicadores da fibra sobre a identidade do grupo. Como já foi comentado oportunamente, esta nào é uma diferença substancial: seria a mesma coisa pedir que as redes fossem a valores na fibra sobre a unidade. No entanto, ao provarmos que um fibrado de Fell sobre um grupo amenable é também amenable, achamos uma rede aproximante que toma seus valores em $Z\left(M\left(B_{e}\right)\right)$ (o centro da álgebra dos multiplicadores). Lembrar que em [4], a autora considera certa propriedade de aproximação, que é satisfeita quando existe uma certa rede de funçòes de tipo positivo (com respeito a uma ação) que tomam valores no centro de uma certa álgebra de von Neumann. Isto pode ser apenas uma coisa casual. mas talvez valha a pena aprofundar neste aspecto. 
Parte II

Ações Envolventes de Ações Parciais 


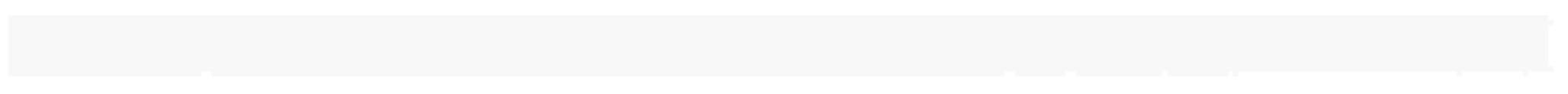

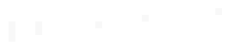

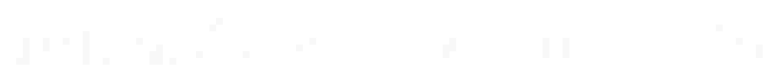

1.

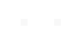


Introdução à Parte II 
A pergunta que motiva esta segunda parte do trabalho é a seguinte: quando é que uma ação parcial a de um grupo $G$ sobre uma $C^{*}$-álgebra $A$ é a restrição a $A$ de uma açào global $\beta$ sobre uma $C^{\prime *}$-álgebra $B$ que contém A como ideal? Para evitar soluçòes redundantes, exigimos também que a $\beta$-órbita de $A$ gere linearmente um subespaço denso de $B$. Uma tal ação $\beta$ será chamada ação envolvente de $a$. Naturalmente, a restrição de uma ação global a um ideal define uma ação parcial sobre este ideal. Junto com todo problema de existencia vem o problema da unicidade da eventual solução, problema que também consideramos para a ação envolvente.

A pergunta acima pode ser considerada também para açōes parciais em outras categorias. e de fato isto é extremamente conveniente. Por exemplo, não podemos esperar que exista uma ação $\beta$ como acima se o problema já nào tem soluçào na categoria de conjuntos e funçòes. Afortunadamente, nesta categoria a ação envolvente existe e é única. Levando em conta a dualidade entre a categoria de espaços topológicos localmente compactos de Hausdorff e funções contínuas próprias com a categoria de $C^{*}$ álgebras comutativas e seus homomorfismos. também nào cabe esperar que exista a desejada $\beta$ se nào existe açào envolvente na categoria de espaços topológicos de Hausdorff. Na verdade, a consideração do problema da açào envolvente nesta categoria determinou um ponto de inflexào no desenvolvimento do trabalho: se bem todla açào parcial contínua o sobre um espaço topológico .Y possui uma única açào envolvente $\dot{\alpha}$, agindo sobre um espaço topológico $\dot{X}$ que contém $X$ como subespaço aberto, exemplos simples mostram que $X$ pode ser cle Hausdorff sem que $\dot{X}$ seja de Hausdorff. Se $X$ é localmente compacto e de Hausdorff, a induz uma ação parcial $a^{*}$ sobre $C_{0}(X)$. Se $a^{*}$ tem ação envolvente $\beta$ agindo sobre a $C^{*}$-álgebra $B$. é fácil ver que $B$ deve ser comutativa, e que $\beta$ induz sobre o espectro $B$ de $B$ uma ação que é ação envolvente de $a$. Daqui concluímos que nem sempre haverá ação envolvente na categoria de $C^{*}$-álgebras.

Esta conclusão pareçe frustrar as nossas expectativas, ou pelo menos nos obriga a reformulá-las. Sendo otimistas e enigmáticos, podemos interpretar o fato anterior assim: nào existe a $C^{*}$-álgebra envolvente $B$, mas sim existe o seu espectro: $\dot{X}$. Em outras palavras, precisamos achar uma outra noção de ação envolvente, mais flexível, de forma que toda açào parcial tenha ação envolvente; se conseguirmos uma tal noção, no exemplo acima $\dot{X}$ deveria ser o espectro da correspondente $C^{*}$ álgebra envolvente. Como achar uma definiçào alternativa de açào envolvente? Por um lado, o conceito considerado no começo parece o único razoável; por outro, os exemplos mostram que a categoria resultou rígida demais para permitir a existencia de ação envolvente em todos os casos. Isto sugere flexibilizar a categoria, por exemplo acrescentando morfismos. Como já foi observado. se queremos uma solução, os espaços topológicos de Hausdorff e as $C^{*}$-álgebras não comutativas devem estar num mesmo nível, no sentido de que o espaço envolvente de uma ação parcial sobre um espaço de Hausdorff possa ser, em geral, uma $C^{*}$-álgebra não comutativa. Isto concorda com o moderno ponto de vista que interpreta a teoria de $C^{*}$-álgebras como uma teoria de espaços topológicos "não comutativos", ou "quanticos". Neste sentido também, deveríamos poder comparar $C^{*}$-álgebras comutativas com $C^{\prime *}$-álgebras nào comutativas, coisa que não podemos fazer com os homomorfismos usuais de $C^{*}$-álgebras: se $B$ é isomorfa a $B^{\prime}$, entào $B$ é comutativa se e somente se $B^{\prime}$ é comutativa. Podemos, no entanto, identificar duas $C^{*}$-álgebras quando elas sejam equivalentes Morita. Assim, por exemplo. o espaço topológico com apenas um ponto pode ser representado tanto pela $C^{*}$-álgebra $\mathbb{C}$ dos complexos, como pela $C^{*}$-álgebra de matrizes $M_{n}(\mathbb{C})$, ou ainda pela $C^{*}$-álgebra $\mathcal{K}$ dos operadores compactos sobre um espaço de Hilbert de dimensão infinita enumerável.

As consideraçōes que fizemos sugerem substituir a definição de ação envolvente inicial pela seguinte: $\beta$ é uma ação envolvente de $\alpha$, a menos de equivalência Morita, se $\beta$ é a ação envolvente de uma açào parcial equivalente Morita com $\alpha$; naturalmente, isto requer definir equivalência Morita entre açòes parciais. Um argumento que reforçou significativamente este ponto de vista, foi um resultado que tínhamos obtido no caso de existir ação envolvente, junto com uma observação que descreveremos a seguir. Por um lado, provamos que se $(\beta, B)$ é uma ação envolvente de $(\alpha, A)$, então os produtos cruzados reduzidos $A \rtimes_{a, r} G$ e $B \rtimes_{\beta, r} G$ sào equivalentes Morita. Por outro lado, supondo que $G$ 
é abeliano, o teorema de Takai implica que $A \rtimes_{\alpha} G \rtimes_{\dot{\alpha}} G$ é equivalente Morita com $B$, onde $\alpha$ é a açào dual de $G$ sobre $A \rtimes_{G} G$ : além disso, $\beta$ é equivalente Morita com a ação bidual $\alpha$ de $G$ sobre $A \rtimes_{a} G \rtimes_{\hat{a}} G$. Isto sugere que, no caso de não existir $(\beta, B)$, o par $\left(\alpha, A \rtimes_{a} G \rtimes_{\hat{a}} G\right)$ pode ser un substituto adequado para $(3, B)$. Se $G$ nào é abeliano, podemos ainda ter esperanças similares. usando os resultados da teoria de clualidade para produtos cruzados por grupos nào comutativos. análogos ao teorema de Takai. Afortunadamente, este ponto de vista resultou acertado: para toda açào parcial de um grupo localmente compacto sobre uma $C^{* *}$-álgebra existe uma ação envolvente a menos de equivalencia Morita, e esta açào envolvente é única a menos de equivalencia Morita. Equivalentemente: toda açào parcial tem uma única açào envolvente na categoria de $C^{* *}$-álgebras com os bimódulos de Hilbert plenos como morfismos.

Antes de descrever a organizaçào do trabalho, gostaríamos de salientar alguns dos resultados e aplicaçòes mais importantes obtidos, além do principal, já mencionado acima. Mostramos que senpre existe e é única a açào envolvente de uma açào parcial na categoria de conjuntos e também na categoria de espaços topológicos. e as caracterizamos através de propriedades universais. Caracterizamos também as açòes parciais solore espaços de Hausdorff, cujo espaço envolvente é de Hausdorff. como aquelas que tem gráfico fechado (no sentido precisado em 4.51). Ainda na cat egoria de espaços topológicos, vemos que, de maneira geral, as propriedades dinamicas de uma açào parcial e de sua ação envolvente são as mesmas. Já no contexto de $C^{*}$-álgebras, vemos que a ação envolvente $(\beta, B)$ da açào parcial $(a, A)$ ć única. sempre que exista. Além disso, provamos que $A \rtimes_{\alpha, r} G$ e $B \rtimes_{B, r}(;$ são equivalentes Morita, c também que $A$ e $B$ são bastante parecidas, no sentido de que $A$ é nuclear. liminal, postliminal (por exemplo), se e somente se $B$ é nuclear, liminal, postliminal respectivamente. Isto implica, em particular, que se $B$ é um fibrado de Fell e $\delta$ é a coaçào dual sobre $C_{r}^{*}(\mathcal{B})$, entào $C_{r}^{*}(\mathcal{B}) \searrow_{\delta, r} G$ é nuclear. liminal, postliminal, se e somente se $B_{\epsilon}$ (a fibra de $\mathcal{B}$ sobre a identidade de $G$ ) é, respectivamente. nuclear. liminal, postliminal. Que saibamos, isto era sabido apenas para fibrados de Fell sobre grupos discretos ([53], [49]).

Um resultado bastante curioso, implicado pelo nosso resultado principal, é o seguinte: se $G$ é um grupo conexo que age parcialmente sobre uma $C^{*}$-álgebra com unidade, entào a açào parcial é, na verdade, uma açào global. Esta é uma generalizaçào bastante agradável, do conhecido resultado sobre sistemas dinamicos que ocorrem em variedades compactas: se $\dot{x}=\mathrm{v}(x)$ é uma equaçào diferencial sobre uma variedade compacta $\mathbb{X}$. entào as soluçòes da equação estào definidas sobre toda a reta real. Com efeito, como mostramos no Capítulo 4, o fluxo de uma tal equaçào diferencial é uma ação parcial de $\mathbb{F}^{\prime}$ sobre a variedade $\mathrm{X}$, e portanto induz uma ação parcial sobre $C_{0}(\mathrm{Y})$. Como .I é compacta, $C_{0}(\mathrm{X})$ tem unidade, e portanto a ação parcial é uma ação global. Isto equivale a dizer que as soluções da equaçào acima estão definidas para todo $t \in \mathbb{R}$.

Suponhamos que $\alpha$ é uma açào parcial contínua de $G$ sobre $A$, e sejam $A$ e Prim( $A)$ o espectro e o espaço de ideais primitivos de $A$, respectivamente. Então $\alpha$ induz açòes parciais contínuas á sobre $A$ e à sobre Prim( $A$ ), e temos o seguinte bonito resultado: se $(\beta, B)$ é uma açào envolvente a menos de equivalència Morita de $(\alpha, A)$, entào $(\hat{\beta}, \dot{B})$ é a ação envolvente de $(\alpha, A)$, e $(\bar{\beta}$. Prim $(B))$ é a ação envolvente de $(\tilde{\alpha}, \tilde{A})$.

Finalmente, mostramos que se $(3, B)$ é a ação envolvente de $(\alpha, A)$, entào $A \rtimes_{a, r} G$ é equivalente Morita com $B \rtimes_{\beta, r} G$.

Esta segunda parte do nosso trabalho está distribuída em quatro capít.ulos.

O primeiro deles, embora importante para o desenvolvimento posterior, é totalmente elementar do ponto de vista das técnicas matemáticas utilizadas. Nele estudamos e resolvemos o problema 
INTRODUÇÃO À PARTE II

da existência e unicidade da ação envolvente nas categorias de conjuntos e de espaços topológicos. Consideramos o exemplo do fluxo de uma equação diferencial mencionado anteriormente, e também apresentamos exemplos elementares de ações parciais sobre espaços de Hausdorff cujos espaços envolventes não são de Hausclorff. ('aracterizamos as ações parciais cujas açòes envolventes atuam sobre espaços de Hausdorff, e comparamos as propriedades dinamicas de uma açào parcial e de sua açào envolvente.

No Capítulo 5, consideramos o problema da ação envolvente na categoria de ('*-álgebras. Essencialmente, o único problema é o da unicidade da ação envolvente no caso em que exista. Vemos. em 5.11, que a açào envolvente é única sempre que exist.a. Neste capítulo mostramos que uma açào parcial sobre $A$ induz açòs parciais sobre o espectro de $A$ e sobre o espaço de ideais primitivos de $A$. Na última parte comparamos algumas propriedades de A e da $C^{\prime *}$-álgebra envolvente $B$. Vemos, por exemplo, que se C' é uma classe de ('ª́lgebras fechada por ideais, quocientes, extensões e limites indutivos, então $A$ pertence a $C^{\prime}$ 'se e somente se $B$ pertence a $C$ '.

O resultado principal do ('apítulo 6 é: se $(\beta, B)$ é uma açào envolvente de $(\alpha, A)$, entào $A \rtimes_{\alpha, r}(i$ e $B \rtimes_{\beta, r} G$ sào equivalentes Morita. Apresentamos depois. bastante rapidamente, alguns exemplos e aplicaçòes. A aplicaçào que pessoalmente nos parece mais interessante ć que toda representaçào parcial de um grupo discreto "amenable” é uma compressào de uma representaçào unitária do grupo. e em particular é uma funçào de tipo positivo. Achamos que este resultado deve ser válido ainda no caso não amenable.

Dedicamos também algumas páginas ao estudo de certa ("*álgebra $E(G)$ de dimensão finit.a ligada naturalmente a um grupo finito $\left(i\right.$. Lembremos que se $C_{i}$ é um grupo discreto. existe uma $C^{*}$-álgebra $C_{p}^{*}(G)$ cuja tcoria de representaçòes reflete a teoria de represcntaçòes parciais do grupo. $C_{p}^{*}(G)$ é um produto cruzado parcial de uma $C^{*}$-álgebra comutativa por uma açào parcial de $G$. No caso de $\operatorname{ser} G$ finito, esta açào parcial tem sempre açào envolvente. e como $G$ é amenable, $C_{p}^{*}(G)$ é equivalente Morita com o correspondente produto cruzado pela ação envolvente, que denotamos por $E(G)$. Sendo equivalentes Morita, $C_{p}^{*}\left(C_{i}\right)$ e $E(G)$ tem a mesma teoria de representaçòes, e portanto a teoria de representaçòes de $E(G)$ também deve refletir a teoria de representaçòes parciais de $G$. Por outro lado, parece-nos que o cálculo de $E(G)$ é mais simples que o cálculo de $C_{p}^{\prime *}(G)$, pelo menos no caso em que $G$ seja comutativo. A única razào para ter dedicado tantas páginas a este exemplo foi mostrar, através da análise de casos particulares, que este é realmente o caso. No ano passado foi provado que $C_{p}^{*}(G)$ é um invariante completo dos grupos abelianos finitos $([20])$. Acreditamos que o mesmo acontece com $E(G)$. com a vantagem de que seu cálculo é mais fácil. Os assuntos principais desta tese impediram que dedicássemos mais tempo ao estudo desta álgebra.

O Teorema 7.38 é o mais importante da Parte II. Nele provamos que toda ação parcial tem uma ação envolvente a menos de equivalencia Morita. Suponhamos que o é uma açào parcial sobre $A$. e seja $\mathcal{B}$ o fibrado de Fell associado a a. En particular, a fibra $B_{\epsilon}$ sobre a identidade de $G$ se identifica $\operatorname{com} A$. Construímos uma $C^{*}$-álgebra $\mathfrak{F}_{r}(\mathcal{B})$, que chamamos $C^{*}$-álgebra reduzida dos núcleos de $\mathcal{B}$, sobre a qual existe uma açào natural 3 de $G$, e mostramos que $(3, \mathbb{k} r(\mathcal{B}))$ é uma açào envolvente a menos de equivalencia Morita de a. Rapidamente descrito, isto é feito assim: $L^{2}(\mathcal{B})$ é um módulo de Hilbert pleno à direita sobre $A$, e portanto induz uma equivalencia Morita entre $A$ e $I_{r}$, onde $I_{r}=\mathcal{K}\left(L^{2}(\mathcal{B})\right)$, a $C^{*}$-álgebra dos operadores compactos do $A$-módulo de Hilbert $L^{2}(\mathcal{B})$. Acontece que $I_{r}$ é um ideal de $\tilde{L}_{r}(\mathcal{B})$. e que $\beta$ é a açào envolvente de $\left.\beta\right|_{I_{r}}$. donde segue, após um certo trabalho. que $\beta$ é uma ação envolvente a menos de equivalencia Morita de $\alpha$. Mostramos, depois, que $\left(\beta, \mathbb{k}_{r}(\mathcal{B})\right)$ é um sistema dinámico isomorfo a $\left(\delta \cdot C_{r}^{*}(\mathcal{B}) \rtimes_{\delta, r} G\right)$. Além da $C^{*}$-álgebra $\mathcal{r}_{r}(\mathcal{B})$, consideramos outra $C^{*}$-álgebra $\mathbb{k}(\mathcal{B})$ associada ao fibrado. definida através de uma construçào universal. Provamos que $\mathbb{E}(\mathcal{B})$ e $\mathbb{k}_{r}(\mathcal{B})$ são naturalmente isomorfas.

Em [54], Quigg mostrou que a dualidade de Takai falhava para produtos cruzados por automorfismos parciais (i.e.. produtos cruzados por certas açōes parciais de $Z$. determinadas por um automorfismo parcial de uma ('x-álgebra; os automorfismos parciais e seus produtos cruzados sào o 
antecedente imediato da teoria de açoes parciais e os correspondentes produtos cruzados). Ele afirma inclusive que a dualidade de Takai tende a fallar tanto mais quanto mais parcial o automorfismo é. Em nossa terminologia, e de maneira pouco formal, isto corresponde ao seguinte fato: se a é uma ação global, então $I_{r}=\mathbb{k}_{r}(B)$ e $\beta$ é equivalente Morita com a (é outra forma de expressar o teorema de Imai-Takai); se a é "muito parcial". entào $I_{r}$ é apenas uma parte "pequena" de $\mathbb{t}_{r}(\mathcal{B})$. Porém. nào tào pequena para que nào possamos recuperar $\mathbb{k}_{r}(\mathcal{B})$ a partir de $I_{r}$ e de $\beta$. Por outro lado, sendo a restrição de $\beta$ a $I_{r}$ equivalente Norita com $\alpha$, vemos que. mais do que falhar, a dualidade de Takai para açōes parciais adquere uma forma diferente, que é a forma descrita neste trabalho: se $\alpha$ é uma ação parcial sobre $A$. entào existe un ideal $I_{r}$ de $A \rtimes_{\alpha, r} G \rtimes_{\delta, r} G$ que é equivalente Morita com $A$. e tal que a ação parcial $\left.\delta\right|_{L}$ é equivalente Norita com $a$.

Finalmente, algumas palavras deveriam ser ditas sobre a escolha de const ruçòes espaciais em lugar de construçōes universais. ou seja, porque trabalhamos com produtos cruzados reduzidos por açōes e coaçòes, e não com produtos cruzados plenos. Mais do que uma preferencia, fomos impulsados a isto pelo Teorema 6.(6. onde mostramos que $A \rtimes_{\alpha, r} G \stackrel{M}{\sim} B \rtimes_{\beta, r} G$. sendo $(\beta, B)$ a açào envolvente de $(a, A)$. Não conseguimos provar um resultado similar para os produtos cruzados plenos. Se tal resultado existe, naturalmente que outras escolhas são possíveis. De fato. na teoria de dualidade de Takai existem as duas versòes. a espacial e a universal (ver [56]). 
CAPíTULO 4

Ações Envolventes na Categoria de Espaços Topológicos 
Estudaremos, neste capítulo inicial da Parte II, o problema da existencia e unicidade da ação envolvente em duas categorias: a categoria de conjuntos e funçōes, e a categoria de espaços topológicos e funções contínuas.

$\mathrm{Na}$ primeira seçào nos concentramos na categoria dos conjuntos e funçòes. Começamos com um estudo das propriedades básicas das ações parciais sobre conjuntos, generalizando a teoria bem conhecida de açōes globais sobre conjuntos. Em particular, mostramos que uma ação parcial transitiva é isomorfa a uma ação parcial por translaçào sobre um conjunto de classes laterais do grupo com respeito ao estabilizador de um elemento (4.21). Este resultado é a chave para mostrar que toda ação parcial tem uma açào envolvente. O Teorema 4.23 mostra que. além de existir. a ação envolvente de uma ação parcial está caracterizada por uma propriedade universal. e em particular é única. Duas caracterizações simples da açào envolvente são dadas em 4.29 e 4.30. Em 4.35. aplicamos os resultados prévios para estudar açòes enrolventes de açòes parciais que comutam.

Na segunda seçào consideramos açōes parciais contínuas sobre espaços topológicos. Começamos apresentando um par de exemplos motivadores. O primeiro é a açào parcial obtida pela restrição de uma ação contínua sobre um espaço topológico a um subconjunto aberto deste espaço (4.38). Este exemplo é um dos fundamentos da noção de açào envolvente que consideraremos nesta categoria. $O$ segundo exemplo provém da teoria de sistemas dinamicos: a ação parcial é o fluxo de uma equação diferencial sobre uma variedade (4.39). A Proposição 4.40 está diretamente relacionada com este exemplo, porque mostra que uma açào parcial contínua de um grupo conexo sobre um espaço topológico compacto é necessarianente uma açào global, resultado que generaliza o bem conhecido fato de que as soluções de uma equaçào diferencial sobre uma variedade compacta estào definidas sobre toda a reta real. Resultados posteriores, dos Capítulos 6 e $\tau$, mostrarào que na verdade, toda ação parcial contínua de um grupo conexo sobre uma $C^{*}$-álgebra cujo espaço de ideais primitivos seja compacto é uma açào global (5.18 e T.50).

Logo depois da Proposiçào 4.40. iniciamos o estudo do problema da açào envolvente nest.e contexto topológico. Neste sentido. o resultado principal é o Teorema 4.44, que mostra a existencia e a unicidade da ação envolvente, e a caracteriza por uma propriedade universal. Apresentamos seguidamente um exemplo de importantes conseqüencias: uma ação parcial sobre um espaço de Hausdorff cujo espaço envolvente não é de Hausdorff (4.50). Est.e exemplo implica que nem sempre existirá ação envolvente de uma ação parcial sobre uma $C^{* *}$-álgebra (na categoria de $C^{*}$-álgebras). Posteriormente caracterizamos as ações parciais sobre espaços de Hausdorff que tem ação envolvente de Hausdorff: sào aquelas cujo gráfico é fechado (4.51).

Em 4.46 e 4.47 damos const ruçōes alternativas das açōes envolventes. tanto no caso de açōes sobre conjuntos como no caso de açòes sobre espaços topológicos (para quem tiver pressa, esta é uma ótima oportunidade para evitar a leitura da primeira parte do capítulo!). Usamos esta construção para descrever o espaço envolvente da ação parcial gerada por um homeomorfismo parcial de um espaço topológico.

Por último, no final do capítulo. comparamos propriedades topológicas da açào parcial e de sua ação envolvente, concluíndo que em geral são as mesmas. Também vemos que os correspondentes espaços de órbitas sào naturalmente homeomorfos. (4.62).

\subsection{Açōes envolventes de ações parciais sobre conjuntos}

4.1.1. Ações parciais sobre conjuntos. Nesta seção lembramos a definição e fazemos um estudo primário das açòes parciais. generalizando a teoria bem conhecida de açòes plenas. Definimos morfismos entre açòes parciais de $G$. de maneira que a classe de açòes parciais de $G$ com estes morfismos é uma categoria que contém como subcategoria plena à categoria de açòes plenas e funções covariantes. O resultado principal da seção é o Lema 4.21, que descreve completamente às ações 
parciais transitivas. Como no caso de açōes plenas, uma ação parcial transitiva é isomorfa a uma ação por translação sobre um conjunto de classes laterais à esquerda segundo um subgrupo de $G$.

DEFINIÇÃo 4.1. Uma açào parcial de um grupo $G$ sobre um conjunto İ é um par $\alpha=\left(\left\{Y_{s}\right\}_{s \in G},\left\{a_{s}\right\}_{s \in C_{i}}\right)$ formado por uma coleção $\left\{X_{s}\right\}_{s \in G}$ de subconjuntos de $\mathrm{Y}$ e uma coleçào de bijeçöes $\left\{a_{s}: X_{s-1} \longrightarrow X_{s}\right\}$, tais que:

1. $\mathrm{H}_{e}=\mathrm{X}$.

2. $\alpha_{s t}$ é uma extensào de $\alpha_{s} \alpha_{t}$, onde o domínio de $\alpha_{s} \alpha_{t}$ é $\alpha_{t}^{-1}\left(\mathrm{Y}_{s-1}\right)$.

Se $a=\left(\left\{\mathrm{H}_{s}\right\}_{s \in G},\left\{\alpha_{s}\right\}_{s \in C_{i}}\right)$ e $\beta=\left(\left\{Y_{s}\right\}_{s \in G},\left\{\beta_{s}\right\}_{s \in G}\right)$ sào açóes parciais de $(i$ sobre conjuntos $\mathrm{I}$ e $Y$, entào um morfismo (ou ( $i$-morfismo) $\phi: \alpha \longrightarrow \beta$ é uma funçào o: $Y \longrightarrow Y$ tal que, $\forall t \in(i$. tem-se que $\phi\left(x_{t}\right) \subseteq Y_{i}$, e o diagrama seguinte é comutativo:

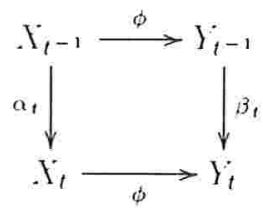

OBservação 4.2. O domínio de $\alpha_{s} \alpha_{t}$ está claramente contido em $X_{t-1} \cap X_{t-1} s^{-1}$, pois $a_{s t}$ estende $\alpha_{s} \alpha_{t}$. Por outro lado, se $x \in X_{t-1} \cap X_{t-1} s^{-1}$, existem $z=\alpha_{s t}(x) . y=n_{t}(x)$. de forma que $x=\alpha_{t-1}(y)$. e como $a_{s}$ estende $\alpha_{s t} \alpha_{t-1}$, temos: $z=\alpha_{s t} \alpha_{t-1}(y)=\alpha_{s}(y)=\alpha_{s} \alpha_{t}(x)$, donde segue que $x$ pertence ao domínio de $\alpha_{s} a_{t}$. Em conclusào, $\operatorname{dom}\left(\alpha_{s} \alpha_{t}\right)=X_{t-1} \cap X_{t-1}-1$.

DEFINIÇÃo 4.3. Diremos que uma açào parcial $\tau=\left(\left\{\mathrm{X}_{s}\right\}_{s \in G},\left\{\tau_{s}\right\}_{s \in G}\right)$ é trivial se $X_{s}=X_{s-1}$ e $\tau_{s}=i d_{\lambda_{s}}, \forall s \in G$. Os casos extremos de açòes parciais triviais sào a açào trivial do grupo e a ação parcial supertrivial, definida como: $\mathrm{Y}_{\epsilon}=\mathrm{K}, \mathrm{X}_{s}=\emptyset$ se $s \neq \epsilon . \tau_{\epsilon}=i d_{\lambda}$. e $\tau_{s}=\emptyset$ se $s \neq c$.

ObservaçÃo 4.4. Se $\beta$ é uma ação parcial de $G$ sobre um conjunto $Y$, e $\tau$ é a ação parcial supertrivial de $G$ sobre o conjunto $\mathcal{X}$, entào qualquer função $0: \mathcal{Y} \longrightarrow \mathcal{Y}$ é um morfismo $\phi: \tau \longrightarrow 3$.

OBSERVAÇÃo 4.5. É muito fácil verificar que a classe de açòes parciais de $G$ com os morfismos definidos acima formam uma categoria $\mathcal{A}_{p}(G)$ que contém à categoria $\mathcal{A}(G)$, de açòes plenas de $G$ e funçôes covariantes, como subcategoria plena. Cada morfismo injetivo é um monomorfismo. Inversamente, cada monomorfismo é uma função injetiva. Com efeito, suponhamos que $\phi$ é um monomorfismo. Quer dizer que se $\psi_{1}, \psi_{2}: \gamma \rightarrow \alpha$ são morfismos tais que $\phi \psi_{1}=\phi \psi_{2}$, entào $\psi_{1}=\psi_{2}$. Consideremos a ação parcial supertrivial $\tau$ de $G$ sobre $\mathrm{I}$ (Definição 4.3). Então cada funçào $\psi: X \longrightarrow X$ é um morfismo $\psi: \tau \longrightarrow \alpha$. Se $\phi$ nào fosse injetora, existiriam $x_{1}, x_{2} \in X$. $x_{1} \neq x_{2}$, tais que $\phi\left(x_{1}\right)=\phi\left(x_{2}\right)$. Tomando $\psi_{1}=i d_{\lambda}, \psi_{2}: X \rightarrow X$ tal que $\psi_{2}(x)=x$ se $x \neq x_{1}, x_{2}$. e $\psi_{2}\left(x_{1}\right)=x_{2}, \psi_{2}\left(x_{2}\right)=x_{1}$, teríamos que o $\psi_{1}=\phi \psi_{2}$, mas $\psi_{1} \neq \psi_{2}$, o que contradiz o fato de $\phi$ ser um monomorfismo. Isto mostra que se $\phi$ é um monomorfismo, entào o é uma função injetiva.

Com a notaçào usada na Definição 4.1. introduzimos a seguinte definição: 
DEFiniçÃo 4.6. Se $\beta$ é uma açào plena sobre o conjunto $Y$, e $0: \alpha \longrightarrow \beta$ é um monomorfismo, diremos que $(\phi, Y, \beta)$. ou simplesmente $\beta$, é uma extensão de $\alpha$.

Exemplo 4.7. Suponhamos que $\beta: G \times Y \rightarrow Y$ é uma ação, e seja $X \subseteq Y$. Para $s \in$ $G$ consideremos $\mathrm{X}_{s^{-1}}=\beta_{s}^{-1}(\mathrm{Y}) \cap \mathrm{Y}$. e $\alpha_{s}: \mathrm{X}_{s-1} \longrightarrow \mathrm{Y}_{s}$ tal que $\alpha_{s}(x)=\beta_{s}(x)$. Então $\alpha=$ $\left(\left\{Y_{s}\right\}_{s \in G},\left\{\alpha_{s}\right\}_{s \in G}\right)$ é uma ação parcial, e a inclusão $\iota: X \hookrightarrow Y$ é um morfismo. Assim, $(\iota, Y, \beta)$ é uma extensão de $a$.

DEFINIÇÃo 4.8. Se $\beta: G \times Y^{\circ} \longrightarrow Y$ é uma ação e $\mathrm{Y} \subseteq Y$. a ação parcial a construída no Exemplo 4.7 acima se chama restriçào de $\beta$ ao conjunto $X$. e se denota $a=\left.B\right|_{X}$.

Observação 4.9. A noçào de restriçào não é exatamente a oposta da noção de extensão. Mais precisamente, se a é uma açào parcial sobre $Y \subseteq Y$, e $\beta$ é uma açào sobre $Y$ tal que $\forall t \in G$ e $x \in Y_{t-1}$ temos $\beta_{t}(x)=\alpha_{t}(x)$. entào é verdade que $(\iota, Y, \beta)$ é uma extensão de $\alpha$. mas nào podemos afirmar que $\alpha=\left.\beta\right|_{X}$. Por exemplo. se $\tau$ é a açào parcial supertrivial de $\mathbb{Z}_{2}$ sobre $\beth_{2}$, e $\beta$ é a ação por translação $\beta: \mathbb{Z}_{2} \times \mathbb{Z}_{2} \longrightarrow \mathbb{E}_{2}$, entào $\left(i d_{\Xi_{2}}, \Sigma_{2}, \beta\right)$ é uma extensão de $\tau$, mas $\alpha \neq 3=\left.3\right|_{\Xi_{2}}$.

Observaç:ão 4.10. (omo mostra o exemplo de 4.9, um morfismo entre açòes parciais pode ser bijetivo sem ser um isomorfismo. Porém, se $\alpha=\left(\left\{X_{t}\right\}_{t \in G},\left\{\alpha_{t}\right\}_{t \in G}\right)$ e $\beta=\left(\left\{Y_{t}\right\}_{t \in G},\left\{\beta_{t}\right\}_{t \in G}\right)$ são ações parciais e $\phi: a \longrightarrow \beta$ é um morfismo bijetivo tal que $\phi\left(X_{t}\right)=Y_{t}, \forall t \in G$, então é fácil ver que $\phi^{-1}$ é um morfismo de $\beta$ em $\alpha$, que é inverso de $\phi$, e assim concluímos que $\phi$ é um isomorfismo.

A seguinte é uma versào puramente conjuntista do Lemma $1.2 \mathrm{em}$ [55].

ProposiçÃo 4.11. Sejam ( $i$ um grupo $e a=\left(\left\{X_{s}\right\}_{s \in G},\left\{a_{s}\right\}_{s \in G}\right)$ um par formado por uma coleção $\left\{X_{s}\right\}_{s \in G}$ de subconjuntos de um conjunto $Y, e \alpha_{s}: X_{s^{-1}} \longrightarrow X_{s}$ uma bijeção, $\forall s \in G$. Então $\alpha$ é uma açào parcial sobre $\mathrm{X}:=\mathrm{I}_{\epsilon}$ se $\epsilon$ somente se as condições seguintes sào satisfeitas, $\forall s, t \in G$ :

1. $\alpha_{e}$ é a identidade sobre $\mathrm{X}, \in \alpha_{s-1}=\alpha_{s}^{-1}$.

2. $\alpha_{s}\left(X_{s^{-1}} \cap \mathrm{X}_{t}\right)=\mathrm{K}_{s} \cap \mathrm{K}_{s t}$.

3. $\alpha_{s} \alpha_{t}: X_{t^{-1}} \cap X_{t^{-1} s^{-1}} \longrightarrow \mathrm{X}_{s} \cap X_{s t}$ é uma bijeção, e $\alpha_{s} \alpha_{t}(x)=\alpha_{s t}(x), \forall x \in X_{t^{-1}} \cap X_{t^{-1} s^{-1}}$.

DemonstraÇȦo. Suponhamos primeiro que as propriedades 1.2 e 3 são verificadas por $\alpha$. Devido à propriedade 3 , só temos que ver que $\mathrm{Y}_{t-1} \cap X_{t^{-1} s^{-1}}$ contém $\alpha_{t}^{-1}\left(\mathrm{Y}_{s^{-1}}\right)$, o domínio de $\alpha_{s} \alpha_{t}$ considerado na Definição 4.1. Agora, se $x \in a_{t}^{-1}\left(X_{s-1}\right)$, temos que $\alpha_{t}(x) \in X_{s-1} \cap X_{t}$, e portanto, usando 2, concluímos que $\alpha_{s} \alpha_{t}(x) \in X_{s} \cap X_{s t}$. Quer dizer que $a_{t}^{-1}\left(X_{s-1}\right) \subseteq\left(\alpha_{s} \alpha_{t}\right)^{-1}\left(X_{s} \cap X_{s t}\right)=$ $X_{t^{-1}} \cap X_{t^{-1} s^{-1}}$, onde a última igualdade se deve à propriedade 3 .

Suponhamos agora que a é uma açào parcial. Por 1 da Definiçào 4.1, temos que $\alpha_{e}$ está definida sobre $X$, e por 2 da Definiçào 4.1. $\alpha_{\epsilon}=\alpha_{e^{2}}$ é uma extensào de $\alpha_{\epsilon}^{2}$. quer dizer, $\alpha_{e}=\alpha_{\epsilon}^{2}$; segue que

$$
i d_{\lambda}=\alpha_{\epsilon}^{-1} \alpha_{e}=\alpha_{e}^{-1} \alpha_{e}^{2}=\alpha_{e} \text {. }
$$

Por 2 de 4.1, e pela conclusão obtida acima. temos que $i d_{\lambda}$ é uma extensão de $\alpha_{s} \alpha_{s^{-1}}$ e de $\alpha_{s^{-1}} \alpha_{s}$, e portanto $\alpha_{s}^{-1}=\alpha_{s-1}$. Isto demonstra 1 . 
Vejamos 2: se $x \in \mathbb{X}_{s-1} \cap X_{t}$, entào $\alpha_{s}(x) \in X_{s}$; por outro lado, $x=\alpha_{t}\left(a_{t-1}(x)\right)$, e portanto

$$
\alpha_{s}(x)=\alpha_{s} \alpha_{t}\left(\alpha_{t-1}(x)\right)=\alpha_{s t}\left(\alpha_{t-1}(x)\right) \text {. }
$$

pois $\alpha_{s t}$ estende $\alpha_{s} \alpha_{t}$. Segue que $\alpha_{s}(x)$ pertence a $X_{s t}$, a imagem de $\alpha_{s t}$. e concluímos que $\alpha_{s}\left(X_{s-1} \cap\right.$ $\left.\mathrm{Y}_{t}\right) \subseteq \mathrm{X}_{s} \cap \mathrm{Y}_{s t}$. Mas entào. usando de novo esta inclusão, com $s^{-1} \mathrm{em}$ lugar de $s$, temos:

$$
X_{s-1} \cap X_{t}=a_{s-1} a_{s}\left(X_{s-1} \cap Y_{t}\right) \subseteq a_{s-1}\left(X_{s} \cap X_{s t}\right) \subseteq X_{s-1} \cap Y_{t}
$$

e portanto $a_{s-1}\left(X_{s} \cap X_{s t}\right)=X_{s-1} \cap X_{t} ; \log 0, X_{s} \cap X_{s t}=a_{s}\left(X_{s-1} \cap X_{t}\right)$. donde segue 3 .

Observaçào 4.12. No conjunto de condiçòes $1,2,3$ do Lema acima, a condiçào 2 pode ser substituída pela condição aparentemente mais fraca

$$
2^{\prime}: \quad \alpha_{s}\left(\mathrm{~K}_{s-1} \cap \mathrm{X}_{t}\right) \subseteq \mathrm{X}_{s} \cap \mathrm{X}_{s t} .
$$

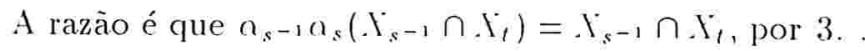

Seja $\alpha=\left(\left\{X_{s}\right\}_{s \in C_{i}},\left\{a_{s}\right\}_{s \in C_{i}}\right)$ unna açào parcial sobre o conjunto $X_{\text {. Definimos }} \Gamma_{\alpha} \subseteq G \times \mathbb{H}$ como:

$$
\Gamma_{a}=\left\{(t, x): x \in \mathrm{Y}_{t-1}\right\}
$$

Denotaremos também por $n$ à funçào $\Gamma_{a} \longrightarrow \mathrm{X}$ tal que $(t, x) \longmapsto(1,1,(x))$. Finalmente, se $x \in \mathcal{X}$. definimos

$$
A_{, r}=\left\{t \in G:(t, x) \in \Gamma_{\alpha}\right\} .
$$

Em outras palavras, $A_{x}$ é o subconjunto de $G$ formado pelos elementos $t$ tais que $x$ está no domínio de $\alpha_{t}$, ou, ainda, $A_{x}$ é o conjunto de elementos de $G$ que agem sobre $x$.

ProposiçÃo 4.13. Sejam a uma ação parcial sobre o conjunto $\mathrm{X}$ \& $A=\cap_{x \in X} A_{x}$. Então A $\dot{\epsilon}$ um submonóide de $G$. Se cada elemento de A tem ordem finita. entào A í um subgrupo de $G$.

Demonstraçào. Sejam $s, t \in A$. Se $x \in X$, então $(t, x) \in \Gamma_{a}$, pois $t \in A$. e pela mesma razão $\left(s, \alpha_{t}(x)\right) \in \Gamma_{\alpha}$. Como $\alpha_{s t}$ estende $\alpha_{s} \alpha_{t}$, concluímos que $(s t, x) \in \Gamma_{\alpha}$. ('omo isso é para todo $x \in X^{\prime}$. vemos que st $\in A$. Pela definiçào de açào parcial, $e \in A_{x}, \forall x \in \mathcal{Y}$, e portanto $A_{x}$ tem unidade. A segunda afirmaçào da Proposição é evidente.

Exemplo 4.14. Seja a a açào parcial de $\mathbb{R}$ sobre $X=[0,+\infty)$. obtida restringindo a ação por translação de $\mathbb{F}$ sobre $\mathbb{P}:(t, x) \longmapsto t+x$. Então $X_{t}=[-t,+\infty) \cap[0, x)$, ou seja, $X_{t}=[0,+\infty)$ se $t \geq 0, X_{t}=[|t|,+\infty)$ se $t<0$. Portanto, se $x \in X$, temos que $A_{r}=[-x+x)$. donde

$$
A=\bigcap_{x \in X} A_{x}=\bigcap_{x \geq 0}[-x,+\infty)=[0,+\infty) .
$$

que é um submonóide mas nào um subgrupo de $\mathbb{R}$.

DEFINIÇÃo 4.15. Seja a uma açào parcial sobre $\mathcal{X}$, e seja $x \in \mathbb{X}$. A órbita de $x$ é $o(x)=\left\{\alpha_{t}(x)\right.$ : $\left.t \in A_{x}\right\}$. Diremos que $x$ é um ponto fixo se $o(x)=\{x\}$, e diremos que a açào é transitiva se existe $x \in X$ tal que $o(x)=\mathrm{Y}$. 
Proposiçào 4.16. Śjam a uma açào parcial sobre $X, \in \mathcal{R}=\{(x, y) \in X \times X: y \in o(x)\}$. Então $\mathcal{R}$ é uma relaçào de equivalência $\mathrm{em} . \dot{\mathrm{Y}}$. cujas classes de equivaléncia são as órbitas da ação parcial.

Demonstraçào. $\epsilon \in \mathcal{A}_{x}, \forall x$, e entào $\left(x, \alpha_{e}(x)\right)=(x, x) \in \mathcal{R}$; portanto, a relaçào é reflexiva. Também é simétrica: se $y \in o(x)$, existe $t \in A_{x}$ tal que $a_{t}(x)=y$, e portanto $y \in \mathrm{F}_{t}$; mas $\alpha_{t-1}(y)=$ $a_{t-1}\left(\alpha_{t}(x)\right)=a_{t-1}(x)=\alpha_{t}(x)=x$, donde $x \in o(y)$. ou seja, $(y, x) \in \mathcal{R}$.

Com respeito à propriedade transitiva. suponhamos que $(x, y),(y, z) \in \mathcal{R}$. Existem $s \in A_{x}, t \in$ $A_{y}$, tais que $\alpha_{s}(x)=y, \alpha_{t}(y)=z$. Logo, $x \in \operatorname{dom}\left(\alpha_{t} \alpha_{s}\right) \subseteq \operatorname{dom}\left(\alpha_{t s}\right)$, e $\alpha_{t s}(x)=\alpha_{t} \alpha_{s}(x)=\alpha_{t}(y)=z$; então $(x, z) \in \mathcal{R}$.

Finalmente, é óbvio pela definiçào de $\mathcal{R}$ que as classes de equivalencia sào as órbitas de $\alpha$.

DefiniçÀo 4.17. Seja $x \in \mathbb{X}$. O estabilizador de $x$ é $G_{x}=\left\{t \in A_{x}: \alpha_{t}(x)=x\right\}$. Se for necessário indicar a dependencia com respeito à ação $a$, o denotaremos $G_{r}^{\text {a }}$; usaremos a mesma convenção para todos os objetos definidos a partir de uma ação parcial.

Proposição 4.18. Śejam a uma ação parcial sobre o conjunto X. e seja $x \in \mathrm{X}$. Então $G_{x}$ é um subgrupo de $G$. Além disso. sé $y=\alpha_{t}(x)$. entào:

1. $G_{y}=t G_{x} t^{-1}$.

2. $A_{y}=A_{x} t^{-1}$.

Demonstraçäo. Notemos primeiro que $e \in G_{x}$ e, se $t \in G_{x}$, entào $x=\alpha_{t}(x) \in \operatorname{dom} \alpha_{t-1}$, e $\alpha_{t-1}(x)=\alpha_{t-1} \alpha_{t}(x)=a_{e}(x)=x$, donde $t^{-1} \in G_{x}$. Sejam agora $s, t \in G_{x}$. Então $\alpha_{s} \alpha_{t}(x)=\alpha_{s}(x)=$ $x$, e como $\alpha_{s t}$ é uma extensào de $a_{s} a_{t}$, vemos que st $\in G_{x}$. Assim, $G_{x}$ é um subgrupo de $G$.

Se $y=\alpha_{t}(x)$. e $s \in G_{x}$. temos que $\alpha_{t} \alpha_{s} \alpha_{t-1}(y)=\alpha_{t} \alpha_{s}(x)=\alpha_{t}(x)=y$, o que mostra que $t G_{x} t^{-1} \subseteq G_{y}$, e também que $t^{-1} G_{y} t \subseteq G_{x}$, pois $x=\alpha_{t-1}(y)$. Logo: $G_{y} \subseteq t G_{x} t^{-1} \subseteq G_{y}$, e concluímos que $G_{y}=t G_{x} t^{-1}$.

Se $y=\alpha_{t}(x)$ e $s \in A_{x}$, entào $\alpha_{s} \alpha_{t-1}(y)=a_{s t-1}(y)$, e portanto $s t^{-1} \in A_{y}$. Logo $A_{x} t^{-1} \subseteq A_{y}$, e da mesma forma $A_{y} t \subseteq A_{x}$, donde segue que $A_{y}=A_{x} t^{-1}$.

ProposiçÃo 4.19. Sejam $x \in \mathrm{X}$ e $R$ um conjunto de representantes das classes laterais à esquerda de $G_{x}$ em $G$. Então:

$$
A_{x}=\biguplus_{r \in R \cap A_{x}} r G_{x}
$$

Demonstração. Sejam $r \in R \cap A_{x}$ e $s \in G_{x}$ : então é claro que $r s \in A_{x}$, e portanto $\biguplus_{r \in R \cap A_{x}} r G_{x} \subseteq$ $A_{x}$. Por outro lado. dado $t \in A_{x}$, existem únicos $r_{t} \in R, s_{t} \in G_{r}$, tais que $r_{t} s_{t}=t$. Isto implica que $r_{t}=t s_{t}^{-1} \in A_{r} G_{x}=A_{r}$. Logo $r_{t} \in R \cap A_{x}$. e portanto $t=r_{t} s_{t} \in r_{t} G_{r} \subseteq \biguplus_{r \in R A_{x}} r G_{x}$, o que encerra a demonstração. 
Proposição 4.20. Sejam $\Lambda=\bigcap_{x \in X} G_{x}$, e A como na Proposição 4.13. Então $N \subseteq A, \epsilon N \dot{\epsilon}$ um subgrupo de $G$ tal que $t N t^{-1} \subseteq N, \forall t \in A$. Em particular $N$ é normal em $A$ quando $A$ é um subgrupo.

Demonstração. Pela Proposiçào 4.19. temos que $G_{x} \subseteq A_{x}, \forall x \in$ X. e portanto

$$
N=\bigcap_{x \in X} G_{x} \subseteq \bigcap_{x \in X} A_{x}=A .
$$

Finalmente, $t N t^{-1} \subseteq N$, pela Proposiçào 4.18.

Lema 4.21. Seja a uma ação parcial sobre $X$, e seja $x \in X$. Entào $\varphi_{x}: o(x) \longrightarrow A_{x} / G_{x}$ tal que $\alpha_{t}(x) \longmapsto t G_{x}$ é um isomorfismo. onde consideramos sobre $A_{x} / G_{x}$ a açào parcial obtida por restrição da ação por translą̧ào $\left(i \times C_{i} / G_{r} \longrightarrow C_{i} / G_{x}\right.$. e sobre o $(x)$ a restriçào de $a$.

Demonstraçào. Notar que se $s, t \in A_{x}$, então $\alpha_{t}(x)=\alpha_{s}(x)$ se e somente se $s^{-1} t \in G_{x} \Longleftrightarrow$ $s G_{x}=t G_{x}$, donde vemos que $o_{r}$ está definida e é injetiva, tomando valores sobre $G / G_{x}$. Além disso, se $t \in A_{x}$, tem-se que $\phi_{x}\left(\alpha_{t}(x)\right)=t G_{r} \in A_{x} / G_{x}$. Se $y=\alpha_{t}(x)$ e $s \in A_{y}$, então

$$
\begin{aligned}
\phi_{x}\left(\alpha_{s}(y)\right) & =\phi_{x}\left(\alpha_{s} \alpha_{t}(x)\right) \\
& =\phi_{x}\left(\alpha_{s t}(x)\right) \\
& =(s t) G_{x} \\
& =s\left(t G_{x}\right) \\
& =s \phi_{x}\left(\alpha_{t}(x)\right) \\
& =s \phi_{x}(y),
\end{aligned}
$$

e portanto $\phi$ é um morfismo. Como é claro que $\phi$ também é sobrejetivo. vemos que é um morfismo bijetivo. Notemos, para terminar. que se $s, t \in A_{x}$, então $s G_{x}=t G_{x}$ se e somente se $t^{-1} s \in G_{x}$, e portanto $\alpha_{t-1} s(x)=x$, e como $a_{s}$ estende $\alpha_{t} \alpha_{t^{-1} s}$, temos que $\alpha_{s}(x)=\alpha_{t}(x)$. Assim, temos definida uma função $\psi_{x}: A_{x} / G_{x} \longrightarrow o(x)$. tal que $t G_{x} \longmapsto \alpha_{t}(x)$. $\psi_{x}$ é um morfismo: sejam $t, s \in A_{x}$, tais que $s t G_{x} \in A_{x} / G_{x} ;$ por $4.19, G_{r} \subseteq A_{x}$, donde $s t \in A_{x}$, e além disso:

$$
\begin{aligned}
\psi_{x}\left(s\left(t G_{x}\right)\right) & =\psi_{x}\left((s t) G_{x}\right) \\
& =\alpha_{s t}(x) \\
& =\alpha_{s} \alpha_{t}(x) \\
& =\alpha_{s}\left(\psi_{x}\left(t G_{x}\right)\right) .
\end{aligned}
$$

Por outro lado, é claro que $\psi_{x}$ é a função inversa de $\phi_{x}$, e que $\psi_{x}$ é um $G$-morfismo. Daí segue que $\phi_{x}$ é um isomorfismo de ações parciais.

Proposição 4.22 (Equaçào das classes). Sejam $\alpha$ uma ação parcial sobre $X e W \subseteq X$ um conjunto de representantes das órbitas. $\epsilon F \subseteq \mathrm{X}$ o conjunto dos pontos fixos. Então:

e portanto:

$$
X=F \biguplus \biguplus_{w \in W \backslash F} o(w),
$$

$$
|\mathrm{Y}|=|F|+\sum_{w \in W \backslash F}\left|A_{w} / G_{w}\right|
$$


Demonstração. A primeira equação é obviamente verdadeira, pois as órbitas são as classes de equivalencia de $\mathrm{X}$, e portanto constituem uma partição de $\mathcal{H}$. Daí segue imediatamente que $|X|=|F|+\sum_{w \in W \backslash F}|o(w)|$ e, usando o Lema 4.21, sabemos que $|o(w)|=\left|A_{w^{\prime}} / G_{w}\right|$, donde a segunda equação segue.

4.1.2. Açōes envolventes. O resultado principal desta seção é o Teorema 4.23, que estende o Lema 4.21, e que mostra que toda ação parcial é a restrição de uma ação plena, que chamaremos envolvente. ${ }^{1}$. Após este teorema, alguns exemplos são considerados, como também alguns critérios (4.29 e 4.30) para decidir quando uma extensão de uma ação parcial é sua ação envolvente.

Teorema 4.23. Seja a uma ação parcial de $G$ sobre $X \dot{X}$. Então existe um par $(\phi, \dot{\alpha})$ formado por uma ação plena à de $G$ sobre um conjunto $\dot{X}$. e um morfismo $\phi: \alpha \longrightarrow \dot{\alpha}$. tal que para todo morfismo $\psi: a \longrightarrow \beta$. onde $\beta$ é uma açào plena, existe um único morfismo $\eta: \dot{a} \longrightarrow \beta$ tal que $\eta \phi=\psi$ :

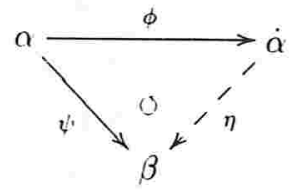

Além disso, o par $(\phi, \dot{a})$ fica unicamente determinado, a menos de isomorfismos canónicos, pela propriedade acima. Tem-se que $(\phi, \dot{\mathcal{X}}, \dot{\alpha})$ é uma extensão de $\alpha, \epsilon \alpha \cong \bar{\alpha}:=\left.\dot{\alpha}\right|_{\phi(X)}$. Desta forma obtemos um funtor $\mathcal{A}_{p}(G) \longrightarrow \mathcal{A}(G)$. que leva $\alpha$ em $\dot{\alpha}$.

Demonstração. Sejam $W \subseteq \mathrm{X}$ um conjunto de representantes das órbitas, $\dot{\mathrm{X}}=\biguplus_{w \in W} G / G_{u}$, e $\phi: X \longrightarrow \dot{X}$ tal que $\phi=\uplus_{w \in W} \phi_{w^{\prime}}$, onde $\phi_{w^{\prime}}: o(w) \longrightarrow A_{w} / G_{w}$ é tal que $\phi_{w}\left(\alpha_{t}(w)\right)=t G_{w}$. Como as $\phi_{w}$ são injetivas (Lema 4.21), temos que $\phi$ é injetiva. Consideremos sobre $\dot{X}$ a ação $\dot{\alpha}: G \times \dot{X} \longrightarrow \dot{X}$ dada por $\dot{\alpha}\left(t, s G_{w}\right)=t s G_{w}$. Se $x \in \mathcal{X}$, existe um único $w \in W$, e algum $t \in G$, tais que $x=\alpha_{t}(w)$. Suponhamos que $s \in A_{x}$; então $\alpha_{s}(x)=\alpha_{s} \alpha_{t}(w)=\alpha_{s t}(w)$, donde

$$
\begin{aligned}
\phi\left(\alpha_{s}(x)\right) & =\phi\left(\alpha_{s t}(w)\right) \\
& =\phi_{w}\left(\alpha_{s t}(w)\right) \\
& =s t G_{w} \\
& =\dot{\alpha}_{s}\left(t G_{w}\right) \\
& =\dot{\alpha}_{s}\left(\dot{\phi}_{w}\left(\alpha_{t}(w)\right)\right) \\
& =\dot{\alpha}_{s}(\phi(x))
\end{aligned}
$$

Logo, $\phi: \alpha \longrightarrow \dot{\alpha}$ é um monomorfismo, e portanto $(\phi, \dot{X}, \dot{\alpha})$ é uma extensão de $\alpha$.

Para ver que $\alpha \cong \bar{\alpha}$, onde $\bar{\alpha}$ é a restrição de $\dot{\alpha}$ a $\phi(X)$, por 4.10 basta ver que $\forall s \in G$, temos $\phi\left(X_{s}\right)=\dot{X}_{s}$, onde $\dot{X}_{s}=\dot{\alpha}_{s}^{-1}(\phi(X)) \cap \phi(X)$. Fixado $x \in X_{s^{-1}}$, existem $w \in W, t \in G$, tais que $x=\alpha_{t}(w)$, e pela Proposiçào 4.18, temos que $A_{x}=A_{w} t^{-1}$. Da mesma forma, $A_{\phi(x)}^{\bar{\alpha}}=A_{\phi(w)}^{\bar{\alpha}} t^{-1}$, pois $\phi(x)=\bar{\alpha}_{t}(\phi(w))$. Por outro lado, $A_{\phi(w)}^{\bar{\alpha}}=\left\{r \in G: r G_{w} \in A_{w} / G_{w}\right\}=A_{w}$. Portanto

\footnotetext{
${ }^{1}$ Em termos categóricos, se $\mathcal{F}: \mathcal{A}(G) \longrightarrow \mathcal{A}_{p}(G)$ é o funtor de esquecimento, entào $\dot{a}$ é o universal de a com respeito a $\mathcal{F}$. e portanto é único a menos de isomorfismos em $\mathcal{A}(G)$
} 
$A_{x}=A_{\phi(x)}^{\bar{\alpha}}, \forall x \in X$. Agora, $Y_{s-1}=\left\{x \in X: s \in A_{x}\right\}=\left\{x \in X: s \in A_{\phi(x)}^{\bar{\alpha}}\right\}$, e portanto $\phi\left(X_{s-1}\right)=\left\{\phi(x): s \in A_{\phi(x)}^{\bar{a}}\right\}=\dot{X}_{s-1}$. Concluímos então que $a \cong \bar{\alpha}$.

Suponhamos agora que $\ell^{\circ}: \alpha \longrightarrow \beta$ é um morfismo. onde $\beta$ é uma ação sobre um conjunto $Y$. Queremos achar um morfismo $\eta: \dot{i} \longrightarrow \beta$ tal que $\eta \phi=\beta$. Como $\forall t \in G$ temos que $t G_{w}=\dot{\alpha}_{t}(\phi(w))$, e $\eta$ deve ser um morfismo, tem que ser $\eta\left(t G_{w}\right)=\beta_{t}\left(\eta\left(o\left(w^{\prime}\right)\right)\right)=\beta_{t}\left(v\left(w^{\prime}\right)\right)$. Portanto, basta ver que se $t G_{w}=s G_{w}$, temos que $\beta_{l}\left(\iota^{\prime}\left(w^{\prime}\right)\right)=\beta_{s}(\psi(w))$ ou. equivalentemente, que $s^{-1} t \in G_{\psi(w)}^{\beta}$; mas $\beta_{s^{-1} t}(\psi(w))=\psi\left(\alpha_{s^{-1} t}(w)\right)=\psi(u)$. pois $s^{-1} t \in G_{w}$.

A unicidade salvo isomorfismos da extensão $(\phi, \dot{Y}, \dot{a})$ se prova da maneira usual.

Definiçào 4.24. Se o é uma açào parcial sobre um conjunto $X$. tocla tripla $(\phi, \dot{\alpha}, \dot{X})$ como a fornecida pelo Teorema 4.23 acima será chamada ação açào envolvente de a. Em geral não faremos referencia a aplicaçào $o$. e cliremos simplesmente que $\dot{\alpha}($ ou $(\dot{\alpha}, \dot{\zeta}))$ é a açào açào envolvente de $\alpha$ (ou $(\alpha, X)$ respectivamente). O espaço $\dot{X}$ será chamado espaço açào envolvente.

OBSERVAÇÃo 4.25. Da construçào da ação envolvente vemos que $|\dot{X}| \leq|G||X / a|$, e haverá igualdade quando os estabilizadores sejam todos triviais. Em particular. $\dot{X}$ será finito quando $G$ e $X$ sejam finitos.

Exemplo 4.26. Suponhamos que $G$ é um grupo que age parcialmente sobre um conjunto $X$ através da ação parcial supertrivial $\tau$ (lembrar 4.3). Notar que se $\gamma: G \times Y \longrightarrow X$ é uma ação, então $\gamma$ é automaticamente una extensào de $\tau$. Seguindo a construçào da açào envolvente feita no Teorema 4.23 , vemos que na verdade a ação envolvente é $(\phi, \dot{X}, \dot{T})$. onde $\dot{X}=G \times X, \phi: X \longrightarrow \dot{X}$ é dada por $\phi(x)=(e, x)$, e $\dot{\tau}_{s}(t, x)=(s t, x), \forall s, t \in G, x \in X$. Para qualquer açào $\gamma$ como acima, $\eta: \dot{X} \longrightarrow X$ tal que $(t, x) \longmapsto x$, é o $(i$-morfismo do Teorema 4.23.

Exemplo 4.2T. É claro que se a $: G \times X \longrightarrow X$ é uma ação, então $\dot{X}=X$ e $\dot{\alpha}=\alpha$. Portanto o funtor $\alpha \longmapsto \dot{\alpha}$ é inverso à direita do funtor de esquecimento $\mathcal{F}$.

Exemplo 4.28. Sejam $X=\{1, \ldots, n\}$ e $\alpha$ a ação parcial de $Z$ sobre $X$ dada pela restriçào da ação por translação de $Z$ sobre $Z$. Temos que todos os estabilizadores sào triviais e que a açào é transitiva, donde, seguindo a prova do Teorema 4.23 , podemos identificar $\dot{X} \operatorname{com} \mathbb{Z}, \phi: X \longrightarrow \mathbb{Z}$ com a inclusão $j \longmapsto j-1$, e então $\dot{a}: \mathbb{Z} \times \mathbb{Z} \longrightarrow \mathbb{Z}$ é a ação por translação.

Uma outra extensào natural que existe para a ação parcial a acima é a seguinte: identifiquemos $X$ com $\mathbb{Z}_{n}$, e definamos $\gamma: \mathbb{Z} \times \bar{Z}_{n} \longrightarrow \Xi_{n}$ tal que $\gamma(k, \bar{j})=\overline{k+j}$. Então deve existir um $\mathbb{Z}$-morfismo $\eta: \mathbb{Z} \longrightarrow \mathbb{Z}_{n}$, ou seja, $\eta\left(\dot{\alpha}_{k}(j)\right)=\gamma_{k}(\eta(j))$; é claro que $\eta$ é precisamente a projeção canonica $\mathbb{Z} \longrightarrow \mathbb{Z}_{n}$.

Nos exemplos considerados acima, achamos as correspondentes açòes envolventes seguindo a prova do Teorema 4.23. A proposiçào a seguir fornece um critério um pouco mais prático para determinar se uma ação que estende uma açào parcial é a ação envolvente ou não. Ver também o Corolário 4.30 
ProposiçÃo 4.29. Sejam a uma açào parcial de $G$ sobre $\mathrm{Y}, \epsilon(\psi, Y, \beta)$ uma extensão de $\alpha$. Então $(\psi, Y, \beta)$ é a açcio envolvente de a se $\epsilon$ somente se as duas condiçòes sfguintes são satisfeitas:

1. A aplicaçào quociente $\dot{Y}: \mathrm{H} / \mathrm{a} \longrightarrow \mathrm{Y} / \beta$ é uma bijeçào.

2. $G_{x}^{\alpha}=G_{\psi(x)}^{\beta}, \forall x \in \mathcal{X}$.

Demonstraçào. Suponhamos primeiro que $\beta$ é a ação envolvente. Entào 3 é isomorfa à ação $\dot{\alpha}$ construída no Teorema 4.23, quem obviamente satisfaz 1 e 2.

Inversamente, suponhamos que a extensão $(\psi, Y, \beta)$ satisfaz 1 e 2 . Vejamos que ela possui a propriedade universal que define à açào envolvente: para todo morfismo $\lambda: 0 \longrightarrow \hat{i}_{i}$. onde $\gamma$ é uma ação plena, existe um morfismo $\eta: \beta \longrightarrow \gamma$ tal que

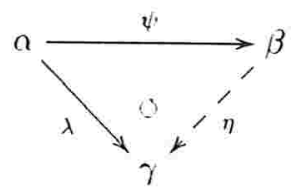

Suponhamos então que $\gamma$ é uma açào plena sobre um conjunto $Z$. Queremos achar $\eta: Y \rightarrow Z$ tal que $\eta\left(\beta_{t}(y)\right)=\gamma_{t}(\eta(y)), \forall y \in Y^{\circ}$. e tal que $\eta \psi=\lambda$. Seja $R$ um conjunto de representantes das a-órbitas. Por $1, \psi(R)$ é um conjunto de representantes das 3 -órbitas. Agora, dado $y \in Y$, existem $t \in G$ e um único $r \in R$ tais que $\beta_{t}(\tau(r))=y$. Se tal $\eta$ existe, devemos ter:

$$
\begin{aligned}
\eta(y) & =\eta\left(\beta_{t}(\psi(r))\right) \\
& =\gamma_{t}(\eta(\psi(r))) \\
& =\gamma_{t}(\lambda(r)) .
\end{aligned}
$$

Agora, se $\beta_{t}(\psi(r))=\beta_{s}(\psi(r))=y$. entào $\beta_{s^{-1} t}(\psi(r))=\psi(r)$, e portanto $s^{-1} t \in G_{(i, r)}^{a}=G_{r}^{a}$, devido a 2. Segue então que:

$$
\lambda(r)=\lambda\left(\alpha_{s-1}(r)\right)=\gamma_{s-1}(\lambda(r)) .
$$

pois $\lambda$ é um $G$-morfismo. Como $\gamma$ é uma ação. então

$$
\gamma_{s}(\lambda(r))=\gamma_{s}\left(\gamma_{s-1}(\lambda(r))\right)=\gamma_{t}(\lambda(r)) .
$$

Isto mostra que podemos definir $\eta$. e é óbvio agora que $\eta$ é um $G$-morfismo. Finalmente, se $x \in \mathrm{Y}$ é tal que $x=\alpha_{t}(r), r \in R$, entào

$$
\begin{aligned}
\eta \psi(x) & =\eta \psi\left(\left(\alpha_{t}(r)\right)\right. \\
& =\eta\left(\beta_{t}(\psi(r))\right) \\
& =\gamma_{t}(\lambda(r)) \\
& =\lambda\left(\alpha_{t}(r)\right) \\
& =\lambda(x)
\end{aligned}
$$

Segue então que $\eta \psi=\lambda$.

Corolário 4.30. Śe $\beta: G \times Y^{*} \longrightarrow Y^{*}$ é uma ação, $X \subseteq Y^{\circ} . \epsilon a=\left.\beta\right|_{X}$. então $\dot{a}=\left.\beta\right|_{\dot{X}}$, onde $\dot{\mathrm{I}}=\bigcup_{x \in X} o^{\beta}(x)$. Em particular. se a $\dot{\epsilon}$ uma ação parcial sobre um conjunto $\mathrm{X}$. $\epsilon$ 3 é uma extensão de a tal que $\alpha=\beta_{\left.\right|_{x}}$, então à é a restrição de $\beta$ à $\beta$-órbita de $\mathrm{X}$. 
Demonstraçào. Se $x \in X$, temos que $G_{x}^{\beta} \subseteq A_{x}^{\alpha}$, e portanto $G_{x}^{\beta}=G_{x}^{\alpha}$. Por outro lado, a definição de $\dot{X}$ implica que se $\iota: X \longrightarrow \dot{X}$ é a inclusão, então o morfismo quociente $\tilde{\imath}: X / \alpha \longrightarrow$ $\dot{X} /\left(\left.\beta\right|_{\dot{X}}\right)$ é sobrejetivo. Finalmente, se $x_{1}, x_{2} \in X$ são tais que $x_{2}=\beta_{t}\left(x_{1}\right)$ para algum $t \in G$, segue que $t \in A_{x_{1}}^{\alpha}$, e que $x_{2}=a_{t}\left(x_{1}\right)$, donde deduzimos que $i$ é também injetiva. Assim obtemos, usando a Proposição 4.29 , que $\dot{a}=\left.\beta\right|_{X}$.

Exemplo 4.31. Seja $C i$ agindo parcialmente por conjugaçào sobre um subconjunto $X$, e sejam $Y=\bigcup_{t \in G} t X t^{-1}$ e $\beta: G \times Y \longrightarrow Y$ a ação por conjugação. Esta extensão é a ação envolvente da ação parcial considerada, devido ao Corolário 4.30.

Exemplo 4.32. Suponhamos que o é uma ação parcial de $G$ sobre $X$, tal que $G_{x}^{\alpha}=\{e\}, \forall: x \in X$ (a ação parcial é "livre"). Indiquemos por $\bar{x}$ a órbita de $x \in X$, e seja $\sigma: X / a \longrightarrow X$ uma seçào. i.e., $\overline{\sigma(\bar{x})}=\bar{x}, \forall \bar{x} \in X / n$. Como $G_{r}^{\mathrm{a}}=\{e\}$, existe um único $t_{r} \in G$ tal que $x=\alpha_{t_{x}}(\sigma(\bar{x}))$, e portanto $t_{\alpha_{s}(x)}=s t_{x}, \forall s \in A_{x}$. Sejam $Y=G \times(X / \alpha), \beta: G \times Y \longrightarrow Y$ tal que $\beta_{s}(t, \bar{x})=(s t, \bar{x})$, e $\phi: X \longrightarrow Y$ tal que $\phi(x)=\left(t_{x}, \bar{x}\right)$. Entào, se $s \in A_{x}$,

$$
\phi\left(a_{s}(x)\right)=\left(t_{\alpha_{s}(x)}, \bar{x}\right)=\left(s t_{x}, \bar{x}\right)=\beta_{s}\left(t_{x}, \bar{x}\right)=\beta_{s}(\phi(x)) .
$$

Se $\phi(x)=\phi(y)$, entào $\bar{x}=\bar{y}, t_{x}=t_{y}$, e portanto $x=t_{x} \sigma(\bar{x})=t_{y} \sigma(\bar{y})=y$. Assim, $(\phi, Y, \beta)$ é uma extensão de $\alpha$.

Claramente temos que $G_{\phi(x)}^{1 \beta}=\{e\}, \forall x \in X, \bigcup_{x \in X} o^{\beta}(\phi(x))=Y$. Se $\phi\left(x_{1}\right)=\phi\left(x_{2}\right)$, então $\bar{x}_{1}=\bar{x}_{2}$, donde $\tilde{\phi}$ é injetora. $\operatorname{Logo}(\phi, Y, \beta)$ é a ação envolvente de a pela Proposiçào 4.29.

Exemplo 4.33. Sejam $G$ um grupo, $\mathrm{X}=\{F \subseteq G: \epsilon \in F\}$, e $\alpha$ a açào parcial de $G$ sobre $X$ tal que $X_{t}=\left\{F \in X: t^{-1} \in F\right\}$, e $\alpha_{t}: X_{t^{-1}} \longrightarrow X_{t}$ tal que $\alpha_{t}(F)=t F$. Então $\alpha=\left.\beta\right|_{X}$, onde $\beta: G \times P(G) \longrightarrow P(G)$ está dada por $(t, E) \longmapsto t E$, e $P(G)$ denota a família de subconjuntos de $G$. Segue de 4.30 que a ação envolvente de $\alpha$ é $(\iota, \dot{X}, \dot{\alpha})$, onde $\dot{X}=\{t F: F \in X, t \in G\}=P(G) \backslash\{\emptyset\}$, e $\iota$ é a inclusão.

Terminamos esta seção com uma aplicação a ações parciais que comutam.

DEFINIÇÃo 4.34. Sejam $\alpha=\left(\left\{Y_{t}^{\alpha}\right\}_{t \in G},\left\{\alpha_{t}\right\}_{t \in G}\right)$ e $\beta=\left(\left\{X_{s}^{\beta}\right\}_{s \in H},\left\{\beta_{s}\right\}_{s \in H}\right)$ duas ações parciais

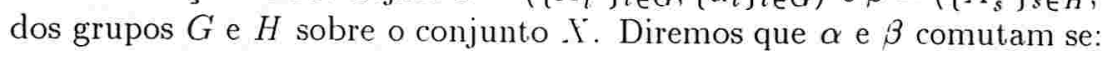

1. $\beta_{s}\left(X_{t}^{\alpha}\right) \cap X_{s}^{\beta}=\alpha_{t}\left(X_{s}^{\beta}\right) \cap X_{t}^{\alpha}, \forall t \in G, s \in H$.

2. Se $t \in G, s \in H$, então $\alpha_{t} \beta_{s}(x)=\beta_{s} \alpha_{t}(x), \forall x \in \beta_{s}^{-1}\left(X_{t-1}^{\alpha}\right) \cap X_{s-1}^{\beta}$.

Proposição 4.35. Se a e B scio açòes parciais que comutam, dos grupos $G$ e $H$ sobre o conjunto $X$, então existem ações à e $\dot{\beta}$ que comutam, de $G$ e $H$ sobre um conjunto $\tilde{X}$, tais que $X \subseteq \hat{X}$. $\alpha=\tilde{\alpha}_{\left.\right|_{X}}$ e $\beta=\tilde{\beta}_{\left.\right|_{X}}, \epsilon . \tilde{X}=\bigcup_{t \in G, s \in H} \tilde{\alpha}_{t} \tilde{\beta}_{s}(X)$. A tripla $(\tilde{\alpha} . \tilde{3}, \tilde{X})$ é única a menos de isomorfismos. 
DemonstraçÃo. Consideremos $\gamma=\left(\left\{X_{(t, s)}\right\}_{(t, s) \in G \times H},\left\{\gamma_{(t, s)}\right\}_{(t, s) \in G \times H}\right)$, onde

$$
X_{(t, s)}=\beta_{s}\left(X_{t}^{\alpha}\right) \cap X_{s}^{\beta}, \gamma_{(t, s)}=\alpha_{t} \beta_{s} .
$$

Então $\gamma$ é uma ação parcial de $G \times H$ sobre $X$ e, se $\iota_{G}: G \longrightarrow G \times H$ e $\iota_{H}: H \longrightarrow G \times H$ são as incluções canónicas, entào $\alpha=\hat{\imath} \iota_{i}$ e $\beta=\gamma \iota_{H}$.

Seja agora $(\dot{\gamma}, X)$ a açào envolvente da açào parcial $\gamma$. Obtemos açòes $\dot{\alpha}:=\dot{\gamma} \iota_{G}$ e $\tilde{\beta}:=\dot{\gamma} \iota_{H}$ de $G$ e $H$ sobre $\dot{X}$, que claramente satisfazem $\alpha=\tilde{\alpha}_{\left.\right|_{x}}$ e $\beta=\tilde{\beta}_{\left.\right|_{x}}$. A unicidade se deduz da unicidade de $\dot{\gamma}$.

ObSERVAÇào 4.36. Notar que à e $\hat{\beta}$ sào ao mesmo tempo extensòes das ações envolventes $\dot{\alpha}$ e $\dot{\beta}$ de $\alpha$ e $\beta$, e coincidem com elas se e somente se $o^{\dot{\alpha}}(X)=\dot{X}$ e $o^{\dot{\beta}}(X)=\dot{X}$.

\subsection{Açōes envolventes de ações parciais topológicas}

Nesta seçào tentaremos obter resultados análogos aos da seção anterior, mas num contexto topológico. De fato. mostraremos que se $G \dot{i}$ é um grupo topológico agindo sobre um espaço topológico $X$ através de uma açào parcial contínua $\alpha$, e se $(\dot{\alpha}, \dot{X})$ é a correspondente açào envolvente, então existe sobre $\dot{X}$ uma única topologia $\dot{\tau}$ tal que $\dot{\alpha}: G \times \dot{X} \longrightarrow \dot{X}$ é contínua, $X \subseteq \dot{X}$ é aberto, e a topologia relativa de $X$ como subespaço de $\dot{X}$ coincide com a topologia original de $X$. Teremos que $(\dot{\alpha}, \dot{X}, \dot{\tau})$ está unicamente determinada a menos de isomorfismos. Veremos também que $\alpha$ e $\dot{\alpha}$ compartilham várias propriedades topológicas, como minimalidade, liberdade topológica, etc. Finalmente, caracterizamos aquelas açōes parciais sobre espaços topológicos de Hausdorff para as quais o espaço envolvente também é de Hausdorff. O fato de existirem açōes parciais sobre espaços de Hausdorff com espaços envolventes não de Hausdorff será de muita importança quando consideremos ações parciais na categoria de $C^{*}$-álgebras.

No que segue, $G$ é um grupo topológico com topologia $\tau_{G}$, $X$ é um espaço topológico com topologia $\tau, \alpha=\left(\left\{X_{t}\right\}_{t \in G},\left\{\alpha_{t}\right\}_{t \in G}\right)$ é uma ação parcial de $G$ sobre $X$, com ação envolvente $\dot{\alpha}$ agindo sobre o espaço envolvente $X$, que contém $X$ como subconjunto. Suporemos ainda que a ação parcial $\alpha$ é contínua, segundo a definição seguinte (ver também 5.3 e 8.3 ):

DEFINIÇÃo 4.37. Sejam $G$ um grupo topológico e $X$ um espaço topológico. Uma ação parcial $\alpha=\left(\left\{X_{t}\right\}_{t \in G},\{\alpha\}_{t \in G_{i}}\right)$ do grupo $G$ sobre $X$ é dita contínua se o domínio de $\alpha$, definido como $\Gamma_{\alpha}=\left\{(t, x) \in G \times X: x \in X_{t^{-1}}\right\}$. é aberto em $G \times X$, e a função $\Gamma_{\alpha} \longrightarrow X$ dada por $(x, t) \longmapsto \alpha_{t}(x)$ é contínua. Identificaremos esta função com a ação parcial $\alpha$, e em particular a denotaremos também pelo símbolo $\alpha$. Notar que esta definição implica que cada $\alpha_{t}: X_{t-1} \longrightarrow X_{t}$ é um homeomorfismo.

Exemplo 4.38. Suponhamos que $\beta: G \times Y \longrightarrow Y$ é uma ação contínua do grupo topológico $G$ sobre o espaço topológico $Y$, e seja $X \subseteq Y$ um subconjunto aberto. Consideremos a ação parcial $\alpha:=\left.\beta\right|_{X}$ (ver $4.8,4.7$ ). Afirmamos que $\alpha$ é uma ação parcial contínua. Só é necessário mostrar que $\Gamma_{\alpha}$ é aberto, porque o resto decorre da continuidade de $\beta$. Agora, seja $(t, x) \in \Gamma_{\alpha}$, de forma que $\alpha_{t}(x) \in X$. Como $X$ é aberto e 3 é contínua, existe uma vizinhança $W$ de $G \times Y$ tal que $\beta(W) \subseteq X$, e portanto $W \subseteq \Gamma_{\alpha}$, pela definiçào de $a$. Portanto $\Gamma_{\alpha}$ é aberto.

Antes de começar a estudar as açòes envolventes de ações parciais contínuas, gostaríamos de apresentar uma situaçào típica em que tem uma ação parcial presente: o fluxo de um sistema dinámico 
Exemplo 4.39. Consideremos uma variedade diferenciável $\mathrm{I}$ e sobre ela um campo vetorial diferenciável $\mathbf{v}: \mathrm{I} \longrightarrow T \mathrm{Y}$. Podemos considerar então a equação diferencial

$$
\dot{x}=\mathrm{v}(x)
$$

Entào o fluxo gerado pela equação (6) é uma ação parcial contínua de $\mathbb{P}$ sobre .Y (de fato: diferenciável).

Vejamos os detalhes com cuidado. Citando 1.48 de [63], podemos afirmar que para cada $x \in \mathrm{I}$ existe uma curva diferenciável $\gamma_{x}$, cujo domínio é um intervalo $\left(a_{x}, b_{x}\right)$, e tal que:

1. $0 \in\left(a_{x}, b_{x}\right)$. e $\gamma_{x}(0)=x, \forall x \in \mathrm{Y}$.

2. $\gamma_{x}$ é uma curva integral de $\mathrm{v}$, ou seja: $\dot{\gamma}_{x}(t)=\mathrm{v}\left(\gamma_{x}(t)\right)$, para todo $x \in \mathrm{X}$ e para todo $t$ no domínio de $i x$.

3. Se $\mu:(c, d) \longrightarrow \mathrm{Y}$ é outra curva integral de $\mathrm{v}$ tal que $\mu(0)=x$, então $(c, d) \subseteq\left(a_{x}, b_{x}\right)$. e $\mu=\left.\gamma_{r}\right|_{(c, d)}$.

4. Para cada $t \in \mathbb{R}$ seja $\mathrm{X}_{-t}=\left\{x \in \mathbb{X}: t \in\left(a_{x}, b_{x}\right)\right\}$, e seja $a_{t}: X_{-t} \rightarrow X^{*}$ tal que $\alpha_{t}(x)=\gamma_{x}(t)$. Então:

(a) Para todo $x \in \mathrm{I}^{*}$ existem $V \subseteq \mathrm{Y}$ aberto e $\epsilon>0$ tais que $x \in V \subseteq \mathrm{X}_{-t}, \forall t \in(-\epsilon, \epsilon)$, e a transformação $(-\epsilon, \epsilon) \times \mathrm{V} \longrightarrow \mathrm{X}$ tal que $(t, y) \longmapsto \alpha_{t}(y)$ é diferenciável.

(b) $\mathrm{Y}_{t}$ é aberto, $\forall t \in \mathbb{P}$.

(c) $\mathrm{X}=U_{t>0} \mathrm{X}_{-t}$.

(d) $\alpha_{t}: \mathrm{X}_{-t} \longrightarrow \mathrm{I}_{t}$ é um difeomorfismo.

(e) Sejam $s, t \in \mathbb{E}$.. Então $\operatorname{dom}\left(\alpha_{s} \alpha_{t}\right) \subseteq X_{-(s+t)}$, mas em geral não são iguais. Se st $>0$. temos que $\operatorname{dom}\left(\alpha_{s} a_{t}\right)=\mathrm{X}_{-(s+t)}$.

Além disto, se $y \in \operatorname{dom}\left(\alpha_{s} \alpha_{t}\right)$, então $\alpha_{s} \alpha_{t}(y)=a_{s+t}(y)$.

Seja $\Gamma_{\alpha}=\left\{(t, x) \in \mathbb{F} \times \mathbb{X}: x \in \mathcal{Y}_{-t}\right\}$. Então $\Gamma_{\alpha}$ é aberto. Com efeito, seja $(t, x) \in \Gamma_{\alpha}$. Por 4.(a), existem $\epsilon>0$ e $V \subseteq D_{s}, \forall s \in(-\epsilon, \epsilon)$, tais que $\alpha_{t}(x) \in V$ e existe $\alpha_{s}(y), \forall(s, y) \in(-\epsilon, \epsilon) \times \Gamma^{*}$ : como $\alpha_{t}$ é contínua e $X_{t}$ é aberto (por 4.(d) e (b), respectivamente), existe $U \subseteq X_{t}$ tal que $x \in I^{\circ}$ e $\alpha_{t}(U) \subseteq V$. Portanto, $U$ está contido no domínio de $\alpha_{s} \alpha_{t}$, que está incluído no domínio de $\alpha_{s+t}$. $\forall s \in(-\epsilon, \epsilon)$. Segue que $U \times(t-\epsilon, t+\epsilon) \subseteq \Gamma_{\alpha}$, e portanto $\Gamma_{\alpha}$ é aberto.

Em conclusão. $a=\left(\left\{\hat{X}_{t}\right\}_{t \in \mathbb{R}},\left\{a_{t}\right\}_{t \in \mathbb{R}}\right)$ é uma ação parcial de $\mathbb{R}$ sobre a variedade $X$. Em geral. esta coleçào de aplicaçòes $\alpha_{t}$, ou a aplicação $\Gamma_{\alpha} \longrightarrow X$ tal que $(t, x) \longmapsto \alpha_{t}(x)$, é chamado fluxo da equação diferencial (6). Consultar por exemplo [39].

Um resultado conhecido de sistemas dinámicos diz que se $\mathbf{v}$ é um campo diferenciável sobre uma variedade compacta $\mathrm{X}$, então as curvas integrais da equação (6) estão definidas para todo $t \in \mathbb{R}$. Na verdade, temos o seguinte resultado mais geral.

Proposiçào 4.40. Seja a uma ação parcial do grupo $G$ sobre o espaço compacto $X$. Então existe um subgrupo aberto $H$ de $G$ tal que a restrita a $H$ é uma ação global (uma ação global é uma ação no sentido usual: colocamos o adjetivo "global" para salientar que cada elemento do grupo age sobre cada elemento do espaço).

Em particular. se Gé conexo, a é uma ação global.

DemonstraçÃo. Para cada $x \in X$, existem um aberto $U_{x} \subseteq X$ que contém $x$ e um aberto simétrico $V_{x} \subseteq G$ que contém a identidade e de $G$, e tais que $V_{x} \times U_{x} \subseteq \Gamma_{\alpha}$. Como $X$ é compacto. existe $\left\{x_{1}, \ldots x_{n}\right\} \subseteq \mathrm{X}$ tal que $\mathrm{Y}=\bigcup_{j=1}^{n} U_{x_{j}}$. Seja $V=\bigcap_{j=1}^{n} \Gamma_{x_{j}}$, de forma que $V$ é uma vizinhança simétrica da identidade. Agora, se $x \in \mathcal{X}$, existe $j$ tal que $x \in U_{x}$, e portanto $V \times\{x\} \subseteq V_{x_{j}} \times$ $U_{x} \subseteq \Gamma_{\alpha}$. Entào $\mathrm{V}$ está contido em cada $A_{x}$, o conjunto de elementos de $G$ que agem sobre $x$. Conseqüentemente. $V \subseteq A=\bigcap_{x \in A} A_{x}$. Como $A$ é um sub-monóide de $G$ (4.13), o monóide $H=$ 
$\bigcup_{n \geq 1} V^{n}$ gerado por $V$ está contido em $A$. Mas como $V$ é simétrico, o monóide $H$ também é simétrico, e portanto um subgrupo de $G$. Como $I$ é aberto, $H$ também é aberto.

Com respeito à última afirmação, é só lembrar que o único subgrupo aberto de um grupo conexo é o próprio grupo (se um subgrupo é aberto, também é aberta cada classe lateral segundo o subgrupo, o que implica, depois de tomar a uniào das classes disjuntas com o subgrupo, que o subgrupo será fechado; mas o único subconjunto nào vazio aberto e fechado de um espaço conexo é o próprio espaço).

Observaçào 4.41. Decorre da demonstração da Proposição 4.40 acima, que se $X$ é um espaço compacto, então $A$ é aberto. Notar que, em geral, $A$ é aberto se e somente se $\epsilon$ é um ponto interior de $A$. O Exemplo 4.14 mostra que $A$ pode ter pontos interiores ainda se $f$ não é ponto interior.

A seguir iniciamos a procura de uma ação envolvente para uma açào parcial contínua. Guiados pelo Exemplo 4.38, tentaremos achar um espaço topológico $Y$ que contenha como subespaço topológico aberto o espaço $X$ onde ocorre nossa açào parcial, e também uma açào continua sobre $Y$, tal que a restrição desta ação a $X$ coincida com a ação parcial. Como já existem un conjunto envolvente $\dot{X}$ e uma ação envolvente $\dot{\alpha}$ da ação parcial. o que temos que achar é uma topologia adequada sobre $\dot{X}$, de forma que $X$ seja um subespaço topológico aberto de $\dot{X}, \dot{\alpha}$ seja contínua sobre $\dot{X}$ com esta topologia, e a restrição de $\dot{a}$ a $X$ coincida $\operatorname{com} \alpha$.

Seja $\bar{\alpha}$ a restriçào de $\dot{\alpha}$ a $G \times X$, ou seja: $\bar{\alpha}: G \times X \longrightarrow \dot{X}$ é tal que $\bar{\alpha}(t, x)=\dot{\alpha}(t, x)=\dot{\alpha}_{t}(x)$.

Proposiçẫo 4.42. A topologia final de $\dot{\mathrm{X}}$ definida por $\bar{\alpha}$ é:

$$
\dot{\tau}=\left\{\bigcup_{t \in G} \dot{\alpha}_{t}\left(V_{t}\right): V_{t} \in \tau\right\} .
$$

Além disso, $X$ é aberto em $\dot{X}$. e a inclusão $\iota:(X, \tau) \hookrightarrow(\dot{X}, \dot{\tau})$ é um homeomorfismo sobre sua imagem.

Demonstraçào. Sejam $t \in G$. $V^{\prime} \in \tau$. Então:

$$
\begin{aligned}
\bar{\alpha}^{-1}\left(\dot{\alpha}_{t}(V)\right) & =\{(s, x) \in G \times H: \bar{\alpha}(s, x)=\dot{\alpha}(t, y), \text { para algum } y \in V\} \\
& =\left\{(s, x) \in G \times H: \bar{\alpha}\left(t^{-1} s, x\right) \in V\right\} \\
& =\left(\bar{\alpha}\left(\lambda_{t}^{-1} \times i d_{X}\right)\right)^{-1}(V),
\end{aligned}
$$

onde $\lambda_{t}: G \longrightarrow G$ é dada por $\lambda_{t}(s)=t s$. Como $\lambda_{t}^{-1} \times i d_{X}: G \times \mathrm{Y} \longrightarrow G \times X$ é um homeomorfismo, $\left(\bar{\alpha}\left(\lambda_{t}^{-1} \times i d\right)\right)^{-1}(V)$ será aberto em $G \times X$ se e somente se $\bar{a}^{-1}(V)$ é aberto; mas como $V \subseteq X, \bar{\alpha}^{-1}(V)=\alpha^{-1}(V)$, que é aberto, pois $\Gamma_{\alpha}$ é aberto em $G \times X$ e a é contínua. Portanto, $\dot{\alpha}_{t}(V) \in \dot{\tau}, \forall t \in G, V \in \tau$.

Inversamente, suponhamos que $W \subseteq \dot{X}$ é aberto na topologia final. Entào:

$$
W=W \cap\left(\cup_{t \in G} \dot{\alpha}_{t}(X)\right)=\cup_{t \in G}\left(W \cap \dot{\alpha}_{t}(X)\right)=\cup_{t \in G} \dot{\alpha}_{t}\left(\dot{\alpha}_{t}^{-1}(I I) \cap X\right) .
$$

Seja $V_{t}=\dot{\alpha}_{t}^{-1}(W) \cap X$. Basta mostrar que $V_{t} \in \tau, \forall t \in G$. Agora:

$$
V_{t}=\{x \in X: \bar{a}(t, x) \in W\}=\left\{x \in X:(t, x) \in \bar{a}^{-1}(W)\right\}=p_{2}\left(\bar{a}^{-1}(W)\right),
$$

onde $p_{2}: G \times X \longrightarrow X$ é a projeçào. Como $W \in \dot{\tau}$, então $\bar{\alpha}^{-1}(W) \in \tau_{G} \times \tau$, e como $p_{2}$ é aberta, concluímos que $V_{t} \in \tau$. 
Fica claro da caracterização acima da topologia $\dot{\tau}$, que cada aberto $V$ de $Y$ também pertence a $\dot{\tau}$, pois $V=\dot{\alpha}_{\epsilon}(V)$. Portanto a inclusão $\iota: X \hookrightarrow \dot{X}$ é aberta. Por outro lado, se $V_{t} \in \tau$, temos:

$$
\begin{aligned}
X \cap \dot{\alpha}_{t}\left(V_{t}\right) & =\left\{x \in X: \exists y \in V_{t} \text { tal que } \dot{\alpha}_{t}(y)=x\right\} \\
& =\left\{x \in X: \exists y \in V_{t} \text { tal que } \alpha_{t}(y)=x\right\} \\
& =\alpha_{t}\left(V_{t} \cap X_{t-1}\right),
\end{aligned}
$$

que pertence a $\tau$. Assim, 1 é um homeomorfismo sobre sua imagem.

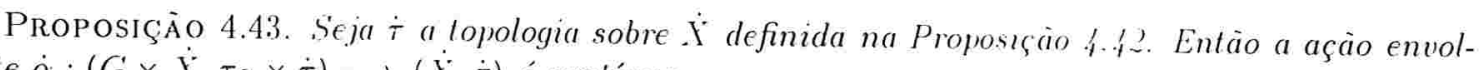
vente $\dot{\alpha}:\left(G \times \dot{Y}, \tau_{G} \times \dot{\tau}\right) \longrightarrow(\dot{X}, \dot{T}) \dot{\epsilon}$ contínua.

Demonstraçào. Temos que ver que para todo $V \in \tau$, e para todo $l \in\left(i\right.$. o conjunto $\dot{\alpha}^{-1}\left(\dot{\alpha}_{t}(V)\right)$ é aberto em $G \times \dot{Y}$. Temos (lembrar a definiçào de $\lambda_{t}$ na Proposiçào 4.42 ):

$$
\begin{aligned}
\dot{\alpha}^{-1}\left(\dot{\alpha}_{t}\left(V^{\prime}\right)\right) & =\{(s, \dot{x}) \in G \times \dot{X}: \dot{\alpha}(s, \dot{x}) \in \dot{\alpha}(\{t\} \times 1)\} \\
& =\left\{(s, \dot{x}) \in G \times \dot{X}: \dot{\alpha}\left(t^{-1} s, \dot{x}\right) \in l\right\} \\
& =\left(\dot{\alpha}\left(\lambda_{t}^{-1} \times i d_{\dot{X}}\right)\right)^{-1}(V) \\
& =\left(\lambda_{t}^{-1} \times i d_{\dot{X}^{\prime}}\right)^{-1}\left(\dot{\alpha}^{-1}(V)\right) .
\end{aligned}
$$

Como $\lambda_{t} \times i d_{\dot{X}}: G \times \dot{X} \longrightarrow G \times \dot{X}$ é um homeomorfismo, basta mostrarmos que $\dot{\alpha}^{-1}(V)$ é aberto.

Seja $\left(s_{0}, \dot{x}_{0}\right) \in \dot{a}^{-1}(V)$, e sejam $t_{0} \in G, x_{0} \in X$ tais que $\bar{\alpha}\left(t_{0}, x_{0}\right)=\dot{x}_{0}$. ('omo $\left(s_{0}, \dot{x}_{0}\right) \in \dot{\alpha}^{-1}(V)$. tem-se que $\alpha\left(s_{0} t_{0}, x_{0}\right) \in V$, e portanto $s_{0} t_{0} \in A_{x_{0}}$. Em outras palarras. $\left(s_{0} t_{0}, x_{0}\right) \in \Gamma_{\alpha}$. Como $\Gamma_{\alpha}$ é aberto em $G \times X$, existem abertos $U \subseteq G$ e $W \subseteq X$ tais que $\left(s_{0} t_{0}, x_{0}\right) \in I^{\circ} \times W^{\prime} \subseteq \Gamma_{\alpha}$. Como $\alpha$ é contínua, podemos supor também que $\alpha(U \times W) \subseteq V$. Agora, o produto é contínuo em $G$, de forma que existem abertos $L_{0}, U_{1} \mathrm{em} G, \operatorname{com} s_{0} \in U_{0}, t_{0} \in U_{1}$, e tais que $l_{0} l_{1} \subseteq U$. Segue então que $\left(U_{0} U_{1}\right) \times W \subseteq \Gamma_{\alpha}$, e $a\left(\left(U_{0} U_{1}\right) \times W\right) \subseteq V$. Mas isto implica que $l_{0} \times \alpha_{t_{0}}(W) \subseteq \alpha^{-1}(V)$ : se $s \in U_{0}$,
$x \in W:$

$$
\dot{\alpha}\left(s, \dot{\alpha}_{t_{0}}(x)\right)=\dot{\alpha}\left(s t_{0}, x\right)=\alpha\left(s t_{0}, x\right) \in \mathrm{l} .
$$
Como $\left(s_{0}, \dot{x}_{0}\right) \in U_{0} \times \dot{\alpha}_{t_{0}}(W)$, aberto em $\dot{\alpha}^{-1}(V)$, concluímos que $\dot{\alpha}^{-1}(V)$ é aberto, e portanto $\dot{\alpha}$ é
contínua.

Teorema 4.44. Seja a uma ação parcial contínua de um grupo topológico $\left(G, \tau_{G}\right)$ sobre um espaço topológico $(X, \tau)$. e seja $\alpha$ a açáa envolvente de $\alpha$, agindo sobre o espaço envolvente $\dot{X}$, com $X \subseteq \dot{X}$. Então $\dot{\tau}$ é a única topologia sobre $\dot{X}$ que satisfaz as seguintes trés propriedades:

1. $X$ é aberto em $\dot{X}$.

2. Se $U \subseteq \mathrm{X}$, então $U \in \tau \Longleftrightarrow U \in \dot{\tau}$, i.e., a topologia $\tau$ coincide com a topologia que $X$ herda de $\dot{\tau}$.

3. $\dot{\alpha}:\left(G \times \dot{X}, \tau_{G} \times \dot{\tau}\right) \longrightarrow(\dot{X}, \dot{\tau}) \dot{e}$ contínua.

Além disso, $(\dot{X}, \dot{\alpha}, \dot{\tau})$ satisfaz a seguinte propriedade universal: se $\iota: a \longrightarrow \beta$ é um morfismo contínuo, onde $\beta$ é uma ação contínua de $G$ sobre um espaço topológico $Y$. então existe um único 
morfismo contínuo $\eta: \dot{\mathrm{X}} \longrightarrow \mathrm{Y}$ tal que $\left.\eta\right|_{X}=\psi:$

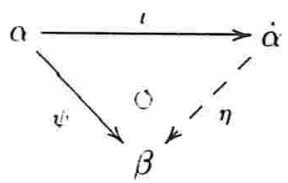

Inversamente, suponhamos que $\left(0, X^{\prime \prime}, \alpha^{\prime}\right)$ é tal que $X^{\prime}$ é um espaço topológico. $\alpha^{\prime}$ é uma ação contínua de $G$ sobre $X^{\prime}, \phi: X \longrightarrow X^{\prime \prime} \epsilon$ um morfismo contínuo de açôes parciais, $\epsilon$ suponhamos que para

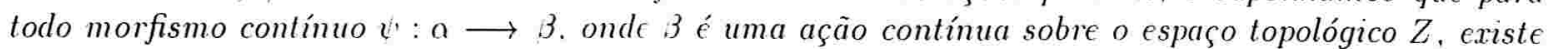
um único morfismo contínuo de açoes parciais $\eta: \alpha^{\prime} \longrightarrow \beta$ tal que $\eta \phi=\psi$. Então existe um único isomorfismo $\theta: \dot{\alpha} \longrightarrow \alpha^{\prime}$ de açóєs parciais que $\dot{\epsilon}$ um homeomorfismo entre $\dot{X} \in X^{\prime}$. e tal que $\theta \iota=\phi$.

Demonstraçào. As Proposiçoes 4.42 e 4.43 mostram que $\dot{\tau}$ satisfaz as condiçōes 1., 2 . e 3 . acima. Suponhamos que $\tau^{\prime}$ é uma topologia que também as verifica. Entào, devido às duas primeiras, cada $I^{\prime} \in \tau$ pertence também a $\tau^{\prime}$. A terceira condição implica que cada $\dot{\alpha}_{t}:\left(\dot{X}, \tau^{\prime}\right) \longrightarrow\left(\dot{X}, \tau^{\prime}\right)$ é um homeomorfismo, e portanto $\dot{\alpha}_{t}\left(V_{i}\right) \in \tau^{\prime} . \forall t \in G, V_{t} \in \tau$. Segue entào que $\dot{\tau} \subseteq \tau^{\prime}$. Mas como $\bar{a}:\left(G \times X, \tau_{G} \times \tau_{\left.\right|_{X}}^{\prime}\right) \longrightarrow\left(\dot{X} \cdot \tau^{\prime}\right)$ é obviamente contínua e $\dot{\tau}$ é a topologia final definida por $\bar{a}$, concluímos que também deve ser $\tau^{\prime} \subseteq \dot{\tau}$. e portanto $\dot{\tau}=\tau^{\prime}$.

Suponhamos agora que $\psi \cdot a \longrightarrow \beta$ é um morfismo contínuo, onde $\beta$ é uma ação contínua de $G$ sobre o espaço topológico $Y$. Pelo Teorema 4.23, sabemos que existe um único morfismo de ações $\eta: \dot{a} \longrightarrow \beta$ tal que $\eta \iota=\psi$, e a única coisa que temos que provar é que $\eta: \dot{X} \longrightarrow Y$ é contínua. Como $\dot{\tau}$ é a topologia final sobre $\dot{X}$ com respeito a $\bar{\alpha}$, temos que $\eta$ é contínua se e somente se $\eta \bar{\alpha}$ é contínua. Notemos que o seguinte diagrama comuta:

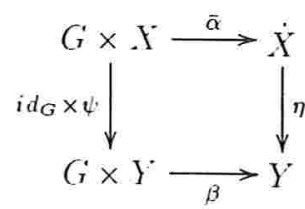

Com efeito, dado $(t, x) \in G \times Y$.

$$
\beta_{t}(\varphi(x))=\eta\left(\dot{\alpha}_{t}(x)\right)=\eta \bar{\alpha}(t, x) .
$$

Portanto, $\beta\left(i d_{G} \times \psi^{\prime}\right)=\eta \bar{\alpha}$, e como $\beta\left(i d_{G} \times \psi^{\prime}\right)$ é contínua, concluímos que $\eta$ é contínua.

Suponhamos agora que $\left(\phi, \mathrm{H}^{\prime}, \mathrm{a}^{\prime}\right)$ satisfaz a propriedade universal. Entào existem únicos morfismos contínuos de ações parciais $\theta: \dot{X} \longrightarrow X^{-1}$ e $\theta^{\prime}: X^{\prime} \longrightarrow \dot{X}$ tais que $\theta \iota=\phi$, e $\theta^{\prime} \phi=\iota$. Então $i d_{X^{\prime}} \phi=\phi=\theta \theta^{\prime} \phi$. e $i d_{\dot{X}^{\prime}}=\iota=\theta^{\prime} \theta \iota$. Como $i d_{X^{\prime}}$ e $\theta \theta^{\prime}$ são morfismos contínuos de ações parciais, concluímos que, pela unicidade na propriedade universal de $(\dot{X}, \dot{\alpha})$, deve ser $i d_{X^{\prime}}=\theta \theta^{\prime}$. Analogamente $i d_{\dot{X}}=\theta^{\prime} \theta$. Segue que $\theta$ é um isomorfismo e um homeomorfismo.

DEFINIÇÃo 4.45. Seja $\alpha$ uma açào parcial do grupo topológico $\left(G, \tau_{G}\right)$ sobre o espaço topológico $\left(X, \tau_{X}\right)$. Diz-se que $\left(\phi, Y, \tau_{Y^{\prime}}, \beta\right)$ é uma ação envolvente de $\alpha$ se: $\beta$ é uma ação contínua sobre o espaço topológico $\left(Y, \tau_{Y}\right),(\phi, Y, \beta)$ é uma açào açào envolvente no sentido da Definição 4.24 ,e $\phi: X \longrightarrow \phi\left(X^{\prime}\right)$ é um homeomorfismo, onde a topologia de $O(X)$ é a herdada de $\tau^{\prime}$. 
4.2.1. Uma construção alternativa do espaço e da ação envolventes. A seguir veremos que $\dot{X}$ pode ser construído como quociente de uma relação de equivaléncia sobre $G \times X$, que $\dot{\alpha}$ é então a ação quociente da ação de $G$ por translação à esquerda na primeira variável de $G \times X$, e que $\dot{T}$ é a correspondente topologia quociente.

ProposiçÃo 4.46. Seja $a=\left(\left\{\hat{Y}_{t}\right\}_{t \in G},\left\{\alpha_{t}\right\}_{t \in G}\right)$ uma ação parcial do grupo $G$ sobre o conjunto $\mathrm{Y}$, e seja $\beta: G \times \mathrm{X} \longrightarrow G \times \mathrm{J}^{*}$ dada por $\beta_{t}(s, x)=(t s, x)$. Definamos sobre $G \times \mathrm{Y}$ a seguinte relação $\sim$ :

Então:

$$
(r, x) \sim(s, y) \Longleftrightarrow s^{-1} r \in A_{x} \text { e } \alpha_{s^{-1} r}(x)=y
$$

1. $\beta$ é uma ação sobre $G \times \mathrm{Y}$. compativel com a relação $\sim$.

2. $\sim$ é uma relaçcio de equivalência sobre $G \times X$.

3. Seja $\frac{\beta}{\sim}: G \times\left(\frac{G \times X}{\sim}\right) \longrightarrow \frac{G \times X}{\sim}$ a açaio quociente, e seja $\phi: X \longrightarrow \frac{G \times X}{\sim}$ a composiçăo da aplicação $x \longmapsto(\epsilon, x)$ de $\mathrm{X} \longrightarrow G \times \mathrm{Y}$ com a projeção $p: G \times \mathrm{X} \longrightarrow \frac{(i \times X}{\sim}$. Entáo $\left(\phi, \frac{G \times X}{\sim}, \frac{\beta}{\sim}\right)$ é a açaio envolvente de $\alpha$.

Demonstração. 1. e 2. são evidentes, de modo que podemos concentrarnos em 3 . Vejamos que $\left(\phi, \frac{G \times X}{\sim}, \frac{\beta}{\sim}\right)$ é uma extensào de $\alpha$. Como $\left(e, \alpha_{s}(x)\right) \sim(s, x), \forall s \in A_{x}$, temos:

$$
\begin{aligned}
\phi\left(\alpha_{s}(x)\right) & =p\left(e, \alpha_{s}(x)\right) \\
& =p(s, x) \\
& =p\left(\beta_{s}(e, x)\right) \\
& =(\beta / \sim)_{s} p(e, x) \\
& =(\beta / \sim)_{s} \phi(x)
\end{aligned}
$$

Por outro lado, se $\phi(x)=\phi(y)$, entào $(c, x) \sim(e, y)$, o que implica que $x=y$. Conseqüentemente, $\phi$ : $\alpha \longrightarrow \frac{\beta}{\sim}$ é uma ext.ensão. Vejamos que $\left(\phi, \frac{G \times X}{\sim}, \frac{\beta}{\sim}\right)$ tem a propriedade universal da ação envolvente (lembrar o Teorema 4.23). o que acabaria a prova. Notemos primeiro que se $\gamma: G \times Y \longrightarrow Y$ é uma ação, e $\psi^{\prime}: X \longrightarrow Y$ é uma função arbitrária, então a função $\eta^{\prime}: G \times X \longrightarrow Y$ tal que $(t, x) \longmapsto \gamma_{t}(\psi(x))$ é um $G$-morfismo, onde estamos considerando a ação 3 sobre $G \times X$. Com efeito:

$$
\eta^{\prime}\left(\beta_{s}(t, x)\right)=\eta^{\prime}(s t, x)=\gamma_{s t}(\psi(x))=\gamma_{s} \gamma_{t}(\psi(x))=\gamma_{s}\left(\eta^{\prime}(t, x)\right)
$$

Suponhamos agora que $\psi^{\prime}: a \longrightarrow \gamma$ é um morfismo, e sejam $(t, x) \sim(s, y)$. Entào, como $s^{-1} t \in A_{x}$. e $\alpha_{s-1}(x)=y$ :

$$
\gamma_{s^{-1}}\left(\eta^{\prime}(t, x)\right)=\gamma_{s^{-1}}\left(\gamma_{t}(\psi(x))\right)=\gamma_{s^{-1} t}(\psi(x))=\psi\left(\alpha_{s^{-1} t}(x)\right)=\psi(y)
$$

Portanto:

$$
\eta^{\prime}(t, x)=\gamma_{s}(\psi(y))=\eta^{\prime}(s, y)
$$

de maneira que $\eta^{\prime}$ passa ao quociente, e obtemos um morfismo $\eta: \frac{G \times X}{\sim} \longrightarrow Y$ tal que:

$$
\eta \phi(x)=\eta^{\prime}(e, x)=\gamma_{e}(\psi(x))=\psi(x) .
$$

Assim, $\eta \phi=\psi$. A unicidade da aplicação $\eta$ é trivial, já que ele é um morfismo.

ProposiçÃo 4.47. Seja a uma açào parcial contínua do grupo topológico $\left(G, \tau_{G}\right)$ sobre o espaço topológico $(X, \tau)$, e seja $\frac{\tau_{G} \times T}{\sim}$ a topologia quociente sobre o espaço quociente $\frac{G \times X}{\sim}$ construído na Proposição 4.46 acima. Entào $\left(\phi, \frac{B}{\sim}, \frac{G \times X}{\sim}, \frac{\tau_{G} \times \tau}{\sim}\right)$ é a ação envolvente de $a$. 
Demonstraçäo. Vamos mostrar que $\left(\frac{G \times X}{\sim}, \frac{\beta}{\sim}, \frac{\tau_{G} \times \tau}{\sim}\right)$ satisfaz a propriedade universal do Teorema 4.44, que caracteriza a topologia do espaço topológico envolvente.

Sejam $\gamma$ uma ação contínua de $G$ sobre $Y$. e suponhamos que $\psi$ : $\alpha$ ’ é um morfismo contínuo. Pela Proposição 4.46, existe um único morfismo $\eta:\left(\frac{G \times X}{\sim}\right) \longrightarrow Y$ tal que:

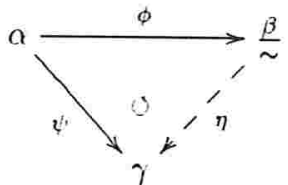

De fato, temos que $\eta$ está determinado por $\eta(p(e, x))=\psi(x)$. Temos que mostrar que $\eta$ é contínua. Para isto, observemos que o diagrama seguinte comuta:

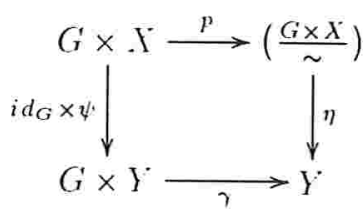

Com efeito:

$$
\begin{aligned}
\gamma(i d \times \psi)(t, x) & =\gamma_{t}(\psi(x)) \\
& =\gamma_{t} \eta(p(e, x)) \\
& =\eta\left(\left(\frac{\beta}{\sim}\right)_{t}(p(e, x))\right) \\
& =\eta\left(p\left(\beta_{t}(e, x)\right)\right) \\
& =\eta(p(t, x)) \\
& =\eta p(t, x) .
\end{aligned}
$$

Como $\gamma\left(i d \times \psi^{\prime}\right)$ é contínua, temos que $\eta p$ também é contínua. Como $\frac{G \times X}{\sim}$ tem a topologia quociente induzida por $p$, isto implica que $\eta$ é contínua.

Existe uma construção conhecida em sistemas dinâmicos, chamada suspensão de um sistema discreto. Suponhamos que $h: X \longrightarrow X$ é um homeomorfismo, de forma que temos uma ação $\alpha: \mathbb{Z} \times X \longrightarrow X$ dada por $\alpha_{n}(x)=h^{n}(x)$. Sobre $\mathbb{R} \times X$ considera-se a seguinte relação de equivalência: $(t, x) \sim(s, y)$ se e somente se $n:=t-s \in=$, e $h^{n}(x)=y$. Sobre o quociente $Y:=(\mathbb{R} \times X) / \sim$ define-se então uma ação de $\mathbb{R}: \beta: \mathbb{R}: Y \longrightarrow Y$ tal que $\beta_{s}(\overline{(t, x)})=\overline{(t+s, x)}$. Notar que $\alpha$ pode ser vista como uma ação parcial de $\mathbb{R}$. sobre $X$ (embora não contínua segundo nossa definição), e portanto $\beta$ é a ação envolvente desta ação parcial. Assim. vemos que a ação envolvente de uma ação parcial é uma generalização da suspensão de uma ação do grupo dos inteiros.

4.2.2. Alguns exemplos. A seguir consideramos alguns exemplos de açōes envolventes, entre eles vários nos quais o espaço envolvente não é de Hausdorff. Também determinamos precisamente as açōes parciais cuja ação envolvente age sobre um espaço de Hausdorff: são aquelas que tem gráfico fechado. Em particular, levando em conta o exemplo do fluxo 4.39, este fato divide os campos vetoriais 
em duas classes, segundo que a correspondente ação parcial tenha gráfico fechado ou não. Veremos que nenhuma destas classes é vazia.

Exemplo 4.48. Considere a açào $\beta_{\theta}: \Xi \times S^{1} \longrightarrow S^{1}$ dada pela rotaçào por um ángulo irracional $\theta: \beta_{k}(z)=e^{2 \pi i k \theta} z, \forall k \in Z, z \in S^{1}$. Seja $\mathbb{X}=\{1\} \subseteq S^{1}$, e consideremos a açào parcial supertrivial $\alpha$ sobre $\mathcal{Y}^{-}$(Definiçào 4.3). É claro que a ação envolvente algébrica de $(\mathcal{Y}, \alpha)$ é $(Z, \dot{\alpha})$, onde $\mathrm{Y}$ se identifica com $\hat{X}^{-1}=\{0\}$, e $\dot{\alpha}(k)=k+1, \forall k \in \mathbb{Z}$. A topologia $\dot{\tau}$ de $Z=\hat{X}$ correspondente segundo o Teorema 4.44 é a topologia discreta, pois $\mathrm{I}=\{0\}$ deve ser aberto em $\dot{\mathrm{X}}$. Obviamente a inclusão $\psi: X \hookrightarrow S^{1}$ tal que $1 \longmapsto 1$ é um morfismo contínuo, e portanto existe um único morfismo contínuo $\eta: \mathbb{Z} \longrightarrow S^{1}$ tal que $\eta(0)=1$. Naturalmente, temos que $\eta(k)=r^{2 \pi i k \cdot t} . \forall k \in Z$.

Exemplo 4.49. Seja $\beta_{\theta}$ como no exemplo acima, e seja $\alpha_{\theta}^{U}$ a restrição de $3_{\theta}$ a um aberto não vazio $U \subseteq S^{1}$ qualquer. Como a ação $\beta_{\theta}$ ć minimal, é claro que $\beta_{\theta}$ é a açào envolvente de $\alpha_{\theta}^{U}$. Este exemplo chama a atençào sobre o fato de uma ação parcial determinar propriedades topológicas profundas de $\dot{X}$, diferentes das propriedades do próprio $X$; por exemplo. o grupo de homotopia $\pi_{1}\left(S^{1}\right)=Z$. enquanto que $\pi_{1}(U)=0$ se $U$ é um arco de circumferencia diferente de $S^{1}$.

O seguinte exemplo mostra que, embora uma açào parcial atue sobre um espaço de Hausdorff, a sua ação envolvente nào tem por que agir sobre um espaço de Hausdorff.

Exemplo 4.50. Consideremos a seguinte ação parcial trivial $\alpha$ de $\ldots . .$. sobre o intervalo $X=[0,1]$ : $\alpha_{0}=i d_{X}, \alpha_{1}=i d_{1}$, onde $\dot{Y}=\left(\frac{1}{3}, \frac{2}{3}\right)$. Seja $\alpha: G \times \dot{X} \longrightarrow \dot{X}$ a ação envolvente de $\alpha$. Sabemos que para todo $x \in V$ temos que $\dot{\alpha}_{0}(x)=x=\dot{\alpha}_{1}(x)$, e que para cada $x \notin 1$, deve ser $\dot{\alpha}_{1}(x) \neq x$. Portanto, como conjunto, $\dot{X}$ pode ser representado assim:

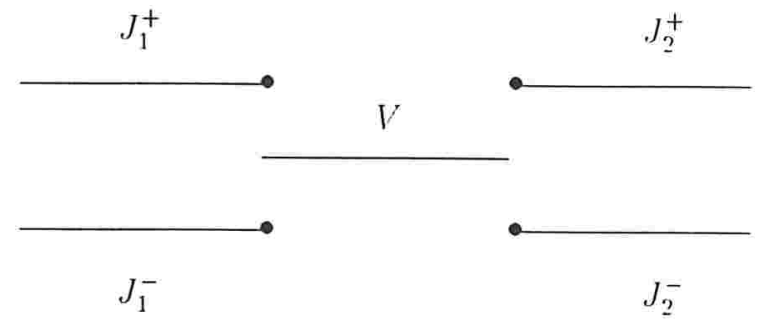

Ou seja, $\dot{X}=V \biguplus \biguplus_{i=1.2} J_{i}^{ \pm}$, onde $J_{1}^{-} . J_{1}^{+}$são cópias disjuntas do intervalo $\left[0, \frac{1}{3}\right]$, e $J_{2}^{-}$, $J_{2}^{+}$são cópias disjuntas do intervalo $\left[\frac{2}{3}, 1\right]$. Se $x \notin V$, usamos a notações $x^{+}$e $x^{-}$para indicar que $x \in J_{i}^{+}$ou que $x \in J_{i}^{-}$respectivamente; é também conveniente pôr $x=x^{+}=x^{-}$quando $x \in V$. Identificamos $X \operatorname{com} J_{1}^{-} \uplus V \biguplus J_{2}^{-}$. Desta forma, $\dot{\alpha}_{1}\left(x^{-}\right)=x^{+}$, e $\dot{\alpha}_{1}\left(x^{+}\right)=x^{-}, \forall x^{-}, x^{+} \in \dot{X}$. Seja $U \subseteq \dot{X}$ tal que $\frac{1}{3}^{ \pm}, \frac{2}{3}^{ \pm} \notin U$. Da descrição da topologia $\dot{\tau}$ na Proposição 4.42, é claro que $U$ é aberto em $\dot{X}$ se e somente se ele é aberto em nossa representação como subconjunto do plano, com a topologia herdada da usual de $\mathbb{F}^{2}$. Agora, as vizinhanças básicas ao redor de $\frac{1}{3}^{+}$sào da forma $\left(a^{+}, b^{+}\right)$, onde $a<\frac{1}{3}<b$, e as vizinhanças básicas de $\frac{1}{3}^{-}$são da forma $\left(c^{-}, d^{-}\right)$. onde $c<\frac{1}{3}<d$. Nestas condições, temos que $\left(a^{+}, b^{+}\right) \cap\left(c^{-}, d^{-}\right)=\left(\frac{1}{3}, \operatorname{mín}\{b . d\}\right) \neq \emptyset$. Portanto $\frac{1}{3}^{+}, \frac{1}{3}^{-}$não tem vizinhanças disjuntas: duas respectivas vizinhanças possuem sempre uma parte comum não vazia. contida em $V$. Da mesma forma podem ser descritos os abertos básicos de $\frac{2}{3}^{+}$e $\frac{2}{3}^{-}$, e concluímos que $\frac{2}{3}^{+}$e $\frac{2}{3}^{-}$também são inseparáveis. 
A obstrução para que o espaço envolvente não seja de Hausdorff é revelada na proposição que segue.

Proposição 4.51. Ś jam a uma ação parcial contínua do grupo topológico $G$ sobre o espaço de Hausdorff $\mathrm{Y}$, e $(\dot{a}, \dot{\mathrm{Y}}, \dot{\tau})$ sua açào emuolvente. Seja $\operatorname{Gr}(\alpha)$ o gráfico de $\alpha$, ou seja: $\operatorname{Gr}(\alpha)=\{(t, x, y) \in$ $\left.G \times X \times X: x \in \mathcal{X}_{t-1}, \alpha_{t}(x)=y\right\}$. Entào $(\dot{X}, \dot{\tau})$ é um espaço de Hausdorff se e somente se $\operatorname{Gr}(\alpha) \dot{\epsilon}$ fechado em $\mathrm{G} \times \mathrm{X} \times \mathrm{H}$.

Demonstração. Suponhamos que $\dot{\mathrm{X}}$ é de Hausdorff, e seja $\left(t_{i}, x_{i}, \alpha\left(t_{i}, x_{i}\right)\right) \longrightarrow(t, x, y) \in$ $G \times \dot{X} \times X$. Em particular, $\alpha\left(t_{i}, x_{i}\right) \longrightarrow y \in \mathcal{X}$. Como $\dot{\alpha}$ é contínua, $\dot{\alpha}\left(t_{i}, x_{i}\right) \longrightarrow \dot{\alpha}(t, x)$ e, devemos ter que $y=\alpha(t, x)$ pois o limite é único por ser $\dot{X}$ de Hausdorff. Segue que $\operatorname{Gr}(\alpha)$ é fechado em $G \times X \times X$.

Inversamente, suponhamos que $\operatorname{Gr}(\alpha)$ é fechado em $G \times \dot{X} \times \dot{K}$, e sejam $\dot{x} \neq \dot{y} \in \dot{X}$. Queremos mostrar que existem abertos disjuntos em $\dot{X}$, cada um dos quais contém $\dot{x}$ ou $\dot{y}$. Como cada $\dot{a}_{t}$ é um homeomorfismo de $\dot{\mathrm{Y}}$, podemos supor que de fato $\dot{x}=x \in \mathrm{X}$. Sejam $y \in X, t \in G$ tais que $\dot{\alpha}_{t}(y)=\dot{y}$. Suponhamos entào que toda vizinhança de $x$ tem interseção não vazia com toda vizinhança de $\dot{y}$. Então, para todo par $(U, V)$ de vizinhanças de $x$ e $y$ respectivamente, contidas em $\mathcal{X}$, existe $x_{u, v} \in I \cap \dot{\alpha}_{t}(V)$, digamos $x_{U, V}=\dot{\alpha}_{t}\left(y_{U, V}\right)$, com $y_{U, V} \in V^{\prime}$. Consideremos a rede $\left\{\left(t, y_{U, V}, x_{U, V}\right)\right\}_{U, V} \subseteq \operatorname{Gr}(\alpha)$. Entào $\left\{\left(t, y_{U, V}, x_{U, V}\right)\right\}_{U, V}$ converge para $(t, y, x)$; mas como $\operatorname{Gr}(\alpha)$ é fechado, temos que $\alpha_{t}(y)=x$, donde $x=\dot{y}$. Portanto, $\dot{\mathrm{X}}$ é de Hausdorff.

Observação 4.52. Se $G$ é discreto e $\alpha$ é uma ação parcial sobre $\mathcal{X}$, então $\operatorname{Gr}(\alpha)$ é fechado em $G \times X \times X$ se e somente se $\operatorname{Gr}\left(\alpha_{t}\right)$ é fechado em $X \times X, \forall t \in G$.

Observação 4.53. Naturalmente. se $\beta: G \times Y \longrightarrow Y$ é uma ação contínua sobre um espaço de Hausdorff $Y$, e $X^{*} \subseteq Y^{*}$ é aberto, e se definimos $\alpha:=\left.\beta\right|_{X}$ (4.8), então obtemos uma ação parcial com gráfico fechado, pois a açào envolvente de $a$ é a ação $\beta$ restrita à $\beta$-órbita de $X$ em $Y$, que é um espaço de Hausdorff. Notar que se $Y$ nào for de Hausdorff mas soubermos que $\bar{X}$ sim é de Hausdorff. então $\operatorname{Gr}(\alpha)$ é fechado, e portanto a $\beta$-órbita de $\mathcal{X}$ é de Hausdorff, pois coincide com $\dot{X}$.

Exemplo 4.54. Suponhamos que $h:[0,1] \longrightarrow[0,1]$ é um homeomorfismo, e seja $U \subseteq[0,1]$ um aberto que não é todo $[0,1]$. Restringindo a $U$ a ação global $\mathbb{Z} \times[0,1] \longrightarrow[0,1]$ dada por $(n, t) \longmapsto h^{n}(t)$, obtemos uma ação parcial $\alpha^{\prime}$ de $\mathbb{Z}$ sobre $U$. Denotemos por $U_{n}^{\prime}$ o domínio de $\alpha_{n}^{\prime}$. Definamos agora $X_{0}=[0,1]$, e $X_{n}=U_{n}^{\prime}, \forall n \neq 0$, e sejam $\alpha_{n}: X_{-n} \longrightarrow X_{n}$ dadas por $\alpha_{n}=\alpha_{n}^{\prime}$ se $n \neq 0, \alpha_{0}=i d_{X_{c}}$. Então $\alpha$ não tem gráfico fechado, porque $\alpha_{1}=\left.h\right|_{U}: U \longrightarrow h(U)$ não pode ter gráfico fechado, já que $U i$ e $h(U)$ sào diferentes de $[0,1]$. Portanto o espaço envolvente correspondente a $\alpha$ não é de Hausdorff.

Exemplo 4.55. Usaremos a construção da Proposição $4.4 \bar{\imath}$ para descrever o espaço envolvente de uma ação parcial gerada por um homeomorfismo parcial de um espaço topológico.

Sejam $\mathrm{I}$ um espaço topológico, $l^{Y}, V \subseteq \mathbb{X}$ abertos, e $h: U \rightarrow V$ um homeomorfismo. Seja $\alpha=\left(\left\{X_{n}\right\}_{n \in \Xi},\left\{a_{n}\right\}_{n \in \Xi}\right)$ a ação parcial de $\mathbb{Z}$ sobre $X$ gerada por $h$, ou seja: $X_{0}=X, \alpha_{0}=i d_{X}$. $X_{-n}=\bigcap_{j=0}^{n-1} h^{-j}\left(L^{i}\right)$ se $n>0, \mathrm{I}_{n}=\bigcap_{j=0}^{n-1} h^{j}(V)$ se $n>0$, e $\alpha_{n}: X_{-n} \longrightarrow X_{n}$ tal que $\alpha_{n}(x)=h^{n}(x)$. 
Dado $(n, x) \in \mathbb{Z} \times \mathbb{X}$, com $n \geq 0$, existe $m \geq 0$ mínimo tal que $(n, x) \sim(m, y)$, para algum $y \in X$; na verdade, este y é único: se $(m, y) \sim\left(m, y^{\prime}\right)$. então $\alpha_{m-m}(y)=y^{\prime}$, ou seja $y=y^{\prime}$. Analogamente, para cada $(n, x) \in Z \times X$, com $n \leq 0$, existe $m \leq 0$ máximo tal que $(n, x) \sim(m, y)$, para algum $y \in \mathrm{Y}$, e de novo temos que $y$ é único. Temos definida assim uma função $\mu: \mathbb{Z} \times X \longrightarrow \mathbb{Z} \times X$, tal que $\mu(n, x)=(m, y)$, definido da forma acima. Notar que $\left.\mu\right|_{\{0\} \times X}$ é a identidade.

Afirmamos que se $(n, x)$ é tal que $\mu(n, x)=(m, y)$, com $m>0$, então $y \in \mathrm{X} \backslash U$ : caso contrário, poderíamos considerar o elemento $(m-1, h(y))$, e teríamos que $\alpha_{m-1-n}(h(y))=a_{m-n}(y)=x$, o que implicaria que $(m-1, h(y)) \sim(n, x)$, contradizendo com isso a minimalidade de $m$. Por outro lado, o mesmo argumento mostra que se $(n, x)$ é tal que $n \geq 0$ e $x \notin U$, então $\mu(n, x)=(n, x)$. Um resultado análogo obtemos para $n \leq 0$ : se $(n, x)$ é tal que $n \leq 0$ e $x \notin V$, entào $\mu(n, x)=(n, x)$, e também se tem que para todo $n \leq 0$ e para todo $x$, se $\mu(n, x)=(m, y)$, então $y \notin V$. Portanto $\mu: \mathbb{Z} \times X^{-} \longrightarrow Z:=\left(Z^{-} \times(X \backslash Y)\right) \times\left(\{0\} \times Y^{-}\right) \times\left(\mathbb{Z}^{+} \times\left(Y^{-} \backslash U\right)\right)$ é sobrejetiva. Por outro lado, é claro que se $\mu\left(n_{1}, x_{1}\right)=\mu\left(n_{2}, x_{2}\right)$, entào $\left(n_{1}, x_{1}\right) \sim\left(n_{2}, x_{2}\right)$. Inversamente, suponhamos que $\left(n_{1}, x_{1}\right) \sim\left(n_{2}, x_{2}\right)$. Se $n_{1}, n_{2} \geq 0$ o $n_{1}, n_{2} \leq 0$, então é claro que $\mu\left(n_{1}, x_{1}\right)=\mu\left(n_{2}, x_{2}\right)$. Si $n_{1} n_{2}<0$, suponhamos que $n_{1}<0<n_{2}$; como existe $\alpha_{n_{2}-n_{1}}$, e $n_{2}-n_{1}>n_{2}$, existe $\alpha_{n_{2}}\left(x_{2}\right)$, e temos então que $\mu\left(n_{2}, x_{2}\right)=\left(0, \alpha_{n_{2}}\left(x_{2}\right)\right)$, porque $\left(n_{2}, x_{2}\right) \sim\left(0, a_{n_{2}}\left(x_{2}\right)\right)$; mas também temos que $\left(n_{1}, x_{1}\right) \sim\left(0, a_{n_{2}}\left(x_{2}\right)\right)$, e portanto $\mu\left(n_{1}, x_{1}\right)=\mu\left(n_{2}, x_{2}\right)$. Em conclusão, $\mu\left(n_{1}, x_{1}\right)=\mu\left(n_{2}, x_{2}\right)$ se e somente se $\left(n_{1}, x_{1}\right)=\left(n_{2}, x_{2}\right)$, de maneira que $(\Xi \times X) / \sim$ se identifica com o conjunto $Z$.

Se $A=C_{0}(\mathrm{Y})$, entào a ação parcial $\alpha$ define uma ação parcial $\beta$ sobre $A$. Esta ação parcial fica determinada unicamente pelo automorfismo parcial $\beta_{1}: C_{0}(V) \longrightarrow C_{0}(U)$, tal que $\beta_{1}(f)=f \circ h^{-1}$. A teoria de produtos cruzados por açòes parciais começou com a definição por Exel de produtos cruzados por automorfismos parciais ([23]). Por exemplo, toda $C^{*}$-álgebra aproximadamente finita é um produto cruzado por um sistema dinamico parcial deste tipo (ver [8] sobre as $C^{*}$-álgebras aproximadamente finitas, e [25] para o resultado mencionado), e o mesmo acontece com as $C^{*}$ álgebras de Bunce-Deddens (introduzidas e estudadas em [12] e [13]; que são produtos cruzados por automorfismos parciais foi provado em [24]). Veremos no Capítulo 7 que existem uma $C^{*}$-álgebra $B$ e uma ação $\gamma$ sobre $B$, naturalmente associadas a $A$, e tais que o espectro de $B$ coincide com o espaço envolvente de $\mathrm{Y}$, e a ação induzida por $\gamma$ sobre o espectro de $B$ é a ação envolvente de $\alpha$. Portanto, a descrição acima do espaço envolvente de $\mathrm{X}$ ilustra sobre o espectro desta $C^{*}$-álgebra $B$. Em alguns casos particulares, por exemplo as $C^{*}$-álgebras aproximadamente finitas ou as $C^{*}$-álgebras de Bunce-Deddens, o espaço topológico X pode ser descrito com bastante detalhe ([25], [2] e [24]).

Finalmente, consideramos alguns casos do exemplo do fluxo de um campo vetorial, dois com gráfico fechado e um com gráfico não fechado.

Exemplo 4.56. Consideremos a ação parcial $\alpha$ sobre $\mathbb{R}^{+}$dada pelo fluxo da equação diferencial

$$
\dot{y}=-y^{2}
$$

Se $\gamma_{x}$ é a solução de (7) tal que $\gamma_{x}(0)=x$, então é imediato que $\gamma_{x}(t)=x /(1+t x)$, e está definida para todo $t>-1 / x$. Portanto $\operatorname{Gr}(\alpha)=\left\{\left(t, x, \frac{x}{1+t x}\right): t>\frac{-1}{x}\right\}$, que é fechado em $\mathbb{R} \times \mathbb{R}^{+} \times \mathbb{R}^{+}$.

ExEmplo 4.57. Seja agora a equação diferencial em $\mathbb{E}^{+}$dada por:

$$
\dot{y}=1 / 2 y
$$

Neste caso, $\gamma_{x}(t)=\sqrt{x^{2}+t}$, definida para todo $t \in \mathbb{R}$, de forma que o gráfico da ação a correspondente é fechado; de fato: $\operatorname{Gr}(a)=\left\{\left(t, x, \sqrt{x^{2}+t}\right): t \in R, x \in \mathbb{R}^{+}\right\}$, que é fechado em $\mathbb{R}^{+} \times \mathbb{P}^{+} \times \mathbb{R}^{+}$. 
Exemplo 4.58. Seja $X=\mathbb{E}^{2} \backslash\{(1,0)\}$, e consideremos sobre $X$ a equaçào diferencial:

$$
\dot{y}=(1,0)
$$

Então, $\gamma_{x}(t)=x+t(1,0)$, definida para todo $t$ se $x \neq(\lambda, 0), \forall \lambda \in \mathbb{R}$. Se $x=(\lambda, 0)$, então o intervalo de definição de $\gamma_{x}$ é $(-\infty, 1-\lambda)$ se $\lambda<1$, e $(1-\lambda, \infty)$ se $\lambda>1$. Sejam $x_{n}=\left(0, \frac{1}{n}\right)$ e $t_{n}=2, \forall n \geq 1$. Então $z_{n}:=\left(t_{n}, x_{n},\left(2, \frac{1}{n}\right)\right) \in \operatorname{Gr}(\alpha), \forall n \geq 1$, e $z_{n} \longrightarrow z$, onde $z=(2,(0,0),(2,0))$. Mas $z \notin \operatorname{Gr}(\alpha)$. porque $\gamma_{0}$ não está definida em $t=2$. Assim, vemos que $\overline{\operatorname{gr}(\alpha)} \neq \operatorname{gr}(\alpha)$.

4.2.3. Propriedades dinâmicas da ação envolvente. A seguir vemos que $\alpha$ e á possuem as mesmas propriedades dinamicas.

DEFiniçào 4.59. Seja a uma açào parcial contínua de $G$ sobre $X$. Entào dizemos que:

1. $\alpha$ é livre se o estabilizador de todo elemento é trivial.

2. $\alpha$ é transitiva se existe apenas uma órbita.

3. $\alpha$ é minimal se toda órbita é densa em $X$.

4. $\alpha$ é topologicamente livre se $\forall t \neq c$ tem-se que $F_{t}=\left\{x \in D_{t^{-1}}: a_{t}(x)=x\right\}$ tem interior vazio.

5. $\alpha$ é topologicamente transitiva se para todo par de abertos nào vazios $l:$ e $V$ de $\mathrm{X}^{\prime}$ existe $t \in G$ tal que $\alpha_{t}\left(U \cap X_{t-1}\right) \cap V \neq \emptyset$.

6. Se $G$ e $X$ são localmente compactos, a é chamada errante se $\forall K \subseteq X$ compacto tem-se que o conjunto $A(K)=\left\{s \in G: a_{s}\left(K \cap X_{s-1}\right) \cap K \neq \emptyset\right\}$ é precompacto.

7. Se $G$ e $X$ são localmente compactos, e $X$ é de Hausdorff, $\alpha$ é chamada própria se a aplicação $\left(\alpha \times i d_{X}\right): \Gamma_{\alpha} \longrightarrow X \times X$ tal que $(t, x) \longmapsto\left(\alpha_{t}(x), x\right)$ é própria (lembrar que uma função contínua $f$ é chamada própria quando $f^{-1}(K)$ é compacto, para todo compacto $K$ ).

Observação 4.60. Notar que a condiçào 6. é equivalente à seguinte propriedade: 6'. para todo par de subconjuntos compactos $K_{1}$ e $K_{2}$ de $X_{\text {, o conjunto }}$

$$
A\left(K_{1}, K_{2}\right):=\left\{s \in G: \alpha_{s}\left(K_{1} \cap X_{s-1}\right) \cap K_{2} \neq \emptyset\right\}
$$

é precompacto. Com efeito, $A\left(K^{*}\right)=A\left(K^{*}, K^{*}\right)$, e $A\left(K_{1}, K_{2}\right) \subseteq A\left(K_{1} \cup K_{2}\right)$.

Observação 4.61. Suponhamos que a é errante, e que $X$ é de Hausdorff. e sejam $K_{1}, K_{2} \subseteq X$ compactos. Então

$$
\begin{aligned}
\left(\alpha \times i d_{X^{-}}\right)^{-1}\left(K_{1} \times K_{2}\right) & =\left\{(t, x): x \in K_{2} \cap X_{t-1} \text { e } \alpha_{t}(x) \in K_{1}\right\} \\
& =\left\{(t, x): x \in \alpha_{t-1}\left(K_{1} \cap X_{t}\right) \cap K_{2}\right\} \\
& =\left\{(t, x): t \in A\left(K_{1}, K_{2}\right)^{-1} \text { e } x \in \alpha_{t-1}\left(K_{1} \cap X_{t}\right) \cap K_{2}\right\} \\
& \subseteq \overline{A\left(K_{1}, K_{2}\right)^{-1}} \times K_{2},
\end{aligned}
$$

onde $A\left(K_{1}, K_{2}\right)$ é como em 4.60 acima. Como $\alpha$ é errante e a inversão no grupo é contínua, temos que $\overline{A\left(K_{1}, K_{2}\right)^{-1}}$ é compacto, e portanto $\overline{A\left(K_{1}, K_{2}\right)^{-1}} \times K_{2}$ também é compacto. Agora, $K_{1} \times K_{2}$ é compacto no espaço de Hausdorff $X$, e portanto é fechado; como $\alpha \times i d_{X}$ é contínua, $\left(\alpha \times i d_{X}\right)^{-1}\left(K_{1} \times\right.$ $K_{2}$ ) é fechado, e daqui compacto, pois está contido no compacto $\overline{A\left(K_{1}, K_{2}\right)^{-1}} \times K_{2}$. Finalmente, se $K \subseteq X \times X$ é compacto, sempre existem $K_{1}, K_{2} \subseteq X$ compactos tais que $K \subseteq K_{1} \times K_{2}$, e portanto $\left(\alpha \times i d_{X}\right)^{-1}(K)$ é um subconjunto fechado do compacto $\left(\alpha \times i d_{X}\right)^{-1}\left(K_{1} \times K_{2}\right)$, e portanto também é compacto. Assim, vemos que uma ação parcial errante sobre um espaço de Hausdorff é própria.

Inversamente, suponhamos que a é própria, e seja $K$ um subconjunto compacto de $X$. Então $\left(\alpha \times i d_{X}\right)^{-1}(K \times K)$ é compacto. ou seja: $\left\{(t, x): t \in A(K)^{-1}\right.$ e $\left.\alpha_{t}(x) \in K^{-}\right\}$é compacto, e portanto 
também é compacta a projeção deste conjunto na primeira coordenada, que é exatamente $A\left(K^{-}\right)^{-1}$. Portanto, $A\left(K^{\circ}\right)$ é compacto, donde segue que a ação $\alpha$ é errante. Portanto. toda ação parcial própria é errante.

ProposiçÃo 4.62. Ś jam a uma ação parcial contínua do grupo topológico $G$ sobre o espaço topológico X́, e seja a a correspondente açào envolvente sobre o espaço envolvente $\dot{\mathrm{X}}$. Entào temos:

1. a é livre se e só se i éliure.

2. a é transitiva se $\epsilon$ só se i í transitiva.

3. a é topologicamente liure se e só se à é topologicamente livre.

4. $\alpha$ é topologicamente transitue se e só se $\dot{\alpha}$ é topologicamente transitiva.

5. a é minimal se ó só se $a$ i mimimal.

6. a é errante sf f só sf $i$ i cremle

7. $\alpha$ é própria e Cirr(a) é fechado sf e só se d é própria.

8. A aplicaçào quociente $i: \hat{H} / 0 \hookrightarrow \dot{X} / \dot{a}$ é um homeomorfismo.

Demonstraçáo. Seja $x \in \dot{X}$ tal que $x=\dot{\alpha}_{t}(x), x \in \mathbb{X}, t \in q_{i}$. Entào temos $G_{\dot{x}}=1 G_{x} t^{-1}$. Sejam $F_{t}^{\alpha}=\left\{x \in X: a_{t}(x)=x\right\} \rho F_{t}^{\dot{a}}=\left\{\dot{x} \in \dot{X}: \dot{\alpha}_{t}(\dot{x})=\dot{x}\right\}$. Entào é claro que $F_{t}^{\alpha}=\hat{X} \cap F_{t}^{\dot{\alpha}}$, $\forall t \in G$. Notar também que, como $\mathrm{X}$ é aberto em $\dot{X}$, o interior de um conjunto $D \subseteq \mathrm{X}$ como subconjunto de $X$ é o mesmo que o interior de $D$ como subconjunto de $\dot{X}$. Denotamos o interior de $D$ com $\operatorname{int}(D)$.

1. É imediato do fato de ser $C_{i}=t G_{r} t^{-1}$, pois o subgrupo conjugado do grupo trivial é também trivial.

2. É conseqüencia imediata da Proposiçào 4.29 .

3. Se $\dot{\alpha}$ é topologicamente livre, a também é topologicamente livre, pois $\operatorname{int}\left(F_{t}^{\alpha}\right)=\operatorname{int}\left(X \cap F_{t}^{\alpha}\right) \subseteq$ $\operatorname{int}\left(F_{t}^{\dot{\alpha}}\right)=\emptyset$. Inversamente, suponhamos que $\alpha$ é topologicamente livre. Se $\dot{x}=\dot{\alpha}_{s}(x) \in F_{t}^{\dot{a}}$. então $t \in G_{\dot{r}}=s G_{x} s^{-1}$, donde $s^{-1} t s \in G_{x}$, e portanto $x \in F_{s^{-1} / s}^{a}$. Suponhamos que $\dot{x} \in$ $\operatorname{int}\left(F_{t}^{\dot{\alpha}}\right)$ : então existe $l \subseteq X$ aberto tal que $\dot{\alpha}_{s}(U) \subseteq F_{t}^{\dot{\alpha}}$, pois $\dot{\alpha}_{s}{ }^{s} \dot{e}$ um homeomorfismo. Mas entào devemos ter que $l \subseteq F_{s^{-1} t s}^{a}$. e portanto $x \in \operatorname{int}\left(F_{s^{-1} t s}^{a}\right)=\emptyset$. o que é contraditório.

4. Suponhamos primeiro que $a$ é topológicamente transitiva. e sejam $\zeta$. $Y$ abertos não vazios de $X$. Como eles sào também abertos em $\dot{\mathrm{X}}$, existe $t \in G$ tal que $\dot{\alpha}_{t}(l) \cap V \neq \emptyset$. Por outro lado, $\dot{\alpha}_{t}(U) \cap V^{*}=\left(\dot{\alpha}_{t}\left(L^{\prime}\right) \cap Y^{\prime}\right) \cap\left(\dot{\alpha}_{t}\left(\mathrm{Y}^{*}\right) \cap \mathrm{X}^{*}\right)$, e portanto:

$$
a_{t}\left(U \cap X_{t-1}\right) \cap V=\left(\dot{a}_{t}(U) \cap V\right) \cap X_{t}=\dot{a}_{t}\left(L^{*}\right) \cap V \neq \emptyset .
$$

Na outra direçào, sejam $l, V$ abertos não vazios de $\dot{X}$. Entào existem abertos não vazios $U^{\prime}$ e $V^{\prime}$ em $X$, e $r, s \in G$, tais que $\dot{a}_{r}\left(U^{\prime}\right) \subseteq U$, e $\dot{a}_{s}\left(V^{\prime}\right) \subseteq I^{\prime}$. Como $\alpha$ é topológicamente transitiva, existe $t \in G$ tal que $a_{t}\left(U^{\prime} \cap X_{t-1}\right) \cap V^{\prime} \neq \emptyset$. Trocando $U^{\prime \prime}$ por $U^{\prime} \cap X_{t-1}$ se for necessário, podemos supor que $U^{\prime} \subseteq \mathrm{X}_{t-1}$. Agora:

$$
\dot{\alpha}_{s t r-1}(U) \cap V \supseteq \dot{\alpha}_{s t r-1}\left(\dot{\alpha}_{r}\left(U^{\prime}\right)\right) \cap \dot{\alpha}_{s}\left(V^{\prime}\right)=\dot{\alpha}_{s}\left(\dot{\alpha}_{t}\left(U^{\prime}\right) \cap V^{\prime \prime}\right)=\dot{\alpha}_{s}\left(\alpha_{t}\left(U^{\prime}\right) \cap V^{\prime}\right) \neq \emptyset .
$$

5. Evidente, pois todas as $\dot{\alpha}$-órbitas tem interseção não vazia com $\mathrm{X}$.

6. Como $X$ e $\dot{X}$ sào localmente homeomorfos, no sentido de que todo aberto de $\dot{X}$ é homeomorfo a algum aberto de $X$ e viceversa, temos que $X$ é localmente compacto se e somente se $\dot{X}$ é localmente compacto.

Suponhamos que à é errante. e seja $I \subseteq \subseteq X$ compacto. I'sando as notações da Definiçào 4.59 e a Observçào 4.60, e colocando um supraíndice que corresponda a ação envolvida, temos claramente que $A^{a}\left(K^{\circ}\right) \subseteq A^{a}\left(K^{\circ}\right)$. Como o conjunto à direita tem fecho compacto, o conjunto à esquerda também.e portanto a é errante. 
Inversamente, supondo que $\alpha$ é errante, seja $K \subseteq \dot{X}$ compacto. Como $\dot{X}$ é localmente compacto e $K$ é compacto. e como cada $\dot{\alpha}_{t}: \dot{X} \longrightarrow \dot{X}$ é um homeomorfismo, vemos que existem $K_{1}, \ldots, K_{l} \subseteq X^{*}$ compactos, e $t_{1}, \ldots, t_{l} \in G$, tais que $K \subseteq \cup_{j=1}^{l} \dot{a}_{t},\left(K_{j}\right)$. Suponhamos que $s \in$ $A^{\dot{\alpha}}(K)$; então exsitem $i, j$ tais que $s \in A^{\dot{\alpha}}\left(\dot{\alpha}_{t_{1}}\left(K_{i}\right), \dot{\alpha}_{t}\left(K_{j}\right)\right)$, ou seja, $t_{j}^{-1} s t_{i} \in A^{\dot{\alpha}}\left(K_{i}, K_{j}\right)=$

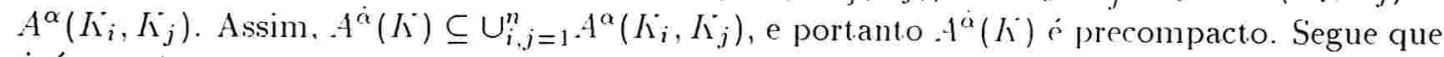
$\dot{\alpha}$ é errante.

T. Já vimos em 5 . que $\mathrm{X}$ é localmente compacto se e somente se $\dot{\mathrm{I}}$ é localmente compacto. Suponhamos que $\dot{\alpha}$ é própria. Entào ela é errante por 4.61 , e portanto a é errante pelo item acima. Como $\dot{Y}$ é de Hausdorff, $Y \dot{y}$ é de Hausdorff e $G r(a)$ deve ser fechado por 4.51. O fato de ser $X$ de Hausdorff e a errante implica que a é própria, de novo por 4.61.

Inversamente, suponhamos que $\alpha$ é própria e de gráfico fechado. ('omo ela é própria, $\dot{\alpha}$ é errante e $\mathrm{Y}$ é de Hausdorff; como $\operatorname{Gr}(\alpha)$ é fechado, $\dot{X}$ é de Hausdorff. Finalmente, como $\dot{\alpha}$ é errante sobre o Hausdorff $\dot{X}$, ela é própria.

8. Pela Proposição 4.29. sabemos que $i$ é uma bijeção. Temos o diagrama comutativo. onde $p_{\alpha} \mathrm{e}$ $p_{\dot{\alpha}}$ são as projeçòes canonicas:

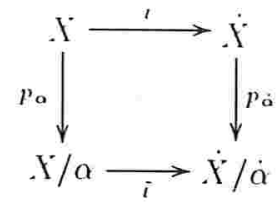

Como X/a tem a topologia quociente. i é contínua se e somente se ipa é contínua. Pela comutatividade do diagrama, isto é equivalente à continuidade de $\mu_{i} \iota$, que é contínua porque $p_{\dot{\alpha}}$ e $l$ são contínuas. Portanto $i$ é contínua. Resta mostrar que $i$ é aberı. Seja $U \subseteq \mathrm{K} / \alpha$ abert.o. Então $\tilde{\imath}(U) \subseteq \dot{\mathrm{X}} / \dot{\alpha}$ é aberto se e só se $p_{a}^{-1}(\tilde{\imath}(U))$ é aberto em $\dot{X}$. Mas $\dot{x} \in p_{a}^{-1}(i(U)) \Longleftrightarrow$ $\exists x \in X, t \in C_{i}$ tais que $p_{\alpha}(x) \in U$ e $\dot{\alpha}_{t}(x)=\dot{x}$. Portanto $p_{\dot{i}}^{-1}\left(i\left(l^{*}\right)\right)=\bigcup_{t \in G_{i}} \dot{\alpha}_{t}\left(p_{a}^{-1}\left(l^{i}\right)\right)$, que é aberto em $\dot{X}$. Concluímos assim que $i$ é um homeomorfismo. 
CAPíTULO 5

Ações Envolventes na Categoria de $C^{*}$-álgebras 
Neste capítulo consideraremos açòes parciais na categoria das $C^{*}$-álgebras, e veremos como alguns dos resultados das seçòes anteriores podem ser usados para obter conclusòes neste contexto. Mostraremos que a ação envolvente de uma ação parcial sobre uma $C^{*}$-álgebra é única a menos de isomorfismos, quando ela existir. Neste caso, veremos também que a álgebra e sua álgebra envolvente compartilham várias propriedades, como nuclearidade, ser CCR, GCR, etc.

Precisamos lembrar a definiçào de ação parcial contínua sobre uma $C^{*}$-álgebra.

Definição 5.1. Sejam $E$ um espaço de Banach, $X$ um espaço topológico e, para cada $x \in X$. $E_{x}$ um subespaço de Banach de $E$. Dizemos que $\left\{E_{x}\right\}_{x \in X}$ é uma família contínua se $\forall U \subseteq E$ aberto. o conjunto $\left\{x \in X: I^{\prime} \cap E_{r} \neq \emptyset\right\}$ é aberto em $X^{\prime}$ (ver [27]).

ObSERVAÇÃo 5.2. Seja $\mathcal{E}=\left\{(x, v) \in X \times E: v \in E_{x}\right\}$, seja $\pi: \mathcal{E} \longrightarrow \mathrm{X}$ a projeção natural. Então $\left\{E_{x}\right\}_{x \in X}$ é contínua se e somente se $\pi$ é aberta.

DEFiniÇÃo 5.3. Sejam $G$ um grupo localmente compacto, e $\alpha=\left(\left\{D_{t}\right\}_{t \in G},\left\{a_{t}\right\}_{t \in G}\right)$ uma açào parcial de $G$ sobre a ("*álgebra $A$. Sejam

$$
\mathcal{B}^{-1}=\left\{(t, b) \in G \times B: b \in D_{t-1}\right\}, \text { e } \mathcal{B}=\left\{(t, b) \in G \times B: b \in D_{t}\right\} .
$$

Dizemos que a é contínua se a família $\left\{D_{t}\right\}_{t \in G}$ é contínua e a aplicação (também chamada) $\alpha$ : $\mathcal{B}^{-1} \longrightarrow \mathcal{B}$ tal que $(t . b) \longmapsto\left(t, \alpha_{t}(b)\right)$ é contínua (notar que sendo $\left\{D_{t}\right\}_{t \in G}$ contínua, $\mathcal{B}$ e $\mathcal{B}^{-1}$ são fibrados de Banach).

Em [27], Exel mostra que se $\alpha=\left(\left\{D_{t}\right\}_{t \in G},\left\{\alpha_{t}\right\}_{t \in G}\right)$ é uma ação parcial de $G$ sobre a $C^{*}$-álgebra $A$, o fibrado de Banach $\mathcal{B}_{\mathrm{a}}=\left\{(t, x): x \in D_{t}\right\} \subseteq G \times A$, com a topologia relativa, é um fibrado de Fell com as seguintes operaçōes (onde $x_{t} \delta_{t}:=\left(t, x_{t}\right) \in \mathcal{B}_{\alpha}$ ):

- $\left(x_{t} \delta_{t}\right) *\left(x_{s} \delta_{s}\right)=a_{t}\left(a_{t}^{-1}\left(x_{t}\right) x_{s}\right) \delta_{t s}$.

- $\left(x_{t} \delta_{t}\right)^{*}=a_{t}^{-1}\left(x_{t}^{*}\right) \delta_{t-1}$.

$\mathcal{B}_{\alpha}$ se chama produto semidireto de $A$ e $G$. O produto cruzado de $A$ por $G$ segundo a ação parcial a é definido como:

$$
A \rtimes_{\alpha} G:=C^{*}\left(\mathcal{B}_{\alpha}\right)
$$

e o produto cruzado reduzido como:

$$
A \rtimes_{\alpha, r} G:=C_{r}^{*}\left(\mathcal{B}_{\alpha}\right)
$$

Para motivar a definição de ação envolvente de uma ação parcial sobre uma $C^{*}$-álgebra que daremos a seguir, consideremos primeiro o caso comutativo. Suponhamos então que $A=C_{0}(X)$ é uma $C^{*}$-álgebra comutativa, e que $a=\left(\left\{D_{t}\right\}_{t \in G},\left\{\alpha_{t}\right\}_{t \in G}\right)$ é uma ação parcial contínua do grupo $G$ sobre $A$. Então existe uma única ação parcial contínua $\alpha=\left(\left\{X_{t}\right\}_{t \in G},\left\{\alpha_{t}\right\}_{t \in G}\right)$ sobre $X$, tal que $D_{t}=C_{0}\left(X_{t}\right)$. e $\alpha_{t}(a)=a \circ \alpha_{t^{-1}}, \forall t \in G, a \in D_{t^{-1}}$. Suponhamos que $\operatorname{Gr}(\dot{\alpha})$ é fechado em $G \times X \times X$. Então o espaço envolvente $\dot{X}$ de $X$ é de Hausdorff pela Proposição 4.51. Seja $B=C_{0}(\dot{X})$, e seja $\dot{a}$ a ação sobre $B$ induzida pela açào envolvente $\dot{\alpha}$ de $\alpha$, ou seja, $\dot{\alpha}_{t}(b)=b \circ \dot{\alpha}_{t^{-1}}, \forall b \in B$. Como $X \dot{e}$ aberto em $\dot{X}$. temos que $A$ é um ideal bilateral fechado em $B$, e é imediato ver que $\alpha=\left.\dot{\alpha}\right|_{A}$. Por 
outro lado, do teorema de Stone-Weierstrass deduzimos que $\operatorname{span}\left\{\dot{\alpha}_{t}(a): a \in A, t \in G\right\}$ é denso en B.

Em geral, se $V$ é um espaço vetorial onde um grupo $G$ está agindo através de uma ação $\gamma$, e se $S \subseteq V$, indicaremos por $\gamma(S)$ a órbita de $S: \gamma(S):=\left\{\gamma_{t}(s): s \in S, t \in G\right\}$. e por $[\gamma(S)]$ a órbita linear de $S:[\gamma(S)]:=\operatorname{span}\left\{\gamma_{t}(s): s \in S, t \in G\right\}$. Se $V$ tem topologia, diremos que $\overline{[\gamma(S)]}$ é a órbita linear fechada de $S$. Com esta notaçào introduzimos a seguinte definiçào:

DEFINIÇÃo 5.4. Seja $n=\left(\left\{D_{t}\right\}_{t \in G} \cdot\left\{\alpha_{t}\right\}_{t \in G}\right)$ uma ação parcial contínua de um grupo topológico $G$ sobre uma $C^{*}$-álgebra $A$, e seja $\beta$ uma ação contínua de $G$ sobre uma $C^{*}$-álgebra $B$ que contém $A$. Diremos que $(\beta, B)$ é uma açào envolvente de $(\alpha, A)$ (na categoria de $C^{*}$-álgebras), se as seguintes trés propriedades sào satisfeitas:

1. $A$ é um ideal bilateral fechado de $B$.

2. $\alpha=\left.\beta\right|_{A}$.

3. $[\alpha(A)]$ é denso em $B$ (Ver a notação acima).

Diremos que $B$ é uma $C^{\prime *}$-álgebra envolvente.

Veremos que, a diferença do que acontece com as ações parciais sobre conjuntos e sobre espaços topológicos, nem sempre haverá ação envolvente de uma ação parcial na categoria das $C^{*}$-álgebras. Porém, se existir, ela é (essencialmente) única.

A Definição 5.4 sugere imediatamente a pergunta seguinte: se $\beta$ é uma açào contínua de um grupo $G$ sobre uma $C^{*}$-álgebra $B$, e $A$ é um ideal bilateral fechado de $B$, então $a:=\left.B\right|_{A}$ é uma ação parcial contínua? Se o grupo $G$ é discreto é fácil ver que sim, mas se o grupo nào for discreto, algumas verificações menos imediatas são necessárias. É estas verificações que faremos a seguir.

DEFiniçÃo 5.5. Seja $\beta=\left(\left\{D_{t}^{\beta}\right\}_{t \in G},\left\{\beta_{t}\right\}_{t \in G}\right)$ uma ação parcial do grupo discreto $G$ sobre a $C^{*}$ álgebra $A$, e seja $I$ um ideal de $A$. Definimos a restrição $\left.\beta\right|_{I}$ de $\beta$ a $I$ como $\alpha=\left(\left\{D_{t}^{\alpha}\right\}_{t \in G},\left\{\alpha_{t}\right\}_{t \in G}\right)$, onde:

- $D_{t}^{\alpha}=\left(I \cap D_{t}^{\beta}\right) \cap \beta_{t}\left(I \cap D_{t-1}^{\beta}\right)$.

- $\alpha_{t}(x)=\beta_{t}(x), \forall i r \in D_{t}^{\alpha}$.

É claro que $\alpha$ é uma ação parcial sobre $I$.

Queremos ver que se $\beta$ é contínua, então $\alpha$ também é contínua. Este fato é uma conseqüencia direta do seguinte lema.

LEMA 5.6. Sejam E E $E^{\prime}$ dois espaços de Banach, $G$ um espaço topológico localmente compacto, $\left\{E_{t}\right\}_{t \in G},\left\{E_{t}^{\prime}\right\}_{t \in G}$ famílias contínuas, $\mathcal{E}=\left\{(t, x) \in G \times E: x \in E_{t}\right\}, \mathcal{E}^{\prime}=\left\{(t . x) \in G \times E^{\prime}: x \in E_{t}^{\prime}\right\}$ os correspondentes fibrados de Banach, $\in \beta: \mathcal{E} \longrightarrow \mathcal{E}^{\prime}$ contínua, tal que $\beta(t, x)=\left(t, \beta_{t}(x)\right), \forall(t, x) \in \mathcal{E}$. Tem-se:

1. Se $\xi: G \longrightarrow \mathcal{E}$ é uma seção contínua. então $\beta \xi$ é uma seção contínua de $\mathcal{E}^{\prime}$

2. Se $F_{t}^{\prime}=\beta_{t}\left(E_{t}\right)$, então $\left\{F_{t}^{\prime}\right\}_{t \in G}$ é uma família contínua.

3. Se $E=E^{\prime}$ é uma $C^{*}$-álgebra, e cada $E_{t}$, $E_{t}^{\prime}$ é um ideal bilateral fechado de $E$, então $\left\{E_{t} \cap\right.$ $\left.E_{t}^{\prime}\right\}_{t \in G}$ é uma família contínua.

Demonstração. 1. Se $\xi$ é seção de $\mathcal{E}$, é claro que $\beta \xi$ é seção de $\mathcal{E}^{\prime}$ : se $\xi$ é contínua, então $\beta \xi$ também é contínua. 
2. Sejam $t_{0} \in G, x_{0}^{\prime} \in F_{t_{0}}^{\prime}$, tais que $x_{0}^{\prime}=\beta_{t_{0}}\left(x_{0}\right)$, para algum $x_{0} \in E_{t_{0}}$. Como $G$ é localmente compacto, existe $\xi: G^{\prime} \longrightarrow \mathcal{E}$, seção contínua, tal que $\xi\left(t_{0}\right)=x_{0}$. Então $\beta \xi$ é uma seção contínua de $\left\{F_{t}^{\prime}\right\}$ tal que $\beta \xi\left(t_{0}\right)=\left(t_{0}, \beta_{t_{0}}\left(x_{0}\right)\right)$. Então $\left\{F_{t}^{\prime}\right\}$ é uma família contínua, por [27], Proposition 3.3 .

3. Seja $x \in E_{t} \cap E_{t}^{\prime}$. Pelo teorema de Cohen-Hewitt, existem $y \in E_{t}, z \in E_{t}^{\prime}$, tais que $x=y z$. Como $G$ é localmente compacto, existem seçōes $\xi$ de $\mathcal{E}$ e $\xi^{\prime}$ de $\mathcal{E}^{\prime}$ tais que $\xi(t)=y, \xi^{\prime}(t)=z$. Como $E_{t}, E_{t}^{\prime}$ são ideais bilaterais, temos $E_{t} E_{t}^{\prime}=\subseteq E_{t} \cap E_{t}^{\prime}$, e portanto $\eta$ tal que $\eta(s)=\xi(s) \xi^{\prime}(s)$ é uma seçào contínuauma seção do fibrado correspondente à família $\left\{E_{t} \cap E_{t}^{\prime}\right\}_{t \in G}$. Como $\eta(t)=\xi(t) \xi^{\prime}(t)=y z=r$. temos que $\left\{E_{t} \cap E_{t}^{\prime}\right\}_{t \in G}$ é uma família contínua, de novo por [27], 3.3 .

Proposição 5.7. Ś jam $\beta=\left(\left\{D_{t}^{\beta}\right\}_{t \in G},\left\{\beta_{t}\right\}_{t \in G}\right)$ uma açcio parcial contínua do grupo $G$ sobre a $C^{*}$-álgebra $A, I$ um ideal de $A$. e $a=\left.\beta\right|_{I}=\left(\left\{D_{t}^{a}\right\}_{t \in G},\left\{\alpha_{t}\right\}_{t \in G}\right)$ a restriçào de $\beta$ ao ideal I. Então $B_{a}$ é um subfibrado de Fell de $B_{\beta}$. $\epsilon$ o é uma ação parcial contínua.

DemonstraÇào. $\left\{D_{t}^{a}\right\}$ é uma família contínua: lembrar que $D_{t}^{a}=\left(I \cap D_{t}^{\beta}\right) \cap \beta_{t}\left(I \cap D_{t-1}^{\beta}\right)$; por 3. do Lema 5.6, $\left\{I \cap D_{t}^{\beta}\right\}$ e $\left\{I \cap D_{t-1}^{\beta}\right\}$ sào famílias contínuas, e por 2 . $\left\{\beta_{t}\left(I \cap D_{t-1}^{\beta}\right)\right\}$ é contínua; então $\left\{D_{t}^{\alpha}\right\}$ é contínua. usando de novo 3 . Em particular, o fibrado de Banach $\mathcal{B}_{\alpha}$ é um subfibrado de Banach de $\mathcal{B}_{\beta}$, e como a é a restriçào de $\beta$. também o produto e a involução de $\mathcal{B}_{\alpha}$ são as restriçōes das correspondentes operaçōes sobre $\mathcal{B}_{\beta}$. A contimuidade da aplicação a é automática, pois $\alpha$ é a restrição da aplicaçào contínua $\beta$ ao subfibrado $\mathcal{B}_{\alpha}$ correspondente a $\alpha$.

ProposiçÃo 5.8. Seja $\alpha=\left(\left\{D_{t}\right\}_{t \in G},\left\{\alpha_{t}\right\}_{t \in G}\right)$ uma açào parcial contínua de um grupo topológico $G$ sobre uma ("álgebra abeliana $A$, e seja $\alpha$ a correspondente ação parcial de $G$ sobre A. Então as condiçòes seguintes são equivalentes:

1. $\operatorname{Gr}(\hat{\alpha})$ é fechado $\mathrm{em} G \times A \times A$.

2. a possui açào envolvente na categoria de $C^{*}$-álgebras comutativas.

3. $\alpha$ possui açào envolvente na categoria de $C^{*}$-álgebras.

Demonstração. É claro que 2. implica 3., e 1. e 2. são equivalentes pela Proposição 4.51, devido à equivalencia entre as categorias de espaços de Hausdorff localmente compactos e de $C^{*}$ álgebras comutativas. Basta portanto mostrar que 3 . implica 2 . Suponhamos então que $(\beta, B)$ é uma ação envolvente da ação parcial a sobre a $C^{*}$-álgebra abeliana $A$. Como $A$ é um ideal comutativo de $B$, então $A$ está contido no centro $Z(B)$ de $B$ : sejam $a \in A, b \in B$, e $\left(\epsilon_{i}\right)_{i \in I} \subseteq A$ uma unidade aproximada de $A$; então:

$$
a b=\lim _{i}(a b) \epsilon_{i}=\lim _{i} a\left(b e_{i}\right)=\lim _{i}\left(b e_{i}\right) a=\lim _{i} b\left(e_{i} a\right)=b a .
$$

Como $Z(B)$ é invariante, temos que $\beta_{t}(A) \subseteq Z(B) . \forall t \in G$, e portanto $[\beta(A)]=\operatorname{span}\left\{\gamma_{t}(a): t \in\right.$ $G, a \in A\} \subseteq Z(B)$. Mas $B=\overline{[\beta(A)]} \subseteq Z(B) \subseteq B$, e concluímos que $B=Z(B)$, ou seja, $B$ é abeliana. 


\subsection{Sobre a unicidade da ação envolvente}

A Proposição 5.8 mostra, junto com os exemplos 4.50, 4.54 e 4.58 , que nem sempre existe ação envolvente no sentido de $C^{*}$-álgebras. Nesta seção mostraremos que, pelo menos, teremos unicidade da ação envolvente quando ela existir. Notar que já sabemos isso no caso comutativo.

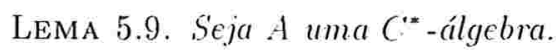

1. Seja $J \triangleleft A \in$ definamos $\|\cdot\| J: A \longrightarrow \mathbb{P}$, tal que $\|a\|_{J}=\sup \{\|a x\|: x \in J,\|x\| \leq 1\}$.

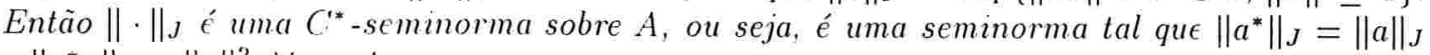
$e\left\|a^{*} a\right\|_{J}=\|a\|^{2}, \forall a \in A$.

2. Sejam $\mathcal{T}$ uma família de ideais de $A, I=\overline{\operatorname{span}}\{J: J \in \mathcal{J}\}, \epsilon\|\cdot\|_{\mathcal{J}}: A \longrightarrow \mathbb{R}$ tal que $\|a\|_{\mathcal{T}}=\sup _{J \in \mathcal{T}}\|a\|_{J}$. Entĩo $\|\cdot\|_{\mathcal{J}}$ é uma $C^{*}$-seminorma sobre $A$, é é uma norma se $\epsilon$ somente se I f́ um ideal essencial de A. Neste caso, tem-se que $\|\cdot\|_{\mathcal{J}}=\|\cdot\|$.

Demonstraçào. É claro que $\|\cdot\|_{J}$ é uma seminorma. Sejam $x \in J, \operatorname{com}\|x\| \leq 1, a, b \in A$. Então, se $b x \neq 0$ :

- $\|a b x\|=\|b x\|\left\|a \frac{b x}{\|b x\|}\right\| \leq\|b\|_{J}\|a\|_{J}$, e portanto $\|a b\|_{J} \leq\|a\|_{J}\|b\|_{J}$.

- $\|a x\|^{2}=\left\|x^{*} a^{*} a x\right\| \leq\left\|a^{*} a x\right\| \leq\left\|a^{*} a\right\|_{J} \leq\left\|a^{*}\right\|\left\|_{J}\right\| a \|_{J}$, donde segue que $\left\|a^{*}\right\|_{J}=\|a\|_{J} \mathrm{e}$ $\|a\|_{J}^{2}=\left\|a^{*} a\right\|_{J}$.

Assim, vemos que $\|\cdot\|_{J}$ é uma $C^{* *}$-seminorma sobre $A$. Como o supremo de $C^{*}$-seminormas é uma $C^{*}$-seminorma, temos também que $\|\cdot\|_{\mathcal{J}}$ é uma $C^{*}$-seminorma sobre $A$. Agora temos:

$$
\begin{aligned}
\|\cdot\|_{\mathcal{J}} \text { é uma } C^{*} \text {-norma } & \Longleftrightarrow\|a\|_{\mathcal{J}}=0 \text { implica que } a=0 \\
& \Longleftrightarrow a x=0 \forall x \in J \text { e } \forall J \in \mathcal{J} \text { implica que } a=0 \\
& \Longleftrightarrow a I=0 \text { implica que } a=0 \\
& \Longleftrightarrow I \text { é um ideal essencial em } A
\end{aligned}
$$

Finalmente, suponhamos que $\|\cdot\|_{\mathcal{I}}$ é uma $C^{*}$-norma sobre $A$, e seja $A_{\mathcal{J}}$ o correspondente completamento de $A$. Seja $\pi: A_{\mathcal{J}} \longrightarrow B(H)$ uma representação fiel de $A_{\mathcal{J}}$; como $\pi$ é fiel, tem-se que $\|a\|_{\mathcal{T}}=\|\pi(a)\|, \forall a \in A_{\mathcal{J}}$. Por outro lado, como $A$ está contido em $A_{\mathcal{J}}$, a restrição de $\pi$ a $A$ é uma representação fiel de $A$, e portanto temos que $\|a\|=\|\pi(a)\|, \forall a \in A$, donde segue que $\|\cdot\|_{\mathcal{J}}=\|\cdot\|$.

Lema 5.10. Seja $(\alpha, A)$ uma ação parcial de $G$, e suponhamos que $(\beta, B)$ e $(\hat{i}, C)$ são ações envolventes de $\alpha$. Então, $\forall a, b \in A, t \in G$, tem-se: $\beta_{t}(a) b=\gamma_{t}(a) b$ (notar que cada um destes produtos é um elemento de $\left.D_{t}\right)$.

Demonstração. Seja $\left(u_{i}\right)$ uma unidade aproximada de $D_{t^{-1}}$. Então $u_{i} a \in D_{t^{-1}}$. $\forall i$, e como $\alpha$ é a restrição a $A$ de $\beta$ e também de $\gamma$ temos:

$$
\beta_{t}(a) b=\lim \beta_{t}\left(u_{i} a\right) b=\lim \alpha_{t}\left(u_{i} a\right) b=\lim \gamma_{t}\left(u_{i} a\right) b=\gamma_{t}(a) b .
$$

Teorema 5.11. Seja $(\alpha, A)$ uma ação parcial de $G$, e suponhamos que $(\beta, B) \in(\hat{i}, C)$ são ações envolventes de $\alpha$. Então existe um único isomorfismo $\phi: B \longrightarrow C$ tal que $\phi \beta_{t}=\hat{i}_{i t} \phi, \forall t \in G, \epsilon$ $\left.o\right|_{A}=i d_{A}$. 
DemonstraçÃo. Para $s \in G$, sejam $\|\cdot\|_{s}: B \longrightarrow \mathbb{R}$ e $\|\cdot\|^{s}: C \longrightarrow \mathbb{R}$ dadas por $\|b\|_{s}:=$ $\sup \left\{\|b x\|: x \in \beta_{s}(A),\|x\| \leq 1\right\}$ e $\|c\|^{s}:=\sup \left\{\|c y\|: y \in \gamma_{s}(A),\|y\| \leq 1\right\}$. Pelo Lema 5.9 , $\|\cdot\|_{s}$ e $\|\cdot\|^{s}$ são $C^{*}$-seminormas, e $\|\cdot\|_{B}=\sup _{s}\|\cdot\|_{s},\|\cdot\|_{C}=\sup _{s}\|\cdot\|^{s}$.

Sejam $t_{1}, \ldots, t_{n} \in G$. e $a_{1} \ldots a_{n} \in A$. Queremos ver que $\left\|\sum_{i} \beta_{t_{i}}\left(a_{i}\right)\right\|_{B}=\left\|\sum_{i} \gamma_{t_{i}}\left(a_{i}\right)\right\|_{C}$. Para isto, basta mostrar que $\left\|\sum_{i} \beta_{l_{1}}\left(a_{i}\right)\right\|_{s}=\left\|\sum_{i} \gamma_{t_{1}}\left(a_{i}\right)\right\|^{s}, \forall s \in G$. Sejam $s \in G$ e $a \in A$. Usando o Lema 5.10 temos:

$$
\left\|\sum_{i} \beta_{t_{i}}\left(a_{i}\right) \beta_{s}(a)\right\|=\left\|\beta_{s}\left(\sum_{i} \beta_{s^{-1} t_{1}}\left(a_{i}\right) a\right)\right\|=\left\|\gamma_{s}\left(\sum_{i} \gamma_{s-1},\left(a_{i}\right) a\right)\right\|=\left\|\sum_{i} \gamma_{t_{i}}\left(a_{i}\right) \gamma_{s}(a)\right\| .
$$

Portanto $\left\|\sum_{i} \beta_{t_{1}}\left(a_{i}\right)\right\|_{s}=\left\|\sum_{i} \gamma_{t_{t}}\left(a_{i}\right)\right\|^{s}, \forall s \in G$, e então $\left\|\sum_{i} \beta_{t_{1}}\left(a_{i}\right)\right\|_{B}=\left\|\sum_{i} \gamma_{t_{1}}\left(a_{i}\right)\right\|_{C}$. Assim, $\phi:[\beta(A)] \longrightarrow[\gamma(A)]$ tal que $\phi\left(\sum_{i} \beta_{t_{1}}\left(a_{i}\right)\right)=\sum_{i} \gamma_{t_{i}}\left(a_{i}\right)$ é uma isometria de um *-ideal denso de $B$ sobre um *-ideal denso de $C$. e portanto se estende por continuidade a um isomorfismo $\phi: B \longrightarrow C$, que claramente satisfaz $\jmath_{t} O=\phi \beta_{t} . \forall t \in G$, e $\left.\phi\right|_{A}=i d_{A}$; além dist.o, é claro que estas duas condições determinam unicamente a 0 .

\subsection{Ação parcial sobre o espectro e o espaço de ideais primitivos}

Queremos ver a seguir como se manifesta a presença de uma ação parcial a sobre uma $C^{*}$-álgebra $A$ no espectro de $A$ e no espaço de ideais primitivos de $A$.

Será conveniente relembrar e resumir os fatos mais importantes relativos ao espectro e ao espaço de ideais primitivos. Para mais detalhes, consultar por exemplo [57], Apendice A.

\section{$\S 10$ espaço de ideais primitivos}

O núcleo de uma representação irredutível de $A$ é chamado primitivo, e o conjunto de tais ideais é denotado por $\operatorname{Prim}(A)$. Todo ideal $I$ de $A$ é a interseção dos ideais primitivos que o contem. Além disso, se $P \in \operatorname{Prim}(A)$ é tal que $P \supseteq I \cap J$, onde $I$ e $J$ são ideais de $A$, tem-se que $P \supseteq I$ ou $P \supseteq J$.

Para cada ideal $I$ de $A$ consideremos o conjunto $\mathcal{O}_{I}=\{P \triangleleft A: P$ é primitivo e $P \nsupseteq I\}$. Então $\left\{\mathcal{O}_{I}: I \triangleleft A\right\}$ é uma topologia sobre o conjunto Prim $(A)$, conhecida como topologia de Jacobson (também "hull-kernel"), e Prim(A) com a topologia de Jacobson é chamado espaço de ideais primitivos de $A$. A correspondència $I \longmapsto \mathcal{O}_{I}$ é uma bijeção, e $\mathcal{O}_{I} \cap \mathcal{O}_{J}=\mathcal{O}_{I \cap J}$.

Suponhamos que $I \triangleleft A$, e seja $r: \mathcal{O}_{I} \longrightarrow \operatorname{Prim}(I)$ a aplicaçào restrição, ou seja: $r(P)=P \cap I$. Então $r$ é um homeomorfismo. Em particular, para cada $Q \in \operatorname{Prim}(I)$ existe um único ideal primitivo $P$ de $A$ tal que $P \cap I=Q$.

Se $\phi: A \longrightarrow A^{\prime}$ é um isomorfismo de $C^{*}$-álgebras, ele induz um homeomorfismo $\hat{\phi}: \operatorname{Prim}(A) \longrightarrow$ $\operatorname{Prim}\left(A^{\prime}\right)$, definido por $\dot{\phi}(P)=\phi(P)$.

\section{$\$ 20$ espectro de $A$}

$\mathrm{O}$ espectro de $A$ está constituído pelas classes de equivalencia unitária de representações irredutíveis de $A$, e é simbolizado por $A$.

Existe uma aplicação natural $\kappa: \dot{A} \longrightarrow \operatorname{Prim}(A)$, dada por $\kappa([\pi])=k e r \pi$, que é sobrejetiva mas em geral não é injetiva.

Sobre $\hat{A}$ se considera a topologia inicial definida por $\kappa$. Com esta topologia, $\hat{A}$ é localmente compacto, e é compacto se $A$ tem unidade ([37], VII-6.11). Assim, os abertos de $\hat{A}$ são da forma $\mathcal{V}_{I}=\kappa^{-1}\left(\mathcal{O}_{I}\right)$. De novo, portanto, estào parametrizados pelos ideais de $A$.

Suponhamos que $I \triangleleft A$. Então temos uma aplicação restrição $r: \mathcal{V}_{I} \longrightarrow I$, tal que $r([\pi])=\left[\left.\pi\right|_{I}\right]$, e $r$ é um homeomorfismo. 
O diagrama abaixo comuta, para todo ideal $I$ de $A$ :

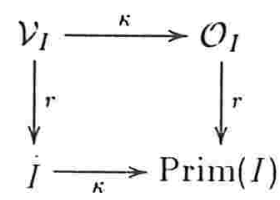

Finalmente, se $0: A \longrightarrow A^{\prime}$ ć um isomorfismo, temos um homeomorfismo $\hat{\phi}: \hat{A} \longrightarrow \hat{A}^{\prime}$ tal que $[\pi] \longmapsto\left[\pi \circ \phi^{-1}\right]$, e o diagrama seguinte comuta:

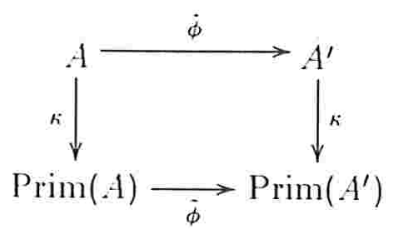

Temos o seguinte resultado:

Proposiçào 5.12. Ścja B : $G \times B \longrightarrow B$ uma açào contínua do grupo localmente compacto $G$ sobre a $C^{*}$-álgebra $B$. Então:

1. $\hat{\beta}: G \times B \longrightarrow B$ tal que $\beta_{t}([\pi])=\left[\pi \circ \beta_{t^{-1}}\right]$ é uma ação contínua.

2. $\tilde{\beta}: G \times \operatorname{Prim}(B) \longrightarrow \operatorname{Prim}(B)$ tal que $\tilde{\beta}_{t}(P)=\beta_{t}(P)$ é uma açào contínua.

Demonstração. Uma demonstração de 1. pode ser achada em [57], 7.1.

Para provar 2., notemos que temos o seguinte diagrama comutativo:

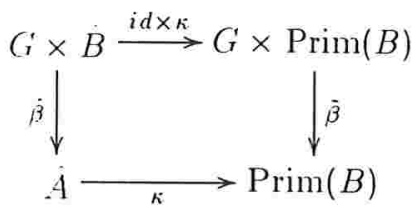

Como $\hat{B}$ tem a topologia inicial induzida da topologia de $\operatorname{Prim}(B)$ através de $\kappa$, então $\operatorname{Prim}(B)$ é precisamente o espaço topológico quociente de $\hat{B}$ com respeito a $\kappa$, e $G \times \operatorname{Prim}(B)$ é o quociente de $G \times \hat{B}$ com respeito a $i d \times \kappa$. Portanto, $\tilde{\beta}$ é contínua se e somente se $\dot{\beta}(i d \times \kappa)$ é contínua; mas $\tilde{\beta}(i d \times \kappa)=\kappa \hat{\beta}$. e $\kappa \dot{3}$ é contínua.

Veremos a seguir que se $\alpha$ é uma ação parcial de um grupo discreto sobre a $C^{*}$-álgebra $A$, então $\alpha$ induz ações parciais contínuas sobre $\operatorname{Prim}(A)$ e $\hat{A}$. Mais tarde veremos que este resultado é válido também para grupos nào discretos.

Proposiçào 5.13. Seja $a=\left(\left\{D_{t}\right\}_{t \in G}, a_{t t \in G}\right)$ uma ação parcial do grupo discreto $G$ sobre a $C^{*}$-álgebra A. Para cada $t \in G$. seja $\mathcal{O}_{t}=\mathcal{O}_{D_{t}}=\left\{P \in \operatorname{Prim}(A): P \nsupseteq D_{t}\right\}$. Então $\tilde{\alpha}=$ $\left(\left\{\mathcal{O}_{t}\right\}_{t \in G},\left\{\tilde{\alpha}_{t}\right\}_{t \in G}\right)$ é uma açìo parcial contínua de $G$ sobre $\operatorname{Prim}(A)$. onde $\tilde{\alpha}_{t}(P)$ está definido como sendo o único ideal primitivo de $A$ tal que $\dot{\alpha}_{t}(P) \cap D_{t}=\alpha_{t}\left(P \cap D_{t-1}\right)$. 
Demonstração. Da própria definição, temos que $\mathcal{O}_{e}=\mathcal{O}_{A}=\operatorname{Prim}(A)$, e $\tilde{\alpha}_{e}=i d$. Por outro lado, a definição de $\tilde{a}_{t}$ implica que o diagrama seguinte é comutativo, onde a flecha horizontal superior está induzida pelo isomorfismo $\alpha_{t}: D_{t^{-1}} \longrightarrow D_{t}$, ou seja, $\alpha_{t}^{*}(P)=\alpha_{t}(P)$, e as verticais sào as aplicações de restrição. Como cada $r$ e $\alpha_{t}^{*}$ são homeomorfismos, temos que $\bar{\alpha}_{t}: \mathcal{O}_{t-1} \longrightarrow \mathcal{O}_{t}$ é um homeomorfismo, e é claro que sua inversa é $\tilde{\alpha}_{t-1}$.

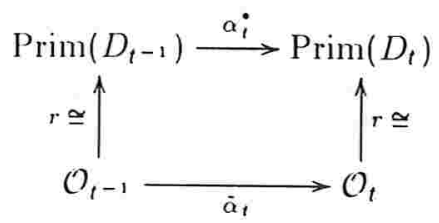

Temos que provar que $\tilde{\alpha}_{t s}$ é uma extensão de $\tilde{\alpha}_{t} \tilde{\alpha}_{s}$.

A primeira coisa que verificamos ć que $\operatorname{dom}\left(\bar{\alpha}_{t} \bar{\alpha}_{s}\right) \subseteq \operatorname{dom}\left(\bar{\alpha}_{t s}\right)=\mathcal{O}_{s^{-1} t-1}$. Suponhamos que $P \in$ $\operatorname{dom}\left(\tilde{\alpha}_{t} \tilde{\alpha}_{s}\right)$. Entào $P \in \mathcal{C}_{s^{-1}}$, e $\tilde{\alpha}_{s}(P) \in \mathcal{O}_{t-1}$. Suponhamos que $P \notin \mathcal{O}_{s-1}-1$, ou seja, suponhamos que $P \supseteq D_{s^{-1} t^{-1}}$; em particular, $P \cap D_{s^{-1}} \supseteq D_{s^{-1} t_{-1}} \cap D_{s^{-1}}$. Como $P \cap D_{s^{-1}} \in \operatorname{Prim}\left(D_{s^{-1}}\right)$. $\alpha_{s}\left(P \cap D_{s-1}\right) \in \operatorname{Prim}\left(D_{s}\right)$, e entào $\alpha_{s}(P) \cap D_{s-1}=\alpha_{s}\left(P \cap D_{s^{-1}}\right) \supseteq a_{s}\left(D_{s^{-1},-1} \cap D_{s^{-1}}\right)=D_{t-1} \cap D_{s}$. Portanto, $\tilde{\alpha}_{s}(P) \supseteq D_{t-1} \cap D_{s}$, e como $\dot{\alpha}_{s}(P)$ é um ideal primitivo, deve ser $\dot{\alpha}_{s}(P) \supseteq D_{t-1}$ ou $\tilde{\alpha}_{s}(P) \supseteq D_{s}$, isto é: ou $\bar{\alpha}_{s}(P) \notin \mathcal{O}_{t_{-1}}$ ou $\tilde{\alpha}_{s}(P) \notin \mathcal{O}_{s}$. Esta é uma contradiçào, que surgiu de supor que $P \notin \mathcal{O}_{s^{-1} t^{-1}}$; portanto $P \in \mathcal{O}_{s^{-1} t-1}$.

Vejamos agora que $\bar{\alpha}_{t} \bar{\alpha}_{s}(P)=\bar{\alpha}_{t s}(P) . \forall P \in \operatorname{dom}\left(\tilde{\alpha}_{t} \tilde{\alpha}_{s}\right)$. Como $P \nsupseteq D_{s^{-1}} \cap D_{s^{-1} t^{-1}}$, então $\bar{\alpha}_{t} \bar{\alpha}_{s}(P) \nsupseteq D_{t} \cap D_{t s}$. Portanto. para ver que $\bar{\alpha}_{t} \bar{\alpha}_{s}(P)=\bar{\alpha}_{t s}(P)$, basta mostrar que suas restriçòes a $D_{t} \cap D_{t s}$ coincidem. Agora:

$$
\begin{aligned}
& \tilde{\alpha}_{t} \tilde{\alpha}_{s}(P) \cap D_{t} \cap D_{t s}=\dot{\alpha}_{t}\left(\bar{\alpha}_{s}(P)\right) \cap a\left(D_{s} \cap D_{t-1}\right) \text { porque a é ação parcial } \\
& =\alpha_{t}\left(\dot{a}_{s}(P) \cap D_{t-1}\right) \cap \alpha\left(D_{s} \cap D_{t-1}\right) \text { pela definição de } \dot{a}_{t} \\
& =\alpha_{t}\left(\dot{\alpha}_{s}(P) \cap D_{t-1} \cap D_{s}\right) \\
& =\alpha_{t}\left(\alpha_{s}\left(P \cap D_{s-1}\right) \cap D_{t-1} \cap D_{s}\right) \text { pela definiçào de } \tilde{a}_{s} \\
& =\alpha_{t}\left(\alpha_{s}\left(P \cap D_{s-1}\right) \cap a_{s}\left(D_{s^{-1} t_{-1}} \cap D_{s^{-1}}\right)\right) \text { porque a é açào parcial } \\
& =a_{t}\left(a_{s}\left(P \cap D_{s^{-1}} \cap D_{s^{-1}} t^{-1}\right)\right) \\
& =\alpha_{t} \alpha_{s}\left(P \cap D_{s-1} \cap D_{s-1} t-1\right) \\
& =\alpha_{t} \alpha_{s}\left(\left(P \cap D_{s^{-1} t^{-1}}\right) \cap\left(D_{s^{-1}} \cap D_{s^{-1} t^{-1}}\right)\right) \\
& =a_{t s}\left(P \cap D_{s^{-1} t^{-1}}\right) \cap D_{t} \cap D_{t s} \text { porque } \alpha \text { é ação parcial } \\
& =\bar{\alpha}_{t s}(P) \cap D_{t} \cap D_{t s} \text { pela definição de } \bar{\alpha}_{t s} \text {. }
\end{aligned}
$$

Proposição 5.14. Seja $\alpha=\left(\left\{D_{t}\right\}_{t \in G},\left\{\alpha_{t}\right\}_{t \in G}\right)$ uma ação parcial do grupo discreto $G$ sobre a $C^{*}$-álgebra $A$. Para cada $t \in G$. seja $\mathcal{V}_{t}=\left\{[\pi] \in \hat{A}:\left.\pi\right|_{D_{t}} \neq 0\right\}$. ou seja, $\mathcal{V}_{t}=k^{-1}\left(\mathcal{O}_{t}\right)$, onde $\mathcal{O}_{t}$ é como em 5.13. Então $\alpha=\left(\left\{\mathcal{V}_{t}\right\}_{t \in G},\left\{\alpha_{t}\right\}_{t \in G}\right)$ é uma ação parcial contínua de $G$ sobre o espaço topológico $A$, onde. se $[\pi] \in V_{t}$. entào $\alpha_{t}([\pi]) \dot{\epsilon}$ a classe da única extensão de $\pi \circ \alpha_{t-1}: D_{t} \longrightarrow B\left(H_{\pi}\right)$ a todo $A$.

Demonstração. Tem-se claramente que $\nu_{e}=A, a_{e}=i d$, e que $a_{t}: \nu_{t-1} \longrightarrow V_{t}$ é uma bijeção com inversa $\hat{\alpha}_{t-1}$. Além disso. $\alpha_{t}$ é contínua, e daqui um homeomorfismo, por causa da comutatividade do diagrama seguinte, junto com o fato de que a topologia de $A$ é a topologia inicial induzida pela 
aplicação $\kappa$ :

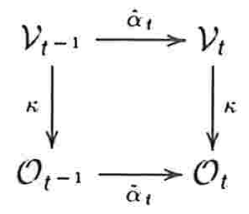

Finalmente, temos que ver que $\alpha_{t s}$ é uma extensão de $\alpha_{t} \alpha_{s}$. Agora, usando de novo o diagrama acima e o fato de que $\dot{\alpha}$ é uma açào parcial:

$$
\begin{aligned}
\operatorname{dom}\left(\alpha_{t} \alpha_{s}\right) & =\mathcal{V}_{s-1} \cap \hat{\alpha}_{s}^{-1}\left(\mathcal{V}_{t-1}\right) \\
& =\mathcal{V}_{s-1} \cap \hat{\alpha}_{s}^{-1} k^{-1}\left(\mathcal{O}_{t-1}\right) \\
& =\mathcal{V}_{s-1} \cap\left(\kappa \alpha_{s}\right)^{-1}\left(\mathcal{O}_{t-1}\right) \\
& =\mathcal{V}_{s-1} \cap\left(\tilde{\alpha}_{s}\right)^{-1}\left(\mathcal{O}_{t-1}\right) \\
& =\mathcal{V}_{s-1} \cap k^{-1} \tilde{\alpha}_{s}^{-1}\left(\mathcal{O}_{t-1}\right) \\
& =\mathcal{V}_{s-1} \cap k^{-1} \tilde{\alpha}_{s}^{-1}\left(\mathcal{O}_{t-1} \cap \mathcal{O}_{s}\right) \\
& =\mathcal{V}_{s-1} \cap k^{-1}\left(\mathcal{O}_{s-1} t_{-1} \cap \mathcal{O}_{s-1}\right) \\
& =\mathcal{V}_{s-1} \cap \mathcal{V}_{s-1} t^{-1} \cap \mathcal{V}_{s-1} \\
& =\mathcal{V}_{s-1} t^{-1} \cap \mathcal{V}_{s-1} \\
& \subseteq \mathcal{V}_{s-1} t^{-1} \\
& =\operatorname{dom}\left(\hat{\alpha}_{t s}\right)
\end{aligned}
$$

Suponhamos agora que $[\pi] \in \operatorname{dom}\left(\hat{\alpha}_{t} \alpha_{s}\right)$. Então $\kappa([\pi]) \nsupseteq D_{s^{-1} t-1} \cap D_{s^{-1}}$. Agora, é fácil ver que $\hat{\alpha}_{t s}([\pi])$ e $\alpha_{t} \dot{\alpha}_{s}([\pi])$ coincidem sobre $D_{s^{-1} t^{-1}} \cap D_{s^{-1}}$ (na verdade: representantes destas classes restritos a $D_{s^{-1} t^{-1}} \cap D_{s^{-1}}$ são unitariamente equivalentes). Portanto, $\alpha_{t s}([\pi])=\alpha_{t} \hat{\alpha}_{s}([\pi])$.

Suponhamos a seguir que a ação parcial $(\alpha, A)$ tem uma açào envolvente $(\beta, B)$. Como $A \triangleleft B$, $\hat{A}$ é naturalmente homeomorfo a um subconjunto aberto de $B$, e portanto a ação $\hat{\beta}$ restrita a este aberto define, via este homeomorfismo, uma ação parcial de $G$ sobre a. Veremos a seguir que esta ação parcial coincide $\operatorname{com} \alpha$. Analogamente, a restrição de $\hat{\beta}$ a $\operatorname{Prim}(A)$ coincide $\operatorname{com} \tilde{\alpha}$.

ProposiçÃo 5.15. Seja a uma ação parcial sobre $A$, e suponhamos que $\beta$ é uma ação envolvente de $\alpha$, agindo sobre $B$. Então $\left(r^{-1}, \operatorname{Prim}(B), \tilde{\beta}\right)$ é a ação envolvente de $(\tilde{a}$. Prim $(A)), e\left(r^{-1}, \hat{B}, \hat{\beta}\right) \dot{\epsilon}$ a ação envolvente de $(\alpha, A)$.

Demonstraç:ão. Identifiquemos $\operatorname{Prim}(A) \operatorname{com} \mathcal{O}_{A}$ através do homeomorfismo $r^{-1}$. Assim, $\tilde{a}=$ $\left(\left\{\mathcal{O}_{t}\right\},\left\{\tilde{\alpha}_{t}\right\}\right)$ tem a forma seguinte: $\mathcal{O}_{t}=\left\{P \in \operatorname{Prim}(B): P \nsupseteq D_{t}\right\}$, e se $P \in \mathcal{O}_{t^{-1}}$, então $\tilde{\alpha}_{t}(P) \in \mathcal{O}_{t}$ é o único ideal primitivo de $B$ tal que $\tilde{\alpha}_{t}(P) \cap D_{t}=\alpha_{t}\left(P \cap D_{t^{-1}}\right)$. Vejamos primeiro que $\operatorname{dom}\left(\left.\tilde{\beta}_{t}\right|_{\mathcal{O}_{A}}\right)=$ 
$\operatorname{dom} \tilde{\alpha}_{t}:$

$$
\begin{aligned}
\left.\operatorname{dom}\left(\tilde{\beta}_{t}\right)\right|_{\mathcal{O}_{A}} & =\left\{P \in \operatorname{Prim}(B): P \in \mathcal{O}_{A} \text { e } \tilde{\beta}_{t}(P) \in \mathcal{O}_{A}\right\} \\
& =\mathcal{O}_{A} \cap\left\{P \in \operatorname{Prim}(B): P \in \tilde{\beta}_{t-1}\left(\mathcal{O}_{A}\right)\right\} \\
& =\mathcal{O}_{A} \cap\left\{P \in \operatorname{Prim}(B): P \in \mathcal{O}_{\dot{\beta}_{t-1}(A)}\right\} \\
& =\mathcal{O}_{A} \cap \mathcal{O}_{\dot{\beta}_{t-1}(A)} \\
& =\mathcal{O}_{A \cap \dot{\beta}_{t-1}(A)} \\
& =\mathcal{O}_{D_{t-1}} \\
& =\mathcal{O}_{t-1} \\
& =\operatorname{dom}\left(\tilde{\alpha}_{t}\right)
\end{aligned}
$$

Agora, se $P \in \mathcal{O}_{t^{-1}}$, temos que $\dot{\beta}_{t}(P)=\beta_{t}(P) \nsupseteq D_{t}$, e portanto $\dot{\beta}_{t}(P) \in \mathcal{O}_{t}$. Mas $\beta_{t}(P) \cap D_{t}=$ $\beta_{t}\left(P \cap \beta_{t^{-1}}\left(D_{t}\right)\right)=\alpha_{t}\left(P \cap D_{t-1}\right)$. Então $\tilde{\beta}_{t}(P)$ coincide com $\tilde{\alpha}_{t}(P)$ devido à definição deste último.

Falta verificar apenas que a $\dot{\beta}$-órbita de $\mathcal{O}_{A}$ é $\operatorname{Prim}(B)$. Suponhamos que existe $P \in \operatorname{Prim}(B)$ tal que $P \neq \tilde{\beta}_{t}(Q), \forall Q \in \mathcal{O}_{A}$. Entào $\tilde{\beta}_{t}(P) \notin \mathcal{O}_{A}, \forall t \in G$, ou seja, $\beta_{t}(P) \supseteq A, \forall t \in G$. Mas então $P \supseteq \beta_{t}(A), \forall t \in G$. e portanto $P \supseteq \overline{[\beta(A)]}=B$, porque $\beta$ é a ação envolvente de $\alpha$. A contradição implica que todo ideal primitivo pertence à $\dot{\beta}$-órbita de algum elemento de $\mathcal{O}_{A}$.

Com respeito a $\beta$ e $\alpha$, identifiquemos $A$ com $\mathcal{V}_{A}$ através de $r^{-1}$. Assim, $\alpha=\left(\left\{\mathcal{V}_{t}\right\},\left\{\hat{a}_{t}\right\}\right)$ tem a forma seguinte: $\mathcal{V}_{t}=\left\{[\pi] \in \dot{B}:\left.\pi\right|_{D_{t}} \neq 0\right\}$. e para $[\pi] \in \mathcal{V}_{t^{-1}}, \alpha_{t}([\pi])$ é a classe da única extensão a $B$ da representação irredutível $\pi \circ \alpha_{t^{-1}}$ de $D_{t}$. Dos cálculos feitos anteriormente segue que $\left.\operatorname{dom}(\hat{\beta})\right|_{\mathcal{V}_{A}}=\mathcal{V}_{t-1}=\operatorname{dom}\left(\alpha_{t}\right)$. Por outro lado, se $[\pi] \in \mathcal{V}_{t^{-1}}$, então $\left(\pi \circ \beta_{t-1}\right)$ é uma extensão a $B$ da representação $\pi \circ \alpha_{t-1}$, e portanto $\dot{\beta}_{t}([\pi])$ coincide $\hat{\alpha}_{t}([\pi])$. Falta ver só que a $\hat{\beta}$-órbita de $\mathcal{V}_{A}$ é todo $\hat{B}$, o que é análogo ao já feito para $\hat{\alpha}$ e $\tilde{\beta}$ : se $[\pi] \notin \dot{\beta}_{t}\left(\mathcal{V}_{A}\right), \forall t \in G$, entào $\left.\pi\right|_{\beta_{t}(A)}=0, \forall t \in G$, e portanto $\pi=0$, o que é absurdo.

Os seguintes tres resultados valem ainda quando a ação parcial $\alpha$ não possui ação envolvente, mas ainda é cedo para demonstrá-los.

Corolário 5.16. Suponhamos que a ação parcial $(\alpha, A)$ tem ação envolvente $(\beta, B)$. Então as ações parciais a sobre $A$ e à sobre $\operatorname{Prim}(A)$ induzidas por a são contínuas.

Demonstraçào. Por 5.15 temos que $\hat{\alpha}$ é a restrição de $\hat{\beta}$ ao subconjunto aberto $\mathcal{V}_{A}$ de $\hat{B}$, e $\tilde{\alpha}$ é a restrição de $\hat{\beta}$ ao subconjunto aberto $\mathcal{O}_{A}$ de $\operatorname{Prim}(B)$. Como $\hat{\beta}$ e $\tilde{\beta}$ são contínuas por 5.12 , temos que $\hat{\alpha}$ e $\tilde{\alpha}$ são contínuas pelo exemplo 4.38 .

Corolário 5.17. Suponhamos que $(\alpha, A)$ é uma açào parcial que tem ação envolvente $(\beta, B)$. Então, se $\hat{A}=\operatorname{Prim}(A)$, também é $B=\operatorname{Prim}(B)$.

Demonstração. Por 5.15 temos que $(\hat{\beta}, \hat{B})$ é a ação envolvente de $\alpha$ e $(\tilde{\beta}, \operatorname{Prim}(B))$ é a ação envolvente de $\tilde{\alpha}$. Mas, como neste caso $\alpha=\hat{\alpha}$, e como a ação envolvente é única, concluímos que $(\hat{\beta}, \hat{B})=(\tilde{\beta}, \operatorname{Prim}(B))$. 
COROlÁrio 5.18. Suponhamos que a é uma ação parcial do grupo localmente compacto $G$ sobre a $C^{* *}$-álgebra $A$. que tem ação envolvente $(3, B)$. Se Prim $(A)$ é compacto, então existe um subgrupo aberto $H$ de $G$ tal que a restrição de a a $H$ é uma ação global. Em particular, se $G$ é conexo, a $\epsilon^{\prime}$ uma açáo global ( $\epsilon$ portanto $\alpha=\beta, A=B$ ). Em particular, isto vale se $A$ tem unidade.

Demonstração. Se A tem unidade. Prim $(A)$ é compacto por [37], VII-6.11. Por 5.16, à é contínua. Portanto, se Prim(A) é compacto. por 4.40 existe um subgrupo aberto $H$ de $G$ tal que à restrita a $H$ é uma açào global sobre Prim(A), e portanto todo ideal primitivo pertence ao domínio de $\bar{\alpha}_{s}, \forall s \in H$. Pela definiçào de $\bar{\alpha}$, isto implica que nenhum ideal primitivo de $A$ contém o ideal $D_{s^{-1}}$, o que implica que $D_{s^{-1}}=A, \forall s \in H$. Portanto $\left.\alpha\right|_{H \times A}$ é uma ação global. Se $G$ é conexo, então $G=H$.

\subsection{Propriedades da álgebra envolvente}

Esta última parte do capítulo será dedicada a comparar propriedades de uma $C^{*}$-álgebra $A$ sobre a qual age uma ação parcial $\alpha$. com a $C^{*}$-álgebra $B$ envolvente de $A$ onde atua a ação envolvente, sempre que esta exista. Veremos que várias propriedades sào comuns às duas álgebras. En toda a seção estaremos supondo que $(B, B)$ é uma ação envolvente de $(\alpha, A)$.

Começamos com um resultado que descreve $B$ como limite indutivo, e que permite afirmar que. sob certas condiçòes na classe de $C^{\prime *}$-álgebras consideradas, $A$ pertence a uma tal classe se e somente se $B$ pertence.

Proposição 5.19. Seja ('. uma classe de $C^{*}$-álgebras fechada por ideais. extensōes, quocientes $e$ limites indutivos. Então $A \in \mathcal{C}$ se $\epsilon$ somente se $B \in \mathcal{C}$.

Demonstraçào. Se $B \in \mathcal{C}$, então $A \in \mathcal{C}$ porque $\mathcal{C}$ é fechada por ideais.

Inversamente. suponhamos que $A \in \mathcal{C}$. Seja $T$ um subconjunto denso de $G$. Para cada conjunto finito $F=\left\{t_{1}, \ldots, t_{n}\right\} \subseteq T$, consideremos $B_{F}=\beta_{t_{1}}(A)+\ldots+\beta_{t_{n}}(A)$. Então $B_{F}$ é um ideal de $B([52], 1.5 .8)$. Por outro lado, $B=\lim _{\longrightarrow} B_{F}$ : se $t \in G, a \in A$, existe uma rede $\left\{t_{i}\right\} \subseteq T$ tal que $t_{i} \longrightarrow t$, e portanto $\beta_{t},(a) \longrightarrow \beta_{t}(a)$, porque $\beta$ é contínua. Assim, $\beta_{t}(a) \in \overline{\bigcup_{i} \beta_{t_{2}}(A) \subseteq \underline{\lim _{\longrightarrow}} B_{F}}$; como $B=\overline{[\beta(A)]}$, temos que $B \subseteq \lim _{\longrightarrow} B_{F}$, e portanto são iguais. Conseqüentemente, basta provar que $B_{F} \in \mathcal{C}, \forall F$, e por indução é suficiente mostrar que, se $I, J \in \mathcal{C}$, com $I \triangleleft B, J \triangleleft B$, então $I+J \in \mathcal{C}$. Agora, temos a seguinte seqüencia exata de $C^{*}$-álgebra:

$$
0 \longrightarrow I \stackrel{I}{\longrightarrow} I+J \stackrel{\pi}{\longrightarrow} J /(I \cap J) \longrightarrow 0,
$$

onde $\iota$ é a inclusão e $\pi$ é a projeção no quociente. Como $J \in \mathcal{C}$ e $\mathcal{C}$ é fechada por quocientes, então $J /(I \cap J) \in \mathcal{C}$. Finalmente, como $I, J /(I \cap J) \in \mathcal{C}$ e $\mathcal{C}$ é fechada por extensòes, temos que $I+J \in \mathcal{C}$.

Lembrando que uma $C^{* *}$-álgebra é chamada aproximadamente finita se ela é o limite indutivo de um sistema direto enumerável de $C^{*}$-álgebras de dimensão finita, temos:

Corolário 5.20. Suponhamos que $B$ é a álgebra envolvente de A. Então temos:

1. A é nuclear se $\epsilon$ somente se $B$ é nuclear

2. Se $G$ é um grupo separável. entào Á $́$ aproximadamente finita se e somente se $B$ é aproximadamente finita. 
DemonstraçÃo. A classe de ('*álgebra nucleares é fechada por ideais (2.11), quocientes ([64], 2.5), extensões ([65], T.6.27) e limites indutivos ([65], T.M). Portanto 1. segue de 5.19. A classe de $C^{*}$-álgebra aproximadamente finitas é fechada por ideais, quocientes, extensōes ([19], III.4.1, III.4.4 e III.6.3 respectivamente) e limites indutivos enumeráveis (segue da própria definição de $C^{*}$-álgebra aproximadamente finita); assim 2. segue da demonstração de 5.19

Lembremos algumas outras propriedades de $C^{*}$-álgebras que compararemos depois em $A$ e $B$. As seguintes definições e resultados podem ser achados no capítulo 6 de [52].

Definição 5.21. Scjam A uma ("'-álgebra e $x \in A$. Então diz-se que:

1. $x$ é abeliano se $\overline{x-A x}$ é una $C^{\prime *}$-álgebra abeliana.

2. A é de tipo $I_{0}$ se ela está gerada pelos seus elementos abelianos.

3. $A$ é de tipo $I$ se para todo ideal $I$ de $A$, tem-se que $A / I$ contém elementos abelianos não nulos.

4. A é liminal (ou C(CR), se para toda representação irredutível $\left(\pi, H_{-}\right)$se tem que $\pi(A)=\mathcal{K}\left(H_{\pi}\right)$

5. $A$ é postliminal (ou $(\mathrm{C} C \mathrm{R})$ se para toda representaçào irredutível $\left(\pi, H_{\pi}\right)$ se tem que $\pi(A) \supseteq$ $\mathcal{K}\left(H_{\pi}\right)$

6. A é antiliminal se $A$ nào contém elementos abelianos nào nulos.

Existem as seguintes relaçōes de implicação:

$$
A \text { abeliana } \Longrightarrow A \text { de tipo } I_{0} \Longrightarrow A \text { liminal } \Longrightarrow A \text { postliminal }=\text { tipo } I \text {. }
$$

Proposição 5.22. Seja $(\alpha, A)$ uma ação parcial que tem ação envoltente $(\beta, B)$. Então:

1. A é de tipo $I_{0}$ se e somente se $B$ é de tipo $I_{0}$.

2. A é liminal se e somente se $B$ é liminal.

3. A é postliminal se $\epsilon$ somente se $B$ é postliminal.

4. A é antiliminal se e somente se $B$ é antiliminal.

Demonstraçào. Começamos observando que se um elemento $x$ de um ideal $I$ de uma $C^{*}$ álgebra $C$ é abeliano em $I$, então é também abeliano em $C$ : se $\left(u_{i}\right)$ é uma unidade aproximada de $I$, e $c \in C$, então $x c x=\lim _{i} x u_{i} c u_{i} x \in \overline{x I x}$, donde segue que $\overline{x C x}=\overline{x I x}$ (na verdade, $\overline{x I x}$ é a $C^{*}$-álgebra hereditária gerada por $x$ em $I$, e portanto também a $C^{*}$-álgebra hereditária gerada por $x$ em $C$, que é exatamente $\overline{x C x}$.

1. Se $A$ é de tipo $I_{0}, \beta_{t}(A)$ é de tipo $I_{0}$, e como $[\beta(A)]$ é denso em $B$, e cada elemento abeliano de $\beta_{t}(A)$ é abeliano em $B$. concluímos que $B$ também é de tipo $I_{6}$. Se $B$ é de tipo $I_{0}$, então $A$ é de tipo $I_{0}$ por [52]. 6.2.10.

2. Se $B$ é liminal, entào [52]. 6.2 .9 implica que $A$ é liminal. Inversamente, suponhamos que $A$ é liminal, e seja $\pi: B \longrightarrow B\left(H_{\pi}\right)$ uma representação irredutível de $B$. Como cada $\beta_{t}(A)$ é liminal, temos que $\left.\pi\right|_{\beta_{t}(A)}=0$ ou $\pi\left(\beta_{t}(A)\right)=\mathcal{K}\left(H_{\pi}\right)$; como $B=\overline{[\beta(A)]}$, concluímos que $\pi(B)=\mathcal{K}\left(H_{\pi}\right)$.

3. Se $B$ é postliminal, entào $A$ é postliminal por [52], 6.2.9. Agora. se $A$ é postliminal, cada $\beta_{t}(A)$ também é postliminal. Portanto, se $\pi: B \longrightarrow H_{\pi}$ é uma representação irredutível, então $\left.\pi\right|_{\beta_{t}(A)}$ é nào nula para algum $t \in G$, e neste caso também é irredutível; portanto $\pi(B) \supseteq \pi\left(\beta_{t}(A)\right) \supseteq \mathcal{K}\left(H_{\pi}\right)$. donde segue que $B$ é postliminal. 
4. Se $B$ é antiliminal. não contém elementos abelianos não nulos, e então, como vimos no começo da prova, $A$ também não tem elementos abelianos não nulos; assim, $A$ é antiliminal. Suponhamos que $A$ é antiliminal, e seja $b \in B$ um elemento abeliano de $B$. Se $a \in A, t \in G$, temos que $\beta_{t}(a) b$ e $b \beta_{t}(a)$ são elementos abelianos de $\beta_{t}(A): \overline{\beta_{t}(a) b \beta_{t}(A) \beta_{t}(a) b} \subseteq \beta_{t}(A) \cap \overline{b B b}$, e portanto $\overline{\beta_{t}(a) b \beta_{t}(A) \beta_{t}(a) b}$ é uma $C^{*}$-álgebra abeliana: da mesma forma, $\overline{b \beta_{t}(a) \beta_{t}(A) b \beta_{t}(a)}$ é uma $C^{* *}$-álgebra abeliana. Assim temos que $\beta_{t}(a) b$ e $b \beta_{t}(a)$ são abelianos, e portanto nulos, já que cada $\beta_{t}(A)$ é antiliminal, donde concluímos que $b$ é um anulador de $\beta_{t}(A), \forall t \in G$. Mas então $b \overline{[\beta(A)]}=0$. o que implica que $b=0$ e então que $B$ é antiliminal. 
CAPíTULO 6

Produtos Cruzados Parcial e Envolvente 
Demonstraremos neste capítulo um dos resultados principais da parte 2: se $(\beta, B)$ é uma ação envolvente da açào parcial $(\alpha, A)$, no sentido de $C^{*}$-álgebras. entào $A \rtimes_{\alpha, r} G \stackrel{M}{\sim} B \rtimes_{\beta, r} G(6.6)$, onde $\stackrel{M}{\sim}$ significa equivalencia Morita. Na verdade, deduziremos este fato de um outro mais geral (6.3), que também será de muita utilidade no seguinte Capítulo. Veremos também que, se a ação parcial a é amenable, entào a ação envolvente $\beta$ também é amenable (6.7).

Posteriormente aplicaremos o Teorema 6.6 à análise de alguns exemplos. Entre as aplicaçōes há um resultado de dilatação de uma representação parcial a uma representação unitária que parece de bastante interesse. Todas estas aplicações sào apenas uma primeira aproximação aos exemplos considerados. e sentimos que bastante mais pode ser feito após um estudo mais profundo.

\subsection{Equivalência Morita dos produtos cruzados parcial e envolvente}

Obteremos a equivalencia Morita entre os produtos cruzados reduzidos (pela ação parcial e pela ação envolvente) como caso particular de um teorema mais geral sobre equivalencia Morita (6.4), que será utilizado também em outras ocasiōes. As hipótese em 6.4 implicam uma "equivalencia Morita" entre os fibrados de Fell $\mathcal{A}$ e $\mathcal{B}$. Tcmos que supor, porém, que $\mathcal{A} \subseteq \mathcal{B}$. Temos tentado achar uma definição geral de equivalencia Morita entre fibrados de Fell, mas para a noção que nos parece adequada não conseguimos provar que $C_{r}^{* *}(\mathcal{A}) \stackrel{M}{\sim} C_{r}^{*}(\mathcal{B})$, nem que $C^{* *}(\mathcal{A}) \stackrel{M}{\sim} C^{* *}(\mathcal{B})$.

Lema 6.1. Seja $f: G \longrightarrow H$ uma funçào contínua entre dois grupos topológicos $G$ e $H$, e seja $K \subseteq G$ compacto. Entào $f \hat{\epsilon}$ uniformemente contínua sobre $K$. no seguinte sentido: dada uma vizinhança $H^{-}$da identidade de $H$. existe uma vizinhança $V$ da identidade de $G$, tal que para todos $r, s \in K$ tais que $r^{-1} s \in V$ e $r s^{-1} \in V$ tem-se que $f(r) f(s)^{-1} \in W^{-}$e $f\left(r^{-1}\right) f(s) \in W$. Em particular. se $f$ tem suporte compacto, ela é uniformemente contínua, no sentido de que podemos substituir $I^{*}$ por $G$ na condiçào acima.

Demonstraçio. Seja $F: G \times G \longrightarrow H \times H$ tal que $F(r, s)=\left(f(r) f(s)^{-1}, f(r)^{-1} f(s)\right)$. Entaoo $F$ é uma funçào contínua, e $F(s, s)=(e, e), \forall s \in G$. Suponhamos que para toda vizinhança $V$ da identidade de $G$ existem $r_{\mathrm{V}^{\prime}}, s_{\mathrm{V}^{\prime}} \in K$ tais que $r_{V^{-1}}^{-1} s_{V} \in V$ e $r_{\mathrm{V}^{\prime}} s_{V^{-1}}^{-1} \in V$ mas $F\left(r_{V}, s_{V^{\prime}}\right) \notin W \times W$. Como $K$ é compacto, a rede $\left(r_{r}\right)$ deve ter uma subrede convergente, e portanto podemos supor que a própria $\left(r_{\mathrm{V}}\right)$ converge a certo ponto $r \in K$. Isto implica que $\left(s_{V}\right)$ também converge, ao mesmo limite $r$, já que $s_{\mathrm{V}} \in r_{\mathrm{V}} V, \forall \mathrm{V}$. Como $F$ é contínua, e $F(r, r)=(e, e)$, deve ser $F\left(r_{V}, s_{V}\right) \in W \times \mathrm{I}^{\mathrm{V}}$. $\forall V \geq V_{0}$.

Sejam $\mathcal{V}$ uma base local de vizinhanças precompactas e simétricas da unidade $e \in G$, dirigido pela relaçào: $V \geq V^{\prime \prime} \Longleftrightarrow V \subseteq V^{\prime \prime},\left\{\left(f_{V}, V\right)\right\}_{V \in \mathcal{V}}$ uma unidade aproximada de $L^{1}(G)$ contida em $C_{c}(G)$, tal que $\sup \left(f_{V}\right) \subseteq V, f_{V} \geq 0$, e $\int_{G} f_{V}(s) d s=1, \forall V \in \mathcal{V}$.

Lema 6.2. Sejam $\mathcal{B}$ um fibrado de Fell sobre o grupo localmente compacto $G, b: G \times G \longrightarrow \mathcal{B}$ uma funçâo contínua de suporte compacto, tal que $b(r, s) \in B_{s}, \forall(r, s) \in G \times G$. Seja $\left(f_{V}\right)_{V \in \mathcal{V}}$ uma unidade aproximada de $L^{1}(G)$ como a descrita acima, e definamos, para cada par $(V, r) \in \mathcal{V} \times G$, a função $b_{V, r}: G \longrightarrow \mathcal{B}$, tal que $b_{V, r}(t)=\int_{G} f_{V}\left(r^{-1} s\right) b(s, t) d s$. Então:

1. $b_{V, r} \in C_{c}^{\prime}(\mathcal{B}) . \forall V \in \mathcal{V}, r \in G$.

2. $\lim _{Y} b_{Y, r}=b_{r}$ na topologia do limite indutivo de $C_{c}(\mathcal{B})$. onde $b_{r}: G \longrightarrow \mathcal{B}$ é dada por $b_{r}(s)=b(r . s)$. 
Demonstração. A função $\mu: G \times G \times G \longrightarrow \mathcal{B}$ tal que $(r, t, s) \longmapsto f_{V}\left(r^{-1} s\right) b(s, t)$ é uma função contínua e tal que $\mu(r, t, s) \in B_{s}, \forall r, s, t \in G$. Portanto, por [37], II-15.19, a função $G \times G \longrightarrow \mathcal{B}$ tal que $(r, t) \longmapsto \int_{G} f_{V^{\prime}}\left(r^{-1} s\right) b(s, t) d s$ é contínua. Em particular, $b_{V, r}$ é contínua, o que prova 1.

Com respeito a 2 .. sejam $K_{1}, K_{2} \subseteq G$ compactos tais que $\sup (b) \subseteq K_{1} \times K_{2}$. Como a função $b$ é contínua, cada $b_{r}: C_{i} \longrightarrow \mathcal{B}$ é uma seção contínua tal que $\sup \left(b_{r}\right) \subseteq K_{2}$, de forma que temos uma função $G \longrightarrow C_{c}^{\prime}(\mathcal{B})$ tal que $r \longrightarrow b_{r}$, cujo suporte está contido em $K_{1}$. Esta função é contínua. Com efeito, seja $r_{0} \in G$. Como a função $G \longrightarrow \mathbb{R}$ tal que $(r, s) \longmapsto\left\|b\left(r_{0}, s\right)-b(r, s)\right\|$ é contínua e se anula para cada $\left(r_{0}, s\right), \operatorname{com} s \in K_{2}$, existem vizinhanças abertas $U_{s}$ de $r_{0}, V_{s}$ de $s$, tais que $\left\|b\left(r_{0}, s^{\prime}\right)-b\left(r, s^{\prime}\right)\right\|<c, \forall r \in I_{s}, s^{\prime} \in V_{s}$. Como os $V_{s}$ cobrem o compacto $K_{2}$, existe uma subcobertura finita $V_{s_{1}}, \ldots, V_{s_{n}}$ de $I_{2}$. Seja $U=\bigcap_{i=1}^{n} U_{s_{1}}$. Então $I$ é uma vizinhança aberta de $r_{0}$. Sejam $r \in U^{\prime}$. $s \in G$. Entào $s \notin K_{2}$, em cujo caso $b_{r}(s)=0, \forall r \in G$, ou $s \in K_{2}$, e portanto $s \in V_{s}$, para algúm $i$, è neste caso $(r, s) \in U_{s_{1}} \times I_{s_{1}}$, donde segue que $\left\|b_{r_{0}}(s)-b_{r}(s)\right\|<\epsilon$, e portanto $\left\|b_{r_{0}}-b_{r}\right\|_{\infty}<\epsilon$. Assim.

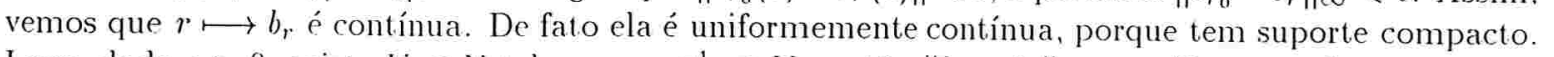
Logo, dado $t>0$. existe $I_{i} \in \mathcal{V}$ tal que se $r^{-1} s \in V_{0}$. entào $\left\|b_{r}-b_{s}\right\|_{\infty}<\epsilon$. Temos assim, para todo $V \in \mathcal{V}$, tal que $V \geq V_{0}$ :

$$
\begin{aligned}
\left\|\left(b_{r}-b_{1, r}\right)(t)\right\| & =\left\|\int_{G} f_{1} \cdot\left(r^{-1} s\right)(b(r, t)-b(s, t)) d s\right\| \\
& \leq \int_{V} f_{V} \cdot\left(r^{-1} s\right)\|b(r, t)-b(s, t)\| d s \\
& =\int_{r \cdot \cdot} f_{1} \cdot\left(r^{-1} s\right)\|b(r, t)-b(s, t)\| d s \\
& =\epsilon \int_{r \cdot} f\left(r^{-1} s\right) d s \\
& =\epsilon
\end{aligned}
$$

Portanto, $\left\|b_{r}-b_{V, r}\right\|_{\infty}<\epsilon . \forall V \geq V_{0}$, e daqui segue que $\lim _{V} b_{V, r}=b_{r}, \forall r \in G$.

Teorema 6.3. Sejam $B=\left(B_{t}\right)_{t \in G}$ um fibrado de Fell sobre o grupo localmente compacto $G$. $\mathcal{E}=\left(E_{t}\right)_{t \in G}$ um ideal à direita de $\mathcal{B}$ (ver 3.8). $\epsilon \mathcal{A}=\left(A_{t}\right)_{t \in G}$ um subfibrado de Fell de $\mathcal{B}$ contido $\mathrm{\epsilon m}$ $\mathcal{E}$. Se $\mathcal{A} \mathcal{E} \subseteq \mathcal{E} \in \mathcal{E} \mathcal{E}^{*} \subseteq \mathcal{A}$, tem-se:

1. $L^{1}(\mathcal{A}) L^{1}(\mathcal{E}) \subseteq L^{1}(\mathcal{E})$

2. $L^{1}(\mathcal{E}) L^{1}(\mathcal{B}) \subseteq L^{1}(\mathcal{E})$.

3. $L^{1}(\mathcal{E}) L^{1}(\mathcal{E})^{*}=L^{1}(\mathcal{A})$

4. Se para cada $t \in G$ tem-se que $\operatorname{span}\left(\mathcal{E}^{*} \mathcal{E} \cap B_{t}\right)$ é denso em $B_{t}$, então $\overline{\operatorname{span}} L^{1}(\mathcal{E})^{*} L^{1}(\mathcal{E})=$ $L^{1}(\mathcal{B})$.

DemonstraÇÃo. Para provar as duas primeiras inclusões acima, é suficiente mostrar que $C_{c}(\mathcal{A}) *$ $C_{c}(\mathcal{E}) \subseteq C_{c}(\mathcal{E})$ e $C_{c}(\mathcal{E}) * C_{c}(\mathcal{B}) \subseteq C_{c}(\mathcal{E})$. Vejamos:

1. Sejam $a \in C_{c}(\mathcal{A}), \xi \in C_{c}^{\prime}(\mathcal{E}), t \in G$; entào:

$$
a * \xi(t)=\int_{G} a(s) \xi\left(s^{-1} t\right) d s \in E_{t},
$$

pois $a(s) \xi\left(s^{-1} t\right) \in A_{s} E_{s-1} \subseteq \mathcal{E} \cap B_{t}=E_{t}, \forall s, t \in G$. Portanto $a * \xi \in C_{c}(\mathcal{E})$.

2. Sejam $\xi \in C_{c}(\mathcal{E}), b \in C_{c}(\mathcal{B}), t \in G$; entào:

$$
\xi * b(t)=\int_{G} \xi(s) b\left(s^{-1} t\right) d s \in E_{t},
$$

pois $\xi(s) b\left(s^{-1} t\right) \in E_{s} B_{s^{-1}} \in \mathcal{E} \cap B_{t}=E_{t} . \forall s, t \in G$. Portanto $\xi * b \in C_{c}(\mathcal{E})$. 
3. Como $\mathcal{A} \subseteq \mathcal{E} \subseteq \mathcal{B}$, temos inclusòes isométricas $L^{1}(\mathcal{A}) \subseteq L^{1}(\mathcal{E}) \subseteq L^{1}(\mathcal{B})$. $L^{1}(\mathcal{A})$ é uma sub-*álgebra de Banach com unidade aproximada de $L^{1}(\mathcal{B})$, e está contida no ideal à direita $L^{1}(\mathcal{E})$ de $L^{1}(\mathcal{B})$ (sabemos que é um ideal à direita por 2.). Portanto, usando 1. e o teorema de Cohen-Hewitt:

$$
L^{1}(\mathcal{A})=L^{1}(\mathcal{A}) * L^{1}(\mathcal{A})^{*} \subseteq L^{1}(\mathcal{E}) * L^{1}(\mathcal{E})^{*} .
$$

Por outro lado, se $\xi, \eta \in C_{c}(\mathcal{E}), t \in G$ :

$$
\begin{aligned}
\xi * \eta^{*}(t) & =\int_{G} \xi(s) \eta^{*}\left(s^{-1} t\right) d s \\
& =\int_{G} \xi(s) \Delta\left(t^{-1} s\right) \eta\left(t^{-1} s\right)^{*} d s \\
& =\int_{G} \Delta\left(t^{-1} s\right) \xi(s) \eta\left(t^{-1} s\right)^{*} d s \in A_{t} .
\end{aligned}
$$

pois $\xi(s) \eta\left(t^{-1} s\right)^{*} \in E_{s} E_{t^{-1} s}^{*} \in \mathcal{A} \cap B_{t}=A_{t} . \forall s, t \in G$. Portanto $\xi * \eta^{*} \in C_{c}(\mathcal{A})$, o que prova a inclusão contrária.

4. Sejam $\xi, \eta \in C_{c}(\mathcal{E}), t \in G$. Entào:

$$
\begin{aligned}
\xi^{*} * \eta(t) & =\int_{G} \xi^{*}(s) \eta\left(s^{-1} t\right) d s \\
& =\int_{G} \Delta\left(s^{-1}\right) \xi\left(s^{-1}\right)^{*} \eta\left(s^{-1} t\right) d s \\
& =\int_{G} \Delta\left(s^{-1}\right) \xi\left(s^{-1}\right)^{*} \eta\left(s^{-1} t\right) d s
\end{aligned}
$$

Consideremos agora $\left\{\left(f_{\mathrm{V}}, V\right)\right\}_{V \in \mathcal{V}}$ uma unidade aproximada como no Lema 6.2. Para $\xi \in C_{c}(\mathcal{E}), V \in \mathcal{V}, r \in G$, seja $\xi_{\mathrm{r} ; r}: G \longrightarrow \mathcal{B}$ tal que

$$
\xi_{1}, r(s)=\Delta\left(s^{-1}\right) f_{V}\left(r^{-1} s^{-1}\right) \xi(s) .
$$

Então $\xi_{V, r} \in C_{c}(\mathcal{E})$, e temos:

$$
\begin{aligned}
\xi_{1, r}^{*} * \eta(t) & =\int_{G} \Delta\left(s^{-1}\right) \Delta(s) f_{V}\left(r^{-1} s\right) \xi\left(s^{-1}\right)^{*} \eta\left(s^{-1} t\right) d s \\
& =\int_{G} f_{V}\left(r^{-1} s\right) \xi\left(s^{-1}\right)^{*} \eta\left(s^{-1} t\right) d s \\
& =\int_{G} f_{V}\left(r^{-1} s\right) \zeta_{\xi, \eta}(s, t) d s
\end{aligned}
$$

onde $\zeta_{\xi, \eta}: G \times G \longrightarrow B$ é tal que $\zeta_{\xi, \eta}(s, t)=\xi\left(s^{-1}\right)^{*} \eta\left(s^{-1} t\right)$. Notar que $\zeta_{\xi, \eta}$ é contínua e de suporte compacto: se $\zeta_{\xi, \eta}(s, t) \neq 0 \longrightarrow s \in(\sup (\xi))^{-1}$ e $s^{-1} t \in \sup (\eta)$, donde segue que $t \in$ $s \sup (\eta) \subseteq(\sup (\xi))^{-1} \sup (\eta)$; portanto, $\sup \left(\zeta_{\xi, \eta}\right) \subseteq(\sup (\xi))^{-1} \times(\sup (\xi))^{-1} \sup (\eta)$, que é compacto. Usando agora o Lema 6.2 , vemos que $\lim _{V} \xi_{V, r}^{*} * \eta=\zeta_{\xi, \eta, r}$ na topologia do limite indutivo de $C_{c}\left(\mathcal{B}_{\beta}\right)$, e portanto também em $L^{1}\left(\mathcal{B}_{\beta}\right)$. Assim, temos que $\left(\overline{\operatorname{span}} L^{1}(\mathcal{E})^{*} L^{1}(\mathcal{E})\right) \bigcap C_{c}(\mathcal{B}) \supseteq Z$, onde $Z=\operatorname{span}\left\{\zeta_{\xi, \eta, r}: \xi, \eta \in C_{c}^{\prime}(\mathcal{E}), r \in G\right\}$.

Para mostrar que $\overline{\operatorname{span}} L^{1}(\mathcal{E})^{*} L^{1}(\mathcal{E})=L^{1}(\mathcal{B})$, basta ver que $Z$ é denso em $C_{c}(\mathcal{B})$ na topologia do limite indutivo; para isso é suficiente, por [37], II-14.6 (ou por 3.14), ver que: (a) $Z(t)$ é denso em $B_{t}, \forall t \in G$, onde $Z(t)=\{\zeta(t): \zeta \in Z\}$ e (b) se $g: G \longrightarrow \mathbb{C}$ é contínua, então $g \zeta \in Z, \forall \zeta \in Z$.

(a) Temos: $Z(t) \supseteq\left\{\zeta_{\xi, \eta, r}: \xi, \eta \in C_{c}(\mathcal{E}), r \in G\right\}=\left\{\xi\left(r^{-1}\right)^{*} \eta\left(r^{-1} t\right): \xi, \eta \in C_{c}(\mathcal{E}), r \in G\right\}$. Portanto, $\overline{Z(t)} \supseteq \overline{\operatorname{span}}\left\{E_{r^{-1}}^{*} E_{r^{-1} t}: r \in G\right\}=\left(B_{t} \bigcap \overline{\operatorname{span}} \mathcal{E}^{*} \mathcal{E}\right)=B_{t}$ pela hipótese 4 . 
(b) Sejam $g: G \longrightarrow \mathbb{C}$ contínua, $\xi, \eta \in C_{c}(\mathcal{E}), r, t \in G$. Definindo $g^{r}: G \longrightarrow \mathbb{C}$ como $g^{r}(s)=g(r s)$ tem-se:

$$
\left(g \dot{\zeta}_{\xi, \eta, r}\right)(t)=g(t) \xi\left(r^{-1}\right)^{*} \eta\left(r^{-1} t\right)=\xi\left(r^{-1}\right)^{*} g^{r}\left(r^{-1} t\right) \eta\left(r^{-1} t\right)=\zeta_{\xi, g^{r} \eta, r}(t) .
$$

Como $\zeta_{\xi, g^{r} \eta, r} \in C_{C}(\mathcal{E})$, concluímos que $\zeta_{\xi, g^{r} \eta, r} \in Z$, o que encerra a prova.

Corolário 6.4. Sejam $B=\left(B_{t}\right)_{t \in G}$ um fibrado de Fell sobre o grupo localmente compacto $G$, $\mathcal{E}=\left(E_{t}\right)_{t \in G}$ um ideal à direita de $\mathcal{B}$ (ver 3.8), e $\mathcal{A}=\left(A_{t}\right)_{t \in G}$ um subfibrado de Fell de $\mathcal{B}$ contido em $\mathcal{E}$. Se $\mathcal{A} \mathcal{E} \subseteq \mathcal{E}$, $\mathcal{E}^{*} \subseteq \mathcal{A}$. tem-se que $C_{r}^{*}(\mathcal{A})$ é uma sub-('* -álgebra hereditária de $C_{r}^{*}(\mathcal{B}), \epsilon$ se $\left(B_{t} \cap \overline{\operatorname{span}} \mathcal{E}^{*} \mathcal{E}\right) \dot{\epsilon}$ denso em $B_{t}$. para cada $t \in G$, entäo $C_{r}^{*}(\mathcal{A}) \in C_{r}^{*}(\mathcal{B})$ sio equivalentes Morita. através do ideal dircito $\left(_{r}^{*}(\mathcal{E}):=\overline{L^{1}(\mathcal{E})} \subseteq C_{r}^{*}(\mathcal{B})\right.$.

Demonstraçào. Por 3.10, sabemos que $C_{r}^{*}(\mathcal{A})$ é naturalmente isomorfa ao fecho de $L^{1}(\mathcal{A})$ em $C_{r}^{*}(\mathcal{B})$. Por $6.32 \ldots L^{1}(\mathcal{E})$ é um ideal à direita em $L^{1}(\mathcal{B})$, e portanto $C_{r}^{*}(\mathcal{E})$, seu fecho em $C_{r}^{*}(\mathcal{B})$, é um ideal direito de $C_{r}^{*}(\mathcal{B})$. Agora, de 3. deduzimos que $C_{r}^{*}(\mathcal{A})=C_{r}^{*}(\mathcal{E}) C_{r}^{*}(\mathcal{E})^{*}$, e portanto $C_{r}^{*}(\mathcal{A})$ é uma sub- $C^{* *}$-álgebra hereditária de $C_{r}^{\prime *}(B)$. Finalmente, a última afirmaçào segue do quarto ponto de 6.3 .

Corolário 6.5. Siejam $B=\left(B_{t}\right)_{t \in G}$ um fibrado de Fell sobre o grupo localmente compacto $G$, $\mathcal{E}=\left(E_{t}\right)_{t \in G}$ um ideal à direita de $\mathcal{B}$ (ver 3.8), e $\mathcal{A}=\left(A_{t}\right)_{t \in G}$ um subfibrado de Fell de $\mathcal{B}$ contido em $\mathcal{E}$. Se $\mathcal{A} \mathcal{E} \subseteq \mathcal{E}$ e $\mathcal{E} \mathcal{E}^{*} \subseteq \mathcal{A}$. e se span $\left(B_{t} \cap \mathcal{E}^{*} \mathcal{E}\right)$ é denso em $B_{t}$, para cada $t \in G$, então $\mathcal{B}$ é amenable sempre que $\mathcal{A}$ seja amenable.

DemonstraçÃo. Suponhamos que $\mathcal{A}$ é amenable, e sejam $\|\cdot\|_{\operatorname{máx}}$ a norma de $C^{*}(\mathcal{B})$ e $\|\cdot\|_{r}$ a norma sobre $C_{r}^{*}(\mathcal{B})$. O fecho de $C_{c}(\mathcal{A})$ dentro de $C_{r}^{*}(\mathcal{B})$ é $C_{r}^{*}(\mathcal{A})$. pela Proposição 3.10. Também temos que o fecho de $C_{c}(\mathcal{A})$ dentro de $C^{*}(\mathcal{B})$ é $C^{*}(\mathcal{A})=C_{r}^{*}(\mathcal{A})$. pois toda representação de $L^{1}(\mathcal{B})$ induz uma representaçào de $L^{1}(\mathcal{A})$ por restriçào, e portanto a norma de $C^{*}(\mathcal{A})$ é maior ou igual que $\|\cdot\|_{\text {máx }}$; a amenabilidade de $\mathcal{A}$ implica que são iguais. Seja $\xi \in C_{c}(\mathcal{E})$. Como $C^{*}(\mathcal{A})=C_{r}^{*}(\mathcal{A})$ por hipótese, e $\xi * \xi^{*} \in C_{c}^{*}(\mathcal{A})$ por 6.3 , temos que:

$$
\|\xi\|_{r}^{2}=\left\|\xi^{*} * \xi\right\|_{r}=\left\|\xi * \xi^{*}\right\|_{r}=\left\|\xi * \xi^{*}\right\|_{\text {máx }}=\left\|\xi^{*} * \xi\right\|_{\text {máx }}=\|\xi\|_{\text {máx }}^{2},
$$

e portanto $\|\xi\|_{r}=\|\xi\|_{\text {máx }}$. Então, os completamentos de $C_{c}(\mathcal{E})$ com respeito a $\|\cdot\|_{\text {máx }}$ e $\|\cdot\|_{r}$ coincidem; denotemos este completamento por $E$. Temos então que $E$ é um módulo de Hilbert pleno sobre $C^{*}(\mathcal{B})$ e também sobre $C_{r}^{*}(\mathcal{B})$. Mas por [68], $E$ só pode ser um módulo de Hilbert pleno sobre uma única $C^{*}$-álgebra: isto implica que $C^{*}(\mathcal{B})=C_{r}^{*}(\mathcal{B})$, e portanto $\mathcal{B}$ é amenable.

Teorema 6.6. Sejam $\beta$ uma açào contínua do grupo localmente compacto $G$ sobre a $C^{*}$-álgebra $B, I \triangleleft B, \alpha=\left.\beta\right|_{I}$. Entcio $I \rtimes_{a, r} G$ é uma subálgebra hereditária de $B \rtimes_{3, r} G$. Sé além disso $[\beta(I)]$ é denso em $B$, i.e. $\beta$ é a ação envolvente de $\alpha$, então $I \rtimes_{\alpha, r} G \in B \rtimes_{B, r} G$ são Morita equivalentes.

Demonstração. Sejam $\mathcal{B}_{B}=\left(B_{t}\right)_{t \in G}$ o fibrado de Fell associado a $3, \mathcal{B}_{\alpha}=\left(A_{t}\right)_{t \in G}$ o fibrado de Fell associado a $a$, e $\mathcal{E}=\left(E_{t}\right)_{t \in G}$, onde $E_{t}=\left\{(t, x) \in \mathcal{B}_{\beta}: x \in I\right\}$. É claro que $\mathcal{B}_{\alpha} \subseteq \mathcal{E} \subseteq \mathcal{B}_{\beta}$ como subfibrados de Banach, e que $\mathcal{B}_{a}$ é um subfibrado de Fell de $\mathcal{B}_{\beta}$. Além disso, se $\left(r, a_{r}\right) \in A_{r}$, $\left(s, x_{s}\right) \in E_{s},\left(t, y_{t}\right) \in E_{t},\left(u, b_{u}\right) \in B_{u}$. tem-se:

- $\left(r, a_{r}\right)\left(s, x_{s}\right)=\left(r s, a_{r}\left(a_{r-1}\left(a_{r}\right) x_{s}\right) \in E_{r s}\right.$, porque $\alpha_{r}\left(a_{r-1}\left(a_{r}\right), x_{s}\right) \in I$. Portanto: $\mathcal{B}_{\alpha} \mathcal{E} \subseteq \mathcal{E}$ 
- $\left(s, x_{s}\right)\left(t, y_{t}\right)^{*}=\left(s, x_{s}\right)\left(t^{-1}, \beta_{t-1}\left(y_{i}^{*}\right)\right)=\left(s t^{-1}, x_{s} \beta_{s t-1}\left(y_{t}^{*}\right)\right) \in A_{s t-1}$, porque $\left.x_{s} \beta_{s t-1}\left(y_{t}^{*}\right)\right) \in$ $I \bigcap \beta_{s t-1}(I)=D_{s t-1}$. Assim. $\varepsilon \varepsilon^{*} \subseteq \mathcal{B}_{\alpha}$

- $\left(s, x_{s}\right)\left(u, b_{u}\right)=\left(s u, x_{s} \beta_{s}\left(b_{u}\right)\right) \in E_{u}$ porque $I$ é um ideal e $x_{s} \in I$. Logo. $\mathcal{E} \mathcal{B}_{\beta} \subseteq \mathcal{E}$.

Portanto, estamos nas condiçòes do Corolário 6.4 , donde concluímos que $I \rtimes_{a, r} G_{r}=C_{r}^{*}\left(\mathcal{B}_{\alpha}\right)$ é uma sub- $C^{*}$-álgebra hereditária de $C_{r}^{*}\left(\mathcal{B}_{\beta}\right)=B \rtimes_{\beta, r} G$.

Suponhamos agora que $\beta$ é a açào envolvente de $\alpha$, ou seja, que $[3(I)] \dot{C}$ denso en $B$. Para ver que $I \rtimes_{\alpha, r} G \stackrel{M}{\sim} B \rtimes_{\beta, r} G$. basta ver que $\left(\overline{\operatorname{span}} \mathcal{E}^{*} \mathcal{E}\right) \cap B_{t}$ é denso 'III $B_{t}$, para todo $t \in G$. Agora: $\left(s, x_{s}\right)^{*}\left(t, y_{t}\right)=\left(s^{-1}, \beta_{s-1}\left(x_{s}^{*}\right)\right)\left(t, y_{t}\right)=\left(s^{-1} t, \beta_{s-1}\left(x_{s}^{*} y_{t}\right)\right)$. Portanto. (span $\left.\mathcal{E}^{*} \mathcal{E}\right) \cap B_{t}=$ $\left\{\left(t, \beta_{v}\left(x^{\prime} y^{\prime}\right)\right): v \in G, x^{\prime}, y^{\prime} \in I\right\}$. Pelo teorema de Cohen -Hewitt. cada $z \in I$ pode ser escrito na forma $z=x^{\prime} y^{\prime}$, para certos $x^{\prime}, y^{\prime} \in I$. Portanto, o conjunto acima é exatamente $\{t\} \times[\beta(I)]$, que é denso em $B_{t}$.

Corolário 6.ī. Ś $\beta$ é a açào emeolvente de uma açào parral amenable a (ver 3.7), entào $\beta$ também é amenable.

Demonstraçào. Segue de 6.5.

\subsection{Aplicaçōes e exemplos}

Como aplicaçōes do Teorema 6.6. a seguir vemos alguns exemplos de ('*-álgebras que são Morita equivalentes a produtos cruzados reduzidos por açòes globais de grupos discretos; também obtemos um resultado de dilatação de representaçòes parciais de grupos discretos amenable.

Exemplo 6.8. Se $\hat{Y}=\{1, \ldots, n\}$. e a é a açào parcial de $\Xi$ por translaçào, então $\dot{X}=\mathbb{Z}$, e $\dot{\alpha}$ é a translação. Portanto, usando o Teorema 6.6. obtemos o resultado bem conhecido seguinte:

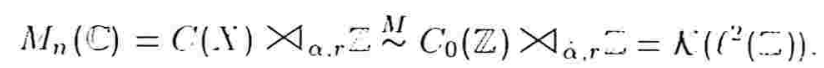

Exemplo 6.9. Sejam $\theta \in \mathbb{P}$ um irracional, $\beta_{\theta}: \mathbb{Z} \times S^{1} \longrightarrow S^{1}$ tal que $\beta_{\theta}(k, z)=e^{2 k \pi i \theta} z$. Se $U \subseteq S^{1}$ é um aberto não vazio, e $\alpha_{\theta}^{l}:=\left.\beta_{\theta}\right|_{U}$. então $\left(S^{1}, \beta_{\theta}\right)$ é a ação envolvente de $\left(U, \alpha_{\theta}^{U}\right)(\tilde{v}$ er o exemplo 4.49). Segue que:

$$
A_{\theta}:=C\left(S^{1}\right) \rtimes_{\beta_{\theta}} \mathbb{Z} \stackrel{M}{\sim} C_{0}(U) \rtimes_{\alpha_{\theta}^{U}}=
$$

\section{ExEMPLo 6.10. ('*-álgebra parcial de um grupo discreto}

Suponhamos que $G$ é discreto, e consideremos a seguinte ação parcial (canonica) $\alpha$ de $G$ sobre

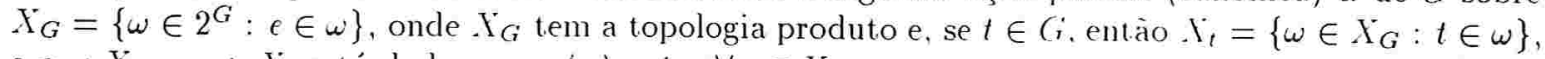
e $\alpha_{t}: X_{t-1} \longrightarrow X_{t}$ está dada por $\alpha_{t}(\omega)=t \omega, \forall \omega \in X_{t-1}$.

Como $\{0,1\}$ é compacto, temos que $2^{G}$ é compacto com a topologia produto, e portanto $X_{G}$, $X_{t}$ são compactos. pois são fechados em $2^{G}$. Isto implica que $\operatorname{Gr}(\alpha)$ é fechado em $G \times X_{G} \times X_{G}$ (ver Observação 4.52), e portanto o espaço envolvente $\dot{X}_{G}$ é de Hausdorff. pela Proposição 4.51. De fato, é claro que $X_{G}$ é também aberto em $2^{G}$, e portanto, levando em conta o Teorema 4.44 , a ação 
envolvente de $\alpha$ é simplesmente $\dot{a}: G \times \dot{X}_{G} \longrightarrow \dot{X}_{G}$ tal que $\dot{\alpha}_{t}(\omega)=t \omega, \forall \omega \in \dot{X}_{G}, t \in G$, onde $\dot{X}_{G}$ é a órbita de $X_{G}$ pela ação por translação à esquerda por elementos de $G$ dentro de $2^{G}$, isto é: $\dot{X}_{G}=\left\{\omega \in 2^{G}: \omega \neq \emptyset\right\}$. Devido à nossa Proposição 4.62 e à Proposition 5.1 de [32], temos que $\dot{\alpha}$ é topologicamente livre se e somente se $G$ é infinito.

Lembremos de [26] (ver também [32]), que a $C^{*}$-álgebra parcial do grupo $G$ é definida como $C_{p}^{*}(G)=C^{\prime}\left(X_{G}\right) \rtimes_{\alpha} G$. A propriedade fundamental de $C_{p}^{*}(G)$ é que as representações parciais de $G$ estão em correspondencia biunívoca com as representaçōes nào degeneradas de $C_{p}^{*}(G)$. Se $G$ é amenable, então $C_{p}^{*}(G)=C\left(Y_{G i}\right) \rtimes_{\alpha} G=C^{\prime}\left(X_{G}\right) \rtimes_{\alpha, r} G$, por [28]. 'Theorem 4.ī. e também temos $C\left(\dot{X}_{G}\right) \rtimes_{\dot{\alpha}} G=C\left(\dot{X}_{G}\right) \rtimes_{\dot{a}, \cdot} G$. Entào, se $G$ é amenable. o Teorema 6.6 implica que temos:

$$
C_{r}^{*}(G) \stackrel{M}{\sim} C\left(\dot{X}_{G}\right) \rtimes_{\dot{\alpha}} G
$$

\section{ExEMPLo 6.11. ( ${ }^{*}$-álgebra parcial de um grupo finito}

A seguir estudamos um caso particular do exemplo acima, a saber. o caso em que $G$ é um grupo finito. Uma referencia suficiente para esta parte é [60]

Como $G$ é finito, temos que $C_{p}^{* *}(G)=C^{\prime}\left(\mathrm{X}_{G}\right) \rtimes_{\alpha} G$ é equivalente Norita com a álgebra envolvente $E(G):=C\left(\dot{X}_{G}\right) \rtimes_{\alpha} G$. O nosso objetivo é achar a álgebra $E(G)$, e compará-la com $C_{p}^{*}(G)$, que vem sendo estudada em [20], inclusive de um ponto de vista puramente algébrico.

Começaremos com algumas considerações gerais sobre produtos cruzados de ('*-álgebras comutativas de dimensào finita por grupos finitos.

Consideremos então um grupo finito $G$ que atua sobre um conjunto finito $X$ através de uma ação $\beta$. Suponhamos inicialmente que $\beta$ é transitiva. Neste caso, é conhecido que $\beta$ é isomorfa à ação por translação à esquerda $\tau: G \times\left(G / G_{x}^{\beta}\right) \longrightarrow G / G_{x}^{\beta}$, tal que $\beta_{r}\left(s G_{x}^{3}\right)=(r s) G_{x}^{\beta}$, onde $G_{x}^{\beta}$ é o estabilizador de qualquer elemento $x \in X$. Por outro lado, é fácil ver que o correspondente produto cruzado é $M_{[G: H]}\left(\mathbb{C} G_{x}^{\beta}\right)$, sendo $\mathbb{C} G_{x}^{\beta}$ a álgebra do grupo $G_{x}^{\beta}$, i.e.:

$$
C(\mathrm{Y}) \rtimes_{\beta} G=C^{\prime}\left(G / G_{x}^{\beta}\right) \rtimes_{\tau} G=M_{\left[G ; G_{x}^{\beta}\right]}\left(\mathbb{C} G_{x}^{3}\right) .
$$

Se a ação $\beta$ é arbitrária, ela será transitiva quando restrita a cada uma das suas órbitas. Portanto, se $R^{\beta}$ é um conjunto completo de representantes das órbitas, e $o(x)$ denota a órbita do elemento $x \in X$, teremos:

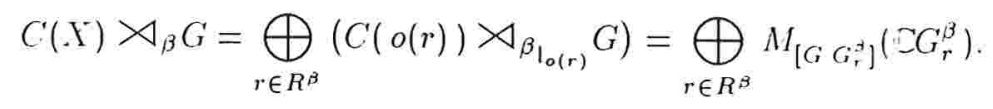

Queremos ir um pouco além desta descrição de $C(X) \rtimes_{\beta} G$, e para isso introduzimos algo de notação. Seja $H$ um subgrupo de $G$; indicaremos esta relação pondo $H \leq G$, e $H<G$ se $H$ for um subgrupo próprio de $G$. Para $H \leq G$, definimos $X_{H}^{\beta}=\left\{x \in X: G_{x}^{\beta}=H\right\}$, e $F_{H}^{\beta}(X)=$ $\left\{x \in X: H \leq G_{x}^{\beta}\right\}$. Indicaremos por $n_{H}^{\beta}$ o cardinal de $X_{H}^{\beta}$. Em geral. $|S|$ denotará o cardinal do conjunto $S$; assim por exemplo, $n_{H}^{\beta}=\left|X_{H}^{\beta}\right|$. Se $t \in G$, denotaremos por $H_{t}$ o subgrupo de $G$ conjugado com $H$ através de $t$, ou seja: $H_{t}=t H t^{-1}$, e por $[H]$ a classe de conjugação de $H$, isto é, $[H]=\left\{H_{t}: t \in G\right\}$. Seja $C=\{[H]: H \leq G\}$ a família de classes de conjugação de subgrupos de $G$. Notar que a função $X \longrightarrow$ \{subgrupos de $G$ \} que a cada elemento $x \in X^{\prime}$ associa seu estabilizador $G_{x}^{\beta}$ passa aos quocientes, donde obtemos uma função Est $: X / \beta \longrightarrow \mathrm{C}$ tal que $o(x) \longmapsto\left[G_{x}^{\beta}\right]$. Dado $H \leq G$, definimos $n_{[H]}^{\beta}=\mid$ Est $^{-1}([H]) \mid$, isto é, $n_{[H]}^{\beta}$ é o número de órbitas cujos elementos têm estabilizador conjugado com $H$. Notar que a quantidade de tais elementos é $n_{[H]}^{3}[G: H]$. Daqui em 
diante, $R^{\beta}$ denotará um conjunto completo de representantes das órbitas e, para $H \leq G$, indicaremos por $R_{[H]}^{\beta}=\left\{r \in R^{\beta}\right.$ : Est $\left.(o(r))=[H]\right\}$. É claro que $n_{[H]}^{\beta}=\left|R_{[H]}^{\beta}\right|$.

Proposição 6.12. Suponhamos que $\beta: G \times X \longrightarrow X$ é uma ação de um grupo finito $G$ sobre um conjunto finito $X$. Entio. com a notaçào acima temos:

1. $C(X) \rtimes_{\beta} G=\bigoplus_{[H] \in \mathcal{C}} M_{[G: H]}(\mathbb{C} H)^{n_{[H]}^{\beta}}$, onde $M_{n}(\mathbb{C} H)^{k}=\underbrace{M_{n}(\mathbb{C} H) \oplus \ldots \oplus M_{n}(\mathbb{C} H)}_{k \text { vezes }}$ (em particular, $\left.M_{n}(\mathbb{C} H)^{0}=0\right)$.

2. Se G é abeliano, entrio $C(\mathrm{Y}) \rtimes_{\beta} G=\bigoplus_{H \leq G} M_{[G: H]}(\mathbb{C})^{|H| n_{[H]}^{\beta}}$.

Demonstraçào. Temos:

$$
\begin{aligned}
C^{\prime}(X) \rtimes_{\beta} G & =\bigoplus_{r \in R^{3}} C(o(r)) \rtimes_{\beta_{l_{o(r)}} G} \\
& =\bigoplus_{r \in R^{3}} M_{\left[G: G_{r}^{\beta}\right]}\left(\mathbb{C} G_{r}^{\beta}\right) \\
& =\bigoplus_{[H] \in \mathcal{C}} \bigoplus_{r \in R_{[H]}^{\beta}} M_{[G: H]}(\mathbb{C} H) \\
& =\bigoplus_{[H] \in \mathcal{C}} M_{[G: H]}(\mathbb{C} H)^{n_{[H]}^{\beta}} .
\end{aligned}
$$

O enunciado para $G$ abeliano segue do fato que $\Xi H=\mathbb{C}^{H \mid}$, para todo subgrupo $H$ de $G$, e de que podemos identificar $H \mathrm{com}[H]$, pois todos os subgrupos são normais.

O Lema seguinte será útil para calcular $E(G)$.

LEMA 6.13. Tem-se:

1. $F_{H}(X)=\biguplus_{H \leq K \leq G} . X_{K}^{\beta}$, e $n_{H}^{\beta}=\left|F_{H}(X)\right|-\sum_{H<K \leq G} n_{K}^{\beta}$

2. $n_{H}^{\beta}=n_{[H]}^{\beta}\left[N_{G i}(H): H\right]$, onde $N_{G i}(H)$ é o normalizador de $H$ em $G$.

DemonstraçÃo. A primeira parte do Lema é trivial, e portanto provaremos apenas a segunda. Para isto, notemos que se $t \in G$, então $t X_{H}^{\beta}=X_{H_{t}}^{-3}$ e, em particular, $\left|X_{H_{1}}^{\beta}\right|=\left|X_{H}^{\beta}\right|$. Portanto:

$$
n_{[H]}^{\beta}[G: H]=\left|\biguplus_{r \in \mathbb{E}_{[H]}^{\beta}} o(r)\right|=\left|\biguplus_{H^{\prime} \in[H]} X_{H^{\prime}}^{\beta}\right|=|[H]|\left|X_{H}^{\beta}\right|=\left[G: N_{G}(H)\right] n_{H}^{\beta} .
$$

Então, $n_{H}^{\beta}=\left(n_{[H]}^{\beta}[G: H]\right) /\left[G: N_{G i}(H)\right]=\left[N_{G}(H): H\right] n_{[H]}^{\beta}$. 
ObservaÇão 6.14 . Notar que. como conjunto, $C(X) \rtimes_{\beta} G$ pode ser identificado $\operatorname{com}\{f: G \longrightarrow$ $C(X)\}$, e portanto $\operatorname{dim}\left(C^{\prime}(X) \rtimes_{\beta} G\right)=|G||X|$. Por outro lado, pela Proposição 6.12 temos:

$$
\begin{aligned}
\operatorname{dim}\left(C(\mathrm{Y}) \rtimes_{B} G\right) & =\operatorname{dim} \bigoplus_{[H] \in \mathrm{C}} M_{[G: H]}(\mathbb{C} H)^{n_{H}^{\beta} /\left[N_{G}(H): H\right]} \\
& =\sum_{[H] \in C}[G: H]^{2}|H| \frac{n_{H}^{\beta}}{\left[N_{G}(H): H\right]} \\
& =\sum_{[H] \in C}|[H]||G| n_{H}^{\beta} .
\end{aligned}
$$

Assim concluímos que, para toda açào $\beta$ de um grupo finito $G$ sobre um conjunto finito $X$, tem-se:

$$
\sum_{[H] \in C}|[H]| n_{H}^{\beta}=\left|X^{-}\right| .
$$

Observaçào 6.15. Seja $c_{G}$ o número de classes de conjugaçào do grupo $G$. Lembrar que $\operatorname{dim}(Z(\mathbb{C} G))=c_{G}$, onde $Z\left(\widetilde{C}(i)\right.$ denota o centrode $G$. Como $C^{\prime}(X) \rtimes_{\beta}\left(i=\bigoplus_{[H] \in \mathcal{C}} M_{[G: H]}(\mathbb{C} H)^{n_{[H]}^{\beta}}\right.$. tem-se que $\operatorname{dim}\left(Z\left(C\left(\mathrm{X}^{*}\right) \rtimes_{3,}(i)\right)=\sum_{[H] \in C}{ }^{{ }^{C} H} n_{[H]}^{\beta}\right.$.

Lema 6.16. Sejam $\beta:(i \times X \longrightarrow X \in \gamma: H \times Y \longrightarrow Y$ açóes dos grupos finitos $G$ e $H$ sobre os conjuntos finitos $X, Y$. Seja $\beta \times i:(G \times H) \times(X \times Y) \longrightarrow X \times Y$ a açáo produto. Então temos. para $G^{\prime} \leq G, H^{\prime} \leq H$ :

$$
\left(X \times Y_{G^{\prime} \times H^{\prime}}^{\beta \times \gamma}=Y_{G^{\prime}}^{\gamma^{\prime}} \times Y_{H^{\prime}}^{\gamma^{\prime}}, \quad n_{G^{\prime} \times H^{\prime}}^{\beta \times \gamma}=n_{G^{\prime}}^{\beta} n_{H^{\prime}}^{\gamma}, \quad n_{\left[G^{\prime} \times H^{\prime}\right]}^{3 \times \gamma}=n_{\left[G^{\prime}\right]}^{\beta} n_{\left[H^{\prime}\right]}^{\gamma} .\right.
$$

Demonstração. Seja $(x, y) \in X \times Y$. Entào $(G \times H)_{(x, y)}^{\beta \times \gamma}=G_{x}^{3} \times H_{y}^{\gamma}$. Segue que $(x, y) \in$ $\left(X \times Y^{\gamma}\right)_{G^{\prime} \times H^{\prime}}^{\beta \times \gamma} \Longleftrightarrow(G \times H)_{(x, y)}^{\beta \times \gamma}=G^{\prime} \times H^{\prime} \Longleftrightarrow G_{x}^{\beta}=G^{\prime}$ e $H_{y}^{\gamma}=H^{\prime} \Longleftrightarrow x \in X_{G^{\prime}}^{-\beta}$ e $y \in Y_{H^{\prime}}^{\gamma}$.

A última igualdade se deduz das duas primeiras e de 2 . do Lema 6.13. além da observação trivial de que $\left[N_{G \times H}\left(G^{\prime} \times H^{\prime}\right): G^{\prime} \times H^{\prime}\right]=\left[N_{G}\left(G^{\prime}\right): G^{\prime}\right]\left[N_{H}\left(H^{\prime}\right): H^{\prime}\right]$.

Podemos agora considerar o caso em que a ação $\beta$ é a açào $a$ envolvente da açào parcial de $G$ sobre $G$ que define $C_{p}^{*}(G)$. Ou seja. $\dot{\alpha}: G \times \dot{X}_{G} \longrightarrow \dot{X}_{G}$ é tal que $\forall t \in G, A \in \dot{X}_{G}$, temos $\dot{\alpha}_{t}(A)=t A$. onde $\dot{X}_{G}=\{A \subseteq G: A \neq \emptyset\}$. Em lugar de por $\left(\dot{X}_{G}\right)_{H}^{\dot{\alpha}}, n_{H}^{\dot{\alpha}}$, etc., poremos simplesmente $X_{H}, n_{H}$, etc. .

Para referencia posterior, e utilizando já o Lema 6.13, reestabelecemos a Proposição 6.12 para o caso da álgebra $E(G)$.

Proposição 6.1T. 1. $E(G)=\bigoplus_{[H] \in \mathrm{C}} M_{[G: H]}(\mathbb{C} H)^{n_{H}} /\left[N_{G}(H): H\right] . \in n_{H} \neq 0, \forall H \leq G$.

2. Se $G$ é abeliano. entào $E(G)=\bigoplus_{H \leq G} M_{[G: H]}(\mathbb{C})^{\left(n_{H}|H|^{2}\right) /|G|}$.

Demonstraçào. A única novidade com respeito à Proposição 6.12 e ao Lema 6.13 , é a afirmação de que $n_{H} \neq 0$, para todo subgrupo $H$ de $G$; mas isto é claro, porque o estabilizador do conjunto $H \in \dot{X}_{G}$ é o subgrupo $H$, e portanto $H \in X_{H}$. 
Observaçío 6.18. Lembrar que se $H$ é um grupo finito e $\mathbb{C} H=\bigoplus_{i=1}^{r} M_{d_{1}}(\mathbb{C})$, então cada $d_{i}$ é um divisor de $H$. Por outro lado, como $n_{H} \neq 0$, para todo subgrupo $H$ de $G$, vemos que na decomposição de $E(G)$ como soma de álgebras de matrizes complexas, os tamanhos das matrizes que aparecem são da forma $[G: H] d \leq|G|$, onde $H$ é um subgrupo arbitrário de $G$, e $d$ é um divisor de $|H|$. Em particular, no caso abeliano os tamanhos das matrizes complexas movolvidas são exatamente os divisores da ordem de $G$.

Antes de calcularmos $E\left(G^{i}\right)$ em alguns exemplos concretos. tentaremos achar uma fórmula para $n_{H}$.

Proposiçio 6.19. 1. Seja $A \in \dot{\mathrm{X}}_{G}, \epsilon$ suponhamos que $H \leq\left(i_{A}\right.$. lintäo eristem $a_{1}, \ldots, a_{l} \in$ A tais que $A=\biguplus_{i=1}^{\prime} H a_{i}$.

2. Seja $A \in \dot{X}_{i}$ tal que $A=\biguplus_{i=1}^{\prime} H a_{i}$. para certo $H \leq G+$ itrtos $a_{1} \ldots \ldots$ al . Quando mde $\left(l_{1},[G\right.$ : $H])=1$. ention $\left(i_{A}=H\right.$.

3. (a) $n_{G}=1$

(b) $\left|F_{H}\left(\dot{X}_{G}\right)\right|=2^{[G: H]}-1$.e $n_{H}=2^{[G: H}-2-\sum_{H<H^{\prime}<r_{i}} n H^{\prime}$.

(c) Se $H<$ G. entaio $n_{H} \geq 2^{[G: H]}-2-\sum_{\{l<[G: H]: m d c(l,[G ; H]\}>1\}}\left(\begin{array}{c}{[(;: H]} \\ l\end{array}\right)$.

4. Se $M<$ G é maximal, então

(a) $n_{M}=2^{[G: M]}-2$.

(b) $n_{[M]}=\left(2^{[G: M]}-2\right) /[G: M]$. se $M$ é normal em (;

(c) $n_{[M]}=2^{[G: M]}-2$. se $M$ nào é normal em $G$

Demonstração. 1. Como $H \leq G_{A}$, temos que $h A=A$. $\forall h \in H$. c portanto $H$ atua sobre $A$ por translação à esquerda. Se $a \in A$, a correspondente órbita é $H a$.

2. Por 1., existem $a_{1}, \ldots, a_{l}, b_{1}, \ldots, b_{m} \in A$ tais que $A=\biguplus_{i=1}^{l} H a_{i}$ e $A=\biguplus_{j=1}^{m} G_{A} a_{j}$. Como $H \leq G_{A}$, temos que $G_{A}=\biguplus_{k=1}^{\left[G_{A}: H\right]} H x_{k}$, para certos $x_{1} \ldots, x_{\left[G_{A} H\right]} \in G_{A}$. Portanto, $A=$ $\biguplus_{j=1}^{m}\left(\biguplus_{k=1}^{\left[G_{A}: H\right]} H x_{k}\right) b_{j}=\biguplus_{j=1}^{m} \biguplus_{k=1}^{\left[G_{A}: H\right]} H x_{k} b_{j}$, donde concluímos que $H x_{k} b_{j}, j=1, \ldots, m$. $k=1, \ldots,\left[G_{A}: H\right]$, sào as órbitas da ação de $H$ sobre $A$ por translaçào à esquerda. Portanto. $l=m\left[G_{A}: H\right]$. e como $\left[G_{A}: H\right]$ divide $[G: H]$, a nossa hipótese implica que $\left[G_{A}: H\right]=1$, ou seja, $G_{A}=H$.

3. (a) Se $A \neq G$ e $t \notin A$, existe $s \in G$ tal que $s a=t$, para algum $a \in A$. Portanto $G_{A} \neq G$. Portanto $n_{G}=1$, sendo o conjunto $G$ o único elemento de $\dot{X}_{G}$ cujo estabilizador é $G$.

(b) Sejam $H t_{1}, \ldots, H t_{[G: H]}$ as classes laterais à direita de $H$ em $G$. Para $l \leq[G: H]$, seja $A=\biguplus_{j=1}^{r} H t_{i_{j}}$, para $\left\{t_{i_{1}}, \ldots, t_{i_{1}}\right\} \subseteq\left\{t_{1}, \ldots, t_{[G: H]}\right\}$. Como $\forall I=1 \ldots,[G: H]$ existem $\left(\begin{array}{c}{[G: H]} \\ i\end{array}\right)$ destes conjuntos, e estes sâo (por 1.) os únicos pontos fixos por $H$, temos que $\left|F_{H}\left(\dot{X}_{G}\right)\right|=\sum_{l=1}^{[G: H]}\left({ }^{[G: H]}\right)=2^{[G: H]}-1$. O resto de 3 .(b) segue de 1. do Lema 6.13.

(c) Se $\operatorname{mdc}(l,[G: H])=1$, temos por 2. acima que $G_{A}=H$. ('omo existem $\left({ }^{[G: H]}{ }_{l}\right)$ destes conjuntos teremos que:

$$
\begin{aligned}
& n_{H} \geq \sum_{\{l \leq[G: H]: \operatorname{mdc}(l,[G: H])=1\}}\left(\begin{array}{c}
{[G: H]} \\
l
\end{array}\right) \\
& =\sum_{l=1}^{[G: H]-1}\left(\begin{array}{c}
{[G: H]} \\
l
\end{array}\right)-\sum_{\{l<[G: H]: \operatorname{mdc}(l,[G: H]\}>1\}}\left(\begin{array}{c}
{[G: H]} \\
l
\end{array}\right) \text {. }
\end{aligned}
$$


4. Se $M$ é maximal entre os subgrupos próprios de $G,[G: M]$ é primo, e portanto o segundo somando em 3.(b) é igual a zero. Para provar (b) e (c), é só observar que, como $M$ é maximal. tem-se que $N_{G}(M)=G$ ou $N_{G}(M)=M$. e depois usar 2. do Lema 6.13.

Já está na hora de ver alguns exemplos, que se calculam facilmentr usando principalmente a Proposição 6.19 e o Lema 6.13.

Exemplo: $E\left(Z_{p}\right), p$ primo

Temos que o grupo trivial $1=\{\epsilon\}$ é um subgrupo maximal $r$ normal de $\tilde{z}_{p}$. Portanto $n_{[1]}=\frac{2^{p}-2}{p}$. Como. por outro lado, $n_{\left[E_{p}\right]}=1$, concluímos que

$$
E\left(Z_{p}\right)=\widetilde{\Psi^{\prime}} M_{p}(\mathbb{C})^{\frac{2^{p}-2}{p}}
$$

Exemplo: $E\left(\bar{w}_{p^{2}}\right), p$ primo

$\mathbb{Z}_{p^{2}}$ é cíclico, e portanto tem exatamente um subgrupo $H$ de ordem $p$, que é maximal e normal. Então $n_{[H]}=\frac{2^{p}-2}{r}$. Por outro lado, $n_{[1]}=\frac{n_{1}}{\left[N_{G(1)}(1]\right.}=\frac{n_{1}}{r^{2}}$. Pelo Lema 6.13, sabemos que $n_{1}=\left(2^{r^{2}}-1\right)-n_{H}-n_{G}=\left(2^{r^{2}}-1\right)-\left(2^{r}-2\right)-1=2 r^{2}-2 r^{r}$. Portanto:

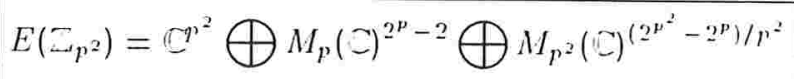

Exemplo: $E\left(\Xi_{p} \times \Xi_{q}\right), p, q$ primos, $p<q$

Existem dois subgrupos maximais: $H$, de ordem $p, K$, de ordem $q$. Portanto $n_{[H]}=\frac{2^{q}-2}{q}$. $n_{[K]}=\frac{2^{p}-2}{p}$. Então $n_{1}=2^{p q}-1-\left(2^{q}-2\right)-\left(2^{p}-2\right)-1=2^{p q}-2^{q}-2^{p}+2$ (notar que nestes primeiros tres exemplos, o valor de $n_{1}$ também podia ter sido obtido usando os valores achados antes junto com o valor da dimensão de $E(G))$. Temos assim:

$$
E\left(\mathbb{Z}_{p} \times \mathbb{Z}_{q}\right)=\mathbb{C}^{p q} \bigoplus M_{p}(\mathbb{C})^{\frac{q}{p}\left(2^{p}-2\right)} \bigoplus M_{q}(\mathbb{C})^{\frac{p}{q}\left(2^{q}-2\right)} \bigoplus M_{p q}(\mathcal{C})^{\frac{1}{p q}\left(2^{p q}-2^{p}-2^{q}+2\right)}
$$

Exemplo: $E\left(\mathbb{Z}_{p} \times \mathbb{Z}_{p}\right), p$ primo

Cada subgrupo de ordem $p$ é maximal, e existem exatamente $p+1$ de tais subgrupos. Se $H$ é um deles, temos $n_{[H]}=\frac{2^{p}-2}{p}$. Então, $n_{1}=\left(2^{p^{2}}-1\right)-(p+1)\left(2^{r}-2\right)-1=2^{p^{2}}-(p+1) 2^{p}+2 p$. Logo:

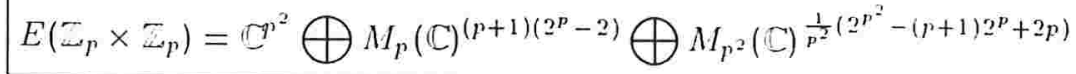


Exemplo: $E\left(S_{3}\right)$, onde $S_{3}$ é o grupo de permutações de três elementos

Sejam $P_{2}$ um 2-Sylow e $P_{3}$ um 3-Sylow de $S_{3}$. Como $P_{3} \triangleleft S_{3}$, temos que $n_{\left[P_{3}\right]}=\frac{2^{2}-2}{2}=1$. $P_{2}$ é maximal, mas nào é normal. Portanto $n_{\left[P_{2}\right]}=2^{3}-2=6$. Por último, como o número de 2-Sylow é exatamente 3: $n_{1}=\left(2^{6}-1\right)-2-3 \times 6-1=42$, e portanto $n_{[1]}=\frac{42}{6}=7$. Por outro lado, $S_{3}$ tem duas representaçōes unidimensionais, a trivial e o sinal, e como $E\left(S_{3}\right)$ não é comutativa e tem dimensão 6 , deduzimos que $\mathbb{C} S_{3}=\mathbb{C}^{2} \oplus M_{2}(\widetilde{Z})$. Em conclusão, temos:

$$
E\left(S_{3}\right)=\mathbb{C}^{2} \bigoplus M_{2}(\mathbb{C})^{4} \bigoplus M_{3}(\mathbb{C})^{12} \bigoplus M_{6}(\mathbb{C})^{7}
$$

Proposiçào 6.20. Seja $\left(i=H \times K\right.$. Consideremos a subálgebra $E_{H}(G)$ de $E(G)$ definida como:

Então temos que

$$
E_{H}\left(G^{\prime}\right)=\bigoplus_{H \leq H^{\prime} \leq G} M_{\left[G: H^{\prime}\right]}\left(\mathbb{C} H^{\prime}\right)^{n}\left[H^{\prime}\right]
$$

$$
E_{H}(G)=\mathbb{C} H \bigotimes E(K) .
$$

Demonstraçào. Se $H \leq H^{\prime}$, entào existe um (único) subgrupo $K^{-1}$ de $K$ tal que $H^{\prime}=H \times K^{-1}$ (a saber: $K^{\prime \prime}=H^{\prime} \cap K^{\prime}$ ). Portanto. temos: $\left[G^{\prime}: H^{\prime}\right]=\left[H \times K^{\prime}: H \times K^{-\prime}\right]=\left[K^{\prime}: K^{-\prime}\right]$ e, pelo Lema 6.16 , $n_{\left[H^{\prime}\right]}=n_{[H \times K]}=n_{[H]}^{H} n_{\left[K^{\prime}\right]}^{K}=n_{\left[K^{\prime}\right]}^{K}$. onde estamos utilizando as notaçoes $n_{[H]}^{H}$ e $n_{\left[K^{\prime}\right]}^{K^{\prime}}$ para indicar que estamos enxergando $H$ como subgrupo de $H$, e $K^{\prime \prime}$ como subgrupo de $K$. Assim, tem-se:

$$
\begin{aligned}
E_{H}(G) & =\bigoplus_{H \leq H^{\prime}} M_{\left[G: H^{\prime}\right]}\left(\mathbb{C} H^{\prime}\right)^{n_{\left[H^{\prime}\right]}} \\
& =\bigoplus_{H \leq H^{\prime}} M_{\left[G: H^{\prime}\right]}\left(\mathbb{C}\left(H \times K^{-\prime}\right)\right)^{n_{\left[H^{\prime}\right]}} \\
& =\bigoplus_{K \leq K^{\prime}}\left(M_{\left[K: K^{\prime}\right]}(\mathbb{C}) \bigotimes \mathbb{C H} \bigotimes \mathbb{C} K^{\prime} \bigotimes \mathbb{C}^{\left.2 K^{\prime} K^{\prime}\right]}\right) \\
& =\mathbb{C}(H) \bigotimes\left[\bigoplus_{K \leq K^{\prime}} M_{\left[K: K^{\prime}\right]}\left(\mathbb{C} K^{-\prime}\right)^{\left.n_{\left[K^{\prime}\right]}^{K}\right]}\right. \\
& =\mathbb{C} H \bigotimes E(K)
\end{aligned}
$$

Corolário 6.21. $\operatorname{dim} E_{H}(G)=|G|\left(2^{[G: H]}-1\right)$

ObservaÇÃo 6.22. Sejam $G$ abeliano, $p$ um primo que divide à ordem de $G, P$ o $p$-Sylow de $G$, e $N \leq G$ tal que $G=P \times N$. Sabemos que $E(G)=\bigoplus_{d|| G \mid} M_{d}(\mathbb{C})^{a_{d}}$, para certos inteiros positivos $a_{d}$. É claro então que $E_{N}(G)=\bigoplus_{p^{j} \leq|G|} M_{p^{j}}(\mathbb{C})^{a_{p^{j}}}$. Em particular, se $G_{1}$. $G_{2}$ são grupos abelianos tais que $E\left(G_{1}\right)=E\left(G_{2}\right)$, com $G_{i}=P_{i} \times N_{i}$, onde $P_{i}$ é o $p$-Sylow de $G_{i}$, temos que $E\left(P_{1}\right)=E\left(P_{2}\right)$ : $\left|G_{1}\right|=\left|G_{2}\right|$ porque $\operatorname{dim} E(G)$ é uma funçào estritamente crescente, e portanto $\left|P_{1}\right|=\left|P_{2}\right|,\left|N_{1}\right|=\left|N_{2}\right|$, donde vemos que $E_{N_{1}}\left(G_{1}\right)=E_{N_{2}}\left(G_{2}\right)$, i.e. $E\left(P_{1}\right)^{\left|N_{1}\right|}=E\left(P_{2}\right)^{\left|N_{2}\right|} ; \operatorname{logo} E\left(P_{1}\right)=E\left(P_{2}\right)$. Segue que se quisermos mostrar que $E(G)$ é um invariante completo dos grupos abelianos finitos, bastaria mostrar que isto é verdadero com respeito aos p-grupos abelianos finitos. Por sua vez. para isto seria suficiente 
provar que a quantidade de subgrupos maximais de dois $p$-grupos abelianos finitos da mesma ordem é diferente se os grupos nào são isomorfos.

\section{ExEMPLO 6.23. ('*álgebras de Wiener-Hopf de grupos ordenados}

Para maiores detalhes sobre as ('*-álgebras que consideramos a seguir, consultar [51], o artigo de Nica onde elas foram introduzidas. Sejam $G$ um grupo discreto e $P \subseteq G$ um subsemigrupo. Temos uma ordem parcial sobre $G$ definida como: $r \leq s \Longleftrightarrow r^{-1} s \in \bar{P}$. Supomos que o par $(G, P)$ satisfaz a condiçào de "quase-lattice": se $r, s \in G$ e $P_{r, s}=\{t \in P: r \leq t, s \leq t\} \neq \emptyset$, entào $P_{r, s}$ tem um elemento mínimo $r \smile s$, chamado mínimo múltiplo comum de $r, s$. Se $P_{r, s}=\emptyset$. definimos $r \smile s=x$. I'ma representação isométrica de $P$ sobre o espaço de Hilbert $H$ é um mapa $V: P \longrightarrow B(H)$ tal que $I_{r} I_{r}=1$. e $V_{r} V_{s}=V_{r s}, \forall r, s \in P$. A representaçào $V$ é chamada covariante se $V_{r} V_{r}^{*} V_{s} V_{s}^{*}=I_{r \sim s} I_{r, s}^{*}, \forall r, s \in P$, com a convenção $V_{\infty}=0$. Para um tal par, Nica define em [51] duas $C^{*}$-álgebras: $C^{*}(C, P)$ e $\mathcal{T}(G, P) . C^{*}(G, P)$ é uma $C^{*}$-álgebra caracterizada por uma propriedade universal: as representaçòes covariantes de $P$ estào em bijeçào com as representações nào degeneradas de $C^{*}\left(C_{i}, P\right)$. A $C^{* *}$-álgebra $\mathcal{T}(G, P)$, chamada de Wiener-Hopf, é a $C^{*}$-álgebra gerada pela representaçào regular esquerda de $P$ sobre $\ell^{2}(P)$, que é covariante.

Em [32], os autores provam que $C^{\prime *}(G, P)$ e $\mathcal{T}(G, P)$ são respectivamente os produtos cruzados pleno e reduzido por uma ação parcial de $G$. Lembremos os ingredientes do sistema dinamico parcial.

Seja $\omega \subseteq G$. Dizemos que $\omega$ é hereditário (com respeito a $P$ ), se $t P^{-1} \subseteq \omega, \forall t \in \omega$. Dizemos que $\omega$ é dirigido se $\forall r, s \in \omega$, existe $t \in \omega \cap P$ tal que $r \leq t, s \leq t$. Usando as notações de 6.10 . seja $X_{\mathcal{N}}=\left\{\omega \in X_{G_{i}}: \omega\right.$ é dirigido e hereditário $\}$. Se $\alpha$ é a ação parcial canonica sobre $X_{G}$, então $\alpha\left(X_{\mathcal{N}}\right) \subseteq X_{\mathcal{N}}$ (Lemma 6.3 de [32]). Além disso, $X_{\mathcal{N}}$ é compacto ([32], Proposition 4.1). Sejam

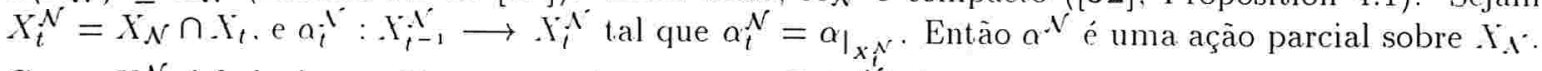
Como $X_{t}^{\mathcal{N}}$ é fechado em $X_{\mathcal{N}}$, temos de novo que $\operatorname{Gr}\left(\alpha^{\mathcal{N}}\right)$ é fechado em $G \times X_{\mathcal{N}} \times X_{\mathcal{N}}$, e portanto $\dot{X}_{\mathcal{N}}$ é de Hausdorff. É claro que, como conjunto, $\dot{X}_{\mathcal{N}}$ é a $\dot{\alpha}$-órbita de $X_{\mathcal{N}}$, onde $\dot{\alpha}$ é a ação envolvente da ação parcial canonica a. Porém. não temos verificado ainda se a topologia de $\dot{X}_{\mathcal{N}}$ é a herdada da topologia produto de $2^{G}$.

Com os elementos introduzidos acima temos ([32], 6.4 e 6.8):

$$
C^{\prime *}(G, P)=C\left(X_{\mathcal{N}}\right) \rtimes_{\alpha^{\mathcal{N}}} G, \quad \text { e } \quad \mathcal{T}(G, P)=C\left(X_{\mathcal{N}}\right) \rtimes_{\alpha^{\mathcal{N}}, r} G
$$

Portanto, pelo Teorema 6.6. temos que $\mathcal{T}(G, P) \stackrel{M}{\sim} C\left(\dot{X}^{\mathcal{N}}\right) \rtimes_{\dot{\alpha}^{\mathcal{N}},{ }_{r} G}$.

\section{EXEMPLo 6.24. ('* -álgebras parciais com relações de grupos discretos}

$\mathrm{Na}$ verdade, os exemplos 6.10 e 6.23 são casos particulares de uma situação mais geral, estudada em [32]. Sem entrar em detalhes, que podem ser consultados em [32], qualquer $C^{*}$-álgebra universal associada a representações parciais, que satisfazem um certo conjunto de relações $\mathcal{R}$, é um produto cruzado parcial da açào parcial canónica restrita a um determinado subconjunto compacto $\alpha$-invariante $\mathrm{X}_{\mathcal{R}}$ de $\mathrm{X}_{G_{i}}$. chamado o espectro das relações $\mathcal{R}$ ([32], 4.4). Sejam $X_{t}^{\mathcal{R}}=X_{\mathcal{R}} \cap X_{t}$. e $\alpha_{t}^{\mathcal{R}}: X_{t^{-1}}^{\mathcal{R}} \longrightarrow \mathrm{Y}_{t}^{\mathcal{R}}$ a restrição de $\alpha_{t}$ a $\mathrm{X}_{t^{-1}}^{\mathcal{R}}$. Como $X_{\mathcal{R}}$ é invariante, e cada $X_{t}$ é aberto em $X_{G}$. entâo cada $X_{t}^{\mathcal{R}}$ é aberto em $X_{\mathcal{R}}$, e $a^{\mathcal{R}}$ é uma ação parcial; como $X_{t}$ é fechado em $X_{G}$, então cada $X_{t}^{\mathcal{R}}$ é fechado em $\mathrm{I}_{\mathcal{R}}$. e portanto $a^{\mathcal{R}}$ tem gráfico fechado em $G \times \mathrm{X}_{\mathcal{R}} \times \mathrm{X}_{\mathcal{R}}$. Portanto $\dot{X_{\mathcal{R}}}$ é de Hausdorff. De novo como conjunto. $\dot{X}_{\mathcal{R}}$ não é outra coisa que a $\dot{\alpha}$-órbita de $X_{\mathcal{R}}$. mas não parece que possamos 
fazer também uma identificaçào topológica entre estes espaços topológicos. Devido ao Teorema 6.6, podemos concluir, como nos exemplos acima, que

$$
C\left(X_{\mathcal{R}}\right) \rtimes_{a}{ }_{, r} G \stackrel{M}{\sim} C\left(\dot{X}_{\mathcal{R}}\right) \rtimes_{\dot{\alpha}^{r}, r} G .
$$

Outro caso particular destas álgebras sào as $C^{\prime *}$-álgebras de Cuntz-lirieger. para matrizes finitas ou infinitas ([32] e [31]).

O último resultado do capítulo diz que toda representação parcial de um grupo discreto amenable pode ser dilatada a uma representaçào unitária do grupo. Em particular. $\dot{C}$ uma função de tipo positivo. Lembremos primeiro a definição de representação parcial de um grupo discreto ([26], 6.2):

DEFINIÇÃo 6.25. Seja $G$ um grupo discreto. Uma representaçào parcial de $G$ sobre o espaço de Hilbert $H$ é uma aplicação $u:(i \longrightarrow B(H)$ tal que, $\forall t, s \in G$ :

1. $u(s) u(t) u\left(t^{-1}\right)=u(s t) u\left(t^{-1}\right)$.

2. $u\left(t^{-1}\right)=u(t)^{*}$

3. $u(e)=i d_{H}$

OBSERVAÇÃo 6.26. É fácil ver que as condiçōes acima implicam que cada $u(t)$ é uma isometria parcial, e também que $u\left(s^{-1}\right) u(s) u(t)=u\left(s^{-1}\right) u(s t), \forall s, t \in G$. lisaremos a notação $u_{t}$ em lugar de $u(t)$.

ProposiçÃo 6.2T. Ṡjam $G$ um grupo discreto amenable, $\epsilon u:(i \rightarrow B(H)$ uma representação parcial. Então existem um espaço de Hilbert $\dot{H}$, que contém $H$ romo sub spuço de Hilbert. e uma representaçâo unitária $\dot{u}: G \longrightarrow B(\dot{H})$. tais que

$$
u_{t}=P_{H} \dot{u}_{t} i_{H}, \quad \forall t \in G,
$$

onde $P_{H}: \dot{H} \longrightarrow H$ é a projeçào ortogonal de $\dot{H}$ sobre $H, e i_{H}: H \longrightarrow H$ é a inclusào natural.

Demonstração. Sejam $X_{t}=\left\{\omega \in 2^{G}: e, t \in \omega\right\}, \alpha_{t}: X_{t-1} \longrightarrow X_{t}$ tal que $\alpha_{t}(\omega)=t \omega$, $\forall \omega \in X_{t^{-1}}$. Entào $\alpha$ é uma ação parcial sobre $X_{G}:=X_{e}$, e $C_{p}^{\prime *}(G)=\left({ }^{\prime}\left(X_{G}\right) \rtimes_{\alpha} G\right.$. Como $G$ é amenable, também temos que $C_{r}^{*}(G)=C\left(X_{G}\right) \rtimes_{\alpha, r} G$. Sejam $\mathcal{B}_{n}$ o fibrado de Fell sobre $G$ definido pela ação parcial $a, 1_{t}$ a função característica de $X_{t}$ e, se $a_{t} \in C_{0}\left(\mathscr{K}_{t}\right)$. o elemento $a_{t} \delta_{t} \in C_{c}\left(\mathcal{B}_{\alpha}\right)$ definido como $a_{t} \delta_{t}(s)=\delta_{t, s} a_{t}$, onde $\delta_{t, s}$ é o símbolo de Kronecker.

Por 6.5 de [26], u define uma única representação $\pi_{u}: C_{p}^{*}(G) \longrightarrow B(H)$. nào degenerada, e tal que $\pi_{u}\left(1_{t} \delta_{t}\right)=u_{t}, \forall t \in G$. Em particular, $\pi_{u}\left(1_{e} \delta_{e}\right)=i d_{H}$.

Pelo Teorema 6.6, temos que $C_{p}^{*}(G)=C\left(X_{G}\right) \rtimes_{\alpha, r} G$ é uma sub-C '“-álgebra hereditária de $C\left(\dot{X}_{G}\right) \rtimes_{\dot{\alpha}, r} G$, que é igual a $C\left(\dot{X}_{G}\right) \rtimes_{\dot{\alpha}} G$ pois $G$ é amenable. Além disto. $C_{p}^{\prime}(G)$ é equivalente Morita a $C\left(\dot{X}_{G}\right) \rtimes_{\dot{\alpha}} G$. Portanto, existem um espaço de Hilbert $\dot{H}$ e uma representação não degenerada $\dot{\pi}_{u}: C\left(\dot{X}_{G}\right) \rtimes_{\dot{\alpha}} G \longrightarrow B(\dot{H})$, tais que $H$ é um subespaço de Hilbert de $\dot{H}$. e $\pi_{u}$ é a compressão de $\dot{\pi}_{u}$ a $H$, isto é: $\pi_{u}(x)=P_{H} \dot{\pi}_{u}(x) i_{H}, \forall x \in C_{p}^{*}(G)$, onde $P_{H}: \dot{H} \longrightarrow H$ é a projeção ortogonal, e $i_{H}=P_{H}^{*}: H \longrightarrow \dot{H}$, a inclusão natural ([37], XI-7.6).

Agora, $\dot{\pi}_{u}=\dot{\phi} \times \dot{u}$, para alguma representação covariante $(\dot{\phi}, \dot{u})$ do sistema dinamico $\left(C_{0}\left(\dot{X}_{G}\right), \dot{\alpha}, G\right)$; em particular, $\dot{u}: G \longrightarrow B(\dot{H})$ é uma representação unitária de $G$.

Notar que, como $\dot{X}_{G}$ é aberto e fechado em $\dot{X}_{G}$, então $1_{e} \in C\left(\dot{X}_{G}\right)$. e podemos calcular, em $C\left(\dot{X}_{G}\right) \rtimes_{\dot{\alpha}, r} G$ :

$$
\left(1_{\epsilon} \delta_{\epsilon}\right)\left(1 \delta_{t}\right)\left(1_{e} \delta_{\epsilon}\right)=\left(1_{e} \delta_{t}\right)\left(1_{e} \delta_{e}\right)=1_{e} \dot{\alpha}_{t}\left(1_{e}\right) \delta_{t}=1_{t} \dot{\delta}_{t}
$$




\section{Portanto:}

$$
u_{t}=\pi_{u}\left(1_{t} \delta_{t}\right)=\left.P_{H} \dot{\pi}_{u}\left(1_{t} \delta_{t}\right)\right|_{H}=\left.P_{H} \dot{\pi}\left(1_{e} \delta_{e}\right) \dot{\pi}\left(1 \delta_{t}\right) \dot{\pi}\left(1_{e} \delta_{e}\right)\right|_{H}=\left.P_{H} \dot{\pi}\left(1_{e} \delta_{e}\right) \dot{u}_{t} \dot{\pi}\left(1_{e} \delta_{e}\right)\right|_{H} .
$$

Agora notemos que $\dot{\pi}\left(1_{\epsilon} \delta_{\epsilon}\right)$ é uma projeção ortogonal tal que $\left.P_{H} \dot{\pi}\left(1, \delta_{\epsilon}\right)\right|_{H}=\pi\left(1_{e} \delta_{\epsilon}\right)=i d_{H}$, e portanto é maior o igual que a projeção ortogonal $Q_{H} \in B(\dot{H})$ com imagem $H$. Assim, temos que $Q_{H} \dot{\pi}\left(1_{e} \delta_{e}\right)=Q_{H}=\dot{\pi}\left(1_{e} \delta_{\epsilon}\right) Q_{H}$. Por outro lado, é claro que $P_{H} Q_{H}=P_{H} . Q_{H}=Q_{H} i_{H}$, e portanto temos

$$
\begin{gathered}
P_{H} \dot{\pi}\left(1_{e} \delta_{\epsilon}\right)=\left(P_{H} Q_{H}\right) \dot{\pi}\left(1_{e} \delta_{e}\right)=P_{H}\left(Q_{H} \dot{\pi}\left(1_{e} \delta_{\epsilon}\right)\right)=P_{H} Q_{H}=P_{H}, \\
\dot{\pi}\left(1_{e} \delta_{\epsilon}\right) i_{H}=\dot{\pi}\left(1_{e} \delta_{\epsilon}\right)\left(Q_{H} i_{H}\right)=\left(\dot{\pi}\left(1_{e} \delta_{e}\right) Q_{H}\right) i_{H}=Q_{H} i_{H}=i_{H} .
\end{gathered}
$$

Concluímos entào que:

$$
u_{i}=P_{H} \dot{\pi}\left(1_{e} \delta_{e}\right) \dot{u}_{t} \dot{\pi}\left(1_{e} \delta_{e}\right) i_{H}=P_{H} \dot{u}_{t} i_{H} .
$$

o que encerra a demonstraçào.

Observação 6.28. A hipótese de amenabilidade do grupo ( $i$ foi necessária porque só sabemos que existe equivalencia Morit a entre os produtos cruzados reduziclos. Se também os produtos cruzados plenos fossem equivalentes Morita, então o mesmo resultado valeria para representaçòes parciais de grupos discretos arbitrários. Achamos que tal resultado deve ser verdadeiro. Um possível caminho de demonstração pode ser tentado levando em conta a observaçào a seguir. Suponhamos que $u$ : $G \longrightarrow H$ é uma representação parcial de $G$, e seja $H_{t}$ o espaço final da isometria parcial $u_{t}$, ou seja, $H_{t}=u_{t} u_{t}^{*}(H)$. Para cada $t$ consideremos $\psi_{t}: H_{t^{-1}} \longrightarrow H_{t}$ tal que $\psi_{t}(x)=u_{t}(x)$. Então $\psi_{t}$ é um isomorfismo de espaços de Hilbert, e nào é muito difícil ver que a coleçào $\iota:=\left(\left\{H_{t}\right\}_{t \in G},\left\{\psi_{t}\right\}_{t \in G}\right)$ é uma ação parcial. Portanto, $\psi$ tem uma açào envolvente $\dot{\psi}$, agindo sobre rerto espaço topológico de Hausdorff (porque cada $\psi$ t tem gráfico fechado). No caso de $G$ amenable. e usando a notação de 6.27 , este espaço de Hausdorff deve estar contido em $\dot{H}$, e deve gerá-lo como espaço de Hilbert. 
CAPíTULO 7

Ações Morita Envolventes 
Vimos que nem sempre existe ação envolvente de uma açào parcial sobre uma $C^{*}$-álgebra. No que segue tentaremos contornar este problema, achando uma solução alternativa. Introduziremos uma noção mais fraca de ação envolvente (7.13), porém conseguindo resultados totalmente análogos aos obtidos no capítulo anterior: comparar por exemplo o Teorema $6.6 \mathrm{com}$ a Proposiçào 7.14 .

A idéia do novo conceito surge da seguinte observação. Suponhamos que ( $i$ é um grupo abeliano. e que $\alpha$ é uma açào parcial de $\left(i\right.$ sobre a $C^{\prime *}$-álgebra $A$, que tem uma açào envolvente $\beta$ agindo sobre a $C^{* *}$-álgebra $B$. Pelo Tcorema 6.6 , temos que $A \rtimes_{a} G \stackrel{M}{\sim} B \rtimes_{B} G$. Temos agora a ação dual $\dot{\beta}: \hat{G} \times\left(B \rtimes_{\beta} G\right) \longrightarrow B \rtimes_{\beta}\left(i\right.$ que. quando restrita à subálgebra $A \rtimes_{\alpha}(i$, é exatamente a ação dual a sobre $A \rtimes_{a} G$. Segue. usando resultados de Combes ([14]) ou de (urto. Muhly e Williams ([18]). que teremos

$$
. \rtimes_{n} G \rtimes_{\dot{\alpha}} G \stackrel{M}{\sim} B \rtimes_{\beta} G \rtimes_{\beta} G
$$

Notar que o lado esquerdo da relaçào (10) pode ser definido ainda no caso ell que o par (B. B) náo exista; notar também que o lado direito de (10) é isomorfo a $B \otimes \Lambda^{*}\left(L^{2}\left({ }^{2}(i)\right)\right.$, pelo teorema de Takai ([61]). Em particular. $A \rtimes_{n}\left(i \rtimes_{a i}(i \cdot B\right.$ sào equivalentes Morita quando $B$ existe. Além disto. podemos considerar a açào bidual n sobre $A \rtimes_{\alpha} G \rtimes_{\bar{\alpha}} G$. e, de novo pelo teorema de Takai, teremos que

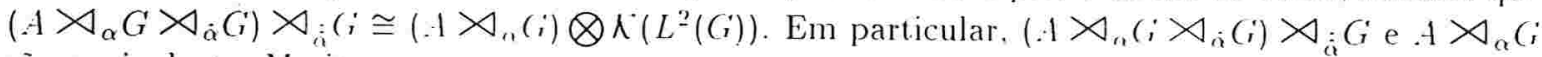
sào equivalentes Morita.

No caso em que ( $i$ nào $e^{\prime}$ abeliano. ainda podemos fazer raciocínios semelhantes, usando desta vez a teoria de dualidade para grupos nào abelianos, concretamente os teoremas de Imai-Takai e de Katayama, que generalizam o teorema de Takai ([40] e [42]: em [6] pode ser achada a versão mais geral de teoremas de dualidade existente no contexto de $C^{*}$-álgebras).

As consideraçōes acima mostram que o par $\left(A \rtimes_{a} G \rtimes_{\dot{a}} G, a\right)$ é um substituto muito adequado para o par $(B, \beta)$ no caso deste nào existir. Porém, gostaríamos de achar uma relaçào mais forte entre estes pares, além dos fatos já notados. ('oncretamente, gostaríamos de ver o par $\left(A \rtimes_{\alpha} G \rtimes_{\dot{\alpha}} G, \alpha\right)$ como a ação envolvente de uma açào parcial "equivalente Morita" com a nossa ação parcial inicial (7.6). Uma tal açào será chamada açào envolvente a menos de equivalencia Morita (7.13). Assim. poderíamos dizer que o problema de achar açòes envolventes de açòes parciais estaria sendo substituído pela seguinte pergunta: toda classe de equivalencia Morita de açóes parciais possui uma classe de equivalência Morita de açōes envolvente? Na primeira seção precisaremos melhor esta pergunta e na segunda veremos que a resposta é afirmativa. Veremos também, na terceira seçào, que a ação envolvente a menos de equivalencia Morita é única, a menos de equivalencia Morita, naturalmente. É um fato conhecido que as ('*álgebras formam uma categoria com os bimódulos de equivalencia como morfismos ([59]). Formalmente. portanto, para um grupo discret.o est amos provando que a açào envolvente de uma açào parcial dada de um grupo discreto sempre existe e é única nesta categoria. $\mathrm{Na}$ quarta seção relacionamos as açōes envolventes a menos de equivalencia Morita com as açōes duais sobre produtos cruzados por coaçōes. conectando a nossa prova da existencia de ação envolvente a menos de equivalencia Morita com a idéia mencionada no começo desta introdução. Finalmente. na última seção estudamos brevemente algumas aplicações; por exemplo. mostramos que uma ação parcial de um grupo conexo sobre uma ('*álgebra com unidade deve ser uma ação global.

\subsection{Açōes parciais equivalentes Morita.}

A seguir definimos açòes parciais equivalentes Morita, e provamos que esta equivalencia Morita é uma relação de equivalencia na classe de açōes parciais contínuas sobre (")-álgebras de um grupo localmente compacto $G$. Também mostramos, usando o Teorema 6.3. que os produtos cruzados reduzidos de açòes parciais equivalentes Morita são, eles também. equivalentes Morita. 
Duas ações parciais $(\alpha, A)$ e $(\beta, B)$ serão ditas equivalentes Morita quando elas sejam induzidas por uma ação parcial sobre um bimódulo de equivalencia entre $A$ e $B$ :

DefiniÇÃo 7.1. Sejam $E$ um $C^{* *}$-anel ternário, e $\alpha=\left(\left\{E_{t}\right\}_{t \in G},\left\{\alpha_{t}\right\}_{t \in G}\right)$ uma ação parcial sobre o conjunto subjacente de $E$. Diremos que $\alpha$ é uma açào parcial do grupo ( $i$ sobre o $C^{*}$-anel ternário $E$ se para todo $t \in G$, tem-se que $E_{t}$ é um ideal de $E$ e $\alpha_{t}: E_{t-1} \longrightarrow E_{t}$ é um isomorfismo de $C^{*}$-anéis ternários. Se $G$ é localmente compacto e $\left\{E_{t}\right\}$ é uma família contínua, e $\mathcal{E}=\left\{(t, x): x \in E_{t-1}\right\} \subseteq$ $G \times E$ com a topologia produto. dizemos que $\alpha$ é uma ação parcial contínua sobre $E$ se a aplicação $\mathcal{E} \longrightarrow E$ tal que $(t, x) \longmapsto \alpha_{t}(x)$ é contínua.

Proposição 7.2 . Si ja $n=\left(\left\{E_{t}\right\}_{\ell \in G},\left\{a_{t}\right\}_{t \in G}\right)$ uma ação parcial do grupo discreto $G$ sobre o $C^{*}$-anel ternário E. Śfjam:

$$
\begin{aligned}
n^{\prime} & =\left(\left\{Z_{l}\left(E_{t}\right)\right\}_{t \in G} \cdot\left\{Z_{l}\left(\alpha_{t}\right)\right\}_{t \in G}\right) \\
n^{r} & =\left(\left\{Z_{r}\left(E_{t}\right)\right\}_{t \in G} \cdot\left\{Z_{r}\left(\alpha_{t}\right)\right\}_{t \in G}\right)
\end{aligned}
$$

Então $\alpha^{l}$ é uma ação parcial de (i sobre $\mathrm{Z}_{l}(E)$. e a ${ }^{r} \dot{\epsilon}$ uma ação parcial de (i sobre $\mathrm{Z}_{r}(E)$.

Demonstraçào. Pela Proposiçào $1.23, Z_{r}\left(E_{t}\right) \triangleleft Z_{r}(E), \forall t \in G$, e pela Proposição 1.18 , temos que cada $Z_{r}\left(\alpha_{t}\right): Z_{r}\left(E_{t-1}\right) \longrightarrow Z_{r}\left(E_{t}\right)$ é um isomorfismo, e $Z_{r}\left(a_{t}\right)=i d_{Z_{r}(E)}$. Usando a Proposiçào 1.24 , é claro que. como $\alpha_{s t}$ ć uma extensào de $\alpha_{s} \alpha_{t}$, entào $Z_{r}\left(\alpha_{s t}\right)$ é uma extensão de $Z_{r}\left(a_{s} \alpha_{t}\right)$. Agora, o domínio de $\alpha_{s} \alpha_{t}$ é $E_{t^{-1}} \bigcap E_{t^{-1} s^{-1}}$, e $\alpha_{s} \alpha_{t}: E_{t-1} \bigcap E_{t^{-1} s^{-1}} \longrightarrow E_{t} \cap E_{s t}$ é um isomorfismo. Por 1.24, o domínio de $Z_{r}\left(a_{s} a_{t}\right)=Z_{r}\left(E_{t-1}\right) \bigcap Z_{r}\left(E_{t-1 s-1}\right)$. Como $\alpha$ é uma açào parcial, também temos por 1.24 que $Z_{r}\left(\alpha_{t}\right)\left(Z_{r}\left(E_{t-1}\right) \cap Z_{r}\left(E_{r}\right)\right)=Z_{r}\left(E_{t}\right) \cap Z_{r}\left(E_{t r}\right)$. Segue daqui que o domínio de $Z_{r}\left(\alpha_{s}\right) Z_{r}\left(\alpha_{t}\right)$ é $Z_{r}\left(E_{t-1}\right) \bigcap Z_{r}\left(E_{t-1} s^{-1}\right)$, e é claro entào que $Z_{r}\left(\alpha_{s}\right) Z_{r}\left(\alpha_{t}\right)=Z_{r}\left(\alpha_{s} \alpha_{t}\right)$. Portanto. $Z_{r}\left(\alpha_{s t}\right)$ é uma extensão de $Z_{r}\left(\alpha_{s}\right) Z_{r}\left(\alpha_{t}\right)$.

OBSERVAÇÃo $T .3$. É claro que toda ação parcial $\gamma$ sobre um ('*-anel ternário $E$ é também uma ação parcial sobre $E^{*}$, o $C^{* *}$-anel ternário adjunto de $E$. Denotando esta ação parcial por $\gamma^{*}$, é claro que $\left(\gamma^{*}\right)^{l}=\gamma^{r}$, e $\left(\hat{\imath}^{*}\right)^{r}=\vartheta^{l}$.

Exemplo 7.4. Se $E$ é um ideal à direita de uma $C^{*}$-álgebra $A$, na qual $G$ age através de uma ação $\beta$, então $\alpha:=\left.\beta\right|_{E}$ é uma açào parcial sobre $E$. e tem-se que $\alpha^{r}=\left.\beta\right|_{\overline{\operatorname{span}} E \cdot E^{*}}$, e $\alpha^{l}=\left.\beta\right|_{\overline{s p a n} E E}$.

Proposição 7.5. Suponhamos que $\gamma=\left(\left\{E_{t}\right\}_{t \in G} \cdot\left\{\alpha_{t}\right\}_{t \in G}\right)$ é uma açào parcial do grupo $G$ sobre $o C^{*}$-anel ternário positivo $E, \epsilon \operatorname{sejam}(\beta, B)=\left(\gamma^{l}, Z_{l}(E)\right),(\alpha, A)=\left(\vartheta^{r}, Z_{r}(E)\right)$. Consideremos as ações parciais $(\tilde{\beta} \cdot \operatorname{Prim}(B)) \in(\bar{\alpha} \cdot \operatorname{Prim}(A))$ induzidas por $\beta$ e a sobre $\operatorname{Prim}(B)$ e $\operatorname{Prim}(A)$ respectivamente, segundo 5.13. Então existe um homeomorfismo natural $h: \operatorname{Prim}(B) \longrightarrow \operatorname{Prim}(A)$ tal que $h: \tilde{\beta} \longrightarrow \tilde{\alpha}$ é um isomorfismo de aşòes parciais. Em particular. $\dot{\beta}$ é uma ação contínua se e somente se $\tilde{\alpha}$ é uma ação contínua. Lim enunciado análogo é válido para as açóes parciais $\hat{\beta}$ e $\hat{\alpha}$ induzidas por $\beta$ e a sobre os respectivos espectros $B \in A$ de $B \in$ A. segundo 5.14 .

Demonstração. Dado $I \triangleleft B$, por $1.23 I$ tem associado um único ideal $h(I)$ de $A: h(I)=$ $Z_{r}(I E)$. É conhecido que se $P \in \operatorname{Prim}(B)$, entāo $h(P) \in \operatorname{Prim}(A)$, e também que é um homeomorfismo (consultar por exemplo 3.3 de [57]; que $h$ é um homeomorfismoé fácil de ver. porque, por $1.24, h$ é um 
isomorfismo entre os reticulados de ideais de $B$ e de $A)$. Em particular, temos que $h\left(Z_{l}\left(E_{t}\right)\right)=Z_{r}\left(E_{t}\right)$, donde segue que $h\left(\mathcal{O}_{t}^{B}\right)=\mathcal{O}_{t}^{A}$. Seja $I \triangleleft B$. Então $\beta_{t}\left(Z_{l}\left(E_{t-1}\right) \cap I\right)$ é um ideal de $Z_{l}\left(E_{t}\right)$, e portanto existe um único $F \triangleleft E_{t}$ tal que $Z_{l}(F)=\beta_{t}\left(Z_{l}\left(E_{t-1}\right) \cap I\right)$. Afirmamos que $F=\gamma_{t}\left(E_{t-1} \cap I E\right)$. Com efeito, $\langle F, F\rangle_{l}=\left\langle\gamma_{t}\left(E_{t^{-1}} \cap I E\right), \gamma_{t}\left(E_{t^{-1}} \cap I E\right)\right\rangle_{l}=\beta_{t}\left(\left\langle E_{t^{-1}} \cap I E, E_{t^{-1}} \cap I E\right\rangle_{l}\right)$, e portanto $Z_{l}(F)=$ $\beta_{t}\left(Z_{l}\left(E_{t-1}\right) \cap I\right)$. Da mesma forma, se $J \triangleleft A$, tem-se que $\alpha_{t}\left(Z_{r}\left(E_{t-1} \cap J\right)=Z_{r}\left(\gamma_{t}\left(E_{t-1} \cap E J\right)\right)\right.$. Agora, se $P \in \mathcal{O}_{t-1}^{B}, \tilde{\beta}_{t}(P)=Q$. onde $Q \in \operatorname{Prim}(B)$ é o único tal que $Q \cap Z_{l}\left(E_{t}\right)=\beta_{t}\left(Z_{l}\left(E_{t-1}\right) \cap P\right)$. Usando de novo o fato de que $h$ é um isomorfismo de reticulados, temos que $h(Q) \cap h\left(Z_{l}\left(E_{t}\right)\right)=h\left(\beta_{t}\left(Z_{l}\left(E_{t-1}\right) \cap P\right)\right.$. Ou seja,

$$
h(Q) \cap Z_{r}\left(E_{t}\right)=Z_{r}\left(E_{t-1} \cap P E\right)=\alpha_{t}\left(Z_{r}\left(E_{t-1}\right) \cap Z_{r}(P E)\right)=\alpha_{t}\left(Z_{r}\left(E_{t-1}\right) \cap h(P)\right) .
$$

Portanto, como $h(Q)$ é primitivo. deve coincidir com $\tilde{\alpha}_{t}(h(P))$. Logo, $h: \dot{3} \longrightarrow \tilde{\alpha}$ é um morfismo de açōes parciais, tal que $h\left(\operatorname{dom}\left(\dot{\beta}_{s}\right)\right)=\operatorname{dom}\left(\tilde{\alpha}_{s}\right), \forall s \in G$, e portanto é um isomorfismo (ver 4.10). Como $h$ é um homeomorfismo, entào $\dot{\beta}$ é contínua se e somente se à é contínua.

A prova do correspondente fato para $\beta$ e $\alpha$ é similar será omitida.

DEFINIÇÃo 7.6. Sejam $a=\left(\left\{f_{t}\right\}_{t \in G_{i}},\left\{\alpha_{t}\right\}_{t \in G}\right)$ e $\beta=\left(\left\{B_{t}\right\}_{t \in G} .\left\{\beta_{t}\right\}_{t \in G}\right)$ açòes parciais contínuas do grupo localmente compacto $G$ sobre as $C^{*}$-álgebras $A$ e $B$ respectivamente. Dizemos que $\alpha$ é equivalente Morita com 3 se existe uma ação parcial contínua $\gamma=\left(\left\{E_{t}\right\}_{t \in G},\left\{\gamma_{t}\right\}_{t \in G}\right)$ sobre um $C^{*}$ anel ternário positivo $E$. tal que $\gamma^{\prime}=\alpha$, e $\gamma^{r}=\beta$. O par $(E, \gamma)$ será chamado sistema parcial de equivalência Morita. Usaremos a notação $\alpha \stackrel{M}{\sim} \beta$ para indicar que $a$ é equivalente Morita com $\beta$.

Observação 7.7. Em [14], Combes definiu ações equivalentes Morita. Quando $\alpha$ e $\beta$ são ações globais na Definiçào 7.6 . ₹ é necessariamente uma ação global, e portanto nossa definição de equivalência Morita coincide com a definição de Combes neste caso. Notar que, de fato, para que $\alpha, \beta$ e $\gamma$ sejam ações globais. é necessário e suficiente que apenas uma delas seja uma ação global.

Lema 7.8. Sejam $E$ um $C^{*}$-anel ternário $\epsilon A=Z_{r}(E)$. SE $\left\{D_{t}\right\}_{t \in G} \dot{\epsilon}$ uma família contínua de ideais bilaterais em $\mathrm{A}$. entào $\left\{E_{t}\right\}_{t \in G}$ é uma família contínua de ideais bilaterais em $E$, onde $E_{t}=E D_{t}, \forall t \in G$.

Demonstração. Sejam $U \subseteq E$ aberto, e $G_{U}=\left\{s \in G: U \cap E_{s} \neq \emptyset\right\}$, e suponhamos que $t \in G_{U}$. Seja $x \in U \cap E_{t}$. Então existem $y \in E, a \in D_{t}$ tais que $x=y a$. Como a ação de $A$ sobre $E$ é contínua, existem $V \subseteq E$. e $W \subseteq A$ abertos, tais que $y \in V, a \in W$, e $V W \subseteq U$. Agora, $a \in W \cap D_{t}$, e como $\left\{D_{s}\right\}_{s \in G}$ é contínua, o conjunto $G_{W}=\left\{s \in G: W \cap D_{s} \neq \emptyset\right\}$ é aberto e contém $t$. Seja, para cada $s \in G_{W}, a_{s} \in W \cap D_{s}$. Então $x a_{s} \in E_{s} \cap V W \subseteq E$, donde segue que $t \in G_{W} \subseteq G_{U}$, e portanto $G_{U}$ é aberto.

Veremos a seguir que a equivalencia Morita de ações parciais é uma relação de equivalência na classe de ações parciais. Lembremos que se $E$ é um $(A-B)$ bimódulo de Hilbert e $F$ é um $(B-C)$ bimódulo de Hilbert, então podemos construir um $(A-C)$ bimódulo de Hilbert $E \bigotimes_{B} F$ chamado produto tensorial interno. A construção deste bimódulo é feita assim: se toma primeiro o produto tensorial algébrico $E \odot F$. e se considera sobre $E \odot F$ a única $C$-forma sesquilinear $\langle\cdot, \cdot\rangle_{r}^{\prime}$ tal que $\left\langle x_{1} \odot y_{1}, x_{2} \odot y_{2}\right\rangle=\left\langle y_{1},\left\langle x_{1}, x_{2}\right\rangle_{B} y_{2}\right\rangle_{C}$, onde $\langle\cdot, \cdot\rangle_{C}$ é o $C$-produto interno sobre $F$, e $\langle\cdot, \cdot\rangle_{B}$ é o $B$-produto interno sobre $E$; esta forma sesquilinear é um semi-produto interno, e então $E \bigotimes_{B} F$ é o completamento de $(E \odot F) / N^{\wedge}$ com respeito ao produto interno induzido no quociente, onde 
$N=\left\{z \in E \odot F:\langle z, z\rangle_{r}^{\prime}=0\right\}$. De forma análoga se procede para definir a estrutura de $A$-módulo de Hilbert sobre $E \bigotimes_{B} F$. O ponto crucial é que o submódulo de $E \odot F$ onde se anula $\langle\cdot, \cdot\rangle_{l}^{\prime}$ coincide com $N$ : de fato, $N=\operatorname{span}\{x b y-x b y: x \in E, y \in F, b \in B\}$ (consultar [59] e [43]). Indicaremos por $x \oslash y$ a projeçào de $x \cdot y$ sobre $E \bigotimes_{B} F$.

Lema 7.9. Sejam $E_{1}, F_{1}(A-B)$ bimódulos de Hilbert. $E_{2}, F_{2} B-(\cdot$ bimódulos de Hilbert. $\epsilon$ sejam $\mu_{1}: E_{1} \longrightarrow F_{1}, \mu_{2}: E_{2} \longrightarrow F_{2}$ morfismos de $C^{*}$-anéis ternários (onde consideramos sobre $E_{1}, E_{2}, F_{1}, \in F_{2}$ as estruturas de $\left(^{\prime *}\right.$-anéis ternários induzidas pelas suas estruturas de bimódulos de Hilbert). Então, se $Z_{r}\left(\mu_{1}\right)=Z_{1}\left(\mu_{2}\right)$. existe um único morfismo de $C^{*}$-anéis ternários $\mu_{1} \otimes_{B} \mu_{2}$ : $E_{1} \otimes_{B} E_{2} \longrightarrow F_{1} \otimes_{B} F_{2}$ tal que $\left(\mu_{1} \ominus_{B} \mu_{2}\right)\left(x_{1} \otimes x_{2}\right)=\mu_{1}\left(x_{1}\right) \odot \mu_{2}\left(x_{2}\right), \forall x_{1} \in E_{1}, x_{2} \in E_{2}$. Se $\mu_{1}$ e $\mu_{2}$ são isomorfismos, entào $\mu_{1} \circlearrowleft_{B} \mu_{2}$ também é um isomorfismo. Além disto, teremos que $Z_{l}\left(\mu_{1} \otimes_{B} \mu_{2}\right)=Z_{l}\left(\mu_{1}\right) . \in Z_{r}\left(\mu_{1} \ominus_{B} \mu_{2}\right)=Z_{r}\left(\mu_{2}\right)$.

Demonstraçío. Seja $\mu_{1} \mu_{2}: E_{1} \odot E_{2} \longrightarrow F_{1} \odot F_{2}$ a única transformação linear tal que $x_{1} \odot x_{2} \longmapsto \mu_{1}\left(x_{1}\right): \mu_{2}\left(x_{2}\right), \forall x_{1} \in E_{1}, x_{2} \in E_{2}$, que é um morfismo de ('*-anéis ternários. Sejam

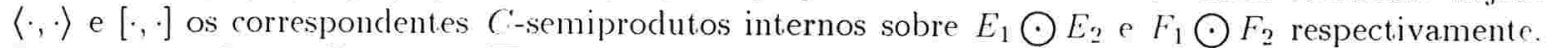
Tomemos $z, z^{\prime} \in E_{1} \odot E_{2}, z=\sum_{i} x_{i}=y_{i}, z^{\prime}=\sum_{j} x_{j}^{\prime} \odot y_{j}^{\prime}$. Entào, com notaçào óbvia, temos:

$$
\begin{aligned}
{\left[\left(\mu_{1} \odot \mu_{2}\right)(z),\left(\mu_{1} \mu_{2}\right)\left(z^{\prime}\right)\right] } & =\left[\sum_{i}\left(\mu_{1} \odot \mu_{2}\right)\left(x_{i} \odot y_{i}\right), \sum_{j}\left(\mu_{1} \odot \mu_{2}\right)\left(x_{j}^{\prime} \odot y_{j}^{\prime}\right)\right] \\
& =\sum_{i, j}\left[\mu_{1}\left(x_{i}\right) \odot \mu_{2}\left(y_{i}\right), \mu_{1}\left(x_{j}^{\prime}\right) \odot \mu_{2}\left(y_{j}^{\prime}\right)\right] \\
& =\sum_{i, j}\left\langle\mu_{2}\left(y_{i}\right),\left\langle\mu_{1}\left(x_{i}\right), \mu_{1}\left(x_{j}^{\prime}\right)\right\rangle_{B}^{F_{1}} \mu_{2}\left(y_{j}^{\prime}\right)\right\rangle_{C}^{F_{2}} \\
& =\sum_{i, j}\left\langle\mu_{2}\left(y_{i}\right), Z_{r}\left(\mu_{1}\right)\left(\left\langle x_{i}, x_{j}^{\prime}\right\rangle_{B}^{E_{1}}\right) \mu_{2}\left(y_{j}^{\prime}\right)\right\rangle_{C}^{F_{2}} \\
& =\sum_{i, j}\left\langle\mu_{2}\left(y_{i}\right), Z_{l}\left(\mu_{2}\right)\left(\left\langle x_{i}, x_{j}^{\prime}\right\rangle_{B}^{E_{1}}\right) \mu_{2}\left(y_{j}^{\prime}\right)\right\rangle_{C}^{F_{2}} \\
& =\sum_{i, j}\left\langle\mu_{2}\left(y_{i}\right), \mu_{2}\left(\left\langle x_{i}, x_{j}^{\prime}\right\rangle_{B}^{E_{1}} y_{j}^{\prime}\right)\right\rangle_{C}^{F_{2}} \\
& =\sum_{i, j} Z_{r}\left(\mu_{2}\right)\left(\left\langle y_{i},\left\langle x_{i}, x_{j}^{\prime}\right\rangle_{B}^{E_{1}} y_{j}^{\prime}\right\rangle_{C}^{E_{2}}\right) \\
& =Z_{r}\left(\mu_{2}\right)\left(\sum_{i, j}\left\langle y_{i},\left\langle x_{i}, x_{j}^{\prime}\right\rangle_{B}^{E_{1}} y_{j}^{\prime}\right\rangle_{C}^{E_{2}}\right) \\
& =Z_{r}\left(\mu_{2}\right)\left(\sum_{i, j}\left\langle x_{i} \odot y_{i}, x_{j}^{\prime} \odot y_{j}^{\prime}\right\rangle\right) \\
& =Z_{r}\left(\mu_{2}\right)\left(\left\langle z, z^{\prime}\right\rangle\right)
\end{aligned}
$$

Tomando $z=z^{\prime}$ na equação (11), e calculando normas, temos

$$
\left\|\left(\mu_{1} \odot \mu_{2}(z)\right)\right\|^{2}=\left\|Z_{r}\left(\mu_{2}\right)(\langle z, z\rangle)\right\| \leq\|z\|^{2} .
$$

Assim, vemos que $\mu_{1} \odot \mu_{2}$ passa aos quocientes, onde é uma contração, e portanto se estende por continuidade a uma aplicaçào $\mu_{1} \Theta_{B} \mu_{2}: E_{1} \otimes_{B} E_{2} \longrightarrow F_{1} \bigotimes_{B} F_{2}$, que é um homomorfismo de $C^{*}$-anéis ternários. e que satisfaz, $\forall z, z^{\prime} \in E_{1} \bigotimes_{B} E_{2}$ :

$$
\left[\left(\mu_{1} \otimes_{B} \mu_{2}\right)(z),\left(\mu_{1} \otimes_{B} \mu_{2}\right)\left(z^{\prime}\right)\right]=Z_{r}\left(\mu_{2}\right)\left(\left\langle z, z^{\prime}\right\rangle\right),
$$

donde concluímos que $Z_{r}\left(\mu_{1} \odot_{B} \mu_{2}\right)=Z_{r}\left(\mu_{2}\right)$; analogamente, tem-se que $Z_{l}\left(\mu_{1} \Theta_{B} \mu_{2}\right)=Z_{l}\left(\mu_{1}\right)$. 
Por último, se $\mu_{1}, \mu_{2}$ são isomorfismos, podemos aplicar a primeira parte aos mapas $\mu_{1}^{-1}$ e $\mu_{2}^{-1}$, e notar que $i d_{E_{1}} \odot i d_{E_{2}}=i d_{E_{1} \otimes_{B} E_{2}}$, e $i d_{F_{1}} i d_{F_{2}}=i d_{F_{1}} \otimes_{B} F_{2}$

Proposição 7.10. A equivaléncia Morita de açòes parciais é uma relação de equivaléncia. 7.3.

Demonstração. A propriedade reflexiva é óbvia, e a propriedade recíproca segue da Observação

Suponhamos agora que $a=\left(\left\{A_{t}\right\} .\left\{a_{t}\right\}\right)$ é uma ação parcial contínua de $G$ sobre $A, \beta=$ $\left(\left\{B_{t}\right\},\left\{\beta_{t}\right\}\right)$ é uma açào parcial contínua de $C_{i}$ sobre $B$, e que $\gamma=\left(\left\{C_{t}\right\}\right.$. $\left.\left\{\gamma_{t}\right\}\right)$ é uma ação parcial contínua de $G$ sobre $\left(\therefore\right.$ tais que $a \stackrel{M}{\sim} \beta$ através da açào parcial contínua $\mu=\left(\left\{E_{t}\right\},\left\{\mu_{t}\right\}\right)$ de $G$ sobre o $C^{*}$-anel ternário $E$. e $\beta \stackrel{M}{\sim} \gamma$ através da ação parcial contínua $\nu=\left(\left\{F_{t}\right\},\left\{\nu_{t}\right\}\right)$ de $G$ sobre o $C^{\prime *}$-anel ternário $F$. Consideremos a família $\mu_{B} \nu=\left(\left\{E_{t} \bigotimes_{B} F_{t}\right\},\left\{\mu_{t} \quad \nu_{B} \nu_{t}\right\}\right.$ ). Como $\mu_{r s}$ estende $\mu_{r} \mu_{s}$ e $\nu_{r s}$ estende $\nu_{r} \nu_{s}$, entào $\mu_{r s} \quad B \nu_{r s}$ estende $\left(\mu_{r} \odot B \nu_{r}\right)\left(\mu_{s} \circlearrowleft_{B} \nu_{s}\right)$. É claro também que $E_{\epsilon} \otimes_{B} F_{\epsilon}=E \bigotimes_{B} F,\left(\mu \nu_{s}=i d_{E \odot B F}\right.$. Por outro lado, $Z_{r}\left(E_{t} \otimes_{B} F_{t}\right)=C_{t}$ :

$$
\begin{aligned}
\overline{\operatorname{san}}\left\langle E_{t} \bigotimes_{B} F_{t}, E_{t} \bigotimes_{B} F_{t}\right\rangle_{C} & =\overline{\operatorname{span}}\left\langle F_{t},\left\langle E_{t}, E_{t}\right\rangle_{B} F_{t}\right\rangle_{C} \\
& =\overline{\operatorname{span}}\left\langle F_{t}, B_{t} F_{t}\right\rangle_{C} \\
& =\overline{\operatorname{span}}\left\langle F_{t}, F_{t}\right\rangle_{C} \\
& =C_{t}
\end{aligned}
$$

Analogamente, $Z_{l}\left(E_{t} \bigotimes_{B} F_{t}\right)=A_{1}$. Por último. pelo Lema 7.9, cada $\mu_{t} \nu_{t}: E_{t}^{-1} \bigotimes_{B} F_{t}^{-1} \longrightarrow$ $E_{t} \otimes_{B} F_{t}$ é um isomorfismo, e $Z_{l}\left(\mu_{t} \otimes_{B} \nu_{t}\right)=Z_{l}\left(\mu_{t}\right)=a_{t}, Z_{r}\left(\mu_{t} \Theta_{B} \nu_{t}\right)=Z_{r}\left(\nu_{t}\right)=\gamma_{t}$, de forma que $\mu \Theta_{B} \nu$ é uma ação parcial sobre $E \bigotimes_{B} F$, e $\left(\mu_{B} \nu\right)^{l}=\alpha,\left(\mu \odot_{B} \nu\right)^{r}=\gamma$.

Só está faltando mostrar que $\mu_{B} \nu$ é contínua. A família $\left\{E_{t} \otimes_{B} F_{t}\right\}_{t \in G}$ é contínua pelo Lema 7.8 , já que $\gamma$ é contínua. Seja $\mathcal{E} \otimes_{B} \mathfrak{F}=\left\{(t, z): z \in E_{t-1} \bigotimes_{B} F_{t-1}\right\}$. Por último, vejamos que $\mu \otimes_{B} \nu$ é contínua. Em primeiro lugar, notar que, se $f \in C_{c}(\mathcal{E}), g \in C_{c}(\mathfrak{F})$, entào $f \widehat{\Theta}_{B} g: G \longrightarrow \mathcal{E} \otimes_{B} \mathfrak{F}$ tal que $\left(f \odot_{B} g\right)(t)=(t, f(t) g(t))$ é uma seçào contínua do fibrado de Banach $\mathcal{E} \bigotimes_{B} \mathfrak{F}$. e para cada

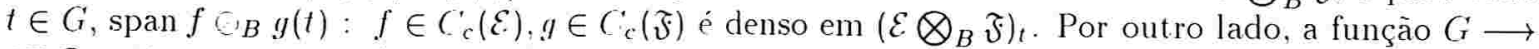
$\left(E \bigotimes_{B} F\right)$ tal que $t \longmapsto\left(t, \mu_{t}(f(t)) \nu_{t}(g(t))\right)$ é contínua, $\forall f \in C_{c}(\mathcal{E}), g \in C_{c}(\Im)$; concluímos, usando [37], II-14.6 e II-13.16, que a aplicaçào $\mathcal{E} \otimes_{B} \mathfrak{J} \longrightarrow G \times\left(E \bigotimes_{B} F\right)$ tal que $(t, x) \longmapsto\left(t,\left(\mu_{t} \otimes \nu_{t}\right)(x)\right)$ é um homomorfismo contínuo de fibrados de Banach, e portanto que $\mu_{B} \nu$ é uma ação parcial contínua.

Assim, temos que $\alpha \stackrel{M}{\sim}$

Proposição 7.11. Sejam $\alpha=\left(\left\{A_{t}\right\}_{t \in G} \cdot\left\{\alpha_{t}\right\}_{t \in G}\right)$ e $\beta=\left(\left\{B_{t}\right\}_{t \in G},\left\{\beta_{t}\right\}_{t \in G}\right)$ ações parciais do grupo localmente compacto $G$ sobre as $C^{*}$-álgebras $A$ e $B$ respectivamente. Se $\alpha \in \beta$ são equivalentes Morita, então $A \rtimes_{\mathrm{a}, r} G \stackrel{M}{\sim} B \rtimes_{B, r} G$.

DemonstraçÃo. Suponhamos que $\alpha$ e 3 sào equivalentes Morita através de $\gamma=\left(\left\{E_{t}\right\},\left\{\gamma_{t}\right\}\right)$; digamos que $\gamma^{\prime}=a$. e $\gamma^{r}=\beta$. Seja $\mathbb{L}(E)$ a álgebra vinculante de $E$ : $\mathbb{L}(E)=\left(\begin{array}{cc}A & E \\ E^{*} & B\end{array}\right)$ (ver a página 21). Como $A$ e $\mathbb{L}(E)$ são equivalentes Morita através do A-módulo de Hilbert à esquerda $A \oplus E$, cada ideal $A_{t}$ de $A$ é Morita equivalente com um certo ideal de $\mathbb{L}(E)$, gerado pelos elementos do tipo $\left(\begin{array}{cc}x_{t} & x_{t}^{\prime} \epsilon \\ 0 & 0\end{array}\right){ }^{*}\left(\begin{array}{cc}y_{t} & y_{t}^{\prime} f \\ 0 & 0\end{array}\right)=\left(\begin{array}{cc}x_{t}^{*} y_{t} & x_{t}^{*} y_{t}^{\prime} f \\ x_{t}^{\prime} e y_{t} & \left\langle x^{\prime} \epsilon \cdot y_{t}^{\prime} f\right\rangle_{r}\end{array}\right)$. Este ideal é $\left(\begin{array}{cc}A_{t} & E_{t} \\ E_{t}^{*} & B_{t}\end{array}\right)$, ou seja, a álgebra vinculante $\mathbb{L}\left(E_{t}\right)$ de $E_{t}$. é claro que a família $\left(-\left(E_{t}\right)\right)_{t \in G}$ é contínua, porque $\left(A_{t}\right)$, $\left(E_{t}\right)$, $\left(E_{t}^{*}\right)$ e $\left(B_{t}\right)$ são contínuas. 
Definamos agora $\mathbb{L}\left(\gamma_{t}\right): \mathbb{L}\left(E_{t-1}\right) \longrightarrow \mathbb{L}\left(E_{t}\right)$, através de

$$
\mathbb{L}\left(\gamma_{t}\right):\left(\begin{array}{ll}
a_{t-1} & x_{t-1} \\
y_{t-1} & b_{t-1}
\end{array}\right) \longmapsto\left(\begin{array}{cc}
\alpha_{t}\left(a_{t-1}\right) & \gamma_{t}\left(x_{t-1}\right) \\
\gamma_{t}\left(y_{t-1}\right) & \beta_{t}\left(b_{t-1}\right)
\end{array}\right) .
$$

É fácil ver que, como $\gamma_{t}\left(a_{t-1} x_{t-1}\right)=\alpha_{t}\left(a_{t-1}\right) \gamma_{t}\left(x_{t-1}\right)$ e $\gamma_{t}\left(x_{t-1} b_{t-1}\right)=\gamma_{t}\left(x_{t-1}\right) \beta_{t}\left(b_{t-1}\right), \forall x_{t-1} \in E_{t-1}$, $a_{t-1} \in A_{t-1}, b_{t-1} \in B_{t-1}$, então $\mathbb{L}(\gamma)=\left(\left\{E_{t}\right\}_{t \in G},\left\{\mathbb{L}\left(\gamma_{t}\right)\right\}_{t \in G}\right)$ é uma ação parcial de $G$ sobre $\mathbb{L}(E)$, e que $\mathbb{L}(\gamma)$ restrita a $\left(\begin{array}{cc}A & 0 \\ 0 & 0\end{array}\right)$ é isomorfa a $\alpha$, restrita a $\left(\begin{array}{ll}0 & E \\ 0 & 0\end{array}\right)$ é isomorfa a $\gamma$, e restrita a $\left(\begin{array}{ll}0 & 0 \\ 0 & B\end{array}\right)$ é isomorfa a $\beta$. Concluímos que a é equivalente Morita com $\mathbb{L}(\gamma)$. Da mesma forma, basta restringir $\mathbb{L}(\gamma)$ a $\left(\begin{array}{cc}0 & 0 \\ E^{*} & B\end{array}\right)$ para ver que ela é equivalente a $\beta$. Diremos que $\mathbb{L}(\gamma)$ é a ação parcial vinculante de $\gamma$.

As consideraçòes acima mostram que podemos supor que $A=p B p$, para certa projeçào $p \in M(B)$. e tal que $a$ e $\gamma$ sào as restriçòes de $\beta$ a $p B p=A$ e a $E=p B$ respectivamente. Sejam agora $\mathcal{A}$ e $\mathcal{B}$ os fibrados de Fell correspondentes a $\alpha$ e $\beta$ respectivamente, e seja $\mathcal{E}=\left(\{t\} \times E_{t}\right)_{t \in G}$. Temos então que $\mathcal{A} \subseteq \mathcal{E} \subseteq \mathcal{B}$, $\mathcal{A}$ é um subfibrado de Fell de $\mathcal{B}$, e $\mathcal{E}$ é um subfibrado de Banach de $\mathcal{B}$. Por outro lado, se $\left(r, a_{r}\right) \in \mathcal{A},\left(s, x_{s}\right),\left(t, y_{t}\right) \in \mathcal{E}$, e $\left(u, b_{u}\right) \in \mathcal{B}$, temos:

- $\left(r, a_{r}\right)\left(s, x_{s}\right)=\left(r s, \beta_{r}\left(\beta_{r}^{-1}\left(a_{r}\right) x_{s}\right)\right) \in\left(r s, \beta_{r}\left(A_{r-1} E_{s}\right)\right) \in \mathcal{E}$.

- $\left(s, x_{s}\right)\left(u, b_{u}\right)=\left(s u, \beta_{s}\left(\beta_{s}^{-1}\left(x_{s}\right) b_{u}\right)\right) \in\left(s u, \beta_{s}\left(E_{s-1} B_{u}\right)\right) \in \mathcal{E}$.

- $\left(s, x_{s}\right)\left(t, y_{t}\right)^{*}=\left(s, x_{s}\right)\left(t^{-1}, \beta_{t-1}\left(y_{t}^{*}\right)\right)=\left(s t^{-1}, \beta_{s}\left(\beta_{s-1}\left(x_{s}\right) \beta_{t-1}\left(y_{t}^{*}\right)\right)\right)$, que pertence a $\left(s t^{-1}, \beta_{s}\left(A_{s-1} \cap A_{t-1}\right)\right) \subseteq\left(s t^{-1}, A_{s} \cap A_{s t-1}\right) \in \mathcal{A}$.

- $\left(s, x_{s}\right)^{*}\left(t, y_{t}\right)=\left(s^{-1}, \beta_{s-1}\left(x_{s}^{*}\right)\right)\left(t, y_{t}\right)=\left(s^{-1} t, \beta_{s^{-1}}\left(x_{s}^{*} y_{t}\right)\right)$. Agora, dado $t \in G$, temos que $\overline{\operatorname{span}}\left\{\beta_{s^{-1}}\left(x_{s}^{*} y_{t}\right): s \in G, x_{s} \in E_{s}, y_{t} \in E_{t}\right\}=B_{t}$ : o membro esquerdo da igualdade acima contém $\overline{\operatorname{span}} E_{e}^{*} E_{t}=\overline{\operatorname{span}} E^{*} E_{t} \supseteq \overline{\operatorname{span}} E_{t}^{*} E_{t}=B_{t}$.

Portanto, estamos nas condições do Corolário 6.4 , donde concluímos que $C_{r}^{\prime *}(\mathcal{A}) \stackrel{M}{\sim} C_{r}^{*}(\mathcal{B})$, ou seja $A \rtimes_{\alpha, r} G \stackrel{M}{\sim} B \rtimes_{\beta, r} G$.

OBservaçȦo 7.12. Parece razoável esperar um resultado similar a 7.11 para os correspondentes produtos cruzados plenos. Porém, não conseguimos provar este resultado. As dificuldades são as mesmas que aparecem quando queremos comparar $A \rtimes_{\alpha} G$ e $\dot{A} \rtimes_{\dot{\alpha}} G$, onde $(\dot{\alpha}, \dot{A})$ é a ação envolvente de $(\alpha, A)$. Morita

Tendo chegado a este ponto, introduzimos a noção de ação envolvente a menos de equivalencia

DEFIniçÃo 7.13. Seja $\alpha$ uma ação parcial contínua do grupo localmente compacto $G$ sobre a $C^{*}$ álgebra $A$. Diremos que uma açào contínua $\beta$ de $G$ sobre uma $C^{*}$-álgebra $B$ é uma ação envolvente a menos de equivaléncia Morita de $\alpha$, se existe um ideal $I \triangleleft B$ tal que $[\beta(I)]$ é denso em $B$ e $\left.\alpha \stackrel{M}{\sim} \beta\right|_{I}$.

Encerramos a seçào provando o resultado correspondente ao Teorema 6.6 no Capítulo 6: se $(\beta, B)$ é uma ação envolvente a menos de equivaléncia Morita de $(\alpha, A)$, então $A \rtimes_{\alpha, r} G \stackrel{M}{\sim} B \rtimes_{\beta, r} G$. 
ProposiçÃo 7.14 . Seja $(A, a)$ uma ação parcial contínua do grupo localmente compacto $G, e$ seja $(B, \beta)$ uma açào envolvente a menos de equivalência Morita de a. Então:

$$
A \rtimes_{\alpha, r} G \stackrel{M}{\sim} B \rtimes_{\beta, r} G .
$$

Demonstração. ('omo $(B, \beta)$ é uma ação envolvente a menos de equivalencia Morita de $a$, existe um ideal $I$ de $B$ tal que $\left.\beta\right|_{I} \stackrel{M}{\sim}$ a, e $\overline{[\beta(I)]}=B$. Pela Proposiçào $7.11 .\left..1 \rtimes_{a, r} G \stackrel{M}{\sim} I \searrow_{\beta}\right|_{I}, r$. Como $\beta$ é a ação envolvente de $\left.\beta\right|_{I}$. temos que $\left.I \rtimes_{\beta}\right|_{I}, r \stackrel{M}{\sim} B \rtimes_{B, r} G$, por 6.6. Como a equivalencia Morita de $C^{*}$-álgebras é transitjva, concluímos que $A \rtimes_{\alpha, r} G \stackrel{M}{\sim} B \rtimes_{\beta, r} C_{r}$.

\section{2. ("'álgebras de núcleos associadas a um fibrado de Fell}

Na próxima seçào provaremos que sempre existe uma ação envolvente a menos de equivalencia Morita de uma açào parcial clada. De fato, se $\alpha$ é uma açào parcial de $G$ sobre a $C^{*}$-álgebra $A$, mostraremos que existe uma forma funtorial de achar uma ação envolvente a menos de equivalência Morita. Descrevamos rapidamente as construções envolvidas. Dada $a$, ela tem um fibrado de Fell associado $B_{\alpha}=\left(B_{t}\right)_{t \in G}(2.15)$. Agora, existem várias álgebras ligadas naturalmente a $\mathcal{B}_{\alpha}$, que sào diversos completamentos de $\mathbb{k}_{c}\left(\mathcal{B}_{\alpha}\right)=\left\{k: G \times\left(i \longrightarrow \mathcal{B}_{\alpha}: k(r, s) \in\right.\right.$ $B_{r s^{-1}}, \forall r, s \in G$, e $k$ é contínua e de suporte compacto\}. Sobre $\mathbb{k}_{c}\left(\mathcal{B}_{a}\right)$ existe uma ação natural $\bar{a}$ de $G:\left.\check{\alpha}_{t}(k)\right|_{(r, s)}=\Delta(t) k(r t . s t)$. onde $\Delta$ é a função modular de $G$. Existe um *-homomorfismo injetivo $\Omega: \mathbb{k}_{c}\left(\mathcal{B}_{\alpha}\right) \longrightarrow \mathcal{L}\left(L^{2}\left(\mathcal{B}_{\alpha}\right)\right)$ : completando $\Omega\left(\mathbb{k}_{c}\left(\mathcal{B}_{\alpha}\right)\right)$ em $\mathcal{L}\left(L^{2}\left(\mathcal{B}_{a}\right)\right)$, obtemos $\mathbb{F}_{r}\left(\mathcal{B}_{\alpha}\right)$, que chamamos $C^{*}$-álgebra reduzida dos núcleos de $\mathcal{B}_{\alpha}$. A ação $\bar{\alpha}$ se estende a $\mathbb{K}_{r}\left(\mathcal{B}_{\alpha}\right)$, e o par $\left(\mathbb{K}_{r}\left(\mathcal{B}_{\alpha}\right), \check{a}\right)$ é uma ação envolvente a menos de equivalencia Morita de $\alpha$.

A construção descrita acima pode ser feita para qualquer fibrado de Fell. Este é o objetivo da presente seção. Seja $\underline{K}_{c}(B)$ o conjunto das funções contínuas e de suporte compacto $k: G \times G \longrightarrow$ $\mathcal{B}$, tais que $k(r, s) \in B_{r, s^{-1}}, \forall r, s \in \mathcal{G}$. Entào $\mathbb{R}_{c}(\mathcal{B})$ é uma *álgebra normada com as operaçōes: $k_{1} * k_{2}(r, s):=\int_{G} k_{1}(r . t) k_{2}(t . s) d t . k^{*}(r, s):=k(s, r)^{*}$, e a norma $\|k\|_{2}^{2}=\int_{C i \times C}\|k(r, s)\|^{2} d(r, s)$. Além disso, cada $k \in \mathbb{R}_{c}(\mathcal{B})$ age sobre $C_{c}(\mathcal{B})$ como um operador integral: $\left.k \xi\right|_{r}=\int_{G} k(r, s) \xi(s) d s, \forall \xi \in C_{c}(\mathcal{B})$. Por esta razão, $k$ é chamado núcleo do fibrado $\mathcal{B}$, e $\mathbb{R}_{c}(\mathcal{B})$ é a álgebra dos núcleos contínuos de $\mathcal{B}$. Existe uma aplicação sesquilinear $\langle\cdot,\rangle_{l}: C_{c}(\mathcal{B}) \times C_{c}(\mathcal{B}) \longrightarrow \mathbb{k}_{c}(\mathcal{B})$, dada por $\langle\xi, \eta\rangle_{l}(r, s)=\xi(r) \eta(s)^{*}$. Seja $I_{c}(\mathcal{B}):=\operatorname{span}\left\langle C_{c}(\mathcal{B}), C_{c}(\mathcal{B})\right\rangle_{l} \subseteq \mathcal{Z}_{c}(\mathcal{B})$. e seja $E=L^{2}(\mathcal{B})$. Então é fácil ver que existe uma inclusão: $I_{c}(\mathcal{B}) \hookrightarrow \mathcal{K}(E)$, de imagem densa. De fato, com um pouco mais de trabalho, é possível mostrar que existe um *-homomorfismo injetivo $\Omega: \mathbb{k}_{c}(\mathcal{B}) \longrightarrow \mathcal{L}(E)$, único tal que $\Omega_{k} \xi=k \xi, \forall k \in \mathbb{k}_{c}(\mathcal{B})$ e $\forall \xi \in$ $C_{c}(\mathcal{B})$. A $C^{*}$-álgebra reduzida dos núcleos de $\mathcal{B}$ é definida entào como: $\mathbb{K}_{r}(\mathcal{B}):=\overline{\Omega\left(\mathbb{R}_{c}(\mathcal{B})\right)} \subseteq \mathcal{L}(E)$. Também usamos a notaçào $I_{r}$ para denotar $\overline{\Omega\left(C_{c}(\mathcal{B})\right)} \subseteq \mathcal{L}(E)$ (i.e. : $I_{r}=\mathcal{E}$ ).

Sobre $\mathbb{k}_{c}(\mathcal{B})$ existe uma açào natural $\beta$ de $G$, dada por $\left.\beta_{t}(k)\right|_{(r, s)}=\Delta(t) k(r t$, st), onde $\Delta$ é a função modular do grupo $G$. Tem-se que a $\beta$-órbita linear de $I_{c}(\mathcal{B})$ é densa em $\mathbb{k}_{c}(\mathcal{B})$ na topologia do limite indutivo. Como 3 pode ser estendida a $\mathbb{k}_{r}(\mathcal{B})$, temos então que $\left(\beta \mathbb{k}_{r}(\mathcal{B})\right)$ é a ação envolvente de $\left.\beta\right|_{I_{r}}$.

Além de $\mathbb{k}_{r}(\mathcal{B})$ existem outros completamentos de $\mathbb{k}_{c}(\mathcal{B})$ : a *álgebra de Banach $H S(\mathcal{B})$ dos operadores de Hilbert-Schmidt de $\mathcal{B}$ (que é o completamento de $\mathbb{E}_{c}(\mathcal{B})$ com respeito à norma $\left.\|\cdot\|_{2}\right)$, e sua $C^{*}$-álgebra envolvente $\mathbb{E}(\mathcal{B})$. Por outro lado, para cada representaçào $\pi: \mathcal{B} \longrightarrow B(H)$ tal que $\left.\pi\right|_{B_{c}}$ é fiel, existe uma representação induzida $\pi_{c}: \mathbb{k}_{c}(\mathcal{B}) \longrightarrow B\left(L^{2}(G, H)\right)$. também fiel. Definimos então $\mathbb{k}_{\pi}(\mathcal{B})$ como o completamento de $\mathbb{F}_{c}(\mathcal{B})$ com respeito à norma $\|k\|:=\left\|\pi_{c}(k)\right\|$. Acontece que $\mathbb{R}_{\pi}(\mathcal{B})$ é naturalmente isomorfa a $\hat{\mathfrak{Z}}_{r}(\mathcal{B})$, e, em particular, não depende de $\pi$. 
A ação natural $\beta$ é contínua na norma $\|\cdot\|_{2}$, e portanto se estende a uma ação sobre $H S(\mathcal{B})$. e daqui a $\mathbb{E}(\mathcal{B})$. Pela propriedade universal de $\mathbb{R}(\mathcal{B})$, existe um epimorfismo natural (que também chamaremos $\Omega) \Omega: \mathbb{R}(\mathcal{B}) \longrightarrow \mathbb{E}_{r}(\mathcal{B})$, tal que $\Omega_{k} \xi=k \xi, \forall k \in \mathbb{E}_{c}(\mathcal{B}), \xi \in C_{c}(\mathcal{B})$. Este epimorfismo também é covariante com respeito as açōes naturais. Seja $I$ o fecho de $I_{c}(\mathcal{B})$ dentro de $\mathbb{E}(\mathcal{B})$. Entào tem-se que $(\beta, \mathbb{R}(\mathcal{B}))$ é a açào envolvente de $\left.\beta\right|_{I}$. Veremos que $\left.\Omega\right|_{I}: I \longrightarrow I_{r}$ é um isomorfismo. donde seguirá, por causa da unicidade da açào envolvente, que $\Omega: \mathbb{R}(\mathcal{B}) \longrightarrow \mathbb{F} r(\mathcal{B})$ é na verdade um isomorfismo.

A seção é encerrada com algumas aplicaçōes. Por exemplo, vemos que $\mathbb{R}(\mathcal{B})$ é nuclear. liminal. postliminal, se e somente se $B_{t}$ é nuclear, liminal, ou postliminal, respectivamente.

7.2.1. Operadores de Hilbert-Schmidt e núcleos de um fibrado de Fell. Seja $B=$ $\left(B_{t}\right)_{t \in G}$ um fibrado de Fell sobre o grupo localmente compacto $(i$. ('onsideremos uma função $k$ : $G \times G \longrightarrow \mathcal{B}$ contínua e de suporte compacto, tal que $k(r, s) \in B_{r s-1} . \forall r, s \in G$. Uma tal funçào será chamada núcleo contínuo de suporte compacto associado a $\mathcal{B}$. Denotamos por $\mathbb{E}_{\mathrm{c}}(\mathcal{B})$ o conjunto de tais núcleos. Veremos mais tarde que cada $k$ pode ser visto como um operador integral, o que justifica o termo "núcleo" que estamos utilizando.

Proposiçào 7.15 . Fo (B) ¿ uma *álgebra normada com as operaçós de soma e produto por escalares ponto a ponto. as operaçòs de produto e involuçào dadas por:

$$
\begin{gathered}
k_{1} * k_{2}(r, s)=\int_{G} k_{1}(r, t) k_{2}(t, s) d t, \forall k_{1}, k_{2} \in \mathbb{R}_{c}(\mathcal{B}) . \\
k^{*}(r, s)=k(s, r)^{*}, \forall k \in \mathbb{k}_{c}(\mathcal{B}) .
\end{gathered}
$$

e a norma $\| k_{2}=\left(\int_{G} \int_{G}\|k(r, s)\|^{2} d r d s\right)^{1 / 2}$.

Demonstra̧̧ão. Seja $\nu: G \times G \longrightarrow G$ tal que $\nu(r, s)=r s^{-1}$, e seja $\mathcal{B}_{\nu}$ a retração de $\mathcal{B}$ com respeito a $\nu$ ([37]. II-13.3). Ent ào $B_{\nu}$ é um fibrado de Banach sobre $G \times G$, e a fibra de $\mathcal{B}_{\nu}$ sobre $(r . s i$ é $\left(r, s, B_{r s^{-1}}\right)$. que podemos identificar com $B_{r s^{-1}}$. Portanto. $\mathbb{E}_{c}(\mathcal{B})=C_{r}\left(\mathcal{B}_{\nu}\right)$ como espaço vetorial.

Consideremos agora a função $\mu: G \times G \times G \longrightarrow \mathcal{B}_{\nu}$ tal que $\mu(t, r . s)=\left(r, s, k_{1}(r, t) k_{2}(t, s)\right)$. Temos que $\mu$ é uma função contínua. de suporte compacto, e tal que $\mu(t, r, s) \in\left(\mathcal{B}_{\nu}\right)_{(r, s)}, \forall t, r, s \in G$. Portanto, estamos em condiçòes de aplicar [37], II-15.19, donde concluímos que a função $(r, s) \longmapsto$ $\int_{G} \mu(t, r, s) d t$ é uma seção contínua de suporte compacto de $\mathcal{B}_{\nu}$; em outras palavras, $k_{1} * k_{2} \in \mathbb{k}_{c}(\mathcal{B})$.

Com respeito a $k^{*}$, temos que $\sup \left(k^{*}\right)$ é compacto, e $k^{*}(r, s)=k(s, r)^{*} \in B_{s-1}^{*}=B_{r s^{-1}}$. Portanto $k^{*} \in \mathbb{k}_{c}(\mathcal{B})$.

Por outro lado, se $k_{1}, k_{2}, k_{3} \in \mathbb{E}_{c}(\mathcal{B})$, temos:

$$
\begin{aligned}
\left(k_{1} * k_{2}\right) * k_{3}(r, s) & =\int_{G}\left(k_{1} * k_{2}\right)(r, t) k_{3}(t, s) d t \\
& =\int_{G} \int_{G} k_{1}(r, u) k_{2}(u, t) k_{3}(t, s) d u d t \\
& =\int_{G} \int_{G} k_{1}(r, u) k_{2}(u, t) k_{3}(t, s) d t d u \\
& =\int_{G} k_{1}(r, u) k_{2} * k_{3}(u, s) d u \\
& =k_{1} *\left(k_{2} * k_{3}\right)(r, s)
\end{aligned}
$$


e

$$
\begin{aligned}
\left(k_{1} * k_{2}\right)^{*}(r, s) & =\left(k_{1} * k_{2}\right)(s, r)^{*} \\
& =\left[\int_{G} k_{1}(s, t) k_{2}(t, r) d t\right]^{*} \\
& =\int_{G}\left[k_{1}(s, t) k_{2}(t, r)\right] d t \quad \text { porque } *: B_{s-1} \longrightarrow B_{r-1} \dot{e} \text { antilinear contínua } \\
& =\int_{G_{i}} k_{2}(t, r)^{*} k_{1}(s, t)^{*} d t \\
& =\int_{G_{i}} k_{2}^{*}(r, t) k_{1}^{*}(t, s) d t \\
& =\left(k_{2}^{*} * k_{1}^{*}\right)(r, s) .
\end{aligned}
$$

Assim, vemos que $\mathbb{Z}_{c}(\mathcal{B})$ é uma *álgebra. Vejamos que também é normada:

$$
\begin{aligned}
& \left\|k_{1} * k_{2}\right\|_{2}^{2}=\int_{G} \int_{G}\left\|\left(k_{1} * k_{2}\right)(r, s)\right\|^{2} d r d s \\
& =\int_{G} \int_{G}\left\|\left[\int_{G} k_{1}(r, t) k_{2}(t, s) d t\right]\right\|^{2} d r d s \\
& \leq \int_{G} \int_{G}\left[\int_{G_{i}}\left\|k_{1}(r, t) k_{2}(t, s)\right\| d t\right]^{2} d r d s \\
& \leq \int_{G} \int_{G}\left[\int_{G}\left\|k_{1}(r, t)\right\|\left\|k_{2}(t, s)\right\| d t\right]^{2} d r d s \\
& \leq \int_{G} \int_{G}\left[\left(\int_{G}\left\|k_{1}(r, u)\right\|^{2} d u\right)^{1 / 2}\left(\int_{G}\left\|k_{2}(v, s)\right\|^{2} d v\right)^{1 / 2}\right]^{2} d r d s \quad \text { por Cauchy-Scwarz } \\
& =\int_{G} \int_{G}\left[\int_{G}\left\|k_{1}(r, u)\right\|^{2} d u \int_{G}\left\|k_{2}(v, s)\right\|^{2} d v\right] d r d s \\
& =\left[\int_{G} \int_{G}\left\|k_{1}(r, u)\right\|^{2} d r d u\right]\left[\int_{G} \int_{G}\left\|k_{2}(v, s)\right\|^{2} d v d s\right] \\
& =\left\|k_{1}\right\|_{2}^{2}\left\|k_{2}\right\|_{2}^{2}
\end{aligned}
$$

Por outro lado:

$$
\left\|k^{*}\right\|_{2}^{2}=\int_{G} \int_{G}\left\|k^{*}(r, s)\right\|^{2} d r d s=\int_{G} \int_{G}\left\|k(s, r)^{*}\right\|^{2} d r d s=\int_{G} \int_{G}\|k(s, r)\|^{2} d r d s=\|k\|_{2}^{2} .
$$

Seja agora $E:=L^{2}(\mathcal{B})$. ou seja, $E$ é o completamento de pré- $B_{e}$-módulo de Hilbert à direita $C_{c}(\mathcal{B})$, com o produto interno $\langle\cdot, \cdot\rangle_{r}: C_{c}(\mathcal{B}) \times C_{c}(\mathcal{B}) \longrightarrow B_{e}$ dado por $\langle\xi, \eta\rangle_{-}=\int_{G} \xi(r)^{*} \eta(r) d r$.

$C_{c}(\mathcal{B})$ tem estrutura de $\xi_{c}(\mathcal{B})$-módulo á esquerda: se $k \in \mathbb{R}_{c}(\mathcal{B}), \xi \in C_{c}(\mathcal{B}) . r \in G$, definimos: $\left.k \xi\right|_{r}=\int_{G} k(r, s) \xi(s) d s$. Temos também um "produto interno" $\langle\cdot, \cdot\rangle_{l}: C_{c}(B) \times C_{c}(\mathcal{B}) \longrightarrow \mathbb{E}_{c}(\mathcal{B})$ tal que $\left.\langle\xi, \eta\rangle\right|_{(r, s)}=\xi(r) \eta(s)^{*}$. Tem-se:

1. $\left.\left(\left\langle\xi_{1}, \xi_{2}\right\rangle_{1} \xi_{3}\right)\right|_{r}=\int_{G} \xi_{1}(r) \xi_{2}(s)^{*} \xi_{3}(s) d s=\xi_{1}(r) \int_{G} \xi_{2}(s)^{*} \xi_{3}(s) d s=\left.\left(\xi_{1}\left\langle\xi_{2}, \xi_{3}\right\rangle_{r}\right)\right|_{r}$, e portanto $\left\langle\xi_{1}, \xi_{2}\right\rangle_{l} \xi_{3}=\xi_{1}\left\langle\xi_{2}, \xi_{3}\right\rangle_{,} . \forall \xi_{1}, \xi_{2}, \xi_{3} \in C_{\mathrm{c}}(\mathcal{B})$. 
2. Se $k \in \mathbb{L}_{c}(\mathcal{B}), \xi, \eta \in C_{c}(\mathcal{B}), r . s \in G$ :

$$
\begin{aligned}
\left.\langle k \cdot \xi, \eta\rangle_{l}\right|_{(r . . s)} & =(k \cdot \xi)(r) \eta(s)^{*} \\
& =\left(\int_{G} k(r, t) \xi(t) d t\right) \eta(s)^{*} \\
& =\int_{G} k(r, t) \xi(t) \eta(s)^{*} d t \\
& =\int_{G} k(r, t)\langle\xi, \eta\rangle_{l}(t, s) d s \\
& =\left.k *\langle\xi, \eta\rangle_{l}\right|_{(r, s)}
\end{aligned}
$$

3. Se $\xi, \eta \in C_{c}(\mathcal{B})$,

$$
\left.\langle\xi, \eta\rangle_{i}^{*}\right|_{(r, s)}=\langle\xi, \eta\rangle_{1}(s, r)^{*}=\left\langle\xi(s) \eta(r)^{*}\right)^{*}=\eta(r) \xi(s)^{*}=\left.\langle\eta, \xi\rangle_{l}\right|_{(r, s)},
$$

e portanto $\langle\xi, \eta\rangle_{i}^{*}=\langle\eta, \xi\rangle_{1}$.

4. Seja $\xi \in C_{c}(\mathcal{B})$. Entào $\langle\xi, \xi\rangle_{l}=0 \Longleftrightarrow \xi(r) \xi(s)^{*}=0, \forall r, s \in G$ e portanto $\langle\xi, \xi\rangle_{l}=0 \Longleftrightarrow$ $\xi=0$.

5. Se $k \in \mathbb{R}_{c}(\mathcal{B}), \xi, \eta \in \mathcal{C}_{c}(\mathcal{B})$,

$$
\begin{aligned}
\langle k \xi, \eta\rangle_{r} & =\int_{G i}\left[\int_{G} k(r, s) \xi(s) d s\right]^{*} \eta(r) d r \\
& =\int_{G} \int_{G} \xi(s)^{*} k(r, s)^{*} \eta(r) d r d s \\
& =\int_{G} \xi(s)^{*}\left(k^{*} \eta\right)(s) d s \\
& =\left\langle\xi, k^{*} \eta\right\rangle_{r}
\end{aligned}
$$

Denotaremos por $I_{c}(\mathcal{B}):=\operatorname{span}\left\langle C_{c}(\mathcal{B}) \cdot C_{c}(\mathcal{B})\right\rangle_{l}$, que é um ${ }^{*}$-ideal bilateral de $\mathbb{E}_{c}(\mathcal{B})$.

Seja $[\cdot, \cdot]: E \times E \longrightarrow Z_{l}(E)$ o produto interno à esquerda correspondente ao $C^{*}$-anel ternário positivo $E$. Notar que existe um *-homomorfismo injetivo natural $I_{c}(\mathcal{B}) \hookrightarrow Z_{l}(E)$, único tal que $\langle\xi, \eta\rangle_{l}=[\xi, \eta], \forall \xi, \eta \in C_{c}(\mathcal{B})$, porque se $k=\sum_{i}\left\langle\xi_{i}, \eta_{i}\right\rangle_{l}=0$, tem-se que $k \zeta=0, \forall \zeta \in C_{c}(\mathcal{B})$, e como

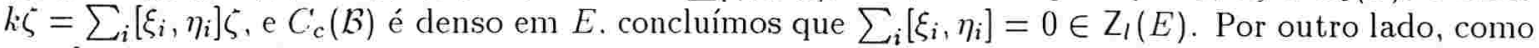
$\operatorname{span}\left[C_{c}(\mathcal{B}), C_{c}(\mathcal{B})\right]$ é denso em $Z_{l}(E)$, temos que $Z_{l}(E)$ é um $C^{*}$-completamento da *álgebra $I_{c}(\mathcal{B})$. Denotaremos este completamento por $I_{r}(\mathcal{B})$ em lugar de $Z_{l}(E)$. Veremos mais tarde que esta inclusão se estende a uma inclusão $\Omega: \mathbb{R}_{c}(\mathcal{B}) \longrightarrow \mathcal{L}(E)$.

No caso em que $\mathcal{B}$ seja $\mathcal{B}_{G}$, o fibrado do grupo $G$, então o completamento de $\mathbb{L}_{c}(\mathcal{B})$ com respeito à norma $\|\cdot\|_{2}$ é exatamente o espaço de operadores de Hilbert-Schmidt do espaço de Hilbert $L^{2}(G)$ ([37], VI-15.21). Isto motiva a definiçào seguinte.

DEFINIÇÃo 7.16. A *-álgebra $\mathbb{R}_{c}(\mathcal{B})$ será chamada álgebra dos núcleos contínuos de suporte compacto do fibrado de Fell $\mathcal{B}$. O completamento $H S(\mathcal{B})$ de $\mathbb{k}_{c}(\mathcal{B})$ com respeito à norma $\|\cdot\|_{2}$ é uma *-álgebra de Banach, que chamaremos álgebra dos operadores de Hilbert-Schmidt do fibrado de Fell B. O fecho de $I_{c}(\mathcal{B})$ dentro de $H S(\mathcal{B})$ será denotado por $I_{2}(\mathcal{B})$.

Definição 7.17 . A $C^{*}$-álgebra envolvente de $H S(\mathcal{B})$ será chamada $C^{*}$-álgebra dos núcleos do fibrado de Fell $\mathcal{B}$. e será denotada por $\mathbb{L}(\mathcal{B})$. O fecho de $I_{c}(\mathcal{B})$ dentro de $\mathbb{E}(\mathcal{B})$ será denotado por $I(\mathcal{B})$. 
Observação 7.18. Notar que, como espaço de Banach, $H S(\mathcal{B})=\mathfrak{L}^{2}\left(\mathcal{B}_{\nu}\right)$ (consultar [37], II15.7-15.9, ou o começo do (apítulo 3).

7.2.2. Ação sobre os núcleos. Temos sobre $\mathbb{k}_{c}(\mathcal{B})$ a seguinte ação natural de $G: \beta: G \times$ $\mathbb{E}_{c}(\mathcal{B}) \longrightarrow \mathbb{E}_{c}(\mathcal{B})$ tal que $\beta_{t}(k)(r, s)=\Delta(t) k(r t, s t)$. Verifiquemos que $\beta$ é uma ação:

$$
\begin{aligned}
\left.\beta_{t}\left(k_{1} * k_{2}\right)\right|_{(r, s)} & =\Delta(t)\left(k_{1} * k_{2}\right)(r t, s t) \\
& =\Delta(t) \int_{G} k_{1}(r t, u) k_{2}(u, s t) d u \\
& =\Delta(t) \int_{G} \Delta(t) k_{1}(r t, v t) k_{2}(v t, s t) d v \\
& =\int_{G} \beta_{t}\left(k_{1}\right)(r, v) \beta_{t}\left(k_{2}\right)(v, s) d v \\
& =\left.\beta_{t}\left(k_{1}\right) * \beta_{t}\left(k_{2}\right)\right|_{(r, s)}
\end{aligned}
$$

e

$$
\left.\left(\beta_{t}\left(k^{*}\right)\right)\right|_{(, r, s)}=\Delta(t) k^{*}(r t, s t)=\Delta(t) k(s t, r t)^{*}=\beta_{t}(k)(s, r)^{*}=\left.\beta_{t}(k)^{*}\right|_{(r, s)}
$$

Esta ação pode ser estendida a $H S(\mathcal{B})$ : fazendo $u=r t, v=s t$ na integral abaixo temos:

$$
\left\|\beta_{t}(k)\right\|_{2}^{2}=\int_{G_{i}} \int_{G_{i}} \Delta(t)^{2}\|k(r t . s t)\|^{2} d r d s=\int_{G} \int_{G} \Delta(t)^{-1} \Delta(t)^{-1} \Delta(t)^{2}\|k(u, v)\|^{2} d u d v=\|k\|_{2}^{2} .
$$

Notar que $\beta$ é uma ação contínua sobre $\mathbb{R}_{c}(\mathcal{B}) \operatorname{com}$ a topologia do limite indutivo, e portanto é contínua sobre $H S(\mathcal{B})$. Como $\mathcal{K}(\mathcal{B})$ é a $C^{*}$-álgebra envolvente de $H S(\mathcal{B}), \beta$ também se estende a uma ação contínua sobre $\_(\mathcal{B})$. Todas estas açòes serão denotadas por $B$.

Proposição T.19. A B-órbita linear de $I_{c}(\mathcal{B})$ é densa em $\mathbb{E}_{c}(\mathcal{B})$ na topologia do limite indutivo.

Demonstração. Lembrar que $\xi_{c}(\mathcal{B})=C_{c}\left(\mathcal{B}_{\nu}\right)$, onde $\mathcal{B}_{\nu}$ é a retraçào de $\mathcal{B}$ com respeito à aplicação $\nu: G \times G \longrightarrow G$ tal que $\nu(r, s)=r s^{-1}$. Portanto, usando o Lema 3.14, basta mostrar que, se $Z$ é a $\beta$-órbita linear de $I_{c}(\mathcal{B})$. então tem-se: $1 . Z(r, s)$ é denso em $B_{r s^{-1}}, \forall r, s \in G$ e 2 . $\forall \varphi_{i}, \psi_{i} \in C_{c}(G)$. e $\forall \zeta \in Z$, a funçào $(r, s) \longmapsto \sum_{i} \varphi_{i}(r) \psi_{i}(s) \zeta(r, s)$ pertence a $Z$.

1. Dado $b \in B_{r s^{-1}}$, por Cohen-Hewitt existem $b_{1} \in B_{e}, b_{2} \in B_{r s^{-1}}$ tais que $b_{1} b_{2}=b$. Também existem seçōes $\xi, \eta \in C_{c}(\mathcal{B})$ tais que $\xi(e)=\Delta(r) b_{1}, \eta\left(s r^{-1}\right)=b_{2}^{*}$. Então temos: $\left.\beta_{r^{-1}}\left(\langle\xi, \eta\rangle_{l}\right)\right|_{(r, s)}=$ $\Delta(r)^{-1} \xi\left(r r^{-1}\right) \eta\left(s r^{-1}\right)^{*}=\Delta(r)^{-1} \Delta(r) b_{1}\left(b_{2}^{*}\right)^{*}=b$.

2. Sejam $\varphi, \psi \in C_{c}(G), \xi, \eta \in C_{c}(\mathcal{B}), r, s, t \in G$. Lembrar que se $\phi: G \longrightarrow \mathbb{C}$. então $\phi_{t}: G \longrightarrow \mathbb{C}$ é definida como sendo $\phi_{t}(s)=\phi(s t)$.

$$
\begin{aligned}
\left.\left(\hat{\varphi} \psi^{\prime}\right)\left(\beta_{t}\left(\langle\xi, \eta\rangle_{l}\right)\right)\right|_{(r, s)} & =\varphi(r) \psi(s) \Delta(t) \xi(r t) \eta(s t)^{*} \\
& =\varphi_{t-1}(r t) \psi_{t-1}(s t) \Delta(t) \xi(r t) \eta(s t)^{*} \\
& =\left.\beta_{t}\left(\left\langle\varphi_{t-1} \xi, \bar{\psi}_{t-1} \eta\right\rangle_{l}\right)\right|_{(r, s)} .
\end{aligned}
$$

donde segue que $\left(\hat{\varphi}_{-} \cdot\right)\left(B_{t}\left(\langle\xi, \eta\rangle_{l}\right)\right) \in Z$, porque $\varphi_{t-1} \xi, \bar{\psi}_{t-1} \eta \in C_{c}(B)$. 
Lembrar que um fibrado de Fell é chamado saturado se para todos $r, s \in\left(i\right.$, tem-se que span $B_{r} B_{s}$ é denso em $B_{r .}$. Acontece que o ideal $I_{\mathrm{c}}(\mathcal{B})$ mede o nível de saturaçào do fibrado:

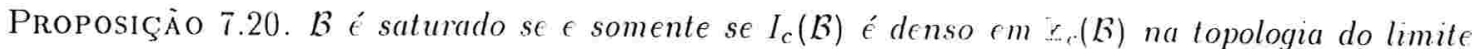
indutivo.

Demonstraçào. Suponhamos primeiro que $\mathcal{B}$ é saturado. Se $\bar{q} \cdot \vartheta \in C_{c}(G), \xi, \eta \in C_{c}(\mathcal{B})$,

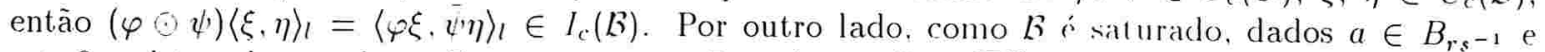
$\epsilon>0$, existem $b_{1} \ldots, b_{n} \in B_{r}, c_{1} \ldots, c_{n} \in B_{s}$, tais que $\left\|b-\sum_{i=1}^{n} b_{i} r_{i}^{*}\right\|<\epsilon$. Além disso, existem seçòes $\xi_{1}, \ldots, \xi_{n}, \eta_{1}, \ldots, \eta_{n} \in C_{c}(B)$ tais que $\xi_{j}(r)=b_{j}$. e $\eta_{j}(s)=c_{j} . \forall j=1, \ldots, n$. Portanto: $\left\|b-\left.\sum_{i=1}^{n}\left\langle\xi_{i}, \eta_{i}\right\rangle_{l}\right|_{(r . .)}\right\|=\left\|b-\sum_{i=1}^{n} \xi_{i}(r) \eta_{i}(s)^{*}\right\|=\left\|b-\sum_{i=1}^{n} b_{i} c_{i}^{*}\right\|<$. L.ogo. usando 3.14, temos que $I_{c}(\mathcal{B})$ é denso em $\mathbb{k}_{c}(\mathcal{B})$ na topologia do limite indutivo.

Inversamente. suponhamos que $B$ nào é saturado: existem $r, s \in\left(i\right.$ tais que $\overline{\text { span }} B_{r} B_{s^{-1}} \neq B_{r^{-1}}$. Seja $b \in B_{r s-1}$ tal que $b \notin \overline{\operatorname{span}} B_{r} B_{s^{-1}}$. e seja $d$ a distancia de $b$ a span $B_{r} B_{s^{-1}}$. Como $G \times G$ é localmente compacto. existe uma seçào contínua de suporte compacto de $B_{v}$, que toma o valor $b \mathrm{em}$ $(r, s)$; em outras palavras, existe $k \in \varkappa_{c}(\mathcal{B})$ tal que $k(r, s)=b$. Agora, se $\xi_{1}, \eta_{t} \in C_{c}(\mathcal{B})$ :

$$
\left\|k-\sum_{i}\left\langle\xi_{i}, \eta_{i}\right\rangle_{l}\right\|_{\infty} \geq\left\|k(r, s)-\sum_{i}\left\langle\xi_{i}, \eta_{i}\right\rangle_{l}(r, s)\right\|=\left\|b-\sum_{i} \xi_{1}(r) \eta(s)^{*}\right\| \geq d,
$$

e portanto $k \notin \overline{I_{c}(\mathcal{B})}$, pois $d>0$.

7.2.3. A $C^{1 *}$-álgebra reduzida dos núcleos de um fibrado de Fell. Nosso próximo objetivo será mostrar que a ação de $Z_{c}(\mathcal{B})$ sobre $C_{c}(\mathcal{B})$ se estende a todo $E$. e que então temos um *-homomorfismo injetivo $\Omega: \mathbb{E}_{c}(\mathcal{B}) \longrightarrow \mathcal{L}(E)$. Para isto, precisaremos representar fielmente $\mathbb{k}_{c}(\mathcal{B})$ como uma *álgebra de operadores sobre um espaço de Hilbert. e representar também $E$ como um anel ternário de operadores.

Proposição 7.21. Seja $\pi: \mathcal{B} \longrightarrow B(H)$ uma representação do fibrado de Fell $\mathcal{B}$ sobre o espaço de Hilbert $H$. Para $k \in \mathbb{E}_{c}(\mathcal{B})$. definimos $\pi_{c}(k): C_{c}(G, H) \longrightarrow C_{c}(G, H)$ tal que $\left.\pi_{c}(k) x\right|_{r}=$ $\int_{G} \pi(k(r, s)) x(s) d s \quad \forall x \in C_{c}(G, H)$. Então tem-se que $\left\|\pi_{c}(k) x\right\|_{2} \leq\|k\|_{2}\|x\|_{2}$, e portanto $\pi_{c}(k)$ se estende a um operador $\pi_{c}(k): L^{2}(G, H) \longrightarrow L^{2}(G, H)$, com $\left\|\pi_{c}(k)\right\| \leq\|k\|_{2}$. Além disso, $\pi_{c}: \mathbb{R}_{c}(\mathcal{B}) \longrightarrow B\left(L^{2}(G, H)\right)$ é uma representação contínua da ${ }^{*}$-álgebra normada $\mathbb{k}_{c}(\mathcal{B})$. Se $\left.\pi\right|_{B_{c}}$ é fiel, então $\pi_{c}$ é fiel: se $\pi$ é não degenerada. então $\pi_{c}$ é não degenerada.

Demonstraçào. Como $x \in C_{c}(G . H)$ e como $\mathcal{B} \times H \longrightarrow H$ tal que $(b . h) \longmapsto \pi(b) h$ é contínua ([37], VIII-8.8), temos que a funçào $G \times G \longrightarrow H$ tal que $(r, s) \longmapsto \pi(k(r . s)) x(s)$ é contínua, e como tem suporte compacto deduzimos que $r \longmapsto \int_{G} \pi(k(r, s)) x(s) d s$ é uma função contínua de suporte 
compacto de $G$ em $H$. e em particular pertence a $L^{2}(G, H)$. Por outro lado:

$$
\begin{aligned}
\left\|\pi_{c}(k) x\right\|_{2}^{2} & =\int_{G}\left\|\pi_{c}(k) x(r)\right\|^{2} d r \\
& =\int_{G}\left\|\left[\int_{G} \pi(k(r, s)) x(s) d s\right]\right\|^{2} d r \\
& \leq \int_{G}\left[\left(\int_{G}\|\pi(k(r, s))\|^{2} d s\right)^{1 / 2}\|x\|_{2}\right]^{2} d r \\
& \leq\left(\int_{G} \int_{G}\|k(r, s)\|^{2} d r d s\right)\|x\|_{2}^{2},
\end{aligned}
$$

donde segue que $\left\|\pi_{c}(k)\right\| \leq\|k\|_{2}$. Portanto, $\pi_{c}(k)$ se estende a um operador contínuo $\pi_{c}(k)$ : $L^{2}(G, H) \longrightarrow L^{2}(G . H)$. e $\pi_{c}$ se estende por continuidade a uma transformação linear contínua $H S(\mathcal{B}) \longrightarrow B\left(L^{2}(G, H)\right)$.

Agora, se $x \in C_{c}(G, H) \cdot k_{1}, k_{2} \in k_{c}(B)$ :

$$
\begin{aligned}
\left.\pi_{C}\left(k_{1} * k_{2}\right) x\right|_{r} & =\int_{G} \pi\left(\left(k_{1} * k_{2}\right)(r, s)\right) x(s) d s \\
& =\int_{G_{i}} \pi\left[\int_{G} k_{1}(r, t) k_{2}(t, s) d t\right] x(s) d s \\
& =\int_{G} \pi\left(k_{1}(r, t)\right)\left[\int_{G} \pi\left(k_{2}(t, s)\right) x(s) d s\right] d t \\
& =\int_{G} \pi\left(k_{1}(r, s)\right)\left[\pi_{c}\left(k_{2}\right) x\right](t) d t \\
& =\left.\pi_{c}\left(k_{1}\right) \pi_{c}\left(k_{2}\right) x\right|_{r}
\end{aligned}
$$

Entào $\pi_{c}$ preserva os produtos. Com respeito à involução, se $k \in \mathbb{E}_{c}(\mathcal{B}), x, y \in C_{c}(G, H)$ :

$$
\begin{aligned}
\left\langle\pi_{c}(k) x, y\right\rangle & =\int_{G}\left\langle\pi_{c}(k) x(r), y(r)\right\rangle d r \\
& =\int_{G} \int_{G}\langle\pi(k(r, s)) x(s), y(r)\rangle d s d r \\
& =\int_{G} \int_{G}\left\langle x(s), \pi\left(k(r, s)^{*}\right) y(r)\right\rangle d s d r \\
& =\int_{G}\left\langle x(s), \int_{G} \pi\left(k^{*}(s, r)^{*}\right) y(r) d r\right\rangle d s \\
& =\int_{G}\left\langle x(s), \pi_{c}\left(k^{*}\right) y(s)\right\rangle d s \\
& =\left\langle x, \pi_{c}\left(k^{*}\right) y\right\rangle,
\end{aligned}
$$

e portanto $\pi_{c}\left(k^{*}\right)=\pi_{c}(k)^{*}$. Assim, temos que $\pi_{c}: \mathbb{R}_{c}(\mathcal{B}) \longrightarrow B\left(L^{2}(G, H)\right)$ é uma representação, contínua na norma $\|\cdot\|_{2}$.

Suponhamos que $\left.\pi\right|_{B_{c}}$ é fiel. Se for $\pi_{c}(k)=0$, para $k \in \mathbb{R}_{c}(\mathcal{B})$, teríamos que, $\forall x \in C_{c}(G, H)$, seria $\int_{G} \pi(k(r, s)) x(s) d s=0$. Em particular, se $\left(f_{V}\right)_{V \in \mathcal{V}}$ é uma identidade aproximada de $L^{1}(G)$ como em 6.2 e $h \in H$, escolhendo $x(s)=f_{V}\left(t^{-1} s\right) h$ e tomando limite em $V$, temos por 6.2 que $\pi(k(r, t)) h=0$, e portanto que $\pi(k(r . s))=0, \forall r, s \in G$. Logo, $\pi_{c}$ também é fiel.

Suponhamos finalmente que $\pi$ é nào degenerada, e seja $y \in L^{2}(G, H)$ tal que $\left\langle\pi_{c}(k) x, y\right\rangle=$ $0, \forall x \in L^{2}(G . H), k \in \mathbb{R}_{c}(\mathcal{B})$. Então temos que $\int_{G}\left\langle\pi_{c}(k) x(r), y(r)\right\rangle d r=0, \forall k \in \mathbb{R}_{c}(\mathcal{B}), \forall x \in$ $C_{c}(G, H)$. Logo, $\int_{G} \int_{G}\langle\pi(k(r, s)) x(s), y(r)\rangle d s d r=0, \forall k \in \mathbb{R}_{c}(\mathcal{B}) . x \in C_{c}(G, H)$. o que implica que 
$\langle\pi(k(r, s)) x(s), y(r)\rangle=0$ em quase todo ponto $(r, s) \in G \times G$, donde segue que $y(r)=0$ para quase todo ponto $r \in G$, porque $\left\{\pi(k(r, s)) x(s): k \in \mathbb{E}_{c}(\mathcal{B}),(r, s) \in G \times G, x \in C_{c}(G, H)\right\}=H$. Com efeito, como $\pi$ é nào degenerada, $\left.\pi\right|_{B_{c}}$ também é não degenerada, e portanto, dado $h \in H$, existem $b \in B_{e}$ e $h^{\prime} \in H$. tais que $\pi(b) h^{\prime}=h$. Agora, pelo teorema de Cohen-Hewitt, existem $b_{1}, b_{2} \in B_{e}$ tais que $b=b_{1} b_{2}^{*}$, e pelo teorema de Douady-dal Soglio Hérault existem seçòes $\eta_{1}, \eta_{2} \in C_{c}(\mathcal{B})$ tais que $\eta_{i}(e)=b_{i}, i=1,2$. Definamos $k: G \times G \longrightarrow \mathcal{B}$ tal que $k(r, s)=\eta_{1}(r) \eta_{2}(s)^{*}$. Então é claro que $k \in \mathbb{t}_{c}(\mathcal{B})$. Por último, tomando $x \in C_{c}(G, H)$ tal que $x(e)=h^{\prime}$, temos que:

$$
\pi(k(\epsilon, \epsilon)) x(e)=\pi\left(\eta_{1}(e) \eta_{2}(e)^{*}\right) h^{\prime}=\pi\left(b_{1} b_{2}^{*}\right) h^{\prime}=\pi(b) h^{\prime}=h .
$$

Assim, concluímos que $\eta=0 \mathrm{em} L^{2}(G, H)$, e portanto $\pi_{c}$ é nào degenerada.

Suponhamos que $\pi: B \longrightarrow B(H)$ é uma representação sobre o espaço de Hilbert $H$, tal que $\left.\pi\right|_{B_{c}}$ é fiel. Pela Proposiçào 7.21 , temos uma representação fiel $\pi_{c}: \mathbb{k}_{c}(\mathcal{B}) \longrightarrow B\left(L^{2}(G, H)\right)$. Seja $\mathbb{Z}_{\pi}(\mathcal{B})$ o completamento de $\mathbb{r}_{c}(\mathcal{B})$ com respeito à norma $\|k\|=\left\|\pi_{c}(k)\right\|$. Então $\beta$ também se estende a uma ação contínua sobre $\mathbb{R}_{\pi}(\mathcal{B})$. Para mostrá-lo. lembrar que a representaçào regular à direita de $G$ sobre o espaço de Hilbert $L^{2}(G, H)$ é definida como $\rho: G \times L^{2}(G, H) \longrightarrow L^{2}(G, H)$, tal que $\left.\rho_{t}(x)\right|_{r}=\Delta(t)^{1 / 2} x(r t), \forall r, t \in G^{\prime}, x \in L^{2}(G, H)$. Agora, se $k \in \mathbb{R}_{c}(\mathcal{B}), x \in C_{c}(G, H):$

$$
\begin{aligned}
\left.\pi_{c}\left(\beta_{t}(k)\right) x\right|_{r}= & \int_{G} \pi\left(\beta_{t}(k)(r . s)\right) x(s) d s \\
& =\int_{G} \Delta(t) \pi(k(r t, s t)) x(s) d s \\
& =\int_{G} \pi(k(r t, u)) x\left(u t^{-1}\right) d u \\
& =\int_{G} \Delta(t)^{1 / 2} \pi(k \cdot(r t, u)) \rho_{t^{-1}}(x)(s) d s \\
& \left.\left(\rho_{t} \pi_{c}(k) \rho_{t-1}\right) x\right|_{r},
\end{aligned}
$$

donde segue que:

$$
\pi_{c}\left(\beta_{t}(k)\right)=\rho_{t} \pi_{c}(k) \rho_{t-1}
$$

e em particular temos que $\left\|\pi_{c}\left(\beta_{t}(k)\right)\right\|=\left\|\pi_{c}(k)\right\|$.

Vamos provar a seguir que se $\pi: \mathcal{B} \longrightarrow B(H)$ é uma representação sobre o espaço de Hilbert $H$, tal que $\left.\pi\right|_{B_{e}}$ é fiel, então $\pi$ induz uma representação fiel de $E$ como um anel ternário de operadores de $H$ em $L^{2}(G, H)$. Lembrar que uma representação de um $C^{*}$-anel ternário positivo sobre os espaços de Hilbert $H$ e $K$ ' é um homomorfismo de $C^{*}$-anéis ternários $E \longrightarrow B(H, K)$ (ver 1.27).

Lema 7.22. Seja $\pi: \mathcal{B} \longrightarrow B(H)$ uma representação do fibrado de Fell $\mathcal{B}$. Entâo existe uma única representação $\pi_{2}: L^{2}(\mathcal{B}) \longrightarrow B\left(H, L^{2}(G, H)\right)$. tal que $\forall \xi \in C_{c}(\mathcal{B}) \in h \in H$ se tem: $\left.\pi_{2}(\xi) h\right|_{r}=$ $\pi(\xi(r)) h$. Se $\left.\pi\right|_{B_{\epsilon}}$ é fiel. então $\pi_{2}$ é fiel. 
Demonstração. $\pi_{2}$ é contínua:

$$
\begin{aligned}
\left\|\left(\pi_{2} \xi\right) h\right\|_{2}^{2} & =\int_{G}\left\langle\left.\left(\pi_{2} \xi\right) h\right|_{r},\left.\left(\pi_{2} \xi\right) h\right|_{r}\right\rangle d r \\
& =\int_{G}\langle\pi(\xi(r)) h, \pi(\xi(r)) h\rangle d r \\
& =\int_{G}\left\langle\pi\left(\xi(r)^{*} \xi(r)\right) h, h\right\rangle d r \\
& =\left\langle\pi\left[\int_{G} \xi\left(r^{*}\right) \xi(r) d r\right] h . h\right\rangle \\
& =\left\langle\pi\left(\langle\xi, \xi\rangle_{r}\right) h, h\right\rangle .
\end{aligned}
$$

Segue que $\pi_{2} \xi \in L^{2}(G, H)$. Por outro lado, como $\pi\left(\langle\xi, \xi\rangle_{r}\right)$ é um operador positivo. tomando supremo sobre a esfera unitária de $H$ na igualdade acima, concluímos que $\left\|\pi_{2} \xi\right\|=\left\|\pi\left(\langle\xi, \xi\rangle_{r}\right)\right\|^{1 / 2}$. Portanto, $\left\|\pi_{2} \xi\right\| \leq\|\xi\|$, e $\left\|\pi_{2} \xi\right\|=\|\xi\|$ se $\left.\pi\right|_{B_{\epsilon}}$ é fiel. Assim, podemos estender $\pi_{2}$ a $L_{2}^{2}($ B) , e esta extensão será uma isometria se $\left.\pi\right|_{B_{\mathrm{c}}}$ é fiel.

Calculemos $\left(\pi_{2} \xi\right)^{*}:$ se $h \in H, x \in L^{2}(G, H)$ :

$$
\left\langle\left(\pi_{2} \xi\right) h, x\right\rangle=\int_{G}\langle\pi(\xi(r)) h, x(r)\rangle d r=\int_{G}\left\langle h, \pi\left(\xi(r)^{*}\right) x(r)\right\rangle d r=\left\langle h, \int_{\sigma_{i}} \pi\left(\xi(r)^{*}\right) x(r) d r\right\rangle,
$$

donde segue que $\left(\pi_{2} \xi\right)^{*} x=\int_{G} \pi\left(\xi(r)^{*}\right) x(r) d r \in H$. Portanto, se $\xi . \eta \zeta \in C_{c}^{*}(\mathcal{B})$. h $, H, r \in G$ :

$$
\begin{aligned}
\left.\pi_{2}(\xi) \pi_{2}(\eta)^{*} \pi_{2}(\zeta) h\right|_{r} & =\pi(\xi(r)) \pi_{2}(\eta)^{*} \pi(\zeta(r)) h \\
& =\left.\pi(\xi(r)) \int_{G} \pi\left(\eta(s)^{*}\right) \pi_{2}(\zeta) h\right|_{s} d s \\
& =\int_{G} \pi\left(\xi(r) \eta(s)^{*}\right) \pi(\zeta(s)) h d s \\
& =\int_{G} \pi\left(\xi(r) \eta(s)^{*} \zeta(s)\right) h d s \\
& =\pi\left(\xi(r)\langle\eta, \zeta\rangle_{r}\right) h \\
& =\left.\pi_{2}\left(\xi\langle\eta, \zeta\rangle_{r}\right) h\right|_{r} .
\end{aligned}
$$

Portanto, $\pi_{2}$ é um homomorfismo de $C^{*}$-anéis ternários positivos.

Teorema 7.23. Seja $\pi: \mathcal{B} \longrightarrow B(H)$ uma representação do fibrado de Fell $\mathcal{B}$, e sejam $\pi_{c}$ : $\mathbb{L}_{c}(\mathcal{B}) \longrightarrow B\left(L^{2}(G, H)\right) \in \pi_{2}: L^{2}(\mathcal{B}) \longrightarrow B\left(H, L^{2}(G, H)\right)$ as representaçóes fornecidas por $7.21 e$ 7.22 respectivamente. Entào temos:

1. Para todos $k \in \mathbb{k}_{c}(\mathcal{B}) . b \in B_{e}, e \xi, \eta \in L^{2}(\mathcal{B})$ :
(a) $\pi_{2}(k \xi)=\pi_{c}(k) \pi_{2}(\xi)$.
(b) $\pi_{c}\left(\langle\xi, \eta\rangle_{1}\right)=\pi_{2}(\xi) \pi_{2}(\eta)^{*}$
(c) $\pi_{2}(\xi b)=\pi_{2}(\xi) \pi(b)$
(d) $\pi\left(\langle\xi, \eta\rangle_{r}\right)=\pi_{2}(\xi)^{*} \pi_{2}(\eta)$.

2. Existe um ${ }^{*}$-homomorfismo injetivo $\Omega: \mathbb{R}_{c}(\mathcal{B}) \longrightarrow \mathcal{L}(E)$. único tal que

$$
\left.\Omega(k) \xi\right|_{r}=\int_{G} k(r, s) \xi(s) d s, \forall k \in \mathbb{k}_{c}(\mathcal{B}) \in \forall \xi \in C_{c}(\mathcal{B}) .
$$


3. Para toda $\pi: \mathcal{B} \longrightarrow B(H)$ tal que $\left.\pi\right|_{B_{e}}$ é fiel, existe um isomorfismo $\mu: \overline{\pi_{c}\left(\mathbb{R}_{c}(\mathcal{B})\right)} \longrightarrow \mathcal{L}(E)$ tal que o diagrama abaixo comuta:

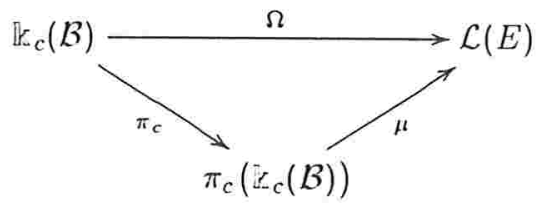

Em particular, $\mathbb{F}_{\pi}(\mathcal{B})$ nào depende da representaçâo $\pi$.

Demonstraçào. Suponhamos que vale 1 ., e seja $\pi: \mathcal{B} \longrightarrow B(H)$ uma representação tal que $\left.\pi\right|_{B_{e}}$ é fiel. Entào a representaçào fiel $\pi_{c}: \mathbb{E}_{c}(\mathcal{B}) \longrightarrow B\left(L^{2}(G, H)\right)$ se estende de forma única a uma representaçào fiel $\tilde{\pi}: \mathbb{F}_{\pi}(\mathcal{B}) \longrightarrow B\left(L^{2}(G, H)\right)$. Por outro lado, $\pi_{2}: E \longrightarrow B\left(H, L^{2}(G, H)\right)$ é um homomorfismo injetivo de $C^{*}$-anéis ternários. Portanto, $\sigma: E \longrightarrow \pi_{2}(E)$ tal que $\sigma(\xi)=\pi_{2}(\xi)$ é um isomorfismo de $C^{*}$-anéis ternários. Assim. a aplicaçào $A d_{\sigma}: \mathcal{L}(E) \longrightarrow \mathcal{L}\left(\pi_{2}(E)\right)$ tal que $T \longmapsto \sigma T \sigma^{-1}$ é um isomorfismo. Agora, por 1.(a) e (d), temos que cada $\tilde{\pi}(k) \in \mathcal{L}\left(\pi_{2}(E)\right)$, e portanto $\operatorname{Ad}_{\sigma}^{-1}(\tilde{\pi}(k)) \in \mathcal{L}(E)$. Além disso, se $\xi \in C_{c}(\mathcal{B}), k \in \mathbb{k}_{c}(\mathcal{B})$, tem-se:

$$
A d_{\sigma}^{-1}\left(\pi_{c}(k)\right)(\xi)=\left(\sigma^{-1} \pi_{c}(k) \sigma\right) \xi=\sigma^{-1}\left(\pi_{c}(k) \pi_{2}(\xi)\right)=\sigma^{-1}\left(\pi_{2}(k \xi)\right)=\sigma^{-1}(\sigma(k \xi))=k \xi .
$$

Isto significa que a aplicaçào $C_{c}(\mathcal{B}) \longrightarrow C_{c}(\mathcal{B})$, tal que $\xi \longmapsto k \xi$, pode ser estendida por continuidade a um operador adjuntável $\Omega(k): \underline{E \longrightarrow E}$. Temos definida assim uma aplicaçào $\Omega: \mathbb{K}_{c}(\mathcal{B}) \longrightarrow \mathcal{L}(E)$. Por outro lado, se definimos $\mu: \overline{\pi_{c}\left(\mathbb{E}_{c}(\mathcal{B})\right)} \longrightarrow \mathcal{L}(E)$ como a restriçào de $A d_{\sigma}^{-1}$ a $\overline{\pi_{c}\left(\mathbb{R}_{c}(\mathcal{B})\right)}$, temos que $\mu \pi_{c}=\Omega$. Como $\mu \pi{ }^{\prime}: \mathbb{t}_{c}(\mathcal{B}) \longrightarrow \mathcal{L}(E)$ é injetiva, tem-se que $\Omega$ é injetiva e que a norma de $k \in \mathbb{k}_{c}(\mathcal{B})$ dentro de $\mathbb{k}_{\pi}(\mathcal{B})$ coincide com $\|\Omega(k)\|$. Isto prova 2. e 3 . .

Logo, somente falta provar 1.

Sejam $h \in H, r \in G, x \in L^{2}(G, H)$ :

- $\pi_{2}(k \xi)=\pi_{c}(k) \pi_{2}(\xi)$ :

- $\pi_{c}\left(\langle\xi, \eta\rangle_{l}\right)=\pi_{2}(\xi) \pi_{2}(\eta)^{*}:$

$$
\begin{aligned}
\left.\left(\pi_{2}(k \xi)\right) h\right|_{t} & =(k \xi)(t) h \\
& =\pi\left[\int_{G} k(r, s) \xi(s)\right] h d s \\
& =\int_{G} \pi(k(t, s)) \pi(\xi(s)) h d s \\
& =\left.\int_{G} \pi(k(t, s))\left(\pi_{2} \xi\right) h\right|_{s} d s \\
& =\left.\pi_{c}(k)\left(\pi_{2} \xi\right) h\right|_{t}
\end{aligned}
$$

$$
\begin{aligned}
\left.\pi_{c}\left(\langle\xi, \eta\rangle_{l}\right) x\right|_{t} & =\int_{G} \pi\left(\langle\xi, \eta\rangle_{l}(t, s)\right) x(s) d s \\
& =\int_{G} \pi\left(\xi(t) \eta(s)^{*}\right) x(s) d s \\
& =\int_{G} \pi(\xi(t)) \pi\left(\eta(s)^{*}\right) x(s) d s \\
& =\pi(\xi(t)) \int_{G} \pi\left(\eta(s)^{*}\right) x(s) d s \\
& =\left.\left(\pi_{2} \xi\right)\left(\pi_{2} \eta\right)^{*} x\right|_{t}
\end{aligned}
$$


- $\pi_{2}(\xi b)=\pi_{2}(\xi) \pi(b)$

$$
\left.\pi_{2}(\xi b) h\right|_{r}=\pi(\xi b(r)) h=\pi(\xi(r) b) h=\left.\pi(\xi(r)) \pi(b) h\right|_{r}=\left.\pi_{2}(\xi) \pi(b) h\right|_{r} .
$$

- $\pi\left(\langle\xi, \eta\rangle_{r}\right)=\pi_{2}(\xi)^{*} \pi_{2}(\eta)$ :

$$
\begin{aligned}
\pi_{2}(\xi)^{*} \pi_{2}(\eta) h & =\left.\int_{G} \pi\left(\xi(r)^{*}\right) \pi_{2}(\eta) h\right|_{r} d s \\
& =\int_{G} \pi\left(\xi(r)^{*}\right) \pi(\eta(r)) h d r \\
& =\int_{G} \pi\left(\xi(r)^{*} \eta(r)\right) h d r \\
& =\pi\left(\langle\xi, \eta\rangle_{r}\right) h
\end{aligned}
$$

DefiniçÃo 7.24. Sejam $\mathcal{B}$ um fibrado de Fell, e $\Omega: \mathbb{k}_{c}(\mathcal{B}) \longrightarrow \mathcal{L}\left(L^{2}(\mathcal{B})\right)$ o homomorfismo definido no Teorema 7.23 acima. Seja $\mathbb{F}_{r}(\mathcal{B}):=\overline{\Omega\left(\mathbb{K}_{c}(\mathcal{B})\right)} \subseteq \mathcal{L}\left(L^{2}(\mathcal{B})\right)$. Diremos que $\mathbb{K}_{r}(\mathcal{B})$ é a $C^{*}$-álgebra reduzida dos núcleos do fibrado de Fell $\mathcal{B}$.

OBSERVAÇÃo 7.25. Notar que se $\pi: \mathcal{B} \longrightarrow B(H)$ é uma representaçào tal que $\left.\pi\right|_{B_{c}}$ é fiel, então $\mathbb{R}_{r}(\mathcal{B}) \cong \mathbb{K}_{\pi}(\mathcal{B})$ naturalmente. Em particular, a ação $\beta$ sobre $\mathbb{k}_{c}(\mathcal{B})$ se estende a $\underline{r}_{r}(\mathcal{B})$.

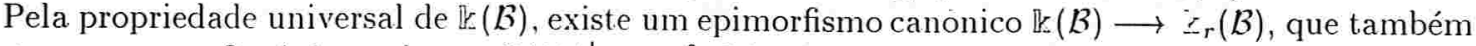
denotaremos por $\Omega$, único tal que $\left.\Omega(k) \xi\right|_{r}=\int_{G} k(r, s) \xi(s) d s, \forall k \in \mathbb{E}_{c}(\mathcal{B})$, e $\forall \xi \in C_{c}(\mathcal{B})$. Este

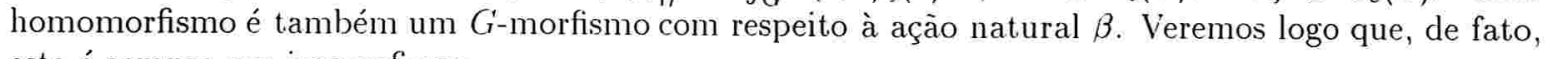
este é sempre um isomorfismo.

OBservaçȦo 7.26. Sejam $k \in \mathbb{R}_{c}(\mathcal{B})$ e $f \in C_{c}(\mathcal{B})$, e definamos $h: G \times G \longrightarrow \mathcal{B}$ tal que $h(r, t)=$ $\int_{G} f(s) k\left(s^{-1} r, t\right) d s$. Notar que $h(r . t) \in B_{r t^{-1}}, \forall r, t \in G$, pois para todos $r . s . t \in G$, tem-se que $f(s) k\left(s^{-1} r, t\right) \in B_{s} B_{s^{-1} r^{-1}} \subseteq B_{r t^{-1}}$. Assim, temos que $h \in \mathbb{k}_{c}(\mathcal{B})$. Agora, considerando $\xi \in C_{c}(\mathcal{B}) \subseteq$ $L^{2}(\mathcal{B})$, temos:

$$
\left.\Lambda_{f} \Omega_{k} \xi\right|_{r}=\int_{G} f(s) \Omega_{k}(\xi)\left(s^{-1} r\right) d s=\int_{G} \int_{G} f(s) k\left(s^{-1} r, t\right) \xi(t) d t d s=\int_{G} h(r, t) \xi(t) d t=\left.\Omega_{h} \xi\right|_{t}
$$

Portanto, concluímos que $C_{r}^{*}(\mathcal{B}) \subseteq M\left(\mathbb{k}_{r}(\mathcal{B})\right)$. Em particular, toda representação de $\mathbb{k}_{r}(\mathcal{B})$ define uma representação de $C_{r}^{*}(\mathcal{B})$.

Corolário 7.27. Sejam $I=I(\mathcal{B})$ o fecho de $I_{c}(\mathcal{B})$ dentro de $\mathbb{k}(\mathcal{B})$, e $I_{r}=I_{r}(\mathcal{B})$ o fecho de $\Omega\left(I_{c}(\mathcal{B})\right)$ em $\mathbb{k}_{r}(\mathcal{B})$. Então:

1. $(\mathbb{k}(\mathcal{B}), \beta)$ é a ação envolvente de $\left(I,\left.\beta\right|_{I}\right)$.

2. $\left(\mathbb{k}_{r}(\mathcal{B}), \beta\right)$ é a açió envolvente de $\left(I_{r},\left.\beta\right|_{I_{r}}\right)$.

DEmonstraÇÃo. As duas partes são conseqüencia direta de 7.19. 
7.2.4. $\mathbb{k}(\mathcal{B})=\mathbb{E}_{r}(\mathcal{B})$, e algumas aplicações. Veremos a seguir que o epimorfismo canônico $\mathbb{R}(\mathcal{B}) \longrightarrow \mathbb{k}_{r}(\mathcal{B})$ é um isomorfismo. De fato, mostraremos que a restrição deste epimorfismo ao ideal $I$ é um isomorfismo $I \cong I_{r}$, e portanto deve ser $\mathbb{k}(\mathcal{B}) \cong \mathbb{R}_{r}(\mathcal{B})$, por causa da unicidade da ação envolvente. $\mathrm{O}$ fato de ser $I \longrightarrow I_{r}$ um isomorfismo decorre do Lema 7.28 abaixo, que permite gerar um módulo de Hilbert sobre $I$ através do processo de completamento duplo (ver a página 23) e do Lema 7.29, que mostra que, sob determinadas circunstancias, duas $C^{*}$-normas comparáveis sobre um *-anel ternário devem ser iguais.

Lema 7.28. Seja $\mathrm{c}:=\overline{\left\{\sum_{i=1}^{n} k_{i} * k_{i}^{*}: n \geq 1, k_{i} \in I_{c}(\mathcal{B})\right\}} \subseteq I_{c}(\mathcal{B})$. onde o fecho é na topologia do limite indutivo. Então, se $\xi \in C_{c}^{\prime}(\mathcal{B})$, tem-se que $\langle\xi, \xi\rangle_{l} \in \mathfrak{c}$.

Demonstraçào. Seja $\xi \in C_{c}^{\prime}(\mathcal{B})$. Como vimos na demonstração do Lema 2.23, existem $\eta \in$ $C_{c}(\mathcal{B}), b \in B_{e}$, tais que $\xi=\eta b$. Seja $\zeta \in C_{c}(\mathcal{B})$ tal que $\zeta(e)=b^{*}$. A função $G \longrightarrow B_{e}$ tal que $t \longmapsto \zeta(t)^{*} \zeta(t)-b b^{*}$ é contínua e se anula em $t=e$. Portanto, dado $\epsilon>0$. existe uma vizinhança $V_{\epsilon}$ de $e$, tal que se $t \in V_{\epsilon}$, entào $\left\|\zeta(t)^{*} \zeta(t)-b b^{*}\right\|<\epsilon$. Seja $\left(f_{V}\right)_{V \in \mathcal{V}}$ uma unidade aproximada de $L^{1}(G)$ como no Lema 6.2, e para cada $V \in \mathcal{V}$ seja $\zeta_{V}=f_{V}^{1 / 2} \zeta$. Então $\zeta_{V} \in C_{c}(\mathcal{B})$. e se $V \geq V_{\epsilon}$ (ou seja: $\left.V \subseteq V_{\epsilon}\right)$ :

$$
\begin{aligned}
\left\|\left\langle\zeta_{V}, \zeta_{V}\right\rangle_{r}-b b^{*}\right\| & =\left\|\int_{G} \zeta_{V}(t)^{*} \zeta_{V}(t) d t-b b^{*}\right\| \\
& =\left\|\int_{G} f_{V}(t)\left(\zeta(t)^{*} \zeta(t)-b b^{*}\right) d t\right\| \\
& \leq \int_{G} f_{V}(t)\left\|\zeta(t)^{*} \zeta(t)-b b^{*}\right\| d t \\
& \leq \epsilon,
\end{aligned}
$$

e portanto $\left\langle\zeta_{V}, \zeta_{V}\right\rangle_{r} \longrightarrow b b^{*}$ tem-se:

Consideremos agora, para cada $V \in \mathcal{V}$, o núcleo $k_{V} \in I_{c}(\mathcal{B})$ dado por $k_{V}=\left\langle\eta, \zeta_{V}\right\rangle_{1}$. Se $V \geq V_{\epsilon}$

$$
\begin{aligned}
\left\|\left.\left(\langle\xi, \xi\rangle_{l}-k_{V} * k_{V}^{*}\right)\right|_{(r, s)}\right\| & =\left\|\xi(r) \xi(s)^{*}-\int_{G} k_{V}(r, t) k_{V}^{*}(t, s) d t\right\| \\
& =\left\|\eta(r) b(\eta(s) b)^{*}-\int_{G} k_{V}(r, t) k_{V}(s, t)^{*} d t\right\| \\
& =\left\|\eta(r) b b^{*} \eta(s)^{*}-\int_{G} \eta(r) \zeta_{V}(t)^{*}\left(\eta(s) \zeta_{V}(t)^{*}\right)^{*} d t\right\| \\
& =\left\|\eta(r) b b^{*} \eta(s)^{*}-\int_{G} \eta(r) \zeta_{V}(t)^{*} \zeta_{V}(t) \eta(s)^{*} d t\right\| \\
& =\left\|\eta(r)\left(b b^{*}-\left\langle\zeta_{V}, \zeta_{V}\right\rangle_{r}\right) \eta(s)^{*}\right\| \\
& \leq \epsilon \eta \|_{\infty}^{2}
\end{aligned}
$$

Portanto, $k_{V} * k_{V}^{*} \longrightarrow\langle\xi, \xi\rangle_{l}$ na topologia do limite indutivo, e portanto $\langle\xi, \xi\rangle_{l} \in \mathfrak{c}$.

Lema 7.29. Sejam $\left(E^{\prime},(\cdot, \cdot, \cdot)\right)$ um *anel ternário, $e\|\cdot\|,|\cdot|$ duas $C^{*}$-normas sobre $E^{\prime}$, com $\|\cdot\| \leq|\cdot|$. Sejam $E$ o completamento de $E^{\prime}$ com respeito a $\|\cdot\|, e\langle\cdot \cdot\rangle: E \times E \longrightarrow Z(E) o$ correspondente produto interno. Se $\operatorname{span}\left\langle E^{\prime}, E^{\prime}\right\rangle$ é um ideal de $\mathrm{Z}(E)$, então $\|\cdot\|=|\cdot|$.

Demonstração. Sejam $F$ o completamento de $E^{\prime}$ com respeito a $|\cdot|$. e $[\cdot, \cdot]: F \times F \longrightarrow Z(F)$ o correspondente produto interno. 
Sejam $x_{1}, \ldots, x_{n}, y_{1}, \ldots, y_{n} \in E^{\prime}$, e suponhamos que $\sum_{i=1}^{n}\left\langle x_{i}, y_{i}\right\rangle=0 \in Z(E)$. Então, $\forall z \in E^{\prime}$ :

$$
0=z \sum_{i=1}^{n}\left\langle x_{i}, y_{i}\right\rangle=\sum_{i=1}^{n}\left(z, x_{i}, y_{i}\right)=z \sum_{i=1}^{n}\left[x_{i}, y_{i}\right],
$$

donde segue que $\sum_{i=1}^{n}\left[x_{i}, y_{i}\right]=0 \in Z(F)$, porque $E^{\prime}$ é denso em $F$. Portanto, podemos definir $\psi: \operatorname{span}\left\langle E^{\prime}, E^{\prime}\right\rangle \longrightarrow Z(F)$ tal que $\psi \cdot\left(\sum_{i=1}^{n}\left\langle x_{i}, y_{i}\right\rangle\right)=\sum_{i=1}^{n}\left[x_{i}, y_{i}\right] . \dot{E}$ imediato verificar que este é um *homomorfismo. Como span $\left\langle E^{\prime} . E^{\prime}\right\rangle$ é um ideal de $Z(E)$, ele é contrativo (1.17). Então temos, $\forall x \in E^{\prime}$ :

$$
|x|^{2}=|[x, x]|=|\psi(\langle x, x\rangle)| \leq\|\langle x, x\rangle\|=\|x\|^{2} \leq|x|^{2},
$$

e portanto $\|\cdot\|=|\cdot|$

Proposição i.30. Para todo fibrado de Fell $\mathcal{B}$ tem-se que $\Omega: \mathbb{R}(\mathcal{B}) \longrightarrow \mathbb{K}_{r}(\mathcal{B})$ é um isomorfismo.

Demonstraçào. Seja c como no Lema 7.28 , ou seja, $\mathfrak{c} \subseteq I_{c}(\mathcal{B})$ é o cone fechado na topologia do limite indutivo gerado pelos elementos da forma $k * k^{*}, \operatorname{com} k \in I_{c}(\mathcal{B})$. Como a convergencia na topologia do limite indutivo implica a convergencia em $H S(\mathcal{B})$, e portanto em $\mathbb{E}(\mathcal{B})$, temos que $\mathfrak{c} \subseteq \mathbb{R}(\mathcal{B})^{+}$. Logo, pelo Lema $\bar{\tau}: 28,\langle\cdot, \cdot\rangle_{l}: C_{c}(\mathcal{B}) \times C_{c}(\mathcal{B}) \longrightarrow \mathbb{E}(\mathcal{B})$ é um produto interno. Consideremos sobre $C_{c}(\mathcal{B})$ a norma $|\cdot|$ induzida por $\mathbb{E}(\mathcal{B})$, ou seja, $|\xi|=\|\langle\xi, \xi\rangle$,$\| , onde estamos vendo o elemento$ $\langle\xi, \xi\rangle_{l}$ como pertencente a $\mathbb{E}(\mathcal{B})$. Em particular, $|\xi| \geq\|\xi\|$, onde esta última é a norma de $\xi$ como elemento de $L^{2}(\mathcal{B})$. Agora, podemos usar o Lema 7.29 acima com $E^{\prime}=C_{c}(\mathcal{B})$, donde concluímos que $\|\cdot\|=|\cdot|$. Portanto $I=Z_{r}(E)=I_{r}$, e como $(\beta, \mathbb{E}(\mathcal{B}))$ e $\left(\beta, \mathbb{K}_{r}(\mathcal{B})\right)$ são respectivamente as açōes envolventes de $\left.\beta\right|_{I}$ e $\left.\beta\right|_{I_{r}}$, entào $\mathbb{E}(\mathcal{B})=\mathbb{E}_{r}(\mathcal{B})$, pela unicidade da ação envolvente.

Em [53], Quigg introduziu a noçào de propriedade ideal de uma $C^{*}$-álgebra

DEFINIÇÃo 7.31. Lima propriedade de uma $C^{*}$-álgebra é chamada ideal se para toda $C^{*}$-álgebra existe um ideal máximo da álgebra que possui esta propriedade, e tal que a propriedade é invariante por equivalência Morita e herdada por ideais.

Exemplos de propriedades ideais são a nuclearidade, a liminalidade, a postliminalidade.

Corolário 7.32. Seja $\mathcal{P}$ uma propriedade ideal. Então $B_{e}$ tem a propriedade $\mathcal{P}$ se e somente se $\mathbb{k}(\mathcal{B})$ tem a propriedade $\mathcal{P}$.

Demonstração. Como $\mathcal{P}$ é invariante por equivaléncia Morita, $B_{e}$ tem $\mathcal{P}$ se e somente se $I$ tem $\mathcal{P}$. Agora, $\mathbb{k}(\mathcal{B})$ tem um ideal máximo que tem a propriedade $\mathcal{P}$. Este ideal deve conter todos os ideais $\beta_{t}(I)$, pois todos eles, sendo isomorfos a $I$, tém a propriedade $\mathcal{P}$. Mas, o menor ideal que contém todos os ideais $\beta_{t}(I)$ é a própria $C^{*}$-álgebra $\mathbb{L}(\mathcal{B})$, devido a 7.27 .

CoRolário 7.33. Suponhamos que $\mathcal{C}$ é uma classe de $C^{*}$-álgebras fechada por ideais, extensões, quocientes, limites indutivos e equivaléncia Morita. Então $B_{\epsilon} \in \mathcal{C} \Longleftrightarrow \mathbb{E}(\mathcal{B}) \in \mathcal{C}$.

Demonstração. Como $B_{\epsilon} \stackrel{M}{\sim} I$. temos que $B_{\epsilon} \in \mathcal{C} \Longleftrightarrow I \in \mathcal{C}$. Agora, como $\beta$ é a ação envolvente de $\left.\beta\right|_{I}$, por 5.19 temos que $I \in \mathcal{C} \Longleftrightarrow \mathbb{k}(\mathcal{B}) \in \mathcal{C}$. Assim, $B_{\epsilon} \in \mathcal{C} \Longleftrightarrow \mathbb{k}(\mathcal{B}) \in \mathcal{C}$. 
Antes de encerrar esta seção, gostaríamos de enfatizar o caráter funtorial das construções realizadas. Suponhamos que $\mathcal{A}=\left(A_{t}\right)$ e $\mathcal{B}=\left(B_{t}\right)$ são fibrados de Fell sobre o grupo localmente compacto $G$, e suponhamos que $\zeta: \mathcal{A} \longrightarrow \mathcal{B}$ é um homomorfismo de fibrados de Fell. Então, se $k \in \mathbb{R}_{c}(\mathcal{A})$, é claro que $o(k) \in \mathbb{r}_{c}(\mathcal{B})$, onde $\left.\phi(k)\right|_{(r, s)}=\phi(k(r, s))$. Por outro lado, é fácil verificar que a aplicação $\phi: \mathbb{R}_{c}(\mathcal{A}) \longrightarrow \mathbb{R}_{c}(B)$ assim definida é um homomorfismo de *álgebras. Além disso, também é claro que $\phi(k) \in H S(B)$. e $\|\phi(k)\|_{2} \leq\|k\|_{2}$, e portanto $\phi$ se estende a um homomorfismo $\phi: H S(\mathcal{A}) \longrightarrow H S(B)$ de *álgebras de Banach. Assim, fica induzido um homomorfismo de $C^{*}$-álgebras $\mathbb{k}(\phi): \mathbb{R}(\mathcal{A}) \longrightarrow \mathbb{E}(\mathcal{B})$.

Se $\phi: \mathcal{A} \longrightarrow \mathcal{B}$ é injetiva, então $\mathbb{E}(\phi): \mathbb{K}(\mathcal{A}) \longrightarrow \mathbb{k}(\mathcal{B})$ também é injetiva: seja $\pi: \mathcal{B} \longrightarrow B(H)$

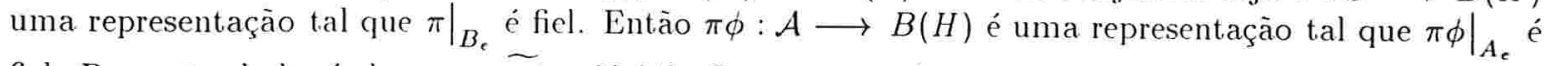
fiel. Por outro lado, é claro que $\widetilde{\pi \varphi}=\bar{\pi} \mathbb{k}(\phi)$. Como o membro esquerdo desta igualdade e injetivo, o membro direito também é. o que mostra que $\mathbb{k}$. $(\phi)$ é injetivo.

\subsection{Sobre a existência da ação envolvente a menos de equivalência Morita}

Nesta seção aplicaremos os resultados prévios ao caso em que o fibrado de Fell $\mathcal{B}$ provém de uma ação parcial $a$. Mostraremos então que a ação natural sobre $\mathbb{k}(\mathcal{B})$ é uma ação envolvente a menos de equivalencia Morita de a. Suponhamos que $\mathcal{B}$ é o fibrado de Fell de uma ação parcial $\alpha=\left(\left\{D_{t}\right\}_{t \in G},\left\{\alpha_{t}\right\}_{t \in G}\right)$. Definimos sobre $E$ a ação parcial $\gamma=\left(\left\{E_{t}\right\}_{t \in G},\left\{\gamma_{t}\right\}_{t \in G}\right)$, onde $E_{t}=E D_{t}$, e $\gamma_{t}: E_{t^{-1}} \longrightarrow E_{t}$ é tal que $\left.\gamma_{t}(\xi)\right|_{r}=\Delta(t)^{1 / 2} \xi(r t)$, para toda $\xi \in E_{t-1} \cap C_{c}(\mathcal{B})$. No Teorema 7.38 , obtemos o resultado principal da Parte II. ao mostrar que $\gamma^{r}=a$ e $\gamma^{\prime}=\dot{\alpha}$, onde $\check{\alpha}$ é a ação natural de $G$ sobre $\mathbb{R}(\mathcal{B})$. Decorre entào que $(\mathbb{K}(\mathcal{B}), \check{\alpha})$ é uma ação envolvente a menos de equivalência Morita de $\alpha$, e portanto toda ação parcial possui uma ação envolvente a menos de equivalência Morita .

LEMA 7.34. Seja $B$ um fibrado de Banach sobre o espaço localmente compacto $X$. Suponhamos que $\Theta \subseteq C_{c}(X)$ é denso $\epsilon m C_{c}\left(X^{*}\right)$ na topologia do limite indutivo, e que $F \subseteq C_{c}(B)$ é um subespaço vetorial tal que $\Theta F \subseteq F$. Então o fecho de $F$ na topologia do limite indutivo é o conjunto: $\{g \in$ $\left.C_{c}(B): g(x) \in \overline{F(x)}, \forall x \in X\right\}$.

DemonstraçÃo. Para cada $x \in \mathrm{Y}$, a aplicação $e_{x}: C_{c}(B) \longrightarrow B_{x}$ tal que $f \longmapsto f(x)$ é linear e contínua na topologia do limite indutivo. Portanto $e_{x}(\bar{F}) \subseteq \overline{F(x)}$. Inversamente, suponhamos que $g \in C_{c}(B)$ é tal que $g(x) \in \overline{F(x)}, \forall x \in X$. Como $X$ é localmente compacto, existe $K \subseteq X$ compacto que contém $\sup (g)$ no seu interior. Agora, para cada $\epsilon>0$, e cada $x \in K^{\circ}$, existe $f_{x} \in F$ tal que $\left\|g(x)-f_{x}(x)\right\|<\epsilon$, e podemos supor que $\sup \left(f_{x}\right) \subseteq K$. Como $g, f_{x}$ e a norma sobre $B$ são funções contínuas, existe uma vizinhança aberta $V_{x}$ de $x$ tal que $\left\|g(y)-f_{x}(y)\right\|<\epsilon, \forall y \in V_{x}$. A família $\left(V_{x}\right)_{x \in K}$ é uma cobertura aberta de $K$, que é compacto, e portanto existe uma subcobertura finita $V_{x_{1}}, \ldots, V_{x_{m}}$. Seja $\left(\iota_{j}\right)_{1}^{m}$ uma partiçào da unidade de $K$ subordinada à cobertura $\left(V_{x_{j}}\right)$, e seja $f=\sum_{j=1}^{m} \psi_{j} f_{x}$. Então temos: se $x \notin \kappa,\|g(x)-f(x)\|=0$ porque ambas funçōes se anulam fora de 
$K$; se $x \in K$, então:

$$
\begin{aligned}
\|g(x)-f(x)\| & =\left\|\sum_{j} \psi_{j}(x) g(x)-\sum_{j} \psi_{j}(x) f_{x_{j}}(x)\right\| \\
& =\left\|\sum_{j: x \in V_{x_{j}}} \psi_{j}(x)\left[g(x)-f_{x_{j}}(x)\right]\right\| \\
& \leq \sum_{j} \psi_{j}(x) \epsilon \\
& =\epsilon .
\end{aligned}
$$

Portanto, só falta mostrar que $f \in \bar{F}$. Para isto é suficiente mostrar que $\psi f^{\prime} \in \bar{F}, \forall \psi \in C_{c}(G)$ e $f^{\prime} \in F$. Mas, como $\Theta$ é denso em $C_{c}(G)$, existe $\psi^{\prime} \in \Theta$ tal que $\left\|\psi-\psi^{\prime}\right\|_{\infty} \leq \varepsilon$, para $\varepsilon$ dado, e portanto $\left\|\psi f^{\prime}-\psi^{\prime} f^{\prime}\right\|_{\infty} \leq \varepsilon\|f\|_{\infty}$; como $\Theta F \subseteq F$, temos que $\psi^{\prime} f^{\prime} \in F$, e portanto $\psi f^{\prime} \in \bar{F}$, o que encerra a prova.

Proposição 7.35. Sejam $\mathcal{B}$ um fibrado de Fell, $\beta$ a ação natural de $G$ sobre $\mathbb{E}_{c}(\mathcal{B})$, e $I_{c}(\mathcal{B})=$ $\operatorname{span}\left\langle C_{c}(\mathcal{B}), C_{c}(\mathcal{B})\right\rangle_{1} o^{*}$-ideal bilateral de $\mathbb{R}_{c}(\mathcal{B})$ definido antes da Proposição 7.19. Para cada $t \in G$, seja $I_{t}^{c}=I_{c}(\mathcal{B}) \bigcap \beta_{t}\left(I_{c}(\mathcal{B})\right)$. Entĩo o fecho de $I_{t}^{c}$ na topologia do limite indutivo é o conjunto $\{k \in$ $\left.\mathbb{E}_{c}(\mathcal{B}): k(r, s) \in \overline{\left(\operatorname{span} B_{r} B_{s}^{*}\right) \cap\left(\operatorname{span} B_{r t} B_{s t}^{*}\right)}, \forall r, s \in G\right\}$.

Demonstração. Seja $k \in I_{c}(\mathcal{B})$. Então exsitem $\xi_{i}, \eta_{i} \in C_{c}(\mathcal{B})$, tais que $k=\sum_{i}\left\langle\xi_{i}, \eta_{i}\right\rangle_{l}$. Em particular, $k(r, s)=\sum_{i} \xi_{i}(r) \eta_{i}(s)^{*} \in \operatorname{span} B_{r} B_{s}^{*}, \forall r, s \in G$. Agora se $k \in I_{t}^{c}$, então $\beta_{t-1}(k) \in I_{c}(\mathcal{B})$, e portanto $\beta_{t^{-1}}(k)(r, s) \in \operatorname{span} B_{r} B_{s}^{*}, \forall r, s \in G$, ou seja, $\Delta(t)^{-1} k\left(r t^{-1}, s t^{-1}\right) \in \operatorname{span} B_{r} B_{s}^{*}, \forall r, s \in G$, o que equivale a dizer que $k(r, s) \in \operatorname{span} B_{r t} B_{s t}^{*}, \forall r, s \in G$. Seja $\Theta=C_{c}(G) \odot C_{c}(G)$. Então $\Theta$ é uma subálgebra densa de $C_{c}(G \times G)$ na topologia do limite indutivo. Se $\phi, \psi \in C_{c}(G), \xi, \eta \in C_{c}(\mathcal{B})$, então $(\phi \otimes \psi)\langle\xi, \eta\rangle_{l}=\langle\phi \xi, \dot{v} \eta\rangle_{l}$, donde segue que $\Theta I_{t}^{c} \subseteq I_{t}^{c}$. Agora o resultado segue do Lema 7.34,

Suponhamos a seguir que $\alpha=\left(\left\{D_{t}\right\}_{t \in G},\left\{\alpha_{t}\right\}_{t \in G}\right)$ é uma ação parcial contínua do grupo localmente compacto $G$ sobre a $C^{*}$-álgebra $A$, e seja $\mathcal{B}_{\alpha}$ o fibrado de Fell associado a $\alpha$. Para cada $t \in G$, consideremos o subconjunto $E_{t}:=\overline{\operatorname{span}} E D_{t}$ de $E$. Pelo teorema de Cohen-Hewitt, $E_{t}=\left\{\xi a: \xi \in E, a \in D_{t}\right\}$. Pela Proposição 1.23, $E_{t}$ é um ideal bilateral de $E$. Se $\xi: G \longrightarrow \mathcal{B}$ é uma seção, existe uma única função $\xi^{\prime}: G \longrightarrow A$ tal que $\xi^{\prime}(r) \in D_{r}, \forall r \in G$, e tal que $\xi(r)=\left(r, \xi^{\prime}(r)\right)$. $\xi$ é contínua e de suporte compacto se e somente se $\xi^{\prime}$ é contínua e de suporte compacto. Suponhamos que $\xi \in C_{c}(\mathcal{B})$, e $a \in D_{t}$. Então $\left.\xi a\right|_{r}=\xi(r)(\epsilon, a)=\left(r, \xi^{\prime}(r)\right)(e, a)=\left(r, \alpha_{r}\left(\alpha_{r-1}\left(\xi^{\prime}(r)\right) a\right)\right)$. Como $\alpha_{r^{-1}}\left(\xi^{\prime}(r)\right) a \in D_{r^{-1}} \cap D_{t}$, temos que $\left.\xi a\right|_{r} \in D_{r} \cap D_{r t}, \forall r \in G$. Portanto $E_{t}$ é o fecho de $E_{t}^{c}:=\left\{\xi \in C_{c}(\mathcal{B}): \xi^{\prime}(r) \in D_{r} \cap D_{r t}, \forall r \in G\right\}$.

Proposição 7.36. Sejam $\alpha=\left(\left\{D_{t}\right\}_{t \in G},\left\{\alpha_{t}\right\}_{t \in G}\right)$ uma ação parcial de $G$ sobre A, com fibrado de Fell associado $\mathcal{B}$. Então temos:

1. $B_{r} B_{s}^{*}=\left\{\left(r s^{-1}, D_{r} \cap D_{r s^{-1}}\right)\right\}, \forall r, s \in G$.

2. $B_{r} B_{s}^{*} \cap B_{r t} B_{s t}^{*}=\left\{\left(r s^{-1}, D_{r} \cap D_{r t} \cap D_{r s-1}\right)\right\}, \forall r, s, t \in G$.

3. $O$ fecho de $\left\langle E_{t}^{c} . E_{t}^{c}\right\rangle_{l}$ na topologia do limite indutivo coincide com o fecho de $I_{t}^{c}$ na topologia do limite indutivo, $e$ portanto $\epsilon$ o conjunto: $\left\{k \in \mathbb{E}_{c}(\mathcal{B}): k(r, s) \in B_{r} B_{s}^{*} \cap B_{r t} B_{s t}^{*}, \forall r, s \in G\right\}$

4. $Z_{l}\left(E_{t}\right)=I_{t}$, onde $I_{t}$ é o fecho de $I_{c}^{t}$ em $I$.

5. Se ờ é a ação natural de $G$ sobre $\mathbb{K}(\mathcal{B})$, então ă $\left.\right|_{I}=\left(\left\{I_{t}\right\}_{t \in G},\left\{\left.\check{\alpha}_{t}\right|_{I_{t}}\right\}_{t \in G}\right)$. 
DemonstraçÃo. 1. Lembrar que $B_{r}=\left(r, D_{r}\right), \forall r \in G$.

$$
\begin{aligned}
\left(r, D_{r}\right)\left(s, D_{s}\right)^{*} & =\left(r, D_{r}\right)\left(s^{-1}, \alpha_{s-1}\left(D_{s}\right)\right) \\
& =\left(r s^{-1}, \alpha_{r}\left(\alpha_{r-1}\left(D_{r}\right) \alpha_{s-1}\left(D_{s}\right)\right)\right) \\
& =\left(r s^{-1}, \alpha_{r}\left(D_{r-1} \cap D_{s-1}\right)\right) \\
& =\left(r s^{-1}, D_{r} \cap D_{r s^{-1}}\right)
\end{aligned}
$$

2. $B_{r} B_{s}^{*} \cap B_{r t} B_{s t}^{*}=\left\{\left(r s^{-1}, D_{r} \cap D_{r s^{-1}}\right)\right\} \cap\left\{\left(r s^{-1}, D_{r t} \cap D_{r s-1}\right)\right\}=\left\{\left(r s^{-1}, D_{r} \cap D_{r t} \cap D_{r s^{-1}}\right)\right\}$

3. Sejam $\xi, \eta \in E$. Temos:

$$
\begin{aligned}
\langle\xi, \eta\rangle_{l}(r, s) & =\xi(r) \eta(s)^{*} \\
& =\left(r, \xi^{\prime}(r)\right)\left(s, \eta^{\prime}(s)\right)^{*} \\
& =\left(r, \xi^{\prime}(r)\right)\left(s^{-1}, a_{s-1}\left(\eta^{\prime}(s)^{*}\right)\right) \\
& =\left(r s^{-1}, \alpha_{r}\left(\alpha_{r-1}\left(\xi^{\prime}(r)\right) a_{s-1}\left(\eta^{\prime}(s)\right)\right)\right)
\end{aligned}
$$

Portanto, se $\xi, \eta \in E_{i}^{c}$, então

$$
\begin{aligned}
&\langle\xi, \eta\rangle_{l} \in\left(r s^{-1} \cdot a_{r}\left(a_{r-1}\left(D_{r} \cap D_{r t}\right) \alpha_{s-1}\left(D_{s} \cap D_{s t}\right)\right)\right) \\
&=\left(r s^{-1}, a_{r}\left(D_{r-1} \cap D_{t} \cap D_{s^{-1}}\right)\right) \\
&=\left(r s^{-1}, D_{r} \cap D_{r t} \cap D_{r s^{-1}}\right) \\
&=B_{r} B_{s}^{*} \cap B_{r t} B_{s t}^{*}, \quad \text { por } 2 .
\end{aligned}
$$

Como $B_{r} B_{s}^{*} \cap B_{r t} B_{s t}^{*}$ é um espaço vetorial fechado, o Lema 7.34 mostra que o fecho na topologia do limite indutivo de $\left\langle E_{t}^{c}, E_{t}^{c}\right\rangle_{l}$ é $\left\{k \in \mathbb{k}_{c}(\mathcal{B}): k(r, s) \in B_{r} B_{s}^{*} \cap B_{r t} B_{s t}^{*}, \forall r, s \in G\right\}$, e por 7.35 , este conjunto coincide com o fecho de $I_{t}^{c}$ na topologia do limite indutivo, o que prova 3 .

4. É conseqüencia direta de 3 .

5. Temos que mostrar que $I \cap \check{\alpha}_{t}(I)=I_{t}$. Como $I_{t}^{c}=I_{c}(\mathcal{B}) \cap \check{\alpha}_{t}\left(I_{c}(\mathcal{B})\right) \subseteq I \cap \check{\alpha}_{t}(I)$, temos que $I_{t} \subseteq I \cap \check{\alpha}_{t}(I)$.

Para provar a inclusão inversa, por 1.23 basta mostrar que $\left(I \cap \check{\alpha}_{t}(I)\right) E \subseteq E_{t}$. Seja $x \in I \cap \check{\alpha}_{t}(I)$. Então $x=\check{\alpha}_{t}(y)$, para algum $y \in I$. Para $\epsilon$ dado, seja $k \in \mathbb{R}_{c}(\mathcal{B})$ tal que $\|y-k\|<\epsilon$. Então $\left\|x-\check{\alpha}_{t}(y)\right\|<\epsilon$, porque $\check{\alpha}_{t}$ é uma isometria. Consideremos agora $\xi \in C_{c}(B)$. Temos:

$$
\left.\check{a}_{t}(k) \xi\right|_{r}=\int_{G} \Delta(t) k(r t, s t) \xi(s) d s \in B_{r t} B_{s t} B_{s} .
$$

Por 1., $B_{r t} B_{s t} B_{s}=\left(r s^{-1}, D_{r t} \cap D_{r s^{-1}}\right)\left(s, D_{s}\right)$, e portanto

$$
\begin{aligned}
\left.\check{\alpha}_{t}(k) \xi\right|_{r} \in & \left(r, \alpha_{r s^{-1}}\left(\alpha_{s r^{-1}}\left(D_{r t} \cap D_{r s^{-1}}\right) D_{s}\right)\right) \\
& =\left(r, \alpha_{r s^{-1}}\left(D_{s r^{-1}} \cap D_{s t} \cap D_{s}\right)\right) \\
& =\left(r, D_{r s^{-1}} \cap D_{r t} \cap D_{r}\right) .
\end{aligned}
$$

donde segue que $\tilde{a}_{t}(k) \xi \in E_{t}^{c}$. Pela continuidade do produto, segue agora que $x E \subseteq E_{t}$, $\forall x \in I \cap \check{\alpha}_{t}(I)$. o que encerra a demonstração.

Consideremos agora, para cada $t \in G$, a aplicaçào $\hat{\jmath}_{t}^{\prime}: C_{c}(G, A) \longrightarrow C_{c}(G, A)$ tal que $\left.\gamma_{t}^{\prime}(\xi)\right|_{r}=$ $\Delta(t)^{1 / 2} \xi^{\prime}(r t)$. Notar que se $\xi^{\prime}$ é tal que $G \longmapsto \mathcal{B}_{\alpha}$ é tal que $r \longmapsto\left(r, \xi^{\prime}(r)\right)$ é um elemento de $E_{t-1}^{c}$, então $r \longmapsto\left(r, \gamma_{t}^{\prime}\left(\xi^{\prime}\right)(r)\right)$ é um elemento de $E_{t}^{c}: \gamma_{t}^{\prime}\left(\xi^{\prime}\right)(r)=\Delta(t)^{1 / 2} \xi^{\prime}(r t) \in D_{r t} \cap D_{r t t-1}=D_{r} \cap D_{r t}$. Portanto, podemos definir $\hat{i t}_{t}: E_{t^{-1}}^{c} \longrightarrow E_{t}^{c}$, tal que $\gamma_{t}(\xi)(r)=\left(r, \gamma_{t}^{\prime}\left(\xi^{\prime}\right)(r)\right)=\left(r, \Delta(t)^{1 / 2} \xi^{\prime}(r t)\right)$. 
Lema 7.37. Sejam $\mathrm{X}, Y$ espaços topológicos, $H$ um grupo topológico, e $f: X \times Y \longrightarrow H$ uma funçào contínua. Se $K \subseteq Y$ é compacto e $W$ é uma vizinhança da identidade em $H$, então cada $x_{0} \in X$ tem uma vizinhança $U$ tal que $\forall x \in U$ e $\forall y \in K$, tem-se que $f(x, y) f\left(x_{0}, y\right)^{-1} \in W$, $\epsilon f(x, y)^{-1} f\left(x_{0}, y\right) \in W$. Em particular. se $H$ é um espaço normado $e f$ tem suporte compacto, a função $X \longrightarrow C_{0}(Y, H)$ tal que $x \longmapsto f_{x}$, onde $f_{x}(y)=f(x, y)$, é contínua na topologia da convergéncia uniforme.

Demonstração. Como o produto e a inversão em $H$ são contínuas, e como $f$ é contínua, para cada $y \in K$ existem vizinhanças $U_{y}$ e $F_{y}$ de $x_{0}$ e de $y$ respectivamente, tais que

$$
f(x, y) f\left(x_{0}, y\right)^{-1} \in W, \text { e } f(x, y)^{-1} l\left(x_{0}, y\right) \in W, \forall x \in U_{y}, y \in V_{y} .
$$

Agora, a família $\left(V_{y}\right)_{y \in K}$ é uma cobertura aberta $d \quad \kappa$, e portanto existe uma subcobertura finita $\left\{V_{y_{1}}, \ldots, V_{y_{n}}\right\}$. Seja $U=\bigcap_{j=1}^{n} U_{y_{1}}$. Então $U$ é uma vizinhança de $x_{0}$, e se $(x, y) \in U \times K^{\circ}$, entào existe $y_{j}$ tal que $(x, y) \in U_{y_{j}} \times V_{y_{j}}$, e portanto $f(x, y) f\left(x_{0}, y\right)^{-1} \in W$, e $f(x, y)^{-1} f\left(x_{0}, y\right) \in W$, como tínhamos afirmado.

Se $H$ é um espaço normado, seu grupo aditivo é um grupo topológico com a topologia induzida pela norma. Por outro lado, como $f$ tem suporte compacto, existem compactos $K_{1} \subseteq X$ e $K_{2} \subseteq Y_{\text {, }}$, tais que $\sup (f) \subseteq K_{1} \times K_{2}$. Pela primeira parte do lema, dado $\epsilon>0$, existe uma vizinhança $\bar{U}$ de $x_{0}$ tal que $\left\|f_{x}(y)-f_{x_{0}}(y)\right\|<\epsilon, \forall x \in I^{\prime}, y \in K_{2}$; e se $y \notin K_{2}, f_{x}(y)=f_{x_{0}}(y)=0$, donde segue que $\left\|f_{x}(y)-f_{x_{0}}(y)\right\|<\epsilon, \forall x \in\left[i, y \in Y^{*}\right.$.

TeOrema 7.38. Toda açio parcial contínua de um grupo localmente compacto sobre uma $C^{*}$ álgebra possui açào envolvente a menos de equivaléncia Morita. Mais exatamente, temos o seguinte. Seja $\alpha=\left(\left\{D_{t}\right\}_{t \in G},\left\{\alpha_{t}\right\}_{t \in G}\right)$ uma ação parcial contínua do grupo localmente compacto $G$ sobre a $C^{*}$-álgebra $A$, e seja $\mathcal{B}$ o fibrado de Fell associado a $\alpha$. Sejam $E_{t}^{c}$ e $\gamma_{t}$ definidos acima. Tem-se:

1. Para todo $t, \gamma_{t}: E_{t-1}^{c} \longrightarrow E_{t}^{c} \epsilon_{\text {uma }}$ isometria, que se estende por continuidade a um isomorfismo $\gamma_{t}: E_{t-1} \longrightarrow E_{t}$ de $C^{*}$-anéis ternários.

2. $\gamma=\left(\left\{E_{t}\right\}_{t \in G},\left\{\gamma_{t}\right\}_{t \in G}\right)$ é uma ação parcial contínua de $G$ sobre $E$.

3. $\gamma^{r}=\alpha$, e $\gamma^{l}=\left.\tilde{a}\right|_{I}$, onde ă $\dot{\epsilon}$ a ação natural de $G$ sobre $\mathbb{k}(\mathcal{B})$. Toda ação parcial contínua de um grupo localmente compacto sobre uma $C^{*}$-álgebra possui açâo envolvente a menos de equivaléncia Morita

Demonstração. Sejam $\xi, \eta \in C_{c}(\mathcal{B})$. Identificando $A$ com a fibra de $\mathcal{B}_{\alpha}$ sobre a unidade, e o núcleo $k \in \mathbb{k}_{c}(\mathcal{B})$ com $k^{\prime}: G \times G \longrightarrow .4$ tal que $k(r, s)=\left(r s^{-1}, k^{\prime}(r, s)\right)$, temos as equações:

$$
\begin{aligned}
\langle\xi, \eta\rangle_{l}(r, s) & =\alpha_{r}\left(a_{r^{-1}}\left(\xi^{\prime}(r)\right) \alpha_{s^{-1}}\left(\eta^{\prime}(s)\right)\right) \\
\langle\xi, \eta\rangle_{r} & =\int_{G} a_{r^{-1}}\left(\xi^{\prime}(r)^{*} \eta^{\prime}(r)\right) d r
\end{aligned}
$$

Pela equação (14), se $\xi, \eta \in E_{t-1}^{c}$, temos que:

$$
\begin{aligned}
\left\langle\gamma_{t}(\xi), \gamma_{t}(\eta)\right\rangle_{r} & =\int_{G} a_{r^{-1}}\left(\gamma_{t}^{\prime}\left(\xi^{\prime}\right)(r)^{*} \gamma_{t}^{\prime}\left(\eta^{\prime}\right)(r)\right) d r \\
& =\int_{G} a_{r^{-1}}\left(\Delta(t)^{1 / 2} \xi^{\prime}(r t)^{*} \Delta(t)^{1 / 2} \eta^{\prime}(r t)\right) d r \\
& =\int_{G} \Delta\left(t^{-1}\right) \Delta(t) \alpha_{t s^{-1}}\left(\xi^{\prime}(s)^{*} \eta^{\prime}(s)\right) d s \\
& =\int_{G^{i}} \alpha_{t} a_{s-1}\left(\xi^{\prime}(s)^{*} \eta^{\prime}(s)\right) d s \\
& =\alpha_{t}\left(\langle\xi, \eta\rangle_{r}\right),
\end{aligned}
$$


onde fizemos a mudança $s=r t$ na terceira igualdade. Isto mostra que $\gamma_{t}$ é uma isometria, e portanto se estende a uma isometria bijetiva $\gamma_{t}: E_{t-1} \longrightarrow E_{t}$, tal que $\left\langle\gamma_{t}(\xi), \gamma_{t}(\eta)\right\rangle_{r}=\alpha_{t}\left(\langle\xi, \eta\rangle_{r}\right), \forall \xi, \eta \in E$. Por outro lado, se $\xi \in E_{t^{-1}}^{c}$, e $a \in D_{t^{-1}}$, temos que $\xi a(r)=\left(r, \xi^{\prime}(r)\right)(e, a)=\left(r, \alpha_{r}\left(\alpha_{r^{-1}} \xi^{\prime}(r) a\right)\right.$. Portanto temos:

$$
\begin{aligned}
\gamma_{t}(\xi a)(r) & =\left(r, \Delta(t)^{1 / 2} \alpha_{r t}\left(\alpha_{r t-1}\left(\xi^{\prime}(r t)\right) a\right)\right. \\
& =\left(r, \Delta(t)^{1 / 2} \alpha_{r t}\left(\alpha_{t-1}-1\left(\xi^{\prime}(r t)\right) \alpha_{t-1}\left(\alpha_{t}(a)\right)\right)\right. \\
& =\left(r, \Delta(t)^{1 / 2} \alpha_{r}\left(\alpha_{r-1}\left(\xi^{\prime}(r t)\right) \alpha_{t}(a)\right)\right. \\
& =\left(r, \Delta(t)^{1 / 2} \xi^{\prime}(r t)\right)\left(e, \alpha_{t}(a)\right) \\
& =\gamma_{t}(\xi) \alpha_{t}(a)(r) .
\end{aligned}
$$

Portanto $\gamma_{t}$ é um isomorfismo de $C^{*}$-anéis ternários, e $Z_{l}\left(\gamma_{i t}\right)=\alpha_{t}$. o que prova 1.

Vejamos que $\gamma$ é uma açào parcial: claramente temos que $E_{r}=E r \gamma_{c}=i d_{E}$. Suponhamos agora que $\xi \in E_{t-1}^{c}$ é tal que $\gamma_{t}(\xi) \in E_{s-1}^{c}$. Entào:

$$
\begin{aligned}
\left.\gamma_{s \hat{\imath} t}(\xi)\right|_{r} & =\left(r, \Delta(s)^{1 / 2}\left(\gamma_{t}^{\prime}\left(\xi^{\prime}\right)\right)(r s)\right) \\
& =\left(r, \Delta(s)^{1 / 2} \Delta(t)^{1 / 2} \xi^{\prime}(r s t)\right) \\
& =\left(r, \Delta(s t)^{1 / 2} \xi^{\prime}(r s t)\right) \\
& =\left.\gamma_{s t}(\xi)\right|_{r} .
\end{aligned}
$$

Assim, $\gamma_{s t}$ é uma extensão de $\gamma_{s} \gamma_{t}$. e portanto $\gamma$ é uma açào parcial sobre $l:$ Logo, 2. está provado. a menos da continuidade de $\gamma$, que provaremos depois.

Como $Z_{l}\left(E_{t}\right)=D_{t}$ por 1.23 , do cálculo que mostrou que cada $\gamma_{t} \dot{\rho}$ uma isometria segue também que $\gamma^{r}=\alpha$, o que demonstra a primeira metade de 3 . Vejamos que $\gamma^{\prime}=\left.i\right|_{l}$. Se $\xi, \eta \in E_{t-1}^{c}$, pela equação (13) temos:

$$
\begin{aligned}
\left\langle\gamma_{t}(\xi), \gamma_{t}(\eta)\right\rangle_{l}(r, s) & =\alpha_{r}\left(\alpha_{r-1}\left(\gamma_{t}^{\prime}\left(\xi^{\prime}\right)(r)\right) \alpha_{s-1}\left(\gamma_{t}^{\prime}\left(\eta^{\prime}\right)(s)\right)\right) \\
& =\alpha_{r}\left(\alpha_{r-1}\left(\Delta(t)^{1 / 2} \xi^{\prime}(r t)\right) \alpha_{s-1}\left(\Delta(t)^{1 / 2} \eta^{\prime}(s t)\right)\right) \\
& =\Delta(t) \alpha_{r t}\left(\alpha_{r t-1}\left(\xi^{\prime}(r t)\right) \alpha_{s t-1}\left(\eta^{\prime}(s t)\right)\right) \\
& =\Delta(t)\langle\xi, \eta\rangle_{l}(r t, s t) \\
& =\check{\alpha}_{t}\left(\langle\xi, \eta\rangle_{l}\right)(r, s)
\end{aligned}
$$

Portanto $\check{\alpha}_{t}$ é uma extensão de $Z_{l}\left(\gamma_{t}\right), \forall t \in G$. Pela Proposição 7.36 . tem-se $Z_{l}\left(E_{t}\right)=I_{t}, \forall t \in G$, donde conluímos que $\gamma^{l}=\left.\check{a}\right|_{I}$, e 3 . está provado.

Só falta provar que $\gamma$ é contínua, porque assim 2. estará demonstrado. e 2. e 3. implicam que a é uma ação envolvente a menos de equivalência Morita de $a$.

Temos que mostrar que se $\xi_{i}$ é uma rede em $E$ que converge a $\xi$. com $\xi_{i} \in E_{t^{-1}}, \xi \in E_{t-1}$, então $\gamma_{t_{i}}\left(\xi_{i}\right) \longrightarrow \gamma_{t}(\xi)$. Dado $\epsilon>0$, vamos mostrar que existe $i_{0}$ tal que $\left\|\gamma_{t_{1}}\left(\xi_{i}\right)-\gamma_{t}(\xi)\right\|<\epsilon, \forall i \geq i_{0}$.

Pelo teorema de Cohen-Hewitt, existem $\xi^{\prime} \in E_{t^{-1}}, a \in D_{t^{-1}}$. tais que $\xi=\xi^{\prime} a$, e podemos supor que $a \neq 0$. Como $E_{t^{-1}}^{c}$ é denso em $E_{t^{-1}}$, existe $\eta \in E_{t^{-1}}^{c}$ tal que $\left\|\xi^{\prime}-\eta\right\|<\epsilon / 4\|a\|$. Portanto,

$$
\|\xi-\eta a\| \leq \epsilon / 4
$$

Como a família $\left\{D_{r}\right\}_{r \in G}$ é contínua, existe $d \in C_{c}(G . A)$ tal que $d\left(t^{-1}\right)=a$. Definamos então $\eta_{s}=\eta d\left(s^{-1}\right)$. Em particular, $\eta_{t}=\eta a$. Notar que $\eta_{s} \in E_{t^{-1}}^{c} \bigcap E_{s-1}^{c}$, porque $\eta \in E_{t-1}^{c}$, e $d\left(s^{-1}\right) \in D_{s-1}$, $\forall s \in G$. Como a açào de $A$ sobre $E$ é contínua, e como $d$ é contínua, temos que $\eta_{s} \longrightarrow \eta_{t}$ em $E$. Portanto, existe $i_{1}$ tal que $\forall i \geq i_{1}$ se tem :

$$
\left\|\xi-\eta_{t,}\right\| \leq \epsilon / 4
$$


(Na verdade, temos ainda que $\eta_{s} \longrightarrow \eta_{t}$ na topologia do limite indutivo, porque $\left\|\eta_{s}(r)-\eta_{t}(r)\right\|=$ $\left.\left\|\eta(r) d\left(s^{-1}\right)-\eta(r) d\left(t^{-1}\right)\right\| \leq\|\eta\|_{\infty}\left\|d\left(s^{-1}\right)-d\left(t^{-1}\right)\right\|\right)$.

Seja $\eta(r)=\left(r, \eta^{\prime}(r)\right)$. Como $\eta_{s}=\eta d\left(s^{-1}\right)$ deve ser: $\left(r, \eta_{s}^{\prime}(r)\right)=\left(r, \eta^{\prime}(r)\right)\left(e, d\left(s^{-1}\right)\right)=\left(r, \alpha_{r}\left(\alpha_{r^{-1}}\left(\eta^{\prime}(r) d\left(s^{-1}\right)\right)\right)\right)$ Logo, $\gamma_{s} \eta_{s}(r)=\left(r, \gamma_{s}^{\prime} \eta_{s}^{\prime}(r)\right)=\left(r, \Delta(s)^{1 / 2} a_{r s}\left(a_{s^{-1} r^{-1}}\left(\eta^{\prime}(r s) d\left(s^{-1}\right)\right)\right)\right)$. Portanto, a função $G \times G \longrightarrow$ $A$ tal que $(s, r) \longmapsto \gamma_{s}^{\prime} \eta_{s}^{\prime}(r)$ é contínua e de suporte compacto. Aplicando o Lema $7.3 \tau$, temos que a função $G \longrightarrow C_{0}(A)$ tal que $s \longmapsto \gamma_{s}^{\prime} \eta_{s}^{\prime}$ é contínua. Logo, $\left\|\gamma_{s} \eta_{s}-\gamma_{t} \eta_{t}\right\|_{\infty} \longrightarrow 0$ quando $s \longrightarrow t$. Então temos também que $\left\|\gamma_{s} \eta_{s}-\gamma_{t} \eta_{t}\right\| \longrightarrow 0$ se $s \longrightarrow t$. Assim, existe $i_{2}$ tal que $\forall i \geq i_{2}$ tem-se que:

$$
\left\|\gamma_{t}, \eta_{t,}-\gamma_{t} \eta_{t}\right\| \leq \epsilon / 4
$$

Por último, como $\xi_{i} \longrightarrow \xi$, existe $i_{3}$ tal que $\forall i \geq i_{3}$ se tem:

$$
\left\|\xi_{i}-\xi\right\|<\epsilon / 4
$$

Seja $i_{0} \geq i_{1}, i_{2}, i_{3}$. Como cada $\imath_{\text {s }}$ é uma isometria, se $i \geq i_{0}$, por (15). (16), (17) e (18) tem-se:

$$
\begin{aligned}
\left\|\gamma_{t_{2}}\left(\xi_{i}\right)-\eta_{1}(\xi)\right\| & \leq\left\|\hat{t}_{t_{i}}\left(\xi_{i}\right)-\gamma_{t_{i}}\left(\eta_{t_{1}}\right)\right\|+\left\|\gamma_{t_{i}}\left(\eta_{t}\right)-\gamma_{t}\left(\eta_{t}\right)\right\|+\left\|\gamma_{t}\left(\eta_{t}\right)-\gamma_{t}(\xi)\right\| \\
& \leq\left\|\xi_{i}-\eta_{t_{1}}\right\|+\epsilon / 4+\|\eta a-\xi\| \\
& \leq\left\|\xi_{i}-\xi\right\|+\left\|\xi-\eta_{t}\right\|+\epsilon / 2 \\
& <\epsilon
\end{aligned}
$$

Isto demonstra que $\gamma$ é uma ação parcial contínua, o que encerra a prova.

\subsection{Sobre a unicidade da ação envolvente a menos de equivalência Morita}

O objetivo desta seçào é mostrar que, a menos de equivalência Morita, a ação envolvente a menos de equivalência Morita é única. Isto é provado na Proposição 7.41. Se $\mathcal{C}_{M}$ é a categoria de $C^{*}$-álgebras com os bimódulos de equivaléncia Morita como morfismos, a Proposição 7.41 junto com o Teorema $\bar{i} .38$ podem ser enunciados assim: para toda ação parcial contínua de um grupo localmente compacto sobre uma $C^{*}$-álgebra existe uma única açào envolvente em $\mathcal{C}_{M}$.

Teorema 7.39. Sejam $\mathcal{B}=\left(B_{t}\right)_{t \in G}$ um fibrado de Fell sobre o grupo localment $\epsilon$ compacto $G$, $\mathcal{E}=\left(E_{t}\right)_{t \in G}$ um ideal à direita de $\mathcal{B}, \in \mathcal{A}=\left(A_{t}\right)_{t \in G}$ um subfibrado de Fell de $\mathcal{B}$ contido em $\mathcal{E}$. Definamos $\mathbb{k}_{c}(\mathcal{E})=\left\{k \in \mathbb{F}_{c}(\mathcal{B}): k(r, s) \in E_{r s^{-1}}, \forall r, s \in G\right\}$, e seja $\mathbb{k}_{r}(\mathcal{E})$ o fecho de $\mathbb{E}_{c}(\mathcal{E})$ dentro de r(B). Suponhamos que $\mathcal{A} \mathcal{E} \subseteq \mathcal{E} \in \mathcal{E} \mathcal{E}^{*} \subseteq \mathcal{A}$. Então temos:

1. $\mathbb{k}_{c}(\mathcal{A}) \mathbb{k}_{c}(\mathcal{E}) \subseteq \mathbb{k}_{c}(\mathcal{E}), \in \mathbb{R}_{r}(\mathcal{A}) \mathbb{k}_{r}(\mathcal{E}) \subseteq \mathbb{k}_{r}(\mathcal{E})$

2. $\mathbb{k}_{c}(\mathcal{E}) \mathbb{k}_{c}(\mathcal{B}) \subseteq \mathbb{k}_{c}(\mathcal{E}), e \mathbb{k}_{r}(\mathcal{E}) \mathbb{k}_{(}(\mathcal{B}) \subseteq \mathbb{R}_{r}(\mathcal{E})$.

3. $\mathbb{k}_{c}(\mathcal{E}) \mathbb{k}_{c}(\mathcal{E})^{*} \subseteq \mathbb{k}_{c}(\mathcal{A}), e \mathbb{k}_{r}(\mathcal{E}) \mathbb{k}_{r}(\overline{\mathcal{E}})^{*}=\mathbb{k}_{r}(\mathcal{A})$. Em particular, $\mathbb{k}_{r}(\mathcal{A})$ é uma sub-C $C^{*}$-álgebra hereditária de $\mathbb{k}(\mathcal{B})$.

4. Suponhamos que para cada $t \in G$ tem-se que $\left(\operatorname{span} \mathcal{E}^{*} \mathcal{E}\right) \cap B_{t}$ é denso em $B_{t}$. Então o fecho de span $\mathbb{k}_{c}(\mathcal{E})^{*} \mathbb{k}_{c}(\mathcal{E})$ em $\mathbb{k}_{c}(\mathcal{B})$ na topologia do limite indutivo é $\mathbb{k}_{c}(\mathcal{B})$, e $\overline{\operatorname{span} \mathbb{k}_{r}}(\mathcal{E})^{{ }^{*} \underline{x}_{r}}(\mathcal{E})=\mathbb{k}(\mathcal{B})$. Em particular, $\mathbb{R}_{r}(\mathcal{A}) \in \mathbb{R}(\mathcal{B})$ são equivalentes Morita.

Além disso, $\mathbb{k}_{r}(\mathcal{E})$ é invariante pela ação natural de $G$ sobre $\mathbb{k}(\mathcal{B}) ;$ na hipótese de 4 . acima, as ações naturais de $G$ sobre $\mathbb{k}_{r}(\mathcal{A})$ e $\mathbb{E}(\mathcal{B})$ sio equivalentes Morita.

Demonstraçào. Pelos comentários finais na subseção 7.2 .2 , sabemos que $\mathbb{k}_{r}(\mathcal{A}) \subseteq \mathbb{k}(\mathcal{B})$.

Sejam $a \in \mathbb{K}_{c}(\mathcal{A}), \xi, \eta \in \mathbb{K}_{c}(\mathcal{E}), b \in \mathbb{R}_{c}(\mathcal{B}), r, s \in G$. Então:

1. $a * \xi(r, s)=\int_{G} a(r, t) \xi(t, s) d t \in A_{r t-1} E_{t s^{-1}} \subseteq \mathcal{E} \cap B_{r s^{-1}}=E_{r s^{-1}}$. Portanto , $\underline{\sqsupset}_{c}(\mathcal{A}) \mathbb{k}_{c}(\mathcal{E}) \subseteq$ $\mathbb{k}_{c}(\mathcal{E})$, donde segue também, tomando fechos, que $\mathbb{k}_{r}(\mathcal{A}) \mathbb{k}_{r}(\mathcal{E}) \subseteq \mathbb{k}_{r}(\mathcal{E})$ 
2. $\xi * b(r, s)=\int_{G} \xi(r, t) b(t, s) d t \in E_{r t^{-1}} B_{t s^{-1}} \subseteq \mathcal{E} \cap B_{r s^{-1}}=E_{r s^{-1}}$, o que prova a primeira afirmação de 2.: de novo, a segunda é uma consequéncia da primeira.

3. $\xi * \eta^{*}(r, s)=\int_{G} \xi(r, t) \eta(s, t)^{*} d t \in E_{r t^{-1}} E_{t s^{-1}} \subseteq \mathcal{A} \cap B_{r s^{-1}}$. Portanto, $\mathbb{R}_{c}(\mathcal{E}) \mathbb{R}_{c}(\mathcal{E})^{*} \subseteq \mathbb{R}_{c}(\mathcal{A})$ e $\mathbb{k}_{r}(\mathcal{E}) \mathbb{R}_{r}(\mathcal{E})^{*} \subseteq \mathbb{i r}_{r}(\mathcal{A})$. A igualdade segue do teorema de Cohen-Hewitt e da inclusào $\mathbb{k}_{r}(\mathcal{A}) \mathbb{k}_{r}(\mathcal{A})^{*} \subseteq \mathbb{R}_{r}(\mathcal{E}) \mathbb{R}_{r}(\mathcal{E})^{*}$

4. Seja $F=\operatorname{span} \mathbb{R}_{c}(\mathcal{E})^{*} \mathbb{Z}_{c}(\mathcal{E}) \subseteq \mathbb{R}_{c}(\mathcal{B})$. Queremos ver que o fecho de $F$ na topologia do limite indutivo é todo $\mathbb{B}_{c}(\mathcal{B})$. Para isso, usaremos mais uma vez o Lema 3.14. Seja $\Theta=C_{c}(G) \odot C_{c}(G)$, subálgebra densa de $C_{c}\left(C \times G^{\prime}\right)$. Se $\varphi, \psi \in C_{C}(G), \xi, \eta \in \mathbb{R}_{c}(\mathcal{E})$, entāo:

$$
\begin{aligned}
\left(\varphi \cdot \zeta^{*}\right) \xi^{*} * \eta(r, s) & =\int_{G} \varphi(r) \psi(s) \xi(t, r)^{*} \eta(t, s) d t \\
& =\int_{G}((\bar{\varphi} \xi)(t, r))^{*}(\imath \cdot \eta)(t, s) d t \\
& =(\bar{\varphi} \xi)^{*} *(\psi \cdot \eta)(r, s) .
\end{aligned}
$$

onde $\varphi \xi(r, s)=\gamma(r) \xi(r, s)$. ('omo $\varphi \xi \in \mathbb{R}_{c}(\mathcal{E}), \forall \varphi \in C_{c}(G), \varepsilon \in \mathbb{R}_{c}(\mathcal{E})$, vemos que $\Theta F \subseteq F$.

Seja agora $a \in B_{r,-1}$ tal que $a=b^{*} c$, com $b \in E_{t r^{-1}}$ e $c \in E_{t s^{-1}}$, para algum $t \in G$. Sejam $\xi, \eta \in \mathbb{R}_{c}(\mathcal{E})=C_{c}\left(\mathcal{E}_{\nu}\right)$ tais que $\xi(t, r)=b, \eta(t, s)=c$, de forma que $\xi(t, r)^{*} \eta(t, s)=b^{*} c=a$. Sejam $\mathcal{V}$ uma base de vizinhanças da identidade de $G$, e $(f \cdot)^{\prime} \in \mathcal{V}$ uma identidade aproximada de $L^{1}(G)$ como a considerada no Lema 6.2. Para cada $\mathcal{V} \in \mathcal{V}$ consideremos $\eta_{V}^{t} \in \mathbb{k}_{c}(\mathcal{E})$ tal que $\eta_{V^{\prime}}^{t}(r, s)=f_{\mathrm{V}} \cdot\left(t^{-1} r\right) \eta(r, s)$. A função $G \longrightarrow B_{r s-1}$ tal que $u \longrightarrow \xi(u, r)^{*} \eta(u, s)$ é contínua de suporte compacto. Portanto, por 6.1 , dado $\epsilon>0$ existe uma vizinhança $V_{\epsilon}^{\prime}$ da identidade em $G$ tal que se $t^{-1} u \in I_{\text {, }}$. entào tem-se que $\left\|\xi(u, r)^{*} \eta(u, s)-\xi(t, r)^{*} \eta(t, s)\right\|<\epsilon$. Assim, se $V \geq V_{\epsilon}$ :

$$
\begin{aligned}
\left\|\xi^{*} * \eta_{\mathrm{Y}}^{t} \cdot(r, s)-a\right\| & =\left\|\int_{G^{\prime}} f_{\mathrm{V}} \cdot\left(t^{-1} u\right)\left[\xi(u, r)^{*} \eta(u, s)-\xi(t, r)^{*} \eta(t, s)\right] d u\right\| \\
& \leq \int_{t \mathrm{Y}} f_{\mathrm{V}^{*}} \cdot\left(t^{-1} u\right)\left\|\xi(u, r)^{*} \eta(u, s)-\xi(t, r)^{*} \eta(t, s)\right\| d u \\
& <\int_{C^{\prime}} f_{\mathrm{V}} \cdot\left(t^{-1} u\right) \epsilon d u \\
& =\epsilon
\end{aligned}
$$

Como por hipótese sabemos que $B_{r^{-1}}=\overline{\operatorname{span}}\left\{E_{u r^{-1}}^{*} E_{u s^{-1}}: u \in G\right\}$, concluímos então que $\operatorname{span} \mathbb{k}_{c}(\mathcal{E})^{*} \mathbb{K}_{c}(\mathcal{E}) \cap B_{r s^{-1}}$ é denso em $B_{r s^{-1}}, \forall r, s \in G$, e portanto span $\mathbb{k}_{c}(\mathcal{E})^{*} \mathbb{R}_{c}(\mathcal{E})$ é denso em $\mathbb{k}_{c}(\mathcal{B})$ na topologia do limite indutivo. Isto, junto com 3 ., prova que $\mathbb{k}_{r}(\mathcal{A})$ e $\mathbb{k}_{c}(\mathcal{B})$ sào equivalentes Morita.

Sejam $\beta$ a açào natural de $G$ sobre $\mathbb{L}_{(}(\mathcal{B})$, e $k \in \mathbb{k}_{c}(\mathcal{E}), t \in G$. Entào, $\left.\beta_{t}(k)\right|_{(r, s)}=\Delta(t) k(r t, s t) \in$ $E_{r t(s t)^{-1}}=E_{s-1}$, e portanto $k \in \mathbb{E}_{c}(\mathcal{E})$. Segue que $\beta_{t}\left(\mathbb{k}_{c}(\mathcal{E})\right)=\mathbb{k}_{c}(\mathcal{E})$, e portanto que $\beta_{t}\left(\mathbb{k}_{r}(\mathcal{E})\right)=$ $\mathbb{R}_{r}(\mathcal{E})$. Isto prova as afirmaçòes finais do teorema.

Corolário 7.40. Sejam a $\in \beta$ açòes parciais contínuas equivalentes Morita do grupo localmente compacto $G$, com fibrados de Fell associados $\mathcal{B}_{\alpha}$ e $\mathcal{B}_{\beta}$ respectivamente. Sejam $\check{\alpha}$ e $\check{\beta}$ as açôes naturais de $G$ sobre $\mathbb{R}_{r}\left(\mathcal{B}_{\alpha}\right) \in \mathbb{E}_{r}\left(\mathcal{B}_{3}\right)$. Então à $\epsilon \check{\beta}$ são equivalentes Morita; em particular, $\mathbb{R}_{r}\left(\mathcal{B}_{\alpha}\right)$ e $\mathbb{R}_{r}\left(\mathcal{B}_{\beta}\right)$ são equivalentes Morita.

Demonstraçìo. Suponhamos que a e $\beta$ são equivalentes Morita através de certa ação parcial $\gamma$. Pela transitividade da equivalencia Morita de ações parciais, podemos substituir $\beta$ pela ação parcial vinculante de $\gamma$ (página 138). Na demonstração da Proposição 7.11 , construímos um ideal direito $\mathcal{E}$ 
de $\mathcal{B}_{\beta}$ tal que $\mathcal{B}_{\alpha}, \mathcal{E}$ e $\mathcal{B}_{\beta}$ satisfazem as hipóteses do Teorema 7.39 (com $\mathcal{B}_{a}$ em lugar de $\mathcal{A}$ e $\mathcal{B}_{\beta}$ em lugar de $\mathcal{B}$ ), incluíndo a hipótese 4 . Agora o resultado decorre de aplicar 7.39 .

ProposiçẪo 7.41. A ação envolvente a menos de equivaléncıa Morita í única. a menos de equivalência Morita.

Demonstração. Como a equivalencia Morita de açōes parciais é transitiva, pelo Corolário 7.40 acima basta provar que, se $(\beta, B)$ é uma ação envolvente da ação parcial $(\alpha, A)$ e $\dot{\alpha}$ é a ação natural sobre $\mathbb{k}_{r}(\mathcal{A})$, onde $\mathcal{A}$ é o fibrado de Fell associado a $\alpha$, entào $\beta$ e $\dot{\alpha}$ são equivalentes Morita.

Agora, sejam $\mathcal{B}$ o fibrado de Fell associado à ação envolvente 3 , e $\overline{3}$ a açào natural de $G$ sobre $\mathcal{E}(\mathcal{B})$. Consideremos o ideal direito $\mathcal{E}$ de $\mathcal{B}$ construído na demonstração do Teorema 6.6 , ou seja, $\mathcal{E}=\{(t, x) \in \mathcal{B}: x \in \mathcal{A}, \forall t \in G\}$. Estamos então nas hipóteses do Teorema $T .39$. donde segue que $\bar{a}$ e $\ddot{\beta}$ são equivalentes Morita. Mas, como $\mathcal{B}$ é um fibrado saturado, temos por $T .20$ que $\mathbb{K}(\mathcal{B})=I$, e portanto $\check{\beta} \stackrel{M}{\sim} \beta$ por 7.38 2. e 3 . Como a equivalencia Morita de açoes parciais é transitiva, concluímos que $\check{\alpha} \stackrel{M}{\sim} \beta$.

\subsection{Açōes envolventes e produtos cruzados por coaçōes}

Relacionamos abaixo o trabalho feito na Seção $2 \mathrm{com}$ os produtos cruzados por coaçòes. Concretamente, provamos que $\mathbb{k}(\mathcal{B})$ é o produto cruzado reduzido de $C_{r}^{\prime *}(\mathcal{B})$ pela coação dual. e que a ação natural $\beta$ sobre $\mathbb{E}(\mathcal{B})$ é a ação dual de $G$ sobre $C_{r}^{*}(\mathcal{B}) \rtimes_{\delta, r} G$. As seguintes sào boas referencias sobre coações e produtos cruzados por coaçòes:[42], [45], [46], e [5].

Se $G$ é um grupo localmente compacto abeliano que atua sobre uma ( ${ }^{*}$-álgebra $A$ através de uma ação $\alpha$, existe uma ação $\alpha$ do grupo dual $G$ sobre o produto cruzado $A \rtimes_{a} G$, e o Teorema de Takai afirma que: $A \rtimes_{\alpha} G \rtimes_{\hat{\alpha}} G \cong A \otimes \hat{\Lambda}\left(L^{2}(G)\right)$, através de um isomorfismo que leva a ação bidual $\alpha$ sobre $A \rtimes_{\alpha} G \rtimes_{\hat{a}} G$ na ação a $\bigcirc A(\rho)$ sobre $A \otimes \mathcal{K}\left(L^{2}\left(G^{\prime} \quad\right.\right.$ nde $\left.\rho_{t}(\xi)\right|_{r}=\rho(r t), \forall \xi \in L^{2}(G)$.

A partir do resultado de Takai. tentou-se achar versi if comutativas do mesmo teorema. O conceito de coação surge de buscar um substituto par : is lual sobre um produto cruzado por um grupo, quando este nào é abeliano ou, melhor ainda, sinł-lesmente do fato de "dualizar" o conceito de ação. A partir do caso abeliano, e via a transformada de iourier, descobrimos quais são as propriedades que uma tal coação deve ter. O fato das $C^{*}$-álgebras não terem em geral elemento unidade (por exemplo $C_{r}^{*}(G)$ se $G$ não é discreto) implica certas dificuldades técnicas, e somos obrigados a considerar as seguintes $C^{*}$-álgebras, um pouco artificiais: se $A$ e $B$ são $C^{*}$-álgebras, definimos $\dot{M}\left(A \bigotimes_{\min } B\right):=$ $\left\{m \in M\left(A \bigotimes_{\min } B\right): m(1 \odot B)+(1 \odot B) m \subseteq A \bigotimes_{\text {min }} B\right\}$. Por exemplo. $\dot{M}\left(A \bigotimes_{\min } C_{0}(G)\right) \cong$ $C_{b}(G, A)$. A primeira coisa a ser dualizada é o produto sobre $G$. Seja $w_{G}: L^{2}\left((i \times G) \longrightarrow L^{2}(G \times G)\right.$ tal que $\left.w_{G}(\xi)\right|_{(r, s)}=\xi\left(r, r^{-1} s\right) ; w_{G}$ é um operador unitário, com adjunto $\left.w_{G}^{*}(\xi)\right|_{(r, s)}=\xi(r, r s)$. A multiplicação sobre $G$ deve ser substituída por uma comultiplicaçào $\delta_{G}$ sobre $C_{r}^{=}(G)$, dada por $\delta_{G}: C_{r}^{*}(G) \longrightarrow \tilde{M}\left(C_{r}^{*}(G) \bigotimes_{\min } C_{r}^{*}(G)\right)$ tal que $\delta_{G}(x)=w_{G}(x \odot 1) u_{G}^{*}, \forall x \in C_{r}^{*}(G)$ (notar que $C_{r}^{*}(G) \subseteq B\left(L^{2}(G)\right)$ por definição).

DEFINIÇÃo 7.42. Uma coação de um grupo localmente compacto $G$ sobre uma $C^{*}$-álgebra $A$ é um homomorfismo injetivo $\delta: A \longrightarrow \dot{M}\left(A \bigotimes_{\min } C_{r}^{*}(G)\right)$ tal que para toda unidade aproximada $\left(e_{i}\right)_{i \in I}$ de $A$ tem-se que $\delta\left(e_{i}\right)$ converge estritamente a $1 \mathrm{em} \tilde{M}\left(A \bigotimes_{\min } C_{r}^{*}(G)\right)$, e tal que

$$
(\delta \odot i d) \delta=\left(i d \otimes \delta_{G}\right) \delta
$$

Em particular, $\delta_{G}$ é uma coação de $G$ sobre $C_{r}^{*}(G)$. 
Se $G$ é abeliano, as ações de $G$ se correspondem bijetivamente com as coações de $G$, via a fórmula:

$$
(a: G \times A \longrightarrow A) \longmapsto\left(\delta: A \longrightarrow \tilde{M}\left(A \bigotimes_{\min } C_{r}^{*}(G)\right)\right)
$$

$\operatorname{com} \delta=\left(\right.$ id $\left.\odot A d\left(\mathfrak{F}^{*}\right)\right) \delta^{\prime}$, onde $\mathfrak{F}: L^{2}(G) \longrightarrow L^{2}(G)$ é a transformada de Fourier: $\left.\mathfrak{F} \xi\right|_{x}=$ $\int_{G} \overline{\langle t, x\rangle} \xi(t) d t, \xi \in L^{2}(G)$, e $x \in G$; a aplicação $\delta^{\prime}: A \longrightarrow . \bar{I}\left(A \bigotimes_{\min } C_{0}(\dot{G})\right) \cong C_{b}(\dot{G}, A)$ é dada por $\left.\delta^{\prime}(a)\right|_{x}=\alpha_{x}(a)$. Esta correspondencia permite traduzir a definição de produto cruzado reduzido por uma ação para o caso de uma coaçào. Temos assim:

DEFINIÇÃo 7.43 . Seja $\delta: A \longrightarrow \dot{M}\left(A \bigotimes_{\operatorname{mín}} C_{r}^{*}(G)\right)$ uma coaçào do grupo localmente compacto $G$ sobre a $C^{*}$-álgebra $A$. O produto cruzado reduzido $A \rtimes_{\delta, r} G$ de $A$ pela coação $\delta$ é:

$$
A \rtimes_{\delta, r} G:=\left\{\delta(a)(1 \odot \varphi): a \in A, \varphi \in C_{0}(G)\right\} \subseteq M\left(A \bigotimes_{\min } \mathcal{K}\right)
$$

onde $\varphi \in C_{0}(G)$ age por multiplicaçào sobre $L^{2}(G)$.

Se $\delta$ é uma coaçào de $G$ sobre $A$, entào existe uma açào natural $\delta$ de $G$ sobre $A \rtimes_{\delta, r} G$. Esta ação é chamada açào dual de $G$ sobre $A \rtimes_{\delta, r} \hat{G}$, e está caracterizada pelo fato de satisfazer:

$$
\delta_{t}(\delta(a)(1 \otimes \varphi))=\delta(a)\left(1 \odot \varphi_{t}\right)
$$

onde, se $\varphi \in C_{0}(G)$, entào $\varphi_{t}(s)=\varphi(s t), \forall s \in G$.

Consideremos agora um fibrado de Fell $\mathcal{B}=\left(B_{t}\right)_{t \in G}$ sobre o grupo localmente compacto $G$, e seja $\mathcal{B}_{G}$ o fibrado do grupo $G(2.13)$. Temos assim o fibrado de Fell $\mathcal{B} \otimes \mathcal{B}_{G}$ sobre $G \times G$, cuja $C^{*}$-álgebra reduzida é naturalmente isomorfa a $C_{r}^{*}(\mathcal{B}) \bigotimes_{\min } C_{r}^{*}(G)$ pela Proposição 3.20 . Também temos que $L^{2}\left(\mathcal{B} \otimes_{\mathcal{B}_{G}}\right) \cong L^{2}(\mathcal{B}) \otimes L^{2}(G)$ (3.18). Seja $w_{\mathcal{B}}: L^{2}\left(\mathcal{B} \otimes \mathcal{B}_{G} \longrightarrow L^{2}\left(\mathcal{B} \otimes \mathcal{B}_{G}\right.\right.$ tal que $\left.w_{\mathcal{B}}(\xi)\right|_{(r, s)}=\xi\left(r, r^{-1} s\right)$. Então $w_{\mathcal{B}}$ é um operador adjuntável, e de fato unitário, com adjunto $w_{\mathcal{B}}^{*}$ tal que $\left.w_{\mathcal{B}}(\xi)\right|_{(r, s)}=\xi(r, r s)$. Podemos considerar agora a aplicaçào $\mathcal{L}\left(L^{2}(\mathcal{B})\right) \longrightarrow \mathcal{L}\left(L^{2}\left(\mathcal{B} \otimes \mathcal{B}_{G}\right)\right)$ tal que $x \longmapsto w_{\mathcal{B}}(x @ 1) w_{\mathcal{B}}^{*}$.

DEFINIÇÃo 7.44. A restriçào da aplicação acima a $C_{r}^{*}(\mathcal{B})$ é uma coação

$$
\delta_{\mathcal{B}}: C_{r}^{*}(\mathcal{B}) \longrightarrow \tilde{M}\left(C_{r}^{*}(\mathcal{B}) \bigotimes_{\min } C_{r}^{*}(G)\right)
$$

chamada coação dual de $G$ sobre $C_{r}^{*}(\mathcal{B})$. 
Suponhamos que $f \in C_{c}(\mathcal{B}), \xi \in C_{c}(\mathcal{B}) \subseteq L^{2}(\mathcal{B})$, e $x \in C_{c}(G) \subseteq L^{2}(G)$. Então temos:

$$
\begin{aligned}
\left.\delta_{\mathcal{L}}\left(\Lambda_{f}\right)(\xi \odot x)\right|_{(r, s)} & =\left.w_{\mathcal{B}}\left(\Lambda_{f} \odot 1\right) w_{\mathcal{B}}^{*}(\xi \odot x)\right|_{(r, s)} \\
& =\left.\left(\Lambda_{f} \odot 1\right) w_{\mathcal{B}}^{*}(\xi \odot x)\right|_{\left(r, r^{-1} s\right)} \\
& =\left.\int_{G}\left(\Lambda_{f(t)} \odot 1\right) w_{\mathcal{B}}^{*}(\xi \odot x)\right|_{\left(t^{-1}, r^{-1}, s\right)} d t \\
& =\left.\int_{G}\left(\Lambda_{f(t)} \odot 1\right)(\xi \odot x)\right|_{\left(t^{-1} r, t^{-1} s\right)} d t \\
& =\int_{G} f(t) \xi\left(t^{-1} r\right) \odot x\left(t^{-1} s\right) d t \\
& =\left.\int_{G}\left(\Lambda_{f(t)} \odot \lambda_{t}\right)(\xi \odot x)\right|_{(r, s)} d t .
\end{aligned}
$$

donde segue que $\delta$ é uma representaçào de $C_{r}^{*}(\mathcal{B})$ que fatoriza a representaçào de $C^{*}(\mathcal{B})$ integrada da representação de $\mathcal{B}$ dada por $b_{t} \longrightarrow \Lambda_{b_{t}} \odot \lambda_{t} \in \mathcal{L}\left(L^{2}(\mathcal{B}) \otimes L^{2}\left(C^{\prime}\right)\right)$. Portanto, supondo que $\pi: C_{r}^{*}(\mathcal{B}) \longrightarrow B(H)$ é uma representaçào fiel, entào $\delta_{\mathcal{B}}\left(\Lambda_{f}\right)=\pi_{\lambda}$. onde $\pi_{\lambda}$ é a representaçào integrada da representaçào $B \longrightarrow B\left(L^{2}(G, H)\right)$ tal que $b_{t} \longmapsto \lambda_{t} \odot \pi\left(b_{t}\right)$.

A seguir vemos que $(\beta, \mathbb{E}(\mathcal{B}))$ e $\left(\delta_{\mathcal{B}}, C_{r}^{*}(\mathcal{B}) \rtimes_{\delta_{B}, r} \hat{G}\right)$ sào $C^{*}$-sistemas dinamicos isomorfos.

Proposição 7.45. Sejam $B=\left(B_{t}\right)_{t \in G}$ um fibrado de Fell sobre o grupo localmente compacto $G$, $\delta$ a coação dual de $G^{\prime}$ sobre $C_{r}^{*}(B)$. $\delta$ a açào dual sobre $C_{r}^{*}(\mathcal{B}) \rtimes_{\delta, r} G \in B$ a ação natural de $G$ sobre $\mathbb{E}(\mathcal{B})$. Então existe um isomorfismo $\sigma: \mathbb{E}(\mathcal{B}) \longrightarrow C_{r}^{*}(\mathcal{B}) \rtimes_{\delta, r} G$ tal que $\sigma_{t} \beta_{t}=\delta_{t} \sigma, \forall t \in G$.

Demonstração. Podemos supor. sem perder generalidade. que $C_{r}^{*}(\mathcal{B}) \subseteq B(H)$ nào degeneradamente, para certo espaço de Hilbert $H$. Portanto, $\mathcal{B} \subseteq B(H)$ também, e $C_{r}^{*}(\mathcal{B}) \rtimes_{\delta, r} G \subseteq$ $B\left(L^{2}(G, H)\right)$.

Por outro lado, por 7.21 , a inclusão $\mathcal{B} \hookrightarrow B(H)$ define uma representação fiel $\sigma: \mathbb{R}(\mathcal{B}) \longrightarrow$ $B\left(L^{2}(G, H)\right)$ tal que, se $k \in \mathbb{E}_{c}(\mathcal{B}), x \in L^{2}(G . H)$, então $\left.\sigma(k)\right|_{r}=\int_{G} k(r, s) x(s) d s$.

Agora, se $f \in C_{c}(\mathcal{B}), \varphi \in C_{c}(G) \subseteq C_{0}(G)$, e se $\xi \in C_{c}(G) \subseteq L^{2}(G)$. $h \in H$, temos:

$$
\begin{aligned}
\left.\delta(f)(1 \odot \varphi)(\xi \odot h)\right|_{r} & =\int_{G} \lambda_{s}(\varphi \xi)(r) f(s) h d s \\
& =\int_{G} \varphi\left(s^{-1} r\right) \xi\left(s^{-1} r\right) f(s) h d s \\
& \left.=\int_{G} \varphi\left(t^{-1}\right) \xi\left(t^{-1}\right) f(r t) h d t \quad \text { (onde se fez } t=r^{-1} s\right) \\
& =\int_{G} \Delta(s)^{-1} \varphi(s) \xi(s) f\left(r s^{-1}\right) h d s \quad \text { (onde se fez } s=t^{-1} \text { ) } \\
& =\left.\sigma\left(k_{\varphi, f}\right)(\xi \odot h)\right|_{r},
\end{aligned}
$$

onde $k_{\varphi, f}(r, s)=\Delta(s)^{-1} \varphi(s) f\left(r s^{-1}\right)$. Portanto, $\sigma\left(k_{\varphi, f}\right)=\delta(f)(1 \odot \varphi)$, o que mostra que $\sigma(\mathbb{R}(\mathcal{B})) \supseteq$ $C_{r}^{*}(\mathcal{B}) \rtimes_{\delta, r} \hat{G}$. Para provar que a inclusão acima é de fato uma igualdade. basta mostrar que $F:=$ $\operatorname{span}\left\{k_{\varphi, f}: \varphi \in C_{c}(G), f \in C_{c}(\mathcal{B})\right\}$ é denso em $\mathbb{K}_{c}(\mathcal{B})$ na topologia do limite indutivo. Para isto usaremos o Lema 3.14. Dadas $\phi, \imath \in C_{c}(G)$, seja $\phi \diamond \psi: G \times G \longrightarrow \mathbb{C}$ tal que $\phi \diamond \psi(r, s)=\phi(s) \psi\left(r s^{-1}\right)$. É claro que $\phi \diamond \psi$ é contínua e de suporte compacto. Seja $\Theta:=\operatorname{span} \psi_{1} \diamond \psi_{2}: \psi_{1}, \psi_{2} \in C_{c}(G)$. Então $\Theta$ é denso em $C_{0}(G \times G)$ pelo teorema de Stone-Weierstrass; em particular. $\Theta$ é um subespaço denso 
em $C_{c}(G \times G)$ na topologia do limite indutivo. Vejamos que $\Theta F \subseteq F$ : se $\psi_{1} \diamond \psi_{2} \in \Theta, k_{\varphi, f} \in F$, t.emos:

$$
\left(\psi_{1} \diamond \psi_{2}\right) k_{\varphi, f}(r, s)=\Delta(s)^{-1} \psi_{1}(s) \psi_{2}\left(r s^{-1}\right) \varphi(s) f\left(r s^{-1}\right)=k_{\psi_{1} \varphi, \psi_{2} f}(r, s) .
$$

Por último, é claro que $F(r, s)=B_{r^{-1}}, \forall r, s \in G$. Isto mostra que $F$ é denso em $\mathbb{k}(\mathcal{B})$, e portanto que $\sigma: \mathbb{E}(\mathcal{B}) \longrightarrow C_{r}^{*}(\mathcal{B}) \rtimes_{\delta, r} G$ é um isomorfismo. Por último, se $t \in G$ temos, por um lado:

$$
\sigma^{-1}\left(\delta_{t}(\delta(f)(1 \otimes \varphi))=k_{\varphi+, f} .\right.
$$

Por outro lado:

$$
\left.\beta_{t}\left(k_{\varphi, f}\right)\right|_{(r, s)}=\Delta(t) k_{\varphi, f}(r t, s t)=\Delta(t) \Delta(s t)^{-1} \varphi(s t) f\left(r s^{-1}\right)=\Delta(s)^{-1} \varphi_{t}(s) f\left(r s^{-1}\right)=\left.k_{\varphi, t f}\right|_{(r, s)} .
$$

e portanto $\sigma \beta_{t}\left(k_{\varphi, f}\right)=\delta_{t} \sigma\left(k_{\varphi, f}\right)$, e como $F$ é denso em $\mathbb{E}(\mathcal{E})$. concluímos que $\sigma \beta_{t}=\hat{\delta}_{t} \sigma$.

Observaçào 7.46 . Como $\mathbb{E}(\mathcal{B})$ é isomorfa a $C_{r}^{*}(\mathcal{B}) \rtimes_{\dot{\partial}, r}(i$, podemos aplicar os Corolários $7.32 \mathrm{e}$ 7.33 ao produto cruzado $C_{r}^{\prime *}(\mathcal{B}) \searrow_{\delta, r}\left(i\right.$. Em particular, temos que $C_{r}^{*}(\mathcal{B}) \rtimes_{\delta, r} G$ é nuclear, liminal. ou postliminal, se e somente se $B_{\epsilon}$ é nuclear, liminal, ou postliminal respectivamente. Isto já era conhecido para grupos discretos ([53] e [49]). Não temos notícia de nenhum resultado anterior ao nosso no caso contínuo.

Também obtemos que se $\mathcal{A}$ e $\mathcal{B}$ estão nas condiçòes do Teorema 7.39 , então $C_{r}^{*}(\mathcal{A}) \rtimes_{\delta, r} G$ é uma sub- $C^{*}$-álgebra hereditária de $C_{r}^{*}(\mathcal{B}) \rtimes_{\delta, r} G$; se também a hipótese do ponto 4 . de 7.39 é satisfeita, então $\left(C_{r}^{*}(\mathcal{A}) \rtimes_{\delta, r} G, \delta\right)$ e $\left(C_{r}^{*}(\mathcal{B}) \rtimes_{\delta, r} \hat{G}, \delta\right)$ são sistemas dinamicos equivalentes Morita. Em particular, se $(\alpha, A)$ e $(\beta, B)$ sào açōes parciais equivalentes Morita, então $\left(A \rtimes_{\alpha, r} G \rtimes_{\delta, r} G, \delta\right)$ e $\left(B \rtimes_{\beta, r} G \rtimes_{\delta, r} G, \delta\right)$ sào equivalentes Morita.

\subsection{Algumas aplicações}

No que segue, a é uma ação parcial contínua de um grupo localmente compacto sobre uma $C^{*}$ álgebra $A, \beta$ é uma ação sobre a $C^{* *}$-álgebra $B$, e $I \triangleleft B$ é tal que $\overline{[\beta(I)]}=B$, e $\alpha$ é equivalente Morita $\left.\operatorname{com} \beta\right|_{I}$; em outras palavras, $\beta$ é uma ação envolvente de a a menos de equivalência Morita. Em particular, $B$ poderia ser $\mathbb{Z}_{r}\left(\mathcal{B}_{\alpha}\right)$, e $\beta$ poderia ser $\check{\alpha}$, a ação natural de $G$ sobre $\mathbb{R}_{r}\left(\mathcal{B}_{\alpha}\right)$.

Proposição 7.47. Seja $\mathcal{P}$ uma propriedade ideal. Entáo A tem a propriedade $\mathcal{P}$ se e soment $\epsilon$ se $B$ tem a propriedade $\mathcal{P}$. Em particular, B é nuclear. liminal, postliminal, se e somente se $A \dot{\epsilon}$ respectivamente nuclear. liminal, postliminal.

Demonstraçào. Como $\mathcal{P}$ é invariante por equivaléncia Morita, $A$ tem $\mathcal{P}$ se e somente se $I$ tem a propriedade $\mathcal{P}$. Agora, $B$ tem um ideal máximo que tem a propriedade $\mathcal{P}$. Este ideal deve conter todos os ideais $\beta_{t}(I)$, pois todos eles, sendo isomorfos a $I$. tem a propriedade $\mathcal{P}$. Mas, o menor ideal que contém todos os ideais $\beta_{t}(I)$ é a própria $C^{*}$-álgebra $B$.

Proposição 7.48. Suponhamos que $\mathcal{C}$ é uma classe $d \epsilon C^{* *}$-álgebras fechada por ideais, extensòes. quocientes, limites indutivos e equivaléncia Morita. Então $\mathrm{A} \in \mathcal{C} \Longleftrightarrow B \in \mathcal{C}$.

DemonstraçÃo. Como $A \stackrel{M}{\sim} I$. temos que $A \in \mathcal{C} \Longleftrightarrow I \in \mathcal{C}$. Agora, como $\beta$ é a ação envolvente de $\left.\beta\right|_{I}$, por 5.19 temos que $I \in \mathcal{C} \Longleftrightarrow B \in \mathcal{C}$. Assim, $A \in C^{?} \Longleftrightarrow B \in \mathcal{C}$. 
Lembrar que se $\gamma$ é uma ação parcial sobre uma $C^{*}$-álgebra $C$, então ela define ações parciais $\gamma$ e $\bar{i}$ sobre $\hat{C}$ e Prim $(C)$ respectivamente (5.14 e 5.13), e que se $\gamma$ é uma ação global contínua, então $\dot{\gamma}$ e $\bar{i}$ são ações contínuas também (5.12).

Corolário 7.49. Tem-se que (3,B) é a ação envolvente de $(\alpha, A)$, e $(\tilde{\beta}, \operatorname{Prim}(B))$ é a ação envolvente de $(\tilde{a}, \operatorname{Prim}(A))$. Em particular, a e à são contínuas.

Demonstraçào. $\left.\beta\right|_{I}$ tem ação envolvente $\beta$, e portanto é contínua. Como, $\alpha$ e $\left.3\right|_{I}$ são equivalentes Morita, a Proposiçào 7.5 implica que $\widehat{\beta}_{I}$ e $\hat{\alpha}$ são açòes parciais isomorfas através de um homeomorfismo, e portanto a é contínua e tem açào envolvente $(\dot{\beta}, B)$. O mesmo argumento prova que $(\tilde{\beta}, \operatorname{Prim}(B))$ é a açào envolvente de à.

Corolário 7.50. Se Prim(A) é compacto $\epsilon$ existe um subgrupo aberto $H$ de $G$ tal que a restrição de a a $H$ é uma ação global. Ś G $G$ é conexo, a é uma ação global. Em particular, isto vale se A tem unidade.

Demonstração. Como $\operatorname{Prim}(B)$ é homeomorfo a Prim(A), que é compacto, e 3 é uma açào envolvente de $\left.\beta\right|_{I}$, o Corolário 5.18 mostra que $\left.\beta\right|_{I}$ é uma açào global; como $\alpha$ e $\left.\beta\right|_{I}$ sào equivalentes Morita, $\alpha$ também deve ser uma açào global (7.7). 
Parte III

Produtos Cruzados Parciais e C*-álgebras de Grupóides 

Introdução à Parte III 
Nesta última parte consideraremos brevemente a relaçào entre as açōes parciais contínuas sobre espaços topológicos e os grupóides topológicos, e também entre as suas respectivas $C^{*}$-álgebras associadas. Dada uma ação parcial contínua $\alpha$, do grupo localmente compacto $G$ sobre o espaço topológico localmente compacto de Hausdorff $X$, veremos que $\alpha$ induz uma ação parcial contínua $\sigma$ de $G$ sobre $C_{0}(X)$. Veremos também que $\alpha$ tem naturalmente associado um grupóide localmente compacto $\mathcal{G}_{\alpha}$, com um sistema de Haar à esquerda canônico. Provaremos entào que $C_{0}(X) \rtimes_{\sigma} G=$ $C^{*}\left(\mathcal{G}_{\alpha}\right)$.

Os grupóides, estruturas mais gerais que os grupos, foram introduzidos em meados da primeira metade deste século para estudar formas quadráticas, e mais tarde, já com estruturas topológicas ou diferenciais, foram utilizados como ferramentas úteis em geometria, topologia diferencial, geometria algébrica, análise, e topologia algébrica. Aplicaçòes importantes dos grupóides, bem atuais, incluem a geometria diferencial e a geometria diferencial não comutativa de Connes. No primeiro caso, vale a pena ler o artigo expositivo [66] de A. Weinstein, que contém também exemplos relativos a outras áreas. No segundo caso, a referencia fundamental é [15]. Neste livro, Connes usa os grupóides para estudar unificadamente as álgebras de operadores, as folhaçōes e a teoria do índice.

Em 1980 foi publicada a monografia de Renault sobre $C^{*}$-álgebras de grupóides ([58]), que continua sendo hoje a referencia mais importante sobre este assunto. Os principais exemplos de grupóides considerados nesta monografia são os grupóides associados a ações contínuas sobre espaços topológicos, os grupóides aproximadamente finitos, e os grupóides de Cuntz. As C**álgebras dos primeiros são os produtos cruzados correspondentes às açòes; as $C^{*}$-álgebras dos segundos sào as $C^{* *}$-álgebras aproximadamente finitas, e dos terceiros as ('ª́lgebras de Cuntz.

Nesta parte, generalizamos a construção do grupóide correspondente a uma ação global ao caso de uma ação parcial. Como as (**álgebras de Cuntz e as $C^{*}$-álgebras aproximadamente finitas são produtos cruzados por açòes parciais ([25]. [31]), da nossa construção segue que temos grupóides cujas $C^{*}$-álgebras são, respectivamente, as álgebras de Cuntz e as álgebras aproximadamente finitas. Uma tarefa que continuaria naturalmente o presente trabalho, seria comparar os grupóides construidos por Renault com os que decorrem da nossa construção. De fato, já sabemos que os grupóides aproximadamente finitos de Renault são isomorfos aos grupóides associados às açōes parciais que definem as $C^{*}$-álgebras aproximadamente finitas (segundo [25]). Não incluimos este resultado neste trabalho.

Além dos exemplos mencionados, segue do Teorema 8.7 que outras $C^{* *}$-álgebras conhecidas sào $C^{*}$-álgebras de grupóides: as álgebras de Bunce-Deddens (devido a [24]). as álgebras de CuntzKrieger para matrizes infinitas (por [31]), as $C^{*}$-álgebras parciais de grupos discretos (por [26]), e de grupos discretos com relaçōes (por [32]).

A Parte III é a menos desenvolvida da tese, e a consideramos apenas como o início de uma interação, esperamos que frutífera, entre as teorias de ações parciais e de grupóides. 
CAPíTULO 8

Grupóide Associado a uma Ação Parcial 
Mostraremos, no presente capítulo, que o produto cruzado de uma ("*-álgebra comutativa por uma ação parcial contínua de um grupo localmente compacto, pode ser obtido como a $C^{*}$-álgebra seccional de um grupóide que se associa naturalmente à ação parcial do grupo sobre o espectro da álgebra pela ação parcial dada.

Na primeira seção estudamos a relaçào entre famílias $\left\{D_{t}\right\}_{t \in G}$ de ideais de $C_{0}(\mathrm{X})$ e certos subconjuntos abertos de $G \times X$, e vemos que $\sigma$ é uma ação parcial contínua sobre ( ${ }_{0}\left(\mathrm{~F}^{*}\right)$ se e somente se $\dot{\sigma}$ é uma ação parcial contínua de $G$ sobre $\mathcal{X}$. onde $\hat{\sigma}$ é a ação parcial fornecicla por 5.14 .

Na segunda seçào construímos o grupóide associado a uma açào parcial contínua sobre um espaço topológico localmente compacto, e na terceira apresentamos um sistema de llaar à esquerda para o grupóide.

Por último, na quarta seçào demonstramos o resultado principal anunciado no primeiro parágrafo.

\subsection{Açōes parciais sobre $\mathrm{I}$ e açöes parciais sobro (}

Nesta seção inicial. mostramos que existe uma bijeção natural ent re as fanílias contínuas de ideais em $C_{0}^{\prime}(X)$, indexadas num grupo localmente compacto $G$, e certos subronjuntos abertos de $G \times . \mathrm{Y}$. Também vemos que uma açào parcial $\sigma$ sobre $C_{0}\left(X^{*}\right)$ é contínua se e somente se $\sigma$ é uma açào parcial contínua sobre $X$, onde $\sigma$ é a açào parcial fornecida por 5.14. Isto justifica a noçào de açào parcial contínua introduzida no Capítulo 4.

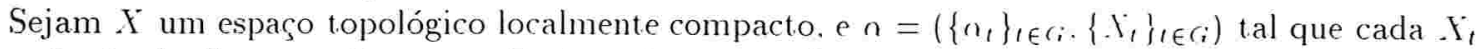
é um aberto de $\mathrm{Y}^{\circ}$, e $a_{t}: \mathrm{Y}_{t-1} \longrightarrow \mathrm{Y}_{t}$ é um homeomorfismo, $\forall t$. Scjam $D=\{(t, x) \in G \times \mathrm{Y}: t \in$ $\left.G, x \in X_{t}\right\}, D^{-1}=\left\{(t, x) \in G \times \mathbb{X}: t \in G, x \in X_{t-1}\right\}$, e a $: D^{-1} \longrightarrow D$ al que $a(t, x)=\left(t, \alpha_{t}(x)\right)$. Sobre $D^{-1}$ e $D$ consideramos a topologia relativa herdada da topologia produto de $G \times X$.

Seja agora $A=C_{0}\left(\mathrm{~K}^{\circ}\right)$. A partir de $a$, construímos a família $\sigma=\left(\left\{\sigma_{t}\right\}_{t \in G},\left\{D_{t}\right\}_{t \in G}\right)$, onde $D_{t}=C_{0}\left(X_{t}\right)$, e $\sigma: D_{t^{-1}} \longrightarrow D_{t}$ está dado por $\sigma(f)=f \alpha_{t-1}, \forall f \in D_{t}$. Sejam, também, $\mathcal{B}=$ $\left\{(t, f) \in G \times A: t \in G, f \in D_{t}\right\}, \mathcal{B}^{-1}=\left\{(t, f) \in G \times A: t \in G ; f \in D_{t-1}\right\}$. e $\sigma: \mathcal{B}^{-1} \longrightarrow \mathcal{B}$ tal que $\sigma(t, f)=\left(t, \sigma_{t}(f)\right)$. Sobre $\mathcal{B}^{-1}$ e $\mathcal{B}$ consideramos as topologias relativas herdadas da topologia produto sobre $G \times A$.

Proposição 8.1. Sejam a e $\sigma$ como acima e consideremos os segumte subespaços de $C_{0}(G, A)$ : $C_{0}(\mathcal{B})=\left\{f \in C_{0}(G, A): f(t) \in D_{t}, \forall t \in G\right\}, \in C_{0}\left(\mathcal{B}^{-1}\right)=\left\{f \in C_{0}(G, A): f(t) \in D_{t-1}, \forall t \in G\right\}$. Então temos:

1. $C_{0}(\mathcal{B})$ e $C_{0}\left(\mathcal{B}^{-1}\right)$ são ideais de $C_{0}(G, A)$.

2. As condiçōes seguintes são equivalentes:
(a) A família $\left\{D_{t}\right\}_{t \in G}$ é contínua.
(b) $\mathcal{B}$ é um fibrado de Banach.
(c) Para todo $t \in G$ tem-se que $D_{t}=\left\{a(t): a \in C_{0}(\mathcal{B})\right\}$.
(d) $O$ espectro de $C_{0}^{\prime}(\mathcal{B})$ é igual a $D$.
(e) Dé aberto.

Demonstração. Como cada $D_{t}$ é um ideal de $A$, é claro que $\mathcal{B}$ e $B^{-1}$ sào ideais de $C_{0}(G, A)$. As equivalèncias entre 2.(a), 2.(b) e 2.(c) seguem de 3.2 e 3.3 de [27].

$(c) \Longrightarrow(\mathrm{d})$ Como $C_{0}(\mathcal{B})$ é um ideal de $C_{0}(G, A) \cong C_{0}(G \times X), \widehat{C_{0}(B)} \dot{C}$ o aberto de $G \times X$ formado pelos pontos $(t, x) \in G \times \mathrm{I}$ tais que existe $f \in C_{0}(\mathcal{B})$ tal que $\left.f(t)\right|_{x} \neq 0$. Suponhamos que $(t, x) \in D$. Então existe $d \in D_{t}$ tal que $d(x) \neq 0$. Como $\mathcal{B}$ é um fibrado de Banach sobre $G$, localmente compacto, existe uma seçào contínua $F: G \longrightarrow \mathcal{B}$ tal que $F(t)=(t$. $d)$. Seja $f: G \longrightarrow A$ tal que $F(s)=(s, f(s))$. Entào $f$ é contínua, e $f \in C_{0}(\mathcal{B})$. Como $\left.f(t)\right|_{x}=d(x) \neq 0$, vemos que 
$(t, x) \in \widehat{C_{0}(\mathcal{B})}$. donde segue que $D \subseteq \widehat{C_{0}(\mathcal{B})}$. Suponhamos agora que $(t, x) \notin D$. Então $d(x)=0$. $\forall d \in D_{t}$, donde segue que $\left.f(t)\right|_{x}=0, \forall f \in C_{0}(\mathcal{B})$. Assim, concluímos que $\widehat{C_{0}(\mathcal{B})}=D$.

(d) $\Longrightarrow$ (e) É claro, porque $\widehat{C_{0}(B)}$ é um aberto de $G \times X$.

(e) $\Longrightarrow$ (a) Se $V^{\prime}$ é um aberto de $A$, definamos $G_{V}=\left\{s \in G: V \cap D_{s} \neq \emptyset\right\}$. Queremos ver que $G_{\mathrm{V}}$ é aberto, para todo $V$ aberto em $A$. Se $\left(V_{i}\right)$ é uma coleção de abertos em $A$ cuja união é $V$, então é claro que $G_{1}=\bigcup_{i} G_{1}$, Portanto, basta provar que $G_{U}$ é aberto para toda bola aberta $U$ em $A$. Sejam $a \in A, \epsilon>0$, e $I^{\prime}=B(a, \epsilon)$. Se $G_{U}$ é vazio, não há nada a provar. Suponhamos, então, que existe $t \in G_{U}$, e seja $d \in U \cap D_{t}$. Existe um compacto $K \subseteq X_{t}$, tal que, $\forall y \notin K$, tem-se que:

$$
|d(y)|<\epsilon-\|d-a\|
$$

Por outro lado, existe uma vizinhança compacta $V$ de $t$, tal que $V \times K \subseteq D$ : como $D$ é aberto. para cada $x \in K$. existem uma vizinhança $U_{x}$ de $x$ e uma vizinhança compacta $V_{x}$ de $t$ tais que $V_{x} \times U_{x} \subseteq D$ : como $K^{\prime}$ c compacto, existem $x_{1}, \ldots, x_{n}$ tais que $K^{\prime} \subseteq \bigcup j=1^{n} U_{x}$, e portanto basta tomar $V=\bigcap_{j=1}^{n} V_{r_{j}}$. Agora, como $\mathrm{I}^{*} \times K$ é um compacto incluído no aberto $D$, existe uma função contínua $g: G \times X \longrightarrow[0,1]$ tal que:

$$
g(s, y)= \begin{cases}1 & \text { se }(s, y) \in V \times K \\ 0 & \text { se }(s, y) \notin D\end{cases}
$$

Seja agora $f: G \times X \longrightarrow \mathbb{C}$ tal que $f(s, y)=d(y) g(s, y)$, e para cada $s \in G$ consideremos $d_{s}: X \longrightarrow Z$ tal que $d_{s}(y)=f(s, y)$. Como $\sup (g) \subseteq D$, temos que $\sup \left(d_{s}\right) \subseteq X_{s}, \forall s \in G$, e portanto $d_{s} \in D_{s}$. $\forall s \in G$.

Por outro lado, se $s \in V$ :

$$
\left|d_{s}(y)-d(y)\right|=|d(y) g(s, y)-d(y)|=|d(y)|(1-g(s, y))<\epsilon-\|d-a\|,
$$

onde a última desigualdade se deve ao fato de que, se $y \in K$, então $g(s, y)=1$ por (21), enquanto que, se $y \notin I . d(x)<\epsilon-\|d-a\|$ por (20). Segue então que:

$$
\left\|d_{s}-a\right\| \leq\left\|d_{s}-d\right\|+\|d-a\|<\epsilon .
$$

Assim, $d_{s} \in D_{s} \bigcap B(a, \epsilon), \forall s \in V$, e portanto $t \in V \subseteq G_{U}$, donde segue que $G_{U}$ é aberto.

Observação 8.2. Como a inversão no grupo $G$ é contínua, é claro que a família $\left\{D_{t}\right\}_{t \in G}$ é contínua se e somente se a família $\left\{D_{t-1}\right\}_{t \in G}$ é contínua, donde segue que as condições equivalentes enumeradas em 8.1 são também equivalentes às três seguintes: (f) a família $\left\{D_{t^{-1}}\right\}_{t \in G}$ é contínua: (g) $\mathcal{B}^{-1}$ é um fibrado de Banach. (h) $\mathrm{O}$ espectro de $C_{0}\left(\mathcal{B}^{-1}\right)$ é igual a $D^{-1}$; (i) $D^{-1}$ é aberto.

Proposição 8.3. Nas condiçōes de 8.1, temos:

1. Se a família $\left\{D_{t}\right\}_{t \in G}$ é contínua, as trés propriedades abaixo são equivalentes:

(a) $\sigma: \mathcal{B}^{-1} \longrightarrow \mathcal{B}$ é um isomorfismo de fibrados de Banach.

(b) $\sigma: C_{0}\left(\mathcal{B}^{-1}\right) \longrightarrow C_{0}(\mathcal{B})$ tal que $\left.\sigma(d)\right|_{t}=\sigma(d(t))$ é um isomorfismo de $C^{*}$-álgebras.

(c) $a: D^{-1} \longrightarrow D$ é um homeomorfismo.

2. $\sigma$ é uma ação parcial contínua sobre $A$ se e somente se a é uma ação parcial contínua sobre $X$.

DemonstraÇÃo. Que 1.(a) implica 1.(b) é claro, porque $C_{0}(\mathcal{B})$ e $C_{0}\left(\mathcal{B}^{-1}\right)$ se identificam naturalmente com as $C^{*}$-álgebras seccionais de $\mathcal{B}$ e $\mathcal{B}^{-1}$ respectivamente. Para ver que 1 .(b) implica 1.(c), notemos que, como $\sigma: C_{0}\left(\mathcal{B}^{-1}\right) \longrightarrow C_{0}(\mathcal{B})$ é um isomorfismo, existe um único homeomorfismo 
$\dot{\sigma}: \widehat{C_{0}\left(\mathcal{B}^{-1}\right)} \longrightarrow \widehat{C_{0}(\mathcal{B})}$ tal que $\sigma(f)=f \sigma^{-1}, \forall f \in C_{0}\left(\mathcal{B}^{-1}\right)$. Pela própria definiçào de $\sigma$, temos que o diagrama abaixo comuta:

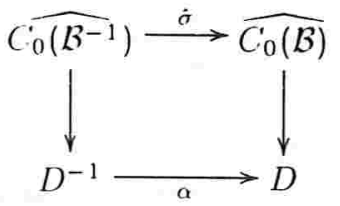

onde as homeomorfismos verticais sào os obtidos através das identificaçōes naturais dos espectros $\widehat{C_{0}\left(\mathcal{B}^{-1}\right)}$ e $\widehat{C_{0}(\mathcal{B})}$ com $D^{-1}$ e $D$ respectivamente, segundo 8.1 e 8.2 . Portanto. a é um homeomorfismo.

1.(c) $\Longrightarrow 1$.(a) Temos que ver que se $\left(t_{i}, d_{i}\right) \longrightarrow(t, d)$ em $\mathcal{B}^{-1}$. então $\left(t_{i}, \sigma_{t},\left(d_{i}\right)\right) \longrightarrow\left(t, \sigma_{t}(d)\right)$. Como $t_{i} \longrightarrow t$, isto é equivalente a mostrar que $\sigma_{t_{t}}\left(d_{i}\right) \longrightarrow \sigma_{t}(d)$ uniformemente sobre $Y_{\text {, sabendo }}$ que $d_{i} \longrightarrow d$ uniformemente sobre $\hat{H}$.

Como $d \in C_{0}\left(X_{t-1}\right)$ e $\alpha_{t-1}: X_{t} \longrightarrow H_{t-1}$ é um homeomorfismo, e como $d_{i} \longrightarrow d$ uniformemente sobre $X$, dado $\epsilon>0$ existem $K \subseteq \mathcal{K}_{t}$ e $i_{0}$ tal que $\forall i \geq i_{0}$ e $\forall y \notin K$ tem-se que:

$$
\max \left\{|d(y)|,\left|d_{i}(y)\right|\right\}<\epsilon / 2
$$

Como já vimos na demonstraçào da Proposiçào 8.1 , existe uma vizinhança compacta $V$ de $t$ tal que $V \times K \subseteq D$. Agora, a função $d o^{-1}$ é contínua sobre $V \times K$, e portanto, usando o Lema 7.37 , a função $V \longrightarrow C(K)$ tal que $s \longrightarrow d_{s}$ é contínua, onde $d_{s}(x)=d\left(\alpha^{-1}(x)\right), \forall x \in K$.

Seja $x \in X$. Se $x \notin K$, e $i \geq i_{0}$, por 22 temos:

$$
\left|\sigma_{t,}\left(d_{i}\right)(x)-\sigma_{t}(d)(x)\right|=\left|d_{i}\left(\alpha_{t_{1}-1}(x)\right)-d\left(\alpha_{t-1}(x)\right)\right|<\epsilon / 2+\epsilon / 2=\epsilon
$$

Como $d_{s} \longrightarrow d_{t}$ em $C\left(K^{*}\right)$ quando $s \longrightarrow t$, existe $i_{1}$ tal que $\left\|d_{t_{1}}-d_{t}\right\|<c / 2, \forall i \geq i_{1}$. Por outro lado, como $d_{i} \longrightarrow d$, existe $i_{2}$ tal que $\left\|d_{i}-d\right\|<\epsilon / 2$. $\forall i \geq i_{2}$. Então, se $i \geq i_{1}$, $i_{2}$ e $x \in K$ :

$$
\begin{aligned}
\left|\sigma_{t_{1}}\left(d_{i}\right)(x)-\sigma_{t}(d)(x)\right| & =\left|d_{i}\left(\alpha_{t_{1}-1}(x)\right)-d\left(\alpha_{t-1}(x)\right)\right| \\
& \leq\left|d_{i}\left(\alpha_{t_{1}^{-1}}(x)\right)-d\left(\alpha_{t_{i}^{-1}}(x)\right)\right|+\left|d\left(\alpha_{t_{1}-1}(x)\right)-d\left(a_{t-1}(x)\right)\right| \\
& \leq\left\|d_{i}-d\right\|+\left|d_{t_{1}}(x)+d_{t}(x)\right| \\
& \leq \epsilon / 2+\left\|d_{t}-d_{t}\right\|<\epsilon
\end{aligned}
$$

Segue que, se $i \geq i_{0}, i_{1}, i_{2}$, entào $\left\|\sigma_{t_{1}}\left(d_{i}\right)-\sigma_{t}(d)\right\|<\epsilon$.

Para provar 2., é necessário provar que $\sigma$ é uma ação parcial se e somente se $\alpha$ é uma ação parcial. Se $\sigma$ é uma ação parcial, por 5.14 tem-se que $\sigma$ é uma ação parcial. Como, pela definição de $\sigma$, $\hat{\sigma}=\alpha$, vemos que $\alpha$ é uma ação parcial. Inversamente, suponhamos que $\alpha$ é uma ação parcial. Como $X_{e}=X$, temos que $D_{e}=A$. Suponhamos que $d \in D_{s^{-1}}$ é tal que $\sigma_{s}(d) \in D_{t-1}$, de forma que podemos calcular $\sigma_{t}\left(\sigma_{s}(d)\right)$. Significa que podemos fazer as composiçōes $d \alpha_{s-1} \alpha_{t-1}$. Como $\alpha_{t s}$ é uma extensão de $\alpha_{t} \alpha_{s}$, temos que $\alpha_{t s^{-1}}(x)=\alpha_{s^{-1}} \alpha_{t-1}(x)$, para todo $x$ onde possa fazer o segundo cálculo. Portanto, $d \alpha_{s-1} \alpha_{t-1}=d \alpha_{t s^{-1}}$, donde segue que $\sigma_{t}\left(\sigma_{s}(d)\right)=\sigma_{t s}(d)$, e portanto $\sigma_{t s}$ é uma extensão de $\sigma_{t} \sigma_{s}$. Logo, $\sigma$ é uma açào parcial.

\subsection{Grupóide associado a uma ação parcial}

Podemos associar um grupóide a uma açào parcial sobre um espaço topológico. Pensado como categoria, este grupóide tem . I como conjunto de objetos, e os morfismos de $y$ a $x$ correspondem aos elementos $t$ de $G$ tais que $\alpha_{t}(y)=x$. Desta seção em diante usaremos a letra a para denotar uma ação parcial como também à função $a: D^{-1} \longrightarrow X$ tal que $\alpha(t, x)=\alpha_{t}(x)$; notar que o significado de $\alpha$ na seção anterior era formalmente diferente. 
A definiçào mais curta de grupóide é a seguinte: um grupóide é uma categoria pequena com inversos. Que a categoria seja pequena significa que a classe de objetos é um conjunto; que seja uma categoria com inversos significa que todo morfismo é um isomorfismo. Assim, por exemplo, um grupo é um grupóide com apenas um objeto. Embora seja esta a definição mais simples e, achamos, a mais sugestiva, nào é a definição mais comum, especialmente no caso em que se considere uma topologia sobre o grupóide. A definição de grupóide em [58] (I-1.1), é a seguinte: um grupóide é um conjunto $\mathcal{G}$ junto com um produto $(x, y) \longmapsto x y$ definido sobre um subconjunto $\mathcal{G}^{(2)} \subseteq \mathcal{G} \times \mathcal{G}$, chamado conjunto dos pares componíveis, e uma inversào $x \longmapsto x^{-1}$, que é uma bijeçào em $\mathcal{G}$. e tal que as seguintes condições são satisfeitas:

1. $\left(x^{-1}\right)^{-1}=x, \forall x \in \mathcal{G}$

2. Se $(x, y),(y, z) \in \mathcal{G}^{(2)}$, entào $(x y, z),(x, y z) \in \mathcal{G}^{(2)}$, e $(x y) z=x(y z)$.

3. $\left(x^{-1}, x\right) \in \mathcal{G}^{(2)}, \forall x \in \mathcal{G}$, e se $(x, y) \in \mathcal{G}^{(2)}$, então $x^{-1}(x y)=y$.

4. $\left(x, x^{-1}\right) \in \mathcal{G}^{(2)}, \forall x \in \mathcal{G}$. e se $(z, x) \in \mathcal{G}^{(2)}$, então $(z x) x^{-1}=z$.

Naturalmente, as duas definiçōes dadas são equivalentes. Se $x \in \mathcal{G}$, o domínio de $x$ é $x^{-1} x$, e o "rangє " é $x x^{-1}$. Temos definidas assim duas aplicaçōes $d, r: \mathcal{G} \longrightarrow \mathcal{G}$, com a mesma imagem, $\mathcal{G}^{(0)}$, chamada espaço das unidades de $\mathcal{G}$. No contexto de categorias, o espaço das unidades não é outra coisa que o conjunto dos objetos.

O grupóide $\mathcal{G}$ é um grupóide localmente compacto se tem uma topologia localmente compacta de Hausdorff, compatível com a estrutura algébrica de $\mathcal{G}$, ou seja:

1. A inversào $\mathcal{G} \longrightarrow \mathcal{G}$ é contínua.

2. O produto $\mathcal{G}^{(2)} \longrightarrow \mathcal{G}$ é contínuo, onde consideramos sobre $\mathcal{G}^{(2)}$ a topologia herdada da topologia produto sobre $\mathcal{G} \times \mathcal{G}$.

Formalmente, definimos a categoria $\mathcal{G}_{\alpha}$ assim: a classe de objetos da categoria é o conjunto $X$. Se $y, x \in X$, definimos:

$$
\operatorname{Mor}(y, x)=\left\{(x, t, y): t \in A_{y}, \text { e } x=\alpha_{t}(y)\right\},
$$

com a lei de composição seguinte:

$$
\begin{gathered}
\operatorname{Mor}(y, x) \times \operatorname{Mor}(z, y) \longrightarrow \operatorname{Mor}(z, x) \\
((x, r, y),(y, s, z)) \longmapsto(x, r s, y) .
\end{gathered}
$$

Lembrar que $A_{y}=\left\{t \in G^{\prime}:(t, y) \in D^{-1}\right\}$. Portanto, como $\alpha_{r s}$ é uma extensão de $\alpha_{r} \alpha_{s}$, temos que se $s \in A_{z}$ e $r \in A_{a_{s}(z)}$, entào $r s \in A_{z}$, e portanto $(x, r s, z) \in \operatorname{Mor}(z, x)$. Sobre cada $x$ temos o morfismo identidade $(x, e, x)$, que podemos identificar com $x$. Com efeito: $(x, e, x)(x, t, y)=(x, t, y)$. e $(y, t, x)(x, \epsilon . x)=(y, t, x)$. Todo morfismo $(x, t, y)$ é inversível, com inverso $(x, t, y)^{-1}=\left(y, t^{-1}, x\right)$. como se verifica imediatamente. Por outro lado, a associatividade da composição de morfismos segue da associatividade da multiplicação no grupo $G$. Em conclusão, $\mathcal{G}_{\alpha}$ é uma categoria pequena com inversos, ou seja, um grupóide. A menos que outra ação parcial esteja envolvida, usaremos a notação $\mathcal{G}$ em lugar de $\mathcal{G}_{\alpha}$. Através da inclusão $\iota: X \longrightarrow \mathcal{G}_{\alpha}$ tal que $x \longmapsto(x, e, x)$, consideraremos $X \subseteq \mathcal{G}$. Temos as funçōes $r: \mathcal{G}_{\alpha} \longrightarrow X$ e $d: \mathcal{G}_{\alpha} \longrightarrow X$ tais que $r(r, t, y)=x$, e $d(x, t, y)=y$. Temos que $r \iota=i d_{X}=d \iota$.

Notar que $\mathcal{G}$ é o gráfico da ação parcial $\alpha$, escrito de uma forma não convencional: em lugar de escrever a imagem de $(t, x)$ à direita de $(t, x)$, a estamos escrevendo à esquerda, e por isso $\mathcal{G}$ está incluído em $X \times G \times X$, e nào em $G \times X \times X$. A razão da nossa escolha é que a multiplicação e a inversão no grupóide são facilmente calculáveis desta maneira.

Sobre $\mathcal{G}$ consideramos a topologia herdada da topologia produto sobre $X \times G \times X$. Segue da continuidade de $a$. que as operaçooes de inversão e produto sobre $\mathcal{G}$ são contínuas.

Temos homeomorfismos $p: \mathcal{G} \longrightarrow D$ e $q: \mathcal{G} \longrightarrow D^{-1}$, com $p(x, t, y)=(t, x)$, e $q(x, t, y)=(t, y)$. De fato, se bem para manipular a multiplicação e a inversão em $\mathcal{G}$ é melhor pensar em $\mathcal{G}$ como foi 
definido, na hora de pensar na $C^{*}$-álgebra do grupóide $\mathcal{G}$ será melhor identificar $\mathcal{G} \operatorname{com} D$ através de p. Assim, a inversão sobre $D$ transladada de $\mathcal{G}$ fica: $(t, x)^{-1}=\left(t^{-1}, \alpha_{t-1}(x)\right)$, e se $(r, x),(s, y) \in D$, então $(r, x)(s, y)$ existe se e somente se $\alpha_{r^{-1}}(x)=y$, e neste caso o produto é igual a $(r s, x)$. Por último, $\iota, r$ e $d$ ficam assim: $\iota(x)=(\epsilon, x), r(t, x)=x$, e $d(t, x)=\alpha_{t-1}(x)$. Na seguinte seção veremos que a vantagem de transladar a estrutura algébrica de $\mathcal{G}$ a $D$ é que assim conseguímos um sistema de Haar natural para o grupóide.

\subsection{Sistema de Haar sobre $\mathcal{G}$}

O nosso próximo objetivo é achar um sistema de Haar esquerdo sobre $\mathcal{G}$, onde $\mathcal{G}$ está identificado com $D$ através da projeção $p$.

Lembrar que um sistema de Haar à esquerda sobre um grupóide localmente compacto $\mathcal{G}$ ([58], I-2.2) é uma família de medidas $\left\{\lambda^{x}: x \in \mathcal{G}^{(0)}\right\}$ sobre $\mathcal{G}$, tais que:

1. $\sup \left(\lambda^{x}\right)=\mathcal{G}^{x}=\{y \in \mathcal{G}: r(y)=x\}$

2. Continuidade: se $f \in \mathcal{C}_{c}(\mathcal{G})$, a transformação $\nu_{f}: \mathcal{G}^{(0)} \longrightarrow \mathbb{C}$ tal que $\nu_{f}(x)=\int_{\mathcal{G}} f(y) d \lambda^{x}(y)$ é contínua

3. Invariáncia à esquerda: para toda $f \in \mathcal{C}_{c}(\mathcal{G})$ e para todo $x \in \mathcal{G}^{(0)}$, tem-se : $\int_{\mathfrak{G}} f(x y) d \lambda^{d(x)}(y)=$ $\int_{\underline{G}} f(y) d \lambda^{r(x)} d(y)$.

Para definir o sistema de Haar que estamos procurando, dado $x \in X$, consideremos a medida $\lambda^{x}$ definida nos borelianos de $\mathcal{G}$ como:

$$
\lambda^{x}(B)=\lambda \times \delta_{x}(B),
$$

onde $B$ é qualquer boreliano, $\lambda$ é a medida de Haar sobre $G$, e $\delta_{x}$ é a medida de Dirac concentrada no ponto $x$. Em outras palavras, $\lambda^{x}$ é a restriçào a $D$ do produto da medida de Haar com a medida concentrada em $x . \lambda^{x}(B)$ mede o "volume" de morfismos em $B$ que chegam ao objeto $x$. Notar que, como $\mathcal{G}$ é aberto em $G \times \mathcal{I}, B \subseteq \mathcal{G}$ é um boreliano de $\mathcal{G}$ se e somente se é um boreliano de $G \times X$.

Sendo $\mathcal{G}$ aberto em $G \times \mathrm{K}$. suporemos daqui em diante que toda $f \in C_{c}(\mathcal{G})$ está definida sobre todo $G \times X$, pondo $f(s, y)=0$ se $(s, y) \notin \mathcal{G}$.

Proposição 8.4. O conjunto $\Lambda=\left\{\lambda^{x}: x \in X\right\}$ é um sistema de Haar sobre $\mathcal{G}$.

DEMONSTRAÇÃO. $\quad \bullet \sup \lambda^{x}=\mathcal{G}^{x}$

Por um lado temos:

$$
\mathcal{G}^{x}=\{(t, y): r(t, y)=x\}=A_{x}^{-1} \times\{x\} .
$$

Por outro lado, sejam $A$ e $B$ abertos não vazios de $G$ e de $X$ respectivamente, tais que $A \times B \subseteq \mathcal{G}$ (lembrar que $\mathcal{G}$ é aberto em $G \times X$ ). Temos:

$$
\lambda^{x}(A \times B)=\lambda(A) \delta_{x}(B)=\chi_{B}(x) \lambda(A),
$$

onde $\chi_{S}$ indica a função característica do conjunto $S$. Como $\lambda(A)>0$, então $\lambda^{x}(A \times B) \neq 0$ se e somente se $x \in B$. Portanto o suporte de $\lambda^{x}$ é igual a $\mathcal{G} \cap(G \times\{x\})=A_{x}^{-1} \times\{x\}$.

- Continuidade

Seja $f \in C_{c}^{\prime}(\mathcal{G})$, e seja $\nu_{f}: \mathrm{Y} \longrightarrow$ - tal que

$$
\nu_{f}(x)=\int_{\underline{G}^{x}} f(z) d \lambda^{x}(z) .
$$


Calculando:

$$
\begin{aligned}
\nu_{f}(x) & =\int_{A_{x}^{-1} \times\{x\}} f(s, y) d \lambda^{x}(s, y) \\
& =\int_{\{x\}} \int_{A_{x}^{-1}} f(s, y) d \lambda(s) d \delta_{x}(y) \\
& =\int_{G} \backslash_{A_{x}^{-1}}(s) f(s, x) d \lambda(s) .
\end{aligned}
$$

Se $K=p_{1}(\sup (f))$, onde $p_{1}: G \times X \longrightarrow G$ é a projeção na primeira coordenada. Entào é claro que:

$$
\nu_{f}(x)=\int_{K} f(s, x) d \lambda(s) .
$$

Se $x \in X$, a funçào $f_{x}: K \longrightarrow \mathbb{C}$ tal que $f_{x}(s)=f(s, x)$ é contínua, e $\xi: X \longrightarrow C\left(K^{*}\right)$ tal que $\xi(x)=f_{x}$, é contínua pelo lema $7.3 i$. Por outro lado,

$$
\begin{aligned}
\lambda_{K}: C^{\prime}(K) & \longrightarrow \mathbb{C} \\
g & \longmapsto \int_{K} g(s) \lambda(s)
\end{aligned}
$$

é um funcional linear contínuo. Como $\nu_{f}=\lambda_{K} \circ \xi$, segue que $\nu_{f}$ é contínua.

\section{- Invariáncia}

Falta mostrar ainda que $\Lambda$ possui a propriedade fundamental de um sistema de Haar, ou seja, que $\forall f \in C_{c}^{\prime}(\mathcal{G})$ e $\forall(t, x) \in \mathcal{G}$ tem-se:

$$
\int_{\underline{G} d(t, x)} f((t, x)(s, y)) d \lambda^{d(t, x)}(s, y)=\int_{\underline{G} r(t, x)} f(s, y) d \lambda^{r(t, x)}(s, y) .
$$

Por um lado, pelo cálculo acima temos:

$$
\int_{\mathfrak{G} r(t, x)} f(s, y) d \lambda^{r(t, x)}(s, y)=\int_{A_{x}^{-1}} f(s, x) d \lambda(s) .
$$

Seja $z=d(t, x)=\alpha_{t-1}(x)$. Temos:

$$
\int_{\mathcal{G}^{d}(t, x)} f((t, x)(s, y)) d \lambda^{d(t, x)}(s, y)=\int_{A_{2}^{-1}} f(x, t s) d \lambda(s)=\int_{G} \lambda_{A_{z}^{-1}}\left(t^{-1} s\right) f(s, x) d \lambda(s) .
$$

Por 4.18 , temos que $A_{z}=A_{x} t$, e portanto

$$
t^{-1} s \in A_{z}^{-1} \Longleftrightarrow s^{-1} t \in A_{x} t \Longleftrightarrow s^{-1} \in A_{x} \Longleftrightarrow s \in A_{x}^{-1} .
$$

Ou seja que $\lambda_{A^{-1}}\left(t^{-1} s\right) \neq 0 \Longleftrightarrow \lambda_{A_{x}^{-1}}(s) \neq 0$.

Segue então que

o que encerra a prova.

$$
\begin{aligned}
\int_{\mathcal{G}^{d}(t, x)} f((t, x)(s, y)) d \lambda^{d(t, x)}(s, y) & =\int_{G} \chi_{A_{x}^{-1}}(s) f(s, x) d \lambda(s) \\
& =\int_{A_{x}^{-1}} f(s, x) d \lambda(s) \\
& =\int_{\mathcal{G}^{r}(t, x)} f(s, y) d \lambda^{r(t, x)}(s, y),
\end{aligned}
$$




\subsection{O Produto Cruzado Parcial e a C*-álgebra do Grupóide}

Nesta seção final, provaremos o resultado principal da Parte III: a C"*álgebra do grupóide $\mathcal{G}$ associado à uma ação parcial contínua do grupo localmente compacto $G$ sobre o espaço localmente compacto $X$ coincide com o produto cruzado $C_{0}(X) \rtimes_{\sigma} G$, onde $\sigma$ é a ação parcial contínua de $G$ sobre $C_{0}(X)$ induzida por $a$.

Em [58], Renault desenvolveu a teoria de $C^{*}$-álgebras associadas a grupóides. A seguir lembramos a construção da $C^{*}$-álgebra associada a um grupóide topológico $\mathcal{G}$ com sistema de Haar $\Lambda$.

No espaço vetorial $C_{c}(\mathcal{G})$ das funçòes complexas contínuas com suporte compacto definidas em $\mathcal{G}$, são definidas uma operaçào de involuçào e um produto que fazem com que este espaço seja uma *-álgebra. Concretamente. se $f, g \in C_{c}^{\prime}(\mathcal{G}), a \in \mathcal{G}$, tem-se:

$$
\begin{gathered}
f \star g(a)=\int_{\mathcal{G}^{d(a)}} f(a b) g\left(b^{-1}\right) d \lambda^{d(a)}(b) \\
f^{\star}(a)=\overline{f\left(a^{-1}\right)}
\end{gathered}
$$

Existem duas normas em $C_{c}(\mathcal{G})$ : se $f \in C_{c}(\mathcal{G})$, então:

$$
\begin{aligned}
& \|f\|_{I, r}=\sup _{a \in \mathcal{G}^{0}} \int_{\mathcal{G}^{a}}|f(b)| d \lambda^{a}(b) \\
& \|f\|_{I, d}=\sup _{a \in \mathcal{G}^{0}} \int_{\mathcal{G}_{a}}|f(b)| d \lambda_{a}(b)
\end{aligned}
$$

onde $\mathcal{G}_{a}=\left(\mathcal{G}^{a}\right)^{-1}$, e $\lambda_{a}(B)=\lambda^{a}\left(B^{-1}\right), \forall B \subseteq \mathcal{G}$ boreliano.

Finalmente, se

$$
\|f\|_{I}=\sup \left\{\|f\|_{I, r},\|f\|_{I, d}\right\},
$$

então \|\|$_{I}$ é uma *-norma em $C_{c}(\mathcal{G}) . C^{* *}(\mathcal{G})$ é definida como a $C^{* *}$-álgebra envolvente do completamento da *álgebra $\left(C_{c}(\mathcal{G})\right.$. \|\|$\left._{I}\right)$. Pela Proposição 1.11 de [58] (pág. 58), $C^{*}(\mathcal{G})$ é um completamento de $C_{c}(\mathcal{G})$ com respeito à norma de $C^{*}(\mathcal{G})$; ou seja: podemos supor que $C_{c}(\mathcal{G}) \subseteq C^{*}(\mathcal{G})$.

Sobre $C_{c}(\mathcal{G})$ existe uma topologia importante, diferente da correspondente à norma $\|\cdot\|_{I}$. Trata-se da topologia do limite indutivo, definida pela família $\{C(K)\}_{K}$, onde $K_{i}$ varia entre os subconjuntos compactos de $\mathcal{G}$. Denotaremos esta topologia por $\tau_{\mathcal{G}}$. Por [58],II-1.22, se $\pi: C_{c}(\mathcal{G}) \longrightarrow B(H)$ é uma representação contínua nas topologias $\tau_{\mathfrak{G}}$ de $C_{c}(\mathcal{G})$ e $W O T$ de $B(H)$, então $\|\pi(f)\| \leq\|f\|_{I}$, e portanto se estende por continuidade a $C^{*}(\mathcal{G})$.

Vejamos como ficam as operaçòes e as normas no caso de um grupóide que provém de uma ação parcial. Sejam $f, g \in C_{c}(\mathcal{G}),(t, x) \in \mathcal{G}$. e seja $z=d(t, x)=\alpha_{t-1}(x)$. Então:

$$
\begin{aligned}
f \star g(t, x) & =\int_{\mathcal{G}^{d(t, x)}} f((t, x)(s, y)) g\left((s, y)^{-1}\right) d \lambda^{d(t, x)}(s, y) \\
& =\int_{A_{z}^{-1}} f(x, t s) g\left(s^{-1}, \alpha_{s}-1 \alpha_{t-1}(x)\right) d \lambda(s) \\
& =\int_{G} \backslash A_{z^{-1}}(s) f(t s, x) g\left((t s)^{-1} t, \alpha_{t s}^{-1}(x)\right) d \lambda(s) \\
& =\int_{G^{-1}} \backslash_{A_{\Sigma}^{-1}}\left(t^{-1} s\right) f(s, x) g\left(s^{-1} t, \alpha_{s}^{-1}(x)\right) d \lambda(s) \\
& =\int_{A_{x}^{-1}} f(s, x) g\left(s^{-1} t, \alpha_{s^{-1}}(x)\right) d \lambda(s)
\end{aligned}
$$




$$
\begin{aligned}
f^{\star}(t, x) & =\overline{f\left(t^{-1}, \alpha_{t-1}(x)\right)} . \\
\|f\|_{I, r} & =\sup _{x \in \lambda} \int_{A_{x}^{-1}}|f(s, x)| d \lambda(s) \\
\|f\|_{I, d} & =\sup _{x \in X} \int_{A_{x}^{-1}}\left|f\left(s^{-1}, a_{s^{-1}}(x)\right)\right| d \lambda(s)
\end{aligned}
$$

Seja $\mathcal{B}$ o fibrado de Banach definido no começo do capítulo. Lembrar que $\mathcal{B}$ é um fibrado de Fell com as operaçòes $(2.15)$ :

$$
\left(t, d_{t}\right)\left(s, d_{s}\right)=\left(t s, \sigma_{l}\left(\sigma_{t-1}\left(d_{t}\right) d_{s}\right)\right) \text { e }\left(t, d_{t}\right)^{*}=\left(t^{-1} \cdot \sigma_{t-1}\left(d_{t}^{*}\right)\right) .
$$

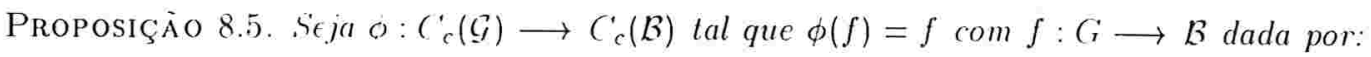

onde

$$
f(t)=\left(t, \frac{1}{\sqrt{\Delta(t)}} f_{t}\right)
$$

$$
f_{t}: \mathrm{X} \longrightarrow \text { ¿́ dada por: } f_{t}(x)=f(t, x) . \forall(t, x) \in G \times \mathrm{X}
$$

Então $\phi$ é um *-morfismo injetivo. Se em $C_{c}(\mathcal{G})$ e em $C_{c}(\mathcal{B})$ se colocam as topologias $\tau_{\mathfrak{G}}$ e $\tau$ dos respectivos limites indutios. entcio $\phi \dot{e}$ contínua com imagem densa.

Demonstraçào. Observemos primeiro que $f$ é realmente um elemento de $C_{c}(\mathcal{B})$ : a função $G \longrightarrow C_{0}(A)$ tal que $t \longmapsto f_{t}$ é contínua pelo Lema 7.37 , já que $f$ tem suporte compacto; como a função modular é contínua e positiva, vemos que $t \longmapsto \sqrt{\Delta\left(t^{-1}\right)} f_{t}$ é contínua. Finalmente, $\sup \left(f_{t}\right) \subseteq$ $X_{t}$, porque $\sup (f) \subseteq \mathcal{G}$. e portanto $\sqrt{\Delta\left(t^{-1}\right)} f_{t} \in D_{t}$.

É claro também que o é linear. e $\phi(f)=0$ implica $f=0$. de modo que $\phi$ é injetiva.

Vejamos agora que $o(f \star b)=\phi(f) * \phi(g)$, e que $\phi\left(f^{\star}\right)=\phi(f)^{*}, \forall a, b \in C_{c}(\mathcal{G})$. Se $h=f \star g$, então:

Portanto:

$$
h(t, x)=\int_{A_{x}^{-1}} f(s, x) g\left(s^{-1} t, \alpha_{s^{-1}}(x)\right) d \lambda(s)
$$

$$
h_{t}(x)=\int_{A_{x}^{-1}} f_{s}(x) g_{s^{-1} t}\left(\alpha_{s^{-1}}(x)\right) d \lambda(s)=\int_{G} \sigma_{s}\left(\sigma_{s-1}\left(f_{s}\right) g_{s^{-1} t}\right)(x) d \lambda(s) .
$$

Em outras palavras:

Por outro lado:

$$
\dot{h}(t)=\left(t, \frac{1}{\sqrt{\Delta(t)}} \int_{G} \sigma_{s}\left(\sigma_{s^{-1}}\left(f_{s}\right) g_{s^{-1}}\right)(x) d \lambda(s)\right)
$$

$$
\begin{aligned}
f * g(t) & =\left(\int_{G} \sigma_{s}\left(t, \sigma_{s^{-1}}\left(\frac{1}{\sqrt{\Delta(s)}} f_{s}\right) \frac{1}{\sqrt{\Delta\left(s^{-1} t\right)}} g_{s^{-1} t}\right)\right) \\
& =\left(t, \frac{1}{\sqrt{\Delta(t)}} \int_{G} \sigma_{s}\left(\sigma_{s^{-1}}\left(f_{s}\right) g_{s^{-1}}\right) d \lambda(s)\right)
\end{aligned}
$$

Portanto, $\hat{h}=f * g$. 
Com respeito à involuçào:

$$
\begin{aligned}
(f)^{*}(t)=\Delta\left(t^{-1}\right) f\left(t^{-1}\right)^{*} & =\Delta\left(t^{-1}\right)\left(t, \sigma_{t}\left(\frac{1}{\sqrt{\Delta\left(t^{-1}\right)}} \overline{f_{t-1}}\right)\right)=\left(t \cdot \frac{1}{\sqrt{\Delta(t)}} \overline{\sigma_{t}\left(f_{t^{-1}}\right)}\right) \\
\widehat{\left(f^{*}\right)(t)} & =\left(t \cdot \frac{1}{\sqrt{\Delta(t)}} f_{t}^{*}\right)=\left(t \cdot \frac{1}{\sqrt{\Delta(t)}} \overline{\sigma_{t}\left(f_{t-1}\right)}\right)
\end{aligned}
$$

Entào $(\hat{f})^{*}=\widehat{\left(f^{*}\right)}$.

Vejamos agora que ó contínua. Seja $\iota: C_{c}(\mathcal{G}) \longrightarrow C_{c}(\mathcal{B})$ tal que $\left.\iota(f)\right|_{t}=(t, f(t))$. Notemos que para todo subconjunto compacto $k$ de $\mathcal{G}$ o diagrama seguinte é comutativo:

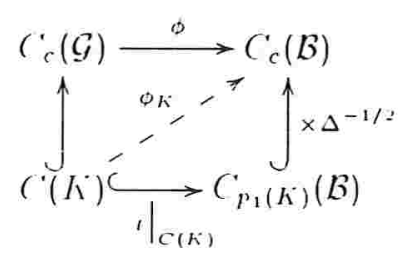

Basta ver que cada $O_{K}$ é contínua. Mas $\varphi_{K}$ é a restriçào a um subconjunto da inclusào $C\left(K^{\circ}\right) \hookrightarrow C_{C}(B)$. que é contínua, multiplicada por una função contínua e limitada: $\left.\Delta\right|_{p_{1}(K)}$. Segue então que $\phi \dot{e}$ contínua.

Falta provar ainda que $o\left(\left(_{c}(\mathcal{G})\right)\right.$ '́ denso em $C_{c}(\mathcal{B})$. Como $o\left(C_{c}(\mathcal{G})\right)=\imath\left(\left(C_{c}(\mathcal{G})\right)\right.$. temos que ver que $\iota\left(C_{c}(\mathcal{G})\right)$ é denso en $\left(\because(\mathcal{B})\right.$ na topologia do limite indutivo. É claro que $\iota\left(C_{c}(\mathcal{G})\right)$ é um sub$C(G)$-módulo de $C_{c}(\mathcal{B})$. e portanto basta. por 14.6 de $[37]$, mostrar que $\left\{\left(t, f_{l}\right): f \in C_{c}(\mathcal{G})\right\}$ é denso em $B_{t}=\{t\} \times D_{t}=\{t\} \times\left({ }_{0}\left(Y_{t}\right)\right.$. Ou seja, temos que ver que $\left\{f_{t}: f \in C_{c}(\mathcal{G})\right\}$ é denso em $D_{t}$. $\forall t \in G$. Seja $a \in C_{c}\left(X_{t}\right)$ : como $\{t\} \times \sup (a) \subseteq \mathcal{G}$ é compacto, existe uma vizinhança $V$ de $\{t\} \times \sup (a)$ tal que $\bar{V} \subseteq \mathcal{G}$, e $\bar{T}$ '́ compacto. Pelo lema de Lrysohn. existe $g \in \mathcal{C}_{c}^{\prime}(\mathcal{G})$ tal que $0 \leq g \leq 1$. $g(t, x)=1, \forall x \in\{t\} \times \sup (a)$. e $g(s, x)=0$ se $(s, x) \notin V$. Finalmente, definamos $f: \mathcal{G} \longrightarrow \mathbb{C}$ tal que $f(s, x)=0$ se $(s, x) \notin \mathrm{I}, f(s, x)=a(x) g(s, x)$ se $(s, x) \in \mathrm{I}^{\prime}$. Assim, $f \in C_{c}(\mathcal{G})$ e $f_{t}=a$. Portanto. $\left\{f_{t}: f \in C_{c}(\mathcal{G})\right\}=C_{c}\left(Y_{t}\right)$. que é denso em $D_{t}$.

Lema 8.6. Seja $K \subseteq\left(i\right.$ um subconjunto compacto e simétrico (i.e. $\left.K^{\cdot}=K^{-1}\right)$. Então, para cada $f \in C_{c}(\mathcal{G})$ tal que sup $(\bar{\varphi}(f)) \subseteq K . t+m-s \epsilon:$

$$
\|f\|_{I} \leq \sqrt{\|\Delta\|_{K}}\|\phi(f)\|_{1} .
$$

Demonstraçào. Seja $f \in C_{c}^{\prime}(\mathcal{G})$ nestas condiçōes. Então:

$$
\|\phi(f)\|_{1}=\|f\|_{1}=\int_{G}\left\|\frac{1}{\sqrt{\Delta(t)}} f_{t}\right\| d \lambda(t) \geq \frac{1}{\sqrt{\|\Delta\|_{K}}} \int_{K}\left\|f_{t}\right\| d \lambda(t) .
$$

Portanto $\|\hat{f}\|_{1} \geq \frac{1}{\sqrt{\|\Delta\|_{K}}}\|f\|_{I, r}$. Como $\|f\|_{1}=\left\|(f)^{*}\right\|_{1}=\left\|\widehat{\left(f^{\star}\right)}\right\|_{1}$, e como $\|f\|_{I, r}=\left\|f^{\star}\right\|_{I, d}$, teremos também: $\|\hat{f}\|_{1} \geq \frac{1}{\sqrt{\|\Delta\|_{K^{-1}}}}\|f\|_{I . d}$. Nas $K=K^{-1}$. donde segue o resultado.

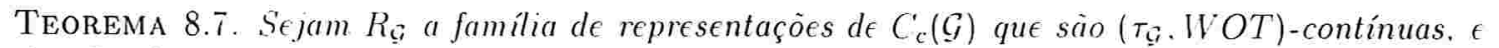
$R$ a familia de representaşôes de $C_{c}(B)$ que são $(\tau$.WOT) contínuas. onde $\tau \dot{c}$ a topologia do limite indutivo sobre $C_{c}(B)$. Então:

1. A correspondéncia $R \longrightarrow R_{G ̧}$ dada por $\pi \longmapsto \pi \circ \phi$ é uma bijeçào.

2. $\phi$ se estende a um isomorfismo de $C^{* *}$-álgebras $C^{*}(\mathcal{G}) \longrightarrow C^{*}(\mathcal{B})$. 
Demonstraçào. É claro que se $\pi \in R$. então $\tilde{\pi}=\pi \circ \phi \in R_{\mathcal{G}}$, pois $\phi$ é $\left(\tau_{\mathcal{G}}, \tau\right)$-contínua. Além disso, se $\pi \circ \phi=\pi^{\prime} \circ \phi$, entào $\pi=\pi^{\prime}$ em $C_{c}(\mathcal{G})$, que é $\tau$-denso em $C_{c}(\mathcal{B})$, e portanto $\pi=\pi^{\prime}$.

Temos que provar agora que para toda $\tilde{\pi} \in R_{\mathfrak{G}}$. existe $\pi \in R$ tal que $\tilde{\pi}=\pi \circ \phi$. Dada $\tilde{\pi}: C_{c}(\mathcal{G}) \longrightarrow$

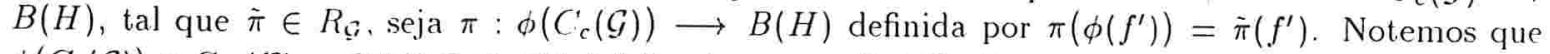
$\phi\left(C_{c}(\mathcal{G})\right) \cap C_{K}(\mathcal{B})=C_{c}(\mathcal{G}) \cap C_{K}(\mathcal{B})$ é \|\|$_{\infty}$-denso em $C_{K}^{\prime}(\mathcal{B})$. Portanto, se $f=\phi\left(f^{\prime}\right), f^{\prime} \in C_{c}(\mathcal{G})$ :

$$
\|\pi(f)\|=\left\|\pi\left(o\left(f^{\prime}\right)\right)\right\|=\left\|\tilde{\pi}\left(f^{\prime}\right)\right\| \leq\left\|f^{\prime}\right\|_{I},
$$

onde a última desigualdade deve-se a II-1.22 de [58]. Mas, pelo lema 8.6. temos que:

$$
\left\|f^{\prime}\right\|_{I} \leq \sqrt{\|\Delta\|_{K}}\left\|\phi\left(f^{\prime}\right)\right\|_{1}=\sqrt{\|\Delta\|_{K}}\|f\|_{1} \leq \sqrt{\|\Delta\|_{K}} \lambda\left(K^{\prime}\right)\|f\|_{\infty} .
$$

Então $\pi$ restrita a $C_{K}(B) \cap C_{C}(\mathcal{G})$ se estende de maneira única a um operador linear limitado $\pi_{K}$ em $C_{K}(\mathcal{B})$. É claro também que se $g \in C_{K}(\mathcal{B})$ e $g \in C_{K^{\prime}}(\mathcal{B})$, entào $\pi_{K}(g)=\pi_{K^{\prime}}(g)$. Assim, obtemos uma aplicação linear e $\tau$-contínua que preserva a involução $\pi: C_{c}(\mathcal{B}) \longrightarrow B(H)$.

Vejamos que $\pi$ preserva o produto. Sejam $f, g \in C_{c}(\mathcal{B})$. Entào existe $K \subseteq C_{r}$ compacto tal que $f, g \in C_{K}(\mathcal{B})$. Dado $c>0$, existem $f^{\prime}, g^{\prime} \in C_{c}(\mathcal{G}), \operatorname{com} p_{1}\left(\sup \left(f^{\prime}\right)\right), p_{1}\left(\sup \left(g^{\prime}\right)\right) \subseteq i$, tais que $\left\|f-\phi\left(f^{\prime}\right)\right\|_{K}+\left\|g-\phi\left(g^{\prime}\right)\right\|_{K}<c$. Notemos que. se $\tilde{f}, \tilde{g} \in C_{K}(\mathcal{B})$, entào $\tilde{f} * \tilde{g} \in C_{K^{2}}(\mathcal{B})$. Temos entào:

$$
\begin{aligned}
\|\pi(f * g)-\pi(f) \pi(g)\| & =\left\|\pi(f * g)-\tilde{\pi}\left(f^{\prime} * g^{\prime}\right)+\tilde{\pi}\left(f^{\prime}\right) \tilde{\pi}\left(g^{\prime}\right)-\pi(f) \pi(g)\right\| \\
& \leq\left\|\pi(f * g)-\pi\left(\phi\left(f^{\prime}\right)\right) \pi\left(\phi\left(g^{\prime}\right)\right)\right\|+\left\|\pi\left(\phi\left(f^{\prime}\right)\right) \pi\left(\phi\left(g^{\prime}\right)\right)-\pi(f) \pi(g)\right\| \\
& \leq \sqrt{\|\Delta\|_{K^{2}}} \lambda\left(K^{\prime 2}\right)\left\|f * g-\phi\left(f^{\prime}\right) \phi\left(g^{\prime}\right)\right\|_{K^{2}}+\left\|\pi\left(\phi\left(f^{\prime}\right)\right) \pi\left(\phi\left(g^{\prime}\right)\right)-\pi(f) \pi(g)\right\|
\end{aligned}
$$

Agora, por um lado:

$$
\begin{aligned}
\left\|\pi\left(\phi\left(f^{\prime}\right)\right) \pi\left(\phi\left(g^{\prime}\right)\right)-\pi(f) \pi(g)\right\| & =\left\|\pi\left(\phi\left(f^{\prime}\right)\right)\left(\pi\left(\phi\left(g^{\prime}\right)\right)-\pi(g)\right)+\left(\pi\left(\phi\left(f^{\prime}\right)\right)-\pi(f)\right) \pi(g)\right\| \\
& \leq\left\|\pi\left(\phi\left(f^{\prime}\right)\right)\right\| \sqrt{\|\Delta\|_{K}} \lambda\left(K^{\prime}\right)\left\|\phi\left(g^{\prime}\right)-g\right\|_{K} \\
& \leq 2 M\|\Delta\|_{K} \lambda\left(K^{\prime}\right)^{2} \epsilon \quad+\|\pi(g)\| \sqrt{\|\Delta\|_{K}} \lambda\left(K^{\prime}\right)\left\|\phi\left(f^{\prime}\right)-f\right\|_{K}
\end{aligned}
$$

onde $M=\operatorname{máx}\left\{\|f\|_{K^{;}},\|g\|_{K^{\prime}},\left\|\phi\left(f^{\prime}\right)\right\|_{K^{\prime}},\left\|\phi\left(g^{\prime}\right)\right\|_{K^{\prime}}\right\}$. Por outro lado:

$$
\begin{aligned}
\left\|f * g-\phi\left(f^{\prime}\right) \dot{\phi}\left(g^{\prime}\right)\right\|_{K^{2}} & =\left\|f *\left(g-\phi\left(g^{\prime}\right)\right)+\left(f-\phi\left(f^{\prime}\right)\right) * \phi\left(g^{\prime}\right)\right\|_{K^{2}} \\
& \leq \epsilon\left(\int_{K}|f(s)| d s+\int_{K}\left|\phi\left(g^{\prime}\right)(s)\right| d s\right) \\
& \leq 2 . M \lambda(K) \epsilon
\end{aligned}
$$

Em conclusão:

$$
\|\pi(f * g)-\pi(f) \pi(g)\| \leq 2 . M \lambda\left(K^{\prime}\right)\left(1+\|\Delta\|_{K} \lambda\left(K^{\circ}\right)\right) \epsilon \longrightarrow 0
$$

quando $\epsilon \longrightarrow 0$. Portanto $\pi(f * g)=\pi(f) \pi(g)$. Agora, pela definição de $\pi$, temos que $\tilde{\pi}=\pi \circ \phi$.

Para provar 2., notemos, por un lado, que $C^{*}(\mathcal{G})$ é o completamento de $C_{c}(\mathcal{G})$ com respeito à norma $\|\cdot\|: C_{c}(\mathcal{G}) \longrightarrow \mathbb{P}$. tal que $\left\|f^{\prime}\right\|=\sup \left\{\left\|\pi\left(f^{\prime}\right)\right\|: \pi \in R_{G}\right\}$; por outro lado, por [37], VIII-13.8. temos que $C^{*}(\mathcal{B})$ é o completamento de $\phi\left(C_{c}(\mathcal{G})\right)$ com respeito à norma: $\|\cdot\|: C_{c}(\mathcal{B}) \longrightarrow \mathbb{R}$ tal que $\|f\|=\sup \{\|\pi(f)\|: \pi \in R\}$. Segue, portanto, que $\left\|f^{\prime}\right\|=\left\|\phi\left(f^{\prime}\right)\right\|, \forall f^{\prime} \in C_{c}^{\prime}(\mathcal{G})$, e então $\phi$ se estende por continuidade a um isomorfismo de $C^{*}(\mathcal{G})$ sobre $C^{*}(\mathcal{B})$. 


\section{Bibliografia}

[1] B. Abadie, R. Exel, Deformation Quantization via Fell bundles, preprint. 1997.

[2] F. Abadie - Vicens, Descripción de las $\mathrm{C}^{*}$-álgebras aproximadamente finitas como productos cruzados. Tesis de Maestría, 1995. Universidad de la República, Montevideo, Uruguay.

[3] F. Abadie - Vicens. Tensor Products of Fell Bundles Over Discrete Groups, preprint (funct-an/9712006), 1997.

[4] C. Anantharaman-Delaroche, Systemes dynamiques non commutatifs et moyennabilité. Math. Ann. 279 (1987). no. $2,303-329$.

[5] S. Baaj, G. Skandalis, $C^{*}$-algébres de Hopf et théorie de Kasparov équivariante, K-Theory 2 (1989), 683-721.

[6] S. Baaj, G. Skandalis. Unitaires multiplicatifs et dualité pour les produits croisés des $C^{*}$-algèbres, Annales Scientifiques de L'École Normale Supérieure, 4e série, 26 (1993), 425-488.

[7] W. Beer, On Morita equivalence of nuclear $C^{*}$-algebras, J. Pure Appl. Math. 26 (1982), 249-267.

[8] O. Bratteli, Inductive limits of finite dimensional $C^{*}$-algebras, Trans. Amer. Math. Soc. 171 (1972) 195-234.

[9] L. G. Brown, Stable isomorphism of hereditary subalgebras of $C^{*}$-algebras, Pacific Journal Math. 71 (1977) 335-348.

[10] L. G. Brown, P. Green and M. A. Rieffel, Stable isomorphism and strong Morita Equivalence of C*-algebras, Pacific J. Math. 71 (1977) 349-363.

[11] L. G. Brown, J. A. Mingo, N. T. Shen, Quasi-Multipliers and Embeddings of Hilbert C*-bimodules, Canad. J. Math. 46 (1994), no. 6, 1150-1174.

[12] J. W. Bunce, J. A. Deddens, $C^{\bullet}$-algebras generated by weighted shifts, Indiana Univ. Math. J. 23 (1973), $257-271$.

[13] J. W. Bunce, J. A. Deddens, A family of simple $C^{*}$-algebras related to weighted shifts, J. Funct. Anal. 19 (1975), $13-24$.

[14] F. Combes, Crossed products and Morita equivalence, Proc. London Math. Soc. (3) 49 (1984), 289-306.

[15] A. Connes, Noncommutative Gieometry, Academic Press, Inc., New York, 1994.

[16] J. Cuntz, Simple $C^{*}$-algebras generated by isometries, Comm. Math. Phys. 57 (197i), 173-185.

[17] J. Cuntz and W. Kirieger, A Class of $C^{*}$-Algebras and Topological Markov Chains, Invent. Math. 56 (1980), 251-268.

[18] R. Curto, P. Muhly. D. Williams. Cross products of strongly Morita equivalent $C^{*}$-algebras, Proc. Amer. Math. Soc. 90(4):528-530, April 1984.

[19] K. R. Davidson, $C^{*}$-algebras by Example, Fields Institute Monographs, Amer. Math. Society, 1996.

[20] M. Dokuchaev, R. Exel, P. Piccione, Partial representations and partial group algebras, preprint, USP, 1998.

[21] S. Doplicher, D. Kastler, D. W. Robinson, Covariance algebras in field theory and statistical mechanics, Comm. Math. Phys. 3 (1966), 1-28.

[22] E. Effros, F. Hahn, Locally compact transformation groups and $C^{*}$-algebras, Memoirs Amer. Math. Soc. 75 (1967).

[23] R. Exel, Circle Actions on $C^{*}$-algebras. Partial Automorphisms and a Generalized Pimsner-Voiculescu Exact Sequence, J. Funct. Anal. 122 (1994), 361-401.

[24] R. Exel, The Bunce-Deddens Algebras and Crossed Products by Partial Automorphisms, Bol. Soc. Bras. Mat. (1994).

[25] R. Exel, Approximately finite $C^{\bullet}$-algebras and Partial Automorphisms. Math. Scand. 77 (1) (1995),

[26] R. Exel, Partial actions of groups and actions of inverse semigroups, Proc. Amer. Math. Soc. 126 (1998), No. $12,3481-3494$.

[27] R. Exel, Twisted Partial Actions. A Classification of Regular $C^{*}$-Algebraic Bundles, Proc. London Math. Soc. 74 (1997), 417-443.

[28] R. Exel, Amenability for Fell Bundles, J. Reine Angew. Math. 492 (1997), 41-43.

[29] R. Exel, Partial representations and amenable Fell bundles over free groups, preprint, Universidade de São Paulo, 1997.

[30] R. Exel, A Note on the Representation Theory of Fell Bundles, preprint, math.OA/9904013, 1999.

[31] R. Exel, M. Laca. Cuntz-Krieger Algebras for Infinite Matrices, preprint, funct-an 9712008, 1997.

[32] R. Exel, M. Laca. J. Quigg, Partial Dynamical Systems and $C^{*}$-algebras generated by Partial Isometries, preprint, funct-an 971200 i. 1997 . 
[33] R. Exel, C. K. Ng, Approximation property of $\mathrm{C}^{*}$-algebraic Bundles, preprint, math. OA/9906070.

[34] J. M. G. Fell, An extension of Mackey's method to Banach *-algebraic bundles, Memoirs Amer. Math. Soc. 90, 1969.

[35] J. M. G. Fell, Banach *-algebraic bundles and induced representations, Actes du Congrès International des Mathématiciens, Nice, 1970, Tome 2. 383-388, Gauthier-Villars, Paris, 1971.

[36] J. M. G. Fell, Induced representations and Banach *-algebraic bundles, Lecture Notes in Math. 582, SpringerVerlag, 1977 .

[37] J. M. Fell, R. S. Doran. Representations of *-algebras, locally compact groups, and Banach *algebraic bundles, Pure and Applied Mathematics vol. 125 and 126, Academic Press, 1988.

[38] J. Glimm, Families of induced representations. Pacific J. Math. 12, 1962, 885-911.

[39] M. W. Hirsh, S. Smale, Differential Equations, Dynamical Systems, and Linear Algebra, Academic Press, 1974.

[40] S. Imai, H. Takai, On a duality for crossed products by a locally compact group, J. Math. Soc. Japan, 30 (1978), 495-504.

[41] G. G. Kasparov, Hilbert $C^{*}$-modules: theorems of Stinespring and Voiculescu. J. Operator Theory 4 (1980) 133-150.

[42] Y. Katayama, Takesaki 's duality for a nondegenerate coaction. Math. Scand. 55 (1985) 141-151.

[43] E. C. Lance, Hilbert C*-modulcs. A toolkit for operator algebraists, London Mathematical Society, Lecture Note Series 210, Cambridge University Press. 1995.

[44] E. M. Landesman, B. Russo. The second dual of a ("*-ternary ring, Canad. Math. Bull. 26(2) (1983), 241-246.

[45] M. B. Landstad, Duality theory for corariant systems, Trans. Amer. Math. Soc. 248 (1979), 223-267.

[46] M. B. Landstad, J. Phillips, I. Raeburn. C. E. Sutherland, Representations of crossed products by coactions and principal bundles. Trans. Amer. Math. Soc. 299 (1987) 747-784.

[47] K. Maclanahan, K-theory for partial crossed products by discrete groups, J. Funct. Anal. 130 (1995), 77-117.

[48] C. K. Ng, $C^{*}$-exactness and crossed products by actions and coactions, J. London Math. Soc. (2), 51 (1995), 321-330.

[49] C. K. Ng, Discrete coactions on $\mathrm{C}^{\prime *}$-algebras, J.Austral.Math.Soc. (Series A) 60 (1996), 118-127.

[50] C. K. Ng, Reduced cross-sectional $C^{*}$-algebras of $C^{*}$-algebraic bundles and coactions, preprint, 1996.

[51] A. Nica, $C^{*}$-algebras generated by isometries and Wiener-Hopf operators, J. Operator Theory 27 (1992), 17-52.

[52] G. K. Pedersen, $C^{*}$-algebras and the ir automorphism groups, Academic Press, London, 1979.

[53] J. C. Quigg, Discrete $C^{*}$-coactions and (**algebraic bundles, J. Austral. Math. Soc. (Series A) 60 (1996), $204-221$.

[54] J. C. Quigg, Crossed product duality for partial $C^{*}$-automorphisms, preprint, 1996.

[55] J. C. Quigg, I. Raeburn, Characterizations of crossed products by partial actions, J. Operator Theory 37 (1997), No. 2, 311-340.

[56] I. Raeburn, On crossed products by conctions and their representation theory, Proc. London Math. Soc. (3) 64 (1992), 625-652.

[5i] I. Raeburn, D. P. Williams, Morita Equivalence and Continuous-Trace $C^{*}$-algebras. Math. Surveys and Monographs, Volume 60, Amer. Math. Society. 1998.

[58] Jean Renault, A groupoid approach to $C^{*}$-algebras, Lecture Notes in Math. 793 (1980), Springer-Verlag.

[59] M. A. Rieffel, Induced Representations of C*-algebras, Advances in Math. 13 (1974) 176-257.

[60] J.- P. Serre, Linear Representations of Finite Giroups, GTM 42, Springer-Verlag, 1977.

[61] H. Takai, On a duality for crossed products of $C^{*}$-algebras, J. Funct. Anal. 19 (1975), 25-39.

[62] T. Turumaru, Crossed products of operators algebras, Tôhoku. Math. J. 10, 1958, 355-365.

[63] F. W. Warner, Foundations of Differentiable Manifolds and Lie Groups, GTM, Springer-Verlag, 1983.

[64] S. Wassermann, Exact $C^{*}$-algebras and Related Topics, Lecture Notes Series 19, Seoul National University, 1994.

[65] N. E. Wegge-Olsen, $K$-Theory and $C^{*}$-algebras, Oxford Science Publications, Oxford University Press, OxfordNew York-Tokyo, 1993.

[66] A. Weinstein, Groupoids: Unifying Internal and External Symmetry, preprint 1996.

[6i] G. Zeller-Meyer, Produits croisés d'une $C^{*}$-algèbre par un groupe d'automorphismes, J. Math. Pures Appl. 47 (1968), 101-239.

[68] H. H. Zettl, A characterization of ternary rings of operators, Adv. in Math. 48 (1983), 117-143. 